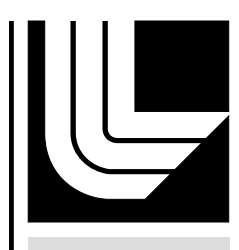

LAWRENCE LIVERMORE N A TIO NAL LABORATORY

Physical Properties of Chemicals in PAC Revision 27 Listing

M. A. Johnson

March 8, 2013 
This document was prepared as an account of work sponsored by an agency of the United States government. Neither the United States government nor Lawrence Livermore National Security, LLC, nor any of their employees makes any warranty, expressed or implied, or assumes any legal liability or responsibility for the accuracy, completeness, or usefulness of any information, apparatus, product, or process disclosed, or represents that its use would not infringe privately owned rights. Reference herein to any specific commercial product, process, or service by trade name, trademark, manufacturer, or otherwise does not necessarily constitute or imply its endorsement, recommendation, or favoring by the United States government or Lawrence Livermore National Security, LLC. The views and opinions of authors expressed herein do not necessarily state or reflect those of the United States government or Lawrence Livermore National Security, LLC, and shall not be used for advertising or product endorsement purposes.

This work performed under the auspices of the U.S. Department of Energy by Lawrence Livermore National Laboratory under Contract DE-AC52-07NA27344. 


\section{Physical Properties of Chemicals in PAC Revision 27 Listing}

\section{Purpose}

The purpose of this chemical physical property listing is to provide data required to apply the DOE SCAPA Protective Action Criteria (PAC) values to calculation of the LLNL Quantity (Q) Value thresholds for facility chemical hazard classification.

This chemical physical property listing based on the DOE SCAPA Protective Action Criteria (PAC) Revision 27 listing Identifies:

1. Physical state at $25^{\circ} \mathrm{C}$ (i.e. solid, liquid, or gas)

2. Vapor pressure of liquids at $25^{\circ} \mathrm{C}$

\section{Summary of Process}

1. Start with PAC Input Data (table 1).

a. Eliminate data columns not required for Q-Value threshold calculations.

b. Add additional, necessary data columns.

2. Modify data format as needed

3. Filter out gases and solids based upon data provided in table 1

4. Utilize reference materials to:

a. Further weed out gases \& solids

b. Identify VP at $25^{\circ} \mathrm{C}$ for liquids

c. Fill in data gaps

\subsection{PAC Input Data (table 1)}

\subsubsection{Eliminate Unnecessary Data Columns}

Only those values necessary to accomplish the stated purpose of this listing are utilized from table 1; these are:

1. PAC Revision 27 Chemical Identification Number (No.); which is required to maintain continuity between listings.

2. Chemical Compound Description; which is required to maintain continuity between listings.

3. Chemical CAS Number (CASRN); which is required for chemical identification.

4. Molecular Weight; which is required for chemical identification.

5. (Physical) State at $25^{\circ} \mathrm{C}$.

6. Melting Point (MP); which is useful in identifying physical state at $25^{\circ} \mathrm{C}$.

7. Boiling Point (BP) (sometimes given with a pressure other than $760 \mathrm{mmHg}$ ).

8. Vapor Pressure (VP).

9. Temperature at which Vapor Pressure has been provided.

\subsubsection{Add in Needed Data Columns}

Some additional data columns have been added; these include:

1. Modified-format CAS numbers (sort-able). 
2. Boiling Point data separated into Boiling Point Temperature $\left({ }^{\circ} \mathrm{C}\right)$ and Boiling Point Pressure ( $\mathrm{mmHg}$ )

3. Added a column to document reference sources for VP at $25^{\circ} \mathrm{C}$.

\subsection{Modify Data Format}

Much of the PAC Revision 27 table 1 content formatting has been modified in this listing to make the data more useful; modifications include:

1. Physical state values simplified (limiting choices to $\mathrm{S}, \mathrm{L}$, or $\mathrm{G}$ )

2. Numerical data reformatted to numerical values (much of the data was in text format).

a. This primarily applies to data for chemicals existing as liquids at $25^{\circ} \mathrm{C}$.

\subsection{Exclusion of Solids and Gases from Further Evaluation}

Auto-filter function of spreadsheet is applied to eliminate from further evaluation all but those chemicals either identified as liquids, or not having an identified physical state.

\subsection{Reference Material Data}

\subsubsection{Application of Referenced Data for Further Exclusion of Solids and Gases}

In some instances, assignment of physical form data (i.e. S, L, or G) provided by SCAPA in table 1 (input data) of the PAC listing is based upon conditions inconsistent with the LLNL Q-value calculation process. In these cases, it is sometimes possible to use additional data to better align physical form of the chemicals to $25^{\circ} \mathrm{C}$. In instances where the chemicals are clearly a solid or gas at $25^{\circ} \mathrm{C}$, these chemicals may be excluded from further evaluation of physical properties.

1. Chemicals assigned a physical state of " $\mathrm{L}$ " in the table 1 listing, but having a melting point equal to or greater than $25^{\circ} \mathrm{C}$ have been reassigned a physical state of "S."

a. This only applies to experimentally derived, published melting point data.

2. Chemicals assigned a physical state of " $L$ " in table 1 but having a boiling point (temperature) at $760 \mathrm{mmHg}$ equal to or less than $25^{\circ} \mathrm{C}$ have been reassigned a physical state of " $\mathrm{G}$."

a. This only applies to experimentally derived, published boiling point data.

3. Chemical assigned a physical state of " $\mathrm{L}$ " in table 1 but found to have a vapor pressure at $25^{\circ} \mathrm{C}$ equal to or greater than $760 \mathrm{mmHg}$ have been reassigned a physical state of "G."

a. This applies to both experimentally derived and estimated vapor pressure data.

\subsubsection{Application of Referenced Data for Vapor Pressure Determinations}

All liquids are required to have a vapor pressure identified at $25^{\circ} \mathrm{C}$; each of these will have a documented reference or source. The hierarchy for referenced data is:

1. First priority is given to values provided by SCAPA in table 1 (input data) of the PAC listing, as these values have been vetted by the DOE SCAPA working group and have been derived from reputable published sources. For those liquids not having a vapor pressure at $25^{\circ} \mathrm{C}$ identified in table 1 of the PAC listing, it is necessary to fill in the gaps utilizing other data sources. Where data ranges are provided, the mean of the range is utilized for calculations.

2. Second priority is given to experimental data derived from other published data sources, including:
a. The EPA's Estimation Program Interface (EPI) Suite Experimental Data Table
b. EPI Suite chemical searches
c. Published Industry technical papers or data sheets
d. MSDSs 
3. Third priority is given to modeled or estimated data. This is arrived at through:

a. The EPA's EPI Suite - either:

i. Through direct use of the EPI Suite program (available from EPA's website: http://www.epa.gov/oppt/exposure/pubs/episuitedl.htm

ii. By proxy, using the Royal Society of Chemistry (RSC) "ChemSpider" free chemical database

b. The ACD I-Lab Physical Chemistry Model - by proxy using the Royal Society of Chemistry (RSC) "ChemSpider" free chemical database

c. Logarithmic interpolation between the chemical's boiling point and a second known vapor pressure \& temperature data set. [see section 3]

d. Aqueous solutions for which the MSDS shows no contribution other than density in physical properties (i.e. VP, BP, MP are that of water) are assigned a hazardous component VP of $1 \mathrm{E}-05$ for calculation purposes.

4. Last priority is given to values approximated from analogous materials. It is understood that such values are approximations only; however these are useful in that (in this PAC revision) these values have been applied only to chemicals having VPs below $1 \mathrm{mmHg}$. In the Q-value threshold calculation process, it is not relevant how far the VP is below $1 \mathrm{mmHg}$.

\subsubsection{Application of Referenced Data to Fill in Gaps}

In many instances, it is possible to utilize data from the sources described in 2.4 .2 to fill in data gaps present in the PAC input table.

1. In all instances where this data is used to re-assign physical form, only experimentally-derived data is applied.

2. In all instances where experimental data is available to fill in gaps, these values will be added to the listing.

3. In instances where EPI Suite-estimated values for MP \& BP are available to fill in gaps, these values will be added to the table only if they are both credible \& consistent with experimentallyderived data.

4. In instances where table 1 data is significantly at odds with published data for a chemical, corrections applied will also be noted in the Reference Documentation field.

\section{Model for Estimation of Vapor Pressure Using Boiling Point and a Known Vapor Pressure Point}

\subsection{Basis and Equations}

The basis for this model is linear interpolation of the natural log of vapor pressure (VP) with the inverse of absolute temperature.

$$
\begin{array}{ll}
\ln (V P)=B \frac{1}{T}+A & \text { Equation 1 } \\
A=\ln \left(B P P-\frac{B}{B P}\right) & \text { Equation 2 } \\
B=\frac{\ln (B P P)-\ln \left(\mathrm{VP}_{1}\right)}{(B P)^{-1}-\left(T_{1}\right)^{-1}} & \text { Equation 3 }
\end{array}
$$

Where

$$
\begin{aligned}
& \mathrm{VP}=\text { Desired Vapor Pressure }[\mathrm{mmHg}] \\
& \mathrm{T}=\text { Temperature for desired vapor pressure }\left[{ }^{\circ} \mathrm{K}\right]
\end{aligned}
$$


$A$ and $B$ are coefficients in equation 1, represented by equations 2 and 3 respectively

And

$$
\begin{aligned}
& \mathrm{BPP}=\text { Boiling Point Pressure }[\mathrm{mmHg}] \\
& \mathrm{BP}=\text { Boiling Point }\left[{ }^{\circ} \mathrm{K}\right] \\
& \mathrm{VP}_{1}=\text { known } \mathrm{VP}[\mathrm{mmHg}] \\
& \mathrm{T}_{1}=\text { Temperature for known } \mathrm{VP}\left[{ }^{\circ} \mathrm{K}\right]
\end{aligned}
$$

\subsection{Sample Calculation}

Ethyl acetate has a VP of $118.3 \mathrm{mmHg}$ at $29.5^{\circ} \mathrm{C}$ and a normal boiling point of $77.1^{\circ} \mathrm{C}$. Vapor pressure at $25^{\circ} \mathrm{C}$ may be estimated as follows:

$$
\begin{aligned}
& B=\frac{\ln (760)-\ln (118.3)}{(77.1+273.15)^{-1}-(29.5+273.15)^{-1}}=-4142.351 \\
& A=\ln \left(760-\frac{(-4142.351)}{(77.1+273.15)}\right)=18.460 \\
& \ln (V P)=(-4142.351) \frac{1}{(25+273.15)}+18.460=4.566 \\
& V P=96.22 \mathrm{mmHg}
\end{aligned}
$$

The published, experimentally-derived VP for ethyl acetate at $25^{\circ} \mathrm{C}$ is $93.2 \mathrm{mmHg}$. The percent error for this method in this instance is:

$$
\frac{96.22-93.2}{93.2} \times 100 \%=3.2 \%
$$

Using the Antoine equation:

$$
\log (V P)=A-\frac{B}{T+C}
$$

Where

$$
\begin{aligned}
& \mathrm{VP}=\text { Desired Vapor Pressure }[\mathrm{mmHg}] \\
& \mathrm{T}=\text { Temperature for desired vapor pressure }\left[{ }^{\circ} \mathrm{C}\right]
\end{aligned}
$$

And (for ethyl acetate)

$$
\begin{aligned}
& A=7.10197 \\
& B=1244.951 \\
& C=217.881 \\
& \log (V P)=7.10197-\frac{1244.951}{25+217.881}=1.976 \\
& V P=94.67 \mathrm{mmHg}
\end{aligned}
$$

With a percent error of:

$$
\frac{94.67-93.2}{93.2} \times 100 \%=1.6 \%
$$

As such this interpolation model appears to be a credible methodology for VP estimation should the coefficients required for the Antoine (or other) methodologies be unavailable. 


\begin{tabular}{|c|c|c|c|c|c|c|c|c|c|c|c|c|c|}
\hline & $A_{-}$ & & & & 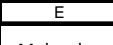 & av state & & $\begin{aligned} \mathrm{H} \\
\mathrm{H}\end{aligned}$ & i & & $x_{\mathrm{K}}$ & & \\
\hline & No. & Chemical Compound & $\begin{array}{l}\text { CAS Number } \\
\text { (CASRN) }\end{array}$ & $\begin{array}{l}\text { Modified } \\
\text { Format CAS }\end{array}$ & $\begin{array}{l}\text { Molecular } \\
\text { Weight (MW) }\end{array}$ & 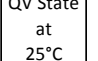 & $\begin{array}{l}\text { MP or FP } \\
{\left[{ }^{\circ} \mathrm{C}\right]}\end{array}$ & $\begin{array}{c}\text { BP Pressure } \\
{[\mathrm{mm} \mathrm{Hg}]}\end{array}$ & $\mathrm{BP}\left[{ }^{[\mathrm{C} C}\right]$ & $\begin{array}{l}\text { Vapor } \\
\text { Pressure } \\
\text { Imm Hg }\end{array}$ & $\begin{array}{c}\text { Tfor VP } \\
{\left[{ }^{\circ C} \mathrm{C}\right]}\end{array}$ & $\begin{array}{c}\text { VP at } 25^{\circ} \mathrm{C} \\
{[\mathrm{mm} \mathrm{Hg}]}\end{array}$ & Vapor Pressure @ @25 C Reference \\
\hline$\frac{2}{2}$ & & Acacia; (Gum arabic) & $9000-01-5$ & $009000-01-5$ & 240000 & & $9.180 \mathrm{E}+01$ & $7.600 E+02$ & $2.800 E+02$ & & $2.4006+01$ & & PAC 27 Listing Table 1 \\
\hline 3 & 2 & Acenaphthene & $83-32-9$ & $000083-32-9$ & 154.22 & $\mathrm{~s}$ & $9.500 E+01$ & $7.600 E+02$ & $2.775 \mathrm{E}+02$ & $1.000 E+01$ & $1.312 E+02$ & & PAC 27 Listing Table 1 \\
\hline$\frac{4}{5}$ & 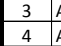 & $\begin{array}{l}\text { Accanapthylene } \\
\text { Acetaldehyde }\end{array}$ & $208-96-8$ & $\frac{000208-99-8}{00007-0}$ & $\frac{152.19}{44.05}$ & $\frac{s}{G}$ & $\frac{9.180 \mathrm{EE}+1}{-1.23 \mathrm{E}+02}$ & $\begin{aligned} 7.600 \mathrm{E}+02 \\
7.600 \mathrm{E} 02 \\
\end{aligned}$ & $2.800 \mathrm{E}+02$ & $\begin{array}{ll}7.500 E-03 \\
7.500 \mathrm{E}+02\end{array}$ & $2.4006+01$ & & $\begin{array}{l}\mid \begin{array}{l}\mid \text { ACC } 7 \text { Lisining Gable } 1 \\
\text { PAC 27 Listing Table } 1\end{array} \\
\end{array}$ \\
\hline 6 & 5 & Acetamide & $60-35-5$ & $000060-35-5$ & 59.067 & $\mathrm{~s}$ & $8.016 \mathrm{E}+01$ & $7.600 \mathrm{E}+02$ & $2.220 \mathrm{E}+02$ & $4.0000-02$ & $2.500 \mathrm{E}+01$ & & $\begin{array}{l}\text { PAC } 27 \text { Listing Table } 1 \\
\end{array}$ \\
\hline$\frac{7}{8}$ & 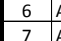 & 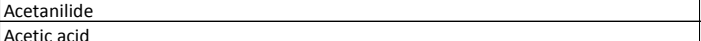 & 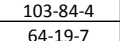 & 些00103-84-4 & $\frac{135.18}{60.05}$ & $\frac{5}{L}$ & $\frac{1.135 E+02}{1.664 \mathrm{E}+01}$ & $\begin{array}{l}7.600 \mathrm{E}+02 \\
7.600 \mathrm{E}+02\end{array}$ & 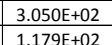 & $\frac{1.0006+00}{1.573 F+01}$ & $\frac{1.140 E+02}{2.500 \mathrm{~F}+01}$ & & 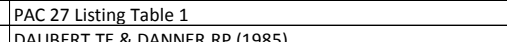 \\
\hline & $\begin{array}{ll}7 \\
8 & \mid\end{array}$ & 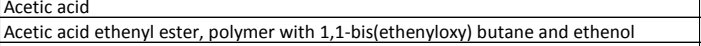 & 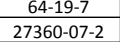 & 000064-19-7 & & $\frac{\mathrm{L}}{\mathrm{s}}$ & 1.664E+ +01 & & $1.179 \mathrm{E}+02$ & & & $1.573 E+01$ & \begin{tabular}{|l} 
DAABERTT,TE \& DANNER,RP (1985) \\
PAC 27 Listing Table 1
\end{tabular} \\
\hline & $\begin{array}{c}8 \\
9\end{array}$ & & & & 100.13 & & & & & $2.720 E+01$ & $2.500 \mathrm{E}+01$ & $2.720 E+01$ & $\begin{array}{l}\text { PAC } \\
\text { DAUBERT,TE \& DANNER,RP (1996) }\end{array}$ \\
\hline$\frac{11}{11}$ & $10 \mid$ & Acetic acid, lithium salt & $\frac{546-89-4}{5}$ & $\frac{000546-89-4}{0.4}$ & $\frac{67}{67}$ & s & $2.8006+02$ & & & & & & 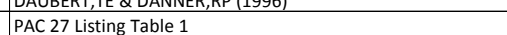 \\
\hline & 111 & Acetic acid, manganese(2+) salt, tetrahydrate & $6156-78-1$ & $006156-78-1$ & & $\mathrm{~s}$ & & & & & & & PAC 27 Listing Table 1 \\
\hline 13 & 12 & \begin{tabular}{|l} 
Acetic acid, manganese(II) salt (2:1) \\
Acctic anbdride
\end{tabular} & $638-38-0$ & $000638-38-0$ & 173.04 & s & $2.100 E+02$ & & & & & & 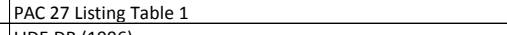 \\
\hline$\frac{14}{15}$ & $\left|\frac{13}{14}\right|$ & $\begin{array}{l}\text { Acectita anhydride } \\
\text { Actone }\end{array}$ & $\frac{108-24-7}{67-64-1}$ & $\begin{array}{l}000008-24-7 \\
00006-64-1\end{array}$ & $\frac{102.1}{58.08}$ & $\frac{L}{L}$ & 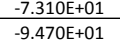 & $\begin{array}{l}7.600 E+02 \\
7.600+502\end{array}$ & $\begin{array}{l}1.396 \mathrm{E}+02 \\
5.605+01\end{array}$ & $\begin{array}{l}5.100 E+00 \\
2.315 E+02\end{array}$ & $\frac{2.500 E+01}{2.500 E+01}$ & $5.1006+00$ & \begin{tabular}{|l|l|} 
LDE,DR (1996) \\
ALARE Y Y AL (1995)
\end{tabular} \\
\hline & & $\begin{array}{l}\text { Acetone } \\
\text { Acetone thiosemicarbazide }\end{array}$ & $\frac{66-1-1-1}{1752-30-3}$ & $\begin{array}{ll}0 \\
000176-12-42-30-3 \\
0\end{array}$ & $\begin{array}{l}58.08 \\
131.22 \\
\end{array}$ & $\frac{L}{s}$ & & & & & & & 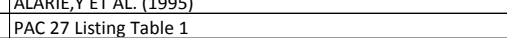 \\
\hline$\frac{10}{17}$ & & Acetone-d6 & $666-52-4$ & $000666-52-4$ & $\frac{15.124}{64.1164}$ & $\frac{L}{L}$ & $-9.3000+01$ & $7.600 E+02$ & 5.550E+01 & $2.320 E+02$ & $2.500 E+01$ & $2.320 E+02$ & FPI Suite - Chemspider Database \\
\hline 18 & 17 & Acetonitrile & $75-05-8$ & $000075-05-8$ & 41.05 & L & $-4.3822+01$ & $7.6006+02$ & $8.165 E+01$ & $8.880 \mathrm{E}+01$ & $2.500 \mathrm{E}+01$ & $8.880 \mathrm{E}+01$ & BouBLLK, ET TAL. (1984) \\
\hline 19 & 18 & Acetonitrile-d3 & $2206-26-0$ & $002206-26-0$ & 44.0707 & $\mathrm{~L}$ & & $7.600 E+02$ & $8.0700+01$ & $8.880 \mathrm{E}+01$ & $2.5000+01$ & $8.880 E+01$ & EPI Suite-chemSpider Database \\
\hline & 19 & Acetophenone & $98-86-2$ & $000098-86-2$ & 120.149 & L & $2.050 \mathrm{E}+01$ & $7.600 E+02$ & $2.020 E+02$ & $3.970 \mathrm{E}-01$ & $2.500 E+01$ & 3.970E-01 & |DAUBERT,TE \& DANNER,RP (1985) \\
\hline 21 & 20 & Acetoxytriphenylstannane & $900-95-8$ & $000900-95-8$ & 409.07 & $\frac{s}{s}$ & 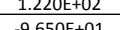 & $76005+02$ & $76705+01$ & 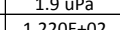 & 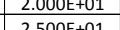 & & 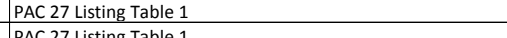 \\
\hline$\frac{22}{23}$ & 21 & 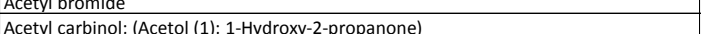 & $\begin{array}{l}506-9-9-1 \\
116-09-6\end{array}$ & 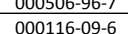 & $\frac{12.948}{74.09}$ & $\frac{S}{L}$ & $\begin{array}{l}-9.650 E+01 \\
-7.000 E+00\end{array}$ & $\begin{array}{l}1.600 E+02 \\
7.600 E+02\end{array}$ & 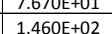 & 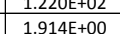 & $2 \frac{2.500 E+01}{2.500 E+01}$ & $1.914 \mathrm{E}+00$ & $\begin{array}{l}\text { PAC } 7 \text { listing Table } 1 \\
\text { ACD Dabs hhysical Chemistry Suite }\end{array}$ \\
\hline$\frac{23}{24}$ & 23 & 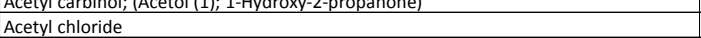 & $\frac{1106-906}{75-36-5}$ & $\begin{array}{l}0000075-36-56 \\
0005\end{array}$ & $\frac{14.09}{78.5}$ & $\frac{L}{L}$ & $\begin{array}{l}-1.00 \mathrm{E}+00 \\
-1.128 \mathrm{E}+02 \\
\end{array}$ & $\begin{array}{l}7.060 \mathrm{E}+02 \\
7.600 \mathrm{E}+02\end{array}$ & $\begin{array}{ll}1.400 E+02 \\
5.070 E+01\end{array}$ & 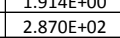 & $\begin{array}{l}2.500 \mathrm{E}+01 \\
2.500 \mathrm{E}+01 \\
\end{array}$ & 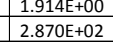 & 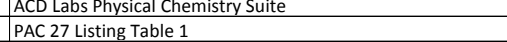 \\
\hline 25 & 24 & Acetyl triethyl citrate & $77-89-4$ & $000077-89-4$ & 318.36 & $\mathrm{~s}$ & & $4.000 E+01$ & $2.140 E+02$ & & & & PAC 27 Listing Table 1 \\
\hline$\frac{26}{27}$ & 25 & Actylaminofluorene, 2- & 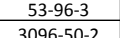 & $000053-96-3$ & 223.27 & $\frac{s}{s}$ & $1.930 \mathrm{E}+02$ & & & & & & $\begin{array}{l}\text { PAC 27 Listing Table } 1 \\
\text { PAC C7 L Lising Table } 1\end{array}$ \\
\hline & 26 & $\begin{array}{l}\text { Actylaminofluorenone, 2- } \\
\text { Actylene }\end{array}$ & $\frac{3096-50-2}{74-86-2}$ & 些003096-58-2 & $\frac{237.27}{22.04}$ & $\frac{S}{G}$ & $-8.070 \mathrm{E}+01$ & $7.600 E+02$ & $8.400 E+01$ & $4.0406+04$ & $2.500 E+01$ & & $\begin{array}{l}\text { PAC 27 Listing a able } 1 \\
\text { PAC 27 listing Table } 1\end{array}$ \\
\hline$\frac{28}{29}$ & 28 & A & 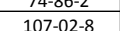 & 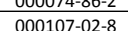 & $\frac{20.04}{56.063}$ & $\frac{G}{L}$ & & $7.600+502$ & $5.260 \mathrm{EF}+01$ & $2700 \mathrm{E}+02$ & 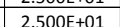 & $27406+02$ & $\begin{array}{l}\text { PAC 2 L Listing labble } 1 \\
\text { PAC } 27 \text { Listing Table } 1\end{array}$ \\
\hline$\frac{29}{30}$ & 29 & $\begin{array}{l}\text { Acrovem } \\
\text { Acrylamide }\end{array}$ & $79-06-1$ & $000079-06-1$ & 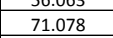 & $\frac{\mathrm{L}}{\mathrm{s}}$ & $8.4506+01$ & $7.600 \mathrm{E}+02$ & $1.926 \mathrm{E}+02$ & $7.5000-01$ & $1.096 \mathrm{E}+02$ & & $\begin{array}{l}\text { PAC } 27 \text { Listing Table } 1 \\
\text { Pand }\end{array}$ \\
\hline 31 & 30 & Acrylamide, N,N'-methylenebisacrylamide copolymer & $25034-58-6$ & $025034-58-6$ & 154.19 & $\mathrm{~s}$ & $185 \mathrm{dec}$ & & & & & & PAC 27 Listing Table 1 \\
\hline$\frac{32}{32}$ & 31 & Acrylic acid & $79-10-7$ & $000079-10-7$ & 72.06 & $\frac{L}{S}$ & $1.250 \mathrm{E}+01$ & $7.600 E+02$ & $1.410 \mathrm{E}+02$ & $3.970 \mathrm{E}+00$ & $2.5000+01$ & $3.970 \mathrm{3}+00$ & DAUBERT,TE \& DANNER,RP (1987) \\
\hline$\frac{33}{34}$ & 32 & $\begin{array}{l}\text { Acrylic acid polymers; (Acrylic polymer or resin) } \\
\text { Acrlonitrile }\end{array}$ & $\frac{9003-01-4}{107-13-1}$ & $\begin{array}{l}0090003-1-1-4 \\
00010-13-1\end{array}$ & $\frac{168.06}{53.063}$ & $\frac{\mathrm{s}}{\mathrm{L}}$ & $-8.348 E+01$ & $7.600 E+02$ & $7,7305+01$ & $1.085 \mathrm{E}+02$ & $2.500 E+01$ & $1.085 \mathrm{E}+02$ & $\begin{array}{l}\text { PAC } 27 \text { listing gable } 1 \\
\text { DAUBERT TE \& DANNER,RP (1985) }\end{array}$ \\
\hline$\frac{34}{35}$ & 34 & $\begin{array}{l}\text { Actryontitue } \\
\text { Acryly chloride; (Acryloyl chloride) }\end{array}$ & $814-68-6$ & & $\begin{array}{l}53.063 \\
90.51\end{array}$ & & & & & 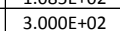 & $250 \mathrm{EF}+1$ & $3000 \mathrm{E}+02$ & 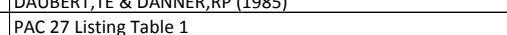 \\
\hline 36 & 35 & 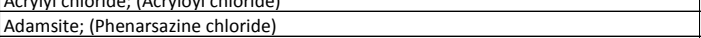 & 578-9949 & $000577-94-9$ & 277.59 & $\frac{5}{s}$ & $1.950 E+02$ & $7.600 \mathrm{et}+02$ & 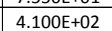 & & & & $\begin{array}{l}\text { PAC } 27 \text { Listing Table } 1 \\
\end{array}$ \\
\hline$\frac{37}{37}$ & 36 & Adipic acid & $124-04-9$ & $000124-04-9$ & 146.16 & $\mathrm{~s}$ & $1.520 \mathrm{E}+02$ & $7.600 E+02$ & $3.375 E+02$ & $1.000 E+00$ & $1.595 E+02$ & & PAC 27 Listing Table 1 \\
\hline 38 & 37 & 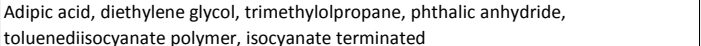 & $68609-57-4$ & 068609-57-4 & & $\mathrm{s}$ & & & & & & & PAC 27 Listing Table 1 \\
\hline 39 & 38 & Adiponitrile & $111-69-3$ & $000111-69-3$ & 108.16 & $\mathrm{~s}$ & $1.000 E+00$ & $7.600 \mathrm{E}+02$ & $2.950 \mathrm{E}+02$ & $6.8000-04$ & $2.500 E+01$ & & PAC 27 Listing Table 1 \\
\hline & 39 & 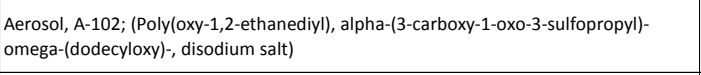 & $39354-45-5$ & $039354-45-5$ & & L & $0.000 E+00$ & $7.600 E+02$ & $1.000 \mathrm{E}+02$ & $1.000 E-05$ & $2.500 E+01$ & $1.000 E=-05$ & 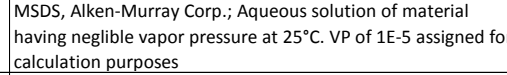 \\
\hline 41 & 40 & Agar & $9002-18-0$ & $009002-18-0$ & & $\mathrm{~s}$ & & & & & & & $\begin{array}{l}\text { PAC } 27 \text { listing Tabses } 1 \\
\text { Pat }\end{array}$ \\
\hline & 41 & Agarose, type VIII (low gelling temperature); (Sepharose) & 9012-36-6 & & & $\mathrm{s}$ & $2.600 E+02$ & & & & & & PAC 27 Li \\
\hline 43 & 42 & Alamine 336 & 68814-95-9 & 068814-95-9 & & $s$ & & & & & & & ting Table 1 \\
\hline 44 & 43 & Albumin (bovine) & 9048-46-8 & $009048-46-8$ & 65000 & $\frac{\mathrm{s}}{\mathrm{s}}$ & & & & & & & PAC 27 Listing Table 1 \\
\hline$\frac{45}{46}$ & $\frac{44}{45}$ & 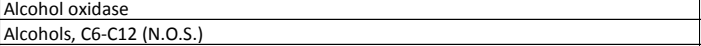 & $\frac{9073-63-6}{68603-15-6}$ & 0090773-33-6 & & $\frac{s}{L}$ & $-4.460 E+01$ & $7.600 \mathrm{E}+02$ & $1.576 E+02$ & $8.810 E-01$ & $2.500 E+01$ & $8.8100-01$ & $\begin{array}{l}\text { PAC } 27 \text { Lising gable } 1 \\
\text { EPP Suite Mean VP of Antoine \& Grain Methods }\end{array}$ \\
\hline & 46 & 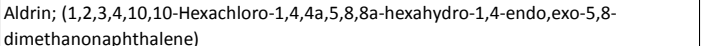 & $309-00-2$ & $000309-00-2$ & 364.91 & $\mathrm{~s}$ & $1.040 \mathrm{E}+02$ & $2.000 E+00$ & $1.450 E+02$ & $1.200 \mathrm{-}-04$ & $2.500 E+01$ & & PAC 27 Listing Table 1 \\
\hline$\frac{47}{48}$ & 47 & 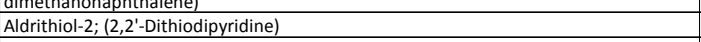 & $2127-03-9$ & $002127-03-9$ & 220.3066 & $\mathrm{~s}$ & $56-60$ & & & & & & PAC 27 Listing Table 1 \\
\hline 49 & 48 & 6; (Adogen 464; Quaternary ammonium compounds, tricC-10)-alkylmethy & 63393-96-4 & $063393-96-4$ & & $\mathrm{~L}$ & & & & $1.880 \mathrm{E}-12$ & $2.500 \mathrm{E}+01$ & $1.880 \mathrm{E}-12$ & EPI Suite Modified Grain Method \\
\hline$\frac{49}{50}$ & & Alizarin; (1,2-Dihydroxyanthraquinone; Turkey red oil) & $72-48-0$ & $000072-48-0$ & 240.22 & $\mathrm{~s}$ & $2.890 E \mathrm{E}+02$ & $7.600 E+02$ & $4.3006+02$ & & & & PAC 27 Listing Table 1 \\
\hline 51 & 50 & Alizarine Red S, Monohydrate; (Sodium alizarin-3-sulfonate) & $130-22-3$ & $000130-22-3$ & 342.26 & $\mathrm{~s}$ & & & & & & 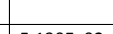 & PAC 27 Listing Table 1 \\
\hline$\frac{52}{53}$ & 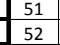 & 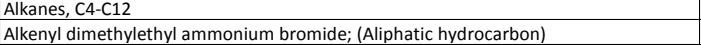 & $\frac{68333-81-3}{-20108}$ & $\begin{array}{ll}068333-381-3 \\
000002-0108\end{array}$ & & $\frac{\mathrm{L}}{\mathrm{s}}$ & & & & $5.130 \mathrm{E}+02$ & $2.500 \mathrm{E}+01$ & $5.130 \mathrm{E}+02$ & \begin{tabular}{|l|} 
PF I I vite Mean V Vof A A \\
PAC L Listing Table 1
\end{tabular} \\
\hline 54 & 53 & Alky benzenes (C8-c9) & 2-0109 & 00000z-0109 & & $\mathrm{L}$ & & & & $2.170 E-03$ & $2.500 \mathrm{E}+01$ & $2.170 \mathrm{E}-03$ & 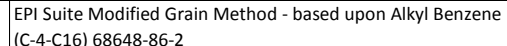 \\
\hline$\frac{34}{55}$ & & Allkyl dimethylbenzyl ammonium chloride; (Benzalkonium chloride) & $8001-54-5$ & $008001-54-5$ & & $\mathrm{~s}$ & $3.437 \mathrm{E}+01$ & & & & & & 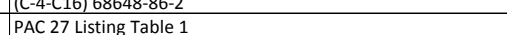 \\
\hline 56 & 55 & Alkylamines & $63231-48-1$ & $063231-48-1$ & & G & & & & & & & PAC 27 Listing Table 1 \\
\hline$\frac{57}{58}$ & & 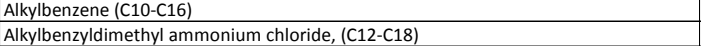 & $\frac{686848-871-3}{68391-01-5}$ & & & $\frac{s}{s}$ & & & & & & & 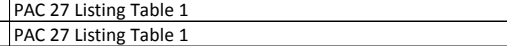 \\
\hline 59 & 58 & Allene; (1,2-2-Propadiene) & $463-49-0$ & $000463-49-0$ & 40.06 & G & $-1.460 \mathrm{E}+02$ & & & & & & PAC 27 Listing Table 1 \\
\hline & & & $107-18-6$ & $000107-18-6$ & 12099 & +1 & 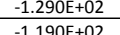 & 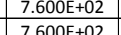 & & 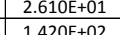 & & & 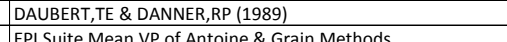 \\
\hline
\end{tabular}




\begin{tabular}{|c|c|c|c|c|c|c|c|c|c|c|c|c|c|}
\hline & No. & Chemical Compound & $\begin{array}{c}\text { cas N Number } \\
\text { (CASPN) }\end{array}$ & $\begin{array}{l}\text { Modified } \\
\text { Format CAS }\end{array}$ & $\begin{array}{l}\text { Molecular } \\
\text { Weight (MW }\end{array}$ & at & $\begin{array}{c}\text { MP or FP } \\
\text { [ic }\end{array}$ & 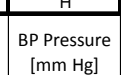 & BP [C] & $\begin{array}{c}\text { Vapor } \\
\text { Pressure }\end{array}$ & $\begin{array}{c}\text { Torvp } \\
\text { [cicl }\end{array}$ & 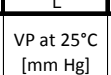 & 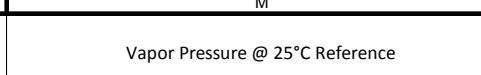 \\
\hline & & Allyy chloride & $\frac{107-05 \cdot 1}{203500}$ & $000107-05-1$ & $\begin{array}{l}76.53 \\
10.054 \\
\end{array}$ & & 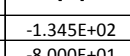 & 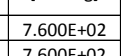 & 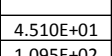 & 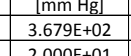 & 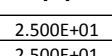 & $\frac{3.699+02}{3.020201}$ & 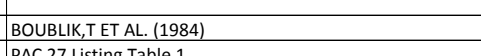 \\
\hline$\frac{63}{64}$ & & $\begin{array}{l}\text { Alyy chlorocabonate } \\
\text { Ally isothicyanate; (Mustard oil) }\end{array}$ & 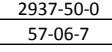 & 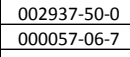 & $\frac{120.54}{99.16}$ & $\frac{L}{L}$ & $\begin{array}{l}-8.8006 \mathrm{E}+01 \\
-8.000+101 \\
\end{array}$ & 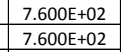 & 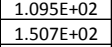 & 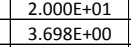 & $\begin{array}{l}2.5000+01 \\
2.500+01\end{array}$ & 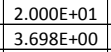 & 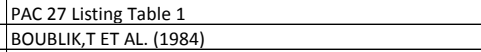 \\
\hline & & lllllamine & & 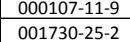 & 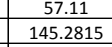 & & & & & & & & 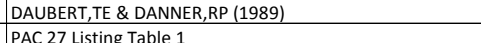 \\
\hline & & lilythrichlorosilane & $\begin{array}{l}107-37 \\
10025\end{array}$ & 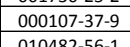 & & & 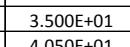 & 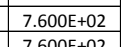 & 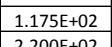 & $5.3005+01$ & $4.7506+01$ & & 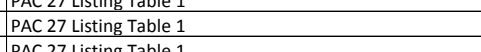 \\
\hline$\frac{66}{69}$ & & 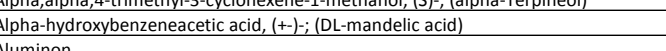 & 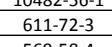 & 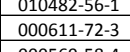 & 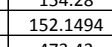 & $\frac{5}{5}$ & 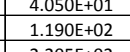 & & & & & & $\begin{array}{llllllll}1 & 1\end{array}$ \\
\hline & & & 429: & 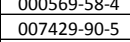 & $\frac{473.43}{26.98}$ & & 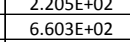 & & 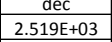 & $1.000=+00$ & $1.284+03$ & & 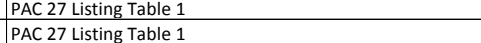 \\
\hline & & 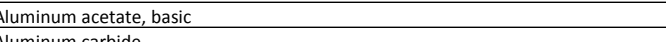 & & .073630.044: & 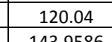 & 3 & & & & & & & ting Table \\
\hline 74 & (1) & 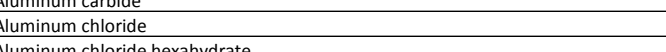 & 74t6-70-0.0 & 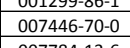 & 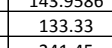 & $\frac{5}{5}$ & $\frac{1.200 \mathrm{cts}}{192.5 \mathrm{~atm}}$ & & 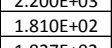 & $1.000 E+00$ & $1.000 E_{0}+22$ & & 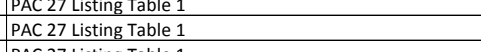 \\
\hline$\frac{76}{76}$ & & 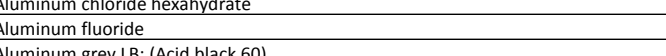 & 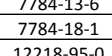 & 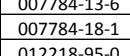 & 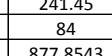 & $\frac{3}{5}$ & 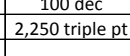 & & $\begin{array}{l}1.2872+252 \\
1.276+03\end{array}$ & $\begin{array}{l}1.0000+700 \\
1.000+00\end{array}$ & $\begin{array}{lll}1.0060202 \\
1.238+03\end{array}$ & & 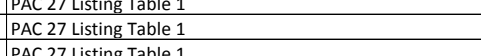 \\
\hline$\frac{78}{79}$ & 77 & 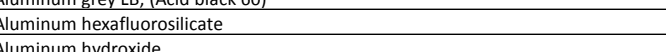 & 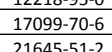 & 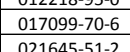 & 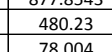 & $\frac{5}{5}$ & 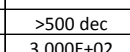 & & & & & & $\begin{array}{l}\text { Listing Table } 1 \\
\text { litsing }\end{array}$ \\
\hline & 79 & Aluminum oxide hydrate & 1333.842. & $001333.84-2$ & 137.99 & $\mathrm{~s}$ & (1058s 20203000 & & & & & & $\begin{array}{l}\text { PAC } 27 \text { Listing Tadele } 1 \\
\text { Pable } 1\end{array}$ \\
\hline$\frac{80}{81}$ & 80 & Aluminum oxide; (Alumina) & ( & $\overline{001344-28-1}$ & 101.96 & $\mathrm{~s}$ & $2.054 \mathrm{E}+03$ & & $2.977 \mathrm{E}+03$ & 7.500E +00 & $2.3511+0.35$ & & 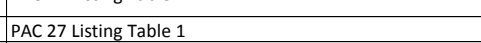 \\
\hline & & 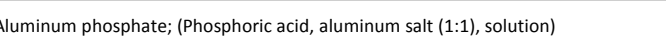 & 7784-30-7 & $007784-30-7$ & 121.95 & $\mathrm{~L}$ & & $7.600 E+02$ & $1.000+ \pm 02$ & $2.900=02$ & $2.000 \mathrm{E}+01$ & $6.427 E-02$ & 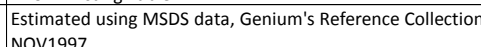 \\
\hline$\frac{83}{83}$ & & Muminum phosphide & $\frac{20859.7 .3 .8}{20323.1}$ & 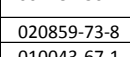 & 57.95 & $s$ & 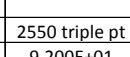 & & & & & & 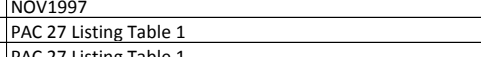 \\
\hline$\frac{65}{85}$ & & 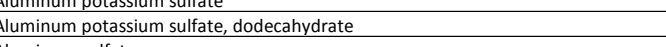 & & 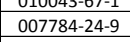 & 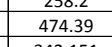 & & 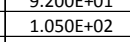 & & & & & & 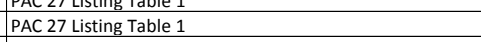 \\
\hline$\frac{86}{87}$ & 85 & luminum mututate & $\begin{array}{l}1.00430 .0-13-3 \\
13530-50-2\end{array}$ & 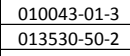 & $\begin{array}{l}\frac{3322.151}{320.9672} \\
3\end{array}$ & $\frac{s}{s}$ & 1000d dec & & & & & & 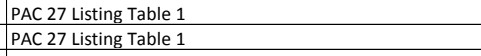 \\
\hline & & 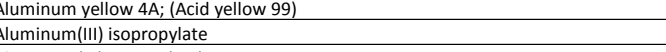 & 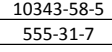 & $\begin{array}{l}0.10345 .58 .5 \\
0.0555-3.2\end{array}$ & 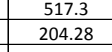 & & & $1.000 E+011$ & $1.250 \mathrm{E}+02$ & & & & 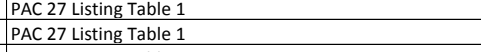 \\
\hline$\frac{90}{90}, 0$ & & 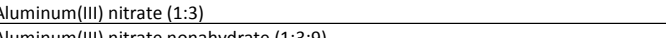 & 13473-90-0 & 013473-9.9-6. & & s. & dec & & 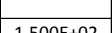 & & & & 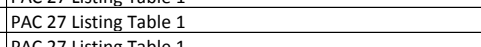 \\
\hline$\frac{91}{92}$ & & 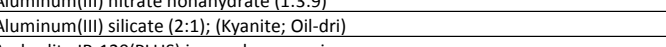 & 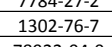 & 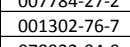 & 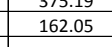 & $\frac{5}{5}$ & 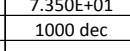 & & $1.500 \mathrm{E}+02$ & & & & 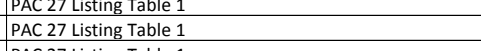 \\
\hline$\frac{38}{994}$ & 烈 & 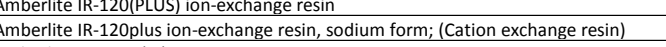 & 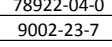 & 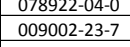 & & $\frac{5}{5}$ & & & & $1.7006+01$ & $2.0005+01$ & & 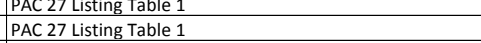 \\
\hline & 94 & 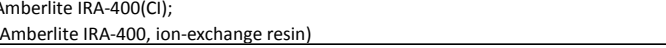 & $9002-24.8$ & $009002-24-8$ & & $\mathrm{~s}$ & & & & & & & PAC 27 Listing Table 1 \\
\hline$\frac{96}{97}$ & 95 & 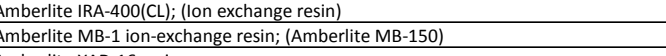 & 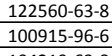 & $\frac{125506-3.8}{100915-96-6}$ & & $\frac{5}{5}$ & $0.000=500$ & & $1.000 E+02$ & & & & $\begin{array}{ll}27 \\
27 \text { Lisiting Tablele } 1 \\
\text { Table } 1\end{array}$ \\
\hline$\frac{96}{999}$ & & 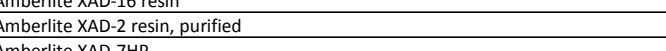 & 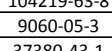 & 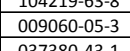 & & $\frac{5}{5}$ & & & & & & & 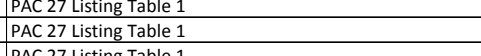 \\
\hline & & Imberlyst 15 ino-exchange resin & $\frac{9037-24.5}{9}$ & 009037 & & $\frac{2}{2}$ & & & & $1.700 E+11$ & $2.000+01$ & & 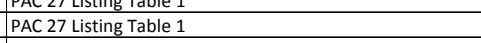 \\
\hline & 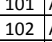 & 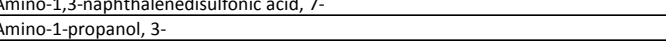 & $\begin{array}{l}\frac{856-7.6}{15.87} \\
\end{array}$ & 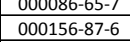 & 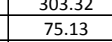 & $\frac{S}{L}$ & 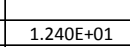 & $\begin{array}{l}7.600 E+02 \\
\end{array}$ & $1.870 \mathrm{E}+022$ & $7.7100-02$ & $2.500+0101$ & $7.7100-0.0$ & 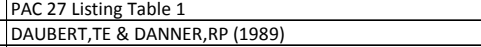 \\
\hline$\frac{104}{105}$ & $\frac{103}{104}$ & 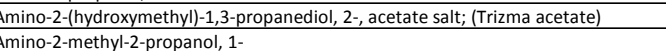 & 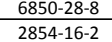 & 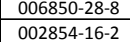 & $\frac{1881.1882}{89816}$ & $s$ & - $13667+01$ & $7.600 E+02$ & $1.510+02$ & $3.3106+000$ & $2.5005+01$ & $3310 E+000$ & 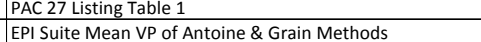 \\
\hline \begin{tabular}{c|ccc}
106 \\
107
\end{tabular} & 105 & 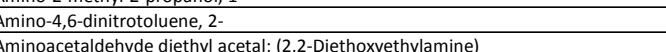 & 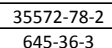 & 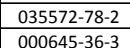 & $\frac{197.17}{133.189}$ & $\frac{s}{1}$ & $.78005+01$ & $76005+02$ & $16000+02$ & $21806+00$ & $25005+01$ & & 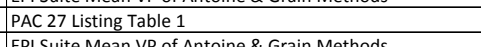 \\
\hline & 107 & 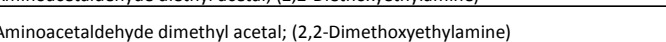 & 2248309.6 .6 & $022483-0.6$ & 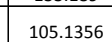 & $\mathrm{L}$ & $.3 .378 \mathrm{E}+01$ & $7.6005+02$ & $1.350 \mathrm{E}+02$ & $1.380 \mathrm{E}+01$ & $2.5000+01$ & $1.380+01$ & 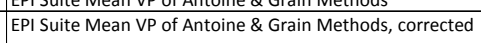 \\
\hline & & Iminoanhthraquinone, 2 . & $\frac{117.79 \cdot 3}{150.9}$ & 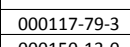 & 223.24 & $\mathrm{~s}$ & $\begin{array}{l}302-306 \\
03600\end{array}$ & & subl & 28000040 & 2500501. & & sting Table 1 1 \\
\hline$\frac{110}{1.0}$ & 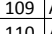 & 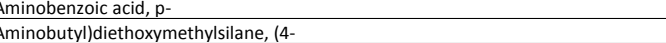 & 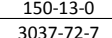 & & 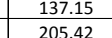 & & 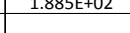 & 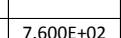 & & 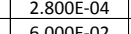 & 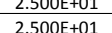 & & \\
\hline 112 & 1111 & 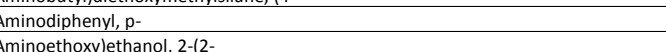 & 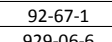 & 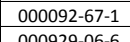 & 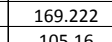 & s & 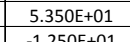 & 2 & 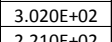 & 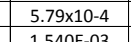 & 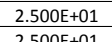 & 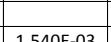 & 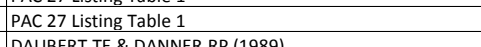 \\
\hline 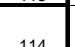 & 113 & 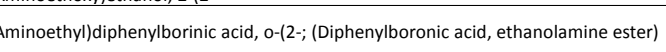 & 524.95.8 & $000524-95-8$ & 225.12 & $\mathrm{~s}$ & & & & & & & PAC 27 L isting Table 1 \\
\hline$\frac{115}{116}$ & $\begin{array}{l}114 \\
115\end{array}$ & 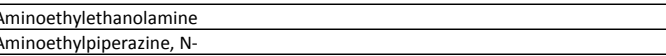 & 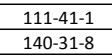 & 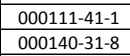 & $\frac{104.18}{129.203}$ & $\frac{L}{L}$ & $1.760 \mathrm{E}+01.10$ & $\begin{array}{l}7.600+502 \\
7.6005+02\end{array}$ & $\begin{array}{l}2.473+502 \\
2.2005+02\end{array}$ & 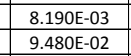 & $\begin{array}{l}2.500+501 \\
2.5005+01\end{array}$ & $\begin{array}{l}8.906-03 \\
9.880-02\end{array}$ & $\begin{array}{l}\text { DAUERRT,TE \& DANNER,RP (1989) } \\
\text { EAI Sutit Modifie Grain Method }\end{array}$ \\
\hline$\frac{117}{10}$ & & 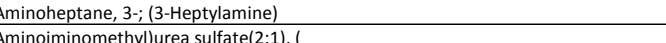 & $\begin{array}{l}\text { 2028292-24-4 } \\
\text { 592-5 }\end{array}$ & 000591-01. & $\begin{array}{l}\frac{11.525}{30256} \\
30265\end{array}$ & $\frac{t}{s}$ & 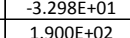 & & 1.438E+02 & & $2.5005+01$ & & EEP I Sute Mean VP of Antoine \& Grain Methods \\
\hline & & & 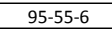 & $000095-55-6 \mathrm{~K}$ & $\begin{array}{ll}109.14 \\
\end{array}$ & & 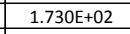 & & subl 174 & 5.000:-04 & $2.5005+01$ & & 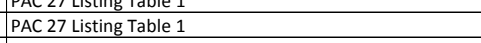 \\
\hline$\frac{120}{12121}$ & $\begin{array}{l}1190 \\
120\end{array}$ & 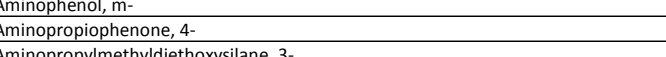 & 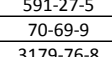 & 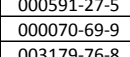 & 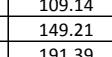 & $\frac{s}{5}$ & 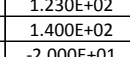 & & 1.640E+02 & 1100501010 & & & 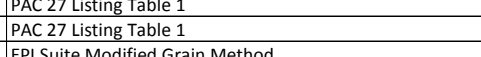 \\
\hline
\end{tabular}




\begin{tabular}{|c|c|c|c|c|c|c|c|c|c|c|c|c|c|}
\hline & №. & Chemical Compound & $\begin{array}{l}\text { CAS Number } \\
\text { (CASRN) }\end{array}$ & $\begin{array}{c}\text { Modififd } \\
\text { Format CAS }\end{array}$ & $\begin{array}{c}\text { Molecular } \\
\text { Weight (MW) }\end{array}$ & \begin{tabular}{|c|} 
QV State \\
at \\
$25^{\circ} \mathrm{C}$
\end{tabular} & $\begin{array}{c}\text { MP or re } \\
\text { [c] }\end{array}$ & $\begin{array}{c}\text { BP Pressure } \\
\text { [mm HE] }\end{array}$ & $\mathrm{BP}\left[{ }^{[\mathrm{C}]}\right]$ & $\begin{array}{l}\text { Vapor } \\
\text { Pressure } \\
\text { [mm Hg] }\end{array}$ & $\begin{array}{c}\begin{array}{c}\text { Tforv VP } \\
\text { [Cc] }\end{array} \\
\end{array}$ & 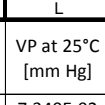 & 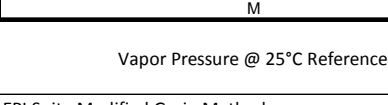 \\
\hline$\frac{123}{1021}$ & $\frac{122}{1023}$ & 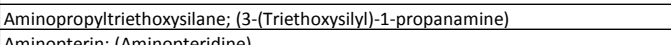 & $\frac{919-30-2}{54626}$ & $\frac{000919-30-2}{00025626}$ & 221.42 & & $-7.0005+01$ & $7.600 \mathrm{E}+02$ & $2.170 \mathrm{E}+02$ & $7.3400-02$ & $2.500 E+01$ & $7.340 \mathrm{E}-02$ & $\begin{array}{l}\text { EPI Suite Modified Grain Method } \\
\text { PAc 207 }\end{array}$ \\
\hline$\frac{124}{1025}$ & $\frac{123}{124}$ & \begin{tabular}{|l|l|} 
Aminopterin; (Aminopteridine) \\
Aminopyraine
\end{tabular} & $\frac{54-62-6}{5096-6-6}$ & $000054-62-6$ & $\frac{440.47}{0.5}$ & $\frac{s}{s}$ & & & & & & & 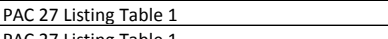 \\
\hline$\frac{125}{109}$ & $\frac{124}{125}$ & Aminopyrazine & $5049-61-6$ & $005049-61-6$ & $\frac{95}{9413}$ & $\frac{s}{s}$ & & & & & & & PAC 27 Listing Table 1 \\
\hline$\frac{126}{127}$ & $\frac{125}{126}$ & $\begin{array}{l}\text { Aminopyrininee, 4;; (4-Pyridinamine) } \\
\text { Amiton oxalate }\end{array}$ & $\begin{array}{l}504424-5 \\
3734-97-2\end{array}$ & $\frac{000500244-5}{003734-97-2}$ & $\begin{array}{l}94.13 \\
359.42\end{array}$ & $\frac{2}{3}$ & $\begin{array}{llll}1.580 E+0202 \\
9.800++01\end{array}$ & 2.000E-01 & $\begin{array}{l}2.730 E+02 \\
1.100 E+02\end{array}$ & $1.000 E-02$ & 8.000E+01 & & $\begin{array}{l}\text { PAC } 27 \text { Litsing Table } 1 \\
\text { PAC } 27 \text { Listing Table } 1\end{array}$ \\
\hline$\frac{2128}{128}$ & & Amiton; (0,0-Diethyl-5-(2-diethylaminoethy)] thiophosphate) & $\frac{78-53-5}{78}$ & $000078-53-5$ & $\frac{269.38}{263}$ & & & $2.0000-01$ & $1.100+502$ & $2.670-04$ & $2.5000+01$ & 2670E-04 $>40$ & FPI Suite Modified Grain Method \\
\hline$\frac{129}{129}$ & 年 & Amitrole & $61-82-5$ & $000061-82-5$ & 84.08 & s & $1.5906+02$ & & & $4.4 \times 10-7$ & $2.5005+01$ & 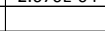 & $\begin{array}{ll}\text { PAC } 27 \text { listing Table } 1 \\
\end{array}$ \\
\hline 130 & 129 & Ammonia & 7664-41-7 & $007664-41-7$ & 17.031 & G & $-7.770 E+01$ & & $-3.330 E+01$ & $7.5000+02$ & -3.360E+01 & & PAC 27 Listing Table 1 \\
\hline 131 & 130 & Ammonia-d3 & $\begin{array}{l}13550-49-7 \\
501-9\end{array}$ & $013550-49-7$ & 20.0487 & 6 & & & & & & & PAC 27 L Listing Table 1 \\
\hline$\frac{132}{133}$ & $\frac{131}{132}$ & $\begin{array}{l}\text { Ammonium mactate } \\
\text { Ammonium aluminum fluoride; (Triammonium hexafluoroaluminate }\end{array}$ & 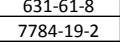 & $\begin{array}{l}0.0631-61-8 \\
007784-19-2\end{array}$ & $\begin{array}{l}797.1 \\
195.13\end{array}$ & $\frac{\mathrm{s}}{\mathrm{s}}$ & 1.140E+02 & & & & & & $\begin{array}{l}\text { PAC } 27 \text { Listing gable } 1 \\
\text { ACC } 27 \text { isting Table } 1\end{array}$ \\
\hline & & 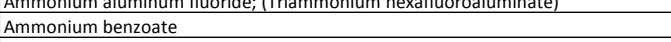 & & 0.1863-63-2-4 & 193.17 & $\frac{2}{3}$ & 198 decom & & 160 sublms & & & & $\begin{array}{l}\text { PAC 27 Ltsting labie } 1 \\
\text { PAC } 27 \text { tisting Table } 1\end{array}$ \\
\hline$\frac{135}{135}$ & 134 & Ammonium bicarbonate & $1066-33-7$ & $001066-33-7$ & 79.1 & $\mathrm{~s}$ & $1.075 E+02$ & & & & & & $\begin{array}{l}\text { PAC } 27 \text { Listing Table } 1 \\
\end{array}$ \\
\hline 136 & 135 & Ammonium bisulfate; (Ammonium hydrogen suffate) & $7803-63-6$. & $007803-63-6$ & 1115.11 & $\mathrm{s}$ & $1.469 \mathrm{E}+02$ & & & & & & PAC 27 Listing Table 1 . \\
\hline 137 & 136 & Ammonium bisulfiti; (Ammonium hydrogen sulfite) & 10192-30-0 & $010192-30-0$ & 99.11 & $\mathrm{~s}$ & dec & & 150 subl & & & & PAC 27 Listing Table 1 \\
\hline 138 & 137 & Ammonium bromide & 12124-97-9 & 012124-97-9 & 97.96 & s & 4.452E+03 & & $\begin{array}{l}325 \text { in } \\
\text { vacuum }\end{array}$ & $1.000 E+00$ & 1.983E+02 & & PAC 27 Listing Table 1 \\
\hline 139 & 138 & Ammonium carbamate; (Carbamic acid, ammonium salt) & $1111-78-0$ & $\begin{array}{llll}001111-78-0 \\
0200695\end{array}$ & 78.09 & 5 & 60 sublms & & $6.000 E+01$ & & & & 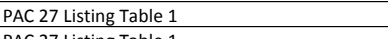 \\
\hline$\frac{140}{141}$ & 1390 & $\begin{array}{l}\text { Ammonium carbonate } \\
\text { Ammonium chloride }\end{array}$ & 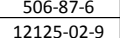 & $\frac{000506-87-6}{0012125-02-9}$ & $\frac{96.11}{5.5}$ & $\frac{s}{s}$ & $\frac{1.075+02}{3.378+5+22}-2$ & & 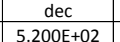 & & & & $\begin{array}{l}\text { PAC } 27 \text { Listing Table } 1 \\
\text { PCA } 27 \text { isting Table } 1\end{array}$ \\
\hline 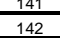 & $\frac{1.40}{141}$ & $\begin{array}{l}\text { Ammonium chlordide } \\
\text { Ammonium chromate }\end{array}$ & 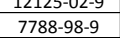 & 0.12158-02-98-9 & $\frac{53.5}{152.1}$ & & 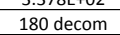 & & & $1.0000+00$ & 100.4 subl & & $\begin{array}{l}\text { PAC 27 IItting Iable } 1 \\
\text { PAC } 27 \text { Listing Table } 1\end{array}$ \\
\hline & 142 & Ammonium citrate & & & 226.18 & $\mathrm{~s}$ & & & & & & & $\begin{array}{ll}\text { PAC } 27 \text { Listing Table } 1 \\
\end{array}$ \\
\hline 144 & 143 & Ammonium citrate tribasic & $3458-72-8$ & $003458-72-8$ & 243.2162 & $\mathrm{~s}$ & & & & & & & PAC 27 Listing Table 1 \\
\hline 145 & 144 & Ammonium citrate, dibasic & $3012-65-5$ & $003012-65-5$ & 226.19 & $\mathrm{~s}$ & & & decom & & & & PAC 27 Listing Table 1 \\
\hline 146 & $\frac{145}{146}$ & Ammonium dichromate & $7789.09 \cdot 5$ & $007789-09-5$ & 252.1 & $\frac{s}{s}$ & $\frac{\operatorname{dec}}{10050}$ & & & & & & 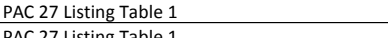 \\
\hline$\frac{147}{148}$ & $\left|\frac{146}{147}\right|$ & $\begin{array}{l}\text { Ammonium dihydrogen phosphate; (Monoaammonium phosphate) } \\
\text { Ammonium ferrous sulfate hexayhdrate }\end{array}$ & 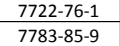 & 些7722-276-1 & $\frac{115.03}{356.09}$ & $\frac{s}{s}$ & $1.900 \mathrm{E}+02$ & & & & & & $\begin{array}{l}\text { PAA } 27 \text { L Listing Table } 1 \\
\text { PCA } 2 \text { Tistig Table } 1\end{array}$ \\
\hline$\frac{148}{149}$ & $\frac{148}{148}$ & \begin{tabular}{|l} 
Amm monium fluoborate \\
Amate nexalyurate
\end{tabular} & 13826-83-0 & $013826-83-0$ & $\begin{array}{lll}1050.09 \\
104.86\end{array}$ & 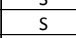 & $2.300 E+02$ & & 238 subl & & & & PAC 27 Listing Table 1 \\
\hline 150 & 149 & Ammonium fluoride & 1212125-01-8 & $012125-01-8$ & 37.05 & $\mathrm{~s}$ & 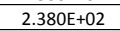 & & & & & & $\begin{array}{ll}\text { PAC } 27 \text { Listing Table } 1 \\
\end{array}$ \\
\hline 151 & 150 & Ammonium formate & 540-69-2 & 0000540-69-2 & 63.07 & $\mathrm{~s}$ & $1.160 \mathrm{E}+02$ & & $180 \mathrm{dec}$ & & & & PAC 27 Listing Table 1 | \\
\hline$\frac{152}{152}$ & $\frac{151}{152}$ & Ammonium hexachlorohydrate (III) & $\frac{z-0001}{10010100}$ & $000000-0001$ & $1781^{\circ}$ & $s$ & & & & & & & $\begin{array}{l}\text { PAC 27 Listing Table } 1 \\
\text { Dap 27 }\end{array}$ \\
\hline$\frac{153}{154}$ & $\frac{152}{153}$ & 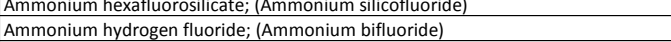 & 136919-19-0 & 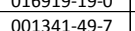 & $\frac{1 / 18.19}{57.06}$ & $\frac{5}{5}$ & $\frac{540102}{1.260 t+02}$ & & $23906+02$ & & & & $\begin{array}{l}\text { PAA } 27 \text { Listing Table } 1 \\
\text { PC } 27 \text { Listing Table } 1\end{array}$ \\
\hline$\frac{154}{155}$ & 154 & Ammonium hydrogen oxalate hemihydrate & 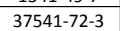 & 0 & 232.146 & $\frac{5}{3}$ & 220 & & $2.300+02$ & & & & $\begin{array}{l}\text { PAC } 27 \text { Listing Table } 1 \\
\text { PAC The }\end{array}$ \\
\hline 256 & 155 & Ammonium hydroxide & 1336-21-6 & 001336-21-6 & 35.046 & $G_{G}$ & 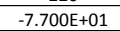 & 7.600E+02 & $\begin{array}{l}3.600 E+01 \\
\end{array}$ & 2.160E+03 & $2.500 E+01$ & & $\frac{1}{\text { PAC } 27 \text { Listing Table } 1}$ \\
\hline 157 & 156 & Ammonium iodide & $12027-06-4$ & 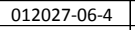 & 145 & $\mathrm{~s}$ & 551 subl & & $220 \mathrm{vac}$ & $1.000 E+00$ & 2.109E+02 & & PAC 27 Listing Table 1 \\
\hline 158 & 157 & Ammonium lactate & $515-98-0$ & $000515-98-0$ & 107.11 & $\mathrm{~s}$ & $9.200 \mathrm{E}+01$ & & & & & & PAC 27 Listing Table 1 \\
\hline$\frac{159}{160}$ & $\frac{158}{159}$ & \begin{tabular}{|l} 
Ammonium ligninin suffonate \\
Ammonium molybdate(VI) tetrahydrate
\end{tabular} & $\begin{array}{l}80015-5-3-8 \\
12054-55-2\end{array}$ & $\frac{008061-1358-8}{012054-85-2}$ & 1235.87 & $\frac{s}{s}$ & $9.000 \mathrm{E}+01$ & & $180 \mathrm{dec}$ & & & & $\begin{array}{l}\text { PAC } 27 \text { L listing Table } 11 \\
\text { AC } 2 \text { listing Table } 1\end{array}$ \\
\hline$\frac{100}{161}$ & 160 & 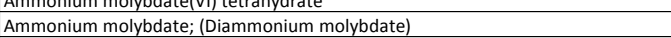 & $\frac{110043-85-2}{13106-76-8}$ & $\frac{131306-76-8}{0130}$ & $\frac{1355.87}{196.04}$ & $\frac{2}{3}$ & dec & & (100uce & & & & $\begin{array}{l}\text { PAC 27 Isting Gabie } 1 \\
\text { PAC } 27 \text { Listing Table } 1\end{array}$ \\
\hline 162 & 161 & Ammonium molybdophosphate & $12026-66-3$ & 012026-66-3 & 1876.35 & $\mathrm{~s}$ & dec. & & & & & & $\begin{array}{lll}\text { ACC } 27 \text { Listing Table } 1 & \\
\end{array}$ \\
\hline 163 & 162 & Ammonium nickel sulfate & $15699-18-0$ & 015699-18-0 & 286.93 & $\mathrm{~s}$ & & & & & & & PAC 27 Listing Table 1 \\
\hline 64 & 163 & Ammonium nitrate & 6484-52-2 & $006484-52-2$ & 80.06 & s & $1.696 \mathrm{E}+02$ & & $200-260 \mathrm{dec}$ & & & & PAC 27 Listing Table 1 \\
\hline 165 & 164 & Ammonium oxalate monohydrate & 5972-73-6. & $005972-73-6$ & 125.08 & $\mathrm{~s}$ & & & & & & & PAC 27 listing Table 1 \\
\hline 166 & 165 & Ammonium oxalate; (Diammonium oxalate monohydrate) & $6009-70-7$ & $006009-70-7$ & 142.11 & s & 70 decom & & & & & & PAC 27 Listing Table 1 \\
\hline$\frac{167}{169}$ & $\frac{166}{167}$ & $\begin{array}{l}\text { Ammonium oxalate; (Erhanedioic acid, diammonium salt) } \\
\text { Ammonium pentaborate }\end{array}$ & 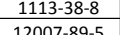 & 001113-38-8 & $\frac{124.12}{2720}$ & $\frac{s}{s}$ & dec & & & & & & $\begin{array}{l}\text { PAC } 27 \text { Listing Table } 1 \\
\text { PCC 27lyting Tathle } 1\end{array}$ \\
\hline$\frac{168}{169}$ & 168 & $\begin{array}{l}\text { Ammmonum pentaborate } \\
\text { Ammonium perchlorate }\end{array}$ & $\begin{array}{l}1.001-89-5 \\
7790-98-9\end{array}$ & 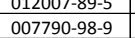 & $\frac{2 / 2.14}{117.5}$ & $\frac{5}{5}$ & dec & & & & & & $\begin{array}{l}\text { PAC 27 I Ltsing Iabie } 1 \\
\text { PAC } 2 \text { Listing Table } 1\end{array}$ \\
\hline & 169 & Ammonium permanganate & $13446-10-1$ & $013446-10-1$ & 136.99 & $\mathrm{~s}$ & expl & & & & & & 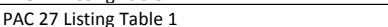 \\
\hline $\begin{array}{lll}171 \\
\end{array}$ & 170 & \begin{tabular}{|l|l|l} 
Ammonium persulfate \\
\end{tabular} & 7727-54-0 & $007727-54-0$ & 228.22 & $\mathrm{~s}$ & $120 \mathrm{dec}$ & & & & & & PAC 27 Listing Table 1 \\
\hline 172 & 171 & Ammonium phosphate dibasic; (Diammonium phosphate) & $7783-28-0$ & 007783-28-0 & 132.08 & $\mathrm{~s}$ & $185 \mathrm{dec}$ & & & & & & PAC 27 Listing Table 1 , \\
\hline 173 & 172 & Ammonium picrate & 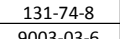 & $000131-74-8$ & 246.135 & 5 & dec & & 423 expl & & & & 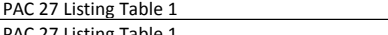 \\
\hline$\frac{174}{175}$ & 174 & $\begin{array}{l}\text { Ammonium molvacryyate } \\
\text { Ammonium sulfamate }\end{array}$ & 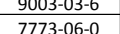 & $\frac{090030-03-6}{007773-06-0}$ & 144.124 & $\frac{5}{5}$ & $1.310 E+02$ & & 160 dec & $0.000 F+00$ & No temp & & $\begin{array}{l}\text { PAC 2/IItsing Iable 1 } \\
\text { PAC 27 Listing Table } 1\end{array}$ \\
\hline 176 & (75) & Ammonium sulfate & $7783-20-2$ & $007783-20-2$ & 132.16 & $\mathrm{~s}$ & $>280$ & & & ( & com & & $\begin{array}{l}\text { PAC } 27 \text { Listing Table } 1 \\
\end{array}$ \\
\hline $\begin{array}{l}2777 \\
177\end{array}$ & 176 & Ammonium sulfide & $12135-76-1$ & 012135-76-1 & 68.14 & $\mathrm{~s}$ & decom & & 1.040E+02 & & & & PAC 27 Listing Table 1 \\
\hline 178 & 177 & Ammonium sulfite & 10196-04-0 & 010196-04-0 & 116.14 & $\mathrm{~s}$ & & & & & & & PAC 27 listing Table 1 \\
\hline 179 & 178 & Ammonium sulfite monohydrate & 7783-11-1 & 007783-11-1 & 134.156 & $\mathrm{~s}$ & $60-70$ & & 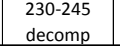 & & & & PAC 27 Listing Table 1 \\
\hline 180 & 179 & Ammonium tartrate; (Butanedioic acid, 2,3-dihydroxy-(2R,3R); ammonium salt) & $14307-43-8$ & $014307-43-8$ & 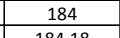 & 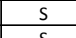 & & & decom & & & & 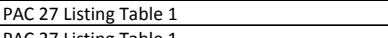 \\
\hline 181 & 180 & Ammonium tartrate; (Diammonium tartrate) & 3164-29-2 & 003164-29-2 & 184.18 & $\mathrm{~s}$ & & & & & & & PAC 27 listing Table 1 | \\
\hline$\frac{182}{183}$ & 182 & $\begin{array}{l}\text { Ammonium thiocyanate } \\
\text { Ammonium thiosulfate; (Ammonium hyposulfite) }\end{array}$ & $\frac{1762-955}{7783-18-8}$ & 000762-255-4 & $\frac{76.13}{148.22}$ & $\frac{2}{2}$ & $\frac{1.496 \mathrm{E}+02}{150 \mathrm{dec}}$ & & $170 \mathrm{dec}$ & & & & $\begin{array}{l}\text { PAC 27 sting Iabie } 1 \\
\text { PAC } 27 \text { Listing Table } 1\end{array}$ \\
\hline 184 & 183 & Ammonium tun & $\frac{11120-25-5}{702556}$ & $011120-25-5$ & $\frac{3042.44}{11690}$ & $\mathrm{~s}$ & & & & & & & 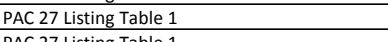 \\
\hline & & Almmonium vanadate; (Ammonium vanadium oxide; Ammonium metavanadate) & & & & & $200 \mathrm{dec}$ & & & & & & PAC 27 listing Table 1 \\
\hline
\end{tabular}




\begin{tabular}{|c|c|c|c|c|c|c|c|c|c|c|c|c|c|}
\hline & & & & & & $\mathrm{F}$ & & H. & & & $\bar{k}$ & & \\
\hline & No. & Chemical Compound & $\begin{array}{c}\text { CAS Number } \\
\text { (CASRN) }\end{array}$ & $\begin{array}{l}\text { Modified } \\
\text { Format CAS }\end{array}$ & $\begin{array}{l}\text { Molecular } \\
\text { Weight (MW) }\end{array}$ & $\begin{array}{c}\text { Q V State } \\
\text { at } \\
25^{\circ} \mathrm{C}\end{array}$ & $\begin{array}{l}\text { MP or FP } \\
{\left[{ }^{\circ C} \mathrm{C}\right]}\end{array}$ & $\begin{array}{c}\text { BP Pressure } \\
{[\mathrm{mm} \mathrm{Hg}]}\end{array}$ & $B P\left[{ }^{[C}\right]$ & $\begin{array}{l}\text { Vapor } \\
\text { Pressure } \\
\text { [mm He }\end{array}$ & $\begin{array}{l}\text { Tfor VP } \\
\text { [C] }\end{array}$ & $\begin{array}{c}\text { VPat } 25^{\circ} \mathrm{C} \\
{[\mathrm{mm} \mathrm{Hg}]}\end{array}$ & Vapor Pressure @ $25^{\circ} \mathrm{C}$ Reference \\
\hline & $185 \mid$ & |Ammonium, hexadecyltrimethyl, bromide; (Hexadecyltrimethylammonium bromide) & $57-09.0$ & $000057-09-0$ & 364.53 & $\mathrm{~s}$ & 237-243 & & & & & & PAC 27 Listing Table 1 \\
\hline$\frac{1.06}{187}$ & $|186|$ & |Ammonium, tetraethyl-bromide: (Tetraethyl ammonium bromide) & $771-91-0$ & $000071-91-0$ & 210.2 & $\mathrm{~s}$ & $286 \mathrm{dec}$ & & & & & & PAC 27 Listing Table 1 \\
\hline 109 & 187 & Ammonium, tetramethyl, bromide; (Tetramethylammonium bromide) & $64-20-0$ & $000064-20-0$ & 154.08 & $\mathrm{~s}$ & dec. .230 & & subl. 3360 & & & & PAC 27 Listing Table 1 \\
\hline$\frac{180}{189}$ & 188 & Amosite & $12172-73-5$ & $012172-73-5$ & 1908.6024 & s & & & & & & & PAC 27 Listing Table 1 \\
\hline 190 & 189 & Amphetamine; (Benzedrine) & $300-62-9$ & $000300-62-9$ & & $\mathrm{~L}$ & & $7.600 E+02$ & 2.030E+02 & $2.400 \mathrm{E}-01$ & $2.500 \mathrm{E}+01$ & $2.4000-01$ & L LAWRENCE,AH ET AL. (1984) \\
\hline$\frac{191}{199}$ & 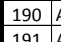 & Amyl acetate & 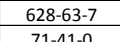 & $\begin{array}{l}000628-63-7 \\
00071-4-0\end{array}$ & $\frac{130.21}{8.87}$ & L & $\frac{-7.850 E+01}{-7900+01}$ & $\begin{array}{l}7.6006+02 \\
7.605 F+02\end{array}$ & $\begin{array}{l}1.484 E+02 \\
13775+02\end{array}$ & $\begin{array}{lll}3.5006+00 \\
22005+00\end{array}$ & $\begin{array}{l}2.5006+01 \\
25050+01\end{array}$ & $3.5006+00$ & DAUBERT,TE \& DANNER,RP (1989) \\
\hline 192 & $|191| t$ & $\begin{array}{l}\text { Amyl alcohol; (1-Pentanon) } \\
\text { Ammy methyl the, tentr; (TAME) }\end{array}$ & 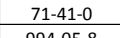 & $000071-41-0$ & $\frac{88.17}{102.2}$ & $\frac{L}{L}$ & & 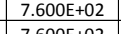 & $1.378 \varepsilon+02$ & $2.2000+00$ & $2.5006+01$ & $2.2006+00$ & RIDDICK,JA ET AL. (1986) \\
\hline & $\frac{192}{193}$ & 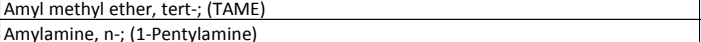 & 994-05-8 & 000994-05-8 & $\frac{102.2}{88.19}$ & & & $7.600 \mathrm{E}+02$ & & $7.520 \mathrm{E}+011$ & $\frac{2.500 \mathrm{EE}+1}{2500 \mathrm{E}+01}$ & $\begin{array}{l}7.520 E+01 \\
3005+01\end{array}$ & 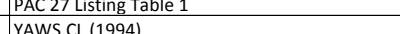 \\
\hline$\frac{1945}{105}$ & 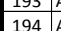 & A & 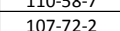 & 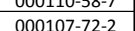 & $\frac{6.19}{205.6}$ & & -26811+01 & $7.600 F+02$ & $1.720 \mathrm{~F}+02$ & 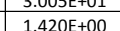 & 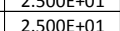 & 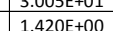 & 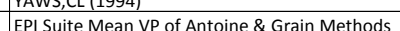 \\
\hline$\frac{195}{196}$ & 195 & Anhydrone; (Magnesium perchlorate) & $10034-81-8$ & $010034-81-8$ & 223.21 & $\frac{t}{s}$ & $251 \mathrm{dec}$ & & & & & & PAC 27 Listing Table 1 \\
\hline 197 & 196 & Aniline & $62-53-3$ & $000062-53-3$ & 93.127 & $\mathrm{~L}$ & $-6.020 \mathrm{E}+00$ & $7.600 E+02$ & $1.842 E+02$ & $4.900--01$ & $2.5006+01$ & 4.900E-01 & DAUBERT,TE \& DANNER,RP (1985) \\
\hline$\frac{198}{109}$ & $|197|$ & Anisidine, $0-$ - & 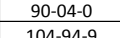 & $0000090-04-0$ & $\frac{123.152}{103.152}$ & $\frac{L}{S}$ & $\frac{6.200 E+00}{570 F+01}$ & $7.600 \mathrm{E}+02$ & $\begin{array}{l}2.240 \mathrm{E}+02 \\
2.430 \mathrm{~F}+02\end{array}$ & $8.000-02$ & $2.5006+01$ & $8.000 E-02$ & \begin{tabular}{|l|} 
PAC 27 Listing Table 1 \\
PA 27 listing Table 1 \\
\end{tabular} \\
\hline$\frac{199}{200}$ & $\begin{array}{l}198 \mid \\
199 \cdot \mid\end{array}$ & $\begin{array}{l}\text { Anisidide, } p \text { - } \\
\text { Aninole (Anisole anhydrous; Methoxybenzene) }\end{array}$ & $\frac{104-94-9}{100-66-3}$ & $000104-94-9$ & $\frac{123.152}{108.15}$ & $\frac{\mathrm{s}}{\mathrm{L}}$ & 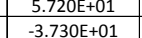 & $7.600 E+02$ & $2.430 E+02$ & 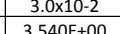 & $\begin{array}{l}2.000 E+01 \\
2500+5+01\end{array}$ & $3540 \mathrm{E}+00$ & PAC 27 Listing Table 1 \\
\hline$\frac{200}{201}$ & 200 & 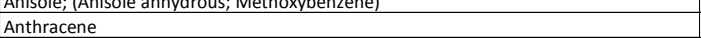 & $\frac{100-60-3}{120-12-7}$ & $000120-12-7$ & $\frac{108.13}{178.229}$ & $\frac{L}{s}$ & 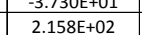 & & 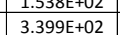 & $\frac{3.540+00}{7.5 \times 10-3}$ & $\begin{array}{llll}2.3 .906 E+01 \\
8.920+01\end{array}$ & & 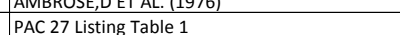 \\
\hline 2002 & 201 & 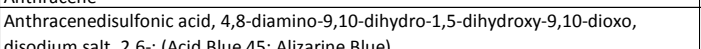 & $2861-02-1$ & $002861-02-1$ & 474.34 & $\mathrm{~s}$ & & & & & & & $\begin{array}{l}\text { PAC } 27 \text { Listing Table } 1 \\
\end{array}$ \\
\hline & 202 & 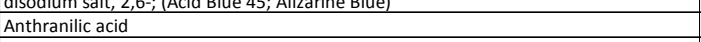 & $118-92-3$ & $000118-92-3$ & 137.15 & $\mathrm{~s}$ & 144-146 & & subl. & & & & PAC 27 Listing Table 1 \\
\hline$\frac{204}{204}$ & & $\begin{array}{l}\text { Anthraquinone } \\
\text { Ans }\end{array}$ & $\frac{2.64-65-1}{8.0}$ & $\frac{000084-65-1}{0.1}$ & 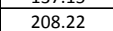 & s & 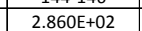 & & 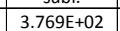 & $1.000 E+00$ & $1.900 E+02$ & & PAC 27 Listing Table 1 \\
\hline 205 & $204 \mid$ & Antimony & $7440-36-0$ & $007440-36-0$ & 121.76 & $\mathrm{~s}$ & $6.306 \mathrm{E}+02$ & & $1.587 \mathrm{E}+03$ & $7.5 \times 10-3$ & $5.340 \mathrm{E}+02$ & & PAC 27 Listing Table 1 \\
\hline 206 & $205 \mid$ & Antimony hydroxide & $\begin{array}{l}39349-74-1 \\
1320.54\end{array}$ & $039349-74-1$ & $\frac{172.7719}{201519}$ & $\frac{s}{s}$ & 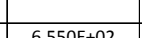 & & 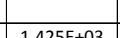 & & & & 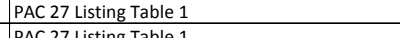 \\
\hline 年 207 & $\begin{array}{ll}206 & 206 \\
07\end{array}$ & $\begin{array}{l}\text { Antimony oxide } \\
\text { Antimony pentachloride }\end{array}$ & $\begin{array}{l}1309964-4 \\
767.9\end{array}$ & $\begin{array}{ll}001309-64-4 \\
007647-18-9\end{array}$ & & $\frac{s}{s}$ & 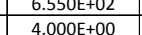 & & $\frac{1.425 E+03}{1.400 E+02}$ & $\frac{7.5 \times 100-3}{1.000+00}$ & $\frac{4.261 E+02}{2.270+01}$ & & $\begin{array}{l}\text { PAC C7 Listing gable } 1 \\
\text { PAC 27 listing Table } 1\end{array}$ \\
\hline 200 & 年 & $\begin{array}{l}\text { Antimony pentachiordice } \\
\text { Antimony pentafluoride }\end{array}$ & 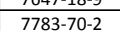 & $\frac{1}{0.077873-30-2.2}$ & $\frac{23.9 .15}{21.67}$ & $\frac{\mathrm{S}}{\mathrm{L}}$ & 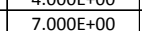 & $7.600 E+02$ & 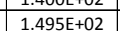 & $\begin{array}{l}1.0060 \mathrm{E}+02 \\
6.6702\end{array}$ & 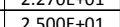 & 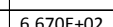 & 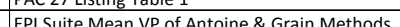 \\
\hline 210 & 2096 & $\begin{array}{l}\text { Antmonyy pentarluondae } \\
\text { Antimony pentasulfide }\end{array}$ & $1315-04-4$ & $001315-04-4$ & $\frac{21.03}{403.8}$ & $\frac{2}{s}$ & 75 decom & 1.000E & & & & ( & PA 27 Listing Table 1 . \\
\hline 211 & 210 & Antimony potassium tartrate trihydrate & $28300-74-5$ & $028300-74-5$ & 667.87 & $\mathrm{~s}$ & 100 lose water & & & & & & PAC 27 Listing Table 1 \\
\hline$\frac{2.42}{2212}$ & $211 \mid$ & Antimony trichloride & $10025-91-9$ & $010025-91-9$ & 228.119 & $\mathrm{~s}$ & $7.300 \mathrm{E}+01$ & & $2.203 E+02$ & $1.000 \mathrm{E}+00$ & $4.9206+01$ & & PAC 27 Listing Table 1 \\
\hline 213 & 212 & Antimony trifluoride & $7783-56-4$ & $007783-56-4$ & 178.75 & $\mathrm{~s}$ & $2.920 \mathrm{E}+02$ & & $3.760 E+02$ & $2.000 E+02$ & $2.920 \mathrm{E}+02$ & & PAC 27 Listing Table 1 \\
\hline 214 & 213 & Antimycin A & $1397-94-0$ & $001397-94-0$ & 548.7 & $\mathrm{~s}$ & & & & $1.000 \mathrm{E}-05$ & $2.500 \mathrm{E}+01$ & & PAC 27 Listing Table 1 \\
\hline$\frac{215}{216}$ & $\left.\frac{214}{215}\right|^{-}$ & 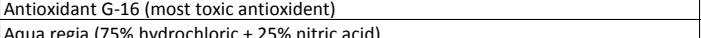 & $\frac{61373-87-3}{8007-56-5}$ & 061373-87-3 & 99.47 & $\frac{s}{L}$ & & $7600 \mathrm{E}+02$ & $8.300 \mathrm{E}+01$ & $4980 \mathrm{9}+01$ & $2500 E+01$ & $4980 E+01$ & $\begin{array}{l}\text { PAC } 27 \text { L Listing Table } 1 \\
\text { ACD Labs }\end{array}$ \\
\hline$\frac{216}{217}$ & 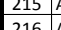 & 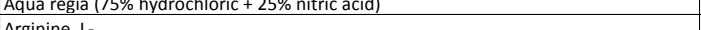 & 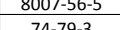 & 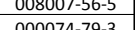 & 1994 & $\frac{L}{s}$ & 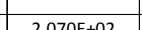 & & & & & & 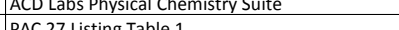 \\
\hline$\frac{217}{218}$ & 217 & Argon, 2 & $7440-37-1$ & $007440-37-1$ & 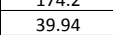 & G & $\begin{array}{l}-.0709+02 \\
-1.892 E+02\end{array}$ & & $-1.857 \mathrm{E}+02$ & & & & 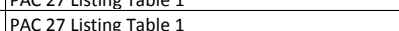 \\
\hline 219 & 218 & 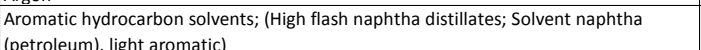 & $64742-95-6$ & $064742-95-6$ & 100 & $\mathrm{~L}$ & $8.020 E+01$ & $7.600 E+02$ & $2.1796+02$ & $4.000 \mathrm{E}-02$ & $2.500 E+01$ & 4.000E-02 & EPI Suite Modified Grain Method \\
\hline 220 & 219 & Aromatic isocyanate mixture "SWD 305A" & $z-0111$ & $00000 z-0111$ & & $\mathrm{~L}$ & & & & $7.760 \mathrm{E}+01$ & $2.500 \mathrm{E}+01$ & $7.760 \mathrm{E}+01$ & MSDS, SWD URETHANE COMPANY AUG93 \\
\hline & $220 \mid$ & $\begin{array}{lll} & \end{array}$ & 7440-38-2 & $007440-38-2$ & 74.92 & $\mathrm{~s}$ & 817 triple point & & \begin{tabular}{c|}
616 \\
sublimation
\end{tabular} & $7.5 \times 10-3$ & $2.800 E+02$ & & PAC 27 Listing Table 1 \\
\hline$\frac{241}{222}$ & 221/ & \begin{tabular}{|l|l|l} 
Arsenic acid; (o-Arsenic acid) \\
\end{tabular} & $77778-39-4$ & $007778-39-4$ & 141.94 & $\mathrm{~s}$ & $3.500 \mathrm{E}+01$ & & $1.600 \mathrm{E}+02$ & & & & PAC 27 Listing Table 1 \\
\hline 223 & 222 & Arsenic pentoxide & $1303-28-2$ & $001303-28-2$ & 229.84 & $\mathrm{~s}$ & $3.150 \mathrm{E}+02$ & & & & & & PAC 27 Listing Table 1 \\
\hline$\frac{224}{225}$ & $223 \mid$ & & $\frac{1327-53-3}{1344-58-9}$ & & $\frac{197.84}{12594}$ & $\frac{s}{G}$ & $2.740 \mathrm{E}+02$ & & 4.600E +02 & $7.5 \times 10-3$ & $1.3377+02$ & & $\begin{array}{l}\text { PAC 27 Listing Table } 1 \\
\text { PAC C7 }\end{array}$ \\
\hline$\frac{225}{226}$ & $224 \mathrm{t}$ & Arsenous acid & $\frac{13464-58-9}{7784-34-1}$ & 013464-485-9 & $\frac{125.94}{18.27}$ & & $-1.600 E+01$ & $7.600 E+02$ & $1.300 E+02$ & $9910++00$ & $2500 \mathrm{E}+01$ & 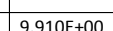 & 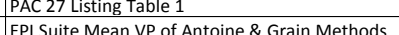 \\
\hline$\frac{227}{227}$ & $\frac{225}{226}$ & $\begin{array}{l}\text { A Arsenous tricnionde } \\
\text { Arsine }\end{array}$ & $77844-42-1$ & 0077884-44-1-1 & $\frac{181.21}{77.95}$ & $\frac{2}{G}$ & $\frac{-1.60 \mathrm{E}+01}{-1.160 \mathrm{E}+02}$ & 1. $.000 E+02$ & $\frac{1.300+02}{-6.250 E+01}$ & $\frac{9.910 t+00}{1.1 \times 10+4}$ & $\frac{2.300 F+01}{2.000++01}$ & . & 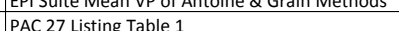 \\
\hline$\frac{228}{228}$ & 227 & Asbestos & $1332-21-4$ & $001332-21-4$ & & $\mathrm{~s}$ & & & & & & & $\begin{array}{lll}\text { PAC } 27 \text { Listing Table } 1 \\
\end{array}$ \\
\hline 229 & 228 & Asbestos (Chrysotile) & $12001-29-5$ & $012001-29-5$ & & $\mathrm{~s}$ & $800-850$ & & & & & & PAC 27 Listing Table 1 \\
\hline$\frac{230}{230}$ & 229 & Ascaridole & $512-85-6$ & $000512-85-6$ & 168.26 & $\mathrm{~L}$ & $3.300 E+00$ & $7.600 E+02$ & 1.965E+02 & $6.110 \mathrm{E}-01$ & $2.500 E+01$ & 6.110E-01 & EPI Suite Mean VP of Antoine \& Grain Methods \\
\hline$\frac{231}{232}$ & $230 \mid$ & $\begin{array}{l}\text { Ascartit; ; Asbestos, 1332-21-4) } \\
\text { Ascorbic acid }\end{array}$ & $\frac{81133-20-2}{50-81-7}$ & $\begin{array}{ll}081133-20-2 \\
00005-81-7\end{array}$ & 176.14 & $\frac{s}{s}$ & $1.920 \mathrm{E}+02$ & & & & & & $\begin{array}{l}\text { PAC 27 Listing Table } 1 \\
\text { PCA } 27 \text { tisting Table } 1\end{array}$ \\
\hline 232 & 232 & Asphalt; (Bitumen) & 8052-42-4a & $008052-42-4 a$ & & $\mathrm{~s}$ & & & $>700 \mathrm{deg} F$ & $0.018 \mathrm{~b} / \mathrm{s} \mathrm{s}$ in $\mathrm{s}$. & $210 \operatorname{deg} \mathrm{F}$ & & PAC 27 Listing Table 1 \\
\hline 234 & $233 \mid$ & Auramine; (4,4'-(Imidocarbony)bisis(N,N-dimethylamine) monohydrochloride) & $2465-27-2$ & $002465-27-2$ & 321.85 & $\mathrm{~s}$ & $2.670 \mathrm{E}+02$ & & & & & & 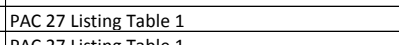 \\
\hline 235 & 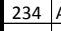 & Azasserine; (L-Serine, diazoacetate (ester)) & $115-02-6$ & $000115-02-6$ & 173.15 & $\mathrm{~s}$ & $1.570 E+02$ & & decom & $1.53 \mathrm{E}-10$ & $2.500 E+01$ & & PAC 27 Listing Table 1 \\
\hline 236 & $235 \mid$ & Azinphos ethyl; (Ethyl guthion) & $2642-71-9$ & $002642-71-9$ & 345.4 & $\mathrm{~s}$ & $5.300 E+01$ & & $111 @ 0.001$ & $3.2000-06$ & $2.000 E+01$ & & PAC 27 Listing Table 1 \\
\hline$\frac{2.50}{273}$ & $236 \mid x$ & Azinphos methy; (Guthio & $86-50-0$ & $000086-50-0$ & 317.34 & 3 & 7.4000E+01 & & & & & & PAC 27 Listing Table 1 \\
\hline 238 & 237 & hylenimine homopolymer; Poly(ethyleneimine)) & $9002-98-6$ & & & $\mathrm{~s}$ & $7.300 E+01$ & & $1.000 E+02$ & $1.754 \mathrm{E}+01$ & $2.000 E+01$ & & Polymers ruspension in w \\
\hline 239 & & Azobis(2-methylpropionitrile), , 2,'- & & $000078-67-1$ & 164.24 & $\mathrm{~s}$ & $107 \mathrm{dec}$ & & & & & & 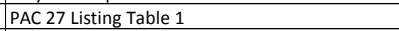 \\
\hline 240 & 239 & 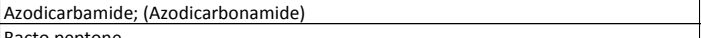 & $\frac{123-77-3}{51102108}$ & $000123-77-3$ & 116.1 & $\frac{s}{s}$ & $2.250 \mathrm{E}+02$ & & & & & & PAC 27 Listing Table 1 \\
\hline & 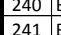 & & 51142-18-8 & $\frac{051142-18-8}{00006-52-7}$ & 1281 & $\mathrm{~s}$ & 50E+02 & & 60 dec & & & & 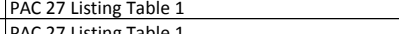 \\
\hline
\end{tabular}




\begin{tabular}{|c|c|c|c|c|c|c|c|c|c|c|c|c|c|}
\hline & No. & chemical Compound & $\begin{array}{c}\text { CAS Number } \\
\text { (CASRN) }\end{array}$ & $\begin{array}{c}\text { Modified } \\
\text { formatc }\end{array}$ & $\begin{array}{l}\text { Molecular } \\
\text { Weight }\end{array}$ & $\begin{array}{c}\mathrm{at} \\
2^{\circ} \mathrm{C} \\
2^{2}\end{array}$ & $\begin{array}{c}\text { Mporfp } \\
\text { [Cc }\end{array}$ & 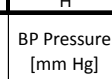 & 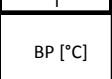 & 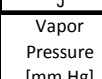 & 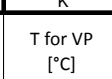 & 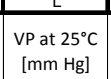 & Vapor Pressure @ $25^{\circ} \mathrm{C}$ Reference \\
\hline$\frac{223}{224}$ & & 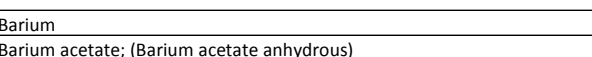 & $7440-393.34$ & $\begin{array}{l}007440-39 \cdot 3 \\
0.0053-30.6\end{array}$ & 137.327 & $\frac{25}{5}$ & $7.270 E+02$ & & $\begin{array}{l}1.8972+03 \\
725 \mathrm{dec}\end{array}$ & 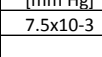 & $6.380+02$ & & 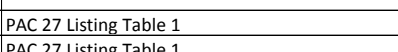 \\
\hline$\frac{2245}{245}$ & & 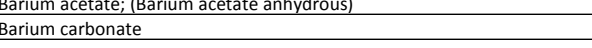 & $\begin{array}{l}534.806 \\
5337.9\end{array}$ & 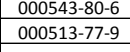 & $\begin{array}{l}255.44 \\
19735 \\
\end{array}$ & $\frac{s}{s}$ & dec & & $725 \mathrm{dec}$ & & & & $\begin{array}{l}\text { PAC2 } 2 \text { Listinin Table } 1 \\
\text { PAC } 27 \text { Liting Table } 1 \\
\end{array}$ \\
\hline & 245 & Barium chloride & 10361-37-2 & 010361-37-2 & 208.233 & s & $9.610 \mathrm{E}+02$ & & 1.560E+03 & $\begin{array}{l}\text { esentially } \\
\text { zeror }\end{array}$ & Notemp & & PAC 27 Listing Table 1 \\
\hline$\frac{247}{248}$ & $\frac{246}{224}$ & $\begin{array}{l}\text { Barium chloride dihydrate } \\
\text { Barium chromate }\end{array}$ & 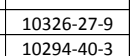 & $\frac{01032627.9}{0.102940 .3}$ & $\frac{2428}{2536}$ & $\frac{s}{s}$ & $9.6205+02$ & & $1.5605+03$ & & & & \begin{tabular}{|l} 
PAC 2 7 Listinin Table 1 \\
PAC 2 Lisin Table 1 \\
\end{tabular} \\
\hline 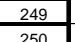 & & $\begin{array}{l}\text { Barium cyanide } \\
\text { Barium dioxide: (Barium peroxide) }\end{array}$ & 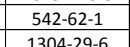 & 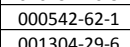 & 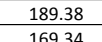 & $\frac{s}{s}$ & 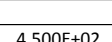 & & sondecer & & & & $\begin{array}{llll}n & 1\end{array}$ \\
\hline & & 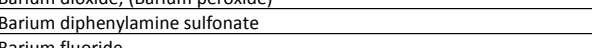 & & & 633.3818 & & & & sovodec & & & & 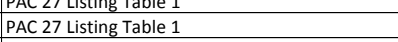 \\
\hline$\frac{252}{253}$ & $\begin{array}{l}\frac{s}{5} \\
x_{2}\end{array}$ & 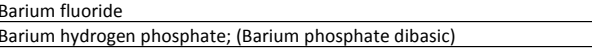 & & 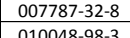 & $\frac{157.324}{12324}$ & $s$ & 1.3.368+03 +03 & & $2.260 \mathrm{E}+03$ & & & & Stiting Table \\
\hline & & 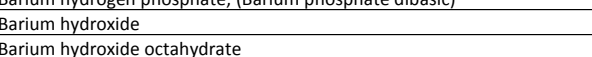 & & 0171994- & & & 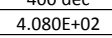 & & & & & & \\
\hline & & $\begin{array}{l}\text { Barium hydroxide octahydrate } \\
\text { Barium metaborate }\end{array}$ & & & & & & & 7.800E 022 & $2.270 \in E+02$ & 7.790E+011 & & Sting Table 1 - \\
\hline & & 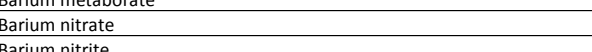 & & 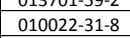 & & & $\frac{5.900+02}{5.902}$ & & & & & & 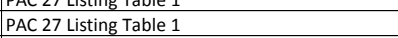 \\
\hline & & $\begin{array}{l}\text { Barium intrite } \\
\text { Barium Oxide }\end{array}$ & & 年13465-99 & & & 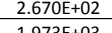 & & & & & & Sting Table \\
\hline$\frac{260}{200}$ & & 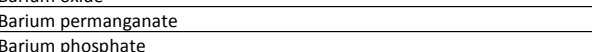 & 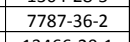 & .007787-36:- & $\frac{375.22}{3.22}$ & 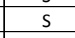 & 200 dec & & & & & & 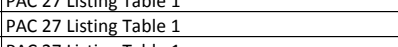 \\
\hline & & 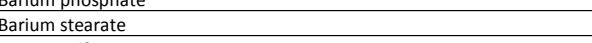 & & $\begin{array}{l}0.1366-620 \\
0.0685-35\end{array}$ & & & $1.000=0202$ & & & & & & \\
\hline 告63 203 & & 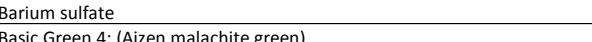 & 7.727-73 & 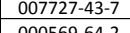 & 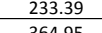 & $s$ & $1.580 \mathrm{E}+03$ & & dec $>1600$ & & & & Sliting Table: \\
\hline & & 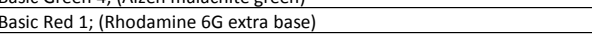 & & & & & & & & & & & \\
\hline $\begin{array}{l}266 \\
267\end{array}$ & & 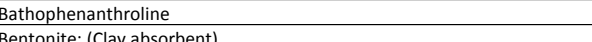 & 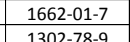 & 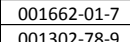 & 175.08 & $s$ & 218.220 & & & & & & 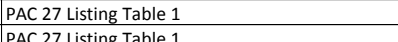 \\
\hline 208 & & 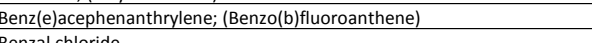 & 205599.9 & $\frac{000205-99}{0.000992}$ & $\begin{array}{l}252.32 \\
1622\end{array}$ & $\mathrm{~s}$ & 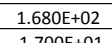 & & & 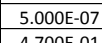 & 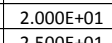 & & $\begin{array}{lll}1 & 1\end{array}$ \\
\hline & & Senzaldethyde & & & & & & 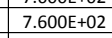 & & & 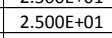 & & 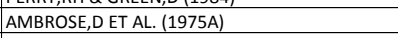 \\
\hline$\frac{271}{272}$ & & Benzamide & 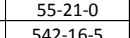 & 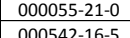 & $\begin{array}{l}121.15 \\
2130\end{array}$ & $\frac{s}{s}$ & $1.3005+02$ & & $2.880 \mathrm{E}+02$ & 1.650:-04 & & & 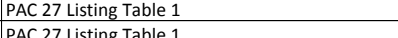 \\
\hline 273 & & 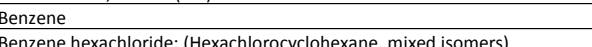 & $\frac{71-4}{60.8}$ & 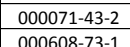 & $\begin{array}{l}78.112 \\
2008 \\
2008\end{array}$ & $\frac{L}{S}$ & 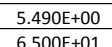 & $7.600+02$ & $8.0095+01$ & $\begin{array}{l}9.480+601 \\
500501\end{array}$ & $\begin{array}{l}2.500+51 \\
6.000+51\end{array}$ & 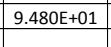 & 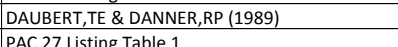 \\
\hline & & 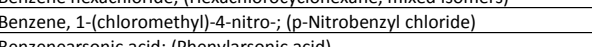 & 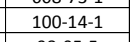 & & $\begin{array}{l}177.59 \\
17.59\end{array}$ & 3 & 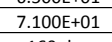 & & $2.300 \mathrm{E}+02$ & 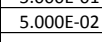 & 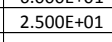 & & 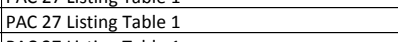 \\
\hline$\frac{260}{227}$ & & 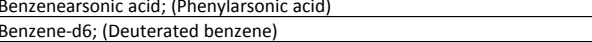 & & 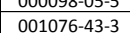 & 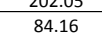 & & $\frac{1.000 \mathrm{cec}}{7.000+00}$ & & 7.900E+01 1 & & & & te \\
\hline 278 & & 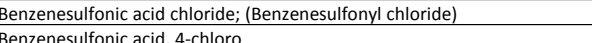 & $\begin{array}{l}98.05 \\
9805\end{array}$ & .000098-09 & $\frac{17}{17}$ & $\mathrm{~L}$ & 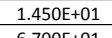 & $7.6005+022$ & $2.5100+022$ & $6.800 E-022$ & 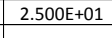 & & Listing Table 1 \\
\hline$\frac{280}{280}$ & & 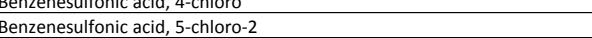 & & & 19262 & 5 & & & & & & & Isting Table 1 . \\
\hline$\frac{281}{281}>2>0$ & & 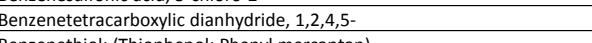 & 89-32-7. & 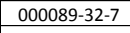 & 228.12 & $\mathrm{~s}$ & 285.3 & & 397-400 & 4.8000:06 & $2.500 E+011$ & & Listing Table 1 . \\
\hline & & 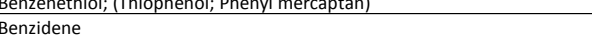 & 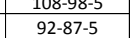 & 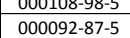 & $\begin{array}{l}10.186 \\
1.4836\end{array}$ & & & 1.:000E+0. & & & & 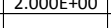 & 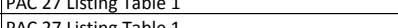 \\
\hline $\begin{array}{lll}2084 \\
2\end{array}$ & & 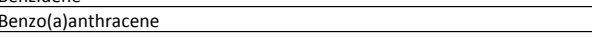 & 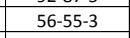 & 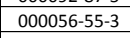 & $\frac{228.29}{2029}$ & $\mathrm{~s}$ & $\frac{1.605+02}{1.052}$ & & & 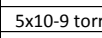 & $2.000 \mathrm{E}+01$. & & 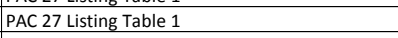 \\
\hline 285 & 284 & Benzo(alapyrene; (Coal tar pitch volatiles) & 50-32-8 & $000050-32-8$ & 252.309 & $\mathrm{~s}$ & $1.811+02$ & & $\begin{array}{l}310.312 @ ~ \\
13 \mathrm{mmHg}\end{array}$ & $5.99 \times 10.9$ & $2.500+501$ & & PAC 27 Listing Table 1 \\
\hline 2866 & 2856 & 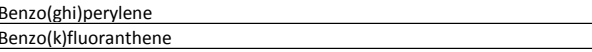 & $\frac{191-24-2}{20-0.9}$ & 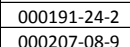 & $\frac{276.34}{253232}$ & 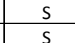 & $\begin{array}{l}2.700+52 \\
21700+0202\end{array}$ & & $\begin{array}{l}5.500+522 \\
4.000++02\end{array}$ & 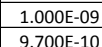 & $\begin{array}{l}2.500+501 \\
25500+5101\end{array}$ & & $\begin{array}{l}\text { PAC 27 Listing Table } 1 \\
\text { PAC C L Listin Table } 1\end{array}$ \\
\hline 288 & 287 & 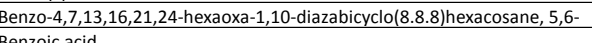 & 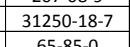 & 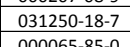 & $\begin{array}{lll}42.54 \\
027.23\end{array}$ & $\frac{s}{5}$ & 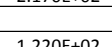 & & & & & & 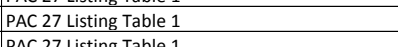 \\
\hline$\frac{2.00}{200}$ & 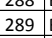 & 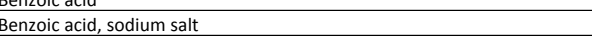 & 532-32-4 & & 告44.11 & $s$ & 1.260utur & & 2,49060202 & $1.000=0$ & $99.600=0+012$ & & \\
\hline$\frac{291}{201}$ & 90 & Benzoin oxime & 441038-3.3. & 000441-38:3: & 227.28 & & & & & & & & Gable 1 \\
\hline$\frac{253}{203}$ & & 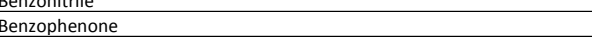 & 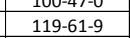 & Door19.6-1.9 & 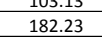 & & 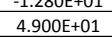 & 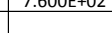 & 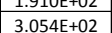 & 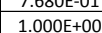 & 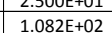 & t. & 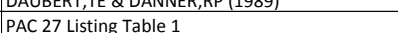 \\
\hline$\frac{294}{205}$ & $293]$ & Benzoquinone, p; (Q) (Quinone) & $\frac{106-51-4}{95-16-9}$ & 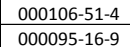 & $\frac{108.1}{135.19}$ & $\frac{s}{4}$ & 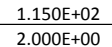 & 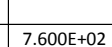 & $\frac{\text { subl. }}{2.3100+02}$ & 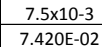 & 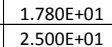 & $7.420=02$ & $\begin{array}{l}\text { PAC } 2 \text { L Listing Table } 1 \\
\text { EFI I Iutete Mean VP of Antoine \& Grain Methods }\end{array}$ \\
\hline 296 & 295 & Benzotrizole & $95-14.7$ & $000095-14-7$ & 19.14 & s & 100 & & $\begin{array}{l}2040 \\
155 m \\
204\end{array}$ & & & & PAC 27 Listing Table 1 \\
\hline$\frac{297}{298}$ & & $\begin{array}{ll}\text { Benzotiffuride } \\
\text { Benzoy l chloride }\end{array}$ & $\begin{array}{l}\frac{23068}{9888.4} \\
9\end{array}$ & 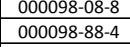 & 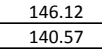 & L & 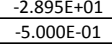 & 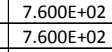 & 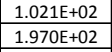 & 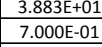 & 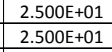 & $\begin{array}{l}\frac{3.838+01}{7.000-01} \\
7\end{array}$ & 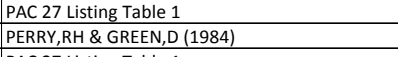 \\
\hline & & 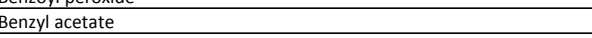 & & 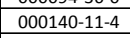 & & & 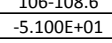 & & 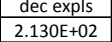 & $1.70=012$ & $2.500=0=112$ & 1./.70=-01] & PAC 27 listing Table 1 \\
\hline & & Benzy alcohol & 100-51-6 & 0.00100:51-6 & 108.138 & $L$ & $-1.500 \mathrm{E}+011$ & 7.600E+022 & $2.053 \mathrm{E}+02$ & $9.400 E-022$ & $2.500 \mathrm{O}+0101$ & 9.400E:02 & DAUUEERT, TE \& D ANNER,RP (1989) \\
\hline $\begin{array}{ll}303 \\
304 \\
04\end{array}$ & & 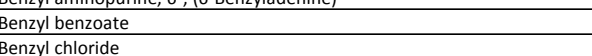 & $\frac{12.51-4}{120.514}$ & 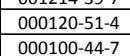 & $\frac{2526}{212.26}$ & & $\frac{2.100 \mathrm{E}+01}{2.501}$ & 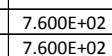 & 3.240E-7. & 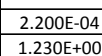 & $00 \mathrm{EE+C}$ & $2.2000-0.0$ & 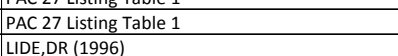 \\
\hline & & & & & & & & & & & & & \\
\hline
\end{tabular}




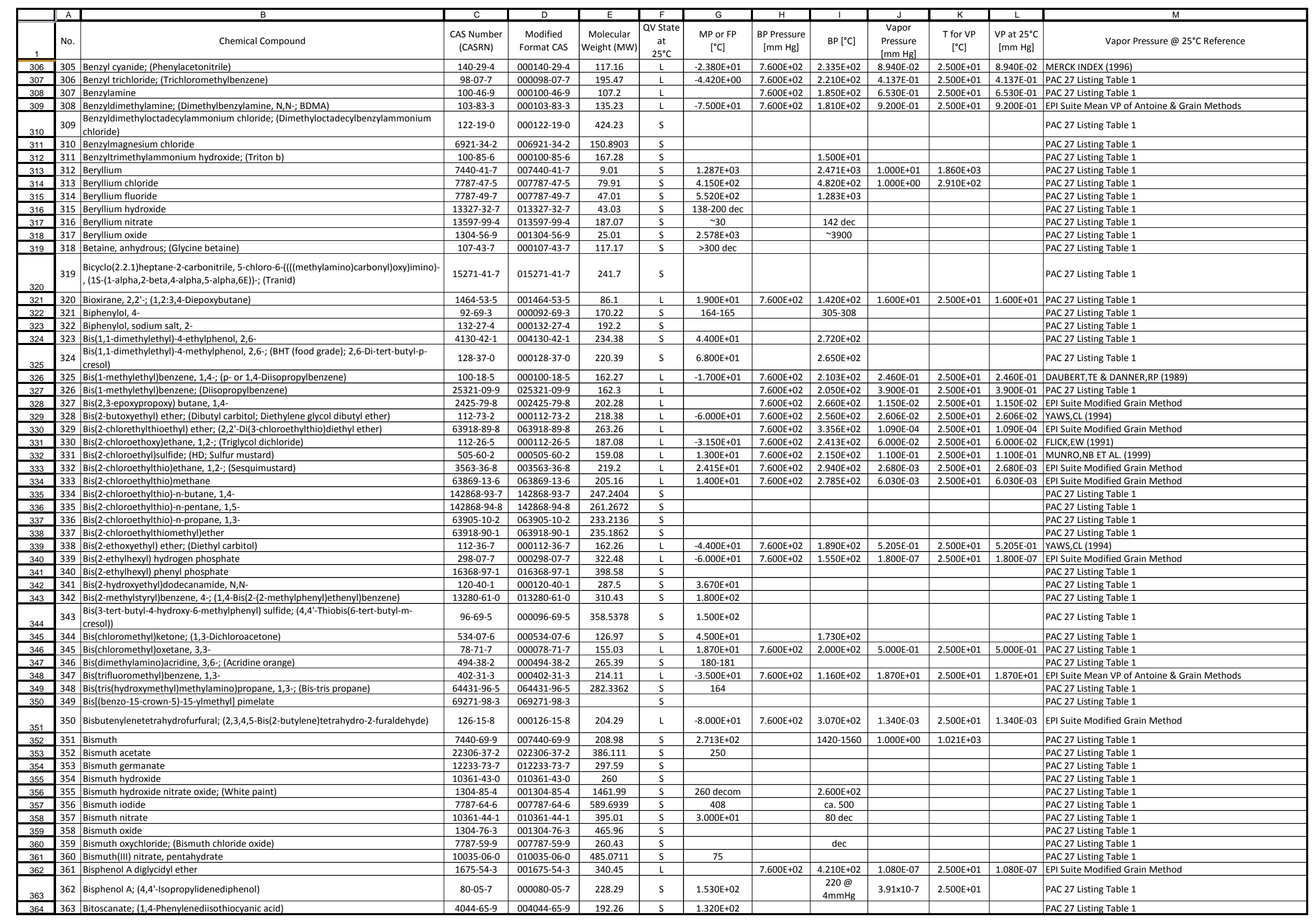




\begin{tabular}{|c|c|c|c|c|c|c|c|c|c|c|c|c|c|}
\hline & & & & & & & & $\overline{-}$ & $-T^{-}$ & & & & \\
\hline & No. & Chemical Compound & $\begin{array}{c}\text { CAS Number } \\
\text { (CASRN) }\end{array}$ & $\begin{array}{l}\text { Modified } \\
\text { Format CAS }\end{array}$ & $\begin{array}{l}\text { Molecular } \\
\text { Weight (MW) }\end{array}$ & $\begin{array}{c}\text { Qv State } \\
\text { at } \\
25^{\circ} \mathrm{C}\end{array}$ & $\begin{array}{c}\text { MP or FP } \\
{\left[{ }^{\circ} \mathrm{C}\right]}\end{array}$ & $\begin{array}{c}\text { BP Pressure } \\
\text { [mm Hg] }\end{array}$ & $\mathrm{BP}\left[{ }^{\circ} \mathrm{C}\right]$ & $\begin{array}{l}\text { Vapor } \\
\text { Pressure } \\
\text { [rmm He }\end{array}$ & $\begin{array}{c}\text { Tfor vP } \\
\text { ["C] }\end{array}$ & $\begin{array}{c}\text { VP at } 25^{\circ} \mathrm{C} \\
{[\mathrm{mm} \mathrm{Hg}]}\end{array}$ & Vapor Pressure @ $2^{\circ} \mathrm{C}$ Reference \\
\hline 365 & 364 & BoBCalix; (Calixx|4]arene-bis(t-octy|benzo-crown-b)) & $\begin{array}{r}z-0110 \\
1220270\end{array}$ & $000002-0110$ & 1149.516 & & $8.000 E+01$ & & & & & & PAC 27 Listing Table 1 \\
\hline 366 & 365 & $\begin{array}{l}\text { Borane methyl sulfide complex; (Methy sulfide complex with borane (1:1)) } \\
\text { Boraneteteranyrofuran }\end{array}$ & $\frac{13292-87-0}{104-65-6}$ & $013292-87-0$ & $\begin{array}{l}75.98 \\
85.93\end{array}$ & L & $-3.700 E+01$ & $\begin{array}{ll}7.600 E+02 \\
7.60 \mathrm{EF}+02 \\
\end{array}$ & 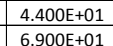 & \begin{tabular}{|l|l|l|l|l}
$5.480 \mathrm{E}+01$ \\
$1.520 \mathrm{E}+02$
\end{tabular} & \begin{tabular}{|lll}
$4.0000+01$ \\
$2.2005+01$
\end{tabular} & $\begin{aligned} 1.525 \mathrm{E}-03 \\
1.50 \mathrm{~T}+02 \\
\end{aligned}$ & 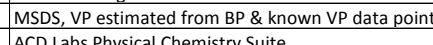 \\
\hline & 367 & 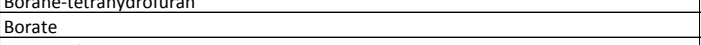 & $14213-97-9$ & $\begin{array}{lll}014444-05-6 \\
014213-9\end{array}$ & $\begin{array}{llll}85.93 \\
58.81\end{array}$ & $\frac{\mathrm{L}}{\mathrm{s}}$ & & & & & & & 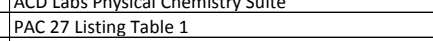 \\
\hline 369 & 368 & Boric acid & $10043-35-3$ & $010043-35-3$ & 61.84 & $\mathrm{~s}$ & $185 \mathrm{dec}$ & & & & & & PAC 27 Listing Table 1 \\
\hline 370 & 369 & Boric acid, tributyly ester; (Tri-in-buty borate) & $688-74-4$ & $000688-74-4$ & 230.2 & L & $\frac{-7.000 E+01}{7.005 .03}$ & $7.600 E+02$ & $2.300 \mathrm{E}+02$ & $5.262 \mathrm{E}-01$ & $2.500 E+011$ & $5.262 \mathrm{E}-01$ & YaWS, CL (1994B) \\
\hline$\frac{371}{372}$ & & & $7440-42-88$ & $007440-42-8$ & $\frac{10.81}{5526}$ & $\frac{s}{5}$ & $\frac{2.190 \mathrm{E}+03}{2.35 \mathrm{E}+03}$ & & $\begin{array}{l}3.660 \mathrm{E}+03 \\
>3500\end{array}$ & & & & $\begin{array}{l}\text { PAC } 2 \text { Lisiting Table } 1 \\
\text { PAC L listing Table } 1\end{array}$ \\
\hline$\frac{3 / 2}{373}$ & 372 & 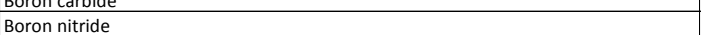 & $10043-11-5$ & 010043-31-8 & $\begin{array}{l}5.26 \\
24.82\end{array}$ & $\frac{2}{3}$ & 2.350E $2.96 \mathrm{E}+03$ & & & & & & $\begin{array}{l}\text { PAC27 Lising tabble } 1 \\
\text { PAC } 27 \text { Listing Table } 1\end{array}$ \\
\hline$\frac{375}{374}$ & 373 & $\begin{array}{l}\text { Beron oxide } \\
\text { Boring }\end{array}$ & $1303-86-2$ & $001303-86-2$ & $\begin{array}{l}2.402 \\
69.62 \\
\end{array}$ & $\frac{5}{5}$ & $\begin{array}{lll}2.3000+03 \\
4.500+02\end{array}$ & & $2.250 E+03$ & & & & PAC 27 listing Table 1 \\
\hline 375 & 374 E & Boron tribromide & 10294-33-4 & $010294-33-4$ & 250.54 & L & $-4.600 E+01$ & $7.600 E+02$ & $9.130 \mathrm{E}+01$ & $6.480 \mathrm{E}+01$ & $2.500 E+01$ & $6.480 \mathrm{E}+01$ & EPP S Suite Mean VP of Antoine \& Grain Methods \\
\hline$\frac{376}{377}$ & 375 & \begin{tabular}{|l} 
Boron trichloride \\
Boron trifluoride.
\end{tabular} & $\frac{10294-34-5}{7677-07-2}$ & $010294-34-5$ & $\frac{117.16}{6781}$ & $\frac{1}{G}$ & $\begin{array}{l}-1.0705+02 \\
-1.2845+02\end{array}$ & $7.600 E+02$ & \begin{tabular}{|l|l|}
$1.250 \mathrm{E}+01$ \\
$-1.000++22$
\end{tabular} & $\begin{array}{l}1.000 \mathrm{E}+00 \\
3.65 \mathrm{~F}+4 . \\
\end{array}$ & 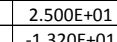 & $1.000 E+00$ & 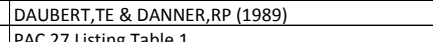 \\
\hline & $\mid \begin{array}{lll}336 \\
377\end{array}$ & $\begin{array}{l}\text { Borono trifluoride } \\
\text { Boron trifluoride dihydrate }\end{array}$ & $7637-07-2$ & $\begin{array}{l}0076377-07-2 \\
013319-5-0 \\
\end{array}$ & 67.81 & $\frac{G}{L}$ & $\frac{-1.2844+02}{6.200 E+00}$ & $1.000 E++00$ & 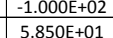 & $\begin{array}{l}3.656 \mathrm{E}+04 \\
5.200 \mathrm{E}+00 \\
\end{array}$ & $\begin{array}{l}-1.320 E+01 \\
2.000 E+01\end{array}$ & $4098++00$ & \begin{tabular}{|l} 
PAC 27 Listing Table 1 \\
Istitanted from "Chemical Book" Data
\end{tabular} \\
\hline & 378 & 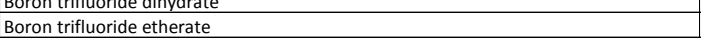 & $\frac{133199-53-0}{109-7}$ & $\begin{array}{l}0.00109-63-7 \\
00019\end{array}$ & 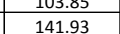 & $\frac{L}{L}$ & $\begin{array}{lll}0.200 E+T O U \\
-6.040 E+01\end{array}$ & 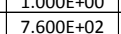 & $\begin{array}{lll}5.850 E+U 1 \\
1.260 E+02\end{array}$ & $\begin{array}{llll}5.200 E+U 0 \\
1.480 E+01\end{array}$ & $\begin{array}{lll}2.000 E+T H \\
2.500 E+01\end{array}$ & $\begin{array}{lll}4.0 .085 E+U 0 \\
1.480 E+01\end{array}$ & $\begin{array}{l}\text { Estimated from "Chemicial oook" Data } \\
\text { EPI Suite Mean VP of Antoine \& Grain Methods }\end{array}$ \\
\hline 380 & 379 & Boron trifluoride-dimethyl ether & $355-2-42-4$ & $000353-2-42-1$ & $\begin{array}{l}111.93 \\
113.89 \\
\end{array}$ & $\frac{L}{L}$ & 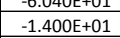 & $\begin{array}{l}7.6000 \mathrm{E}+02 \\
.02\end{array}$ & \begin{tabular}{|l|l}
$1.2000 \mathrm{~L}+26$ \\
$1.260 \mathrm{E}+02$ \\
\end{tabular} & $\begin{array}{l}1.400 \mathrm{E}+11 \\
\end{array}$ & $\begin{array}{l}2.30000+01 \\
2.500+01\end{array}$ & 2..400E+ 201 & PAC 27 Listing Table 1 . \\
\hline 381 & 380 & Botulinum toxin-A & 93384-43-1 & $093384-43-1$ & $150 \mathrm{kDa}$ & $\mathrm{s}$ & & & & & & & PAC 27 Listing Table 1 \\
\hline$\frac{382}{383}$ & $\mid \frac{381}{382}$ & \begin{tabular}{|l|} 
Botulinum toxin-B \\
Botulinum toxin-F
\end{tabular} & $\frac{9338444-2}{107231-25-2}$ & $\frac{093384-44-2}{107231-52}$ & & $\frac{s}{5}$ & & & & & & & $\begin{array}{l}\text { PAC } 27 \text { Listing Table } 1 \\
\text { PAC C Listin Table } 1\end{array}$ \\
\hline$\frac{384}{384}$ & 383 & Botulinum, clostridium & z-0119 & $000002-0119$ & & $\frac{5}{5}$ & & & & & & & PAC 27 Listing Table 1 1 \\
\hline 385 & 384 & 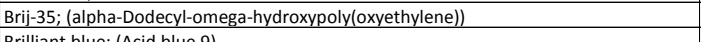 & $9002-92-0$ & $\begin{array}{ll}009002-92-0 \\
0250182\end{array}$ & 230.225 & $\frac{L}{S}$ & $4.000 \mathrm{E}+01$ & $7.600 E+02$ & $3.182 \mathrm{E}+02$ & $1.770 \mathrm{E}-05$ & $2.500 E+01$ & $1.7700-05$ & EPI Suite Modified Grain Method \\
\hline$\frac{386}{387}$ & $\left|\begin{array}{l}385 \\
386\end{array}\right|$ & $\begin{array}{l}\text { Brirliant blue; (Acid blue 9) } \\
\text { Bromadiolone }\end{array}$ & $\frac{2650-18-2}{28772-56-7}$ & $\frac{002650-18-2}{028772-56-7}$ & $\frac{783.01}{527.11}$ & $\frac{s}{s}$ & $200-210$ & & & & & & 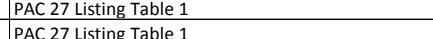 \\
\hline & & & $\frac{287 / 72-56-1}{7726-95-6}$ & $\begin{array}{ll}0 \\
0.028 / 726-56-1\end{array}$ & $\frac{527.11}{159.81}$ & & 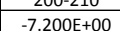 & $7.600 E+02$ & $5.880 \mathrm{E}+01$ & 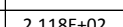 & $25005+01$ & $2.118 \varepsilon+02$ & 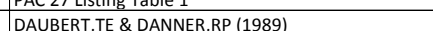 \\
\hline$\frac{3.06}{389}$ & 年 & Bromine chloride & 13863-41-7 & 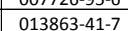 & 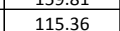 & $\frac{5}{s}$ & & 1.00utur & 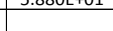 & 2.110tout & 2.00uttor & 2.110trot & 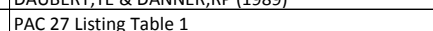 \\
\hline$\frac{500}{3900}$ & 389 & Bromine pentaffluoride & $7789-30-2$ & $007789-30-2$ & 174.91 & $\mathrm{~L}$ & $-6.050++01$ & $\begin{array}{l}7.6000 \mathrm{E}+02 \\
\end{array}$ & $4.0506+01$ & $3.280 \mathrm{E}+02$ & $2.000 E+01$ & $4.0699+02$ & $\begin{array}{l}\text { Esstimated using data from PAC } 27 \text { listing Table } 1 \\
\end{array}$ \\
\hline 391 & 390 & Bromine trifluoride & $7787-71-5$ & $007787-71-5$ & 136.91 & $\mathrm{~L}$ & $8.800 E+00$ & $7.600 E+02$ & $1.270 \mathrm{E}+02$ & $1.800 \mathrm{E}+01$ & $3.900 E+01$ & $8.095 \mathrm{E}+00$ & Estimated using data from PAC 27 Listing Table 1 \\
\hline 392 & 391 & 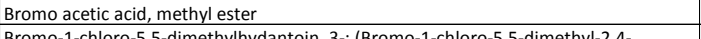 & $96-32-2$ & $000096-32-2$ & 152.99 & & & $7.600 E+02$ & $1.450 \mathrm{E}+02$ & $9.140 \mathrm{E}+00$ & $2.500 E+01$ & $9.140 E+00$ & EPI Suite Mean VP of Antoine \& Grain Methods \\
\hline 393 & 392 & 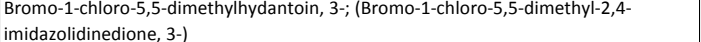 & $126-06-7$ & 000126-06-7 & 241.47 & $\mathrm{~s}$ & $1.620 \mathrm{E}+02$ & & & $6.6 \times 10-6$ & $2.500 E+01$ & & PAC 27 Listing Table 1 \\
\hline 200 & 393 & Bromo-2-chloro-1,1,2-triffuoroethane, 1;; (1,1,2-Trifluoro-1-bromo-2-chloreethane) & $354-06-3$ & 000354-06-3 & 197.39 & $\mathrm{G}$ & $-1.680 E+02$ & & $-5.7800+01$ & $>760$ & $2.000 E+01$ & & PAC 27 Listing Table 1 \\
\hline & 394 & Bromo-3-chloro-5,5-dimethylhydantoin, 1; (1-Brom-3-chloro-5,5-dimethy-2,4- & $16079-88-2$ & 016079-88-2 & 241.49 & $\mathrm{~s}$ & 163-164 & & & $6.61 \times 10-6$ & $2.5000+01$ & & PAC 27 Listing Table 1 \\
\hline$\frac{395}{396}$ & 395 & 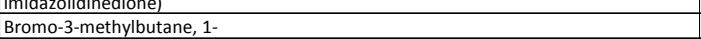 & $\begin{array}{l}107-82-4 \\
\end{array}$ & $000107-82-4$ & 151.05 & L & $-1.120 \mathrm{E}+02$ & $7.6000+02$ & $1.200 E+02$ & $3.462 \mathrm{E}+01$ & $2.500 \mathrm{E}+01$ & $3.4622+01$ & HINE, \& MOOKERIEE,PJ (1975) \\
\hline 397 & 396 & Bromoacetaldehyde diethyl acetal & $2032-35-1$ & $002032-35-1$ & 197.07 & $\mathrm{~s}$ & & & $170-172$ & & & & PAC 27 Listing Table 1 \\
\hline$\frac{398}{399}$ & 397 & Bromoacetic acid & $\frac{79-08-3}{598-3-2}$ & $\frac{000079-08-3}{000098-31-2}$ & $\frac{138.96}{136.98}$ & & 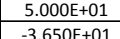 & 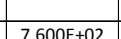 & $2.080 \mathrm{E}+02$ & & & $90005+01$ & PAC 27 Listing Table 1 \\
\hline & 3998 & $\begin{array}{l}\text { Bromoocetone } \\
\text { Bromaanisole 3; (1-Bromo-3-methoxybenzene) }\end{array}$ & 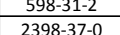 & $000598-3-31-2$ & $\frac{136.98}{187.03}$ & $s$ & $-3.050 \mathrm{E}+01 \mathrm{I}$ & $7.600 E+022$ & $\frac{1.380 E+02}{2110+0202}$ & $9.000 E+011$ & $2.500 \mathrm{E}+01$ & & 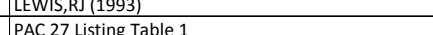 \\
\hline$\frac{140}{401}$ & 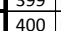 & 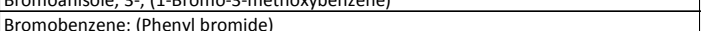 & 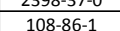 & 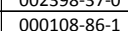 & $\frac{10.35}{157.01}$ & $\frac{3}{4}$ & $-3.072 E+01$ & $7.600 \mathrm{C}+02$ & 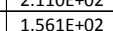 & $4.182 E+00$ & $2500 \mathrm{~F}+01$ & 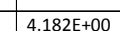 & 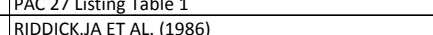 \\
\hline 402 & 401 & Bromochlorobenzenen, $m$ - & $108-37-2$ & $000108-37-2$ & 191.45 & L & $-2.150 \mathrm{E}+01$ & $7.6006+02$ & $1.960 \mathrm{E}+02$ & $4.400 \mathrm{E}-01$ & $2.5000 \mathrm{E}+01$ & $4.4000-01$ & EPP Suite Mean VP of Antoine \& Grain Methods \\
\hline & 402 & Bromochlorobenzene, $\mathrm{p}$ - & $\begin{array}{ccc}106-39-8 \\
703-8\end{array}$ & 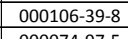 & 191.45 & $\frac{5}{5}$ & & & & & & & PAC 27 Listing Table 1 \\
\hline 404 & 403 & Bromochloromethane & & $000074-97-5$ & 129.39 & & & $7.600 E+02$ & $6.780 \mathrm{E}+01$ & $1.425 \mathrm{E}+02$ & $2.5000 E+01$ & 1.425E+02 & DAUBERT, TE \& DANNER,RP (1989) \\
\hline 405 & 404 & 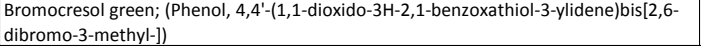 & $76-60-8$ & $000076-60-8$ & 698.05 & $\mathrm{~s}$ & $2.185 \mathrm{E}+02$ & & & & & & PAC 27 Listing Table 1 \\
\hline 406 & 405 & 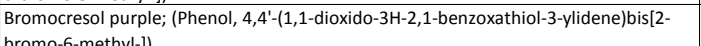 & $115-40-2$ & $000115-40-2$ & 540.222 & $\mathrm{~s}$ & $2.415 E+02$ & & & $1.15 \times 10 \mathrm{E}-15$ & $2.500 E+01$ & & PAC 27 Listing Table 1 \\
\hline$\frac{407}{407}$ & 406 & Bromocyclohexanol, cis-2- & $16536-57-5$ & $016536-57-5$ & 179.0563 & $\mathrm{~L}$ & $1.182 \mathrm{E}+01$ & $7.600 E+02$ & $2.215 E+02$ & $1.980 \mathrm{E}-02$ & $2.5000+01$ & $1.9800-02$ & EPP Suite Mean VP of Antoine \& Grain Methods \\
\hline & 407 & Bromodecane, 1- & $112-29 \cdot 8$ & $000112-29-8$ & 221.22 & L & $-2.920 \mathrm{E}+01$ & $7.600 \mathrm{E}+02$ & 2.406E+02 & 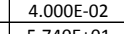 & & 4.000E-02 & RIDDICK,JA ET AL. (1986) \\
\hline$\frac{c^{430}}{440}$ & 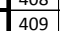 & Bromododecane 1 . & 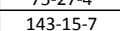 & (0001433-15.7 & $\begin{array}{l}20.03 \\
242.23\end{array}$ & $x_{1}$ & 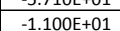 & 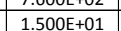 & $\begin{array}{ll} & \\
0\end{array}$ & 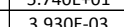 & 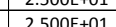 & 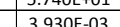 & 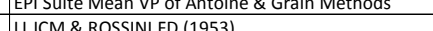 \\
\hline & 410 & Bromoethane: (Ethyl bromide) & & & & & & & & & $2.500 E+01$ & & \\
\hline$\frac{412}{412}$ & 411 & Bromoethanol, 2; (Ethylene bromohydrin) & $540-51-2$ & $000540-51-2$ & 124.98 & $\frac{L}{L}$ & $-8.000 E+01$ & $7.600 E+02$ & $1.5006 \mathrm{E}+02$ & $1.350 \mathrm{E}+00$ & $2.5006+01$ & 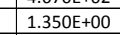 & EPI Suite Mean VP of Antoine \& Grain Methods \\
\hline 413 & 412 & Bromoethylmagnesium & $925-90-6$ & $000925-90-6$ & 133.27 & $\mathrm{~s}$ & & & & & & & PAC 27 listing Table 1 \\
\hline$\frac{414}{415}$ & $\frac{413}{414}$ & $\begin{array}{l}\text { Bromoffluorobenzene, 4; 4; (p-Fluorobromobenzene) } \\
\text { Bromoform; T(Tibromomethane) }\end{array}$ & $\frac{460-00-4}{77-25-2}$ & $\frac{000460-00-4}{000075-25-2}$ & 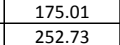 & $\frac{L}{L}$ & $\begin{array}{l}-1.790 E+01 \\
8.690 E+00\end{array}$ & $\begin{array}{l}7.600 E+02 \\
7.600 E+02\end{array}$ & $\begin{array}{l}1.515 \mathrm{E}+02 \\
1.491 \mathrm{E}+02\end{array}$ & $\frac{3.74}{5.40}$ & $2.500 \mathrm{E}+01$ & $\begin{array}{l}3.740 E+00 \\
5.400+000\end{array}$ & Antoine \& Gra \\
\hline$\frac{445}{416}$ & $\frac{414}{415}$ & $\begin{array}{l}\text { Bromotorm } \\
\text { Bromohexa }\end{array}$ & $\frac{1525-2}{111-25-1}$ & $000111-25-1$ & $\frac{22.1 .13}{165.1}$ & $\mathrm{~L}$ & 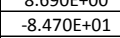 & $\begin{array}{llll}7.006 E+02 \\
7.600+02\end{array}$ & 1.453 & 3.84 & 2.500EE+01 & & \\
\hline 417 & 416 & 0-1,3,5-trimethylbenzene) & $576-83-0$ & & 199.087 & $\mathrm{~L}$ & $-1.000 E+00$ & $7.600 E+02$ & $2.250 E+02$ & $1.010 \mathrm{E}-01$ & $2.5000 \mathrm{E}+01$ & $1.010 E-0.0$ & \\
\hline 418 & 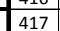 & (1) & 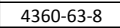 & $004360-63-8$ & & $\mathrm{~L}$ & $-1.517 \mathrm{E}+01$ & & $8.000 \mathrm{E}+01$ & $2.3400+00$ & $2.500 E+01$ & $2.340 E+0+0$ & \\
\hline 419 & 418 & Bromonaphthalene & $90-11-9$ & $000090-11-9$ & 207.08 & & & & & & & & PAC 27 Listing Table 1 \\
\hline 420 & 419 & Bromonitromethane & $563-70-2$ & $000563-70-2$ & 139.95 & $L$ & 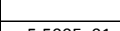 & $7.6000 E+02$ & $1.470 E+02$ & $3.100 E+00$ & $2.500 E+01$ & 3.100E+ +00 & various reduced boil pts \\
\hline & & 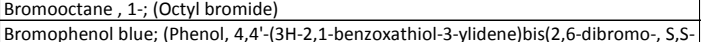 & $111-83-1$ & $000111-83-1$ & 193.16 & 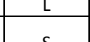 & $-5.500 \mathrm{E}+01$ & & & & & & LI, CM \& ROSSIN, FD (1953) \\
\hline 422 & | & 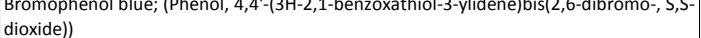 & 115-39-9 & $000115-399-9$ & 669.99 & $\mathrm{~s}$ & $279 \mathrm{dec}$ & & & & & & PAC 27 Listing Table 1 \\
\hline & 422 & \begin{tabular}{|l|} 
Bromophenyl phenyl ether, 4- \\
Bromononane 1.
\end{tabular} & 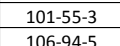 & 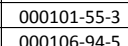 & 249.11 & 2 & $\frac{1.872 E+01}{-1.100+502}$ & $\begin{array}{ll}7.600 E+02 \\
7.60 \mathrm{~F}+02 \\
\end{array}$ & $\begin{array}{ll}3.1011+02 \\
7700++12 \\
\end{array}$ & \begin{tabular}{c|c|c|}
$1.500 E-03$ \\
$1.108+502$ \\
\end{tabular} & 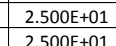 & \begin{tabular}{c|c|c|}
$1.500 E-03$ \\
$108+502$ \\
\end{tabular} & CALLAHAN,MA ET AL. (1979) \\
\hline
\end{tabular}




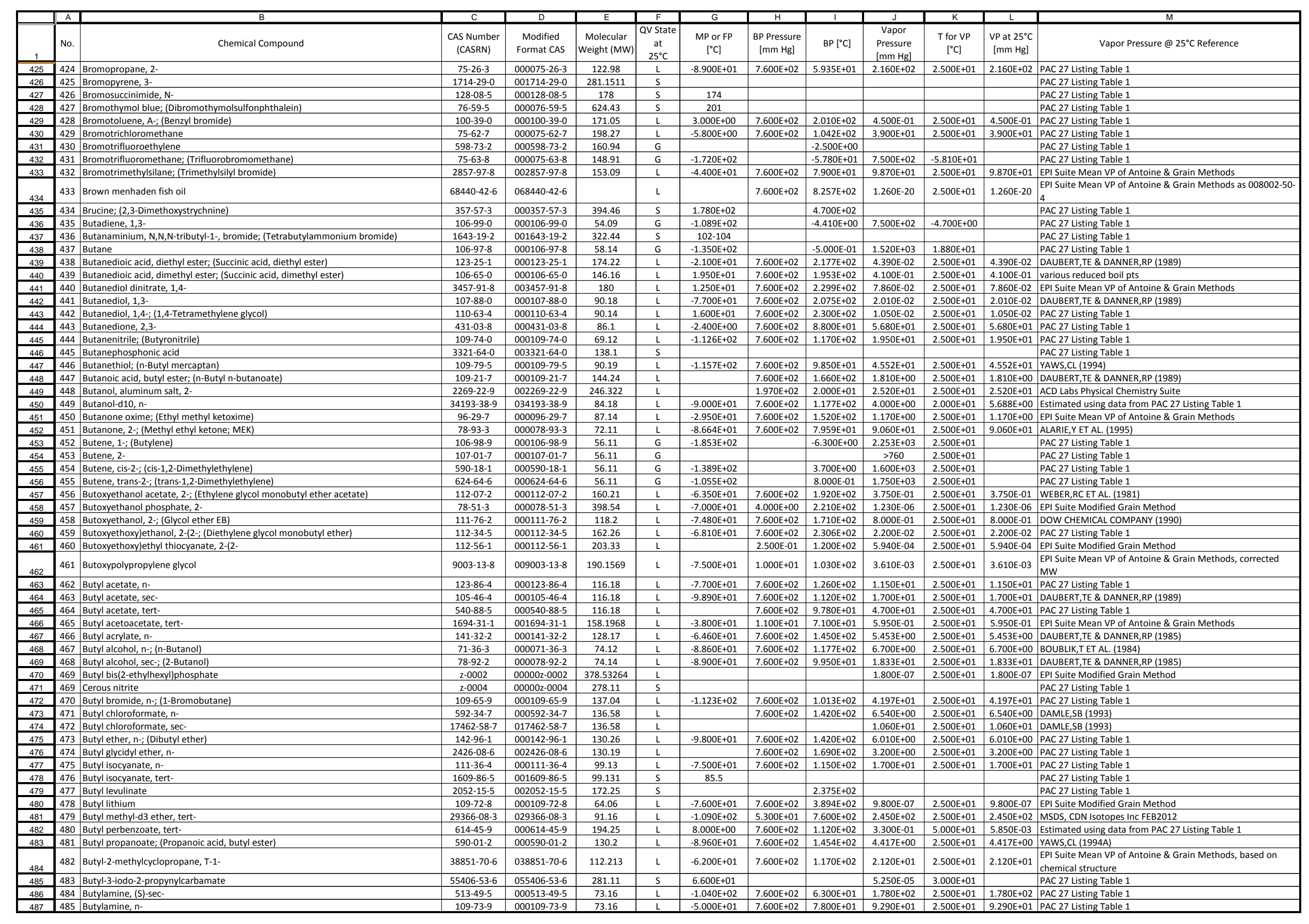




\begin{tabular}{|c|c|c|c|c|c|c|c|c|c|c|c|c|c|}
\hline & No. & Chemical Compound & $\begin{array}{l}\text { cas N Number } \\
\text { (CASPN) }\end{array}$ & $\begin{array}{l}\text { Modified } \\
\text { Format CAS }\end{array}$ & $\begin{array}{l}\text { Molecular } \\
\text { Weight MW }\end{array}$ & 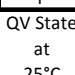 & 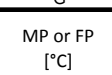 & $\begin{array}{l}\text { BP Pressure } \\
\text { [mm M g }\end{array}$ & вр [ст] & 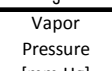 & $\begin{array}{c}\text { Torvp } \\
\text { [cicl }\end{array}$ & 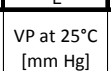 & Vapor Pressure $@ 25^{\circ} \mathrm{C}$ Reference \\
\hline & 1866 & Butrlamine, sec. & $13952-84.6$ & $013952-8446$ & 73.16 & & $-1.0406+022$ & $7.600 €+02$ & $6.3006=0.12$ & 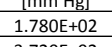 & $2.500=0+012$ & $1.780 €+02$ & 7 ustising Table 1 . \\
\hline $\begin{array}{ll}\frac{289}{4900} \\
490\end{array}$ & & 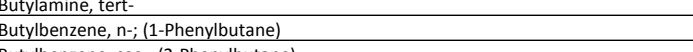 & 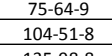 & 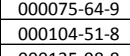 & $\begin{array}{l}\frac{7.16}{134.21} \\
\end{array}$ & $\frac{L}{L}$ & 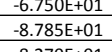 & 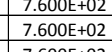 & 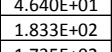 & 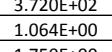 & 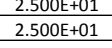 & 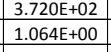 & 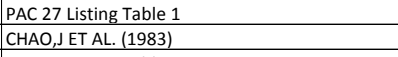 \\
\hline & & & & & & & 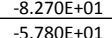 & & & & & & \\
\hline 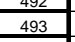 & 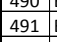 & 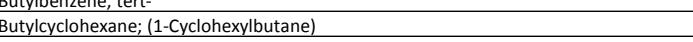 & 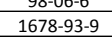 & 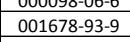 & $\frac{192.268}{1.0268}$ & $\frac{L}{L}$ & & $\begin{array}{l}7.600+0202 \\
7.600++02\end{array}$ & 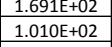 & 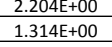 & 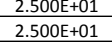 & 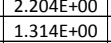 & 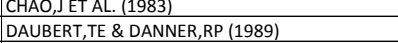 \\
\hline e & 492 ! & Butylyclohexanone, p.tert- & 98.53-3 & 000099:53.3 & 154.28 & $\mathrm{~s}$ & 49.50 & & 90:92@ & & & & PAC 27 listing Table 1 \\
\hline & & 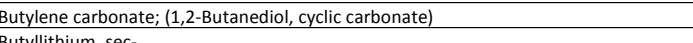 & $\frac{4437.85 .8}{60930.7}$ & 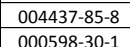 & $\frac{116.13}{6406}$ & $\frac{s}{4}$ & & & & & & 17200.06 & 2 \\
\hline$\frac{2.467}{497}$ & & 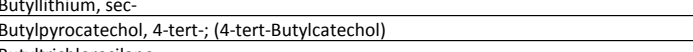 & 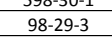 & 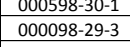 & 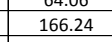 & $\frac{s}{s}$ & 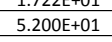 & t.000602 & 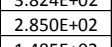 & 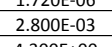 & & & 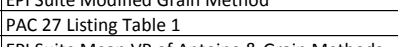 \\
\hline & & 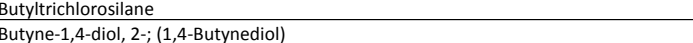 & 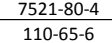 & 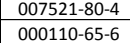 & $\frac{189.95}{86.09}$ & $\frac{1}{5}$ & $\begin{array}{l}-3.398 \mathrm{E}+11 \\
5.000 \mathrm{0}+01\end{array}$ & $7.600 E+02$ & $\frac{1.485 E+22}{2.380+0202}$ & 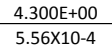 & 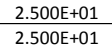 & 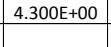 & 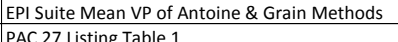 \\
\hline$\frac{500}{501}$ & & Sultraldenhyde & $\frac{123.72 .8}{1.52 .8}$ & 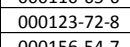 & $\frac{5.02}{72.12}$ & $i$ & 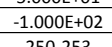 & $7.600 €+02$ & 7.47705+01 & $1.1144+02$ & $2.5006+01$ & $1.1144+02$ & 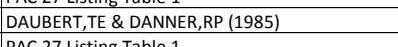 \\
\hline $\begin{array}{ll}502 \\
502\end{array}$ & 500 & 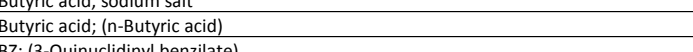 & 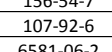 & 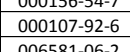 & $\frac{11.11}{38.12}$ & $\frac{1}{L}$ & 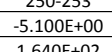 & $7.6005+022$ & $1.635 \mathrm{E}+022$ & $\begin{array}{l}1.6500+00 \\
20000\end{array}$ & $\begin{array}{l}2.500+501 \\
.2500 .51\end{array}$ & $1.650+500$ & 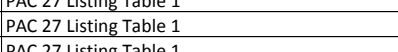 \\
\hline${ }^{503}$ & $\frac{502}{502}$ & 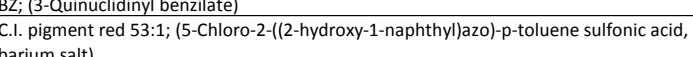 & 658506-2.2 & 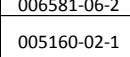 & 344.45 & $\frac{s}{s}$ & $\frac{1.6606+02}{343-34}$ & & & & & & 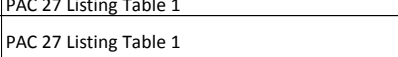 \\
\hline 505 & 503 & 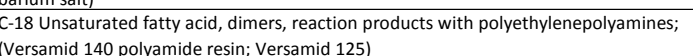 & 68410-23-1 & $068410-23-1$ & 777.28 & $\mathrm{~L}$ & & $7.600+02$ & $1.0655+03$ & 2.410E:27. & $2.500=01$ & $2.4106 \cdot 27$. & EPI Suite Modified Grain Method \\
\hline$\frac{506}{507}$ & & 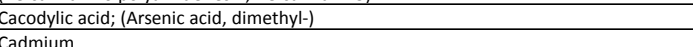 & $\frac{75.6 .5}{7440.03 .9}$ & 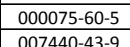 & $\frac{138}{11221}$ & $\frac{s}{s}$ & $\frac{1.950 E+52}{3.2010+52}$ & & $\frac{2200}{76005020}$ & $20005+00$ & 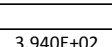 & & 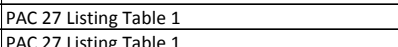 \\
\hline$\frac{508}{508}$ & & cambium bromide & 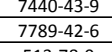 & 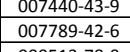 & $\frac{124.41}{272.22}$ & $\frac{s}{5}$ & 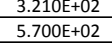 & & $\begin{array}{l}7.6600+02 \\
8.630+02\end{array}$ & & & & 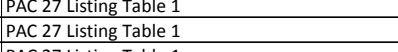 \\
\hline$\frac{509}{510}$ & $\begin{array}{l}07 \\
08\end{array}$ & 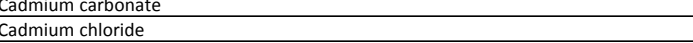 & $\begin{array}{l}\frac{51378-0}{10108.62} \\
\end{array}$ & 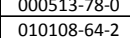 & 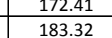 & $\frac{s^{5}}{s}$ & $5.6805+022$ & & 9640502020 & 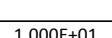 & 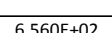 & & 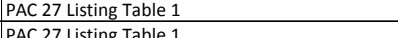 \\
\hline${ }_{511}$ & 500| & 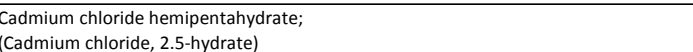 & $7790-78.5$ & $007790-78.5$ & 455.708 & $\mathrm{~s}$ & 568 & & & & & & PAC 27 Listing Table 1 \\
\hline$\frac{512}{513}$ & & $\begin{array}{l}\text { Cadmium fluoride } \\
\text { Cadmium hydroxide }\end{array}$ & $\begin{array}{l}7790-99.6 \\
201019-9.2\end{array}$ & $\begin{array}{l}007790-79 \cdot 6 \\
0.2104-195.2\end{array}$ & $\frac{150.4}{146.63}$ & $\frac{s}{s}$ & $1.075+03$ & & 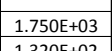 & $1.000++00$ & $1.112+03$ & & PAC 27 Listing Table 1 \\
\hline$\frac{5134}{514}$ & $\frac{11}{12}$ & 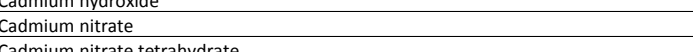 & 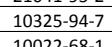 & $\frac{010325.94-7}{0.1002-6.2}$ & $\begin{array}{lll}236.42 \\
30.48\end{array}$ & $\frac{s}{s}$ & 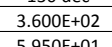 & & $1.320+502$ & & & & 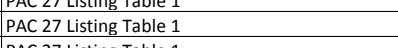 \\
\hline & & 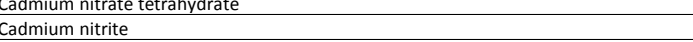 & 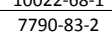 & 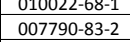 & & & & & & & & & \\
\hline 517 & & camalum oxide & 1306-19.- $\mathrm{C}$ & 001306-19-4 & 128.41 & $s$ & & & 1559 subl & $7.5 \times 10-3$ & 7.7.00E+02 & & \\
\hline$\frac{218}{519}$ & 157 & 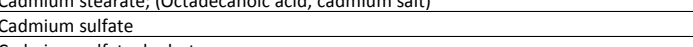 & 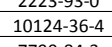 & 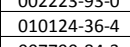 & 208.46 & $\frac{5}{5}$ & 1.000503 & & & & & & 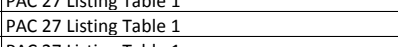 \\
\hline$\frac{201}{521}$ & & $\begin{array}{l}\text { Cadmium suffate, } \\
\text { Cadmium surate } \\
\text { sufide }\end{array}$ & $\begin{array}{ll}77900-8 \cdot 3-3 \\
1306-23-6\end{array}$ & 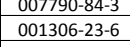 & 1264.49 & & $\frac{105}{1750}$ & & & & & & 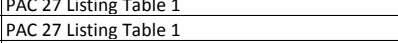 \\
\hline$\frac{522}{523}$ & & 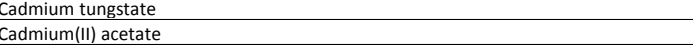 & 7970-58-4.-8 & 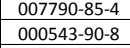 & $\begin{array}{l}36.26 \\
230.5 \\
23\end{array}$ & $\begin{array}{c}\mathrm{s} \\
\mathrm{s} \\
\mathrm{c}\end{array}$ & $2.505+02$ & & & & & & 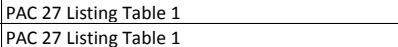 \\
\hline$\frac{524}{555}$ & & Caclium & 74400-70.2. & 000740-70-2.2 & $\begin{array}{l}40.08 \\
10.08 \\
\end{array}$ & $s$ & $8.4206+02$ & & 1.4845 +03 & $7.5 \times 10.3$ & $5.9010+0.022$ & & 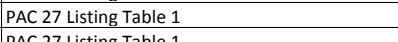 \\
\hline 526 & & $\begin{array}{c}\text { Calcium arsenate } \\
\text { calce }\end{array}$ & $7778-44-1$ & 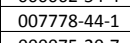 & 398.08 & $\mathrm{~s}$ & 1.4555+000 & & & & & & 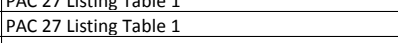 \\
\hline$\frac{257}{528}$ & & Calcium chloride & 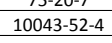 & 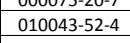 & $\frac{54.1}{110.98}$ & $\frac{5}{5}$ & $\begin{array}{l}2.3000+03 \\
7.750+0202\end{array}$ & & $1.935 E+03$ & & & & 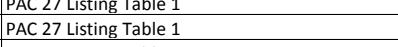 \\
\hline & & 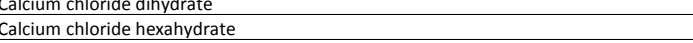 & & 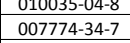 & & $\frac{5}{5}$ & $\frac{11 \text { soce }}{300}$ & & & & & & 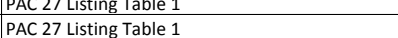 \\
\hline$\frac{51}{552}$ & & $\begin{array}{l}\text { calcium chloride hydrate } \\
\text { lctum }\end{array}$ & 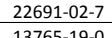 & 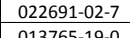 & 129 & $s$ & & & & & & & sting Table 1 1 \\
\hline$\frac{532}{533}$ & & 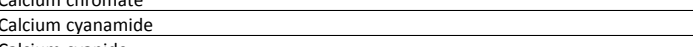 & 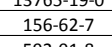 & 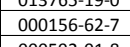 & 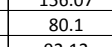 & $\frac{5}{5}$ & 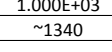 & & subl & & & & 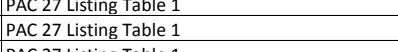 \\
\hline$\frac{2355}{5355}$ & & 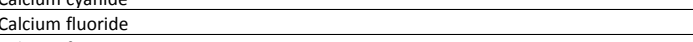 & 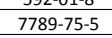 & 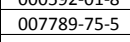 & $\frac{78.08}{78.08}$ & s & $1.4188+03$ & & $2.500=03.03$ & & & & 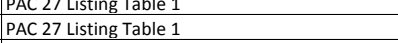 \\
\hline $\begin{array}{l}536 \\
557\end{array}$ & 年4 & 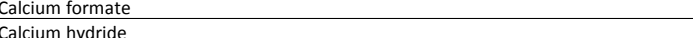 & S44-17.2 & 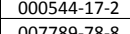 & 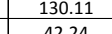 & 5 & 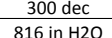 & & 675 dec $\mathrm{C}, \mathrm{C}$ & & & & 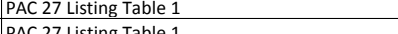 \\
\hline$\frac{258}{538}$ & & $\begin{array}{l}\text { Calcium hydroxide } \\
\end{array}$ & $1305 \cdot 62-0$ & Co1305:-2:- & & $\mathrm{s}$ & 580 dec & & 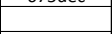 & & & & isting Table 1 1 \\
\hline$\frac{539}{550}$ & & 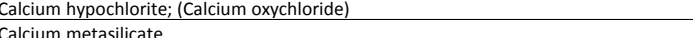 & $\begin{array}{l}7775.543 .3 \\
10103.0\end{array}$ & 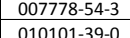 & 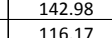 & 5 & $1.000 E+02$ & & & & & & $\begin{array}{ll}\text { able } 1 \\
\text { ble }\end{array}$ \\
\hline$\frac{541}{542}$ & 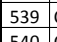 & 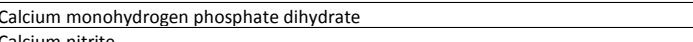 & 77899-77-7.7. & 007789-77-7.7 & $\begin{array}{l}136.06 \\
\end{array}$ & s. & 36 & & & & & & listing Table 1 . \\
\hline$\frac{542}{543}$ & & Calcium oximete & 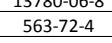 & 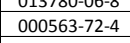 & 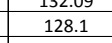 & & & & & & & & 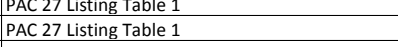 \\
\hline$\frac{5445}{5445}$ & 543 & $\begin{array}{l}\text { Caclium oxalate, hyorate } \\
\text { Calcim oxide }\end{array}$ & 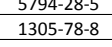 & 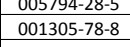 & 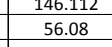 & $\frac{\mathrm{s}}{\mathrm{s}}$ & 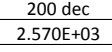 & & $2.8500+03$ & & & & 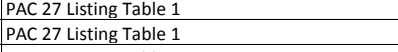 \\
\hline $\begin{array}{ll}5.50 \\
549 \\
549\end{array}$ & $\begin{array}{lll}544 \\
545\end{array}$ & 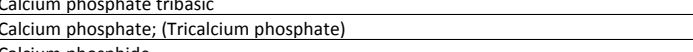 & 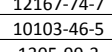 & 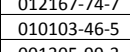 & 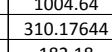 & $\frac{5}{5}$ & & & & & & & 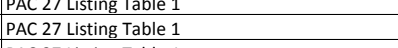 \\
\hline$\frac{-48}{549} \mathrm{t}$ & 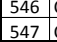 & 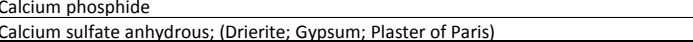 & 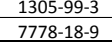 & 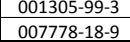 & 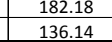 & $\frac{5}{5}$ & 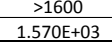 & & & & & & 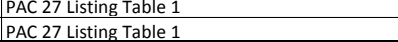 \\
\hline
\end{tabular}




\begin{tabular}{|c|c|c|c|c|c|c|c|c|c|c|c|c|c|}
\hline & No. & Chemical Compound & $\begin{array}{c}\text { cas Number } \\
\text { (caANA) }\end{array}$ & $\begin{array}{c}\text { Modfifed } \\
\text { fermatcas }\end{array}$ & $\begin{array}{l}\text { Molecurar } \\
\text { Weight }\end{array}$ & 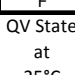 & $\begin{array}{c}\text { MP or fP } \\
\text { pccl }\end{array}$ & 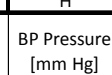 & вр [ссс] & $\begin{array}{c}\text { vapor } \\
\text { Pressure }\end{array}$ & $\begin{array}{l}\text { Tforve } \\
\text { scl }\end{array}$ & 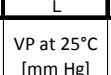 & Vapor Pressure @ $@ 25^{\circ}$ Reference \\
\hline 550 & & 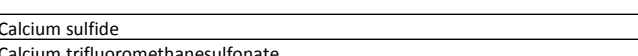 & $\frac{20548.54-3}{5527.757}$ & 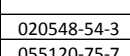 & $\frac{72.14}{3232083}$ & $\frac{25}{5}$ & $2.524+03$ & & dec & & & & 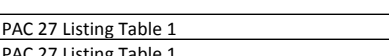 \\
\hline$\frac{551}{552}$ & 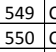 & 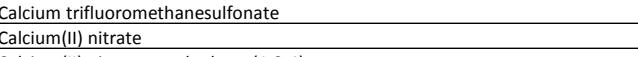 & $\begin{array}{l}5152027-7-7 \\
1024-57.5\end{array}$ & 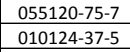 & 38.2.288 & $\frac{s}{s}$ & $5.6105+02$ & & dec & & & & 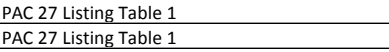 \\
\hline$\frac{-553}{-554}$ & 552 & 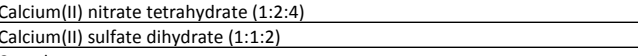 & 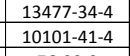 & 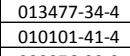 & $\begin{array}{l}\frac{236.18}{172.18} \\
\end{array}$ & $\frac{5}{5}$ & 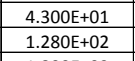 & & $\frac{d e c}{1.630+02}$ & & & & 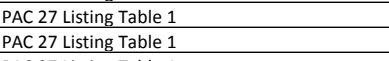 \\
\hline$\frac{555}{556}$ & & $\begin{array}{l}\text { Camphor } \\
\text { Camphoruffonic acid, (1R)-(-)-10- }\end{array}$ & $\begin{array}{l}\frac{762.22 .2}{3595320.3} \\
3\end{array}$ & 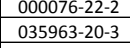 & $\frac{152.26}{232.3}$ & $\frac{s}{s}$ & 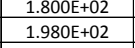 & & $20000+502$ & & & & 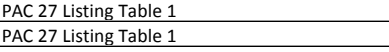 \\
\hline$\frac{5758}{5558}$ & 556 & Cantrandinin (dust) & 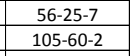 & 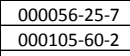 & $\frac{19.62}{13.16}$ & $\begin{array}{c}5 \\
\\
\end{array}$ & 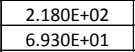 & & 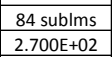 & $\begin{array}{l}2.0005-02 \\
7.5010 .3\end{array}$ & $\begin{array}{l}2.5000+51 \\
.3680+01\end{array}$ & & 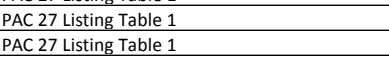 \\
\hline$\frac{559}{560}$ & & 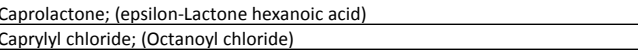 & $\begin{array}{l}50.244 .3 \\
111.64 .8\end{array}$ & 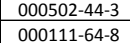 & $\frac{114.16}{162.68}$ & & 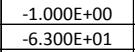 & $\begin{array}{l}7.600+522 \\
7.6005+02\end{array}$ & 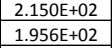 & $\begin{array}{l}1.3 .500-01 \\
.200 E+00\end{array}$ & 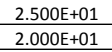 & 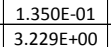 & 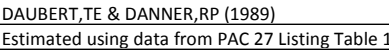 \\
\hline & & $\begin{array}{l}\text { Caspo } \\
\text { Castan }\end{array}$ & 734663:39.5 & 0737463-39:5 & 237.3.134 & & $\frac{270-274}{27-2792}$ & & & Oamos & & & 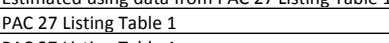 \\
\hline$\frac{562}{563}$ & $5601 \mathrm{c} c \mathrm{c}$ & 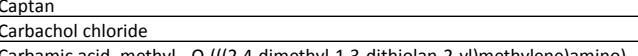 & 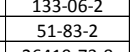 & 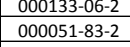 & $\begin{array}{l}30.59 \\
182.68\end{array}$ & $\frac{5}{5}$ & 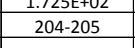 & & & $9 \times 10.8$ & $2.500 \mathrm{E}+01$ & & 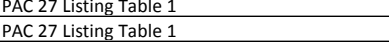 \\
\hline 564 & 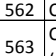 & 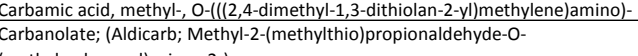 & $26419 \cdot 7 \cdot 3 \cdot 8$ & 0260119-93-86-3 & 239.36 & $\frac{\mathrm{s}}{\mathrm{s}}$ & $9.9005+01$ & & $>100 \mathrm{dec}$ & $9.700: 05$ & $2.0000+01$ & & $\begin{array}{l}\text { PAC } 2 \text { L LStsing Tabble } 1 \\
\text { PCA } 2 \text { Lising Table } 1\end{array}$ \\
\hline$\frac{565}{566}$ & $\frac{564}{655}$ & 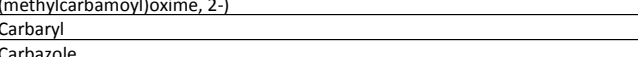 & $\frac{63.25 .2}{68.728}$ & 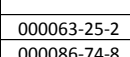 & $\frac{201.22}{1622}$ & $\frac{s}{s}$ & $\frac{1.45060252}{2.585 .02}$ & & $\frac{\text { dec }}{3557+52}$ & $\frac{1.3610 .6}{10000.62}$ & 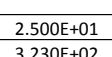 & & 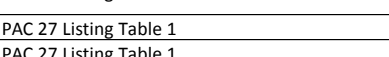 \\
\hline$\frac{568}{568}$ & 666 650 & catbazole vilotet & $\frac{6037830-1}{6537302}$ & $\frac{00358380.1}{0.1}$ & 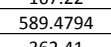 & $\frac{s}{s}$ & & & & & & & 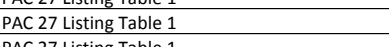 \\
\hline $\begin{array}{ll}\frac{596}{550} \\
57\end{array}$ & $\frac{568}{568 c}$ & 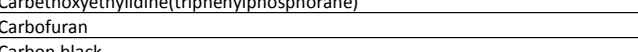 & 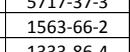 & 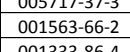 & 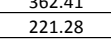 & $\frac{5}{5}$ & $150-152$ & & & $2.000-05$ & $3.300+\div 01$ & & 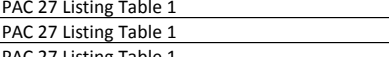 \\
\hline 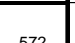 & $570 \mathrm{c}$ & Carbonon dioxide & $122438-9$ & $000124-38.9$ & 44.01 & $\mathrm{G}$ & $-5.6665+01$ & & 78.464 subl & $7.500=+02$ & $.7 .860++01$ & & PAC 27 Listing Table 1 \\
\hline 573 & $\frac{571}{721}$ & Carbon disulfide & 75.15 .0 & $0.00075-15.0 .0$ & 76.14 & 2 & $-1.12121+02$ & $7.600 E+02$ & 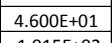 & $\begin{array}{l}3.5006+02 \\
.50600\end{array}$ & $2.5000+01$ & 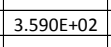 & 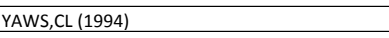 \\
\hline$\frac{5745}{575}$ & 572 & & 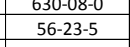 & 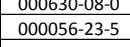 & $\begin{array}{l}28.32 \\
153.82\end{array}$ & $\frac{G}{L}$ & 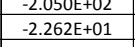 & 7.0006+02 2 & 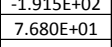 & $\begin{array}{llll}1.15501008 \\
1.150+02\end{array}$ & $\begin{array}{l}2.5000+201 \\
2.505+01\end{array}$ & $1.160+02+2$ & 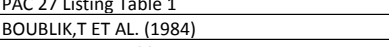 \\
\hline & & 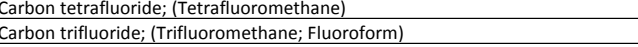 & & & & & 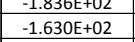 & & & 3.530E+04 & $2.500 \mathrm{E}+01$ & & $\begin{array}{l}\text { Litsinin Table } 1 \\
\text { Listing Table } 1\end{array}$ \\
\hline$\frac{578}{579}$ & 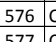 & 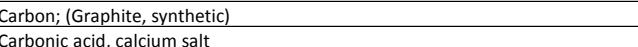 & 7440.04.0.0 & 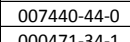 & 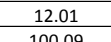 & $s$ & & & 3.642E $E+03$ & $1.000 E+00$ & 3.5865+033 & & 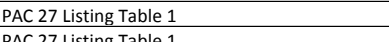 \\
\hline $\begin{array}{ll}580 \\
59 \\
509\end{array}$ & 5878 & 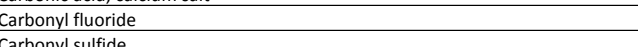 & 355.50.4. & .000335:50.4 & 66.01 & 6 & $-1.1400+50202$ & & 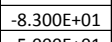 & $>760$ & - & & Listing Table 1 . \\
\hline & & 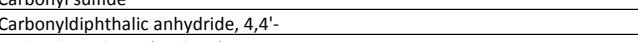 & & 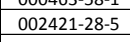 & 322.230202 & $\mathrm{s}$ & $\frac{226}{226}$ & & & & & & \\
\hline$\frac{5534}{584}$ & $\begin{array}{l}581 \\
882\end{array}$ & 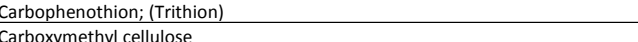 & $\begin{array}{l}\frac{786-196}{900-1.7} \\
9007\end{array}$ & $\begin{array}{l}0.0078-19 .-1 \\
0.000-11.5\end{array}$ & $\begin{array}{l}\frac{34287}{76588} \\
7658\end{array}$ & $\frac{L}{s}$ & & 1.000E-01 & 8.200E+01 & 3.000E:07 & $2.500 E+01$ & 00E -0 & RETA A... (1988 \\
\hline 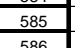 & 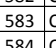 & 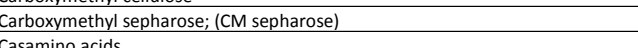 & $6889407-7.5$ & 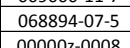 & & $\frac{s}{s}$ & & & & & & & 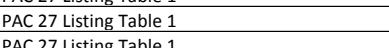 \\
\hline $\begin{array}{ll}5.507 \\
587\end{array}$ & 555 c & 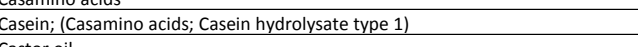 & 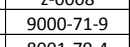 & 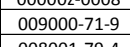 & & $\mathrm{s}$ & & $76005+022>0$ & & 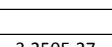 & & & $\begin{array}{llll}-1 & 1\end{array}$ \\
\hline $\begin{array}{ll}\frac{588}{589} \\
589\end{array}$ & $587 \mathrm{coc}$ & 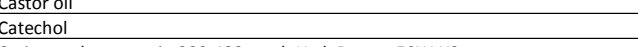 & 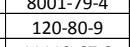 & 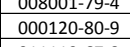 & 110.11 & 5 & $1.046 \mathrm{E}+02$ & 1.:000E=02 & $2.4505+02$ & . & 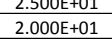 & & 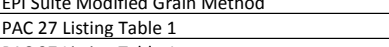 \\
\hline 501 & & 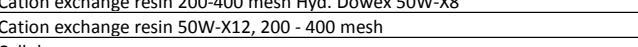 & $\begin{array}{l}10056-0.5 \\
9050.5\end{array}$ & 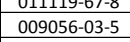 & & $\mathrm{s}$ & & & & & & & 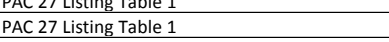 \\
\hline 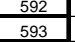 & 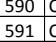 & $\begin{array}{l}\text { Cellllase } \\
\text { Cellulose }\end{array}$ & 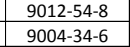 & 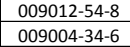 & 160000 & $\frac{5}{s}$ & & & & & & & 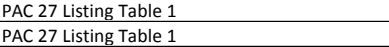 \\
\hline$\frac{594}{5955}$ & 5933 & 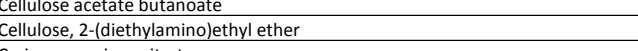 & 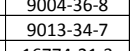 & 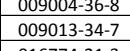 & 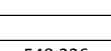 & $\frac{5}{5}$ & & & & & & & 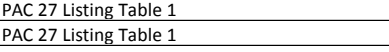 \\
\hline & & $\begin{array}{l}\text { Ceric a ammonium nitrate } \\
\text { Ceric ammonium suffate }\end{array}$ & $1050-0558$ & & $\begin{array}{l}5842.26 \\
60.532\end{array}$ & & & & & & & & \\
\hline$\frac{598}{598}$ & 596 & $\begin{array}{l}\text { Ceric ammonium sulfate, dihydrate } \\
\text { Coic ovido }\end{array}$ & 10337-47-9.9 & 0.10378.47.5. & 632.53 & s & 130 & & & & & & Stsing Table 1 1 \\
\hline 年959 & 998 & 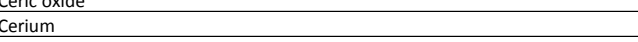 & 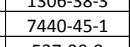 & 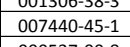 & 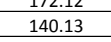 & $\frac{5}{5}$ & $\begin{array}{ll}2.000753 \\
8.006+02\end{array}$ & & $3.433+03$ & & & & $\begin{array}{l}\text { sithing Table } 1 \\
\text { The }\end{array}$ \\
\hline$\frac{601}{602}$ & 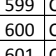 & 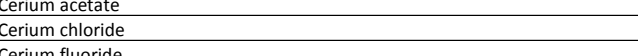 & 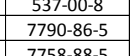 & 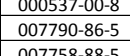 & 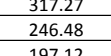 & $\begin{array}{c}5 \\
5 \\
5\end{array}$ & 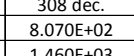 & & 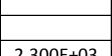 & & & & 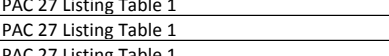 \\
\hline 604 & 502 & Cerium hydroxide & 15775.09 .8 & $0.1575-0.9 .8$ & 191.14 & s & & & & & & & 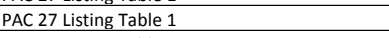 \\
\hline & & 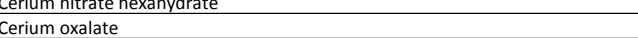 & 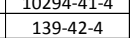 & 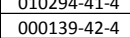 & 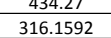 & & & & & & & & 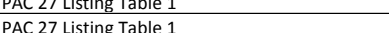 \\
\hline 607 & 505 & $\begin{array}{l}\text { Cerium sulfte } \\
\text { Cuthe }\end{array}$ & 1.1550:82:-4 & 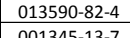 & 年332.24 & $s$ & 1.6925033 & & & & & & 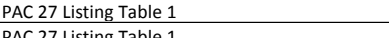 \\
\hline$\frac{609}{610}$ & $607\left[\begin{array}{ccc}6 \\
668\end{array}\right.$ & 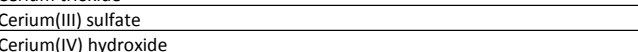 & 13454949.9 & 13454-9.9.9 & $\begin{aligned} 568.42 \\
208.1452 \\
2\end{aligned}$ & $\begin{array}{c}s \\
s \\
\end{array}$ & 250 dec & & & & & & 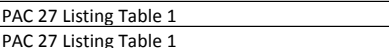 \\
\hline 611 & 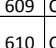 & $\begin{array}{l}\text { Cerium(IV) nitrate } \\
\text { Ceroun sitate- Cerium(II) nitrate) }\end{array}$ & $10108.73 \cdot 3$ & 010108.73 .3 .3 & 392.1708 & $\frac{s}{s}$ & 150 lose water & & 200 dec & & & & \begin{tabular}{|l} 
PAC 27 L Lsting Table 1 \\
\end{tabular} \\
\hline
\end{tabular}




\begin{tabular}{|c|c|c|c|c|c|c|c|c|c|c|c|c|c|}
\hline & №. & Chemical Compound & $\begin{array}{l}\text { CAS Number } \\
\text { (CASBN) }\end{array}$ & $\begin{array}{l}\text { Modified } \\
\text { Format CAS }\end{array}$ & $\begin{array}{c}\text { Molecular } \\
\text { Weight (MW) }\end{array}$ & $\begin{array}{c}\text { QV state } \\
\text { at } \\
25^{\circ} \mathrm{C}\end{array}$ & 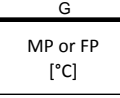 & $\begin{array}{c}\text { BP Pressure } \\
{[\mathrm{mm} H \mathrm{Hg}]}\end{array}$ & $\mathrm{BP}\left[{ }^{[\mathrm{C}]}\right]$ & $\begin{array}{l}\text { Vapor } \\
\text { Pressure } \\
{[\mathrm{mm} H \mathrm{Hgl}]}\end{array}$ & $\begin{array}{c}\text { Tor VP } \\
\text { ["C] }\end{array}$ & $\begin{array}{l}\text { VP at } 25^{\circ} \mathrm{C} \\
{[\mathrm{mm} \mathrm{Hg]}}\end{array}$ & Vapor Pressurue @ $2^{\circ} \mathrm{C}$ Reference \\
\hline 613 & 612 & Cesium & $\frac{7440-46-2}{524-8}$ & $007440-46-2$ & 132.91 & 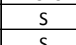 & $2.850 \mathrm{E}+01$ & & $6.680 \mathrm{E}+02$ & $1.000 \mathrm{E}+00$ & $2.790 \mathrm{E}+02$ & & $\begin{array}{l}\text { PAC } 27 \text { Listing Table } 1 \\
\text { PAC 27l liting Tater }\end{array}$ \\
\hline$\frac{614}{6615}$ & 613 & \begin{tabular}{|l} 
Cesium carbonate \\
Cesium chloride \\
\end{tabular} & $\begin{array}{l}534-17-8 \\
75677-1.8\end{array}$ & $\begin{array}{l}000534-17-8 \\
0.0764-87-8\end{array}$ & $\begin{array}{ll}325.83 \\
1683\end{array}$ & $\frac{\mathrm{s}}{\mathrm{s}}$ & & & $12005+03$ & & & & $\begin{array}{l}\text { PAC } 27 \text { Listing Table } 1 \\
\text { PC } 27 \text { liting Tablo }\end{array}$ \\
\hline$\frac{615}{616}$ & $\begin{array}{ll}614 \\
615\end{array}$ & \begin{tabular}{|l} 
Cesium chloride \\
Cesium fluoride
\end{tabular} & $\begin{array}{l}7647-1-8 \\
13400-13-0\end{array}$ & $\begin{array}{l}076767-17-8 \\
013400-13-0\end{array}$ & $\frac{168.36}{15.91}$ & $\frac{s}{s}$ & 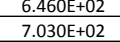 & & $\begin{array}{l}1.209 \mathrm{E}+03 \\
1.251 \mathrm{E} 03\end{array}$ & & & & $\begin{array}{l}\text { PAC } 27 \text { Listing gable } 1 \\
\text { ACC } 27 \text { isting Table } 1\end{array}$ \\
\hline$\frac{616}{617}$ & 616 & esium hydroxide & $21351-79-1$ & $021351-79-1$ & 149.9127 & $\mathrm{~s}$ & $3.150 \mathrm{E}+02$ & & & & & & Pact \\
\hline & 617 & & & & & & & & $1.2806+03$ & & & & 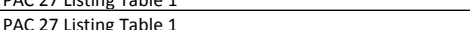 \\
\hline$\frac{319}{619}$ & 618 & esium metaborate & $92141-86-1$ & $092141-86-1$ & 175.715 & $\mathrm{~s}$ & $7.320 E+02$ & & 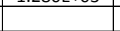 & & & & $\begin{array}{l}\text { PAC } 27 \text { Listing Table } 1 \\
\end{array}$ \\
\hline 620 & 619 & esium nitrate & $7789-18-6$ & $007789-18-6$ & 194.92 & $\mathrm{~s}$ & $4.140 \mathrm{E}+02$ & & dec. & & & & PAC 27 Listing Table 1 \\
\hline$\frac{621}{629}$ & $\frac{620}{621}$ & $\begin{array}{l}\text { Cesium nitrite } \\
\text { Cosium oxide }\end{array}$ & $13454-83-6$ & 013454-83-6 & 178.9097 & $\mathrm{~s}$ & & & & & & & PAC 27 L Listing Table 1 \\
\hline$\frac{622}{623}$ & $\frac{621}{622}$ & $\begin{array}{l}\text { Cesium oxide } \\
\text { Charcoal, activated } \\
\end{array}$ & 202018-0.0-9 & 些20281365-11-9 & $\frac{227.81}{12.01}$ & $\frac{s}{s}$ & $\frac{4900}{3.500+03}$ & & $4.827++03$ & & & & $\begin{array}{l}\text { PAC 27 Listing Table } 1 \\
\text { PAC } 27 \text { Listing Table } 1\end{array}$ \\
\hline$\frac{623}{624}$ & 623 & thloramben; (Amiben; 3-Amino-2,5-dichlorobenzoic acid) & 133-100-4 & $\begin{array}{ll}0 \\
0.063636-11-3 \\
00013-90-4 \\
\end{array}$ & $\frac{12.01}{206.03}$ & $\frac{2}{2}$ & 2.0.000E+ +02 & & 4.827 +03 & 7.000E-03 & 1.000E+02 & & $\begin{array}{l}\text { PAC } 27 \text { Listing Table } 1 \\
\text { PAC The }\end{array}$ \\
\hline$\frac{625}{625}$ & 624 & hloramphenicol & $56-75-7$ & $000056-75-7$ & 323.1322 & $\mathrm{~s}$ & $1.510 \mathrm{E}+02$ & & subl & $1.700 \mathrm{E}-12$ & $2.500 E+01$ & & PAC 27 Listing Table 1 \\
\hline 626 & 625 & chlordane & $57-74-9$ & $000057-74-9$ & 409.78 & $\mathrm{~s}$ & $1.060 \mathrm{E}+02$ & & $\begin{array}{l}175 @ \\
1 \mathrm{mmg}\end{array}$ & $9.75 \times 10-6$ & $2.500 E+01$ & & PAC 27 Listing Table 1 \\
\hline & 626 & chlorfenvinfos & $470-90-6$ & 0000470-90-6 & 359.58 & & $-2.300 E+01$ & 8.000E-03 & $1.250 \mathrm{E}+02$ & 7.500E-06 & $2.500 E+01$ & 7.500E-06 T/ & TOMLIN,C (1997) \\
\hline 628 & 627 & chloric acid, sodium salt; (Sodium chlorate) & $7775-09 \cdot 9$ & $007775-09-9$ & 106.44 & $\mathrm{~s}$ & 264 & & dec & & & & PAC 27 Listing Table 1 \\
\hline 629 & 628 & |chloride; (Chloride(1-1); chloride ions) & $16887-00-6$ & $016887-00-6$ & 35.453 & G & & & & & & & PAC 27 Listing Table 1 \\
\hline$\frac{630}{631}$ & 629 & $\begin{array}{l}\text { Chlorinated polyolefins } \\
\text { Chlorine }\end{array}$ & 68410-99-1 & -068710-09-1 & & $\frac{L}{G}$ & & $7.600 \mathrm{E}+02$ & $\frac{1.0000 E+02}{-3.304+01}$ & 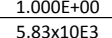 & 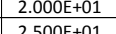 & $1.680 \mathrm{E}+00$ & 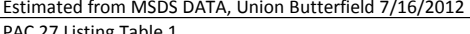 \\
\hline$\frac{0.31}{632}$ & 631 & \begin{tabular}{|l|l} 
Chlorine dioxide \\
\end{tabular} & $\begin{array}{ll}\frac{1}{1022-00-5}-04-4 \\
1049-4\end{array}$ & $\begin{array}{l}0.001 / 8-50-5 \\
010049-04-4 \\
\end{array}$ & $\frac{70.960}{67.45}$ & $\frac{G}{G}$ & 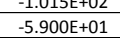 & & $\begin{array}{l}-3.444+F+11 \\
1.100 E+01\end{array}$ & & $2.500 \mathrm{E}+01 \mathrm{~T}$ & & $\begin{array}{l}\text { PAC 2 / IIIting lable } 1 \\
\text { PAC } 27 \text { Listing Table } 1\end{array}$ \\
\hline & 632 & Chlorine Hi dry granular (as Cl) & $2-0006$ & $00000 z-0006$ & 35.453 & & & & & & & & PAC 27 Listing Table 1 \\
\hline 634 & 633 & & 13637-63-3 & $013637-63-3$ & 130.445 & $G$ & $-1.030 \mathrm{E}+02$ & & $-1.310 \mathrm{E}+01$ & & & & PAC 27 Listing Table 1 \\
\hline 635 & 634 & 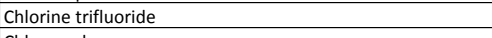 & $7790-91-2$ & $007790-91-2$ & 92.45 & $G$ & $-7.634 \mathrm{E}+01$ & & $1.175 E+01$ & $7.500 E+02$ & $1.140[+01$ & & PAC 27 Listing Table 1 \\
\hline 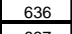 & 635 & Chhormephos & $24934-91-6$ & $024934-91-6$ & 234.7 & $\frac{1}{s}$ & & $1.000 E-01$ & $8.200 \mathrm{E}+01$ & $5.7000-02$ & $2.500 E+01$ & $5.700 \mathrm{E}-02 \mathrm{~T}$ & TOMLIN,C (1994) \\
\hline \begin{tabular}{|c|c|}
637 \\
638 \\
\end{tabular} & $\frac{636}{637}$ & 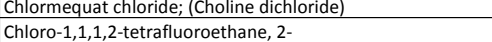 & $\begin{array}{l}\frac{99991.5}{2837-89-0}-0 \\
20\end{array}$ & $\begin{array}{l}000999-81-5 \\
00287-89-0\end{array}$ & $\frac{158.09}{136.4765}$ & $\frac{S}{G}$ & $\frac{245 \mathrm{dec}}{-1.990+52}$ & & $1.200 E+01$ & $\begin{array}{l}7.500 E E-08 \\
2.888+03\end{array}-13$ & $\frac{2.000 E+01}{2.500++01}$ & & $\begin{array}{l}\text { PAA } 27 \text { Listing Table } 1 \\
\text { PCC } 27 \text { isting Table } 1\end{array}$ \\
\hline \begin{tabular}{|l|l|}
630 \\
639
\end{tabular} & 638 & 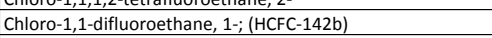 & 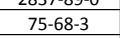 & $\begin{array}{ll}0.00075-68-3-3 \\
0007\end{array}$ & $\begin{array}{ll}150.4705 \\
100.5 \\
\end{array}$ & $\frac{0}{G}$ & 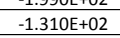 & & 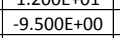 & $\frac{2.000+05}{7760}$ & 2.30uctut & & PAC 27 Listing Tauble 1 \\
\hline 6640 & 6399 & (chloro-1,2-propanedediol, 3; ; (chlorohyddrin) & $96-24-2$ & $000096-24-2$ & 110.55 & L & & $7.600 E+022$ & $2.130 E+02$ & $2.000 E-01$ & $2.5000+01$ & $2.0005 \mathrm{E}-\mathrm{C}$ & $\begin{array}{ll}\text { LANDE,SS ET AL. (1980) } \\
\end{array}$ \\
\hline 641 & 640 & Chloro-1-butanol, 4- & $928-51-8$ & 0000928-51-8 & 108.58 & L & $-2.994 E+01$ & $1.600 E+01$ & $8.400 E+01$ & $3.520 \mathrm{E}-01$ & $2.5000+01$ & 3.520E-01 & EPI Suite Mean VP of Antoine and Grain Methods \\
\hline & 641 & |chloro-2,4-dinitrobenzene, 1- & 97-00-7 & $000097-00-7$ & 202.56 & $\mathrm{~s}$ & $\begin{array}{l}\text { alpha }=51 \\
\text { beta }=43 ;\end{array}$ & & $3.150 E+02$ & & & & PAC 27 Listing Table 1 \\
\hline 643 & 642 & Chloro-2-methyl-1-propene, 3 - & $563-47-3$ & 000563-47-3 & 90.56 & $\mathrm{~L}$ & $8.000 E+01$ & 7.600E+02 & 7.217E+01 & 1.017 +02 & $2.500 E+01$ & $1.017 \mathrm{E}+02 \mathrm{~F}$ & PARMEGGIANI,L (1983) \\
\hline & & Chloro-2-methyl-4-isothiazolin-3-one, 5- & 26172-55-4 & 026172-55-4 & 149.6 & & $5.200 E+01$ & & & & & & PAC 27 Listing Table 1 \\
\hline 645 & 644 & Chloro-2-methylpropane, 2; (Tert-butyly chloride) & 507-20-0 & 000507-20-0 & 92.58 & $\mathrm{~L}$ & $-2.5600++01$ & $7.600 \mathrm{E}+02$ & 5.0906+01 & 3.020E+02 & $2.500 E+01$ & 3.020EE+202 & DAUBERT, TE \& DANNER,RP (1989) \\
\hline 646 & 645 & 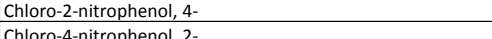 & $89-64-5$ & $000089-64-5$ & 173.554 & $s$ & 88.5 & & & & & & $\begin{array}{lll}\text { PAC } 27 \text { Listing Table } 1 \\
\text { Pac }\end{array}$ \\
\hline \begin{tabular}{|c|c|}
648 \\
648
\end{tabular} & 647 & chloroacetaldehyde & $107-20-0$ & $000107-20-0$ & 78.5 & $\mathrm{~L}$ & $-1.630 \mathrm{E}+01$ & $7.600 E+02$ & $8.500 \mathrm{E}+01$ & 6.427E+01 & $2.500 E+01$ & $6.427 E+01$ & DAUBERT,TE \& DANNER,RP (1994) \\
\hline 649 & 648 & \begin{tabular}{|l|} 
Chloroacetaldehyde dimethyl acetal \\
\end{tabular} & $97-97-2$ & $000097-97-2$ & 124.5669 & $\frac{1}{c}$ & $-7.300 E+01$ & $7.600 E+02$ & $1.275 E+02$ & $1.120 E+01$ & $2.500 E+01$ & $1.120 \mathrm{E}+01$ & 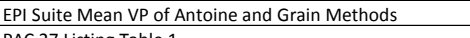 \\
\hline 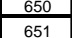 & {$\left[\begin{array}{c}659 \\
650\end{array} \mid\right.$} & 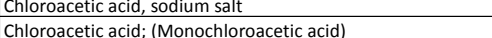 & 3926-62-3 & $\begin{array}{ll}003922-62-3 \\
000079-11-8\end{array}$ & $\frac{116.48}{94.5}$ & $\frac{s}{s}$ & $6.300 E+01$ & & $1.893 E+02$. & $7.500 E+00$ & $7.840 \mathrm{E}+01$ & & $\begin{array}{l}\text { PAC 27 listitig Table } 1 \\
\text { PAC } 27 \text { Listing Table } 1\end{array}$ \\
\hline \begin{tabular}{|c|c|}
651 \\
652
\end{tabular} & 651 & 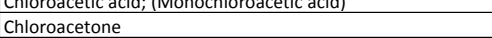 & $\frac{79-11-8}{78-95-5}$ & o. $000078-95-5$ & 94.53 & $\mathrm{~L}$ & $-4.4506+5101$ & 7.600E+02 & 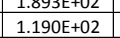 & $\begin{array}{l}7.500+E 0 \\
1.200 E+01\end{array}$ & $\frac{2.5006+01}{2.501}$ & & AANN,RWJIR \& IENSEN,PA (1977) \\
\hline 653 & 652 & chloroacetonititile & $107-14-2$ & 000107-14-2 & $\frac{25.5}{75.5}>0>0$ & L & & 7.600E+02 & 1.265E+02 & $1.500 E+01$ & $2.500 E+01$ & $1.500 E+01$. & $\begin{array}{ll}\text { NEAST,RC ET AL. (1985) } \\
\end{array}$ \\
\hline 654 & 653 & Chloroacetylymloride & $79-04-9$ & $000079-04-9$ & 112.94 & L & 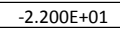 & $7.600 \mathrm{E}+02$ & $1.060 E+02$ & 2.520E+01 & $2.500 E+01$ & $2.520 \mathrm{E}+01[\mathrm{C}$ & DAUBERT, TE \& DANNER,RP (1993) \\
\hline 655 & 654 & |chloroally)|-3,5,7-triaza-1-azoniaadamantane chloride, 1-(-3- & $4080-31-3$ & 004080-31-3 & 251.16 & s & $178-210$ & & & $<1.0 \times 10-7$ & $2.500 \mathrm{E}+01$ & & PAC 27 Listing Table 1 \\
\hline \begin{tabular}{|c|c|}
656 \\
657
\end{tabular} & 655 & Clhloroaniline, p- & 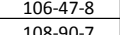 & $000106-47-8$ & $\frac{127.57}{11256}$ & $\frac{s}{1}$ & 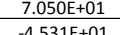 & & $\begin{array}{l}2.320+02 \\
13377+502\end{array}$ & $\begin{array}{l}7.100 E-02 \\
10707501\end{array}$ & $2.500 E+01$ & & 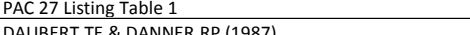 \\
\hline \begin{tabular}{|c|c|}
658 \\
658
\end{tabular} & 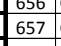 & 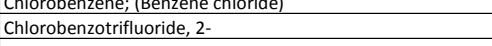 & $\frac{108-10-1}{88-16-4}$ & 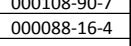 & $\begin{array}{ll}118.26 \\
180.56\end{array}$ & $\frac{L}{L}$ & $\begin{array}{l}-4.531+01 \\
-6.000 E+00\end{array}$ & $\begin{array}{lll}1.6000+62 \\
7.600 E+02\end{array}$ & $1.522+02+02$ & $5.083 E+00$ & $\begin{array}{l}2.2500+101 \\
2.500 E+01 \\
\end{array}$ & 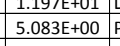 & 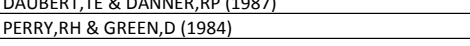 \\
\hline & 658 & Chlorobenzylate; (4,4'-Dichlorobenzilic acid ethyl ester) & $510-15-6$ & $000510-15-6$ & 325.19 & $\mathrm{~s}$ & 3.700E+01 & & $\begin{aligned} 157 @ \\
0.7 \mathrm{mmHg}\end{aligned}$ & $2.2 \times 10-6$ & 2.000E+01 & & PAC 27 Listing Table 1 \\
\hline 660 & 659 & Chlorobenzylidene malononitrile, o; (Tear Gas) & $2698-41-1$ & $002698-41-1$ & 188.62 & $\mathrm{~s}$ & $9.500 \mathrm{E}+01$ & & $3.130 \mathrm{E}+02$ & $3.000 \mathrm{E}-05$ & $2.000 E+01$ & & PAC 27 Listing Table 1 \\
\hline 661 & 660 & $\begin{array}{l}\text { Chlorobutane, 1;; (Butyly chloride) } \\
\text { Chrobtion, }\end{array}$ & $\frac{109-69-3}{9709}$ & $000109-69-3$ & 92.58 & $\mathrm{~L}$ & $-1.231 \mathrm{E}+02$ & $7.600+02+02$ & $7.850 \mathrm{E}+011$ & $\frac{1.013 E+02}{1575502}$ & $2.5000+011$ & $1.013 \mathrm{E}+022$ & 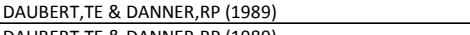 \\
\hline \begin{tabular}{|c|}
662 \\
663
\end{tabular} & $\frac{661}{662}$ & 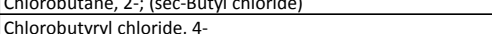 & $\frac{18-866-4}{4635-59-0}$ & $\frac{00000 / 8-86-4}{004655-59-0}$ & $\frac{92.58}{141}$ & $\frac{L}{L}$ & $-1.400 E+02$ & $\frac{7.600+0202}{7.600+202}$ & $\frac{6.800+101}{17355+02}$ & $\frac{1.566+E+02}{1320+200}$ & $\frac{2.500+0+01}{2.500+01}$ & 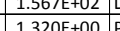 & 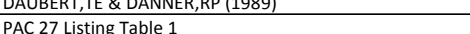 \\
\hline$\frac{0.05}{664}$ & 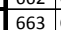 & Chlorocyclohexanolet trans-2.2. & 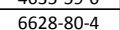 & 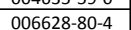 & $\frac{134.61}{134}$ & $\frac{i}{s}$ & $2.900 E+01$ & 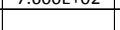 & 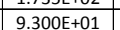 & 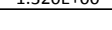 & & & PAC 27 Tistisg Table 1 \\
\hline 665 & 664 & Chlorocycylohexene; (44-Chlorococyclohexene) & $930-65-4$ & $000930-65-4$ & 116.5901 & $\frac{\mathrm{L}}{\mathrm{L}} \mathrm{C}$ & $-5.5944+01$ & 7.600E +02 & $1.361 \mathrm{E}+02$ & $7.580 E+00$ & $2.500 E+01$ & $7.580 \mathrm{E}+00 \mathrm{E} E \mathrm{E}$ & EP S Suite Mean UP of Antoi \\
\hline 666 & 665 & Chhorodecane, 1 - & $1002-69-3$ & 001002-69-3 & 176.7289 & $\mathrm{~L}$ & $-3.4000+01$ & 7.600E+02 & $2.230 \mathrm{E}+02$ & $9.840 \mathrm{E}-03$ & $2.500 E+01$ & 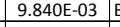 & BOUBLIK, TET AL. (1984) \\
\hline 667 & 666 & Chlorodiethylaluminum; (Diethylaluminum chloride) & 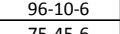 & $000096-10-6$ & $\frac{120.57}{80.67}$ & $s$ & $-5.000 \mathrm{E}+01$ & & $2.080 \mathrm{E}+02$. & & & & PAC 27 Listing Table 1 \\
\hline$\frac{668}{669}$ & 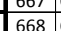 & $\begin{array}{l}\text { Chlorodifluoromethana,; Freon 22; CFC 22) } \\
\text { Chloroethanesulffonyl chloride, 2- }\end{array}$ & $\frac{75-455-6}{1622-8}$ & 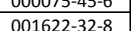 & $\frac{86.47}{16302}$ & $\mathrm{~s}$ & $-1.5 / 4 t+02$ & & 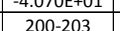 & 1.500ut+U2 & -4.1.1UEE +01 & & 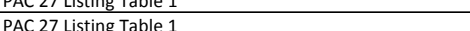 \\
\hline 670 & $669+1$ & chloroethyl chloroformate & $\frac{267-11-2}{627-2}$ & $000627-11-2$ & 142.97 & L & & 7.600E +02 & $1.577+02$. & 1.300E +01 & 4.800E+01 & $3.726 \mathrm{E}+00 \mathrm{E}, \quad 2 \mathrm{E}$ & Estimated using data from $\mathrm{PAC} 27$ Listing Table 1 \\
\hline$\frac{671}{671}$ & 670 & chloroethyl phenyy sulfide, 2 - & 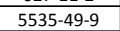 & $005535-49-9$ & 172.6721 & $s$ & $1.040 E+02$ & 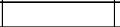 & 2.450E+02 & 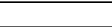 & 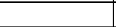 & $t_{p}$ & \begin{tabular}{|l} 
PAC 27 listing Table 1 \\
\end{tabular} \\
\hline 672 & 671 & Chloroethyl vinyl lether, 2;; (Ethene, 2-chloroethoxy-) & $110-75-8$ & $000110-75-8$ & 106.56 & L & $-7.030 E+01$ & 7.600E+02 & $1.080 \mathrm{EE}+02$ & $\begin{array}{l}3.000 E+01 \\
\end{array}$ & $2.500 E+01$ & 3.000E $+011_{\mathrm{P}}$ & ng Table 1 \\
\hline$\frac{673}{674}$ & $\frac{672}{673}$ & $\begin{array}{l}\text { Chlhoroethylchloromethylsulfide, } 2- \\
\text { chloroform }\end{array}$ & $\frac{2625-76-5}{67-66-3}$ & 0026256-76-5 & $\frac{145.0464}{11938}$ & $\frac{L}{L}$ & $\frac{-3.876 E+01}{-6.34 E+01}$ & 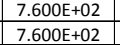 & $\frac{1.799+02}{6.117++01}$ & $\frac{1.020 E+00}{1.970+502}$ & $\frac{2.5500 E+101}{2.500+01}$ & $\frac{1.020 \mathrm{EE}+00}{1.902 \mathrm{E} E \mathrm{E}}$ & $\begin{array}{l}\text { EPP suiti Mean VP of Antoine and Grain Methods } \\
\text { BOUBLKT,T ET AL (1984) }\end{array}$ \\
\hline
\end{tabular}




\begin{tabular}{|c|c|c|c|c|c|c|c|c|c|c|c|c|c|}
\hline & No. & Chemical Compound & $\begin{array}{l}\text { CAS Number } \\
\text { (CASRN) }\end{array}$ & $\begin{array}{c}\text { Modified } \\
\text { Format CAS }\end{array}$ & \begin{tabular}{|c|} 
Molecular \\
Weight (MW)
\end{tabular} & \begin{tabular}{|c|} 
F \\
Q S State \\
at \\
$25^{\circ} \mathrm{C}$ \\
\end{tabular} & $\begin{array}{c}\text { MP or FP } \\
{\left[{ }^{\circ} \mathrm{C}\right]}\end{array}$ & $\begin{array}{c}\mathrm{H} \\
\text { BP Pressure } \\
{[\mathrm{mm} \mathrm{Hg}]}\end{array}$ & $B P\left[{ }^{[C}\right]$ & $\begin{array}{l}\text { Vapor } \\
\text { Pressure } \\
{[\mathrm{mm} H \mathrm{Hg}]}\end{array}$ & $\begin{array}{c}T \text { for VP } \\
{\left[{ }^{\circ} \mathrm{C}\right]}\end{array}$ & 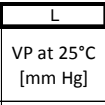 & Vapor Pressure @ 25ㄷ Reference \\
\hline 675 & 674 & Chloroform-d; (Deuterated chloroform) & \begin{tabular}{|l|l|}
$865-49-6$ \\
5057
\end{tabular} & $\begin{array}{ll}000865-49-6 \\
00250-67\end{array}$ & \begin{tabular}{l|l|}
120.4 \\
\end{tabular} & & $\frac{-6.400 E+01}{-6.05501}$ & $7.600 E+02$ & $6.0906+01$ & & $2.500 \mathrm{E}+01$ & $1.970++02$ & 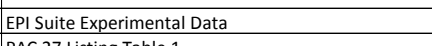 \\
\hline$\frac{676}{677}$ & 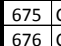 & $\begin{array}{l}\text { Chloro-m-cresol, 4- } \\
\text { Chloromethyl methyl ether }\end{array}$ & $\begin{array}{c}59-50-7 \\
10-70-2\end{array}$ & $\begin{array}{l}000059-50-7 \\
000100-70-2\end{array}$ & $\frac{142.59}{80.51}$ & $\frac{S}{L}$ & $\frac{5.550 \mathrm{E}+01}{-1.035 \mathrm{E}+22}$ & $76005+02$ & $\begin{array}{l}2.350 E+02 \\
5.950 F+01\end{array}$ & & $2500=01$ & 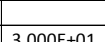 & $\begin{array}{l}\text { PAC } 27 \text { Listing Table } 1 \\
\end{array}$ \\
\hline$\frac{678}{678}$ & $677 \mathrm{c}$ & chloromethyl(trichloros)silane & $1558-25-4$ & $001558-25-4$ & 183.92 & $\mathrm{~L}$ & $1.110 \mathrm{E}+02$ & $7.600 E+02$ & 1.1.180E+02 & 1.710E+01 & $2.5006+01$ & $1.710 \mathrm{E}+01$ & EPI Suite MMan VP of Antoine and Grain Methods \\
\hline 679 & 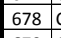 & Chloronaphthalene, 1; (alpha-Chloronaphthalene) & $90-13-1$ & $000090-13-1$ & 162.62 & L & $-2.500 E+00$ & $7.600 E+02$ & $2.590 \mathrm{E}+02$ & $2.9000-02$ & $2.500 \mathrm{E}+01$ & $2.900 \mathrm{E}-02$ & SCHOENE,K ET AL. (1984) \\
\hline 680 & $6790 \mathrm{c}$ & 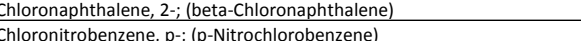 & 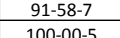 & $000091-58-7$ & $\frac{162.62}{157.56}$ & $\frac{s}{s}$ & $5.800 E+01$ & & 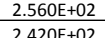 & $\frac{1.220 E-02}{7.5 \times 50.3}$ & $\begin{array}{l}2.500 E+01 \\
1.50+5+01\end{array}$ & & $\begin{array}{l}\text { PAC 27 listing Table } 1 \\
\text { PAC 2listing Tazale } 1\end{array}$ \\
\hline & $\frac{680}{681}$ & 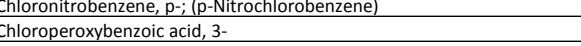 & $\begin{array}{l}100-00-5 \\
937-14-4\end{array}$ & $\begin{array}{l}00100-00-5 \\
000937-14-4\end{array}$ & 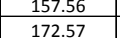 & $\frac{s}{s}$ & 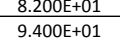 & & & & & & \\
\hline & & & 3591-35-8 & $003691-35-8$ & $\frac{172.7 / 3}{374.83}$ & $\frac{2}{3}$ & & & & & & & $\begin{array}{l}\text { PAC } 27 \text { Listing Table } 1 \\
\text { Pats }\end{array}$ \\
\hline$\frac{685}{684}$ & 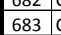 & Chlorophentuluol, 4 ; (p-Chlorophenol) & 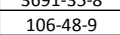 & $000106-48-9$ & $\begin{array}{l}37.403 \\
128.56\end{array}$ & s & 4.280E+01 & & $2.200 E+02$ & $7.5000-01$ & & & PAC 27 Listing Table 1 \\
\hline 685 & 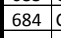 & Chlorophenol, $m$ - & $108-43-0$ & $000108-43-0$ & 128.56 & $\mathrm{~s}$ & $3.300 E+01$ & & $210-214$ & $1.000 E+00$ & $4.420 \mathrm{E}+01$ & & PAC 27 listing Table 1 \\
\hline 686 & 685 c & $\begin{array}{l}\text { Chlorophenol, o- } \\
\text { Chlorophenyl }\end{array}$ & $95-57-8$ & $000095-57-8$ & 128.56 & L & $9.400 E+00$ & $7.600 E+02$ & 1.749E+02 & $2.5306+00$ & $2.500 \mathrm{E}+01$ & $2.530 \mathrm{E}+00$ & DAUBERT,TE \& DANNER,RP (1991) \\
\hline$\frac{687}{6888}$ & $\begin{array}{l}686 \\
687\end{array}$ & $\begin{array}{l}\text { CClorophennyl phenyl ther, 4- } \\
\text { Chlorophenyl thiourea, 2- }\end{array}$ & $\frac{7005-72-3}{5344-82-1}$ & $007005-72-3$ & $\frac{204.65}{186.67}$ & $\frac{L}{S}$ & $\begin{array}{l}-8.000 E+00 \\
1140 \mathrm{E}+02\end{array}$ & & $\begin{array}{l}2.840 \mathrm{E}+02 \\
3.20 \mathrm{~F}+02\end{array}$ & $2.700 E-03$ & $2.500 \mathrm{EE}+01$ & $2.700 E_{-03}$ & $\begin{array}{l}\text { PAC C 27 Listing Table } 1 \\
\text { PA 27 ilsting Table } 1\end{array}$ \\
\hline & 688 & 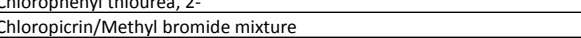 & 8354-804-09-9 & 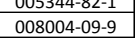 & $\begin{array}{lll}256.61 \\
259.31\end{array}$ & $\frac{S}{G}$ & & & & & & & Liquified gas gas treate 1 as gas for Quantity Value Process \\
\hline$\frac{000}{690}$ & & Chloropicrin/Methyl chloride mixture & $2-0007$ & $00000 z-0007$ & 85 & G & & & & & & & Liquified gas - treat as gas for Quantity Value Process \\
\hline 691 & 690 C 690 & Chloropicrin; (Trichloronitromethane) & $76-06-2$ & $000076-06-2$ & 164.38 & $\mathrm{~L}$ & $-6.4000+01$ & $7.600 E+02$ & & $2.380 E+01$ & $2.500 E+01$ & $2.380 E+01$ & MARTIN, H\& WORTHING, CR (1977) \\
\hline 692 & 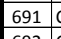 & Chloroprene; (beta-Chloroprene; Neoprene) & $126-99-8$ & $000126-99-8$ & 88.54 & L & $-1.300 E+02$ & $7.600 E+02$ & $5.940 \mathrm{E}+01$ & $2.155 \mathrm{E}+02$ & $2.500 E+01$ & $2.155 \mathrm{E}+02$ & BOUBLLK,T ET AL. (1984) \\
\hline$\frac{693}{694}$ & 6925 & 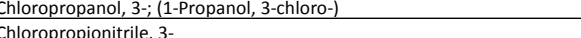 & 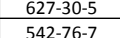 & 000627-30-5 & $\frac{94.55}{8.93}$ & $\frac{L}{L}$ & $\begin{array}{l}-2.0006+01 \\
-5,100 F+01\end{array}$ & $\begin{array}{l}7.600 \mathrm{E}+02 \\
7.60 \mathrm{~F}+02\end{array}$ & $\frac{1.650 \mathrm{E}+02}{1.70 \mathrm{E}+02}$ & $5.770 \mathrm{E}-01$ & $2.500 E+01$ & $5.770 E-01$ & $\begin{array}{l}\text { EPI Suite Mean VP of Antoine and Grain Methods } \\
\text { PAC 27 } 7 \text { isting Table } 1\end{array}$ \\
\hline & $\begin{array}{ll}693 \\
694\end{array}$ & 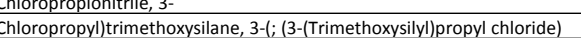 & $2532-6-17-2$ & (1) & 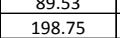 & $\frac{L}{L}$ & & 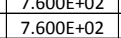 & $\frac{1.1 .906 \mathrm{E}+02}{1.950+02}$ & $\begin{array}{lll}2.500+0+00 \\
5.000++00\end{array}$ & $\frac{2.500 E+01}{2.500 E+01}$ & $\begin{array}{ll}2.500 E+00 \\
5.000 E+00\end{array}$ & $\begin{array}{l}\text { PAA 27 IIIting lable 1 } \\
\text { PAC 27 listing Table } 1\end{array}$ \\
\hline$\frac{396}{696}$ & 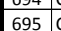 & chloropropylene, 2- & 557-98-2.2 & $000557-98-2$ & 76.53 & $\frac{L}{G}$ & $-1.386 E+02$ & & $2.265 \mathrm{E}+01$ & $8.190 \mathrm{E}+02$ & 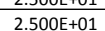 & & PAC 7 Listing Table 1 \\
\hline 697 & 696 & chloropropyy-n-octylyulfoxide, 3- & $3569-57-1$ & $003569-57-1$ & 238.85 & L & & $7.6000 \mathrm{E}+02$ & $3.380 \mathrm{C}+02$ & $2.000 E-05$ & $2.500 E+01$ & $2.000 E-05$ & PAC 27 Listing Table 1 \\
\hline 698 & 697 & Chloro-p-toluenesulfonamide sodium salt, $\mathrm{N}$; ( (Chloramine T) & $127-65-1$ & $000127-65-1$ & 227.65 & $s$ & $167-170$ & & & & & & PAC 27 Listing Table 1 \\
\hline 699 & 698 & 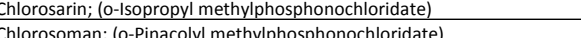 & $\frac{14455-76-7}{7000-57-5}$ & $001445-76-7$ & 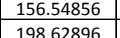 & $L$ & -4.0686E+01 & $\begin{array}{l}7.600 \mathrm{E}+02 \\
76020\end{array}$ & $1.700+5+02$ & 1.500+E+00 & $\begin{array}{l}2.500 \mathrm{E}+01 \\
250 \mathrm{~F} 1\end{array}$ & $1.5400+00$ & EPI Suite Mean VP of Antoine and Grain Methods \\
\hline & 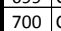 & Chlorosulfonic acid; (Chlorosusufuric acid) & 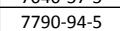 & $\frac{007790-94-5}{0}$ & $\frac{116.52}{116.52}$ & L & $-8.000 E+01$ & 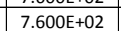 & 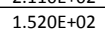 & $\begin{array}{l}7.5000-01 \\
7\end{array}$ & 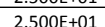 & $\frac{2.500-01}{7.50-01}$ & 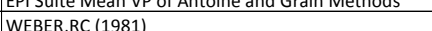 \\
\hline 702 & & Chlorothalonil; (Tetrachloroisophthalonititile) & $1897-45-6$ & $001897-45-6$ & & $\mathrm{~s}$ & & & & & & & PAC 27 Listing Table 1 \\
\hline 703 & 702 C & chlorotoluene, 2; (0-Chlorotoluene) & $95-49 \cdot 8$ & $000095-49 \cdot 8$ & 126.58 & L & $-3.5806+01$ & $7.600 E+02$ & $1.5906+02$ & $3.430 \mathrm{E}+00$ & $2.5006+01$ & $3.4306+00$ & DAUBERT,TE \& DANNER,RP (1985) \\
\hline 704 & 703 c & Chlorotoluene, 4-; (p-Tolyl chloride) & $106-43-4$ & $000106-43-4$ & 126.58 & $\mathrm{~L}$ & $7.500 E+00$ & $7.600 E+02$ & $1.624 \mathrm{E}+02$ & $2.6906+00$ & $2.5006+01$ & $2.6906+00$ & DAUBERT, TE \& DANNER,RP (1989) \\
\hline 705 & $704 \mathrm{c}$ & 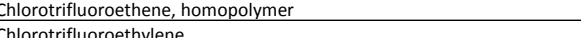 & $9002-83-9$ & $009002-83-9$ & & $\frac{s}{G}$ & & & & & & & PAC 27 Listing Table 1 \\
\hline 706 & $\frac{705}{706} \mathrm{C}$ & $\begin{array}{l}\text { Chlorotrifiluoreothylelene (CF-13) } \\
\text { Chlorotrifluoromethane; (CFC-13) }\end{array}$ & & $\begin{array}{l}000079-88-9 \\
000075-72-9\end{array}$ & $\frac{116.47}{104.46}$ & $\frac{G}{G}$ & 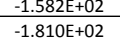 & & $\begin{array}{l}-2.788 \mathrm{EE}+01 \\
-8.10 \mathrm{E}+01\end{array}$ & $\frac{7.50 \mathrm{E} E+02}{2.140 \mathrm{E}+04}$ & $2.2 .800 \mathrm{EE}+01$ & & $\begin{array}{l}\text { PAA 27 Listing Gable } 1 \\
\text { PAC } 27 \text { Listing Table } 1\end{array}$ \\
\hline 708 & $\frac{707}{707} \mathrm{c}$ & chlorovinylarsine dichloride; (Lewisite 1) & $\frac{541-25-3}{54-3}$ & $\frac{000541-25-3}{0.3}$ & 207.31 & $\mathrm{~L}$ & 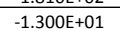 & $7.600 E+02$ & 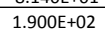 & 5.800e-01 & $\frac{2.5006+01}{2.01}>0$ & 5.800E-01 & TUNRO,NB ET TAL (1999) \\
\hline $\begin{array}{ll}7099 \\
7\end{array}$ & 708 & chloroxuron & $1982-47-4$ & $\frac{001982-47-4}{2}$ & 290.77 & $\mathrm{~s}$ & 151-152 & & & $3.9000-09$ & $2.000 E+01$ & & 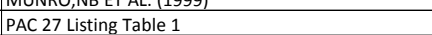 \\
\hline 710 & 709 & Chlorpyrifios; (Dursban) & $2921-88-2$ & $002921-88-2$ & 350.59 & $\mathrm{~s}$ & $42-43.5$ & & & & & & PAC 27 Listing Table 1 \\
\hline 711 & 710 c & chlorsulfuran & $64902-72-3$ & $064902-72-3$ & 357.8 & $\mathrm{~s}$ & 174-178 & & $192 \mathrm{dec}$ & 8.131E-01 & $2.500 E+01$ & & PAC 27 listing Table 1 \\
\hline 712 & $\begin{array}{lll}711 \\
712\end{array}$ & $\begin{array}{l}\text { Chlorthiophos } \\
\text { Chromates }\end{array}$ & $21923-23-9$ & 021923-23-9 & $\frac{361.25}{11597}$ & $\frac{\mathrm{L}}{\mathrm{s}}$ & 2.4000E+01 & $7.600 \mathrm{E}+02$ & $1.500 \mathrm{E}+02$ & 3.970E-01 & $2.500 \mathrm{E}+01$ & 3.970E-01 & $\begin{array}{l}\text { EPP Sutute Modified Grain Method } \\
\text { PAC }\end{array}$ \\
\hline & $\begin{array}{ll}\frac{112}{713} \mathrm{c} \\
\mathrm{c}\end{array}$ & Chromices catate: (Chromium (III) actate) & 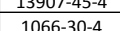 & 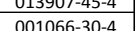 & $\frac{113.37}{22915}$ & $\frac{3}{5}$ & & & & & & & 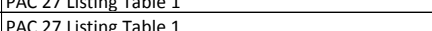 \\
\hline$\frac{715}{715}$ & 714 714 & Chromic chloride; (Chromium(IIII) chloride) & $10025-73-7$ & 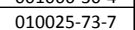 & 158.35 & $\mathrm{~s}$ & $1.152 E+03$ & & 1300 subl & & & & $\begin{array}{l}\text { PAC } 27 \text { Listing Table } 1 \\
\end{array}$ \\
\hline 716 & 715 & Chromic oxide; (Chromium(III) oxide; Chromium sesquioxide) & $1308-38-9$ & $001308-38-9$ & 151.99 & $\mathrm{~s}$ & $2.3206+03$ & & $\sim 3000$ & & & & PAC 27 Listing Table 1 \\
\hline 717 & 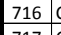 & Chromic sulfate; (Chromium(IIII) sulfate (2:3)) & $10101-53-8$ & $010101-53-8$ & 392.18 & $s$ & & & & & & & $\begin{array}{l}\text { PAC } 27 \text { Listing Table } 1 \\
\end{array}$ \\
\hline 718 & $\begin{array}{lll}717 \\
718\end{array}$ & Chromict riixoxide; (Chromiumm(VI) oxide (1:33)) & $\frac{1333-82-0}{1308-14-1}$ & 001333-82-0 & $\frac{99.99}{10302}$ & $\frac{5}{5}$ & $1.90 \mathrm{OL}+02$ & & 250 dec & & & & \\
\hline 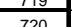 & 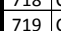 & 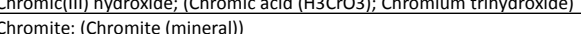 & $\frac{1308-141-1}{1308-31-2}$ & 001308-31-2 & $\frac{10.302}{223.85}$ & 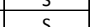 & & & & & & & 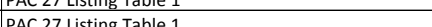 \\
\hline 2721 & 720 c & Chromium & $7440-47-3$ & $007440-47-3$ & 52 & $\mathrm{~s}$ & $1.907 F+03$ & & $2.6711+03$ & $7.5 \times 10-3$ & $1.3833 E+03$ & & PAC 27listing Table 1 \\
\hline 722 & $721 \mathrm{C}$ & chromium nitrate & $10103-47-6$ & $010103-47-6$ & 493.14 & $\mathrm{~s}$ & & & & & & & C27 Listing Table 1 \\
\hline 723 & $722 \mathrm{c}$ & Chromium perchlorate, hydrated & $13537-21-8$ & $013537-21-8$ & 350.35 & $\mathrm{~s}$ & & & & & & & PAC 27 Listing Table 1 , \\
\hline 724 & $\begin{array}{lll}723 \\
724\end{array}$ & $\begin{array}{l}\text { Chromium(III) acetate hydroxide } \\
\text { Chromium(III) chloride hexayhdrate }\end{array}$ & $39430-51-8$ & 039030-51-8 & $\frac{250}{265.17}>20$ & 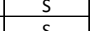 & 82 & & & & & & PAC 27 Listing Table 1 \\
\hline 726 & 725 & Chromium (III) fluoride & $7778-97-8$ & 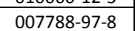 & $\frac{109}{109}$ & $s$ & 1400 & & & & & & PAC 27 Listing Table 1 \\
\hline & 726 & & & & & $\mathrm{s}$ & $6.000 E+01$ & & 100 dec & & & & PAC 27 Listing Table 1 \\
\hline $\begin{array}{ll}728 \\
{ }^{2}\end{array}$ & $727 \mathrm{C}$ & chromium(III) oxide hydroxide; (Chromium oxhhydroxide) & $20770-05-2$ & $020770-05-2$ & 85.0027 & $\mathrm{~s}$ & & & & & & & PAC 27 Listing Table 1 \\
\hline 729 & $728 \mathrm{c}$ & Chromium(III) potassium sulfate (1:1:22), dodecahydrate & $7788-99-0$ & $007788-99-0$ & 499.41 & $\mathrm{~s}$ & 89 & & & & & & PAC 27 Listing Table 1 \\
\hline 730 & $729 \mathrm{c}$ & 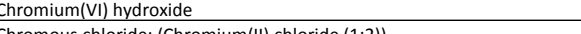 & $12626-43-6$ & $012626-43-6$ & $\frac{154.0399}{1209}$ & s & & & 1300502725 & & & & Listing Table 1 \\
\hline${ }^{732}$ & 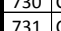 & $\begin{array}{l}\text { Chromous chloride; ; Chromium (III) chloride (1:22)) } \\
\text { Chrysenen (coal tar volatilie) }\end{array}$ & $\frac{1}{21800-05-3}$ & 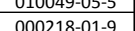 & $\frac{122.9}{2289}$ & $\frac{3}{5}$ & 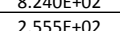 & & 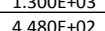 & $6.23 \times 10-9$ & $25006+01$ & & 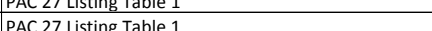 \\
\hline 733 & 732 C & cinnamaldehyde & $104-55-2$ & $000104-55-2$ & & L & $-7.5000+000$ & $7.6006+02$ & $2.530 \mathrm{E}+02$ & $2.8911-02$ & $2.500 E+01$ & 2.891E-02 & RH\& GREEN,D (1984) \\
\hline $\begin{array}{ll}734 \\
734\end{array}$ & 733 C & cinnamaldehyde, (E); (trans-Cinnamaldehyde) & $14371-10-9$ & & 132.17 & $\mathrm{~L}$ & $-7.500 E+00$ & $7.600 E+02$ & $2.520 E+02$ & $2.8900-02$ & $2.500 E+01$ & $2.890 E-02$ & EPr Suite Experimental Data \\
\hline 735 & 734 C & $-{ }_{2}$ & $77-92-9$ & $000077-92-9$ & 192.12 & $\mathrm{~s}$ & $1.530 \mathrm{E}+02$ & & dec & $1.78 \times 10-9$ & 2.500E+01 & & PAC 27 Listing Table 1 . \\
\hline 736 & 735 c & Citric acid monohydrate & 5949-29-1 & 005949-29-1 & 210.16 & s & 1.530E +02 & & dec & & & & PAC 27 Listing Table 1 | \\
\hline 738 & 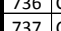 & 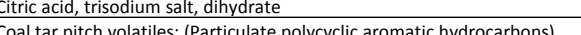 & $6132-04-3$ & 006132-04-3 & 294.1 & $s$ & 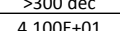 & & & & & & Listing Table 1 \\
\hline 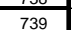 & 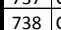 & Coal atra aerosol & $\frac{3507-45-2}{80}$ & $\frac{0.08007-45-2}{0.2}$ & 78.11 & $L$ & 5.500 & 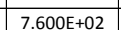 & $00 E+01$ & $0 E+01$ & & & EPI Suitt \\
\hline
\end{tabular}




\begin{tabular}{|c|c|c|c|c|c|c|c|c|c|c|c|c|c|}
\hline & A & & & & $\mathrm{E}$ & 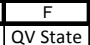 & & $\mathrm{H}$ & 1 & & K & & \\
\hline & No. & Chemical Compound & $\begin{array}{c}\text { CAS Number } \\
\text { (CASRN) }\end{array}$ & $\begin{array}{l}\text { Modified } \\
\text { Format CAS }\end{array}$ & \begin{tabular}{|c} 
Molecular \\
Weight (MW)
\end{tabular} & $\begin{array}{c}\text { avstate } \\
\text { at } \\
25^{\circ} \mathrm{C}\end{array}$ & $\begin{array}{l}\text { MP or FP } \\
{\left[{ }^{\circ} \mathrm{C}\right]}\end{array}$ & $\begin{array}{c}\text { BP Pressure } \\
{[\mathrm{mm} \mathrm{Hg}]}\end{array}$ & $\mathrm{BP}\left[{ }^{[\mathrm{C} C}\right]$ & $\begin{array}{l}\text { Vapor } \\
\text { Pressure } \\
\text { Imm Hg }\end{array}$ & $\begin{array}{c}\text { Tfor VP } \\
{\left[{ }^{\circ C} \mathrm{C}\right]}\end{array}$ & $\begin{array}{c}\text { vp at } 25^{\circ} \mathrm{C} \\
{[\mathrm{mm} \mathrm{Hg}]}\end{array}$ & Vapor Pressure @ @25 C Reference \\
\hline 740 & 7396 & Cobalt & $7440-48-4$ & $007440-48-4$ & 58.93 & & $1.495 \mathrm{E}+03$ & & $2.927 \mathrm{E}+03$ & & $1.517 \mathrm{E}+03$ & & PAC 27 Listing Table 1 \\
\hline 741 & $740 \mathrm{~s}$ & Cobalt acetate tetrahydrate; (Cobaltous acetate tetrahydrate) & $6147-53-1$ & $006147-53-1$ & 249.11 & L & & $7.600 E+02$ & $1.171 \mathrm{E}+02$ & $1.390 \mathrm{E}+01$ & $2.500 E+01$ & $1.3906+01$ & EPI Suite ACD Labs Physical Chemistry Suite \\
\hline$\frac{\frac{772}{743}}{74}$ & $\frac{771}{742} \mathrm{C}$ & $\begin{array}{l}\text { Cobalt carbonyl } \\
\text { cobatlt chloride }\end{array}$ & $\frac{102010-688-1}{7646-9}$ & 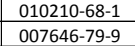 & 341.94 & $\frac{s}{s}$ & $\begin{array}{l}5.1006+01 \\
7.350+02\end{array}$ & & 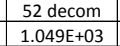 & & & & $\begin{array}{l}\text { PAC C 2 Listing Table 1 } \\
\text { PAC 27 isting Table 1 }\end{array}$ \\
\hline & & Cobalt hydrocarbonyl & $\begin{array}{l}76664-79-9 \\
1682-03-8 \\
\end{array}$ & 0016464-979-9 & $\frac{129.83}{17.98}$ & & 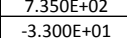 & & $\begin{array}{l}1.049 E+03 \\
1000+01\end{array}$ & & & & $\begin{array}{l}\text { PAC C2 Listing Table } 1 \\
\text { PAC 27 Listing Table } 1\end{array}$ \\
\hline$\frac{744}{775}$ & & & $\frac{16842-23-83}{21021-93-0}$ & o168442-303-8 & $\frac{171.98}{92.95}$ & $\frac{G}{S}$ & -3.30uet+U1 & & & & & & $\begin{array}{l}\text { PAC 27 Listing Table } 1 \\
\text { PAC 27 isting Table } 1\end{array}$ \\
\hline$\frac{745}{746}$ & $7456 \mathrm{c}$ & Cobalt nitrate hexahydrate; (Cobaltous nitrate hexahydrate) & $10026-22-9$ & $\begin{array}{lll}-10 \\
010026-22-9\end{array}$ & 291.07 & $\frac{2}{3}$ & $\sim 55$ & & $>74$ & & & & $\begin{array}{l}\text { PAC 27 Listing Table } 1 \\
\text { Pal }\end{array}$ \\
\hline 747 & 746 & Cobalt nitrate; (Cobalt(II) nitrate) & $10141-05-6$ & $010141-05-6$ & 182.95 & $\mathrm{~s}$ & $5.500 \mathrm{E}+01$ & & $75 \mathrm{dec}$ & & & & PAC 27 Listing Table 1 \\
\hline 748 & $747 \mathrm{c} c \mathrm{c}$ & 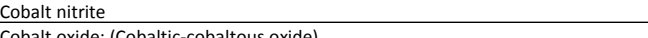 & $32486-57-0$ & $032486-57-0$ & $\begin{array}{r}150.93 \\
24079\end{array}$ & $\frac{s}{s}$ & opodec & & & & & & $\begin{array}{l}\text { PAC } 27 \text { Listing Table } 1 \\
\text { PaC 27listiog Thble1 } 1\end{array}$ \\
\hline$\frac{779}{750}$ & 748 & $\begin{array}{l}\text { Cobalt toxide; ; Cobaltic-cobaltous oxide) } \\
\text { Cobalt sulfate }\end{array}$ & $\frac{1308-066-1}{10124-43-3}$ & 001308-06-1 & 240.79 & $\frac{s}{s}$ & 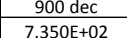 & & dec & & & & $\begin{array}{l}\text { PAA 27 Listing gable } 1 \\
\text { PAC 27 isting Table } 1\end{array}$ \\
\hline & & Cobalt sulfifate heptahydrate; (Cobalt(III) sulfate(1:1), heptahydrate) & $\frac{10144-433-3}{10026-24-1}$ & & 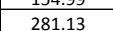 & s & $\frac{1.3560+2}{7.35 \mathrm{E}+02}$ & & & & & & PAC 7 Listing Table 1 \\
\hline$\frac{191}{752}$ & $751 \mathrm{c}$ & 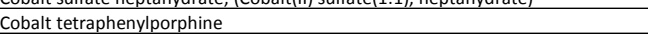 & 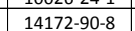 & $\frac{2014172-90-8}{0140}$ & & & & & & & & & $\begin{array}{l}\text { AC } 27 \text { Listing Table } 1 \\
\text { PA }\end{array}$ \\
\hline 753 & $752 \mathrm{c}$ & Cobalt(III) chloride hexahydrate & $7791-13-1$ & $007791-13-1$ & 237.95 & $\mathrm{~s}$ & $8.6006+01$ & & $1.049 E+03$ & & & & PAC 27 Listing Table 1 \\
\hline 754 & 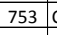 & Cobalt(III) oxide & $1307-96-6$ & $001307-96-6$ & 74.93 & $\mathrm{~s}$ & $1.935 \mathrm{E}+03$ & & & & & & PAC 27 Listing Table 1 \\
\hline$\frac{755}{756}$ & $\begin{array}{ll}754 \\
755 & C \\
C\end{array}$ & $\begin{array}{l}\text { CobattIII) perchlorate, enaxhydrate } \\
\text { Cobalt(II) sulfate hydrate }\end{array}$ & $\frac{13478-333-6}{60459-08-7}$ & 013477-833-6 & $\begin{array}{lll}365.95 \\
173\end{array}$ & $\frac{\mathrm{s}}{\mathrm{s}}$ & & & & & & & $\begin{array}{l}\text { PAA } 27 \text { Litsinin Table } 1 \\
\text { PCC } 27 \text { isting Table } 1\end{array}$ \\
\hline & & 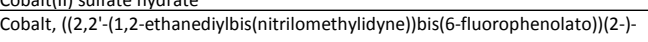 & & & $1 / 3$ & & & & & & & & \\
\hline 757 & 756 & 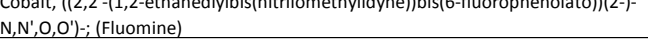 & $62207-76-5$ & $062207-76-5$ & 361.23 & $\mathrm{~s}$ & & & & 1.000E-05 & $2.5000+01$ & & PAC 27 Listing Table 1 \\
\hline 758 & $757 \mathrm{C}$ & Cobaltous bromide; (Cobalt(III) bromide) & $7789-43-7$ & $007789-43-7$ & 218.75 & $\mathrm{~s}$ & $6.780 \mathrm{E}+02$ & & & & & & PAC 27 Listing Table 1 \\
\hline 759 & $758 \mathrm{c}$ & Cobaltous carbonate & $513-79-1$ & $000513-79-1$ & 118.94 & $\mathrm{~s}$ & $280 \mathrm{dec}$ & & & & & & PAC 27 Listing Table 1 \\
\hline$\frac{760}{7761}$ & $759 \mathrm{c}$ & Colchicine & $\frac{64-86-8}{7400-50-8}$ & 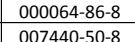 & 399.48 & $\frac{s}{s}$ & $\frac{155-157}{15055+03}$ & & 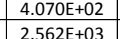 & $\frac{1.000 E-05}{7.5 \times 10.3}$ & $2.500 \mathrm{E}+01$ & & 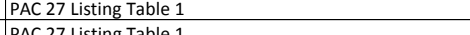 \\
\hline & $761 \mathrm{cc}$ & $\begin{array}{l}\text { Copper } \\
\text { Copper cyanide; (Copper(I) cyanide; Cuprous cyanide) }\end{array}$ & $7440-502-8$ & $\begin{array}{ll}0.00744-050-8 \\
00054-92-3\end{array}$ & $\frac{3.55}{89.56}$ & $\frac{s}{s}$ & 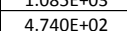 & & 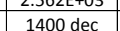 & & & & $\begin{array}{l}\text { PAC C7 Listing gable } 1 \\
\text { PAC 27 listing Table } 1\end{array}$ \\
\hline$\frac{106}{773}$ & & 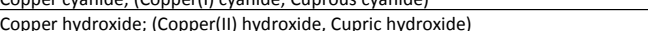 & $\frac{544-92-3}{20427-59-2}$ & $\frac{1003427-35}{0202.2}$ & $\frac{89.96}{97.56}$ & 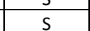 & & & & & & & 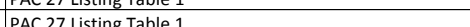 \\
\hline 764 & 763 & Copper nitrate; (Cupric nitrate) & $3251-23-8$ & $003251-23-8$ & 187.56 & $\frac{2}{2}$ & $2.550 E+02$ & & subl & & & & $\begin{array}{l}\text { PAC 27 Listing Table } 1 \\
\end{array}$ \\
\hline 765 & 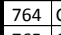 & $\begin{array}{l}\text { Copper oxide; (Copper(I) oxide) } \\
\text { Coper }\end{array}$ & $1317-39-1$ & $\begin{array}{llll}001317-39-1 \\
00758027\end{array}$ & 143.08 & $\mathrm{~s}$ & $1.235 \mathrm{E}+03$ & & $1800 \mathrm{dec}$ & & & & 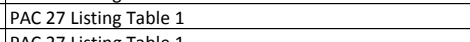 \\
\hline$\frac{766}{6777}$ & $765 \mathrm{c}$ & Copper sulfate; (Copper(III) sulfate) & $7758-98-7$ & $007758-98-7$ & 159.6 & $\frac{5}{5}$ & $\frac{2.000 E+02}{430 \mathrm{E}+2}$ & & $14005+0^{3}$ & 10005000 & 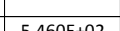 & & PAC 27 Listing Table 1 \\
\hline$\frac{767}{768}$ & $\begin{array}{ll}1060 \\
767 \mathrm{C}\end{array}$ & $\begin{array}{l}\text { Copper(I) chloride; (Cuprous chloride) } \\
\text { Copner(l) suffide }\end{array}$ & $7758-89-6$ & $007758-89-6$ & 98.99 & $\frac{5}{5}$ & $\frac{4.300+02}{1.27}$ & & $1.400 \mathrm{E}+03$ & $1.000 E=+00$ & $5.460 \mathrm{E}+02$ & & 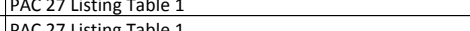 \\
\hline$\frac{106}{769}$ & 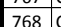 & 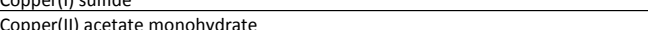 & $\frac{22405-55-4}{6046-93-1}$ & $\begin{array}{llll}0.2205-45-4 \\
006046-93-1\end{array}$ & $\frac{159.14}{199.66}$ & $\frac{2}{3}$ & $1.150 \mathrm{~F}+02$ & & & & & & Pach \\
\hline$\frac{770}{770}$ & $\frac{76996}{769}$ & Copper(III) carbonate hydroxide (2:1:2) & $12069-69-1$ & $0.12069-69-1$ & 221.11 & $\mathrm{~s}$ & $200 \mathrm{dec}$ & & & & & & $\begin{array}{ll}\text { PAC } 27 \text { Listing Table } 1 \\
\end{array}$ \\
\hline 771 & $770 \mathrm{C}$ & $\begin{array}{l}\text { Copper(II) chloride (1:2); (Cupric chloride) } \\
\end{array}$ & $7447-39-4$ & $007447-39-4$ & 134.45 & $\mathrm{~s}$ & $5.980 E+022$ & & $9.930 E+022$ & & & & PAC 27 Listing Table 1 \\
\hline 772 & 771 c & Copper(III) chloride dihydrate; (Cupric chloride) & $\frac{10125-13-0}{814-9-5}$ & 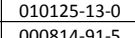 & $\frac{170.48}{153.58}$ & $\frac{s}{s}$ & $1.000 \mathrm{E}+02$ & & $993 \mathrm{dec}$ & & & & PAC 27 Listing Table 1 \\
\hline$\frac{773}{774}$ & $\frac{712}{7736} \mathrm{c}$ & $\begin{array}{l}\text { Coppe(III) xalate } \\
\text { Copper(II) perchlorate, dihydrate }\end{array}$ & $\frac{814-91-5}{17031-32-2}$ & 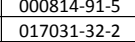 & $\frac{153.58}{298.48}$ & $\frac{s}{s}$ & & & & & & & $\begin{array}{l}\text { PAC } 27 \text { Listing Table } 1 \\
\text { PAC } 27 \text { isting Table } 1\end{array}$ \\
\hline זרק & 774 c & Copper(III) sulfate pentahydrate & $7758-99-8$ & $007758-99-8$ & 249.7 & $\mathrm{~s}$ & 110 lose $4 \mathrm{H} 2 \mathrm{O}$ & & 150 dec & & & & PAC 27 Listing Table 1 \\
\hline 776 & 775 & Copper(II) sulfide & $1317-40-4$ & $001317-40-4$ & 95.6 & $\mathrm{~s}$ & 103 trans. & & $2.000 E+02$ & & & & PAC 27 Listing Table 1 \\
\hline 777 & $\mid \begin{array}{ccc}776 \\
777\end{array}$ & $\frac{\text { Corn oil }}{\text { Coumaghos }}$ & $\frac{8001-30-7}{55-72-4}$ & 008001-30-7 & 883.45 & $\frac{L}{S}$ & $\frac{-1.000 E+01}{9.500 \mathrm{~F}+01}$ & $7.600 E+02$ & $8.330 \mathrm{E}+02$ & $\begin{array}{l}8.0106-21 \\
0.7050 .08\end{array}$ & $2.5006+01$ & $8.0106-21$ & 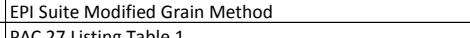 \\
\hline & & & & & & & & & & 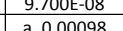 & & & \\
\hline 779 & | $778 \mid \mathrm{C}$ & Coumarin & 91-64-5 & $000091-64-5$ & 146.15 & $\mathrm{~s}$ & 70-71 & & 291-301.7 & 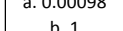 & 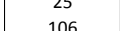 & & PAC 27 Listing Table 1 \\
\hline 780 & 779 & Coumatetralyl; (Endrocide) & $5836-29-3$ & $005836-29-3$ & 292.35 & $\mathrm{~s}$ & $172-176$ & & & & & & PAC 27 Listing Table 1 \\
\hline 781 & $780 \mathrm{C}$ & Creosote (coal tar) & $8001-58-9$ & $008001-58-9$ & & L & & $7.600 E+02$ & 3.000E+02 20.20 & $8.000 E+01$ & $1.0006+02$ & $1.579 E+01$ & Estimated based upon MSDS data, US 614838 3-24-2005 \\
\hline 782 & 781 c & Cresols & $\frac{1319-77-3}{1051-54-1}$ & $001319-77-3$ & $\frac{108.14}{321.1239}$ & $\frac{\mathrm{L}}{\mathrm{s}}$ & $1.100 \mathrm{E}+01$ & $7.600 \mathrm{E}+02$ & $1.910 \mathrm{E}+02$ & & $2.500 E+01$ & 2.9900-01 & PAC 27 Listing Table 1 \\
\hline & $783 \mathrm{c}$ & Crimidine; (Castrix) & $535-89-7$ & $000535-89-7$ & 321.534 .65 & $\mathrm{~s}$ & $8.7006+01$ & & 140-147@ & & & & PAC 27 Listing Table 1 \\
\hline$\frac{784}{785}$ & $784 \mathrm{c}$ & Cristobalite & $14464-46-1$ & $014464-46-1$ & 60.09 & $\mathrm{~s}$ & $1.722 \mathrm{E}+03$ & & $\frac{4 \mathrm{~mm}}{29505+03}$ & & & & PAC 27 Listing Table 1 \\
\hline 786 & $785 \mathrm{C}$ & Crocidolite & 12001-28-4 & $012001-28-4$ & 765.98 & $\mathrm{~s}$ & & & & & & & $\begin{array}{ll}\text { PAC } 27 \text { Listing Table } 1 \\
\end{array}$ \\
\hline 787 & $786[\mathrm{C}$ & Crotonaldehyde & $4170-30-3$ & 004170-30-3 & 70.09 & $\mathrm{~L}$ & $-7.600 E+01$ & $7.600 E+02$ & $1.022 E+02$ & $3.000 E+01$ & $2.5006+01$ & 3.000E+01 & PAC 27 Listing Table 1 \\
\hline 788 & {$[787[\mathrm{c}$} & Crotonalddeh & $123-73-9$ & $000123-73-9$ & 70.1 & & $-6.900 \mathrm{E}+01$ & & & & & & 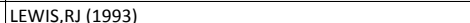 \\
\hline 789 & 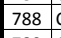 & Crotonic Acid & $3724-65-0$ & $003724-65-0$ & & $\mathrm{~s}$ & $7.200 \mathrm{E}+01$ & & $1.850 \mathrm{E}+02$ & $1.900 \mathrm{E}-01$ & $2.000 \mathrm{E}+01$ & & PAC 27 Listing Table 1 \\
\hline 790 & $789 \mathrm{~s}$ & Crystal violet; (Aniline violet) & $548-62-9$ & $000548-62-9$ & 408.03 & $\mathrm{~s}$ & $2.150 \mathrm{E}+02$ & & & & & & PAC 27 Listing Table 1 \\
\hline & 790 & CS7SB; (1-(2,2,3,3-Tetrafluoropropoxy)-3-(4-sec-buty|phenoxyy-2-2propanol) & 308362-88-1 & 308362-88-1 & 338.341612 & $\mathrm{~L}$ & & $7.600 E+02$ & $3.380 E+02$ & $2.550 \mathrm{E}-06$ & $2.500 E+01$ & $2.550 \mathrm{E}-06$ & EPI Suite Modified Grain Method based upon chemical \\
\hline 792 & $791 \mathrm{c}$ & Cube Resin other than Rotenone & z-0122 & $000002-0122$ & & $\mathrm{~s}$ & & & & & & & 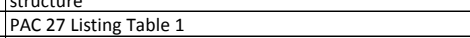 \\
\hline & 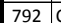 & Cumene hydroperoxide; (Isopropylbenzene hydroperoxide) & & & & $\mathrm{L}$ & & $7.6006+02$ & $1.530 E+02$ & $3.2700-03$ & $2.500 E+01$ & $3.270 \mathrm{E}-03$ & E \& DANNER,RP (1989) \\
\hline 794 & $793[\mathrm{C}$ & Cumene; (Isopropyl benzene) & $98-82-8$ & $000098-82-8$ & 120.19 & L & $-9.602 E+01$ & $7.6000+02$ & $1.524 E+02$ & 4.500E+ +00 & $2.500 E+01$ & 4.500E+o0 & DAUBERT,TE \& DANNER, RP (1985) \\
\hline 795 & 794 ( & Cumenol methylcarbamate, m; ; (3-1-Methylethy)|phenol methylcarbamate) & $64-00-6$ & $000064-00-6$ & 193.27 & $\mathrm{~s}$ & $72-74$ & & $1.430 E+02$ & 4.000E-01 & 2.500E+01 & & PAC 27 Listing Table 1 . \\
\hline 796 & $795[$ [ & Cupferron; (Ammonium--N-itrosophenyyhhydroxylamine) & $135-20-6$ & $000135-20-6$ & 155.19 & $s$ & 163-164 & & & & & & ting Table 1 \\
\hline$\frac{197}{798}$ & 790 & $\begin{array}{l}\text { Cupric acctate, anhyydrous; (Copper acetate) } \\
\text { Curic nitrate heminentanydrate }\end{array}$ & $\frac{142-71-2}{19004-19-4}$ & 000142-71-2 & $\frac{181.64}{24.6}$ & s & $1.150 \mathrm{~F}+02$ & & $170 \mathrm{dec}$ & & & & 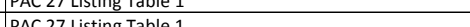 \\
\hline soc & 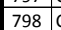 & Cupric nitrite & 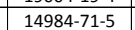 & 019984-71-5 & 155.54 & $\mathrm{~s}$ & & & & & & & 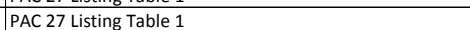 \\
\hline & 799 /c & $\begin{array}{l}\text { Cupric ox } \\
\end{array}$ & $1317-38-0$ & $001317-38-0$ & 79.54 & $\mathrm{~s}$ & $1.326 \mathrm{E}+03$ & & & & & & $\begin{array}{l}\text { PAC } 27 \text { Listing Table } 1 \\
\end{array}$ \\
\hline
\end{tabular}




\begin{tabular}{|c|c|c|c|c|c|c|c|c|c|c|c|c|c|}
\hline & No. & Chemical Compound & $\begin{array}{l}\text { CAS Number } \\
\text { (CASRN) }\end{array}$ & $\begin{array}{c}\text { Modified } \\
\text { Format CAS }\end{array}$ & $\begin{array}{c}\text { Molecular } \\
\text { Weight (MW) }\end{array}$ & \begin{tabular}{|l} 
F \\
Qv State \\
at \\
$25^{\circ} \mathrm{C}$
\end{tabular} & $\begin{array}{c}\text { MP or } F P \\
{\left[{ }^{\circ} \mathrm{C}\right]}\end{array}$ & $\begin{array}{c}\mathrm{H} \\
\begin{array}{c}\text { BP Pressure } \\
{[\mathrm{mm} \mathrm{Hg}]}\end{array}\end{array}$ & $\mathrm{BP}\left[{ }^{\circ} \mathrm{C}\right]$ & $\begin{array}{l}\text { Vapor } \\
\text { Pressure } \\
\text { [mm Hg] }\end{array}$ & $\frac{K}{T}$ & 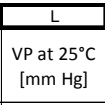 & 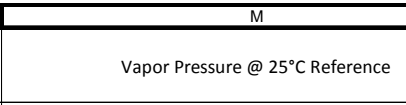 \\
\hline on: & $800 \mid$ & Cyanamide & 420-04-2 & 000420-04-2 & 42.04 & $s$ & 4.556E+01 & & $140 @$ & $3.75 \times 10-3$ & $2.000 E+01$ & & PAC 27 Listing Table 1 \\
\hline 802 & 801 & Cyanic acid; (Hydrogen cyanate) & $420-05-3$ & $000420-05-3$ & 43.025 & $G$ & $-8.600 E+01$ & & $\frac{1.300 \mathrm{E}+01}{2.30}$ & & & & PAC 27 Listing Table 1 \\
\hline 803 & 802 & Cyanide & $57-12-5$ & $000057-12-5$ & 26.02 & $\mathrm{~s}$ & & & & & & & PAC 27 Listing Table 1 \\
\hline 804 & 803 & Cyanoacetamide & $107-91-5$ & $000107-91-5$ & 84.08 & $\mathrm{~s}$ & $1.215 \mathrm{E}+02$ & & dec & & & & PAC 27 Listing Table 1 \\
\hline 805 & 804 & Cyanogen & 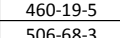 & $000460-19-5$ & 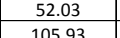 & $\frac{G}{s}$ & $\begin{array}{l}-2.783 \mathrm{E}+01 \\
52005+01\end{array}$ & & $\frac{-2.110 \mathrm{E}+01}{6.60 \mathrm{C}+01}$ & $\begin{array}{l}7.5000+02 \\
1.2025+02\end{array}$ & $\frac{-2.140 E+01}{25005+01}$ & & $\begin{array}{l}\text { PAC 27 Listing Table } 1 \\
\text { PAC C7 }\end{array}$ \\
\hline$\frac{806}{807}$ & $\frac{805}{806}$ & $\begin{array}{l}\text { Cyanogen bromide } \\
\text { Cyanogen chloride }\end{array}$ & 506-68-3-4 & $\begin{array}{l}000006-68-3 \\
000506-7-4\end{array}$ & $\frac{105.93}{61.47}$ & $\frac{S}{G}$ & $\frac{5.200 \mathrm{E}+01}{6.500 \mathrm{E}+00}$ & & $\frac{6.160 E+01}{1.310 E+01}$ & $\frac{1.220 \mathrm{EE}+02}{1.010 \mathrm{E}+03}$ & $\frac{2.500 E \mathrm{E}+01}{2.000 \mathrm{E}+01}$ & & $\begin{array}{l}\text { PAC 27 Listing Gable } 1 \\
\text { PAC } 27 \text { Litsing Table } 1\end{array}$ \\
\hline 808 & & Cyanogen iodide & $5006-78-5$ & $000506-78-5$ & $\frac{15.47}{152.92}$ & $\mathrm{~s}$ & 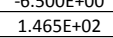 & & & $1.000 E+00$ & $\begin{array}{lll}2.000 E+U 1 \\
2.520 E+01\end{array}$ & & 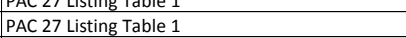 \\
\hline 型 & 808 & Cyanoguanidene; & $461-58-5$ & $000461-58-5$ & 84.08 & $\mathrm{~s}$ & $2.095 E+02$ & & & $3.000 \mathrm{E}+00$ & & & PAC 27 Listing Table 1 \\
\hline & & & $2636-26-2$ & 002636-26-2 20 & 243.23 & & 1.400E+ +01 & $7.600 E+02$ & & $7.8800-04$ & $2.500 E+01$ & $7.880 \mathrm{E}-04$ & TOMLLN,C (1994) \\
\hline$\frac{811}{811}$ & 810 & Cyanuric acid; (1,3,5-Triazine-2,4,6-triol) & $108-80-5$ & $000108-80-5$ & $\begin{array}{l}243.20 \\
129.09\end{array}$ & $\mathrm{~s}$ & 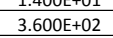 & 1.00uttor & $\frac{1.100 \mathrm{cut}}{380 \text { subl }}$ & 1.000e-04 & 2. $.000 \mathrm{ct}+1$ & 1.000t=-04 & $\begin{array}{l}\text { PAC 27, Listing Table } 1 \\
\text { Pall }\end{array}$ \\
\hline 812 & 811 & Cyanuric chloride & $108-77-0$ & $000108-77-0$ & 184.41 & $\mathrm{~s}$ & $146^{\circ \mathrm{C}}$ & & 194 @ ( 764 & $2.000 E+00$ & $7.000 E+01$ & & PAC 27 Listing Table 1 \\
\hline$\frac{813}{813}$ & 812 & Cyanuric fluoride; (2,4,6-Trifluoro-s-triazine) & $675-14-9$ & $000675-14-9$ & 135.06 & $\mathrm{~s}$ & $-3.800 E+01$ & & $\frac{\operatorname{minfg}}{7.240 \mathrm{E}+01}$ & & & & PAC 27 Listing Table 1 \\
\hline 814 & 813 & cyclohexane & $110-82-7$ & $000110-82-7$ & 84.16 & $G$ & $6.5900++00$ & & $8.073 E+01$ & 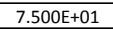 & $1.930 E+01$ & & PAC 27 Listing Table 1 \\
\hline 815 & 814 & 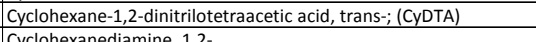 & $13291-61-7$ & $\begin{array}{ll}013291-61-7 \\
025-027\end{array}$ & $\frac{346.3364}{11202}$ & $\frac{s}{s}$ & $213-216$ & & & & & & PAC 27 Listing Table 1 \\
\hline$\frac{816}{817}$ & $\frac{815}{816}$ & $\begin{array}{l}\text { Cyclohexanediaminin, } 1,2-\text { - } \\
\text { Cyclohexanedimethanol cis and trans, } 1.4 \text { - }\end{array}$ & $694-83-7$ & $\begin{array}{l}000694-83-7 \\
0000105-08-8\end{array}$ & $\frac{114.22}{144.24}$ & $\frac{s}{s}$ & $\begin{array}{l}\frac{4.000 E+01}{41-61} \\
41\end{array}$ & 1.800E +01 & $\frac{9.2006 \mathrm{E}+01}{2.70 \mathrm{E}+02}$ & & & & $\begin{array}{l}\text { PAC C } 7 \text { Listing gable } 1 \\
\text { PAC 27 listing Table } 1\end{array}$ \\
\hline$\frac{817}{818}$ & $\frac{8117}{817}$ & 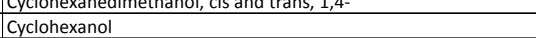 & $\frac{105-08-8}{108-93-0}$ & $\begin{array}{ll}-0000105-08-8 \\
000108-93-0\end{array}$ & $\frac{144.24}{100.16}$ & $\frac{S}{L}$ & $\frac{41-61}{2.400 E+01}$ & $7.600 E+02$ & 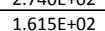 & $8.000 E-01$ & $2.500 E+01$ & 3.000E-0 0 & 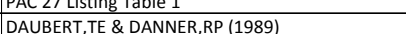 \\
\hline$\frac{26}{819}$ & 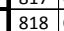 & $\begin{array}{l}\text { Cyclohexanonone; (Ketohexamethylene) } \\
\text { Cyction }\end{array}$ & $\frac{108-94-1}{108}$ & $000108-94-1$ & $\frac{0.0 .10}{98.14}$ & $\frac{\mathrm{L}}{\mathrm{L}}$ & 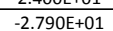 & $\frac{1.9006 \mathrm{~F}}{7.602}$ & 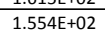 & 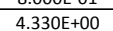 & $\frac{2.50 \mathrm{E}+1}{2.50 \mathrm{E}+1}$ & $\frac{0.0306+0}{4.30+0}$ & D \\
\hline$\frac{820}{820}$ & 819 & Cyclohexene & $110-83-8$ & $000110-83-8$ & 82.15 & & 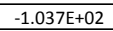 & $7.600 \mathrm{E}+02$ & $\begin{array}{l}8.300 E+01 \\
\end{array}$ & $8.9006+01$ & $2.500 E+01$ & $8.9006+01$ & DAUBERT,TE \& DANNER,RP (1989) \\
\hline 821 & 820 & Cycloheximide & $66-81-9$ & $000066-81-9$ & 281.39 & $\mathrm{~s}$ & 1199-121 & & & $6.000 \mathrm{E}-03$ & $2.500 \mathrm{E}+01$ & & PAC 27 Listing Table 1 \\
\hline$\frac{822}{8023}$ & $\frac{821}{802}$ & 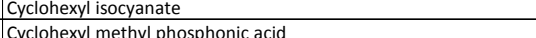 & $\frac{3173-53-3}{1932-60-1}$ & $003173-53-3$ & $\frac{125.19}{10717}$ & $\frac{s}{s}$ & $\frac{168-170}{012032}$ & & & & & & 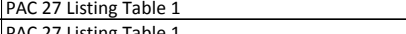 \\
\hline 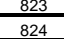 & $\frac{822}{823}$ & $\begin{array}{l}\text { Cyclohexyl methyl phosphonic acid } \\
\text { Cyclohexylamine }\end{array}$ & $\frac{1932-60-1}{108-91-8}$ & $\begin{array}{l}001932-60-1 \\
000108-91-8 \\
\end{array}$ & $\frac{178.17}{99.17}$ & $\frac{S}{L}$ & $\begin{array}{l}41.243 .8 \\
-1.7805+01\end{array}$ & $7.600 E+02$ & $1.340 \mathrm{E}+02$ & $1.010 E+01$ & $2.500 E+01$ & $1.010 \mathrm{E}+01$ & 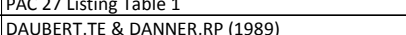 \\
\hline$\frac{84}{825}$ & $\frac{824}{824}$ & 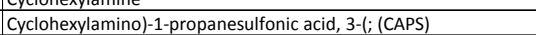 & $\frac{1130-9-0.6}{113-40-6}$ & $001135-40-6$ & $\frac{95.17}{221.35}$ & S & $\frac{-1.1000+01}{300}$ & & & & & & 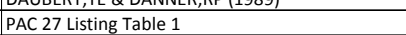 \\
\hline 826 & 825 & Cyclohexylethanol, 2- & $4442-79-9$ & 004442-79-9 & 128.24 & $\mathrm{~L}$ & $8.7006+01$ & $7.600 E+02$ & $2.080 E+02$ & $1.6600-01$ & $\begin{array}{l}2.500 E+01 \\
\end{array}$ & $1.6600-01$. & PERRY,RH \& GREEN,D (1984) \\
\hline 827 & 826 & Cyclooctadiene, $1,5-$ & 1111-78-4 & $000111-78-4$ & 108.2 & $\mathrm{~L}$ & $-6.900 E+01$ & $7.600 E+02$ & $1.508 E+02$ & $4.952 E+00$ & $2.5006+01$ & $4.952 E+00$ & YAWS, CL (1994) \\
\hline$\frac{828}{889}$ & 487 & 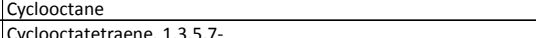 & 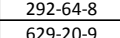 & $000292-64-8$ & $\frac{112.213}{104.1512}$ & $\frac{\mathrm{L}}{\mathrm{L}}$ & $\frac{1.500 E+01}{7.0005+00}$ & $7.600 \mathrm{E}+022$ & & 5.510EF+00 & & 5.510F地0 & OHE,S (1976) \\
\hline$\frac{829}{830}$ & tos & $\begin{array}{l}\text { Cyclooctatetraene, } 1,3,5,7- \\
\text { Cyclooctene cis- }\end{array}$ & $\frac{629-20.9}{938-37}-3$ & $0006291-20-9$ & $\frac{104.1512}{110197}$ & it & 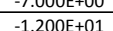 & 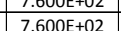 & $1.402+02$ & 1.9006 & 2.500E EU1 & $\frac{1.900 \mathrm{E}+00}{8600}$ & 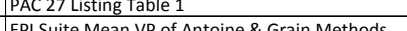 \\
\hline$\frac{300}{801}$ & 烈 & 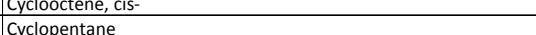 & 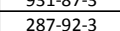 & 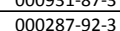 & $\frac{1.10 .137}{70.13}$ & 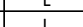 & 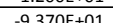 & 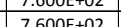 & $1.400+02$ & 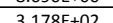 & 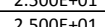 & 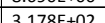 & 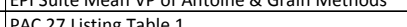 \\
\hline 832 & (831 & Cyclopentanone & $120-92-3$ & $000120-92-3$ & 84.12 & $\mathrm{~L}$ & 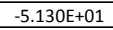 & $7.600 E+02$ & $1.306 \mathrm{E}+02$ & $1.140 E+01$ & $2.500 E+01$ & $1.140 E+01$ & $\begin{array}{l}\text { PAC 27 Listing Table } 1 \\
\text { Pal }\end{array}$ \\
\hline 833 & 832 & Cyclopropane & $75-19-4$ & $000075-19-4$ & 42.09 & G & $-1.266 \mathrm{E}+02$ & & & & & & PAC 27 Listing Table 1 \\
\hline$\frac{834}{835}$ & $\frac{383}{834}$ & 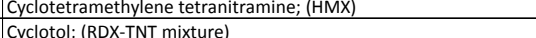 & $2691-41-0$ & $002691-41-0$ & $\frac{296.2}{449.3}$ & $\frac{s}{s}$ & $2.1900 \mathrm{E}+02$ & & $\operatorname{exp@Q2/9}$ & & & & $\begin{array}{lll}\text { PAC } 27 \text { Listing Table } 1 \\
\text { PAC 27lițing Tazle } 1\end{array}$ \\
\hline$\frac{336}{836}$ & 年 & Cyclotrimethy-enetrinitramine; (RDX or Cyclonite) & 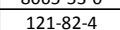 & & & & $2.020 E+02$ & & expl & $4.000=04$ & $1100 \mathrm{0}=02 \mathrm{O}$ & & 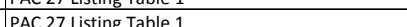 \\
\hline 8377 & 836 & Cytridine monophosshatate; (Cytidinine 5 -monophosphhate free acid) & 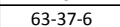 & $000063-37-6$ & 323.23 & $\mathrm{~s}$ & $233 \mathrm{dec}$ & & & & & & PAC 27 Listing Table 1 \\
\hline 838 & 837 & 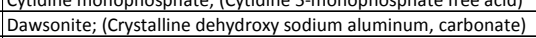 & $12011-76-6$ & $012011-76-6$ & 143.995187 & $\mathrm{s}_{\mathrm{s}}$ & (2) 50 & & & & & & $\begin{array}{l}\text { PAC } 27 \text { Listing Table } 1 \\
\end{array}$ \\
\hline 839 & 838 & DDD; (1,1-bis(4-Chloropheny)|-2,2-dichloroethane) & $72-54-8$ & $000072-54-8$ & 320.04 & $\mathrm{~s}$ & $1.095 \mathrm{E}+02$ & & $193 @$ & $1.35 \times 10-6$ & $2.500 E+01$ & & PAC 27 Listing Table 1 \\
\hline$\frac{800}{840}$ & & DDE; (2,2-2-bis(p-Chlorophenyy)|-1,-1-dichloroethylene) & $72-55-9$ & $000072-55-9$ & 318.03 & $\mathrm{~s}$ & $8.900 E+01$ & & & $6.0 \times 10-6$ & $2.500 E+01$ & & PAC 27 Listing Table 1 \\
\hline 841 & 840 & DDT; (Dichlorodiphenyltrichloreethane) & $50-29-3$ & $000050-29-3$ & 354.49 & $\mathrm{~s}$ & $1.085+02$ & & $2.600 \mathrm{E}+02$ & $1.6 \times 10-7$ & $2.000 E+01$ & & PAC 27 Listing Table 1 \\
\hline 842 & 841 & DEAE Sepharose CL-6B & $62610-50-8$ & 062610-50-8 & & $\frac{s}{s}$ & OQ60F 01 & & 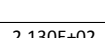 & & & & $\begin{array}{l}\text { PAC } 27 \text { Listing Table } 1 \\
\text { PAC 27| }\end{array}$ \\
\hline$\frac{843}{894}$ & $\frac{842}{883}$ & $\begin{array}{l}\text { |lecaborane } \\
\text { Decafluoropentane } 1,1,1,2,4,4,5,5.5\end{array}$ & $\frac{17702-251-9}{138495-8}$ & $\begin{array}{l}017702-41-9 \\
13895-42-8\end{array}$ & $\frac{122.24}{25207}$ & & & $76005+02$ & $\begin{array}{l}2.130 \mathrm{E}+02 \\
5.50 \mathrm{~T}+01\end{array}$ & $5.000-02$ & $2.500 E+01$ & $226050202-3250$ & $\begin{array}{l}\text { PAC } 27 \text { Listing Table } 1 \\
\text { PACZ2 }\end{array}$ \\
\hline$\frac{344}{85}$ & (8) & De & 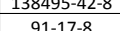 & 000091-1-1-8 & 138 & 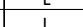 & $-4,3005+01$ & 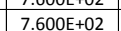 & 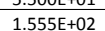 & 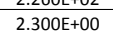 & 250 $250=1$ & $\begin{array}{ll}2 \\
2300 \mathrm{~F}\end{array}$ & 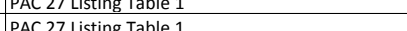 \\
\hline 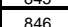 & (854) & Decabydronaphthalene cis-_(cis-Decalin) & 493-01-6 & $000493-01-6$ & 13824 & L & 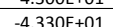 & $7600+52$ & $1965 \mathrm{E}+02$ & $23000+00$ & $2500 \mathrm{~F}+01$ & $2300 \mathrm{E}+00$ & $\begin{array}{l}\text { PAC } \\
\text { PA Listitis Table } 1\end{array}$ \\
\hline$\frac{847}{847}$ & 846 & Decahydronaphthalene, trans;; (trans-Decalin) & $493-02-7$ & $000493-02-7$ & 138.28 & $\mathrm{~L}$ & $-3.070 E+01$ & $7.600 E+02$ & $1.873 \mathrm{E}+02$ & 1.220E+00 & $2.5006+01$ & 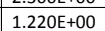 & 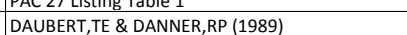 \\
\hline 848 & 847 & Decamethylicyclopentasiloxane & $541-02-6$ & $000541-02-6$ & 370.77 & $\mathrm{~L}$ & $-3.8000+01$ & $7.600 E+02$ & $2.1006+02$ & $2.000 E-01$ & $2.5006+01$ & $2.000 E-01$ & FLANINGAM,OL (1986) \\
\hline$\frac{849}{0.905}$ & $f_{848}^{848}$ & 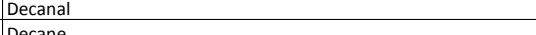 & $112-31-2$ & $000112-31-2$ & $\frac{156.3}{14202}$ & $\frac{L}{L}$ & $-5.0006+00$ & $\begin{array}{l}7.600 E+02 \\
7.6005+02\end{array}$ & $2.085 E+02$ & $1.030 \mathrm{E}-01$ & $2.500 E+01$ & $\begin{array}{l}1.030 E-01 \\
1.4305000\end{array}$ & $\begin{array}{l}\text { PAC 27 Listing Table } 1 \\
\text { PAC }\end{array}$ \\
\hline$\frac{350}{851}$ & $f_{850}^{0.94}$ & $\begin{array}{l}\text { Jecane } \\
\text { Decene 1- homonolymer hydrogenated }\end{array}$ & $\frac{120-18-5}{68037-01-4}$ & $\begin{array}{l}0008242-1-1-5 \\
068037-01-4\end{array}$ & 142.282 & L & $\begin{array}{l}2 \\
-6.500 \mathrm{~F}+01 \\
-6.01\end{array}$ & 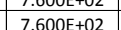 & 3.750F & 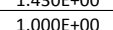 & 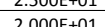 & $1.420+6+00$ & $\begin{array}{l}\text { PAC 27 Listing Table } 1 \\
\text { Estimated using data from PAC } 27 \text { listing Table } 1\end{array}$ \\
\hline$\frac{152}{852}$ & 851 & Devy alcoholi (1-Decanol) & 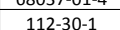 & $0000112-30-11$ & 158.32 & L & $7.0006+00$ & $7.6006+02$ & $2.3296+02$ & $8.510 \mathrm{E}-03$ & $2.500+5+01$ & $8.50=03$ & 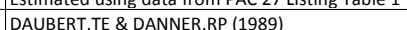 \\
\hline 853 & 852 & Demeton & 8065-48-3 & $008065-48-3$ & 516.72 & L & $-1.3000 \mathrm{E}+01$ & $2.000 E+00$ & 1.340E+02 & $3.000 E-03$ & 2.500E+01 & 3.000E & ChemSpider Experimental Data \\
\hline 854 & 853 & Demeton-S-methyl & $919-86-8$ & $000919-86-8$ & 230.3 & L & & 1.500E-01 & 8.900E +01 & 3.000E-04 & & 3.000E-04 & TOMLIN, (2003) \\
\hline 855 & 854 & Deoxyribonucleic acid; (DNA) & $9007-49-2$ & 0099007-99-2 & 523.3692 & $\frac{S}{G}$ & 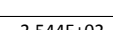 & & 20055007 & & & & $\begin{array}{l}\text { PAC } 27 \text { Listing Table } 1 \\
\text { Pa C27lition }\end{array}$ \\
\hline$\frac{566}{857}$ & $\int_{856}^{835}$ & 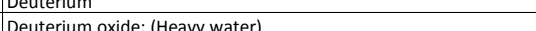 & 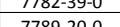 & 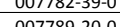 & $\frac{2.03}{2003}$ & $\mathrm{G}$ & 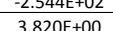 & $76005+02$ & 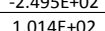 & $2060 \mathrm{E}+01$ & $2500 \mathrm{E}+01$ & $2060 \mathrm{E}+01$ & 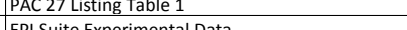 \\
\hline$\frac{358}{885}$ & (857) & Deuterochloric acidd. Deuterium chloride) & $7698-0.5-7$ & $007698-05-7$ & 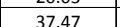 & $G$ & 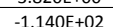 & & $-8.500 \mathrm{~F}+01$ & & & & PAC C7 listing Tazle 1 \\
\hline 859 & 858 & Dextran & 9004-54-0 & $009004-54-0$ & 200000 & $\mathrm{~s}$ & & & & & & & 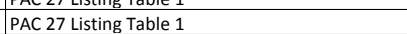 \\
\hline 860 & 859 & Dextran sulfate sodium & 9011-18-1 & 009011-18-1 & & $\mathrm{s}$ & & & & & & & PAC 27 Listing Table 1 \\
\hline 62 & $\begin{array}{ll}8060 & 0\end{array}$ & 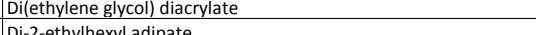 & 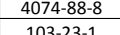 & & 214.24 & +1 & $\begin{array}{l}-3.473 \mathrm{E}+01 \\
-6.7805+01\end{array}$ & 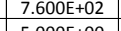 & 2.0000E+02 & 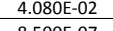 & 钟+01 & 4.0.000 & $\begin{array}{l}\text { EFI Suite Mean VP of Anto } \\
\text { EF The }\end{array}$ \\
\hline
\end{tabular}




\begin{tabular}{|c|c|c|c|c|c|c|c|c|c|c|c|c|c|}
\hline & No. & Chemical Compound & $\begin{array}{l}\text { CAS Number } \\
\text { (CASRN) }\end{array}$ & $\begin{array}{c}\text { Modified } \\
\text { Format CAS }\end{array}$ & \begin{tabular}{|c} 
Molecular \\
Weight (MW)
\end{tabular} & \begin{tabular}{|c|} 
ov state \\
at \\
$25^{\circ} \mathrm{C}$
\end{tabular} & $\begin{array}{c}\text { MP or FP } \\
\text { ["CC] }\end{array}$ & $\begin{array}{c}\text { BP Pressure } \\
{[\mathrm{mm} \mathrm{Hg}]}\end{array}$ & $\mathrm{BP}\left[\mathrm{I}^{\mathrm{C} C}\right]$ & $\begin{array}{l}\text { Vapor } \\
\text { Pressure } \\
{[\mathrm{mm} H \mathrm{Hg}]}\end{array}$ & $\begin{array}{c}T \text { for VP } \\
{\left[{ }^{\circ} \mathrm{C}\right]}\end{array}$ & $\begin{array}{l}\text { VPat } 25^{\circ} \mathrm{C} \\
{[\mathrm{mm} \mathrm{Hg}]}\end{array}$ & Vapor Pressure @ $25^{\circ} \mathrm{C}$ Reference \\
\hline 863 & 862 [ & Diacetoxydibutyly stannane & 1067-33-0 & $001067-33-0$ & 351.05 & & $1.000 E+01$ & $7.600 E+02$ & $1.300 E+02$ & $1.300 E+00$ & $2.000 E+01$ & $1.923 E+00$ & Estimated using data from PAC 27 Listing Table 1 \\
\hline & 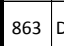 & Diacetyl peroxide; (Acetyl peroxide) & $110-22-5$ & $000110-22-5$ & 118.04 & $\mathrm{~s}$ & $3.000 E+01$ & & 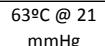 & & & & PAC 27 Listing Table 1 \\
\hline 865 & 8640 & Dialifor & $10311-84-9$ & $010311-84-9$ & 393.846 & $\mathrm{~s}$ & $6.800 E+01$ & & & $6.200 \mathrm{E}-08$ & $2.500 \mathrm{E}+01$ & & PAC 27 Listing Table 1 \\
\hline 866 & 865 D & Dially phthalate & $131-17-9$ & $000131-17-9$ & 246.28 & L & $-7.000 E+01$ & $5.000 E+00$ & $1.660 \mathrm{E}+02$ & $1.1600-03$ & $2.500 \mathrm{E}+01$ & $1.160 \mathrm{E}-03$ & EPI Suite Modified Grain Method \\
\hline 867 & $\begin{array}{lll}866-\mathrm{D} \\
867 \mathrm{D}\end{array}$ & \begin{tabular}{|l}
$\mid$ Dilllyldimethyllammonium chloride \\
Diallyldimethylammonium chloride polymer
\end{tabular} & $\frac{7398-6998}{2602-79-3}$ & 007398-69-9-8 & 161.7 & $\frac{s}{s}$ & & & & & & & $\begin{array}{l}\text { PAA 27 listing Table } 1 \\
\text { PCC } 2 \text { listing Table } 1\end{array}$ \\
\hline & $8688^{0}$ & 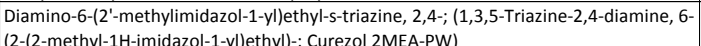 & $38668-46-1$ & $038668-46-1$ & 219.2486 & $\mathrm{~s}$ & & & & & & & PAC 27 Listing Table 1 \\
\hline 869 & $8699^{6}$ & 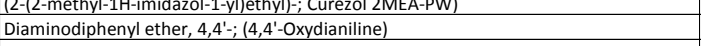 & $101-80-4$ & $000101-80-4$ & 200.26 & $\mathrm{~s}$ & $1.870 \mathrm{E}+02$ & & $>300$ & $3.000 E-07$ & $2.500 \mathrm{E}+01$ & & PAC 27 Listing Table 1 \\
\hline 871 & 870 & Diaminodiphenylsulfone; ; 4,4 - S-Sulfonyldianiline) & $80.08 \cdot 0$ & $000080.08-0$ & 248.32 & s & $1.760 \mathrm{E}+02$ & & & & & & $\begin{array}{l}\text { PAC } 27 \text { Listing Table } 1 \\
\end{array}$ \\
\hline$\frac{872}{873}$ & $\begin{array}{lll}871 \\
872[D\end{array}$ & $\begin{array}{l}\text { |liaminodidropplamine, 3,3;; (Aminobis(propylamine)) } \\
\text { Diaminopyridine, } 2,6-\text {. }\end{array}$ & $\frac{56-188.8}{141-86-6}$ & 些00056-188-8 & $\frac{131.26}{10.15}$ & $\frac{t}{s}$ & $\frac{-1.450 E+01}{121.5}$ & $7.600 \mathrm{E}+02$ & $2.355 \mathrm{EE}+02 \mathrm{~F}$ & 8.620E-02 & $2.5000++01$ & 8.620E-02 & $\begin{array}{l}\text { EPA Suite Mean V V of Antoine \& Grain Methods } \\
\text { PAC 27 listing Table } 1\end{array}$ \\
\hline t & 873 & $\begin{array}{l}\text { Diammonium dimolybdate; } \\
\text { (Ammonium molybdenum oxide) }\end{array}$ & $27546-07-2$ & $027546-07-2$ & 337.9135 & $\mathrm{~s}$ & & & & & & & PAC 27 Listing Table 1 \\
\hline 875 & 874 & $\begin{array}{l}\text { (Antimonium moryouenum oxice) } \\
\text { Diamond }\end{array}$ & $7782-40-3$ & 007782-40-3 & 12.01 & $\mathrm{~s}$ & $4440 @ 12.4$ & & & & & & PAC 27 Listing Table 1 \\
\hline 876 & 875 品 & Dianisidine dihydrochloride, $0 ;$; (3,3,'-Dimethoxybenzidine dihydrochloride) & $20325-40-0$ & $020325-40-0$ & 317.24 & $s$ & $1.370 \mathrm{E}+02$ & & & $8.800 \mathrm{E}-09$ & $2.5000++01$ & & 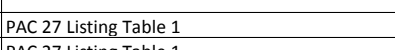 \\
\hline 877 & $\begin{array}{ll}876 \\
877[0\end{array}$ & 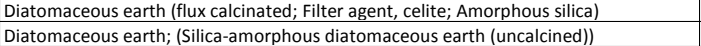 & $\frac{68859-54-9}{61790-53-2}$ & 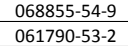 & 60.1 & $\frac{\mathrm{s}}{\mathrm{s}}$ & $\frac{1.083 \mathrm{E}+03}{1.00 \mathrm{E}+03}$ & & $2.595 \mathrm{E}+03$ & & & & $\begin{array}{l}\text { PAA } 27 \text { Listing Table } 1 \\
\text { PAC } 27 \text { isting Table } 1\end{array}$ \\
\hline 879 & 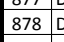 & Diatomaceous silica, calcined & $\begin{array}{ll}019053-39-2 \\
91053-3\end{array}$ & 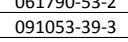 & & $\frac{2}{3}$ & & & & & & & $\begin{array}{l}\text { PAC } 27 \text { Listing Table } 1 \\
\text { Pable } 1\end{array}$ \\
\hline 880 & & Diazabicyclo(2,2,2)|coctane, 1,4- & $280-57-9$ & $000280-57-9$ & 112.2 & s & 158 & & $1.740 E+02$ & & & & PAC 27 Listing Table 1 \\
\hline 881 & $880[\mathrm{D}$ & Diazabicyclo(5.4.0)|undec-7-ene, 1,8- & 6674-22-2 & $006674-22-2$ & 152.24 & L & $-7.000 E+01$ & $6.000 \mathrm{E}-01$ & 8.000E +01 & 5.300E+00 & 3.770E+01 & 1.150E+01 & Estimated using data from PAC 27 listing Table 1 \\
\hline 882 & 881 D & Diazoacetic acid, ethyl ester; (Ethyl diazoacetate) & 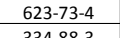 & $000623-73-4$ & $\frac{114.12}{10.04}$ & $\mathrm{~L}$ & $-2.200 E+01$ & $7.2006+02$ & 1.410E+02 & $2.800 E+00$ & $2.500 E+01$ & 2.800E+00 & MERCK INDEX (1996) \\
\hline 883 & 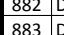 & $\begin{array}{l}\text { Diazomethane } \\
\text { Dibenzanananthracene }\end{array}$ & $\begin{array}{l}334-88-3 \\
53-70-3\end{array}$ & 000334-88-3 & $\frac{42.04}{27.35}$ & $\frac{G}{S}$ & 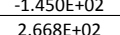 & & $\begin{array}{l}-2.300 E+01 \\
5.240+02\end{array}$ & & & & 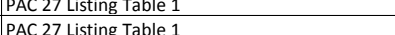 \\
\hline$\frac{684}{885}$ & 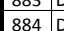 & 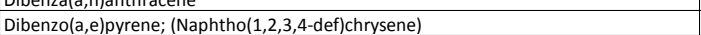 & $\begin{array}{l}53-70-3 \\
192-65-4\end{array}$ & $\begin{array}{ll}0 \\
000053-102-65-4 \\
00019\end{array}$ & $\frac{28.35}{302.38}$ & s & 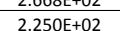 & & & & & & $\begin{array}{l}\text { PAC } \\
\text { PAC Listing Table } 1\end{array}$ \\
\hline$\frac{2066}{886}$ & 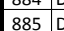 & Dibenzofuran & $132-64-9$ & $000132-64-9$ & 168.2 & s & 8 & & $2.870 E+02$ & $4.4000-03$ & $2.500 E+01$. & & PAC 27 Listing Table 1 \\
\hline 887 & $886\left[\begin{array}{c}0 \\
8\end{array}\right.$ & Dibenzo-p-dioxin & $262-12-4$ & $000262-12-4$ & 184.2 & $\mathrm{~s}$ & $1.190 \mathrm{E}+02$ & & & 4.125E-04 & $2.500 E+01$ & & PAC 27 Listing Table 1 \\
\hline 888 & 887 [D & Diborane & $19287-45-7$ & $019287-45-7$ & 27.67 & G & $-1.649 \mathrm{E}+02$ & & $-9.249 E+01$ & $7.500 E+02$ & $-9.260 \mathrm{E}+01$ & & PAC 27 Listing Table 1 \\
\hline 889 & $\begin{array}{lll}888 & 0 \\
889 & D\end{array}$ & 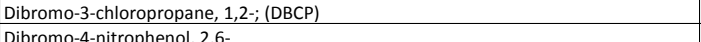 & $96-12-8$ & $000096-12-8$ & 236.33 & L & $5.000 E+00$ & $7.600 E+02$ & $1.960 \mathrm{E}+02$ & 5.800E-01 & $2.500 E+01$ & 5.800E-001 & 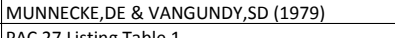 \\
\hline 890 & 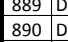 & $\begin{array}{l}\text { Dibromo-4n-itifophenol, 2,6- } \\
\text { Dibromochloromethane; (Chlorodibromomethane) }\end{array}$ & $\begin{array}{l}99-28-5 \\
124-48-1 \\
\end{array}$ & $\begin{array}{l}-1 \\
000099-28-5 \\
000124-1 \\
\end{array}$ & 296.98 & $\frac{S}{L}$ & $\begin{array}{l}145 \mathrm{dec} \\
-2.000 \mathrm{E}+01 \\
\end{array}$ & $7.600 E+02$ & $1.200 E+02$ & & & $5.540 E+00$ & $\begin{array}{l}\text { PAC } 27 \text { 7istitigg Table } 1 \\
\text { PAC Listing Table } 1\end{array}$ \\
\hline 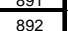 & (891]D & Dibromohexane, 1,6 - & $\begin{array}{l}124-40-1 \\
629-03-8\end{array}$ & 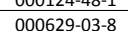 & 201.93 & L & 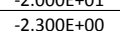 & 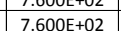 & 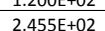 & $\begin{array}{l}3.5450-02 \\
5.50-02\end{array}$ & 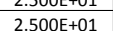 & 5.550-0.02 & $\begin{array}{l}\text { PAC I LItsing Iable } 1 \\
\text { EPI Suite Mean VP of Antoine \& Grain Methods }\end{array}$ \\
\hline 893 & (892]0 & Dibromomethane; (Methylene dibromide) & 74-95-3 & $000074-95-3$ & $\begin{array}{lll}173.85 \\
17.85\end{array}$ & $\mathrm{~L}$ & $-5.270 E+01$ & $7.600 \mathrm{E}+02$ & 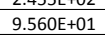 & 4.440E+01 & $2.500 \mathrm{E}+01$ & $4.440 E+01$ & $\begin{array}{l}\text { PAC } 27 \text { Listing Table } 1 \\
\text { 1 }\end{array}$ \\
\hline 894 & 893 ए0 & Dibromophenol, 2,6- & $608-33-3$ & $000608-33-3$ & 251.905 & $\mathrm{~s}$ & & & & & & & PAC 27 listing Table 1 \\
\hline 895 & 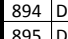 & Dibromopropane, 1,3- & 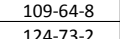 & $000109-64-8$ & 201.91 & L & $\begin{array}{l}-3.600 E+01 \\
-1.120 E+02\end{array}$ & $\begin{array}{l}7.600 E+02 \\
7.600+5+02\end{array}$ & $\begin{array}{l}1.665 \mathrm{E}+02 \\
4.730 \mathrm{~F}+01\end{array}$ & $\begin{array}{l}1.360 \mathrm{E}+00 \\
3.250 \mathrm{~F}+02\end{array}$ & $\begin{array}{l}2.500 E+01 \\
2.500+5+01\end{array}$ & $\begin{array}{l}1.360 E+00 \\
3.250 F+02\end{array}$ & HINE, \& MOOKERJE,PK (1975) \\
\hline$\frac{896}{897}$ & $\begin{array}{l}895 \\
8966 \\
896\end{array}$ & 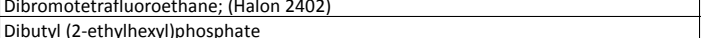 & 124-0011-2 & $\begin{array}{l}0.00124-73-2 \\
000002-0011\end{array}$ & $\frac{2299.84}{322.42512}$ & $\frac{L}{L}$ & & & & $\begin{array}{l}3.250 \mathrm{E}+02 \\
1.800 \mathrm{E}-07\end{array}$ & $\begin{array}{l}2.500+20101 \\
2.500+01\end{array}$ & $\begin{array}{lll}3.250 \mathrm{E}+02 \\
1.800 \mathrm{E}-07\end{array}$ & 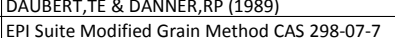 \\
\hline 898 & (896 890 & Dibutyl butylyhosphononate & $\frac{-2011}{78-46-6}$ & $00078-46-6$ & $\begin{array}{lll}32 L .42512 \\
250.36\end{array}$ & $\frac{L}{L}$ & & $7600 \mathrm{~F}+02$ & 1.600E+02 $>202020$ & $7.130-0.04$ & 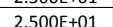 & $7.130 \mathrm{E}-04$ & 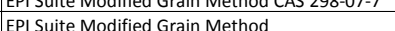 \\
\hline $\begin{array}{lll}899 \\
899\end{array}$ & $898 \mid \mathrm{D}$ & Dibutyl peroxide, tert- & $\frac{110-05-4}{10}$ & $0000110-05-4$ & 146.26 & $\mathrm{~L}$ & $-4.000 E+01$ & $7.600 \mathrm{E}+02$ & 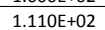 & $2.510 \mathrm{E}+01$ & $2.500 \mathrm{E}+01$ & $2.5106+01$ & $\begin{array}{l}\text { PAC } 27 \text { L Listing Table } 1 \text {. } \\
\text { Putentiou }\end{array}$ \\
\hline 900 & 8990 & Dibutyl phosphate & $107-66-4$ & $000107-66-4$ & 210.2 & $\mathrm{~L}$ & & $5.000 \mathrm{E}-02$ & $1.350 \mathrm{E}+02$ & $1.000 E+00$ & $2.500 E+01$ & $1.000 E+00$ & ChemSpider Experimental Data \\
\hline 9019 & {$\left[\begin{array}{ccc}9001 \\
901 \\
D\end{array}\right.$} & $\begin{array}{l}\text { Dibuty phosphite } \\
\text { Dibuty / phthalate }\end{array}$ & $\frac{1809-19-4}{84-74-2}$ & 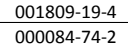 & $\frac{194.24}{27.34}$ & $\frac{L}{L}$ & $\frac{1.738 E+01}{-3.500 E+01}$ & $\frac{1.000 \mathrm{E}+00}{7.600+202}-1$ & 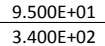 & $\begin{array}{l}2.430 \mathrm{E}-02 \\
2.0005-05\end{array}$ & 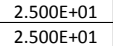 & $\begin{array}{l}2.430 E-02 \\
2.0010-05\end{array}$ & 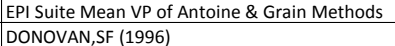 \\
\hline 903 & 9020 & Dibutylboron trifilate; (Dibutylyory) trifluoromethanesulfonate) & $60669-69-4$ & $060669-69-4$ & 274.11 & $\frac{\mathrm{L}}{\mathrm{s}}$ & 4.44 & & & & & & $\begin{array}{l}\text { DUNOVAA,SF (I96) } \\
\text { PAC } 27 \text { Listing Table } 1\end{array}$ \\
\hline 904 & 903 D & Dibutyhhexamethylenediamine, $\mathbb{N}^{N} \mathrm{~N}^{\prime}$ - & $\begin{array}{ll}4835-11-4 \\
77-60-7\end{array}$ & $004835-11-4$ & 228.48 & $\mathrm{~L}$ & & $7.600 E+02$ & $2.050 \mathrm{E}+02$ & $\begin{array}{l}4.000 E-04 \\
.06000\end{array}$ & 2.500e+01 & $4.000 E-04$ & $\begin{array}{l}\text { PAC } 27 \text { Listing Table } 1 \\
\text { Ca }\end{array}$ \\
\hline 905 & $\begin{array}{l}904 \\
905\end{array}$ & 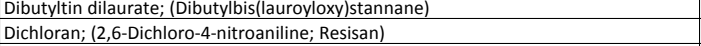 & $\begin{array}{l}7-58-7 \\
99-30.9 \\
\end{array}$ & $\begin{array}{ll}0.00077-58-7 \\
000099-30-9\end{array}$ & $\frac{63.1 .65}{207.02}$ & $\frac{\mathrm{L}}{\mathrm{s}}$ & 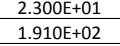 & & & $\begin{array}{lll}4.460 \mathrm{E}-09 \\
0.16 \mathrm{mPa}\end{array}$ & $\begin{array}{l}2.500+E+01 \\
2.000+01\end{array}$ & & $\begin{array}{l}\text { EI I I uite Modified drain Method } \\
\text { PAC } 27 \text { Listing Table } 1\end{array}$ \\
\hline & $9060^{0}$ & 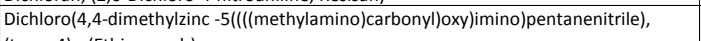 & 58270-08-9 & $058270-08-9$ & 333.54 & $\mathrm{~s}$ & $120-125$ & & & $1.000 \mathrm{E}-05$ & $2.500+01$ & & PAC 27 Listing Table 1 \\
\hline$\frac{907}{908}$ & $9077^{1 / 2}$ & 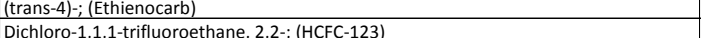 & $306-83-2$ & 0000306-83-2 & 152.93 & & $-1.070 \mathrm{E}+02$ & $7600 E+02$ & $2.782 E+01$ & $7.180 E+02$ & 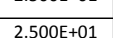 & $180 E+02$ & PAC 27 Listing Table 1 \\
\hline & & 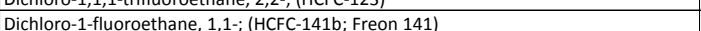 & 1717-50-6 & & 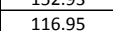 & L & 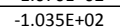 & $7.600 \mathrm{E}+02$ & $3.200 \mathrm{E}+01$ & & $2.500 \mathrm{E}+01$ & 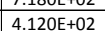 & 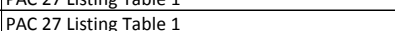 \\
\hline$\frac{510}{9010}$ & 1009090 & Dichloro-2-butene, 1,4- & $\begin{array}{ll}1174-41-0 \\
764-0\end{array}$ & $000764-41-0$ & 125 & $\frac{\mathrm{L}}{\mathrm{L}}$ & $3.500 E+00$ & $7.600 \mathrm{E}+02$ & $1.580 \mathrm{t}+02$ & $3.000 \mathrm{E}+00$ & $2.500 \mathrm{E}+01$ & $3.000++00$ & PAC C L Listing Table 1 \\
\hline & $910 \mid 0$ & Dichloro-2-propanol, 1,3- & $96-23-1$ & $000096-23-1$ & 128.99 & $\mathrm{~L}$ & $-4.0006+00$ & $7.600 E+02$ & 1.740E+02 & 7.500E-01 & 2.500E+01 & 7.500E-01 & WEBER,RC ET AL. (1981) \\
\hline 912 & 9110 & Dichloro-2-trifluoromethylbenzimidazole, 4,5; (Chloroflurazole) & $3615-21-2$ & $003615-21-2$ & 255.03 & $\mathrm{~s}$ & $212-214$ & & & $\begin{array}{l}4.000 E-05 \\
.000\end{array}$ & $2.250 E+01$ & & PAC 27 Listing Table 1 \\
\hline 913 & $912 \mid \mathrm{D}$ & $\begin{array}{l}\text { Dichloroacectic acid } \\
\text { Dichloroacety chloride }\end{array}$ & 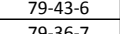 & $000079-43-6$ & $\frac{128.95}{1073}$ & & & 7.600+E02 & $1.990+60202$ & & $2.5000+1011$ & & DAUBERT,TE \\
\hline & $\begin{array}{lll}913 \\
914]_{0}^{D}\end{array}$ & $\begin{array}{l}\text { Dicilhoracectyt chloride } \\
\text { Dichloroacetylyene }\end{array}$ & 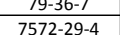 & & 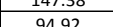 & t & $65005+01$ & 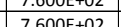 & $\begin{array}{ll} & \end{array}$ & & 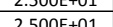 & & 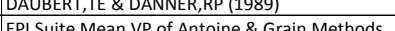 \\
\hline$\frac{150}{916}$ & 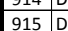 & Dichloramine: (Chlorimide) & 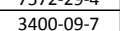 & 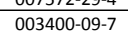 & 85.92 & L & & $7.600 \mathrm{E}+02$ & $4.900+102$ & $6.6306-10$ & $2.500 \mathrm{E}+01$ & $\frac{6.630 E-10}{6.030}$ & The \\
\hline 917 & $\begin{array}{lllll}916 & 0 \\
0\end{array}$ & Dichlorobenzenen, $m$ - & 541-73-1 & 000541-73-1 & 147 & $\begin{array}{ll}\mathrm{L} \\
\end{array}$ & $-2.4800+01$ & $7.6006+02$ & 1.730E+02 & 2.150E+00 & $2.500 E+01$ & 2.150 & T,TE\& DANNER \\
\hline 918 & 917 [0 & Dichlorobenzene, o- & $95-50-1$ & $000095-50-1$ & 147 & L & $-1.700 E+01$ & 7.600E+02 2 & $1.800 \mathrm{E}+02$ & 1.360E +00 & $2.500 \mathrm{E}+01$ & $1.360 E+00$ & DAUBERT,TE \& DANNER, \\
\hline 919 & $\begin{array}{lll}18 & 18 \\
1\end{array}$ & Dichlorobenzzene, $\mathrm{p}$ - & $106-46-7$ & $000106-46-7$ & 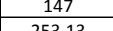 & $\mathrm{s}_{\mathrm{s}}$ & 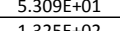 & & $1.700+50202$ & & & & Sting Table 1 \\
\hline$\frac{321}{921}$ & 1920 & Dichlorocyclchexane & $\frac{2108-92-1}{2208}$ & 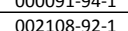 & 153.35 & & 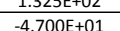 & & 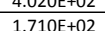 & $0 E+00$ & & & \\
\hline 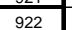 & 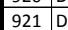 & Dichlorocyclohexane tran & $822-86-6$ & 0008222-86-6 & 153.05 & & $-1.500 E+00$ & & $0695+0^{2}$ & & & & \\
\hline & 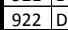 & |Dichlorodiff & $75-71-8$ & $000075-71-8$ & 120.91 & $G$ & $\frac{-1.571 E+02}{-15}$ & & $-2.980 E+01$ & & OOFE+O & & \\
\hline
\end{tabular}




\begin{tabular}{|c|c|c|c|c|c|c|c|c|c|c|c|c|c|}
\hline & No & Chemical Compound & $\begin{array}{c}\text { cas N Number } \\
\text { (CASSN) }\end{array}$ & $\begin{array}{l}\text { Modified } \\
\text { formatc CAS }\end{array}$ & 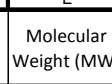 & 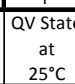 & 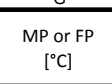 & 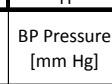 & ${ }_{B P}[\mathrm{CC}]$ & $\begin{array}{l}\text { Vaporor } \\
\text { Pressure } \\
\text { [m H HEg }\end{array}$ & $\begin{array}{c}\text { Torvyp } \\
\text { pcc] }\end{array}$ & 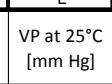 & Vapor Pressure $@ 25^{\circ}$ C Reference \\
\hline$\frac{294}{2025}$ & & 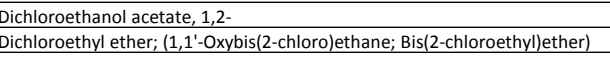 & $\frac{10140-8}{111-4}$ & 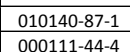 & 13201 & & 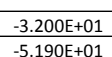 & $\begin{array}{l}3.300+501 \\
.000+52\end{array}$ & 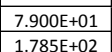 & 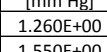 & 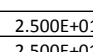 & 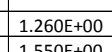 & 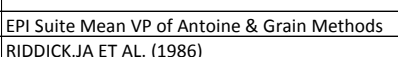 \\
\hline$\frac{326}{926}$ & & 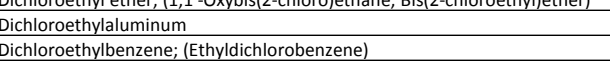 & 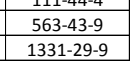 & 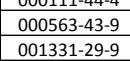 & $\begin{array}{l}\frac{12.65}{129.95} \\
17.06\end{array}$ & $\frac{5}{5}$ & 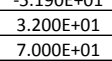 & $7.6000+02$ & 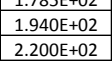 & & & & 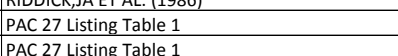 \\
\hline 928 & & 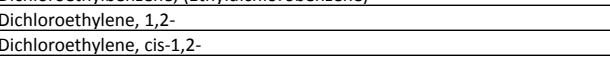 & $\begin{array}{c}540-59 .-0 \\
15.59-2\end{array}$ & 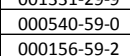 & $\begin{array}{c}96.95 \\
96.94\end{array}$ & $\frac{L}{L}$ & 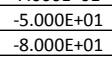 & 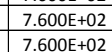 & 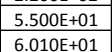 & 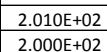 & 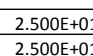 & $\begin{array}{c}2.0100+0+0 \\
2.000++0\end{array}$ & 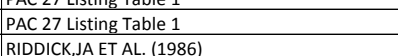 \\
\hline & & & & & & & & & & & & & 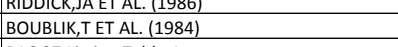 \\
\hline$\frac{931}{932}$ & & 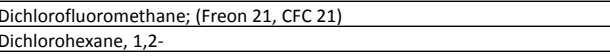 & 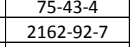 & 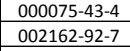 & $\frac{102.92}{155.068}$ & L & $\begin{array}{l}-1.3040+62 \\
-4.2955+01\end{array}$ & $7.600+0202$ & 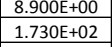 & 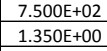 & 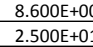 & 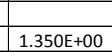 & 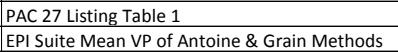 \\
\hline$\frac{9.93}{9.34}$ & & $\begin{array}{l}\text { Dichlorohexane, } 1,6- \\
\text { Dichlorisoisopiople ther }\end{array}$ & $\begin{array}{l}211330.0-0.0 \\
108-60-1\end{array}$ & 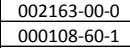 & 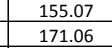 & $\frac{L}{L}$ & 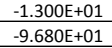 & $\begin{array}{l}1.500+101 \\
7.600+02\end{array}$ & 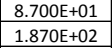 & 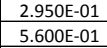 & 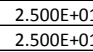 & 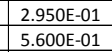 & 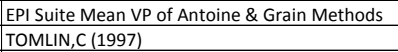 \\
\hline 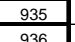 & & 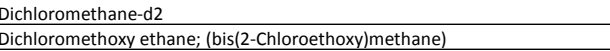 & $\frac{1665-00-5}{119-11-1}$ & 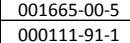 & $\frac{86.94}{17.05}$ & & $\begin{array}{l}-9.700+10+1 \\
-3.280+0101\end{array}$ & 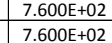 & 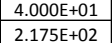 & 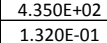 & 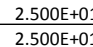 & $\frac{1.300-0}{1.200}$ & $\begin{array}{l}\text { EPI Suite Mean VP of Antoine \& Grain Methods } \\
\text { EPBY }\end{array}$ \\
\hline & & Dichloromethy & 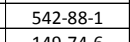 & 000542-88.1.1. & & & & & & & & & 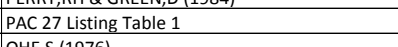 \\
\hline$\frac{938}{939}$ & & 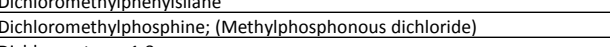 & (677-83-5. & 0.006767-83.5.5 & 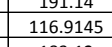 & $\frac{t}{L}$ & 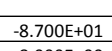 & 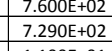 & 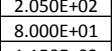 & 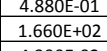 & 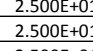 & 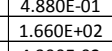 & 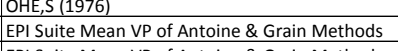 \\
\hline & & 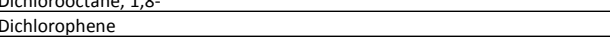 & 2162-29.4 & 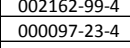 & 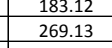 & $\frac{\mathrm{L}}{\mathrm{s}}$ & $\begin{array}{l}-8.000+600 \\
1.7805+02\end{array}$ & & & & & & 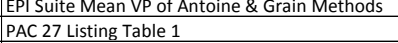 \\
\hline$\frac{9.92}{903}$ & 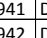 & 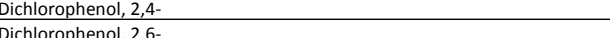 & 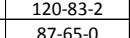 & 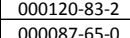 & 年3 163 & 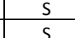 & 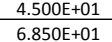 & & 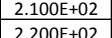 & 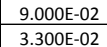 & 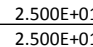 & & 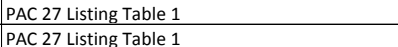 \\
\hline 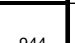 & 943 & Dichlorophenoxyyacetic acid, 2,4; (2,4-D) & $94-75.7$ & $000094-75-7$ & 221.04 & $\mathrm{~s}$ & 1.005e+02 & & 1600 & $8.25 \times 10-8$ & $2.000=0.0$. & & PAC 27 Listing Table 1 \\
\hline & & 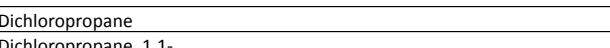 & $26638-19 \cdot 7$ & $\begin{array}{l}026638-19-7 \\
0.0078090\end{array}$ & $\frac{112.99}{11209}$ & & & & & & & 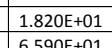 & 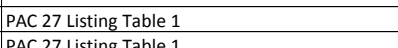 \\
\hline & & 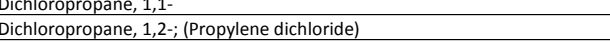 & $\begin{array}{l}\frac{139.98}{78.87} \\
78\end{array}$ & 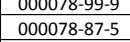 & 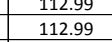 & $\frac{i}{L}$ & & 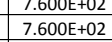 & & & & & $\begin{array}{l}2.27 \text { lisinititg Table } 1 \\
2.2 \text { Liting Table } 1 \\
\end{array}$ \\
\hline$\frac{948}{949}$ & & 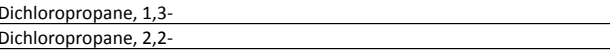 & $\frac{142 \cdot 28.4}{59920.6}$ & 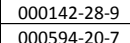 & $\frac{112.29}{111.29}$ & $\frac{L}{L}$ & 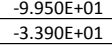 & 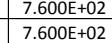 & & & & SOEt & 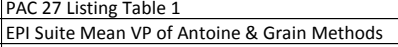 \\
\hline & & 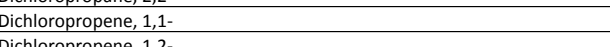 & 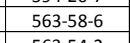 & .000563-5.5. & $\frac{110.97}{10.97}$ & $L$ & & & & & & & BER RCETAL (1981) \\
\hline & & 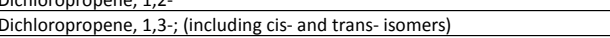 & & & $\frac{10.97}{110.97}>0$ & & & & & & & & 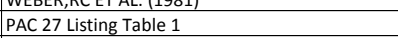 \\
\hline$\frac{953}{0.54}$ & & 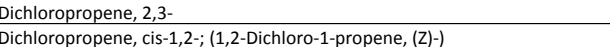 & $\frac{78.83 .6}{6923-20 .}$ & 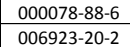 & $\frac{110.97}{110.97}$ & $\frac{L}{L}$ & $\frac{1.000}{-5.000}$ & $\begin{array}{l}7.600 \mathrm{Et} \\
7.600 \mathrm{t}+\mathrm{t}\end{array}$ & 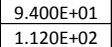 & 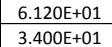 & $\frac{2.500+ \pm 0}{20.050+0}$ & & 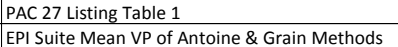 \\
\hline & & Dichlorosilane & & 004109 & & & & & 8.300 & & & & $\begin{array}{l}\text { Listing Table } 1 \\
\text { Lith }\end{array}$ \\
\hline 9577 & & 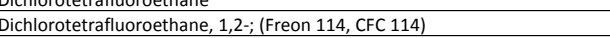 & & & & $e^{6}$ & 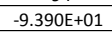 & & & & & & \\
\hline$\frac{958}{959}$ & & $\begin{array}{l}\text { Dichlorovoss; (jichlorvos) } \\
\text { Dicrotophos }\end{array}$ & $\frac{627.7-7}{1416.2 .2}$ & 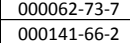 & $\frac{220.98}{237.22}$ & $\frac{L}{L}$ & & 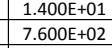 & $\frac{1.200 \mathrm{E}}{4.000 \mathrm{E}+}$ & 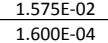 & 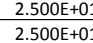 & 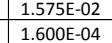 & 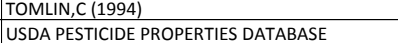 \\
\hline$\frac{990}{961}$ & & 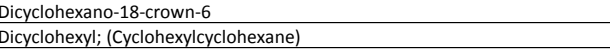 & $\frac{160993.36}{92-5.3}$ & 0.16009:- & 372.56 & $\frac{5}{L}$ & 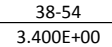 & $7.600=0202$ & 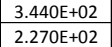 & 1.080E:01 & 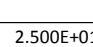 & 1.008E: & 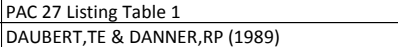 \\
\hline & & & & & & 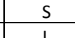 & 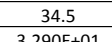 & 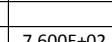 & 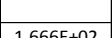 & 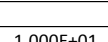 & 年 & 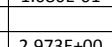 & 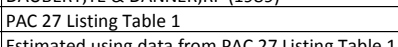 \\
\hline$\frac{393}{964}$ & & $\begin{array}{l}\text { ilicylopentadiene } \\
\text { iidecylimethylammonium chloride }\end{array}$ & & 007173. & & $\frac{5}{5}$ & 3.20060101 & 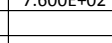 & 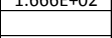 & LOWUEETI & 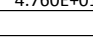 & 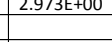 & 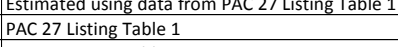 \\
\hline$\frac{965}{965}$ & & Dieldrin & $\frac{60.571}{2-0.12}$ & 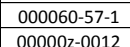 & 380.91 & $\frac{s}{L}$ & .755E+02 & & & 5.89x10- & $5006 \mathrm{~S}$ & & 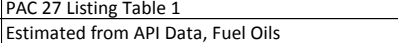 \\
\hline & & 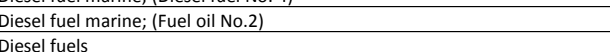 & 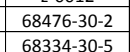 & 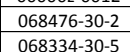 & & $\frac{L}{L}$ & 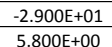 & 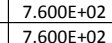 & & & & & 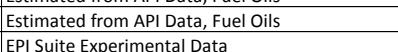 \\
\hline$\frac{2069}{969-1}$ & & & & 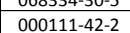 & 105.14 & & $\frac{2.8006+01}{2.801}$ & & 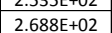 & & 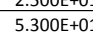 & & liting Table 1 L \\
\hline 9 & $369 \mid$ & 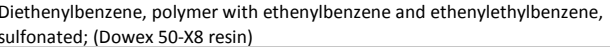 & $69011-20-7$ & $069011-20-7$ & & $\mathrm{~s}$ & & & & & & & PAC 27 Listing Table 1 \\
\hline & & Diethoxydimethysliane & 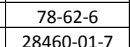 & 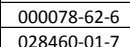 & 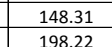 & 2 & & & 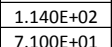 & 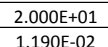 & 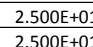 & 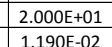 & |ORDAN,TE (1954) \\
\hline & & 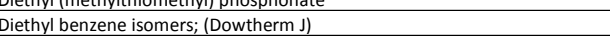 & 25530.17-4 & 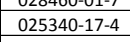 & & & 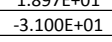 & 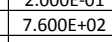 & $\begin{array}{l}1.18385+02 \\
\text { 1. }\end{array}$ & 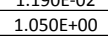 & 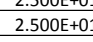 & & 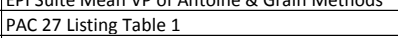 \\
\hline$\frac{974}{975}$ & & $\begin{array}{l}\text { Dietetyl Carbonate } \\
\text { Diethyl chlorophosphate }\end{array}$ & $\begin{array}{l}105.58 \cdot 8 \\
814 \cdot 49 \cdot 3 \\
\end{array}$ & 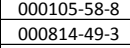 & $\frac{118.15}{172.56}$ & $\frac{\mathrm{L}}{\mathrm{L}}$ & 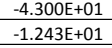 & $\begin{array}{l}7.600+522 \\
1.500++01\end{array}$ & 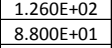 & 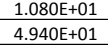 & 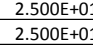 & 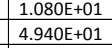 & 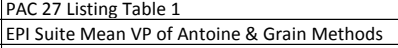 \\
\hline & & $\begin{array}{l}\text { Dietethy lethylphosphhonate } \\
\text { Diethyl malonat; ; (thylyl malonate) }\end{array}$ & 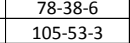 & $\begin{array}{l}0.0078 .88 \\
000015-53\end{array}$ & $\frac{166.16}{160.19}$ & & $-4.980 E+01.11$ & $\begin{array}{l}7.600+5022 \\
7.6000+02\end{array}$ & 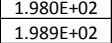 & 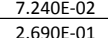 & 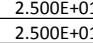 & & 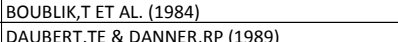 \\
\hline 978 & & Diethy mercury & & 000627-44. & 258.71 & & $-3.1955+011$ & $7.600 E+02$ & $1.500 \mathrm{E}+022$ & $3.5100+022$ & $2.500 \mathrm{E}+0.0$ & & lean V V of Antoine \& G Grin Methods \\
\hline $\begin{array}{ll}9990 \\
9980 \\
\end{array}$ & & $\begin{array}{l}\text { Diethy methylphosphonate; (DEEMP) } \\
\text { Diethyl oxalate }\end{array}$ & 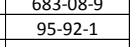 & 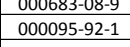 & 年15.15 & $\frac{L}{L}$ & $-4.060+5011$ & 7.600E+ +22 & 1.8544t+0.2 & $\begin{array}{l}1.3300 .02 \\
4.1400=02\end{array}$ & 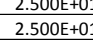 & 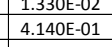 & 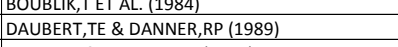 \\
\hline & & 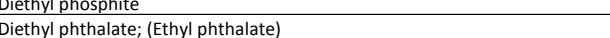 & $\frac{8466-2}{846}$ & & $\frac{122.24}{222.24}$ & & -4.0000E+U1 & 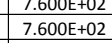 & & 2.11006-0. 03 & 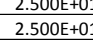 & $\begin{array}{l}1.1135 t-0.0 \\
2.100-0.0\end{array}$ & Pat \\
\hline$\frac{993}{983}$ & & 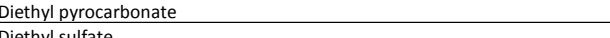 & 1069-47.8 & 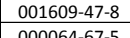 & 162.16 & & 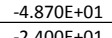 & 7.6000+0202 & 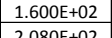 & 1.9700:012 & 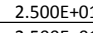 & & FP S Sute Mean VP of A Antoine \& Grain Methods \\
\hline 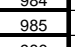 & & Diethyl tartrate & 87.91-2 & 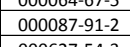 & 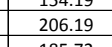 & L & 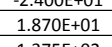 & $7.000 E+02$ & 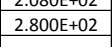 & $2.5900-04$ & 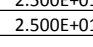 & $2.590=04$ & 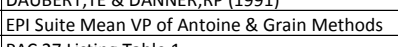 \\
\hline $\begin{array}{ll}9.96 \\
097\end{array}$ & & & 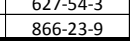 & 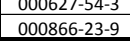 & & & & $1.400 E+11$ & $1.3006+02$ & $9.300=03$ & $2.500=0+0.02020$ & $9.300=03$ & 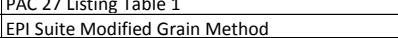 \\
\hline
\end{tabular}




\begin{tabular}{|c|c|c|c|c|c|c|c|c|c|c|c|c|c|}
\hline & & & & & & & & & & & & & \\
\hline & No. & Chemical Compound & 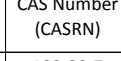 & 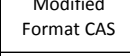 & $\begin{array}{l}\text { Wocenur } \\
\text { Weight (MW }\end{array}$ & 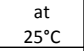 & 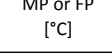 & $\begin{array}{l}\text { Bpipersure } \\
\text { [mm Hel }\end{array}$ & ${ }^{B P[C]}[\mathrm{c}]$ & $\begin{array}{l}\text { Pressure } \\
{[m \text { HIg }}\end{array}$ & 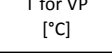 & (Imm Hz] & Vapoo Pressure @ $25^{\circ} \mathrm{C}$ Reference \\
\hline 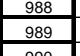 & & 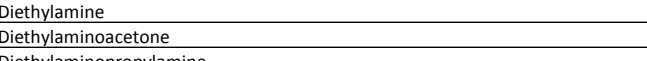 & 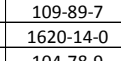 & 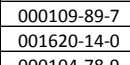 & $\frac{7.316}{129}$ & $\frac{t}{t}$ & 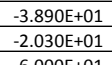 & 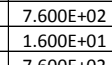 & 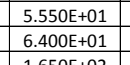 & 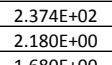 & 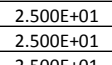 & 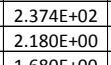 & 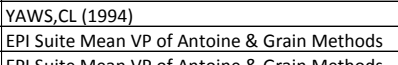 \\
\hline 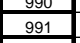 & & 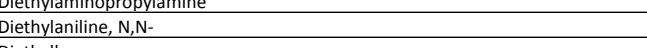 & $\frac{10478-9}{91-66-7}$ & 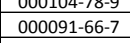 & 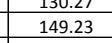 & $\frac{L}{L}$ & 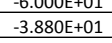 & 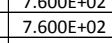 & 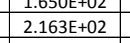 & 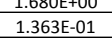 & 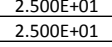 & $\begin{array}{l}1.6860700 \\
1.363=01\end{array}$ & 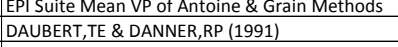 \\
\hline & & 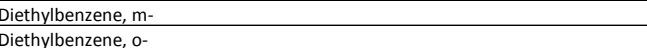 & $\begin{array}{l}141-93-5 \\
135-101.3\end{array}$ & 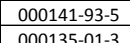 & $\frac{13424}{132424}$ & & 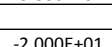 & 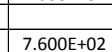 & 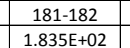 & $1050=500$ & 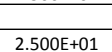 & 然 & 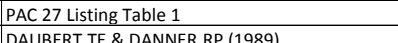 \\
\hline & & 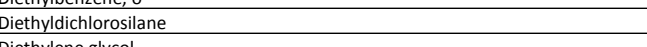 & & & & & 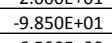 & 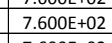 & 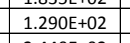 & 4.040E+ +01 & $4.80060+201$ & 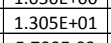 & 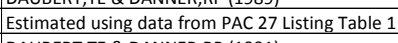 \\
\hline$\frac{995}{996}$ & $\begin{array}{l}9949 \\
995\end{array}$ & 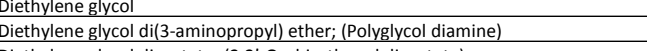 & 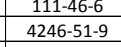 & 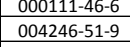 & 2010.14 220.36 & $\frac{L}{L}$ & $6.5000+00$ & $\begin{array}{l}7.600+02 \\
7.600+02\end{array}$ & 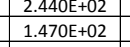 & $\begin{array}{l}5.7 .150: 03 \\
2.150-04\end{array}$ & $\begin{array}{l}2.5000+51 \\
2.500+01\end{array}$ & 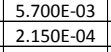 & $\begin{array}{l}\text { DAABERT, EE \& D DNNERRRPR (1991) } \\
\text { EPI Suite Modified Grain Method }\end{array}$ \\
\hline & & 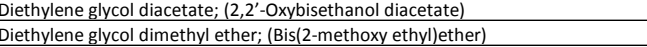 & & & 134.48 & $\frac{s}{L}$ & 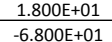 & & 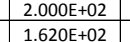 & $2960 \notin+00$ & & & A \\
\hline & & 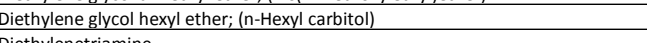 & $\frac{112.59 \cdot 4}{110.40}$ & 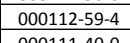 & 190.32 & & 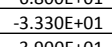 & 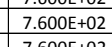 & 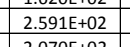 & 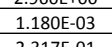 & 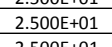 & 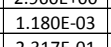 & 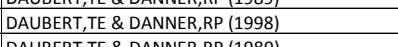 \\
\hline$\frac{10001}{1001}$ & 10000 & 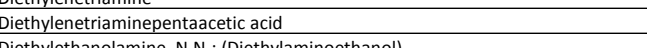 & $67-43 \cdot 6$ & $000067-43-6$ & $\begin{array}{lll}303.35 \\
-3932\end{array}$ & s & 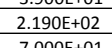 & 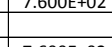 & & $2.317=0.11$ & ( & 2517=02 & 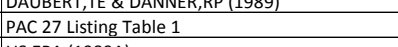 \\
\hline & 1.002 & Diethylyhosshatoethyltriethoxy siane & $757-44.8$ & $000757-44.8$ & 328.47 & $\mathrm{~s}$ & & & 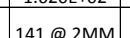 & & & & Pac 27l lising Table 1 \\
\hline & & & & & & & & & & & & & \\
\hline & & & $\begin{array}{l}30.53 .1 \\
105.55\end{array}$ & 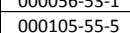 & $\begin{array}{l}268.38 \\
132.25\end{array}$ & $\frac{3}{5}$ & 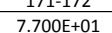 & & & & & & 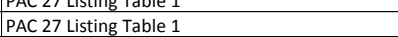 \\
\hline & & Diethylurea, 1,3-3. & $\frac{6237-7.7}{55-20.0}$ & 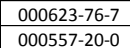 & $\frac{116.19}{123.51}$ & $\frac{5}{5}$ & 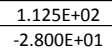 & $7.600 E+02$ & 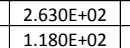 & $080= \pm 0+0$ & & $208080+011$ & 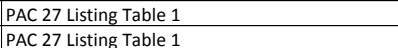 \\
\hline$\frac{1008}{1009}$ & & 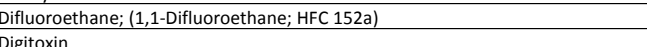 & $\frac{753-376}{71736.6}$ & 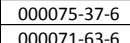 & $\begin{array}{ll}66.06 \\
7655\end{array}$ & 5 & $-\frac{1.170 E+02}{-256-257}$ & & $2.6506 \mathrm{E}$ & & $2.5000+01010$ & & 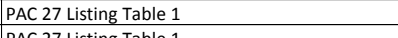 \\
\hline & & 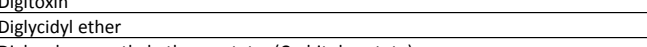 & & & & & & & & & & & 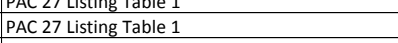 \\
\hline$\frac{10101}{1012}$ & & 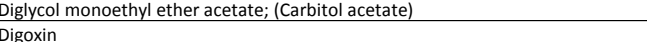 & 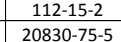 & 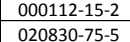 & $\begin{array}{l}\frac{172.21}{18105} \\
8\end{array}$ & $\frac{1}{5}$ & $\begin{array}{l}-2.500501 \\
205050\end{array}$ & $7.600+02$ & $2.1855+022$ & $9.9000-02$. & 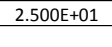 & 900:-02 & 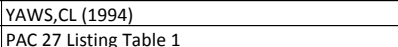 \\
\hline (1013 & 1012 & 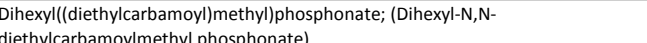 & 7369-66-6 & $007369 \cdot 66-6$ & 363.48 & $\mathrm{s}$ & & & & 3.000E:01 & 1.6800+02 & & PAC 27 Listing Table 1 \\
\hline & & 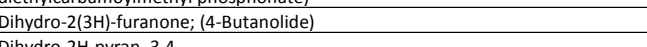 & 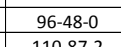 & 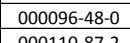 & 86.1 & t & 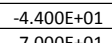 & 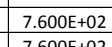 & 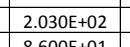 & $\begin{array}{l}4.5000 .01 \\
7.050 .01\end{array}$ & $2.500 \mathrm{E}+01$. & 4.5000-01 & DAUBERT, TE \& DANNER,RP (1989) \\
\hline & 1015 & 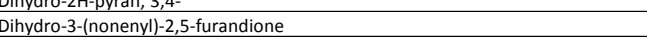 & 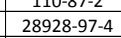 & 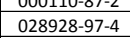 & & $\frac{5}{s}$ & & & & & & & 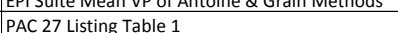 \\
\hline$\frac{1017}{1019}$ & 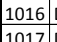 & 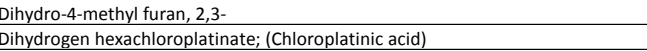 & 343148.83.-5 & 034334483.5 & $\begin{array}{l}84.12 \\
409.82\end{array}$ & $\frac{t}{s}$ & 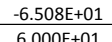 & 7.6005 +022 & & $5.260+501$ & $2.500++01$ & 5.2605+01 & 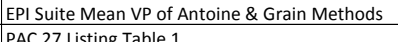 \\
\hline 1019 & 1018 & 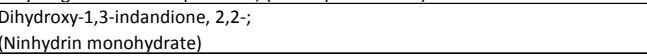 & $485 \cdot 47-2$ & $000485-47-2$ & 178.15 & $\mathrm{~s}$ & $\begin{array}{l}241.3 \\
\text { dec }\end{array}$ & & & $2.3600-07$ & $2.500 \mathrm{E}+01$ & & PAC 27 Listing Table 1 \\
\hline & & 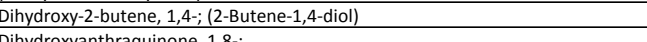 & & & & & 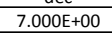 & & $340 E+t$ & 4.620E-0 & $2.500 E+01$ & & \\
\hline 1021 & 10200| & 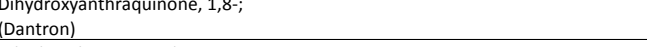 & $17-10-2$ & $000117-10-2$ & 240.22 & s & $1.930+02$ & & & & & & PAC 27 Listing Table 1 \\
\hline$\frac{1022}{1023}$ & 1022 & 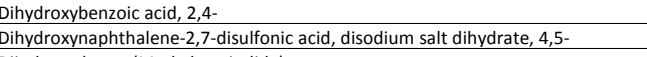 & 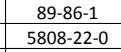 & 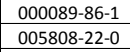 & 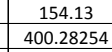 & $\frac{s}{5}$ & $\begin{array}{l}218-219 \\
300 \text { dec }\end{array}$ & & & & & & $\begin{array}{l}\text { PAA } 2 \text { listing Table } 1 \\
\text { PAC } 7 \text { Listing arale } 1 \\
\end{array}$ \\
\hline$\frac{1024}{1025}$ & & Diisomementaninine & 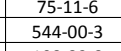 & 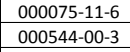 & 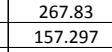 & & $\begin{array}{l}5.000+500 \\
-4.4000+01\end{array}$ & 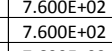 & $1.88060+0=0$ & 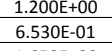 & 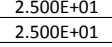 & $\begin{array}{l}1.2000+500 \\
.65300-01\end{array}$ & 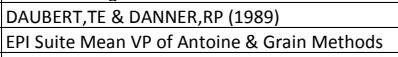 \\
\hline & & 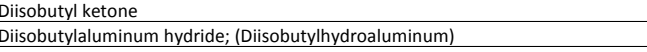 & 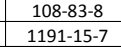 & 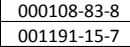 & $\frac{142.27}{122.25}$ & $\frac{L}{L}$ & $8.000 E+01$ & $\begin{array}{l}7.600+5202 \\
.4000+00\end{array}$ & 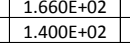 & 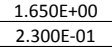 & 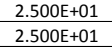 & 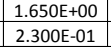 & 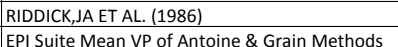 \\
\hline 1028 & & Disisobutrlamine & 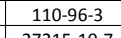 & & $\frac{129.28}{128} \mathrm{C}$ & & $-7.000 E+011$ & & $1.3906+202$ & & 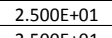 & & PAC 27 lisiting Table 1 | \\
\hline 1030 & 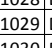 & 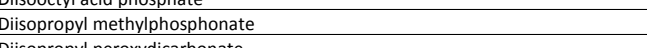 & 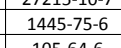 & 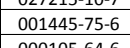 & 180.18 & & 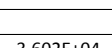 & 7.600E+02 & $1.2111++02$ & $2.8000-01$ & $2.500 \mathrm{E}+01$ & $2.800 E_{0.1}$ & 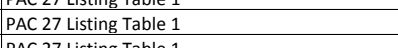 \\
\hline$\frac{1034}{1032}$ & & 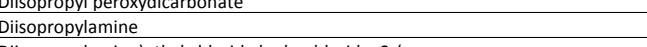 & 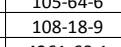 & 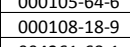 & $\begin{array}{lll}200.22 \\
101.22\end{array}$ & $\frac{1}{3}$ & 3.002 & $7.6005+02$ & $\begin{array}{l}\frac{1.200+201}{8.300+01} \\
8\end{array}$ & $7.900 \mathrm{~F}+01$ & $2.500 €+01$ & $7.9006+01$ & DAUBERT,TE \& DANNER,RP (1989) \\
\hline 10334 & (033 & 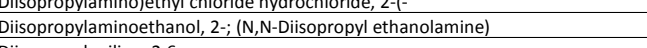 & 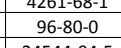 & 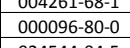 & 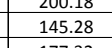 & $i$ & & & & $8.000=02$ & $2.500 E+01$ & $8.000=02$ & 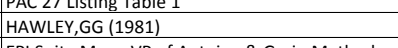 \\
\hline & & 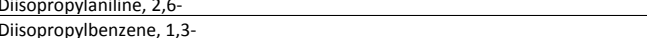 & & & & & 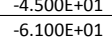 & 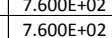 & 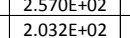 & & & & 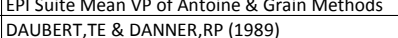 \\
\hline 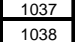 & 036 & 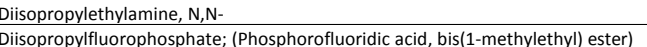 & $\begin{array}{l}7087-68.5 \\
555-91-4\end{array}$ & 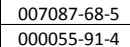 & 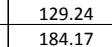 & $\frac{1}{5}$ & $\begin{array}{l}-5.000+5+1 \\
-8.2000+01\end{array}$ & $7.600 E+022$ & $\begin{array}{l}1.2655+02 \\
1.5500+02\end{array}$ & $\begin{array}{l}1.1700+51 \\
5.8000 .01\end{array}$ & $\begin{array}{l}2.500+501 \\
2.5000+01\end{array}$ & 1.170et+11 & EPI Suite Mean VP of Antoine \& Grain Methods \\
\hline$\frac{1039}{1.039}$ & 1038: & 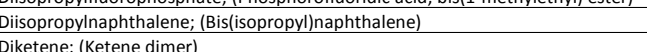 & 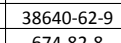 & 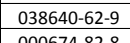 & $\begin{array}{l}212.36 \\
8407\end{array}$ & $s$ & & 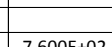 & 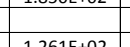 & 107250.11. & 年 & (S) & 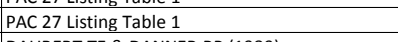 \\
\hline & & Dilauroy peroxide & $105 \cdot 74.8$ & & & $s$ & $\begin{array}{l}4.000+0+1 \\
\end{array}$ & we 5 & 62 dece & & 2:SOUE & 1.viletort & DAUGERT,TE\& DANNERR,RPG \\
\hline & & 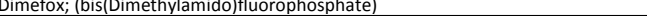 & & & & & & 4.000E +000 & $6.00=50101$ & & & 1:100E:01 & \\
\hline${ }_{1043}$ & $1042 \mid$ & oimethoate & $60051-5$ & 000060-51-5 & 229.27 & $\mathrm{~s}$ & $51-52$ & & 107@0.05 & $1.1 \mathrm{mpa}$ & $2.500+01$ & & PAC 2 Z L siting Table 1 \\
\hline$\frac{1044}{1045}$ & 1043 1044 & 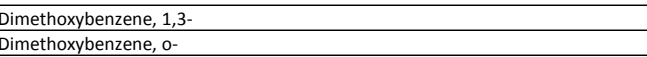 & 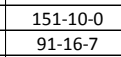 & 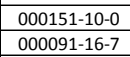 & 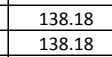 & $\frac{\mathrm{L}}{\mathrm{s}}$ & 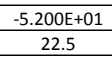 & $7.600+02$ & $\begin{array}{l}2.1756+0202 \\
.2060+02\end{array}$ & 1.4906-01 & $2.500+\div 1$ & 1.49060 .01 & 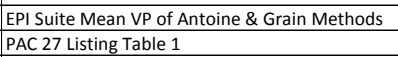 \\
\hline$\frac{1046}{1047}$ & 1005: & 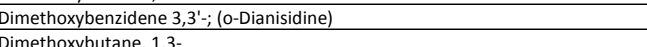 & 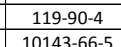 & 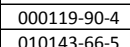 & $\frac{24.29}{21.82}$ & 5 & 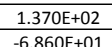 & 10005+00 0 & 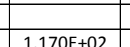 & 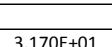 & & & 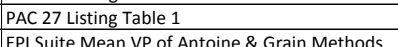 \\
\hline & & & & 003453-9.9.4 & & & & $7.600 E+02$ & 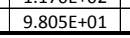 & 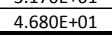 & 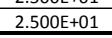 & & 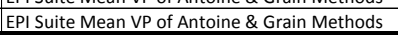 \\
\hline
\end{tabular}




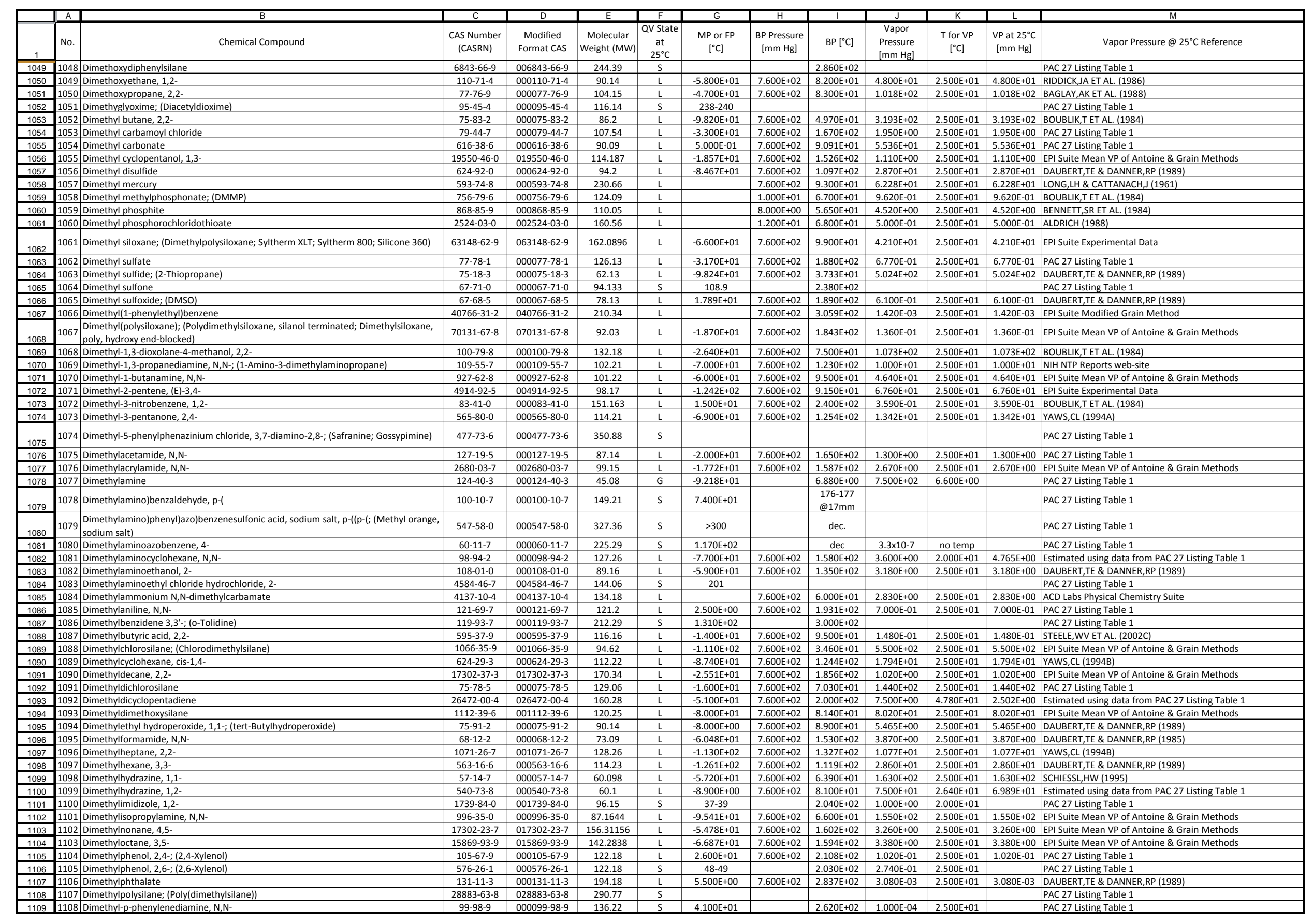




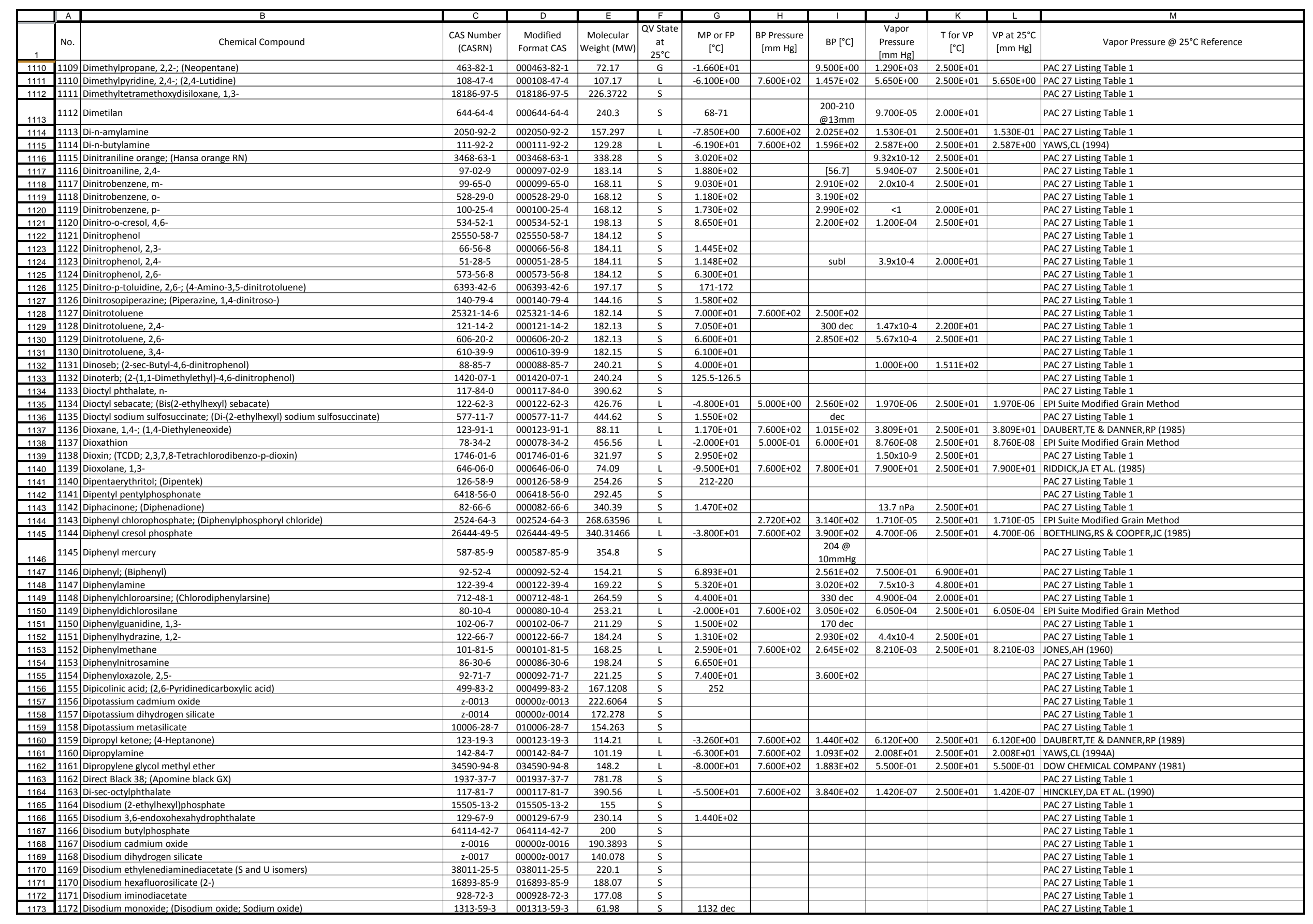




\begin{tabular}{|c|c|c|c|c|c|c|c|c|c|c|c|c|}
\hline & A & & & 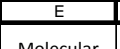 & $\frac{F}{Q V \text { State }}$ & 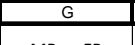 & $\begin{array}{ll}\mathrm{H} \\
\mathrm{H}\end{array}$ & +2 & & $\mathrm{k}$ & & \\
\hline & Chemical Compound & $\begin{array}{l}\text { CAS Number } \\
\text { (CASRN) }\end{array}$ & $\begin{array}{l}\text { Modified } \\
\text { Format CAS }\end{array}$ & $\begin{array}{c}\text { Molecular } \\
\text { Weight (MW) }\end{array}$ & $\begin{array}{c}\text { avstate } \\
\text { at } \\
25^{\circ} \mathrm{C}\end{array}$ & $\begin{array}{c}\text { MP or FP } \\
{\left[{ }^{\circ} \mathrm{C}\right]}\end{array}$ & $\begin{array}{c}\text { BP Pressure } \\
{[\mathrm{mm} \mathrm{Hg}]}\end{array}$ & $\mathrm{BP}\left[{ }^{[\mathrm{C} C}\right]$ & $\begin{array}{l}\text { Vapor } \\
\text { Pressure } \\
\text { Imm Hg }\end{array}$ & $\begin{array}{l}\text { Tfor VP } \\
\text { ['C] }\end{array}$ & $\begin{array}{c}\text { VP at } 25^{\circ} \mathrm{C} \\
{[\mathrm{mm} \mathrm{Hg}]}\end{array}$ & 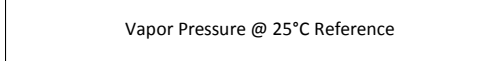 \\
\hline 1174 & 1173 Disulfiram & $97-77-8$ & $000097-77-8$ & 296.56 & $\mathrm{~s}$ & $7.000 E+01$ & & $1.170 E+02$ & & & & PAC 27 Listing Table 1 \\
\hline 1175 & $1174 \mid$ Disulfoton & $298-04-4$ & $000298-04-4$ & 274.42 & $\mathrm{~s}$ & $>-25$ & & $\begin{array}{c}132-133 @ \\
15 \mathrm{~mm}\end{array}$ & $5.400 E-05$ & $2.000 E+01$ & & PAC 27 Listing Table 1 \\
\hline$\frac{1176}{11177}$ & 1175 Di-tert-buty dicarbonate & $24424-99-5$ & $\begin{array}{ll}024424-99-5 \\
002958-1\end{array}$ & 218.28 & L & $2.3006+01$ & $5.000 \mathrm{E}-01$ & $5.600 \mathrm{E}+01$ & $2.880 \mathrm{E}-02$ & $2.500 \mathrm{E}+01$ & $2.880 \mathrm{E}-02$ & EPI Suite Mean VP of Antoine \& Grain Methods \\
\hline & 1176 Di-tert-butyhydroquinone, $2,5-$ & 88-58-4 & $000088-58-4$ & 222.35 & $\mathrm{~s}$ & 213.5 & & & & & & PAC 27 Listing Table 1 \\
\hline$\frac{1178}{11179}$ & 11777|Ditithizanaine iodide; (3,3-2-Diethylpentamethineth & 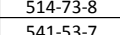 & $000514-73-8$ & $\frac{519.51}{1352}$ & $\frac{s}{s}$ & $2.480 E+02$ & & dec & 1.000E-05 & $2.5000+01$ & & 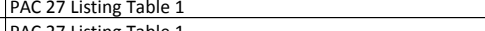 \\
\hline$\frac{1119}{1180}$ & 1179 Dithiodiethanol, 2,2; (Dithiodiglycol) & $\frac{54-3-3-1}{1892-29-1}$ & $001892-29-1$ & $\frac{135.22}{154.26}$ & $\frac{2}{3}$ & 2.600t+01 & $7.600 \mathrm{E}+02$ & $\frac{\text { dec }}{1.600++02}$ & & & & $\begin{array}{l}\text { PAC 27 L Ltsing a able } 1 \\
\text { PAC } 27 \text { isting Table } 1\end{array}$ \\
\hline & & & & & & $\begin{array}{l}8.300 E+01 \\
\end{array}$ & $2.000 E+00$ & & & & & \\
\hline 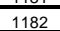 & 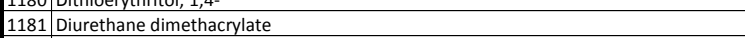 & 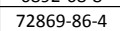 & $072869-86-4$ & $\frac{140.56}{470.5}$ & s & & & 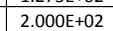 & & & & 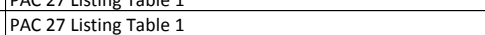 \\
\hline 1183 & 1182 Divinylbenzene, $m ;$; (m-Vinylstyrene) & $108-57-6$ & $000108-57-6$ & 130.2 & $\mathrm{~L}$ & $-8.700 E+01$ & $7.600 E+02$ & $1.950 \mathrm{E}+02$ & $5.790 \mathrm{E}-01$ & $2.500 E+01$ & $5.790 \mathrm{E}-0$ & PAC 27 Listing Table 1 \\
\hline$\frac{1184}{11995}$ & 1183 1183ininylibenzene, mixed isomers; (Vinylstyrene) & $\frac{1321-74-0}{54-097-6}$ & $001321-74-0$ & $\frac{130.2}{44502}$ & $\frac{L}{S}$ & $-8.700 E+01$ & $7.600 E+02$ & $1.950 \mathrm{E}+02$ & $5.7900-01$ & $2.500 E+01$ & $5.7900-01$ & $\begin{array}{l}\text { PAC 27 Listing Table } 1 \\
\text { PAC C27 listing Table }\end{array}$ \\
\hline$\frac{1185}{1186}$ & $\begin{array}{l}\frac{11844 \text { Dodecamethylcyclohexasiloxane }}{1185} \text { Dodecane } \\
\end{array}$ & $\frac{540-97-6}{112-40-3}$ & $\begin{array}{l}000540-97-6 \\
000112-40-3 \\
\end{array}$ & $\frac{4450.02}{170.38}$ & & $-9.600 E+00$ & $7.6006+02$ & $2.163 \mathrm{E}+02$ & $1.350 \mathrm{E}-01$ & $2.500 E+01$ & & $\begin{array}{l}\text { PAC C } 7 \text { Listing gable } 1 \\
\text { PAC 27 listing Table } 1\end{array}$ \\
\hline & $\begin{array}{l}185 \text { SDodecane } \\
1186 \text { Dodecanethiol, 1; (Dodecyl mercaptan; Lauryl mercaptan) }\end{array}$ & $\frac{1112-40-3}{112-55-0}$ & $\begin{array}{ll}0.00112-40-3 \\
000112-55-0 \\
\end{array}$ & $\frac{110.028}{202.44}$ & $\frac{L}{L}$ & $\begin{array}{lll}-9.600 E=00 \\
-7.000 E+00\end{array}$ & $\frac{1.6006 \mathrm{~F}}{7.62}$ & $\frac{2.1052-145}{142}$ & $\begin{array}{l}8.5255-03 \\
\end{array}$ & & & $\begin{array}{l}\text { PAC 2/ LItSing Iable I } \\
\text { YAWS, CL (1994) }\end{array}$ \\
\hline$\frac{1188}{1188}$ & & $19780-11-1$ & $019780-11-1$ & 266.38 & $\frac{5}{s}$ & 41.43 & & & $<1$ & 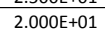 & & PAC 27 listing Table 1 \\
\hline 1189 & 1188 Dodecenyl succine anhydrdide; (Tetrapropenylsuccinic anhydride) & $26544-38-7$ & $026544-38-7$ & 266.38 & $\mathrm{~L}$ & & $7.600 \mathrm{E}+02$ & $\frac{.25006+02}{3.5005}$ & $6.110 \mathrm{E}-05$ & $2.5006+01$ & $6.110 \mathrm{E}-05$ & EPI Suite Modified Grain Method \\
\hline 1190 & $\mid$ & $\frac{25377-73-5}{11252-5}$ & $\begin{array}{llll}025377-73-5 \\
02012529\end{array}$ & 266.38 & $L^{L}$ & & $5.000 \mathrm{E}+00$ & $1.800 \mathrm{E}+02$ & $6.110 \mathrm{E}-05$ & $2.500 \mathrm{E}+01$ & $6.110 E-05$ & 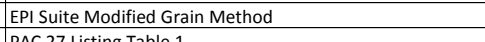 \\
\hline$\frac{1191}{1102}$ & $\begin{array}{l}|1190| \text { Dodecyl alcohol } \\
11191 \text { Dodocely methacrylate }\end{array}$ & $112-53-8$ & $000112-53-8$ & $\frac{186.38}{254.46}$ & $\frac{\mathrm{s}}{\mathrm{L}}$ & $\frac{2.400 E+01}{-2.900 E+11}$ & 5 & \begin{tabular}{|c|c|}
$145-148$ \\
$1420+02$
\end{tabular} & $8.4800-04$ & $2.5000 \mathrm{E}+01$ & 1006503 & PAC 27 Listing Table 1 \\
\hline$\frac{1192}{1193}$ & 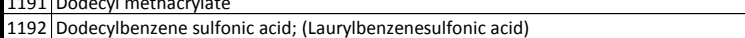 & $\frac{142-90-5}{27176-87-0}$ & $\begin{array}{l}0000142-90-5 \\
027176-87-0\end{array}$ & $\begin{array}{llll}25246 \\
326.54\end{array}$ & $\frac{L}{s}$ & & 4.000et+00 & & & & $1.906 E_{-03}$ & $\begin{array}{l}\text { Estimated ussing data from PAC } 2 \text { / Listing lable } 1 \\
\text { PAC } 27 \text { Listing Table } 1\end{array}$ \\
\hline$\frac{1139}{1194}$ & 1193 Dodecylphenol, 4 - (mixture of i isomers) & $27193-86-8$ & $\begin{array}{ll}02710-019-0 \\
027193-8\end{array}$ & 202.048 & $\frac{2}{3}$ & $6.600 E+01$ & $7.600 \mathrm{E}+02$ & $1.750 \mathrm{E}+02$ & & & & $\begin{array}{l}\text { PAC } 27 \text { Listing Table } 1 \\
\text { Pats }\end{array}$ \\
\hline & 11944 Dodecylsarcosine sodium salt, N; (N-Lauryl Sarcosine sodium salt) & & 007631-98-3 & & & & & & & & & PAC 27 listing Table 1 \\
\hline 11966 & 11955 Dodecyttrichlorosilane & $4484-72-4$ & $004484-72-4$ & 303.81 & $\mathrm{~L}$ & & 7.600E +02 & $2.880 \mathrm{E}+02$ & $2.540 \mathrm{E}-03$ & 2.500E +01 & $2.540 E-03$ & EPI Suite Modified Grain Method \\
\hline 1197 & 11966 [Dowex 50WX4 (50-400 ion exchange resin) & $11113-61-4$ & $011113-61-4$ & 196.04 & $\mathrm{~s}$ & & & & & & & PAC 27 Listing Table 1 \\
\hline 1109 & 1197 Dv methyl ester; (Methy 3-(2,2-dichlorovinyy)|-2,2-dimethylycylopropanecarboxylate) & 61898-95-1 & 061898-95-1 & 223.09 & $\mathrm{~L}$ & & $2.000 E-01$ & 6.400E+01 & $6.440 \mathrm{E}-02$ & $2.500 E+01$ & $6.440 \mathrm{E}-02$ & EPI Suite Modified Grain Method \\
\hline & 1198 Dysprosium & $7429-91-6$ & $007429-91-6$ & 162.5 & $\mathrm{~s}$ & $1.409 \mathrm{E}+03$ & & $2.335 \mathrm{E}+03$ & & & & PAC 27 Listing Table 1 \\
\hline 1200 & 1199 Dysprosium nickelide & $12175-27-8$ & $012175-27-8$ & 221.19 & $\mathrm{~s}$ & & & & & & & PAC 27 Listing Table 1 \\
\hline 1201 & 1200| Dysprosium nitrate & 10143-38-1 & $010143-38-1$ & 348.6 & $\mathrm{~s}$ & 8.860E+ +01 & & & & & & PAC 27 Listing Table 1 \\
\hline 1202 & 12011 Dysprosium oxide; (Dysprosium(III) oxide) & $1308-87-8$ & 001308-87-8 & 373 & $\mathrm{~s}$ & 2.4088E+03 & & & & & & PAC 27 Listing Table 1 \\
\hline 1203 & $1202 \mid$ Ecolite & z-0018 & $00000 z-0018$ & & $\mathrm{~L}$ & & $7.600 E+02$ & $3.000 E+02$ & $1.000 \mathrm{E}+00$ & $2.000 E+01$ & $1.2566+00$ & Estimated based upon MSDS data, MP Biomedicals 882475 \\
\hline 1204 & 1203 Emetine dihydrochloride, $\mathrm{I}-$ & $316-42-7$ & $000316-42-7$ & 553.63 & $\mathrm{~s}$ & $2.550 \mathrm{E}+02$ & & & $1.000 E-05$ & $2.500 \mathrm{E}+01$ & & PAC 27 Listing Table 1 \\
\hline 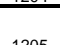 & 1204 Endosulfan & $115-29-7$ & $000115-29-7$ & 406.93 & $\mathrm{~s}$ & $1.060 \mathrm{E}+02$ & & $106 @$ & $6.2 \times 10-6$ & $2.000 E+01$ & & PAC 27 Listing Table 1 \\
\hline & 1205 Endothion & $2778-04-3$ & $002778-04-3$ & 280.25 & $\mathrm{~s}$ & $9.600 E+01$ & & & & & & PAC 27 Listing Table 1 \\
\hline $\begin{array}{l}1207 \\
1207\end{array}$ & $|1206|$ Endrin & $72-20-8$ & $000072-20-8$ & 380.91 & $\mathrm{~s}$ & $245 \mathrm{dec}$ & & & $3 \times 10-6$ & $2.000 E+01$ & & PAC 27 Listing Table 1 \\
\hline 1208 & 1207| Eppibatidine & 140111-52-0 & 140111-52-0 & 208.69 & L & & $7.600 E+02$ & 3.086E+02 & 2.120E-04 & 2.500E+01 & 2.120E-04 & EPI Suite Modified Grain \\
\hline$\frac{1209}{1210}$ & $\begin{array}{l}\frac{12288 \text { Eppibromohohdrin }}{1209} \text { Evichlorohydrin } \\
\end{array}$ & $3132-64-7$ & $003132-64-7$ & $\frac{136.99}{9.53}$ & $\frac{L}{L}$ & 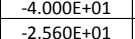 & 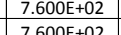 & $\mid \frac{1.350 \mathrm{O}+02}{1.179+\mathrm{P}+02}$ & 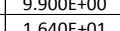 & & 1600501 & |FP S Sunte Mean VP Anto \\
\hline & 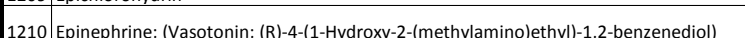 & $51-43-4$ & $000051-43-4$ & 18323 & $\mathrm{~s}$ & $2.1606+02$ & & & & & & 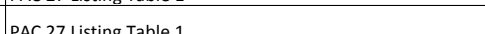 \\
\hline$\frac{1211}{1212}$ & 1211 EPN; (0-Ethyl-0-(4-itrophenyl) phenylthiophosphonate) & $2104-64-5$ & $002104-64-5$ & 323.32 & $\mathrm{~s}$ & $3.6006+01$ & & $215 @ 5 \mathrm{~mm}$ & $0.126 \mathrm{mPa}$ & $2.5000+01$ & & PAC 27 listing Table 1 \\
\hline & 1212| Epoxy r resin (EPON 1001) & 25068-38-6a & $025068-38-6 a \mathrm{a}$ & & $\mathrm{s}$ & & & & & & & PAC 27 Listing Table 1 \\
\hline$\frac{1214}{1215}$ & 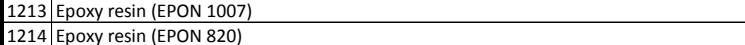 & $25068-38-66$ & 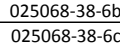 & & $\frac{\mathrm{s}}{\mathrm{s}}$ & & & & & & & 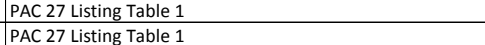 \\
\hline$\frac{1416}{1216}$ & 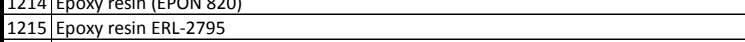 & 25068-38-6c-6d & 0250608-38-60 & & s & & & & & & & $\begin{array}{l}\text { PAC } 27 \text { Listing Table } 1 \\
\text { Pats }\end{array}$ \\
\hline${ }_{1217}$ & $\begin{array}{l}1216 \text { Epoxy resin, curued; (4,4'-(1-Methylethylidene)biscyclohexanol, polymer with } \\
\text { (chloromethyl) oriane) }\end{array}$ & $30583-72-3$ & 030583-72-3 & & s & & & & & & & PAC 27 Listing Table 1 \\
\hline 1218 & 1217 Epoxy resin; (Bisphenonol A-Bisphenol A diglycidyl lether polymer) & $25036-25-3$ & $025036-25-3$ & & & & & & & & & PAC 27 Listing Table 1 \\
\hline$\frac{1219}{1220}$ & 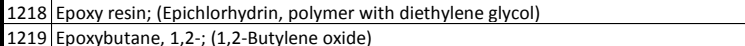 & $\frac{25928-94-3}{1068-7}$ & $\frac{025928-94-3}{0000106-8-7}$ & 72.12 & $\frac{\mathrm{s}}{\mathrm{L}}$ & $-1.500 E+02$ & $7.6006+02$ & $6.330 E+01$ & $1.800 E+02$ & $2.500 E+01$ & $1.800 E+02$ & $\begin{array}{l}\text { PAC } 27 \text { Listing Table } 1 \\
\text { PAC } 7 \text { Listin Table } 1\end{array}$ \\
\hline$\frac{1220}{1221}$ & 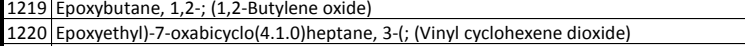 & $\begin{array}{ll}106-88-7 \\
106-87-6 \\
\end{array}$ & $\frac{0000106-87-7}{0.67}$ & $\frac{12.12}{140.18}$ & $\mathrm{~L}$ & $-5.500 \mathrm{E}+01$ & $7.6000 \mathrm{E}+02$ & $2.270 \mathrm{E}+02$ & $1.000 \mathrm{E}-01$ & 2.3000E+U1 & $\begin{array}{l}1.800 \mathrm{E} 02 \\
1.436 \mathrm{E}-01 \\
\mathrm{n}\end{array}$ & $\begin{array}{l}\text { PAC C } 7 \text { Litsinin Table } 1 \\
\text { Estimated using data from PAC } 27 \text { Listing Table } 1 \\
\end{array}$ \\
\hline 1222 & 1221| Erbium nitrate pentahydrate & 10031-51-3 & $010031-51-3$ & 443.35 & $\mathrm{~s}$ & & & & & & & PAC 27 listing Table 1 \\
\hline & & $\frac{10168-80 \cdot 6}{1207705-6}$ & $010168-80-6$ & 353.29 & s & Mes & & & & & & PAC 27 Listing Table 1 \\
\hline 1224 & 1223 Erbium(III) nitrate hexahydrate & 134tro-055-6 & 013476-05-6 & 461.41 & 点 & & & & & & & PAC 27 Listing Table 1 \\
\hline & 1224 Erbium(III) o & $12061-16-4$ & $012061-16-4.4$ & 382.52 & $\mathrm{~s}$ & $2.344 E+03$ & & 3.920E +03 & & & & ting Table 1 \\
\hline 1226 & 1225 Ergocalciferol & 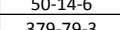 & 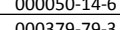 & $396 . / 22$ & $\frac{s}{c}$ & 215318 & & & & & & \\
\hline$\frac{1227}{1228}$ & 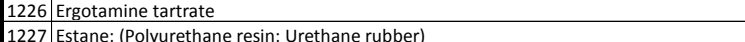 & 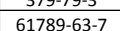 & $\frac{1}{0.061799-63-37}$ & 150.50 & s & & & & & & & $\begin{array}{l}\text { PAA 27 listing Table } 1 \\
\text { PAC } 27 \text { Listing Table } 1\end{array}$ \\
\hline 烈 & 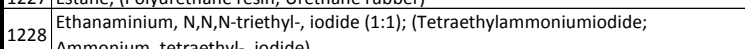 & $68-05-3$ & $000068-05-3$ & 257.19 & $\mathrm{~s}$ & 300 dec & & & & & & PAC 27 Listing Table 1 \\
\hline$\frac{1240}{1020}$ & 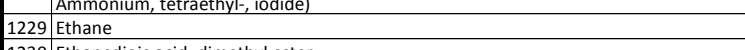 & $74-84-0$ & $000074-84-0$ & 30.08 & G & $-1.720 E+02$ & & $-8.860 \mathrm{E}+01$ & $7.600 \mathrm{E}+02$ & & & PAC 27 Listing Table 1 \\
\hline
\end{tabular}




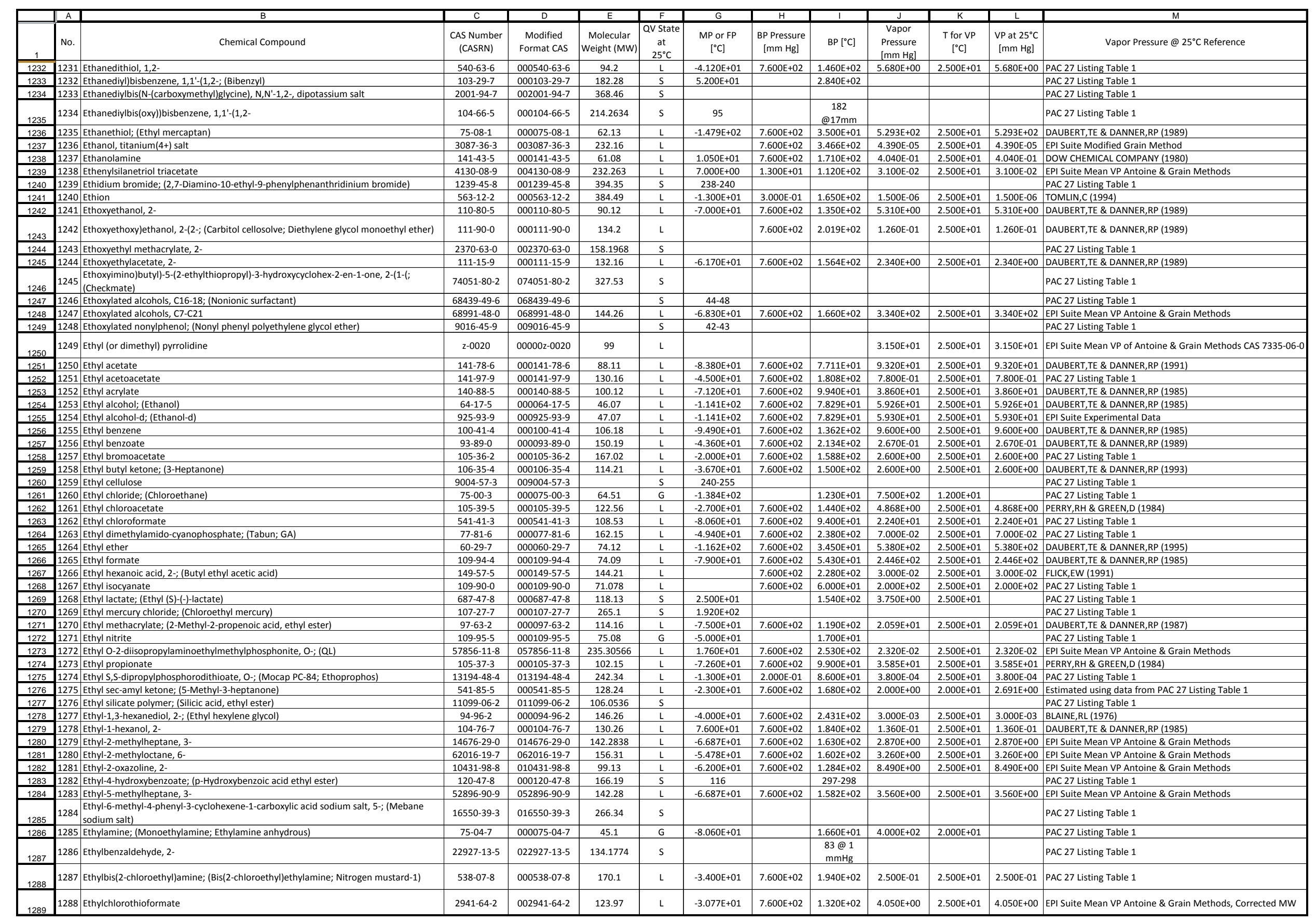




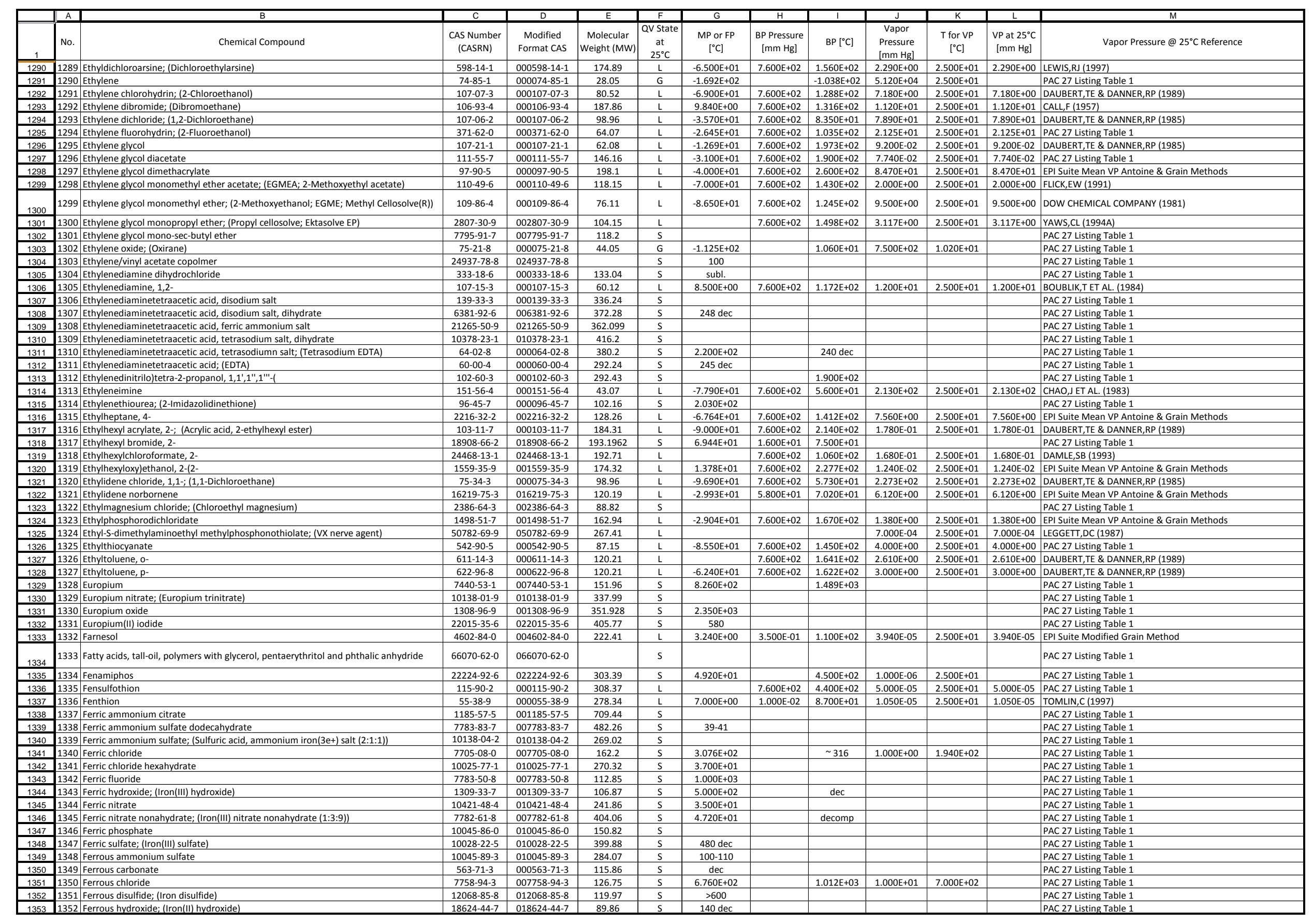




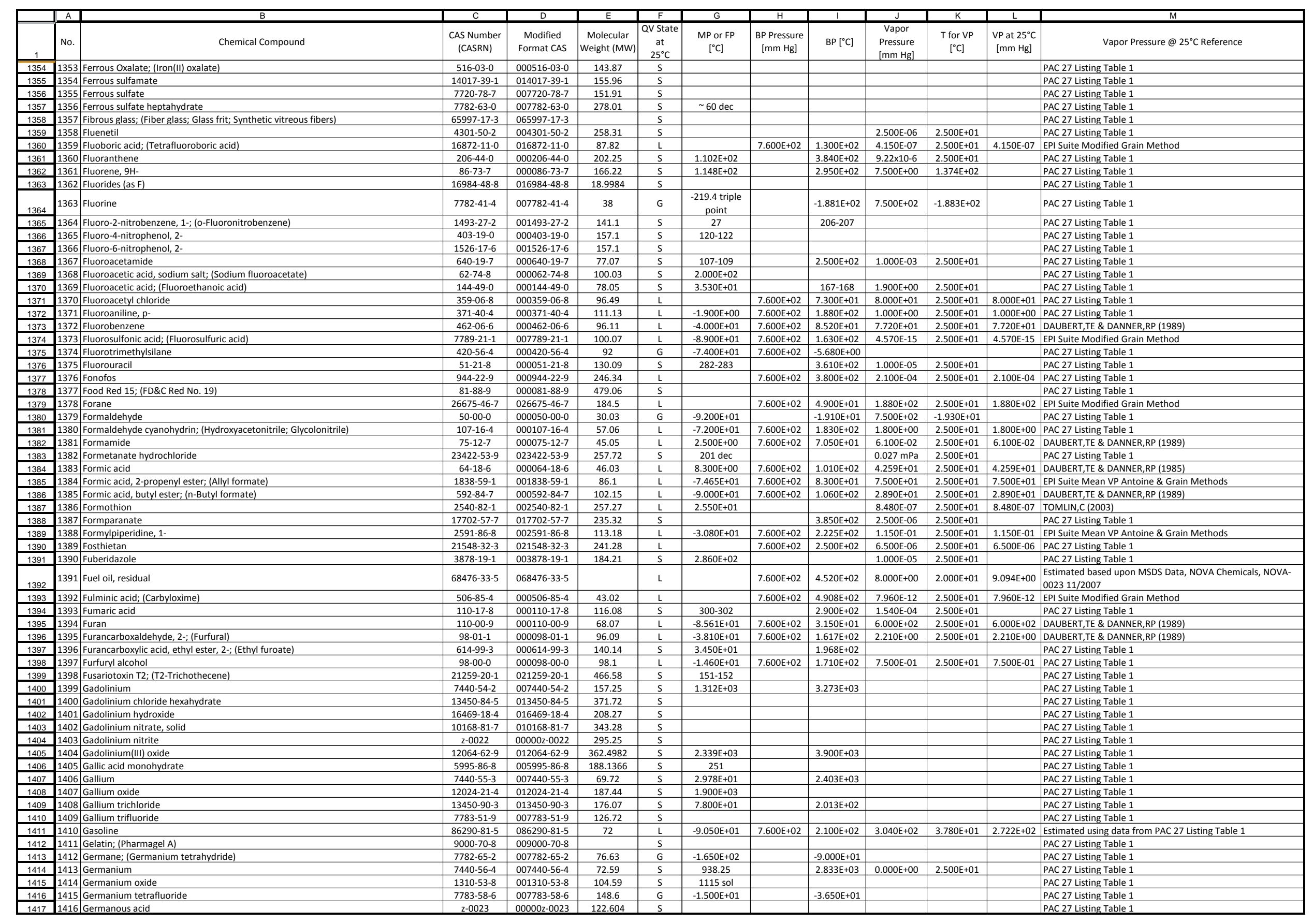









\begin{tabular}{|c|c|c|c|c|c|c|c|c|c|c|c|c|}
\hline & Chemical Compound & $\begin{array}{c}\text { CAS Number } \\
\text { (CASRN) }\end{array}$ & $\begin{array}{c}\text { Modified } \\
\text { Format CAS } \\
\end{array}$ & \begin{tabular}{|c} 
Molecular \\
Weight (MW)
\end{tabular} & \begin{tabular}{|c|} 
Qv state \\
at \\
$25^{\circ} \mathrm{C}$
\end{tabular} & $\begin{array}{c}\text { MP or FP } \\
{\left[{ }^{\circ} \mathrm{C}\right]}\end{array}$ & $\begin{array}{c}\text { BP Pressure } \\
{[\mathrm{mm} H \mathrm{Hg}]}\end{array}$ & $\mathrm{BP}\left[{ }^{\mathrm{C} C}\right]$ & $\begin{array}{l}\text { Vapor } \\
\text { Pressure } \\
{[[m m ~ H g]}\end{array}$ & $\begin{array}{c}\begin{array}{c}\text { T for VP } \\
\text { [Cc] }\end{array} \\
\end{array}$ & 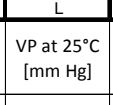 & Vapor Pressure @ $25^{\circ} \mathrm{C}$ Reference \\
\hline & 1477 Hexachloronaphthalene & $1335-87-1$ & $001335-87-1$ & 334.74 & s & 1.372E+02 & & $\begin{array}{l}343.3- \\
387.8\end{array}$ & $3.3 \times 10-6$ & 2.5006+ +01 & & PAC 27 Listing Table 1 \\
\hline 1479 & \begin{tabular}{|l|l|l|l|}
1478 & Hexachlorophene \\
\end{tabular} & $70-30-4$ & $000070-30-4$ & 406.9 & $\mathrm{~s}$ & $1.665+02$ & & & & & & PAC 27 Listing Table 1 \\
\hline$\frac{1480}{1191}$ & $\mid \frac{14790 \text { Hexachloropropene }}{1480 \text { Hexadecanamine, } 1-}$ & $\frac{1888-71-7}{10327-71}$ & $\begin{array}{ll}001888-71-7 \\
000132-271\end{array}$ & $\frac{248.73}{24152}$ & $\frac{L}{S}$ & $\frac{-7.290 E+01}{462}$ & $7.600 \mathrm{E}+02$ & $\begin{array}{l}2.0906+02 \\
3.225 \mathrm{E}+02\end{array}$ & $2.440 \mathrm{E}-01$ & $2.500 \mathrm{E}+01$ & $2.440 \mathrm{E}-01$ & $\begin{array}{l}\text { PAC } 27 \text { Listing Table } 1 \\
\text { PAC 27 lising Table } 1\end{array}$ \\
\hline$\frac{1481}{1482}$ & 1881 Hexadecane & $\frac{143-2-1-1}{544-76-3}$ & $\begin{array}{ll}0 \\
0000544-2-7-1 \\
0005-3\end{array}$ & $\frac{24.123}{226.5}$ & $\frac{\mathrm{L}}{\mathrm{L}}$ & $\frac{40.2}{1.814 E+01}$ & $7.600 E+02$ & 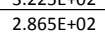 & 1.430E-03 & $2.5006+01$ & $1.430 \mathrm{E}-03$ & DAUBRT,TE \& DANNER,RP (1989) \\
\hline 1483 & 1482 Hexadecanonoic acid; (Palmititic acid) & $\frac{54-10 \cdot 3}{57-3}$ & $000057-10-3$ & 256.48 & $\mathrm{~s}$ & $\frac{1.004-64}{63-64}$ & & 271.50100 & 1.430ecos & & & 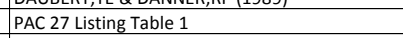 \\
\hline 1484 & 1483 Hexadecanol, 1 - & $36653-82-4$ & $036653-82-4$ & 242.5 & $\mathrm{~s}$ & $5.000 \mathrm{t}+01$ & & $178-182$ & & & & PAC 27 listing Table 1 \\
\hline 1485 & $\begin{array}{l}1484 \text { | Hexadecene, } 1 \text { - } \\
1095\end{array}$ & $629-73-2$ & $000629-73-2$ & 224.48 & $\mathrm{~L}$ & $4.1006+00$ & $7.600 \mathrm{E}+02$ & $2.844 E+02$ & $2.6400-03$ & $2.500 \mathrm{E}+01$ & $2.640 \mathrm{E}-03$ & 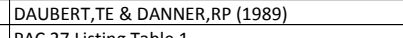 \\
\hline$\frac{1486}{1487}$ & $\begin{array}{l}18455 \text { Hexadecypprifinium chloride, } 1 ; \text {; (Cepacol chloride) } \\
1486 \text { Hexadecyltrimethylammonium chloride }\end{array}$ & $\begin{array}{l}\frac{123303-5}{112-02-7} \\
\end{array}$ & $\begin{array}{l}0.00123-30-5-5 \\
000112-02-7 \\
\end{array}$ & $\begin{array}{l}340.05 \\
3220.07\end{array}$ & $\frac{\mathrm{s}}{\mathrm{s}}$ & & & & & & & \begin{tabular}{|l|} 
PAA 27 Listing Table 1 \\
PC 27 litsing Table 1
\end{tabular} \\
\hline & 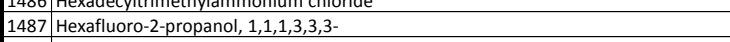 & $\begin{array}{l}\frac{112202-7}{920-66-1} \\
9\end{array}$ & $\begin{array}{ll}0.000112-20-7 \\
00092-66-1 \\
\end{array}$ & $\frac{32.017}{168.05}$ & & $-2.000 E+00$ & $7.600 E+02$ & & 1.591E+02 & & 1.591E+02 & 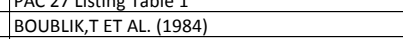 \\
\hline 1489 & 1488 Hexafluoroacetone & $684-16-2$ & $000684-16-2$ & 166.02 & $G$ & $-1.255 \mathrm{E}+02$ & t.ovuctur & $-2.740 \mathrm{E}+01$ & $7.500 \mathrm{E}+02$ & $-2.7606+01$ & & PAC 27 listing Table 1 \\
\hline & 1489 Hexafluorobenzene & $392-56-3$ & $000392-56-3$ & 186.06 & $\mathrm{~L}$ & $3.000 \mathrm{E}+00$ & $7.600 E+02$ & $8.1006+01$ & $8.450 \mathrm{E}+01$ & $2.500 \mathrm{E}+01$ & $8.450 \mathrm{E}+01$ & DAUBERT,TE \& DANNER,RP (1989) \\
\hline 1491 & 1990| Hexafluorobutyne-2; (1,1,1,4,4,4-Hexafluoro-2-butyne) & $692-50-2$ & $000692-50-2$ & 162.04 & $G$ & $-1.174 \mathrm{E}+02$ & & 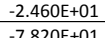 & & & & PAC 27 Listing Table 1 \\
\hline$\frac{1492}{1493}$ & 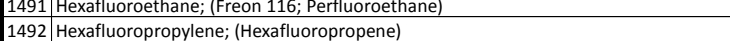 & $\frac{77-16-4}{116-15-4}$ & $\frac{000076-16-4}{00011-15-4}$ & $\frac{138.02}{150.02}$ & $\frac{G}{G}$ & $-1.565 \mathrm{E}+02$ & & 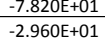 & & & & 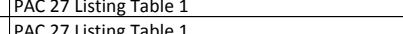 \\
\hline 1494 & 1993 Hexafluoropopropylene-vinulidene fluoride polymer; (Viton) & $\frac{1.01104}{9011-0}$ & & & & $1.500 t+2$ & & & 7. $.000 \mathrm{E}+02$ & $-3.060 \mathrm{E} E+1$ & & $\begin{array}{l}\text { PAC 27 Listing Table } 1 \\
\text { Pand }\end{array}$ \\
\hline & & $541-05-9$ & $000541-05-9$ & 222.46 & $\mathrm{~s}$ & $6.450 \mathrm{E}+01$ & & $1.340 \mathrm{E}+02$ & & & & \\
\hline 1496 & 1495 Hexamethydisisilazane & $\begin{array}{l}999-97-3 \\
\end{array}$ & 0000999-97-3 & 161.44 & $\mathrm{~L}$ & & $7.600 E+02$ & $1.250 €+02$ & 1.379E+01 & $2.500 E+01$ & $1.379 E+01$. & YAWS,CL (1994A) \\
\hline 1497 & |1496- Hexamethyldisiloxane & 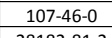 & 000107-46-0 & 162.42 & $\mathrm{~L}$ & $-5.900 E+01$ & $7.600 E+02$ & $1.010 \mathrm{E}+02$ & $4.210 \mathrm{E}+01$ & $2.500 \mathrm{E}+01$ & $4.210 \mathrm{E}+01$ & FLANINGAM,OL (1986) \\
\hline$\frac{1498}{11099}$ & 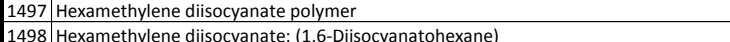 & $\frac{28182-81-2}{822-06-0}$ & $028182-81-2$ & & $\frac{s}{L}$ & & & & & & & 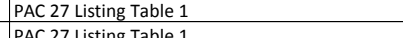 \\
\hline$\frac{1499}{1500}$ & $\begin{array}{l}19488 \text { Hexamethylene disiscyanate; (1,6-Disiscyanatohexane) } \\
11999 \text { Hexamethyleneimine }\end{array}$ & $\frac{822-0-6-0}{111-49-9}$ & $\begin{array}{ll}0.000222-0-0-0 \\
000111-49-9 \\
\end{array}$ & $\frac{168.19}{99.2}$ & $\frac{L}{L}$ & $\frac{-6.700 E+01}{-3.700 E+01}$ & $\begin{array}{ll}\frac{1.000 \mathrm{E} E+01}{7.600 \mathrm{E}+02} \\
\end{array}$ & 1.220E+ 1.302 & $\begin{array}{l}5.0006-01 \\
8.005 E+00\end{array}$ & $\frac{2.500 \mathrm{E}+01}{2.500 \mathrm{E}+01}$ & $\begin{array}{lll}5.000 E=01 \\
8.090+000\end{array}$ & $\begin{array}{l}\text { PAC 2 L Lsing g a able 1 } \\
\text { PAC } 27 \text { Listing Table } 1\end{array}$ \\
\hline & 1500 Hexamethylentetetraamine hydrochloride & $\frac{11743-12-6}{58713-6}$ & $058713-21-6$ & & & & $7.600 E+02$ & $4.068 \mathrm{E}+02$ & 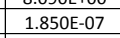 & $2.500 \mathrm{E}+01$ & 1.850E-07 & EPI Suite Modified Grain Method \\
\hline 1502 & 1501 Hexamethylenetetraamine; (Methenamine) & $100-97-0$ & $000100-97-0$ & 140.19 & $\mathrm{~s}$ & $>250$ & & subl & $4.0 \times 10-3$ & $2.5000 \mathrm{E}+01$ & 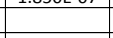 & PAC 27 listing Table 1 \\
\hline 1503 & \begin{tabular}{|l|l|l|l|}
1502 & Hexamethylphosphoramide \\
1502
\end{tabular} & $680-31-9$ & $000680-31-9$ & 179.2 & $\mathrm{~L}$ & $7.200 E+00$ & 7.600E+02 202 & $2.325 E+02$ & $3.000 \mathrm{E}-02$ & $2.500 \mathrm{E}+01$ & $\begin{array}{lll}3.000 E-02 \\
\end{array}$ & PAC 27 listing Table 1 \\
\hline 1504 & $\begin{array}{l}1503 \text { Hexamethyltetracosane, } 2,6,10,15,19,23 ; \text {; (Squalane) } \\
1.504 \mid\end{array}$ & $\frac{111-01-3}{6-25-1}$ & $000111-01-3$ & $\frac{422.92}{10018}$ & $L$ & $\begin{array}{l}-3.8000+01 \\
-5.6305+01\end{array}$ & 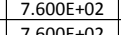 & $\frac{3.500 E+02}{132050.02}$ & $2.750 E-04$ & $2.5006+01$ & $2.750 E-04$ & 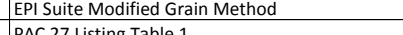 \\
\hline$\frac{1505}{1506}$ & $\begin{array}{l}\frac{1504}{1504 \text { Hexanal }} \\
105 \text { Hexane }\end{array}$ & $\frac{6-625-1}{112-54-3}$ & $\begin{array}{ll}000066-25-1 \\
000110-54-3\end{array}$ & $\frac{100.18}{86.18}$ & $\frac{L}{L}$ & 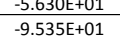 & $\begin{array}{l}7.600 E+02 \\
7.600+02\end{array}$ & $\frac{1.3110 E E+02}{6873+01}$ & $\frac{1.130 E E+1}{1513+02}$ & $\frac{2.500 E \mathrm{E}+11}{2500 \mathrm{E}+01}$ & 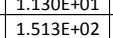 & 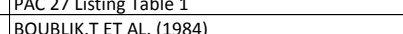 \\
\hline & 1506 Hexananediol diacrylate, 1,6- & $\frac{110-3-3-3}{13048-33-4}$ & $013048-33-4$ & $\frac{00.10}{226.3}$ & $\frac{L}{L}$ & 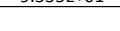 & T.000ut 02 & & 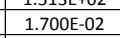 & $2.5000++01$ & 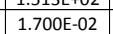 & 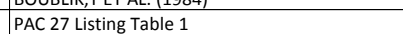 \\
\hline 1508 & 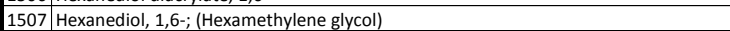 & 629-11-8 & 000629-11-8 & 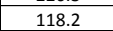 & $\mathrm{s}$ & 42 & & $2.500 E+02$ & 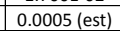 & $\frac{2.5006+01}{2.501}$ & & $\begin{array}{l}\text { PAC 27 Listing Table } 1 \\
\text { Pand }\end{array}$ \\
\hline 1509 & 1508 Hexanehexol, 1,2,3,4,4,5,6; (Mannitol) & $69-65-8$ & $000069-65-8$ & 182.2 & $\mathrm{~s}$ & $1.670 E+02$ & & $290-295$ & & & & PAC 27 Listing Table 1 \\
\hline 1510 & 1509| Hexanenitrile & $628-73-9$ & $000628-73-9$ & 97.18 & $\mathrm{~L}$ & & $7.6006+02$ & $1.636 \mathrm{E}+02$ & $2.850 \mathrm{~F}+00$ & $2.500 \mathrm{E}+01$ & $2.850 \mathrm{E}+000$ & DAUBERT,TE \& DANNER,RP (1989) \\
\hline$\frac{1512}{1512}$ & 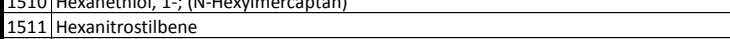 & $\frac{111-3-1-9}{20062-22-0}$ & $020062-22-0$ & $\frac{118.26}{450.23}$ & $\mathrm{~s}$ & (2) & f.ovotur & $1.511+02$ & 4.202tutur & & 4.202E+00 & 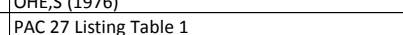 \\
\hline $\begin{array}{lll}1513 \\
1513\end{array}$ & 1512 | Hexanoic acid & $142-62-1$ & $0000142-62-1$ & 116.18 & & $-1.500 E+00$ & 7.600E+02 & $2.050 \mathrm{E}+02$ & 4.350E-02 & 2.500E+01 & & DAUBERT,TE \& DANNER,RP (1989) \\
\hline 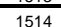 & $\frac{1513 \text { Hexanol, 2, 2; (2-Hydroxyhexane) }}{1513}$ & $\frac{1.46-93-7}{626-3}$ & $000026-93-7-7$ & $\frac{1020}{1022}$ & L & & 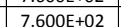 & 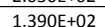 & 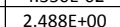 & $2.500 E+01$ & $\frac{1.638+00}{2488+00}$ & YAWS CL (1994AA \\
\hline 1515 & \begin{tabular}{|l|l}
1514 Hexanol, $n-;(n-H e x y l$ alcoholl) \\
\end{tabular} & $\begin{array}{ll}111-27-3 \\
\end{array}$ & $000111-27-3$ & 102.2 & $\mathrm{~L}$ & $-4.670 E+01$ & $7.600 E+02$ & $1.572 E+02$ & $9.280 \mathrm{E}-01$ & $2.500 E+01$ & $9.280 E-01$ & DAUBERT,TE \& DANNER,RP (1989) \\
\hline 1516 & \begin{tabular}{|l|l}
1515 & Hexanone, 2; (Methyl n-butyl ketone) \\
151
\end{tabular} & $591-78-6$ & $\begin{array}{ll}000591-78-6 \\
000-020.0\end{array}$ & 100.18 & $\mathrm{~L}$ & $-5.690 \mathrm{E}+01$ & $7.600 E+02$ & $1.272 E+02$ & $1.1606+01$ & $2.500 E+01$ & $1.160 \mathrm{E}+01$ & AMBROSE, D ET AL. (1975) \\
\hline$\frac{1517}{1518}$ & 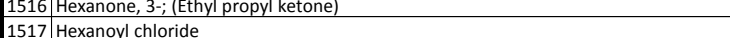 & $\frac{5892-38-8}{142-6-0}$ & $\begin{array}{ll}0005899-38-8 \\
000142-6-0\end{array}$ & $\frac{100.18}{134.603}$ & $\frac{L}{L}$ & & $\frac{7.600 \mathrm{E}+02}{77.60 \mathrm{E}+02}$ & $\frac{1.240 \mathrm{EE}+02}{1.20 \mathrm{E}+02}$ & $\frac{1.394 E+01}{4.490 E+00}$ & $\frac{2.500 E+01}{2.500+01}$ & $\frac{1.394++01}{4.490+500}$ & \begin{tabular}{|l}
$\mid$ YAWS, CL (1994A) \\
EPI Sunte Mean VP Antoine \& Grain Methods
\end{tabular} \\
\hline$\frac{1518}{1519}$ & 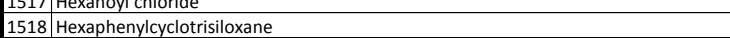 & $\frac{142-6-1-0}{512-63-0}$ & $\begin{array}{lll}0000542-6-63-0 \\
000512-63-0\end{array}$ & $\frac{134.603}{594.8892}$ & $\frac{L}{s}$ & $\frac{-8.700 E+01}{184-188}$ & & 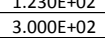 & & & & 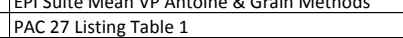 \\
\hline$\frac{1520}{1520}$ & 1519. Hexene, 1 . & 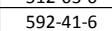 & $\frac{000592-41-6}{0.030}$ & $\frac{5.94 .158}{80.48}$ & & 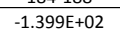 & 7.600E +02 & 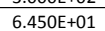 & $1.840 E+02$ & $2.500 E+01$ & $1.800 E+02$ & 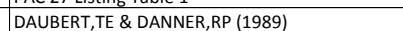 \\
\hline 1521 & 1520 Hexylene glycol & 107-41-5 & $0000107-41-5$ & 118.2 & $\mathrm{~L}$ & $-5.000 E+01$ & $7.600 E+022$ & $1.971 E+02$ & $1.300 E-02$ & $2.500 E+01$ & $1.3000-02$ & DAUBERT,TE \& DANNER,RP (1989) \\
\hline 1522 & 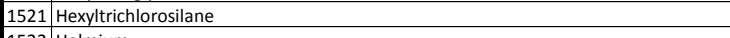 & $928-65.4$ & $000928-65-4$ & 219.63 & & & $7.600 \mathrm{E}+02$ & $1.900 E+02$ & 5.920E-01 & $2.500 E+01$ & 5.920E-01 & EPP Suite Mean VP Antoine \& Grain Methods \\
\hline 1523 & 1522 Holmium & 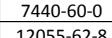 & $\frac{007440-60-0}{012055-6-8}$ & $\frac{164.93}{337.859}$ & $\frac{s}{s}$ & & & $2.7206+03$ & $2.000 \mathrm{E}+00$ & $1.630 \mathrm{E}+03$ & & PAC 27 Listing Table 1 \\
\hline$\frac{1524}{1525}$ & $\begin{array}{l}15233 \text { Holmum trioxide } \\
1524 \text { Humic acid, sodium salt }\end{array}$ & $\frac{12055-62-8}{68131-04-4}$ & 01205351-6-2-8 & $3 / 1.859$ & $\frac{s}{s}$ & $\frac{2.45 \mathrm{t}+03}{>300}$ & & & & & & $\begin{array}{l}\text { PAC/ ILtsing gable 1 } \\
\text { PAC 27 Listing Table } 1\end{array}$ \\
\hline & S & 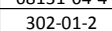 & 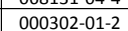 & 32.05 & $\frac{\mathrm{L}}{\mathrm{L}}$ & 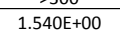 & $7.600 E+02$ & 1.136E+02 202020 & $1.440 E+01$ & $2.500 E+01$ & 1.440E+01 & PAC 7 Listing Table 1 \\
\hline 1527 & 1526 Hydrazine hydrate; (Hydrazine monohydrate; & $7803-57-8$ & $007803-57-8$ & 50.08 & $\mathrm{~L}$ & 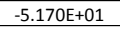 & $7.600 E+022$ & $1.190 E+022$ & $2.070 E+01$ & $2.500 E+01$ & $2.070 \mathrm{E}+01$ & \begin{tabular}{|c|c|} 
AcD Labs Physical Chemistry Suite \\
\end{tabular} \\
\hline 1528 & 1527] Hydrazine hydrochloride; (Hydrazine monochloride) & $2644-70-4$ & $002644-70-4$ & 68.52 & $s$ & $1.9806+02$ & & $200 \mathrm{dec}$ & & & & PAC 27 L Listing Table 1 \\
\hline$\frac{1529}{1520}$ & $\begin{array}{l}1528 \text { Hydrazine nitrate; (Hydrazinium nitrate) } \\
1529 \text { Hydranino sulfote }\end{array}$ & $13464-97-6$ & $013464-97-6$ & $\frac{95.06}{13014}$ & $\frac{\mathrm{s}}{\mathrm{c}}$ & $\begin{array}{l}7.0006+01 \\
205 \text { detem }\end{array}$ & & & & & & $\begin{array}{lll}\text { PAC 27 Listing Table } 1 \\
\text { Pac }\end{array}$ \\
\hline$\frac{1530}{1531}$ & $\begin{array}{l}12599 \text { Hydraine esuffate } \\
1530 \text { Hydrazine, dihydrochloride }\end{array}$ & $\frac{10034-93-2}{5341-61-7}$ & 010034-93-2 & $\frac{130.14}{104.98}$ & $\frac{s}{s}$ & $\frac{254 \text { decom }}{197 \text { dec }}$ & & & & & & $\begin{array}{l}\text { PAC C L Lising a able } 1 \\
\text { PAC 27 listing Table } 1\end{array}$ \\
\hline & 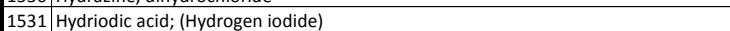 & $\frac{10034-85-2}{1003}$ & $\frac{010034-85-2}{0.03}$ & & & $-5.0800 \mathrm{E}+01$ & & $-3.538 E+011$ & $5.9406+03$ & $2.500 E+01$ & & $\frac{1}{\text { PAC } 27 \text { Listing Table } 1}$ \\
\hline 1533 & 1532 |Hydrobromicic acid; (Hydrogen bromide) & $\begin{array}{l}10035-10 \cdot 6 \\
\end{array}$ & $010035-10-6$ & 80.92 & $\mathrm{G}_{\mathrm{G}}$ & $-8.700 E+01$ & & 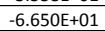 & 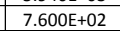 & $\frac{6.6505+01}{6}$ & & PAC 27 Listing Table 1 \\
\hline 1534 & 1533 Hydrogen & $1333-74-0$ & 001333-74-0 & 2.02 & $G_{G}$ & $-2.592 E+02$ & & $-2.528 E+02$ & $1.240 E+06$ & $2.500 E+01$ & & PAC 27 Listing Table 1 \\
\hline 1535 & 1534 Hydrogen chloride; (Hydrochloric acid) & $7647-01-0$ & 007647-01-0 & 36.46 & $G$ & $-1.142 E+02$ & & $-8.500 E+01$ & & & & $\frac{1}{\text { PAC } 27 \text { Listing Table } 1}$ \\
\hline 1536 & 15355 Hydrogenen cyanide; (Hydrocyanic acid) & $74490-8$ & $000074-90-8$ & 27.03 & $G$ & $-1.3292 \mathrm{E}+01$ & & $\begin{array}{l}2.600 E+01 \\
\end{array}$ & $7.500 E+02$ & $2.540 \mathrm{E}+01$ & & PAC 27 Listing Table 1 \\
\hline 1537 & 1536 Hydrogen fluoride; (Hydrofluoric acid) & $\begin{array}{l}7664-399-3 \\
\end{array}$ & $007664-39 \cdot 3$ & 20.01 & $G$ & $-8.336 \mathrm{E}+01$ & & 2.000E+01 & $7.5000 \mathrm{E}+022$ & $1.920 E+01$ & & PAC 27 Listing Table 1 . \\
\hline 1538 & 1537 Hydrogen peroxide & $7722-84-1$ & $007722-84-1$ & 34.02 & L & $-4.300 E-01$ & $7.6000 E+022$ & $1.502 E+02$ & $1.970 \mathrm{E}+00$ & $2.5000 E+01$ & 1.97 & DAUBERT,TE \& DANNER,RP (1989) \\
\hline 1539 & 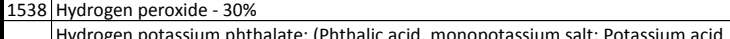 & 7722-84-1a & 007722-84-1a & & & $-1.100 \mathrm{E}+01$ & $7.600 E+022$ & $1.502 E+02$ & $1.970 E+00$ & $2.500 \mathrm{E}+01$ & & |FP Suite Experimental Data \\
\hline 1540 & 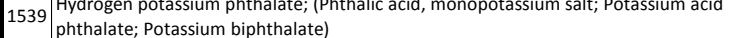 & 877-24-7 & 000877-24-7 & 204.23 & $\mathrm{~s}$ & dec & & & & & & PAC 27 Listing Table 1 \\
\hline & $1540 \mid$ Hydrogen selenide & 7783-07-5 & $000783-07-5$ & 80.98 & $G$ & $-6.4006+01$ & & 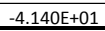 & $7.6000 \mathrm{E}+03$ & $2.300 E+01$ & & PAC 27 Listing Table 1 \\
\hline
\end{tabular}




\begin{tabular}{|c|c|c|c|c|c|c|c|c|c|c|c|c|c|}
\hline & A & & & Medified & Melecular & $\frac{F}{\text { av state }}$ & 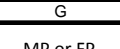 & $\mathrm{H}$ & 1 & & $\frac{\mathrm{k}}{T-T_{0}}$ & & $\mathrm{M}$ \\
\hline & No. & Chemical Compound & $\begin{array}{l}\text { CAS Number } \\
\text { (CASRN) }\end{array}$ & $\begin{array}{l}\text { Modified } \\
\text { Format CAS }\end{array}$ & $\begin{array}{l}\text { Molecular } \\
\text { Weight (MW) }\end{array}$ & at $\begin{array}{c}\text { at } \\
25^{\circ} \mathrm{C}\end{array}$ & $\begin{array}{c}\text { MP or FP } \\
{\left[{ }^{\circ} \mathrm{C}\right]}\end{array}$ & $\begin{array}{c}\text { BP Pressure } \\
{[\mathrm{mm} \mathrm{Hg}]}\end{array}$ & $B P\left[{ }^{[C}\right]$ & $\begin{array}{l}\text { Vapor } \\
\text { Pressure } \\
\text { Imm Hgat }\end{array}$ & $\begin{array}{c}\text { Tfor VP } \\
{\left[{ }^{\circ C} \mathrm{C}\right]}\end{array}$ & $\begin{array}{c}\text { VP at } 25^{\circ} \mathrm{C} \\
{[\mathrm{mm} \mathrm{Hg}]}\end{array}$ & 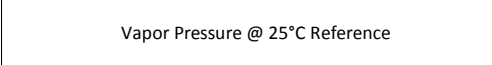 \\
\hline 1542 & $\mid 1541+\mathrm{H}$ & Hydrogen sulfide & $7783-06-4$ & $007783-06-4$ & 34.08 & & $-8.550 E+01$ & & $-5.955 \mathrm{E}+01$ & & -6.050E+01 & & PAC 27 Listing Table 1 \\
\hline 1543 & $\mid \frac{1542}{1562}$ & Hydrogenated terphenyl & $61788-32-7$ & 061788-32-7 & 298 & $\mathrm{~L}$ & & $7.6000+02$ & $3.400 E+02$ & $7.300 \mathrm{E}-05$ & $2.500 E+01$ & $7.3000-05$ & EPI Suite Modifified Grain Method \\
\hline 1544 & 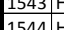 & $\begin{array}{l}\text { Hydroquinone } \\
\text { Hydrotrated (mild \& severe) heavy paraffinic distillates }\end{array}$ & 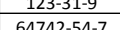 & 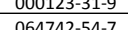 & 110.12 & $\frac{3}{1}$ & 1.720EE+02 & $76005+02$ & $2.850 \mathrm{EE}+02$ & $1.000+100$ & $1.324+0202$ & 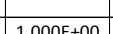 & $\begin{array}{l}\text { PAC 27 Listing Table } 1 \\
\text { MSPS }\end{array}$ \\
\hline$\frac{1534}{1546}$ & $1545 \mathrm{H}$ & Hydrotreated middle distillate (Petroleum base oil) & 64/742-54-1 & $\begin{array}{lll}0.0644-2-4-1-1 \\
064742-46-7\end{array}$ & 212.42 & $\mathrm{~L}$ & $9.900 E+00$ & $7.600+502$ & & 3..430E-0.3 & 2.5000+to1 & 3. & $\begin{array}{l}\text { WISUS Data, snel U8.0.2.2011 } \\
\text { EPI Suite Experimental Data }\end{array}$ \\
\hline & & Hydroxy-2-methylpropanoic acid, 2-; (2-Methyllactic acid) & $\frac{594-61-6}{596}$ & & & & $8.250 \mathrm{E}+01$ & & 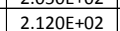 & & & & \\
\hline$\frac{2548}{1548}$ & $1547 \mid \mathrm{H}$ & Hydroxy-4-'-hydroxyethoxy-2-methylpropiophenone, $2-$ & $106797-53-9$ & 106797-53-9 & 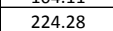 & $\mathrm{s}$ & & & & & & & PAC 27 Listing Table 1 \\
\hline 1549 & 1548 | & Hydroxy-4-methyl-2-pentanone, 4; (Diacetone alcohol) & $123-42-2$ & $000123-42-2$ & 116.18 & $\mathrm{~L}$ & $-4.700 E+01$ & $7.600 E+02$ & $1.640 \mathrm{E}+02$ & $1.7106+00$ & $2.500 E+01$ & $1.710 \mathrm{E}+00$ & DAUBERT, TE \& DANNER,RP (1989) \\
\hline$\frac{1550}{1550}$ & 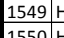 & Hydroxyapatite; (Calcium hydroxyapatite) & $1306-06-5$ & $001306-06-5$ & 502.31 & $\frac{s}{s}$ & & & & & & & 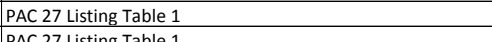 \\
\hline$\frac{1551}{1552}$ & $\begin{array}{l}1550 \mathrm{H} \\
1551 \mathrm{H}\end{array}$ & 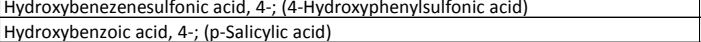 & $\frac{98-67.9}{99-96-7}$ & $\begin{array}{l}000098-67-9 \\
00009-96-7\end{array}$ & $\frac{174.18}{138.1226}$ & $\frac{\mathrm{s}}{\mathrm{s}}$ & $2.145 E+02$ & & & 19000-07 & $2.5006+01$ & & $\begin{array}{l}\text { PAC C2 L Listing Table } 1 \\
\text { PAC 27 Listing Table } 1 \\
\end{array}$ \\
\hline & & 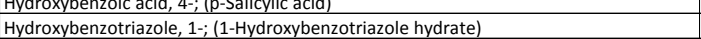 & 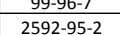 & & $\frac{183.1226}{135.14}$ & s & 1.157.1.02 & & & & & & 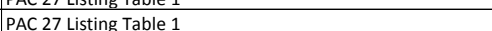 \\
\hline$\frac{2554}{1554}$ & $1553 \mathrm{th}$ & 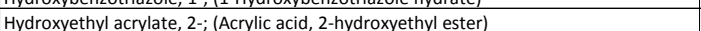 & 818-61-1. & $\frac{000818-61-1}{0.01}$ & $\frac{116.13}{113.13}$ & $\frac{\mathrm{L}}{\mathrm{L}}$ & & $7.600 E+02$ & $2.200 E+02$ & $5.2300-02$ & $2.5000+01$ & $5.230 \mathrm{E}-02 \mathrm{a}$ & 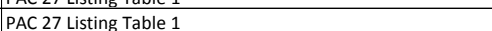 \\
\hline 1555 & $1554 \mid \mathrm{H}$ & Hydroxyethyl methacrylate, 2 - & $868-77-9$ & $000868-77-9$ & 130.14 & L & $-1.200 E+01$ & $7.600 E+02$ & $6.700 \mathrm{E}+01$ & $1.2600-01$ & $2.5000+01$ & $1.2600-01$ & PAC 27 Listing Table 1 \\
\hline 1556 & 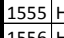 & |Hydroxyethy)|-1-piperazininethanesulfonic acid, $4-\left(2^{-2}-\right.$ & $7365-45-9$ & $007365-45-9$ & 238.24 & $\frac{s}{c}$ & & & & & & & PAC 27 Listing Table 1 \\
\hline & & & & & & & 160.5 dec & & & & & & PAC 27 Listing Table 1 \\
\hline 1558 & $|1557|_{1}^{\mid}$ & $\begin{array}{l}\text { Hydroxyethyidene-1,1-diphosphonic acid, 1;- (Hydroxyethylidine bisphosphonic acid, } \\
1-1\end{array}$ & 2809-21-4 & $002809-21-4$ & 206.03 & $\mathrm{~s}$ & $1.050 \mathrm{E}+02$ & & & & & & PAC 27 Listing Table 1 \\
\hline 1559 & $1558 \mathrm{H}$ & Hydroxxlamine & $7803-49-8$ & $007803-49-8$ & 33.04 & L & $3.400 E+01$ & $7.600 E+02$ & $1.100 E+02$ & $1.000 E+01$ & $4.7206+01$ & $1.398 \varepsilon+00$ & Estimated using data from PAC 27 Listing Table 1 \\
\hline 1560 & $\left.1559\right|^{4}$ & $\begin{array}{l}\text { Hydroxylamine chloride; } \\
\text { Hyydroxlamine hydrochloride) }\end{array}$ & 5470-11-1 & $005470-11-1$ & 69.5 & $\mathrm{~s}$ & $1.5906+02$ & & dec & & & & PAC 27 Listing Table 1 \\
\hline & $1560 \mid \mathrm{H}$ & Hydroxylamine nitrate & $13465-08-2$ & $013465-08-2$ & 96.04 & $\mathrm{~L}$ & & $7.600 E+02$ & $1.000 E+02$ & $1.000 \mathrm{E}-05$ & $2.500 E+01$ & $1.000 E-05$ & $\begin{array}{l}\text { MSDS Data, negligable Aqueous solution of material having } \\
\text { neglible vapor pressure at } 25^{\circ} \text { C. VP of } 11-5 \text { assigned for } \\
\text { chlalutation purposes }\end{array}$ \\
\hline$\frac{1562}{1562}$ & $1561 \mathrm{H}$ & Hydroxylamine sulfate; (Oxammonium sulfate) & $10039-54-0$ & $010039-54-0$ & $\begin{array}{lll}164.16 \\
\end{array}$ & $\mathrm{~s}$ & $1.770 \mathrm{E}+02$ & & & & & & $\begin{array}{l}\text { Ceacuatulon purposes } \\
\text { PAC } 27 \text { Listing Table } 1\end{array}$ \\
\hline 1560 & 1562 & 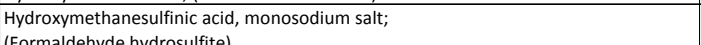 & $149-44-0$ & $000149-44-0$ & 119.1 & $\mathrm{~s}$ & 63 & & & & & & PAC 27 Listing Table 1 \\
\hline 1564 & $1563+\mathrm{H}$ & Hydroxy-N-phenylbenzamide, N;; (Phenylbenzohydroxamic acid, $\mathrm{N}-$-) & $304-88-1$ & $000304-88-1$ & 213.2354 & $\mathrm{~s}$ & $118-120$ & & & & & & PAC 27 Listing Table 1 \\
\hline & $|1564| \mathrm{H}$ & Hydroxypheny)|benzothiazole, 2-(2-- & 3411-95-8 & 003411-95-8 & 227.28 & $\mathrm{~s}$ & $131-133$ & & & & & & PAC 27 Listing Table 1 \\
\hline 1566 & $1565 \mid \mathrm{H}$ & Hydroxyphenylacetic acid, alpha- & $90-64-2$ & $000090-64-2$ & 152.16 & $\mathrm{~s}$ & 117-119 & & dec & & & & PAC 27 Listing Table 1 \\
\hline$\frac{1566}{1568}$ & 1567/H & $\begin{array}{l}\text { Hyycroxypropyy celluluse } \\
\text { Hydroxyquinoline sufate, 8- }\end{array}$ & $\begin{array}{lll}9004-64-2 \\
134-31-6\end{array}$ & $000134-31-6$ & $\frac{800.9296}{388.42}$ & $\frac{2}{3}$ & 177.5 & & $2.670 E+02$ & $1.660 \mathrm{E}-03$ & $2.5000+01$ & & $\begin{array}{l}\text { PAC L Lsting abole } 1 \\
\text { PAC 27 Listing Table } 1\end{array}$ \\
\hline 1569 & $1568 / \mathrm{H}$ & Hypophosphorous acid-d3; (Hydrophosphorus acid-d3 solution, 50 wt.\% in D2O) & $57583-56-9$ & $057583-56-9$ & 69.01 & $\mathrm{~L}$ & (3) & $7.600 E+02$ & $4.800 \mathrm{E}+02$ & $2.060 \mathrm{E}-08$ & $2.5006+01$ & $2.060 \mathrm{E}-08$ & EPI Suite Modififed Grain Method \\
\hline 1570 & $1569+\mathrm{H}$ & Hypophosphorus acid; (Phosphononic acid) & 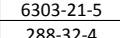 & $006303-21-5$ & $\frac{66}{6609}$ & $\frac{\mathrm{s}}{\mathrm{s}}$ & $\begin{array}{l}2.650 \mathrm{E}+01 \\
90.91\end{array}$ & & $2570=502$ & & & & PAC 27 Listing Table 1 \\
\hline$\frac{151}{1572}$ & 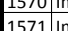 & Iminadore hydrochloride & 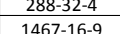 & 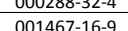 & $\frac{18.09}{1045}$ & $\frac{3}{5}$ & 势- & & 2.5TUE+U2 & & & & 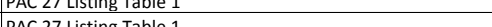 \\
\hline 1572 & 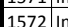 & Iminondicacticicaid & 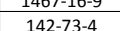 & $000142-73-4$ & 133.12 & $\mathrm{~s}$ & $\frac{220-250}{220}$ & & $247.5 \mathrm{dec}$ & & & & 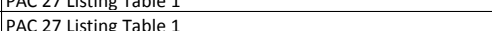 \\
\hline$\frac{1574}{1574}$ & 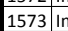 & Iminodiacetic acid, disodium salt hydrate & $17593-73-6$ & $017593-73-6$ & 195.08254 & $\mathrm{s}$ & 180 & & & & & & $\begin{array}{l}\text { PAC } 27 \text { Listing Table } 1 \\
\end{array}$ \\
\hline 1575 & 15744|[n & Indan & $496-11-7$ & 000496-11-7 & 118.19 & $\mathrm{~L}$ & $-5.1406+01$ & $7.600 E+02$ & 1.765E +02 & 1.467€ +00 & $2.500 E+01$ & 1.467 E +00 & BOUBLLK, ET ALL. (1984) \\
\hline 1576 & 1575 |lin & Indene & $\frac{95-13-6}{1033.5}$ & $000095-13-6$ & $\frac{116.16}{27634}$ & $\frac{L}{S}$ & $-\frac{1.5000+00}{161-1655}$ & 7.600E +022 & 1.820E+02 2 & 1.1.00E+00 & & 1.100E+ +00 & DAUBERT,TE \& DANNER,RP \\
\hline$\frac{1578}{1578}$ & 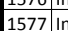 & 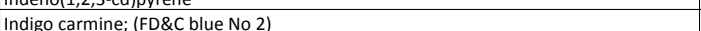 & 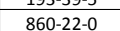 & 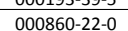 & $\frac{26.36}{46.636}$ & s & & & & & & & 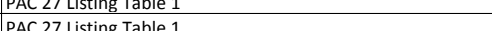 \\
\hline $\begin{array}{ll}1579 \\
15\end{array}$ & $1578 \ln 3(2)$ & Indium & $7440-74-6$ & $007440-74-6$ & 114.82 & $\mathrm{~s}$ & $1.566 \mathrm{E}+02$ & & $2.0806+03$ & & & & PAC 27 listing arable 1 \\
\hline$\frac{2580}{1500}$ & 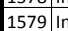 & Indium oxide (vapor) & $1312-43-2 b$ & $\frac{001312-43-2 b}{0.5}$ & 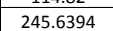 & $\mathrm{G}_{\mathrm{G}} \mathrm{C}, \mathrm{C}$ & & & & & & & PAC 27 Listing Table 1 \\
\hline 1581 & $1580 \mid \ln$ & Indium sulfate & $13464-82-9$ & $013464-82-9$ & 517.82 & $\mathrm{~s}$ & & & & & & & PAC 27 Listing Table 1 \\
\hline$\frac{1582}{1583}$ & 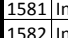 & Indium trichloride & $\frac{10025-82-8}{1312-32-2 a}$ & 010025-82-8 & $\frac{221.17}{2776}$ & $\frac{s}{s}$ & $\frac{586 \text { subl }}{1913 \mathrm{EF} 03}$ & & $6.000 \mathrm{E}+02$ & & & & $\begin{array}{l}\text { PAC 27 Listing Table } 1 \\
\text { PAC 27 listing Table } 1\end{array}$ \\
\hline 1584 & 1583] In & Indole-3-carboxaldehyde, 1H-; (3-Formylindole) & $487-89-8$ & 000487-89-8 & 145.17 & $\mathrm{~s}$ & & & & & & & $\begin{array}{l}\text { PAC 27 Listing Table } 1 \\
\text { Pat }\end{array}$ \\
\hline 1585 & $1584 \mid 100$ & lodic acid & $7782-68-5$ & $007782-68-5$ & 175.91 & $\mathrm{~s}$ & $110 \mathrm{dec}$ & & & & & & PAC 27 Listing Table 1 \\
\hline$\frac{1586}{1529}$ & 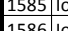 & lodine & 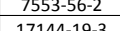 & & 253.51 & $\frac{s}{c}$ & & & $1.844 E+02$ & 7.500E=-01 & 3.590E E01 & & PAC 27 L Listing Table 11 \\
\hline$\frac{1588}{158}$ & $15877_{10}$ & lodobenzzene & 591-50-4 & & 204.01 & $\mathrm{~L}$ & $-2.9006+01$ & $7.6006+02$ & $1.880 E+02$ & $1.060 \mathrm{E}+00$ & $2.5006+01$ & $1.060 \mathrm{E}+000$ & DAUET TS EANANR \\
\hline$\frac{2589}{1599}$ & $\left.1588\right|_{10}$ & lodoethane & $75-03-6$ & $000075-03-6$ & 155.97 & $\mathrm{~L}$ & 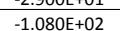 & 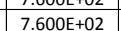 & 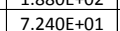 & 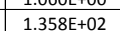 & $\frac{2.5006+1}{2.501}+1$ & 1.3.386+02 & DAUEETT,TE DANANER \\
\hline 1590 & $\mid 15899_{10}$ & 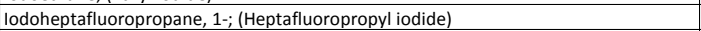 & $754-34-7$ & $000754-34-7$ & 295.93 & L & 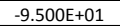 & $7.600 E+02$ & 4.100E+01 & 4.240E+02 2 & 2.500E+01 & 4.240E+02 & EPP Suite Mean VP Anto \\
\hline 1591 & $1590 / 10$ & lodopentane, 1 - & $628-17-1$ & $000628-17-1$ & 198.06 & $\mathrm{~L}$ & 8.560E+ +01 & $7.600 E+02$ & $1.570 E+02$ & 4.3900E+00 & $2.500 E+01$ & & LI,CMM \& ROSSIN, $\mathrm{FD}(19$ \\
\hline$\frac{1592}{1502}$ & 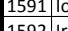 & Jotalamic acid & 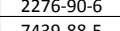 & & 613.92 2020 & $s_{5}^{3}$ & 285 Secc. & & & & & & 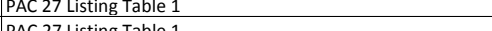 \\
\hline$\frac{2594}{1594}$ & $1593 \mid$ |ir & 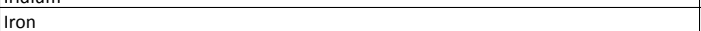 & $7439-89-6$ & $007439-89-6$ & $\frac{5.85}{55.85}$ & $\mathrm{~s}$ & $1.5385+03$ & & $2861+1+03$ & $7.5 \times 10-3$ & $1.455 \mathrm{E}+03$ & & PAC 27 listing Ta \\
\hline$\frac{1595}{1595}$ & 1594 |ir 20 & Iron cat & $12011-67-5$ & $012011-67-5$ & 179.55 & $\mathrm{~s}$ & & & & & & & PAC 27 Listing Tabble \\
\hline $\begin{array}{ll}1596 \\
1596\end{array}$ & 1595 |rir & |ron hydr. & 20344-49-4 & 02034 & 88.85 & $s$ & & & & & & & Ig Table 1 \\
\hline 1597 & $1596 \mid$ |r & Iron oxide; (Ferric oxide) & $1309-37-1$ & $001309-37-1$ & 159.69 & $\mathrm{~s}$ & $1.539 \mathrm{Et}$ & & & & & & PAC 27 Listing Table 1 \\
\hline$\frac{1598}{1509}$ & & 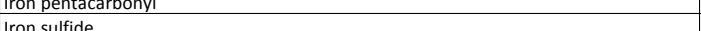 & $\frac{13456-40-6}{137-3-9}$ & 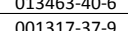 & $\frac{195.9}{891}$ & $\frac{L}{s}$ & 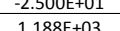 & 1.600UE+02 & $1.0300+202$ & $4.000 \mathrm{E}+01$ & $3.030 E+01$ & 3.051Lt017 & 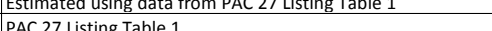 \\
\hline $\begin{array}{ll}1600 \\
1600\end{array}$ & & Iron(II) chloride tetrahydrate & $13478-10-9$ & $013478-10-9$ & 198.83 & 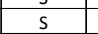 & $105 \mathrm{dec}$ & & & & & & ting Table 1 \\
\hline \begin{tabular}{l|l|}
1601 \\
\end{tabular} & & Iron(III) oxide; ; (Ferrous oxide) & $1345-25-1$ & $001345-25-1$ & 73.85 & $\mathrm{~s}$ & $1.377 E+03$ & & & & & & PAC 27 Listing Table 1 \\
\hline & & & $13520-69-9$ & $013520-69-9$ & 353.3378 & & & & & & & & PAC 27 Listing Table 1 \\
\hline
\end{tabular}




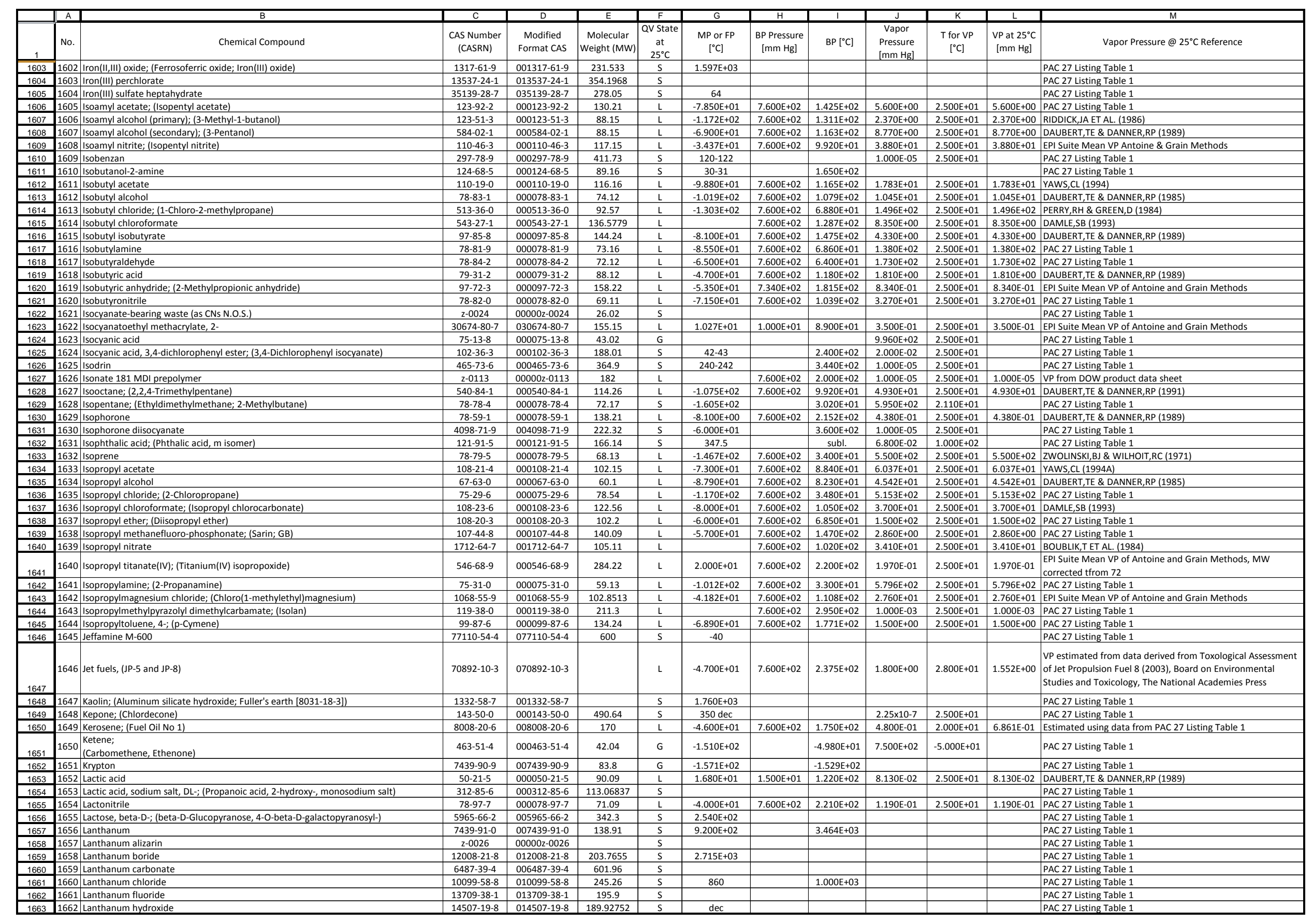




\begin{tabular}{|c|c|c|c|c|c|c|c|c|c|c|c|c|c|}
\hline & No. & Chemical Compound & $\begin{array}{c}\text { cas N Number } \\
\text { (CASPN) }\end{array}$ & $\begin{array}{c}\text { Modified } \\
\text { Format CAS }\end{array}$ & $\begin{array}{l}\text { Molecurar } \\
\text { Weight }\end{array}$ & 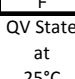 & 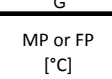 & $\begin{array}{llll}\text { Bep ressure } \\
\text { [mm M }\end{array}$ & ${ }_{B P}[\mathrm{CC}]$ & $\begin{array}{c}\text { vapor } \\
\text { pressurue }\end{array}$ & $\begin{array}{c}\text { Torverp } \\
\text { crla }\end{array}$ & 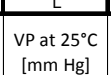 & Vapor Pressure @ $25^{\circ}$ C Reference \\
\hline$\frac{1664}{1.665}$ & & Lantanum nitrate & $\begin{array}{l}\frac{10099 \cdot 5 \cdot 9 \cdot 9}{1312-81-8} \\
\end{array}$ & $\begin{array}{l}010099-59-9.9 \\
001312-81-8\end{array}$ & $\begin{array}{l}324.94 \\
325.52\end{array}$ & $\begin{array}{l}\frac{15}{5} \\
\text { s }\end{array}$ & $\frac{\sim 40}{2.315 E+03}$ & & $\frac{\sim 126}{4.200 E+03}$ & & & & 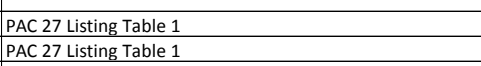 \\
\hline 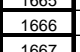 & & 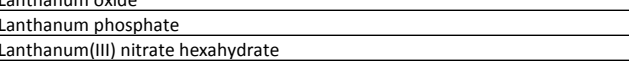 & 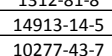 & 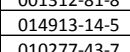 & 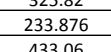 & $\frac{5}{5}$ & ${ }^{940}$ & & 4.20uectos & & & & \\
\hline$\frac{2068}{1.668}$ & 667 66 & 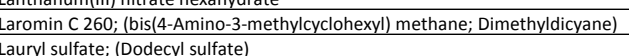 & 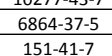 & 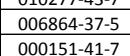 & 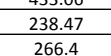 & $\frac{\mathrm{L}}{\mathrm{s}}$ & & $7.600 \pm+02$ & 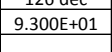 & $6.950 E+00$ & $2.500++01$ & $6.950+500$ & 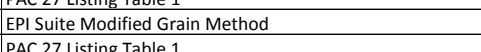 \\
\hline & 669 & 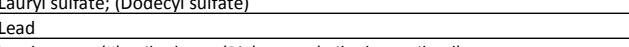 & 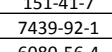 & 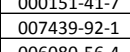 & 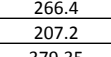 & $\frac{5}{5}$ & $3.275+02$ & & $1.799 E+03$ & $7.5 \times 10.3$ & $7.050 \mathrm{~F}+02$ & & 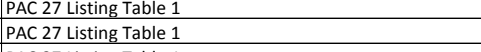 \\
\hline$\frac{1671}{1672}$ & 6771 & 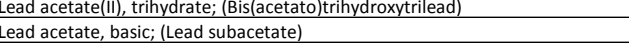 & $\begin{array}{l}6080.56-4 \\
1335-32 \cdot 6\end{array}$ & 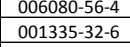 & 807.75 & $\frac{s}{s}$ & $\frac{75 \mathrm{dec}}{\mathrm{dec}}$ & & $200 \mathrm{dec}$ & & & & $\begin{array}{l}\text { PAC27 Listinit Table } 1 \\
\text { PAC 27 Listin Table } 1\end{array}$ \\
\hline 367 & 1672 & Lead acetate; (Lead diacetate) & 301-04:-2 & $000301-04-2$ & 325.29 & $\mathrm{~s}$ & $7.500 \mathrm{E}+01$ & & 200 & & & & PAC 27 Listing Table 1 \\
\hline 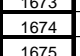 & & 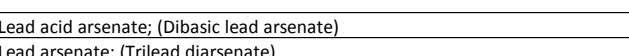 & $\frac{778440.9}{368723}$ & 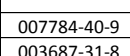 & $\frac{347.1}{8094}$ & $\frac{s}{s}$ & $\frac{280 \mathrm{dec}}{1020 \mathrm{coc}}$ & & & & & & 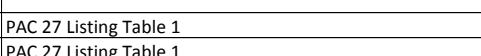 \\
\hline 1676 & 75 & tead bedremide & 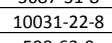 & 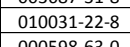 & 367.01 & $\mathrm{~s}$ & 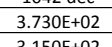 & & $9.160 \mathrm{E}+02$ & & & & 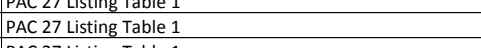 \\
\hline$\frac{167}{1678}$ & & 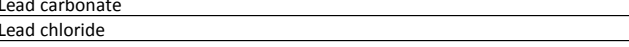 & 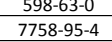 & 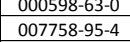 & 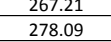 & 5 & $\begin{array}{l}3.1 .060+0202 \\
5.010+02\end{array}$ & & 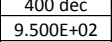 & $1.000+500$ & $5.4700+02$ & & 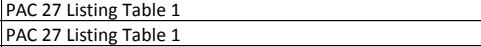 \\
\hline$\frac{1679}{1600}$ & 6789. & lead hromate & $\begin{array}{l}77589.7-6 \\
130960.0\end{array}$ & 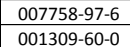 & $\frac{3332}{2332}$ & $\begin{array}{c}\frac{5}{5} \\
5\end{array}$ & $\begin{array}{lll}8.400+02 \\
200 \mathrm{dec}\end{array}$ & & dec & & & & 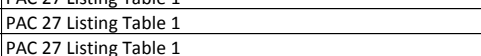 \\
\hline 20 & 1680 & Lead fluoborate & 13814966.5 & $013814-96 \cdot 5$ & 380.81 & L & & & & 1.000E:05 & $2.500 \mathrm{E}+01$ & $1.000=05$ | & 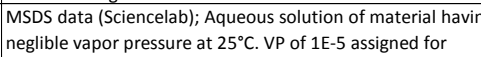 \\
\hline$\frac{1681}{1682}$ & 681 & Lead fluoride & $7773-46-2$ & 0.77878-46-2.2 & 245.2 .2 & s & $8.550+02$ & & $1.200+03$ & $1.000++01$ & $9.0400+ \pm 02$ & & 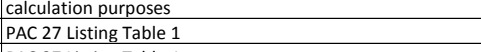 \\
\hline & 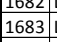 & $\begin{array}{l}\text { lead yntroxde } \\
\text { tead iodide }\end{array}$ & 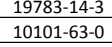 & 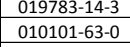 & 2441.21 24.01 & s & $4.020 \mathrm{E}+02$ & & $9.500 \mathrm{E}+022$ & & & & 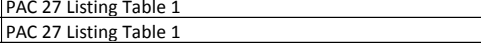 \\
\hline$\frac{1665}{1.685}$ & 684. & lead intrate & $\begin{array}{c}10099-7.8 \\
13826-55.8\end{array}$ & 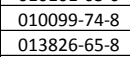 & $\frac{331.2}{299.2}$ & $\begin{array}{cc}\frac{s}{s} \\
\end{array}$ & $4.700+02$ & & & & & & $\begin{array}{l}\text { sting Trable } 1 \\
\text { sting arele } 1\end{array}$ \\
\hline 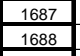 & 6887] & Lead oxalate & $\begin{array}{l}819.937 .7 \\
1317-36 \cdot 8\end{array}$ & 001317-36-8 & 295.1966 & $\frac{s}{s}$ & $8.8805+02$ & & & 7.55x10.3. & 7.2406 +02 & & 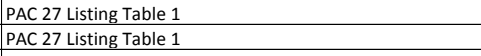 \\
\hline 1689 & 6889] & $\begin{array}{l}\text { tead hosphate } \\
\text { eads suftete }\end{array}$ & 7446-67-7.-7 & 007446-67-7-7 & 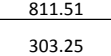 & $\frac{s}{s}$ & $\begin{array}{ll}1.0 .1440+03 \\
1.770+03\end{array}$ & & & & & & 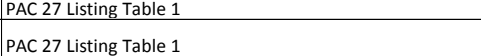 \\
\hline$\frac{1690}{1691}$ & & 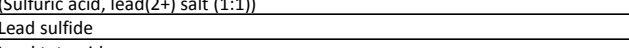 & $1314-87.0$ & $000131487-0$ & 239.25 & $\mathrm{~s}$ & $1.114+ \pm 03$ & & $1.281 E+03$ & $1.000+500$ & $8.5020+02$ & & PAC 27 Listing Table 1 \\
\hline$\frac{1692}{1693}$ & 692 & $\begin{array}{l}\text { tead tetoride } \\
\text { teadilisenite }\end{array}$ & 13034-14-13.7 & $\begin{array}{l}010131441-6 \\
0.1031-13-7\end{array}$ & $\frac{685.6}{421}$ & $\frac{s}{s}$ & & & & & & & 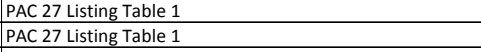 \\
\hline$\frac{1694}{1695}$ & & 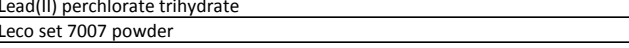 & 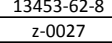 & & & & & & & & & & Listing Table 1 \\
\hline$\frac{1696}{1697}$ & & 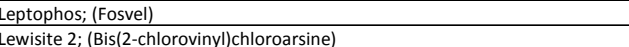 & 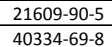 & 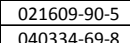 & $\frac{412.07}{233.35}$ & $\frac{s}{L}$ & $\frac{70.270 .4}{10.18501}$ & $7.6005+0.02020$ & 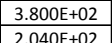 & $\frac{2.000 E=06}{20060-51}$ & $\frac{2.500+501}{20500+01}$ & 2.9600 .01 & 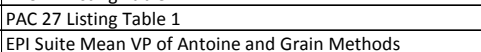 \\
\hline $\begin{array}{ll}1698 \\
1699 \\
\end{array}$ & & 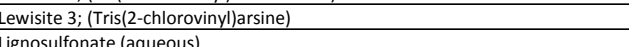 & 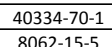 & 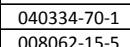 & 259394 & & & 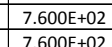 & $\begin{array}{l}2.4715+02 \\
6.959+52\end{array}$ & $\begin{array}{lll}2.7006-02 \\
1.700520\end{array}$ & 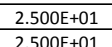 & 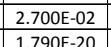 & 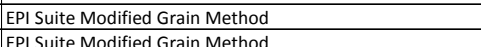 \\
\hline 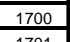 & $\frac{99}{90}$ & 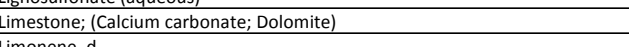 & 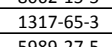 & 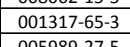 & 100.09 & $\frac{1}{5}$ & & 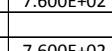 & 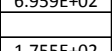 & 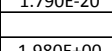 & 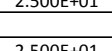 & 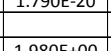 & 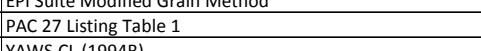 \\
\hline & & 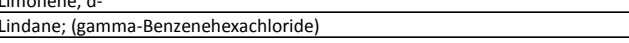 & & & & & $1.1255+02$ & & & & & & 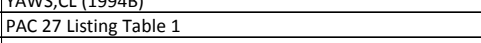 \\
\hline$\frac{103}{1704}$ & & 留seded of & 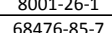 & 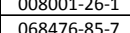 & & & 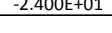 & 1.:00070202 & 3.160E+02 & 3.2300:-21 & $2.500 E+01$ & 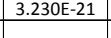 & 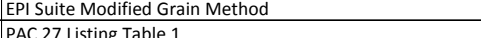 \\
\hline$\frac{1705}{1705}$ & & tuthium & 74399-93-2.2 & 0074399.9: & 6.94 & $s$ & $1.8055+02$ & & 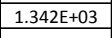 & 5.9200:-13 & $1.270 \mathrm{E}+02$ & & sting table - \\
\hline$\frac{1706}{1707}$ & & $\begin{array}{l}\text { tithium acetate ed ihyyrate } \\
\text { thtium aluminate }\end{array}$ & 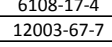 & 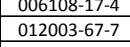 & $\frac{120.06}{6592034}$ & $\frac{s}{5}$ & & & ${ }^{2}$ & ${ }^{2}$ & $x_{0}$ & & 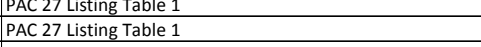 \\
\hline & & 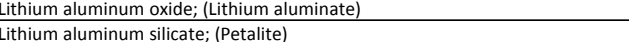 & $\begin{array}{l}101098987.7 \\
13026-5\end{array}$ & 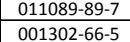 & & & $1000-2000$ & & & & & & \\
\hline 17100 & & lithium azide & $\begin{array}{l}19597-69-4 \\
10501095\end{array}$ & 019597-69. & 48.96 & $\mathrm{~s}$ & $115-298$ & & & & & & 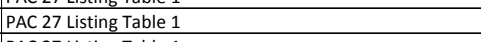 \\
\hline$\frac{1714}{1712}$ & +1 & 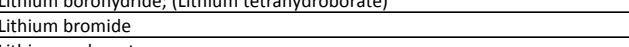 & 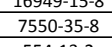 & 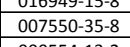 & (20.00 & 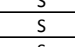 & $5.5006+02$ & & 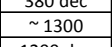 & $7.5 \times 10-2$ & $6.300+02$ & & 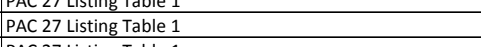 \\
\hline & & 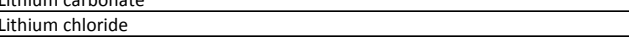 & $\frac{1447-41.8}{774}$ & $\frac{107447-41}{0.42}$ & & 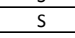 & 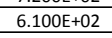 & & 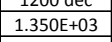 & $1.000+000$ & $5.4700+02$ & & ting \\
\hline$\frac{1715}{175}$ & & 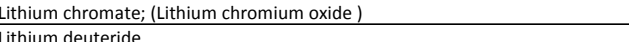 & 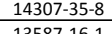 & 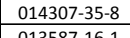 & $\frac{129.98}{1088}$ & s & $\frac{75 \mathrm{dec}}{6800+52}$ & & $x_{2}$ & & & & able: \\
\hline 17177 & & 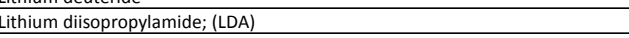 & 告111-54-0 & .00411-54- & 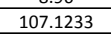 & & & & & & & & 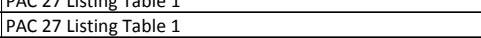 \\
\hline & & - & 7.7982:24.4 & 0.07789-24-4 & & & $\begin{array}{l}8.480+02 \\
6090202\end{array}$ & & $1.676 \in+03$ & $1.000 E+00$ & 1.047 +03 & & tring Table \\
\hline 17720 & & & B11 & & & - & $\frac{1.53062}{4.700+02}$ & & $1.626 \notin+03$ & & & & \\
\hline$\frac{21714}{1722}$ & 1 & $\begin{array}{l}\text { tithium hydroxide monohydrate } \\
\text { then }\end{array}$ & 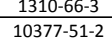 & 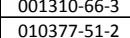 & 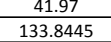 & $\frac{5}{s}$ & 469 & & $1.1711 \pm 03$ & 2 & & & 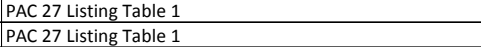 \\
\hline$\frac{1 / 23}{1724}$ & te & tithium metaborate, anhydrous & 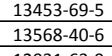 & 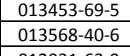 & $\begin{aligned} 4.951 \\
173.82 \\
\end{aligned}$ & $\frac{s}{5}$ & 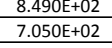 & & 1.7.79E+o3 & & & & 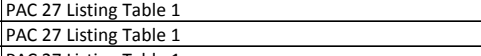 \\
\hline
\end{tabular}




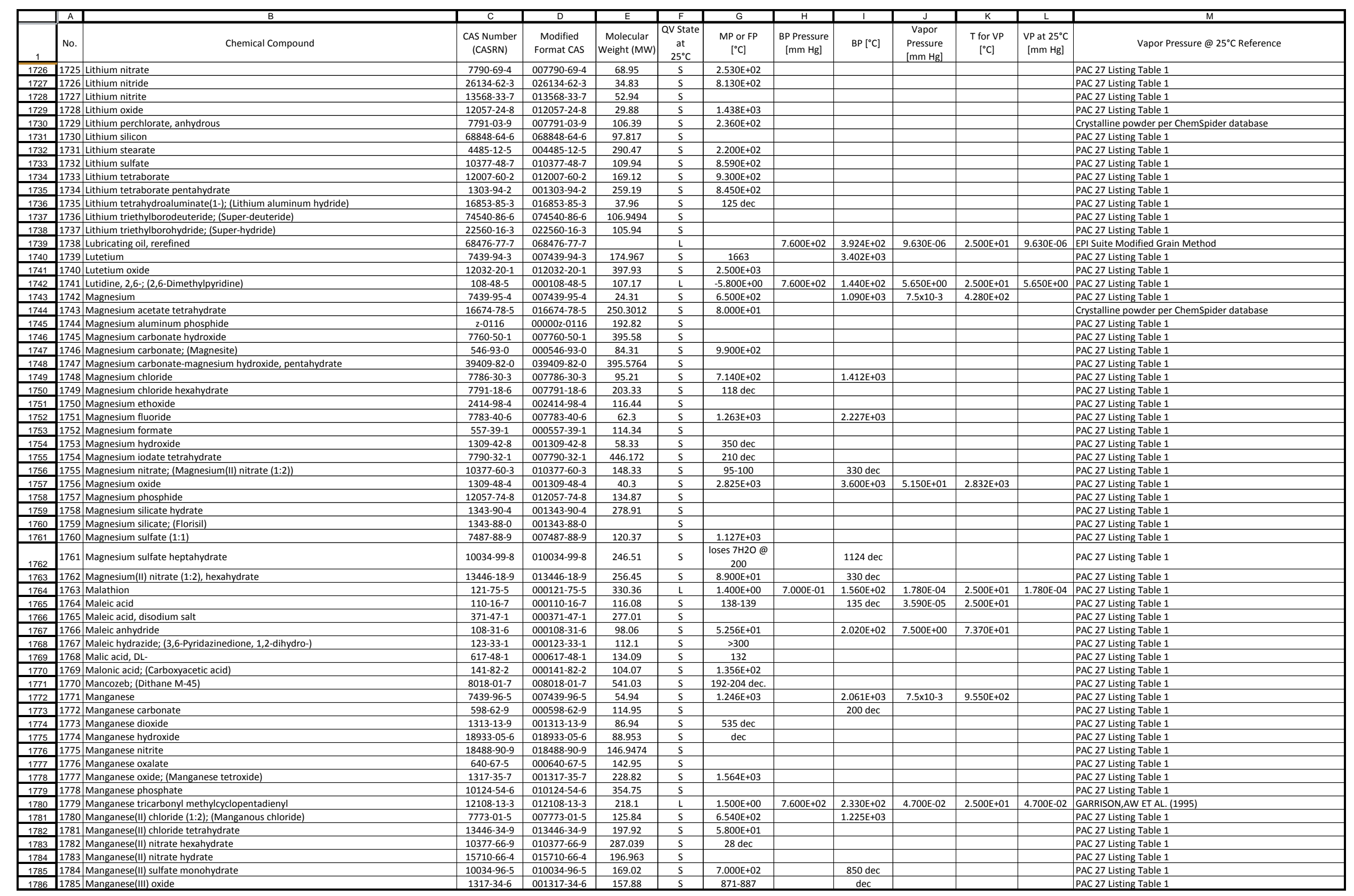




\begin{tabular}{|c|c|c|c|c|c|c|c|c|c|c|c|c|}
\hline & Chemical Compound & $\begin{array}{l}\text { CAS Number } \\
\text { (CASRN) }\end{array}$ & $\begin{array}{c}\text { Modified } \\
\text { Format CAS }\end{array}$ & $\begin{array}{c}\text { Molecular } \\
\text { Weight (MW) }\end{array}$ & $\begin{array}{c}\frac{F}{\text { Qv State }} \text { at } \\
25^{\circ} \mathrm{C}\end{array}$ & $\begin{array}{c}\text { MP of } F P \\
{\left[{ }^{\circ C}\right]}\end{array}$ & 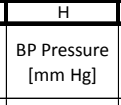 & $B P\left[{ }^{[C}\right]$ & $\begin{array}{l}\text { Vapor } \\
\text { Pressure } \\
{[\mathrm{mm} H \mathrm{Hg}]}\end{array}$ & 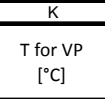 & 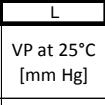 & Vapor Pressure @ @25 C Reference \\
\hline & 1786 Manganese(VIII) oxide & $12057-92-0$ & $012057-92-0$ & 221.88 & L & $5.900++00$ & & & 1.500E+02 & $2.500 \mathrm{E}+01$ & $1.500 E+02$ & 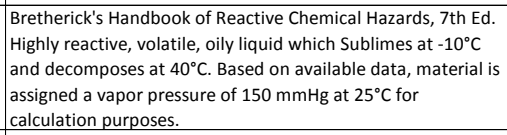 \\
\hline & $\begin{array}{l}1787 \text { Manganous oxide; (Manganesese(II) oxide) } \\
11788 \text { Manganous suffate }\end{array}$ & $\begin{array}{c}1344-43-0 \\
\end{array}$ & $001344-43-0$ & 70.94 & $\mathrm{~s}$ & $1.650 \mathrm{E}+03$ & & & & & & 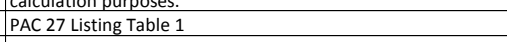 \\
\hline$\frac{1789}{1790}$ & 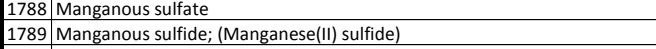 & $\frac{7785-877-7}{1882-29-6}$ & $\frac{007785-87-7}{01820-29-6}$ & $\frac{151}{87}$ & $\frac{s}{5}$ & $\frac{7.009 E+02}{3.990 \mathrm{E}+00}$ & & $850 \mathrm{dec}$ & & & & $\begin{array}{l}\text { PAC 27 Listing gable } 1 \\
\text { PAC 27 Litsing Table } 1\end{array}$ \\
\hline$\frac{1 / 190}{1791}$ & & $\frac{18802-29-6}{61789-92-2}$ & $0181789-29-6-2$ & & $\frac{5}{5}$ & & & & & & & $\begin{array}{l}\text { PAC 27 Listing gabie } 1 \\
\text { Pable } 1\end{array}$ \\
\hline$\frac{1792}{1792}>0>0$ & $\begin{array}{l}1791 \mid \text { Melamine } \\
179\end{array}$ & $\frac{108-78-1}{108}$ & 000108-78-1 & 126.15 & $\mathrm{~s}$ & $3.4706+02$ & & subl. & $5.000 E+01$ & $3.1506+02$ & & $\begin{array}{l}\text { PAC 27 Listing Table } 1 \\
\text { Pand }\end{array}$ \\
\hline 1793 & 1792 Memtetrahydrophthalic anhydride; (Tetrahydrophthalic anhydride) & $85-43-8$ & $000085-43-8$ & 152.1494 & $\mathrm{~s}$ & 101.9 & & $195 @ 50 \circ 0$ & $5.7000-02$ & $2.500 E+01$ & & PAC 27 Listing Table 1 \\
\hline$\frac{1744}{1705}$ & 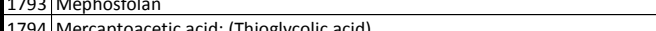 & 950-10-7 & $000950-10-7$ & 269.34 & $\frac{s}{L}$ & $1.5050+01$ & & 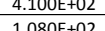 & $\frac{1.000-10}{860502}$ & $2.500=E+01$ & 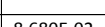 & 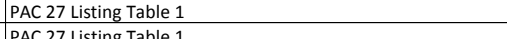 \\
\hline 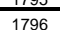 & 1795 Mercaptobenzoic acid, o- & 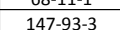 & 0000147-193-3 & $\frac{25.12}{154.19}$ & $\frac{\mathrm{L}}{\mathrm{s}}$ & 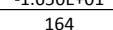 & $1.500 \mathrm{E}+01$ & 1.080E+ +02 & 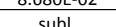 & & $8.680 \mathrm{E}-02$ & $\begin{array}{l}\text { PAC 2/ Llsting labbe } 1 \\
\text { PAC 27 isting Table } 1\end{array}$ \\
\hline & \begin{tabular}{|l|l|l|l|l}
1796 & Mercaptobenzonzothiazole, 2; ; (2-Benzothiazolethiol) \\
\end{tabular} & $149-30-4$ & & & $\mathrm{~s}$ & $1777-179$ & & & & & & \\
\hline 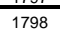 & 11797 Mercaptoethanol, 2- & $\frac{240-24 \cdot 2}{60-2}$ & 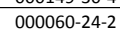 & $\frac{20.14}{77.14}$ & $\frac{\mathrm{L}}{\mathrm{L}}$ & & $7.6000+02$ & $1.570 E+02$ & $1.7566+00$ & $2.5006+01$ & $1.756 E+00$ & DAUBERT, E \& P DNNER,RP (1996) \\
\hline & 1798 Mercaptopropionic acid, 3- & $107-96-0$ & $000107-96-0$ & 106.15 & & $1.680 \mathrm{E}+01$ & $7.6006+02$ & $1.150 \mathrm{E}+02$ & $\begin{array}{ll}4.9844-02 \\
\end{array}$ & $2.5006+01$ & $4.9844-02$ & DAUBRT,TE \& DANNER,RP (1989) \\
\hline 1800 & 1799 Mercuric acetate & $1600-27-7$ & 001600-27-7 & 318.69 & $\mathrm{~s}$ & $178-180$ & & decom & & & & PAC 27 listing Table 1 \\
\hline$\frac{1801}{1802}$ & 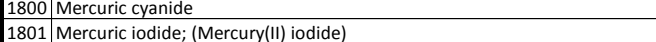 & $\frac{592-044-1}{7774-29-0}$ & $\frac{000592-04-1}{007774-29-0}$ & $\frac{252.63}{454.4}$ & $\frac{s}{s}$ & $\frac{320 \text { decom }}{2.590 \mathrm{E}+02}$ & & & & & & $\begin{array}{l}\text { PAC 27 Listing Table } 1 \\
\text { PAC 27 fisting Table } 1\end{array}$ \\
\hline$\frac{1802}{1803}$ & 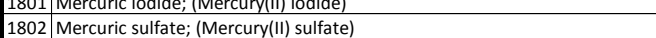 & $7783-295-0$ & $007783-25-0$ & $\frac{434.4}{296.65}$ & $\frac{s}{s}$ & $\frac{2.590 \mathrm{E}+02}{\text { decom }}$ & & & & $2.618 E+02$ & & $\begin{array}{l}\text { PAAC / ILsting Iable } 1 \\
\text { PAC 27 isting Table } 1\end{array}$ \\
\hline$\frac{1050}{1804}$ & 10 & 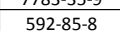 & $\frac{0.0053-35-8}{00059-8}$ & $\frac{19.059}{316.99}$ & $\frac{3}{s}$ & & & & & & & PAC C L Listing Table 1 \\
\hline & 1804|Mercuric trifluoroacetate & $13257-51-7$ & $013257-51-7$ & 426.62 & $\mathrm{~s}$ & 171-173 & & & & & & $\begin{array}{ll}\text { PAC } 27 \text { Listing Table } 1 \\
\end{array}$ \\
\hline 1806 & 1805 /Mercurol; (Mercury nucleate) & $12002-19-6$ & $012002-19-6$ & & $s$ & & & & & & & PAC 27 Listing Table 1 \\
\hline$\frac{1807}{1808}$ & 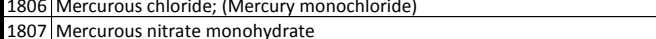 & 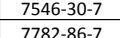 & $007546-30-7$ & $\frac{236.04}{281.62}$ & $\frac{s}{s}$ & $\frac{400 \text { subl }}{79.1 \mathrm{del}}$ & & & & & & $\begin{array}{l}\text { PAC 27 Listing Table } 1 \\
\text { PAC 27 jutsing Table1 }\end{array}$ \\
\hline$\frac{1809}{1809}$ & 1808 (Mercurous nitrate; (Mercurvu(I) nitrate(1:1)) & $10425-75-5$ & $010415-80-75-5$ & $\frac{2026.6}{262.6}$ & s & $\frac{15 \text { decom }}{70 \text { decom }}$ & & & & & & 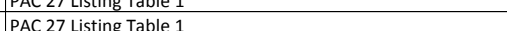 \\
\hline$\frac{1059}{1810}$ & 1809 | Mercurousus oxide; ; (Mercurcury(l) oxide) & $15829-53-5$ & & 41718 & $\mathrm{~s}$ & 100 dec & & & & & & 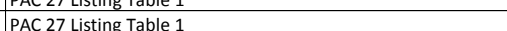 \\
\hline 1811 & 1810 Mercury hydroxide & 121235-13-6 & 012135-13-6 & 234.605 & $\mathrm{~s}$ & & & & & & & PAC 27 Listing Table 1 \\
\hline 1812 & $1811 \mid$ |Mercurry nitrate; (Mercury(II) nitrate (1:2)| & $10045-94-0$ & $010045-94-0$ & 324.6 & $\mathrm{~s}$ & 7.900E +01 & & & & & & PAC 27 Listing Table 1 \\
\hline$\frac{1813}{1814}$ & 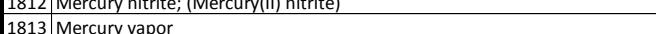 & 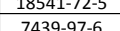 & 1018341-12-5 & $\begin{array}{l}292.29 \\
2009\end{array}$ & $\frac{5}{1}$ & $38005+01$ & $76005+0^{2}-20$ & $35675+02$ & 200050.03 & $25005+01$ & $20000-03$ & $\begin{array}{lll}\text { PAC } 27 \text { Listing Table } 1 \\
\text { PAC 27lițing Table } 1\end{array}$ \\
\hline$\frac{1815}{1815}$ & 1814|Mercury(I) chloride; (Dimercury dichloride) & $\frac{1}{10112-911-1}$ & 0101012-91-1. & $\frac{25.30}{472.08}$ & $\frac{5}{5}$ & 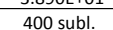 & & & 2.000e-0s & & & PA 27 listing gable 1 \\
\hline & 1815 |Mercury(III) chloride; ; Mercury bichloride) & $7487-94-7$ & 007487-94-7 & 271.5 & $\mathrm{~s}$ & $2,770 \mathrm{E}+02$ & & $3.040 \mathrm{E}+02$ & $7.5 \times 10-3$ & $6.4406+01$ & & 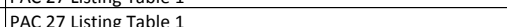 \\
\hline $\begin{array}{l}1817 \\
1817\end{array}$ & $\begin{array}{l}1816 \mid \text { Mercurur(II) nitrate monohydrate } \\
180\end{array}$ & $77783-34-8$ & $0007783-34-8$ & $\frac{342.62}{342 .}>2>0$ & $\mathrm{~s}$ & 79 & & & & & & $\begin{array}{l}\text { PAC } 27 \text { Listing Table } 1 \\
\text { Pand }\end{array}$ \\
\hline 1818 & 1817 |Mercury(III) oxide; (Mercuric oxide) & $21908-53-2$ & $021908-53-2$ & 216.59 & $\mathrm{~s}$ & $500 \mathrm{dec}$ & & & & & & PAC 27 Listing Table 1 \\
\hline 1819 & 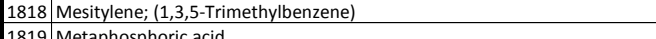 & $\frac{108-67-8}{272-6060}$ & $000108-67-8$ & $\frac{120.19}{7799}$ & $\frac{L}{S}$ & $-4.472 E+01$ & $7.600 E+02$ & $1.647 E+02$ & $2.4806+00$ & 2.500e+01 & $2.480 \mathrm{E}+0$ & DAUERET,TE \& DANNER,RP (1989) \\
\hline & 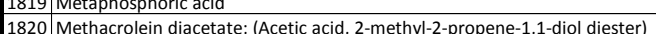 & $\frac{3 / 266-86-0}{1046-95-6}$ & $\frac{03 / 26-8-86-0}{010476-95-6}$ & $\frac{13.50}{172.2}$ & & & $7.600 E+022>020$ & 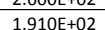 & & & 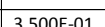 & $\begin{array}{l}\text { PACL/ Ltsting gable 1 } \\
\text { PAC 27 isting Table } 1\end{array}$ \\
\hline 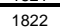 & 1821 Methacrylaldehyde & $78-85-3$ & $000078-85-3$ & 70.1 & t & $-8.1000+01$ & $\frac{1.600 E+02}{7.02}$ & 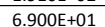 & 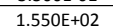 & $\frac{2.5006+01}{2.501}$ & 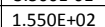 & YAWS, LL (19964) \\
\hline 1823 & 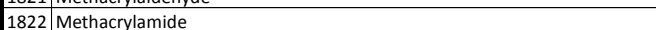 & $799-39-0$ & $000079-39-0$ & 85.12 & $\mathrm{~s}$ & 105-107 & & 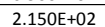 & & & & PAC 2 L Listing Table 1 \\
\hline 1824 & 1823] Methacrylic acid & $79-41-4$ & $000079-41-4$ & 86.1 & $\mathrm{~L}$ & $\frac{1.600 \mathrm{E}+01}{1.01}$ & $7.600 E+02$ & $1.630 \mathrm{E}+02$ & $9.9000-01$ & $2.500 E+01$ & $9.900 E-01$ & $\begin{array}{ll}\text { DAUBERT,TE \& DANNER,RP (1997) } \\
\end{array}$ \\
\hline 1825 & \begin{tabular}{|l|l|l|l|l|}
1824 & Methacrylic acid, diester with triethylene glycol; (Polyester TGM3)
\end{tabular} & $\begin{array}{ll}109-16-0 \\
10-0\end{array}$ & $000109-16-0$ & 286.36 & $\mathrm{~L}$ & $5.760 \mathrm{E}+00$ & $5.0006+00$ & $1.710 E+02$ & $9.440 E=04$ & $2.500 \mathrm{E}+01$ & $9.440 \mathrm{E}-04$ & EPI Suite Modified Grain Method \\
\hline$\frac{1826}{1827}$ & 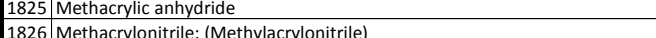 & $760-93-0$ & $000760-93-0$ & $\frac{154.18}{671}$ & & $-36005+01$ & $\begin{array}{l}7.600 E+02 \\
7.600 E+02\end{array}$ & $\begin{array}{l}2.400 E+02 \\
90030+01\end{array}$ & & & $\frac{1.000 E-02}{71120 E+01}$ & 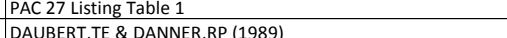 \\
\hline & 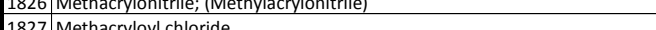 & 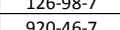 & 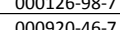 & $\frac{6.1}{10.14}$ & $L_{1}$ & 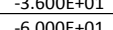 & 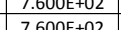 & 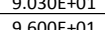 & T.1.120t+01 & 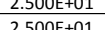 & T.120Et+01 & 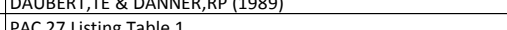 \\
\hline$\frac{1829}{1829}>2$, & 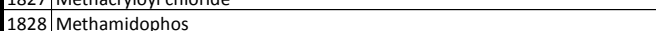 & $\frac{92-4-4-1}{102-6}-6$ & $010265-92-6$ & 141.14 & s & $\frac{1.0006+01}{4.01}$ & & 9.00urtut & $3.0006-04$ & 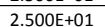 & & 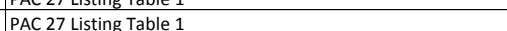 \\
\hline 1830 & 1829 Methan & $74-82-8$ & & 16.04 & $\frac{G}{G}$ & $-1.825 E+02$ & & $-1.615 E+022$ & $7.500 E+02$ & $-1.617 E+02$ & & $\begin{array}{llll}\text { PAC } 27 \text { listing Table } 1 \\
\end{array}$ \\
\hline 1831 & 1830|Methane-d3 & $676-80-2$ & $000676-80-2$ & 19.07 & G & $-1.830 \mathrm{E}+022$ & & $-1.630 \mathrm{E}+02$ & & & & PAC 27 Listing Table 1 | \\
\hline$\frac{1832}{1032}$ & 1831 1 Methanesulfonic acid & 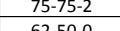 & $000075-75-2$ & 96.01 & $\frac{L}{L}$ & 2.000E +01 & $\frac{1.000 E+01}{7600+02}$ & $1.670+102$ & 4.2.200-0.44 & $\frac{2.500+0+1}{20019}>0$ & 4.208E-04 & EPI Suite Experimental Data \\
\hline$\frac{1034}{1834}$ & 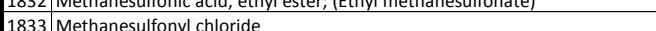 & 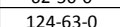 & 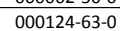 & $\frac{114.55}{11.55}$ & & $-3.200 E+01$ & 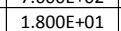 & 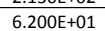 & $\begin{array}{lll}3.2006 \mathrm{~F} \\
2.200\end{array}$ & 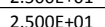 & 2.200 & 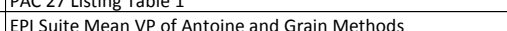 \\
\hline 1835 & 1834/Methane & 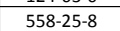 & 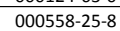 & $\frac{198.1}{98.1}$ & th & ( & $\frac{1.506+2}{7.605+02}$ & $\frac{0.2406+02}{1.202}>20$ & $\frac{2000+01}{1000+01}$ & 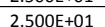 & $\frac{1000+501}{100+01}$ & PAC C7 listing Table 1 \\
\hline 1926 & 1835 Methidathion; (0,0-Dimethyl-s-15-methoxy-1,3,4-thiadiazolinyl-3- & $950-37-8$ & $000950-37-8$ & 302.34 & $\mathrm{~s}$ & $39-40$ & & & $1.000 \mathrm{E}-06$ & $2.500 \mathrm{E}+01$ & & PAC 27 Listing Table 1 \\
\hline & potodimethur) & $2032-65-7$ & $002032-65-7$ & 225.33 & 5 & $117-118$ & & +02 & $1.000 E-04$ & $2.5006+01$ & & PAC2 \\
\hline 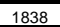 & 1837 Methon & $16752-77-5$ & 016752-77-5 & 162.23 & 5 & $78-79$ & & 3.350 & 5.000E-05 & $2.500 E+01$ & & ing Tab \\
\hline 1839 & Enzaldehyde; (p-Anisaldehyde) & 123-11-5 & $000123-11-5$ & 136.15 & $\mathrm{~L}$ & $0.000 E+00$ & $7.600 E+02$ & 2.495E+02 & 3.290E-02 & $2.500 E+01$ & 3.290E-02 & OHE,S \\
\hline 1840 & 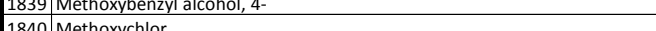 & 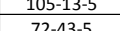 & 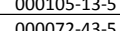 & 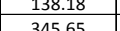 & s & 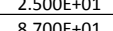 & & & $42 \times 10-5$ & $25005+01$ & & 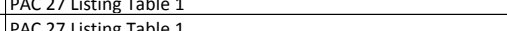 \\
\hline 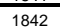 & 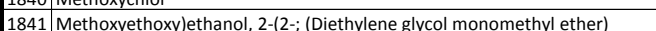 & 111-177-3 & & $\begin{array}{l}120.17 \\
120.17\end{array}$ & L & 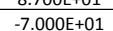 & & & 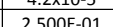 & 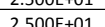 & & Dowe \\
\hline & & & & & & $-8.2006+01$ & & & 4.640E+01 $>2>0$ & & & in VP of Ant \\
\hline 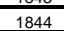 & $\begin{array}{l}1843 \text { Methoxyethylmercuric acetate } \\
18\end{array}$ & 151-38-2 & $\frac{000151-38-2}{0}$ & 31.87 & $\frac{5}{s}$ & 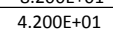 & & & $1.0000-05$ & $2.5000++01$ & & gig Table 1 \\
\hline 1845 & \begin{tabular}{|l|l|l|}
1844 & Methoxyphenol, p- \\
\end{tabular} & $150-76-5$ & $000150-76-5$ & 124.15 & $\mathrm{~s}$ & 52.5 & & $0 \mathrm{E}+02$ & & & & AAC 27 Listing Table 1 \\
\hline$\frac{1846}{1847}>0$ & 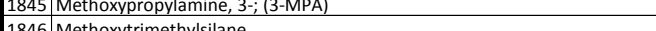 & $\begin{array}{l}53327-750 \\
182-0\end{array}$ & 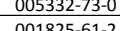 & $\frac{89.16}{1012}$ & $L$ & 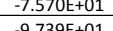 & 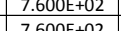 & $\begin{array}{l}1.1600+02 \\
500+21\end{array}$ & 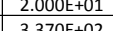 & 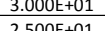 & 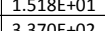 & 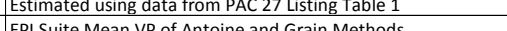 \\
\hline
\end{tabular}




\begin{tabular}{|c|c|c|c|c|c|c|c|c|c|c|c|c|}
\hline & Chemical Compound & $\begin{array}{c}\text { cas N Number } \\
\text { (CASPN) }\end{array}$ & $\begin{array}{c}\text { Modified } \\
\text { frrmat CAS }\end{array}$ & $\begin{array}{l}\text { Molecurar } \\
\text { Weight }\end{array}$ & at & $\begin{array}{c}\text { MP or FP } \\
\text { [ic }\end{array}$ & 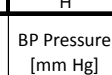 & вр [ст] & 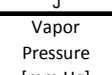 & $\begin{array}{c}\text { Torvp } \\
\text { [cicl }\end{array}$ & 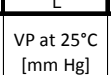 & Vapor Pressure @ $95^{\circ}$ C Reference \\
\hline $\begin{array}{lll}1848 \\
1000\end{array}$ & 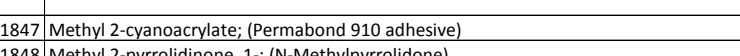 & 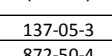 & 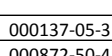 & $\frac{11.11}{9091}$ & & 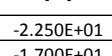 & 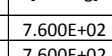 & 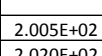 & 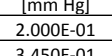 & 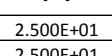 & 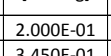 & 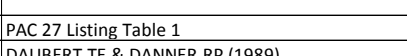 \\
\hline 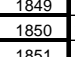 & 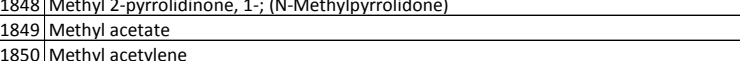 & $\begin{array}{l}\frac{8725.9}{79-2.9} \\
749.9-9\end{array}$ & 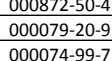 & 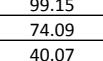 & $\frac{2}{6}$ & 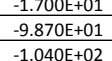 & 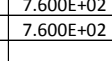 & 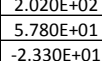 & 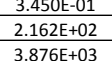 & 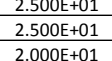 & $\begin{array}{l}3.460-01 \\
2.162+02\end{array}$ & 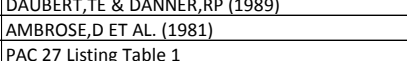 \\
\hline$\frac{1515}{1.952}$ & 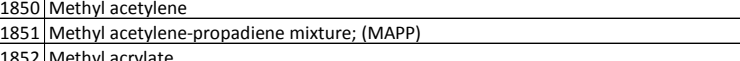 & 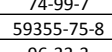 & 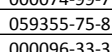 & $\begin{array}{l}80.19 \\
80.19\end{array}$ & $\frac{6}{6}$ & 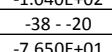 & & 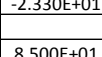 & & & 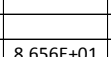 & 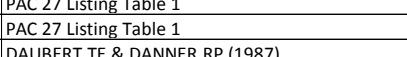 \\
\hline$\frac{1853}{1054}$ & 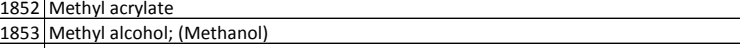 & & & & & & $\begin{array}{l}7.600+522 \\
7.600+02\end{array}$ & 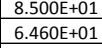 & $\begin{array}{l}8.666+501 \\
1.270+0202\end{array}$ & $\frac{2.500+501}{2.500 E+01}$ & 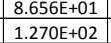 & $\begin{array}{l}\text { DAABBRT, E \& DANNRER,RP (1987) } \\
\text { BOUBLK, ET AL. (1984) }\end{array}$ \\
\hline$\frac{1655}{1056}$ & 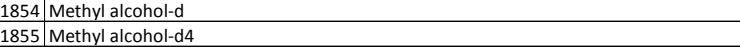 & 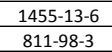 & 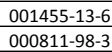 & 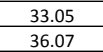 & $\frac{L}{L}$ & $\begin{array}{l}1.1000+501 \\
1.005+01\end{array}$ & $\begin{array}{l}7.600+522 \\
7.0000+02\end{array}$ & 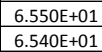 & $\begin{array}{l}1.2705+02 \\
1.7205+02\end{array}$ & $\begin{array}{l}2.500+5+1 \\
2.5000+01\end{array}$ & $\begin{array}{l}1.2700+52 \\
1.7200+02\end{array}$ & 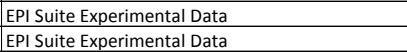 \\
\hline$\frac{1957}{1958}$ & 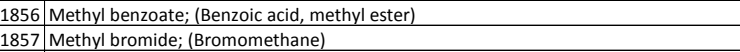 & & 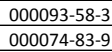 & $\begin{array}{l}136.16 \\
94.94\end{array}$ & & $\begin{array}{l}-1.250 E+1 \\
-9.368+01\end{array}$ & $7.600+02$ & 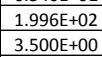 & $\begin{array}{l}3.800=01 \\
7.5000+02\end{array}$ & $\begin{array}{l}2.5000+01 \\
.3000+00\end{array}$ & & 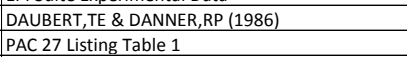 \\
\hline & 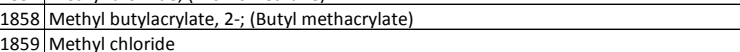 & & 0.00097-88 & 142.22 & & & 7.600E+02 & & 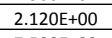 & $2.500 \mathrm{t}+01010$ & $2.120 E+0$ & $\begin{array}{llllll}n & 1\end{array}$ \\
\hline $\begin{array}{l}\frac{1600}{1681} \\
181\end{array}$ & 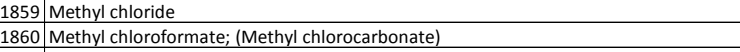 & & 0.0007-47.:-2: & $\frac{50.49}{99.5}$ & & & $7.600 \mathrm{~F}+02$ & 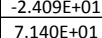 & 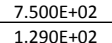 & 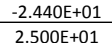 & 1.2005: & $\begin{array}{l}\text { ting Table } 1 \\
\text { tean VP of Antoine and } G \text { : }\end{array}$ \\
\hline 1962 & 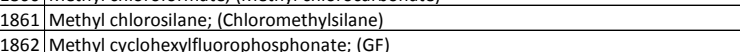 & $\begin{array}{l}993-00-0 \\
320.097\end{array}$ & 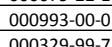 & 80.59 & 6 & $-1.350+5022$ & & 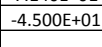 & 4000.02 & & $4000=0$ & 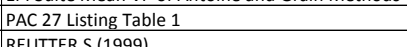 \\
\hline 1864 & 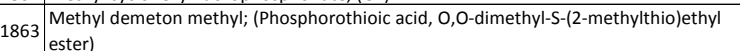 & 2587-909-8 & 002587.90 .8 & 216.27 & L & & 7.600E+02 & $2.300 \mathrm{~F}+02$ & $2.340-03$ & $2.500++01$ & $2.300 \mathrm{0}: 0$ & PI Suite Modfified Grain Method \\
\hline$\frac{1865}{1866}$ & 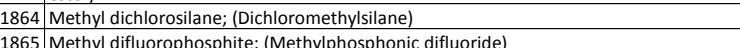 & 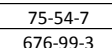 & $\begin{array}{l}0.0075 .5 \\
00076.65\end{array}$ & $\frac{115.04}{1001}$ & $\frac{t}{4}$ & $-9.300 E+01$. & $\begin{array}{l}7.600+522 \\
7.600+52\end{array}$ & 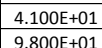 & $\frac{4.2906+52}{20000+02}$ & $\frac{2.500+\div 01}{20500+01}$ & $\frac{4.290 \mathrm{Et}}{2000 \mathrm{E}}$ & $\begin{array}{l}\text { PAC 27 Listing Table } 1 \\
\text { EPPSSite ean Veap of Ant }\end{array}$ \\
\hline$\frac{1666}{1.667}$ & 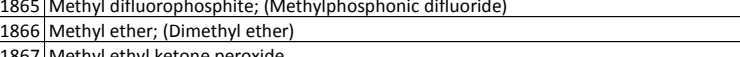 & 167.-9 & 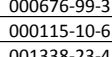 & $\frac{10.01}{\frac{10.08}{67.01}}$ & $\frac{\frac{1}{6}}{6}$ & $-1.3855+02$ & & 9.8000 & 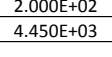 & 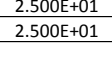 & & 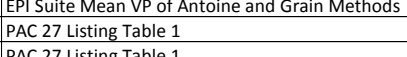 \\
\hline & 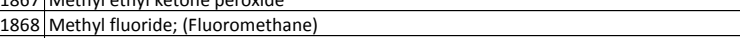 & & & & & $-1.433 E+022$ & & & .5000+t & $7.860 \mathrm{E}$ & & 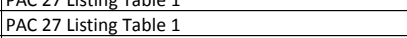 \\
\hline$\frac{188}{108}$ & 689| Methy y luorocactetate & & 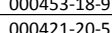 & $\frac{-2.08}{12.11}$ & & & 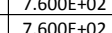 & & & & & 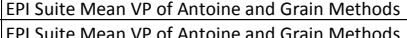 \\
\hline$\frac{18872}{1.872}$ & 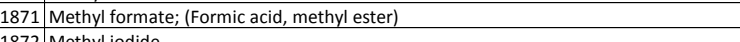 & & D.00107-3. & & & 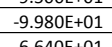 & $\frac{1.600 \mathrm{E}+}{7.6005}$ & & .8.577 & & 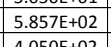 & 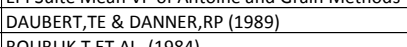 \\
\hline$\frac{1874}{1.74}$ & 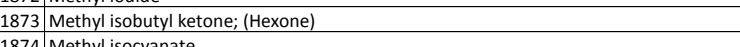 & $\frac{108-1}{10}$ & 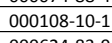 & $\frac{14.1 .44}{100.16}$ & $\frac{t}{L}$ & 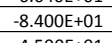 & $7.600 \mathrm{Et}$ & 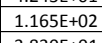 & $9866+0+0$ & & & 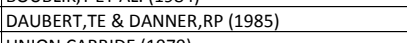 \\
\hline & 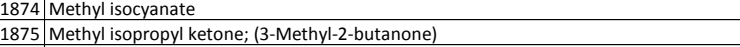 & & & & & & 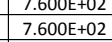 & & & & & 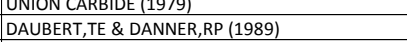 \\
\hline$\frac{1877}{1877}$ & 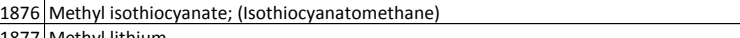 & & 0.00556-61 & $\frac{73.12}{73.90}$ & $s$ & 3.600E+01 & 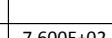 & 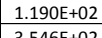 & 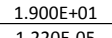 & & m & Listing Table 1 . \\
\hline $\begin{array}{ll}1879 \\
1879\end{array}$ & 78 Menthyl mercaptan & & & 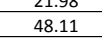 & 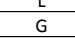 & $-1.230 E+022$ & & 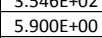 & 7.500E+0. & .700E & & 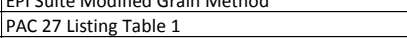 \\
\hline & 79. Methyl mercury & & & & & & & & & & & String Table 1 \\
\hline & (1) & & & 114.19 & & 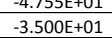 & 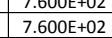 & 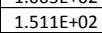 & & & & 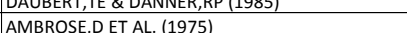 \\
\hline 1883 & 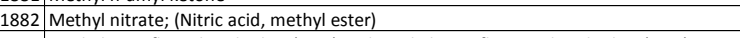 & 598.5 & 000598-58: & & & $-8.3000+011$ & $7.600 E+02$ & & $1.600 \mathrm{E}+0202$ & $2.500 E+0$ & & $\begin{array}{l}\text { te Mean V P o f A A thtoine and Grain Meth } \\
\text { te }\end{array}$ \\
\hline 1884 & 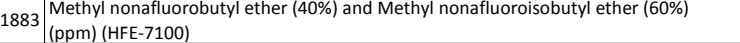 & 16370208. & 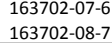 & 250.063727 & ${ }^{6}$ & & & & & & & PAC 27 listing Table 1 \\
\hline & 1884 | Methyl parathion & 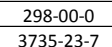 & 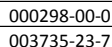 & $\begin{array}{l}263.21 \\
349.25\end{array}$ & $\frac{\mathrm{s}}{\mathrm{L}}$ & 3.800E+01 & $7.600 E+022$ & 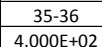 & $\begin{array}{cccc}0.20 \mathrm{~Pa} \\
3.5000 .06\end{array}$ & $\begin{array}{l}2.000+51 \\
2.5000+01\end{array}$ & & $\begin{array}{l}\text { PAC } 27 \text { Listing Table } 1 \\
\text { PAC 27 Lising Tatle } 1\end{array}$ \\
\hline$\frac{1087}{1.809}$ & 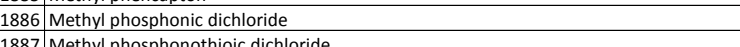 & 676.9 & 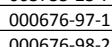 & $\frac{132.91}{1497}$ & 5 & 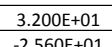 & $76005+20^{2}$ & 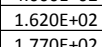 & 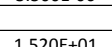 & 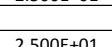 & & 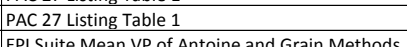 \\
\hline & 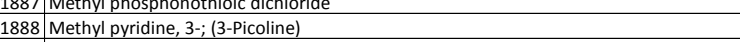 & 108-99 & & & & & 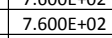 & & & & & 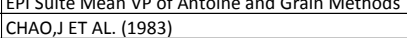 \\
\hline 1.980 & 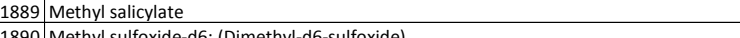 & $1199 \cdot 36$ & 0.001919.36:8 & 152.16 & $\mathrm{~L}$ & $-8.6000=000$ & 7.600E+022 & $2.33 \mathrm{BE}+022$ & 3.4300:02 & 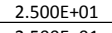 & 3.430E-0.0. & Ac 27 listing Table 1 - \\
\hline 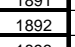 & 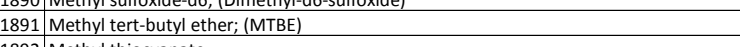 & 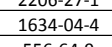 & 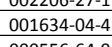 & $\begin{array}{l}88.15 \\
87.15\end{array}$ & L & 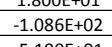 & 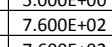 & 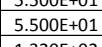 & 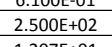 & 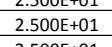 & 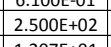 & D. \\
\hline & 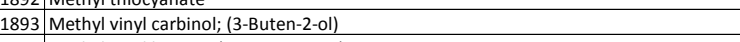 & 598-3. & .005598:32: & & & -8.000t=01 & 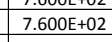 & 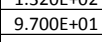 & & 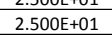 & & 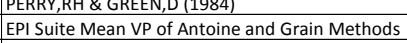 \\
\hline$\frac{1905}{1095}$ & 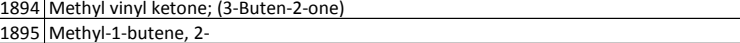 & 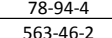 & & 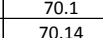 & 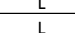 & $\begin{array}{l}-7.000+50 \\
-1355+52\end{array}$ & $\begin{array}{l}7.6000+02 \\
7000202\end{array}$ & 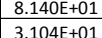 & 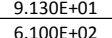 & 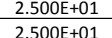 & 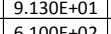 & 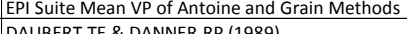 \\
\hline 1987 & 1896 Methy-1H-benzotrizale & & 2029355:43: & & 5 & & & & & & & ting Table 1 \\
\hline$\frac{1988}{1099}$ & 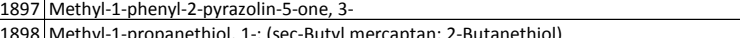 & $\begin{array}{l}8.925 \\
53.53\end{array}$ & $\begin{array}{l}0.00089 .25 .5 \\
0.0003\end{array}$ & $\begin{array}{l}172.42 \\
019\end{array}$ & 5 & $\begin{array}{l}1.285602 \\
-1650+22\end{array}$ & & 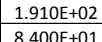 & & & & 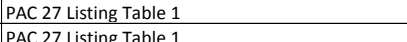 \\
\hline & 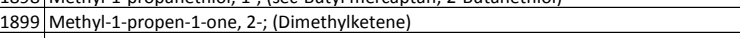 & & c00598-26: & & & 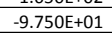 & $\frac{7.600+02}{7.002}$ & & .412E+to2 & & 5.412E+t0 & hysical Chemistry Suite \\
\hline$\frac{1901}{1002}$ & 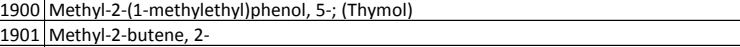 & $\begin{array}{l}\frac{89.8 .8}{553.9} \\
5535.9\end{array}$ & 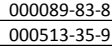 & 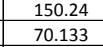 & $\frac{s}{2}$ & $\frac{51}{-1.377+02}$ & $7.600 E+02$ & 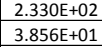 & 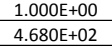 & 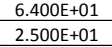 & $4.680+0202$ & $\begin{array}{l}\text { PAA } 27 \text { listing Table } 1 \\
\text { PCC } 27 \text { Listing Table } 1\end{array}$ \\
\hline$\frac{1903}{1904}$ & 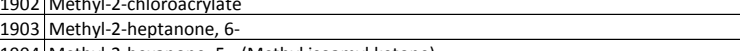 & 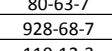 & 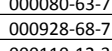 & 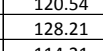 & $i$ & $-4.200 E+011$ & $\begin{array}{l}7.6000+02 \\
7.600+02\end{array}$ & 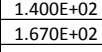 & $\begin{array}{lll}1.20060+01 \\
2.400 E+00\end{array}$ & 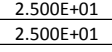 & 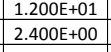 & 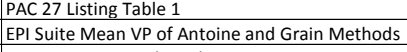 \\
\hline & 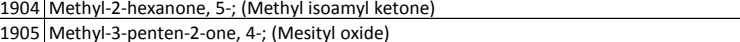 & & 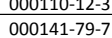 & & & 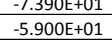 & 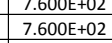 & 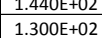 & 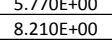 & 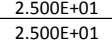 & & 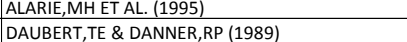 \\
\hline 1007 & 006 06/Methy-4-penten-2-one, 4- & 374402023. & 0.3744-02:3. & 98.14 & & 7.2.2606+01 & & & 1.508Et+121 & 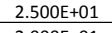 & & RDDDCC,AAET AL. (1986) \\
\hline$\frac{1006}{1009}$ & 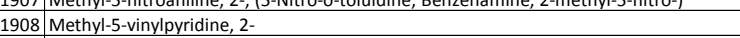 & 190- & 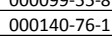 & 疽119.18 & 3 & 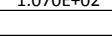 & $7.600 E+02$ & $.810 E+02$ & 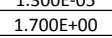 & 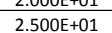 & $1.700 E+0$ & 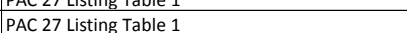 \\
\hline$\frac{1910}{1910}$ & 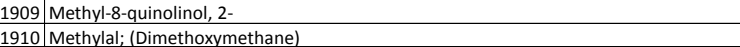 & $\begin{array}{l}826.8-1.3 \\
10987.5\end{array}$ & 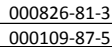 & $\frac{159.2}{77.1}$ & $\frac{S}{L}$ & 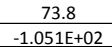 & $7.600+52$ & $\begin{array}{l}2.670+502 \\
4.2000+01\end{array}$ & $3.97 \mathrm{E}+022$ & $2.500=0101$ & $3.975+022$ & $\begin{array}{l}\text { PAA } 27 \text { listiting Table } 1 \\
\text { DUUBERT, }\end{array}$ \\
\hline
\end{tabular}




\begin{tabular}{|c|c|c|c|c|c|c|c|c|c|c|c|c|}
\hline & A & & & Anecouar & Qv state & & $\begin{aligned} \mathrm{H} \\
\mathrm{H}\end{aligned}$ & +2 & & $\mathrm{k}$ & & \\
\hline & Chemical Compound & $\begin{array}{l}\text { CAS Number } \\
\text { (CASRN) }\end{array}$ & $\begin{array}{c}\text { Modified } \\
\text { Format CAS }\end{array}$ & $\begin{array}{c}\text { Molecular } \\
\text { Weight (MW) }\end{array}$ & $\begin{array}{c}\text { avstate } \\
\text { at } \\
25^{\circ} \mathrm{C}\end{array}$ & $\begin{array}{l}\text { MP or FP } \\
{\left[{ }^{\circ} \mathrm{C}\right]}\end{array}$ & $\begin{array}{c}\text { BP Pressure } \\
{[\mathrm{mm} \mathrm{Hg}]}\end{array}$ & $B P\left[{ }^{[C}\right]$ & $\begin{array}{l}\text { Vapor } \\
\text { Pressure } \\
\text { Imm Hg }\end{array}$ & $\begin{array}{l}\text { Tfor VP } \\
\text { ['C] }\end{array}$ & $\begin{array}{c}\text { VP at } 25^{\circ} \mathrm{C} \\
{[\mathrm{mm} \mathrm{Hg}]}\end{array}$ & 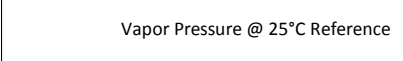 \\
\hline 1912 & 1911 Methylamine hydrochloride & $593-51-1$ & $000593-51-1$ & 67.53 & $\mathrm{~s}$ & $232-234$ & & $227 @ 15 \mathrm{C}$ & & & & $\begin{array}{l}\text { PAC 27 Listing Table } 1 \\
\end{array}$ \\
\hline 1913 & $\begin{array}{l}1912 \text { Methylaminolethanol, } 2-1 \\
1913\end{array}$ & $\frac{109-83-1}{100-18}$ & $000109-83-1$ & 75.11 & L & $\begin{array}{lll}-4.5000+00 \\
.5 .0050101\end{array}$ & 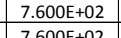 & $\begin{array}{l}1.560 \mathrm{E}+02 \\
1.90 \mathrm{EF}+02\end{array}$ & $\begin{array}{l}1.083 E+00 \\
4.530 E-01\end{array}$ & $2.500 \mathrm{EE}+01$ & $\begin{array}{l}1.083 E+00 \\
4.530-01\end{array}$ & 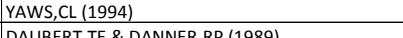 \\
\hline$\frac{1914}{1915}$ & 1014 Methylazirididine, 1- & $1072-44-2$ & $001072-44-2$ & 57.11 & $\mathrm{~L}$ & - & $7.600 \mathrm{E}+02$ & 2. $2.740 \mathrm{t}+\mathrm{E}+01$ & $\begin{array}{ll}4.5300-01 \\
6.930 E+02 \\
\end{array}$ & 2.500E+01 & 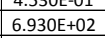 & EPI Suite Mean VP of Antoine and Grain Methods \\
\hline 1916 & \begin{tabular}{|l|l|}
1915 & Methylbutanamide, 3-; (Isovaleramide) \\
\end{tabular} & $541-46-8$ & $000541-46-8$ & 101.15 & $\mathrm{~s}$ & $1.370 E+02$ & & $2.260 \mathrm{E}+02$ & & & & PAC 27 listing Table 1 \\
\hline 1917 & $1916 \mid$ Methylbutylamine, N- & & & 87.19 & & $-7.500 E+01$ & $7.600 E+02$ & $9.100 E+01$ & $5.500 \leftarrow+01$ & $2.5000+01$ & $5.500 \mathrm{E}+01$ & EPI Suite Mean VP of Antoine and Grain Methods \\
\hline 1918 & \begin{tabular}{|l|l|}
1917 & Methylcellulose \\
\end{tabular} & $9004-67-5$ & 009004-67-5 & 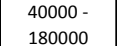 & $\mathrm{s}$ & $290-305$ & & & & & & PAC 27 Listing Table 1 \\
\hline 1919 & 1918 Methylchlorodisiliane; (Chloromethyldisiliane) & $68937-17-7$ & 068937-17-7 & 110.75 & G & & & & & & & PAC 27 Listing Table 1 \\
\hline T & $\mid 1919$ Methylcholanthrene, 3- & $56-49-5$ & $000056-49-5$ & 268.35 & $\mathrm{~s}$ & $1.800 E+02$ & & $280 @$ & $4.3 \times 10-8$ & $2.500 E+01$ & & PAC 27 Listing Table 1 \\
\hline & \begin{tabular}{|l|l|l|}
1920 & Methylcyclohexane \\
\end{tabular} & $108-87-2$ & $000108-87-2$ & 98.21 & & $-1.264 \mathrm{E}+02$ & $7.600 E+02$ & $\begin{array}{lll}0.003 \mathrm{E}+02 \\
1.02\end{array}$ & 4.600E+01 & $2.500 E+01$ & & DAUBERT,TE \& DANNER,RP (1989) \\
\hline & & $\frac{1331-22-2}{130}>20$ & 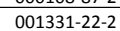 & & L & & & & & $2.5006+01$ & $3.010 \mathrm{E}+00$ & EP Suite Mean VP of Antoine and Grain Methods \\
\hline & 1922 Methylcyclohexanone, 2; (0-Methylycylohexanone) & $583-60-8$ & $000583-60-8$ & 112.19 & $\mathrm{~L}$ & $-1.400 E+01$ & $7.6006+02$ & $1.651 E+02$ & $2.250 \mathrm{E}+00$ & $2.500 \mathrm{E}+01$ & $2.250++00$ & WEBER,RC ET AL. (1981) \\
\hline 1924 & 1923 Methylcyclopentane & 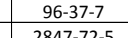 & $000096-37-7$ & 84.18 & $L$ & 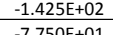 & 7.600E+02 & 7.180다 01 & $1.375 E+02$ & 2.500E+01 & $1.375 E+02$ & BOUBLL, T ET AL. (1984) \\
\hline & $\begin{array}{l}19244 \text { Methyndocenane, } 4 \text { - } \\
1925 \text { Methyldichlorarsine: (Dichloromethylarsine) }\end{array}$ & $\frac{2847-1 / 2-5}{593-89-5}$ & 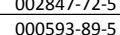 & $\frac{156.11}{160.86}$ & $\frac{L}{L}$ & 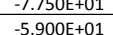 & 1.600+t02 & 1.800+02 & 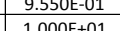 & $\frac{2.500+01}{2.40 E+01}$ & 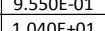 & 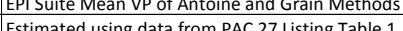 \\
\hline & 1926 Methylene bis(2-chloroaniline), $44^{4} ;$; (MBOCA) & 101-14-4 & $000101-14-4$ & & $\mathrm{~s}$ & $1.100 E+02$ & & & & & & \\
\hline$\frac{1928}{1928}$ & 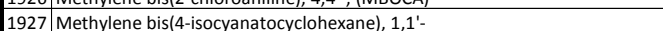 & $\frac{12124-30-1}{5}$ & $\frac{005124-30-1}{0.03}$ & $\frac{262.39}{2639}>20$ & $\mathrm{~L}$ & & $1.500 E+00$ & 1.680E+02 & $\begin{array}{l}2.3330-05 \\
2.305\end{array}$ & 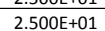 & $2.3300-05$ & EPI Suite Modififid Grain Method \\
\hline 1929 & 1928 Methylene chloride; (Dichloromethane) & $75-09-2$ & $000075-09-2$ & 84.93 & $\mathrm{~L}$ & $-9.720 \mathrm{E}+01$ & $7.600 \mathrm{E}+02$ & $4.000 E+01$ & $4.350 \mathrm{E}+02$ & $2.500 \mathrm{E}+01$ & $4.350 \mathrm{E}+02$ & BOUBLLK, ET AL. (1984) \\
\hline 1930 & 1929 Methylene diphenyl disiscyanate; (Diphenylmethane dissocyanate; MDII) & $101-68-8$ & $000101-68-8$ & 250.25 & $\mathrm{~s}$ & $3.700 E+01$ & & $\begin{array}{c}196 @ \\
5 \mathrm{mHHg}\end{array}$ & $5.0 \times 10-6$ & $2.500 \mathrm{E}+01$ & & PAC 27 listing Table 1 \\
\hline 1931 & 1930 Methylene fluoride; (Difluoromethane; HFC-32) & $75-10-5$ & $000075-10-5$ & 52.03 & & & & & $1.264 \mathrm{E}+04$ & $2.500 E+01$ & & DAUBERT,TE \& DANNER,RP (1989) \\
\hline 1932 & 1931 Methylenebisis(socyanato-benzene)e, 1,1'; (Diphenyl methane disiscocyanate) & $26447-40-5$ & $026447-40-5$ & 250.26 & $\mathrm{~s}$ & & & & & & & PAC 27 Listing Table 1 \\
\hline 1933 & 1932 Methylenebisacrylamide, $N, N^{\prime}-$ _. & $\frac{110-26-9}{101779-9}$ & $000110-26-9$ & $\frac{154.19}{10025}$ & $\frac{s}{s}$ & $\frac{185}{0.050101}$ & & dec & & & & PAC 27 Listing Table 1 \\
\hline$\frac{1934}{1935}$ & 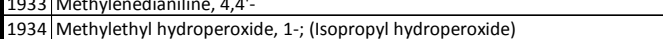 & $\frac{10117-9}{3031-75-2}$ & $\begin{array}{l}00001017-7-9 \\
003031-75-2 \\
\end{array}$ & $\frac{198.26}{76.09}$ & $\frac{5}{5}$ & & & $\begin{array}{l}3.980 \mathrm{E}-02 \\
107-109\end{array}$ & $2.03 \times 10-7$ & $2.500 \mathrm{E}+01$ & & $\begin{array}{l}\text { PAC27 Listing Gable } 1 \\
\text { PAC 27 Listing Table } 1\end{array}$ \\
\hline$\frac{19356}{1936}$ & 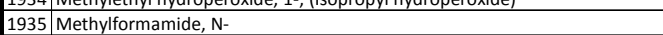 & $\frac{123-39-7}{123-7}$ & $000123-39-7$ & 59.08 & $\frac{3}{L}$ & $-5.4000 E+00$ & $7.600 \mathrm{E}+022$ & $\frac{107-15}{1.800 E+02}$ & $2.530 \mathrm{E}-01$ & $2.500 E+01$ & 2.530 & $\begin{array}{l}\text { PAC } 27 \text { Listing Table } 1 \\
\text { Pats }\end{array}$ \\
\hline $\begin{array}{l}1937 \\
1937\end{array}$ & \begin{tabular}{l|l|}
1936 & Methylfuran, $2-$ \\
\end{tabular} & $534-22-5$ & $000534-22-5$ & 82.11 & L & $-8.870 \mathrm{E}+01$ & $7.600 \mathrm{E}+02$ & 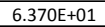 & & $2.5000+01$ & & CHAO, IET AL. (1983) \\
\hline 1938 & 1937 /Methylheptane, $2-$ & 592-27-8 & 000592-27-8 & 114.23 & $\mathrm{~L}$ & $-1.0906+022$ & $7.6000+022$ & 1.1.180E+02 & 2.070E+01 & $2.500 E+01$ & $2.070 E+01$ & DAUBERT,TE \& DANNER,RP (1989) \\
\hline & (19) & & & & L & & & & 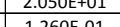 & & & 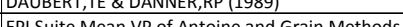 \\
\hline$\frac{1904}{1994}$ & 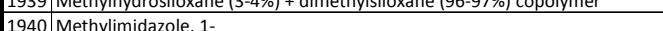 & (61-47-7. & 0000616-4-7-7 & $\frac{28.64}{88.12}$ & 5 & 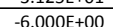 & $\frac{1.000 \mathrm{E}+02}{7.02}$ & $\begin{array}{lll} & \end{array}$ & & & & 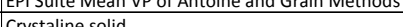 \\
\hline$\frac{1944}{1942}$ & 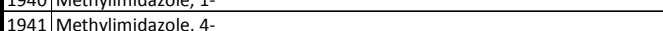 & 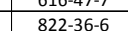 & 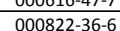 & $\frac{6.12}{8.22}$ & $\frac{3}{5}$ & $\frac{5.600 \mathrm{~F}+01}{501}$ & 1.000Ev+UL & 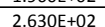 & & & & 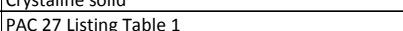 \\
\hline 1943 & 1942 Methyllactic acid ethyl ester, 2;; (Ethyl 2-hydroxyisobutyrate) & $80-55-7$ & $\begin{array}{l}000080-55-7 \\
\end{array}$ & 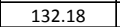 & L & $-1.257 \mathrm{E}+01$. & $7.600 E+02$ & $1.500 E+02$ & $1.350 E+00$ & $2.500 E+01$ & $1.350 €+00$ & $\begin{array}{l}\text { EPP Suite Mean VP of Antoine and Grain Methods } \\
\end{array}$ \\
\hline 1994 & 1943 [Methyllactonitrile, 2;; (Acetone cyanohydrin) & $75-86-5$ & $000075-86-5$ & 85.11 & L & $-1.900 E+01$ & 1.000E+02 & $9.500 E+01$ & 3.4100E-01 & & 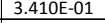 & DAUBERT,TE \& DANNER,RP (1989) \\
\hline$\frac{1945}{1946}$ & 19445 Mathethylyagnesumum bromide, $3.0 \mathrm{~m}$ In diethyl ether & 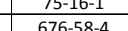 & 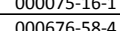 & $\frac{119.26}{74793}$ & 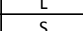 & $-2.828 E+01$ & 7.600E+02 & 1.1.189t+02 & & $2.500 \mathrm{E}+01$ & & 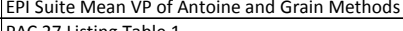 \\
\hline & 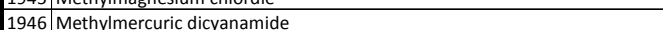 & $\frac{2502.39-6}{503}$ & $\frac{100502-59 \cdot 6}{000056}$ & 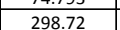 & & $1.560 \mathrm{E}+02$ & & & $3.000 E-05$ & $2500=+01$ & & 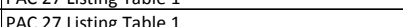 \\
\hline 1998 & 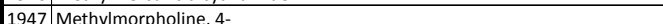 & $109-02-4$ & $000109-02-4$ & 10117 & th & 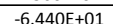 & & & & & & EPI Suite Mean VP of Antoine and Grain Methods \\
\hline$\frac{2949}{1994}$ & $\begin{array}{l}1948 \text { Methyinaphthalene, } 1 \text { - } \\
190\end{array}$ & 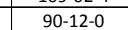 & 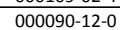 & $\frac{2142.21}{142}$ & L & $\frac{1.2006+01}{-2.205+01}$ & 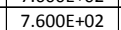 & 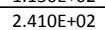 & $\frac{1.700 \mathrm{0}-02}{6}$ & $\frac{2.5006+01}{2.501}>0$ & 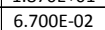 & $\begin{array}{l}\text { PAC } 27 \text { Listing Table } 1 \\
\end{array}$ \\
\hline & 1949 Methylnaphthalene, 2 - & $91-57-6$ & $000091-57-6$ & 142.2 & $\mathrm{~s}$ & $3.460 \mathrm{E}+01$ & & $2.411 \mathrm{E}+02$ & $7.500 E_{-01}$ & 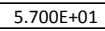 & & PAC 27 Listing Table 1 \\
\hline 1951 & 1950|Methylhitrosopiperidine, 3; (Piperidine, 3-methyl-1-nitroso-) & $13603-07-1$ & 013603-07-1 & $\frac{128}{1028}$ & $\mathrm{~L}$ & $1.412 E+01$ & $7.600 \mathrm{E}+02$ & $2.428 E+02$ & $6.3500-02$ & 2.500E+01 & $6.3500-02$ & EPP Suite Mean VP of Antoine and Grain Methods \\
\hline 19952 & 1951] Methylnonane, 4 - & 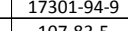 & $0.17301949-99$ & 142.28 & & 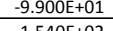 & & $1.657+\mathrm{E}+02$ & $2.540 \mathrm{E}+00$ & & & 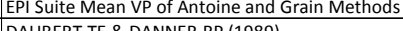 \\
\hline$\frac{1935}{105}$ & 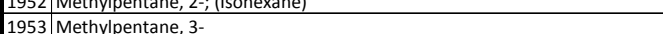 & $\frac{10-78-5}{96-14-0}$ & 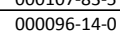 & $\frac{86.2}{86.18}$ & L & 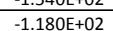 & $\frac{1.00060 \mathrm{~F}}{7.602}$ & 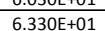 & 1.900F 102 & 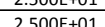 & 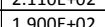 & 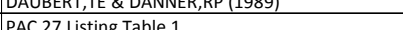 \\
\hline$\frac{1935}{1955}$ & 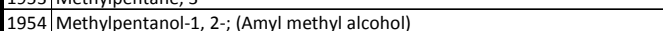 & $\frac{105-30-6}{103}$ & 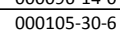 & $\frac{c_{0.10}}{102.2}$ & L & (1.tovitur & $\frac{1.6006 \mathrm{~F}}{7.62}$ & $\frac{0.30601}{1.300+02}$ & 1.900 & $\frac{2.5000 \mathrm{t}}{2.50 \mathrm{E}+1}$ & 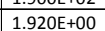 & DAUERRT, TE \& A ANAER,RP (1989) \\
\hline 1956 & 1955 (Methylphenol, 2; (0-Cresol) & $95-48-7$ & $000095-48-7$ & 108.15 & & $3.000 E+01$ & & $1.9106+02$ & $1.000 E+00$ & $3.820 E+01$ & & Listing Table 1 \\
\hline 1957 & \begin{tabular}{|l|l|l|l|l|l}
1956 & Methylphenol, 3; (m-Cresol) \\
\end{tabular} & $108-39-4$ & $000108-39-4$ & 108.15 & $\mathrm{~L}$ & $1.0906 \mathrm{E}+01$ & $7.600 \mathrm{E}+02$ & $2.028 E+02$ & $1.100 \mathrm{E}-01$ & $2.500 E+01$ & $1.100 E-01$ & DAUBERT,TE \& DANNER,RP (1989) \\
\hline$\frac{1958}{1090}$ & 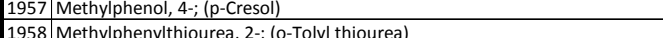 & $\frac{106-44 \cdot 5}{614798}$ & $000106-44-5$ & $\frac{108.15}{11625}$ & $\frac{s}{s}$ & $\frac{3.550 \mathrm{E}+01}{151.155}$ & & $2.018 \mathrm{E}+02$ & $\frac{1.0000+00}{20000}+00$ & $5.3000+01$ & & 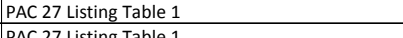 \\
\hline$\frac{1959}{1960}$ & 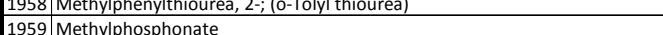 & $\frac{61447-88}{993-13-5}$ & $0000143-78-8$ & $\frac{166.26}{96.2246}$ & $\frac{s}{5}$ & $\frac{151-152}{108.5}$ & & $\begin{array}{ll}d e c \\
\text { a }\end{array}$ & $2.000 \mathrm{E}=06$ & & & $\begin{array}{l}\text { PAC C7 Listing gable } 1 \\
\text { PAC 27 listing Table } 1\end{array}$ \\
\hline$\frac{2961}{1961}$ & 1956 196ethylphosphhonothioic acid, O-(4-nitropheny) O-t & 2665-30-7 & 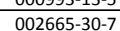 & 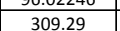 & (5) & & $7.600 E+02$ & 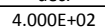 & $1.000 E=05$ & $2.500 E+01$ & & PAC \\
\hline $\begin{array}{ll}1962 \\
192\end{array}$ & 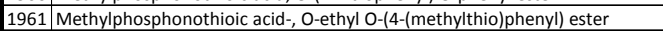 & 2703-13-1. & 002703-13-1 & 262.34 & & & $7.600 E+022$ & $2.980 E+02$ & $1.000 E-04$ & 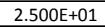 & & PAC 27 \\
\hline 1963 & 1962 |Methylphosphonothioic acid, S-2-(dimethylamino)ethyl) O-ethyl ester & 20820-80-8 & $020820-80-8$ & 211.29 & L & & $7.600 \mathrm{E}+02$ & $2.805 \mathrm{E}+02$ & $5.4700-03$ & $2.5006+01$ & $5.470 \mathrm{E}-0$ & EPI Suite Modified Grain Method \\
\hline 1964 & 1963 Methyl|propane, 2; (Isobutane) & 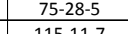 & $000075-28-5$ & $\frac{58.12}{56.12}$ & $G$ & $-1.5994+02$ & & & & & & PAC 27 lisiting \\
\hline$\frac{1966}{1066}$ & 10 & 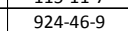 & 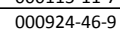 & $\frac{10.16}{102.16}$ & L & 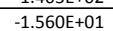 & 4.000E+01 & $\frac{0.1006+01}{9.101}$ & 5.620E-01 & 2.500E+01 & 5.620E-01 & EPP Sute Mean NP of Antoine and Grain Methods \\
\hline (1000 & $\begin{array}{l}1 \\
1\end{array}$ & 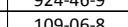 & 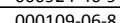 & $\frac{10.10}{9314}$ & & 7. & 760050 & 120050 & & $\frac{1500}{250501}$ & 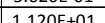 & 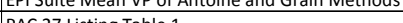 \\
\hline (1960) & 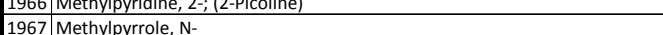 & $\frac{1050-6}{99-84}$ & $\frac{1000095-5.8}{0.8}$ & $\frac{3.14}{81.12}$ & L & 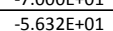 & $\frac{1.000602}{7.60 \mathrm{E}+02}$ & 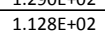 & 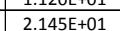 & 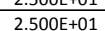 & 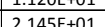 & 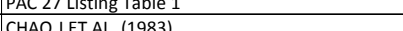 \\
\hline 1969 & 1968 | Methylpyrrolidine; (Methylpyrrolidine, 1-) & $\frac{50-040}{120-94-5}$ & $000120-94-5$ & 85.15 & L & $\frac{-9.000 E+01}{1}$ & $7.600 \mathrm{E}+02$ & 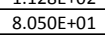 & $\frac{2.1400+101}{1.000+02}$ & $2.500 \mathrm{E}+01$ & 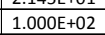 & DAUBERT, IE \& DANNER,RP (1989) \\
\hline & 1969 Methylstyrene, alpha- & & & & & $-2.400 E+01$ & $7.600 E+02$ & & $1.900 \mathrm{E}+00$ & 2.50065 & $1900 \mathrm{E}+00$ & TET AL (1984) \\
\hline$\frac{1971}{1971}$ & 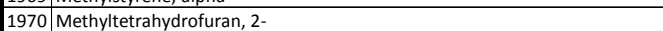 & $96-47-9$ & 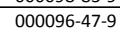 & 86.15 & $\mathrm{~L}_{\mathrm{L}}$ & & 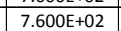 & $8.0006+01$ & 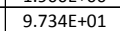 & $\frac{1.5006+01}{2.501}$ & $9.734 \mathrm{E}+01$ & 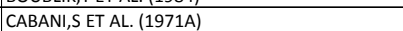 \\
\hline 072 & 1971 Methyltriacetoxysilane & 4253-34-3 & 004253-34-3 & 220.25 & $\mathrm{~s}$ & 4.050E+01 & & 1111@ & & & & PAC 27 Listing Table 1 \\
\hline & 1972 Methyltrichlorosilane; (Trichloromethyl silane) & $75-79-6$ & $000075-79-6$ & 149.48 & & $-9.000 E+01$ & $7.600 E+02$ & $\frac{1.5 m i m b}{6.56 \mathrm{~F}+1}$ & $1.670 E+02$ & $2.5000+01$ & $1.670 \mathrm{E}+02$ & |RIDDICK,A ET AL. (1986) \\
\hline
\end{tabular}




\begin{tabular}{|c|c|c|c|c|c|c|c|c|c|c|c|c|}
\hline & chemical Compound & $\begin{array}{c}\text { cas Number } \\
\text { (CASNR) }\end{array}$ & $\begin{array}{c}\text { Modified } \\
\text { Format CAS }\end{array}$ & $\begin{array}{l}\text { Molecurar } \\
\text { Weight MW }\end{array}$ & atc & 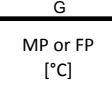 & $\left.\begin{array}{l}\text { Bp persurer } \\
\text { [mm Mag }\end{array}\right]$ & ${ }_{\text {вр }}[\mathrm{CC}]$ & 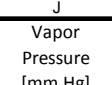 & $\begin{array}{l}\text { Tfor VP } \\
\text { [cc }\end{array}$ & 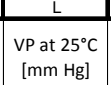 & Vapor Pressure @ $@ 25^{\circ}$ C Reference \\
\hline 型 & 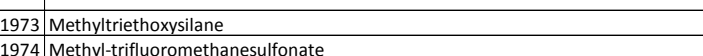 & $\begin{array}{l}2031-67.6 \\
333.277 \\
3\end{array}$ & 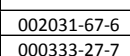 & $\frac{178.34}{164.151}$ & & 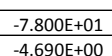 & $\begin{array}{l}7.600+022 \\
7000+02\end{array}$ & 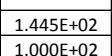 & 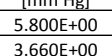 & $\begin{array}{l}2.500+501 \\
2.500+51\end{array}$ & $\begin{array}{l}5.8000+ \pm 00 \\
3.660+00\end{array}$ & $\begin{array}{l}\text { ite Mean V P o f Antoine and Grain Methods } \\
\text { it }\end{array}$ \\
\hline 1996- & 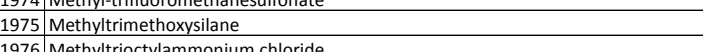 & 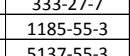 & Do1185-55:- & 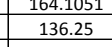 & $\mathrm{L}$ & & $7.00002+02$ & 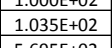 & 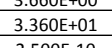 & 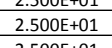 & 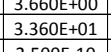 & 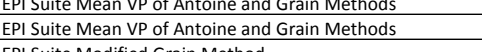 \\
\hline & 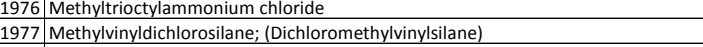 & & 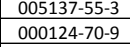 & 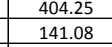 & & & $\begin{array}{l}7.600+0202 \\
7.600+02\end{array}$ & .6956ten2 & $\begin{array}{l}2.5000-10 \\
5.160++01\end{array}$ & 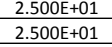 & & 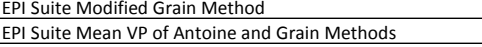 \\
\hline 1.979 & 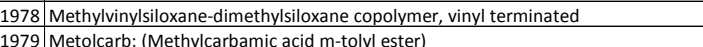 & 680833-1.19 & 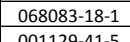 & & & $-6.3306+5000$ & & 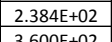 & 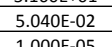 & 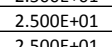 & 5.0060:02 & 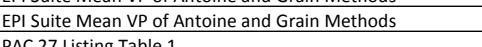 \\
\hline$\frac{1980}{1.981}$ & 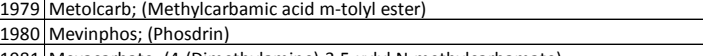 & 17296-1.5 & 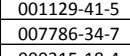 & 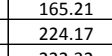 & $\frac{s^{5}}{5}$ & $\frac{76-700}{6.700+000}$ & & $\begin{array}{l}3.6000+02 \\
2.800+02\end{array}$ & $\begin{array}{l}1.0000 .05 \\
2.006+00\end{array}$ & 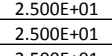 & & 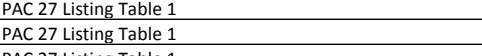 \\
\hline & 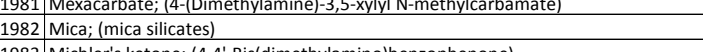 & 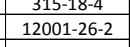 & 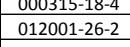 & 每398.3. & & $\begin{array}{l}8.500+21 \\
1.387+03\end{array}$ & & & & & & \\
\hline 1.1989 & 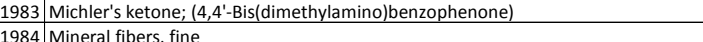 & 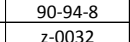 & 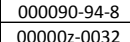 & 268.39 & & 1.7900+02 & & 360 deconn & & & & \\
\hline$\frac{1986}{1986}$ & 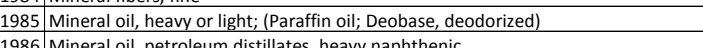 & 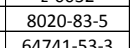 & 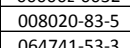 & & L & & 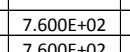 & $\frac{1.7406+02}{1.9050+52}$ & $\begin{array}{l}1.0000 .02 \\
1.002\end{array}$ & $2.000 E+01$ & $1.7288-02$ & 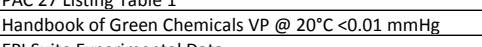 \\
\hline$\frac{1988}{1988}$ & 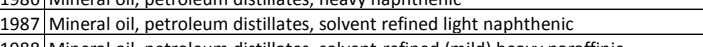 & 64771.97. & 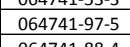 & $\frac{25232}{252}$ & 2 & & $7.6000+52$ & 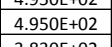 & 5.4900-09 & 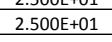 & 5.49060.09 & 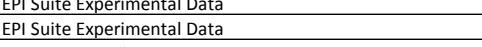 \\
\hline & 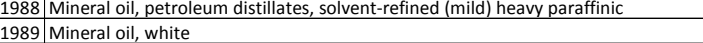 & & & & & $5.6800 €+11$. & $\begin{array}{l}7.600+502 \\
.7 .000+02\end{array}$ & & & & & 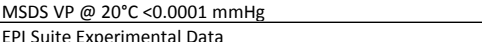 \\
\hline$\frac{1991}{109}$ & 1990|(Minerata oli:; (Oil mist, mineral) & 8012-25: & 008012-2-95. & $\begin{array}{l}170.34 \\
10.34\end{array}$ & & $9.6 .000+000$ & $7.600 E+02$. & $2.163 \mathrm{E}+02$ & 1.3500-01 & 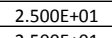 & 1.350:-01. & expeerimental \\
\hline$\frac{1992}{1993}$ & 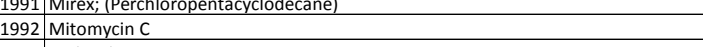 & $\begin{array}{lll}2835088-5 \\
50-07-7\end{array}$ & 0.00055-0.07. & $\begin{array}{l}\frac{54.54}{33437} \\
337\end{array}$ & $\frac{5}{5}$ & 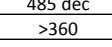 & & $5.340 E+02$ & 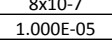 & $\begin{array}{l}2.5000+201 \\
2.500+01\end{array}$ & & 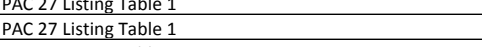 \\
\hline & 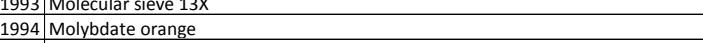 & & & & & & & & & & & \\
\hline$\frac{1996}{1096} \mathrm{C}$ & 1995 Molybdenum & 7339.98.9.8 & 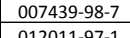 & 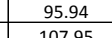 & & 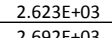 & & 4.6395+03 & 7.5x10:-3 & $2.4695+03$ & & \\
\hline 1998 & 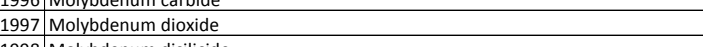 & 18868-43.-4 & 018868-43.4. & $\frac{12794}{12794}$ & s & 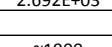 & & 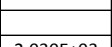 & & & & 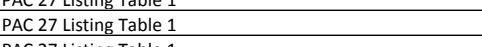 \\
\hline & 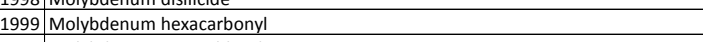 & 1.93930.06: & 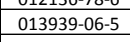 & $\frac{264}{264}$ & & 1900 & & $2.030 \mathrm{E}+03$ & & & & 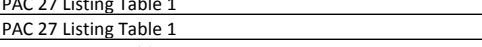 \\
\hline 2000 & 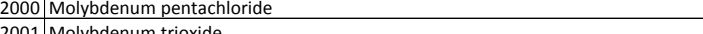 & & 年1024-1.25 & 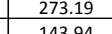 & $\frac{3}{c}$ & 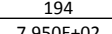 & & $2.6800+02$ & & & & \\
\hline 2003 & 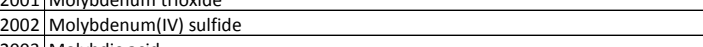 & $1317-33$ & $001317-33$ & 160.07 & $\mathrm{~s}$ & $1.1855+03$ & & & & & & ting Table 1 1 \\
\hline$\frac{2004}{2005}$ & 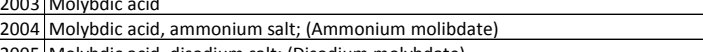 & 1182-998-4-4:- & 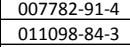 & $\frac{169.9344}{224.28}$ & $\frac{6}{5}$ & & & & & & & 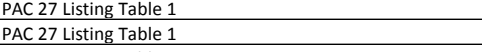 \\
\hline 2006 2007 & 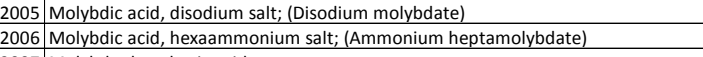 & $\begin{array}{l}763195-1 \\
12027-67-\end{array}$ & 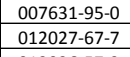 & 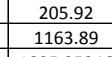 & $\frac{s}{5}$ & 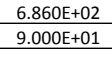 & & dec & & & & 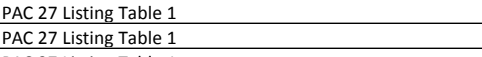 \\
\hline $\begin{array}{ll}\frac{2008}{2009} & 0\end{array}$ & 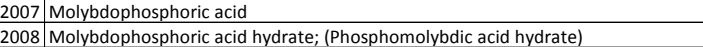 & & & & & 1.040E+02 & & & & & & \\
\hline 2010 & 009 Monobutyl phosphite & 16456-56-7.7 & 0.16456-56-: & 138.12 & L & 1.1.266+01 & $7.6000+022$ & 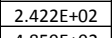 & 5.35060:-33 & 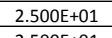 & 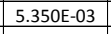 & Sutut Mean V V o o f A Antoine and G. \\
\hline$\frac{2012}{2012}$ & 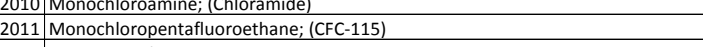 & 每-15.3. & 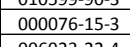 & $\begin{array}{lll}154.47 \\
15270\end{array}$ & $\frac{6}{6}$ & 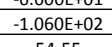 & & 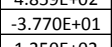 & 70000065 & 25005010 & & 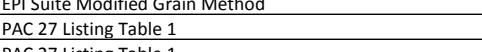 \\
\hline 2014 & 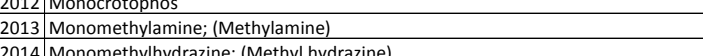 & $\begin{array}{ccc}7489.5 \\
60.34\end{array}$ & 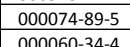 & $\begin{array}{l}31.06 \\
44.07\end{array}$ & 6 & 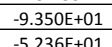 & 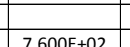 & 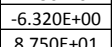 & 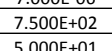 & 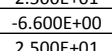 & $50005+0.0$ & 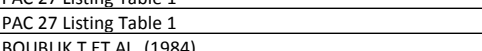 \\
\hline & & 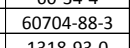 & & 102.87 & sc & & & & & & & \\
\hline 2018 & 017) Morpholine & 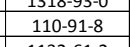 & 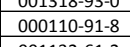 & 87.14 & $i^{2}$ & $-4.900+500$ & $7.600 E+02$ & $1.2895+02$ & $1.008++01$ & $2.500++01$ & $1.008 \mathrm{E}+0$ & RiDOICK,IA ET AL. (1986) \\
\hline & 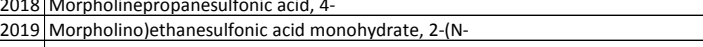 & 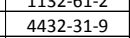 & 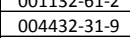 & 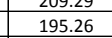 & s & ${ }^{243}$ & & & & & & 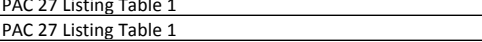 \\
\hline 2021 & 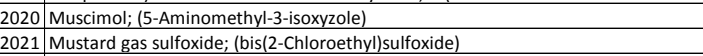 & 2763:-96-4. & 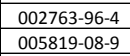 & 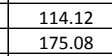 & $\frac{5}{5}$ & 174-176 & & & $1.000=05$ & $2.500+501$ & & 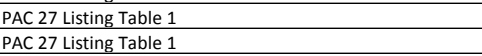 \\
\hline$\frac{2023}{2024}$ & 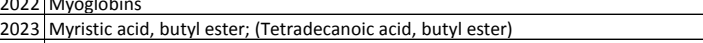 & 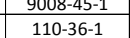 & 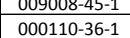 & 284.54 & $\frac{3}{1}$ & & $1.800+01$ & $1.9506+02$ & 9.470E-05 & $2.500+51.1$ & 9.4706 .05 & 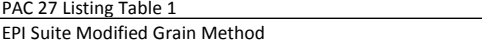 \\
\hline 2025 & 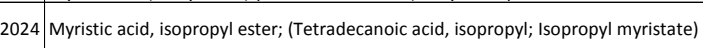 & 110.27.0 & $000110-27.0$ & 270.46 & L & $3.000+500$ & $2.000 €+01$ & $1.926+02$ & $9.350=05$ & $2.500 \mathrm{E}+01$ & 9.350E-05 & PAC 27 L Lsting Table 1 \\
\hline 202027 & 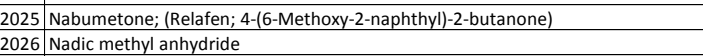 & 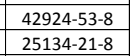 & 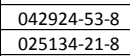 & $\frac{228.29}{1782.2}$ & $\frac{5}{\mathrm{~s}}$ & $\frac{8.000+501}{1.0005+01}$ & $1.000+01$ & 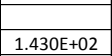 & $1.510 E-02$ & $2.500 \mathrm{E}=01 \mathrm{1}$ & 1.5100:02 & 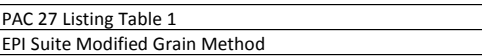 \\
\hline & 2027 Naphtha (coal tar); (Naphtha Ipetroleum] Iight aliphatica Aliphatic naphtha) & 8030-00.6 & 0080303030.6 & 100 & $\mathrm{~s}$ & & & \%150- & $<5$ & & & PAC 27 Listing Table 1 \\
\hline 2029 & 2028 Naphth ( Petroloum), heaver catalytic cracked & 64741.544.4 & 066741.54.4 & 112.22 & & $-6.984+501$ & $7.600+02$ & & $1.650 \mathrm{E}+01$ & $2.500+501$ & $1.650+01$ & inie and Gra \\
\hline 20031 & 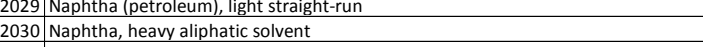 & 647472-69-4-7 & 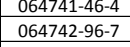 & $\frac{100}{184.37}$ & $\frac{G}{L}$ & -9.610E€+00 0 & $7.600 E+02$ & 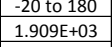 & $9.210 \mathrm{E}-02$ & $2.5005+01$ & $9.210 \mathrm{E}=02$ & 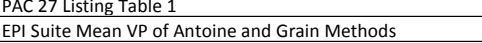 \\
\hline 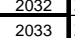 & 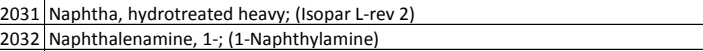 & 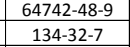 & 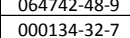 & 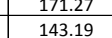 & L & 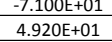 & & $\begin{array}{l}6.50000701 \\
3.007+02\end{array}$ & 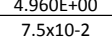 & 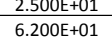 & & 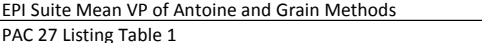 \\
\hline$\frac{2034}{2035}$ & 0334 Naphthalene & $\frac{91-2 \cdot 3 \cdot 3}{86-8 \cdot 2}$ & 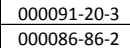 & 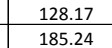 & $\frac{5}{5}$ & $8.0266+011$ & & $\begin{array}{l}2.1799+502 \\
181 \text { subl }\end{array}$ & 7.5x10.2. & $2.410+011$ & & $\begin{array}{l}\text { PAC 27 Listing Table } 1 \\
\text { PAC } 27 \text { Listin Table } 1\end{array}$ \\
\hline 2036 & 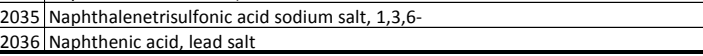 & 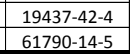 & 10937-42-4 & 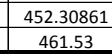 & s & $000+000$ & & & 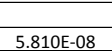 & & & 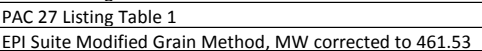 \\
\hline
\end{tabular}




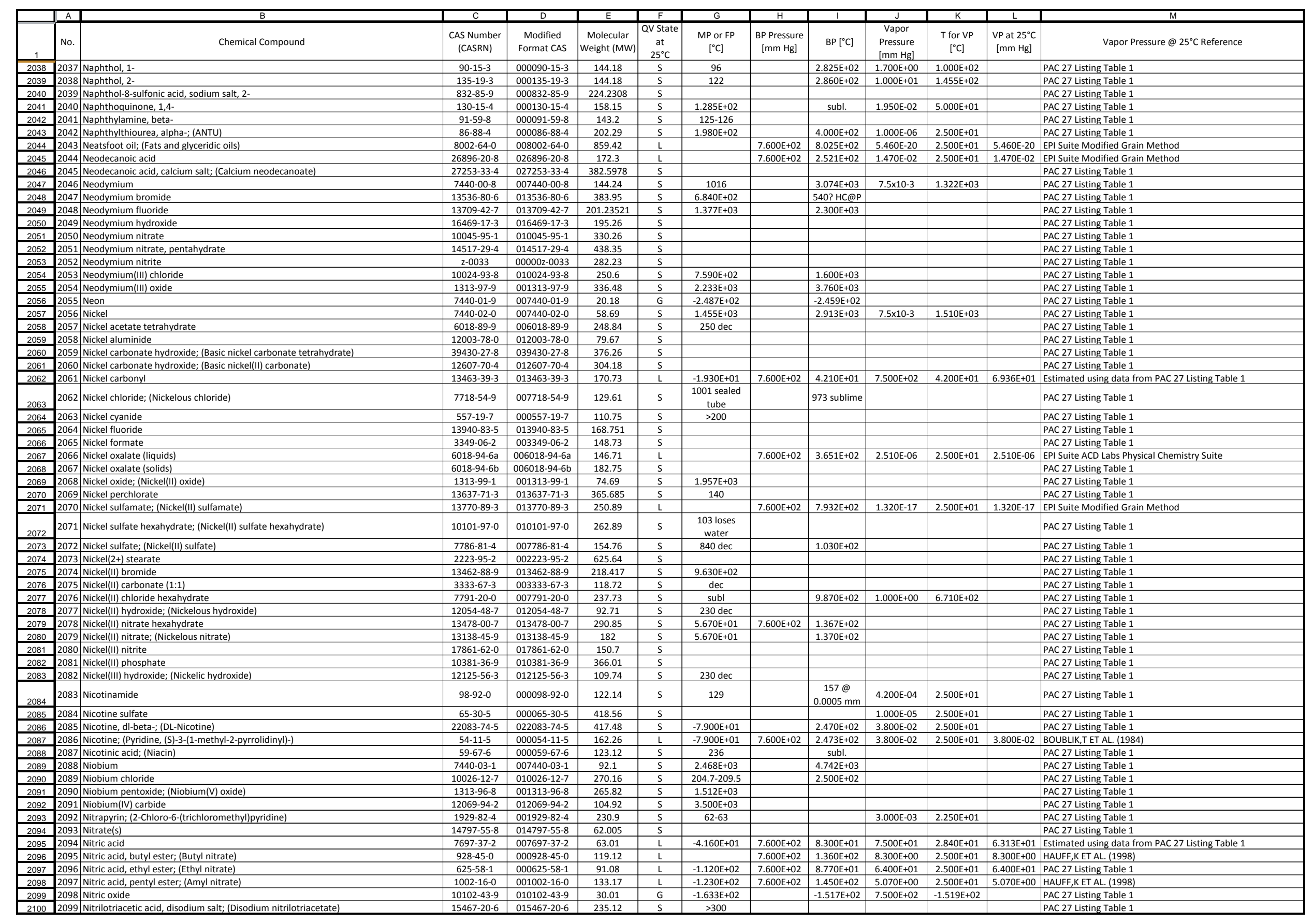




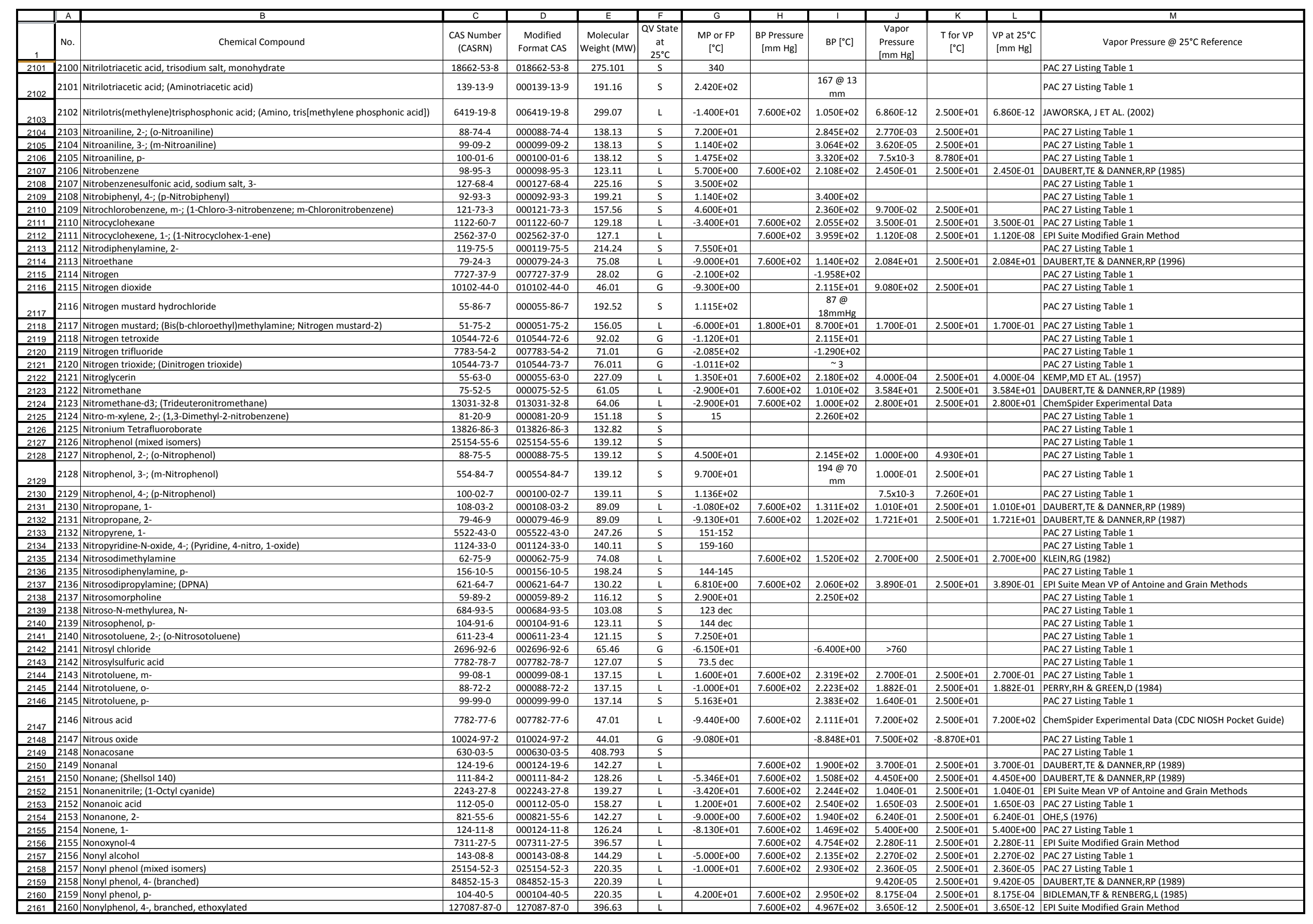




\begin{tabular}{|c|c|c|c|c|c|c|c|c|c|c|c|c|}
\hline & Chemical Compound & $\begin{array}{c}\text { CAS Number } \\
\text { (CASRN) }\end{array}$ & $\begin{array}{l}\text { Modified } \\
\text { Format CAS }\end{array}$ & $\begin{array}{l}\text { Molecular } \\
\text { Weight (MW) }\end{array}$ & \begin{tabular}{|c} 
QV State \\
at \\
$25^{\circ} \mathrm{C}$
\end{tabular} & $\begin{array}{c}\text { MP or FP } \\
{\left[{ }^{\circ C]}\right]}\end{array}$ & $\begin{array}{c}\text { BP Pressure } \\
{\left[\begin{array}{cc}\mathrm{mm} \mathrm{Hg} \\
\mathrm{mg}]\end{array}\right.}\end{array}$ & $\mathrm{BP}\left[{ }^{[\mathrm{C} C}\right]$ & $\begin{array}{l}\text { Vapor } \\
\text { Pressure } \\
{[\mathrm{mm} H \mathrm{Hg}]}\end{array}$ & 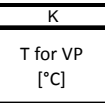 & $\begin{array}{l}\text { VPat } 25^{\circ} \mathrm{C} \\
{[\mathrm{mm} \mathrm{Hg}]}\end{array}$ & Vapor Pressure @ $25^{\circ}$ C Reference \\
\hline 2162 & 2161 Nonylphenoxypolyethoxyethanol & $68412-54-4$ & $068412-54-4$ & & & & & & & & & PAC 27 listing Table 1 \\
\hline 2163 & $\begin{array}{ll}2162 & \text { Nonyytrichlorosilane } \\
21133 & \text { Norbormide } \\
\end{array}$ & $\frac{5283-67-0}{991-24-4}$ & $\begin{array}{ll}005283-67-0 \\
000911-4-4\end{array}$ & $\frac{261.72}{511161}$ & $\frac{L}{S}$ & $\frac{1.923 \mathrm{E}+01}{190.198}$ & $7.600 \mathrm{E}+02$ & $2.337 \mathrm{E}+02$ & $6.4000-02$ & $2.5006+01$ & $6.400 E-02$ & $\begin{array}{l}\text { EPI Suite Mean VP of Antoine and Grain Methods } \\
\text { PAA 27 Litting Table } 1\end{array}$ \\
\hline$\frac{2164}{2165}$ & 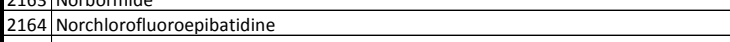 & $2-0034$ & $000002-0034$ & & $\frac{\mathrm{s}}{\mathrm{s}}$ & & & & & & & $\begin{array}{l}\text { PAA 27 Listing Table } 1 \\
\text { PAC } 27 \text { isting Table } 1\end{array}$ \\
\hline 2166 & 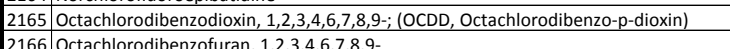 & $3268-87-9$ & $003268-87-9$ & 459.72 & $\frac{\mathrm{s}}{\mathrm{c}}$ & $330-332$ & & & $8.000 E-13$ & $2.5006+01$ & & 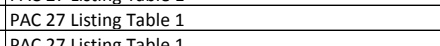 \\
\hline 200 & 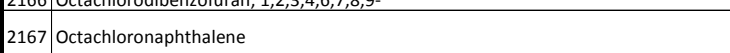 & $2234-13-1$ & $002234-13-1$ & 403.73 & $\mathrm{~s}$ & $1.975 \mathrm{E}+02$ & & $441 @$ & $1.1 \times 10-8$ & $2.5000+01$ & & $\begin{array}{l}\text { PAC } 27 \text { Listing Table } 1 \\
\text { PAC } 27 \text { Listing Table } 1\end{array}$ \\
\hline & \begin{tabular}{|l|l|l|l|l|l|l}
2168 & octacosane \\
\end{tabular} & $630-02-4$ & $000630-02-4$ & 394.76852 & $\mathrm{~s}$ & $57-62$ & & 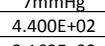 & $<1$ & $2.0006+01$ & & PAC 27 Listing Table 1 \\
\hline$\frac{2170}{2170}$ & 2169 Octadecane, $n$ - & $\begin{array}{l}593-45-3 \\
\end{array}$ & $000593-45-3$ & 254.495 & $\mathrm{~s}$ & 28.2 & & $3.163 \mathrm{E}+02$ & $x_{1}$ & & & $\begin{array}{l}\text { PAC } 27 \text { Listing Table } 1 \\
\text { Pand }\end{array}$ \\
\hline 2171 & 2170 octadecanoic acid, n; (Stearic acid) & $57-11-4$ & $000057-11-4$ & 284.48 & $\mathrm{~s}$ & $6.930 \mathrm{E}+01$ & & $350 \mathrm{dec}$ & $7.5 \times 10-2$ & $1.530 \mathrm{E}+02$ & & PAC 27 Listing Table 1 \\
\hline$\frac{2172}{21732}$ & 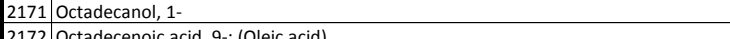 & $112-92-5$ & $000112-92-5$ & 270.56 & $\mathrm{~s}$ & $5.800 \mathrm{E}+01$ & & $\frac{2.0206+02}{2.8050+02}$ & $\begin{array}{l}2.600 E=-06 \\
1.0050 .06\end{array}$ & $\begin{array}{l}2.5006+01 \\
2.505+01\end{array}$ & & $\begin{array}{l}\text { PAC } 27 \text { Listing Table } 1 \\
\text { DERPY }\end{array}$ \\
\hline$\frac{2173}{2127}$ & 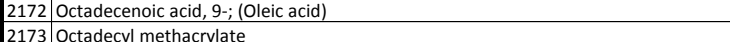 & $\frac{112-80-1}{3230-05-7}$ & $000112-80-1$ & $\frac{282.52}{33.5726}$ & $\frac{L}{L}$ & $\frac{1.630 \mathrm{E}+01}{1.800 \mathrm{E}+01}$ & $\frac{1.000 \mathrm{E}+02}{6.000 \mathrm{E}+00}$ & $\frac{2.860 \mathrm{E}+02}{1.90 \mathrm{E}+02}$ & $\begin{array}{l}1.400 E=06 \\
4820-06\end{array}$ & $\frac{2.5006+01}{2500+501}$ & $\frac{1.400 E-06}{4.820 E-06}$ & 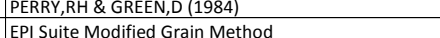 \\
\hline 2175 & 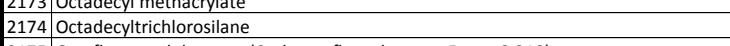 & 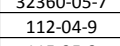 & 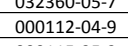 & $\frac{338.5726}{387.99}$ & $\frac{L}{L}$ & & $\begin{array}{ll}6.0 .000 E+00 \\
1.300 E+01 \\
\end{array}$ & $\begin{array}{llll}1.950 E+02 \\
1.600 E+02\end{array}$ & $\begin{array}{ll}4.8006-00 \\
5.000+00\end{array}$ & $2.0000 E+01$ & $\begin{array}{lll}4.8206-06 \\
5.254 E+00\end{array}$ & 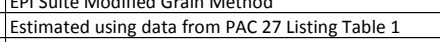 \\
\hline$\frac{2176}{2176}$ & 2175 Octaffluorocycyclobutane; (Cycloctaflluorobutane; Freon C-318) & $\begin{array}{lll}115-25-3 \\
\end{array}$ & $000015-25-3$ & & & $-4.140 E+01$ & & & & & & \\
\hline 2177 & 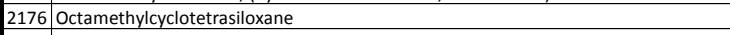 & 556-67-2 & $000556-67-2$ & 296.68 & $\mathrm{~L}$ & $1.7506+01$ & $7.600 E+02$ & $1.7506+02$ & $1.050 E+00$ & $2.500 E+01$ & & FLANINGAM, OL (1986) \\
\hline 2178 & 2177 |ctamethyldiphosphoramide; (Octam methylpyrophosphoramide) & $152-16-9$ & $000152-16-9$ & 286.3 & $\mathrm{~L}$ & $2.000 E+01$ & $2.000 E+00$ & $1.540 \mathrm{E}+02$ & $1.0000-03$ & $2.500 E+01$ & $1.000 \mathrm{E}-03$ & PAC 27 Listing Table 1 \\
\hline$\frac{2179}{2180}$ & 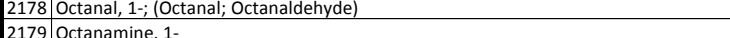 & $\frac{124-13-0}{111-86-4}$ & $000124-13-0$ & $\frac{128.24}{10228}$ & $\frac{L}{L}$ & $-2.300 \mathrm{C}+01$ & $\begin{array}{l}7.6000 \mathrm{E}+02 \\
76020502\end{array}$ & $\frac{1.634 \mathrm{E}+02}{1850 \mathrm{E}+02}$ & & $2.500 E+01$ & $\frac{1.180 \mathrm{E}+00}{0.600501}$ & 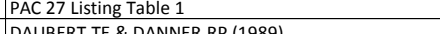 \\
\hline$\frac{2180}{2181}$ & 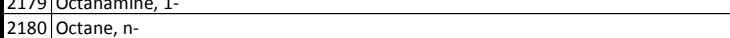 & $\frac{1111-8-6-4}{111-65-9}$ & $000001111-65-9$ & $\frac{129.88}{114.26}$ & $\frac{L}{L}$ & & 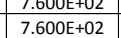 & $\frac{1.850 \mathrm{E}+02}{1.258 \mathrm{E}+02}$ & $\begin{array}{l}9.690 \mathrm{E}-01 \\
1.405 \mathrm{E}+01\end{array}$ & $2.500 \mathrm{EEOT+1}$ & $\begin{array}{l}9.690 E-01 \\
1.405 E+01\end{array}$ & $\begin{array}{l}\text { DAABRR, III \& DANN RR,RP (1989) } \\
\text { |YAWS, (1994) }\end{array}$ \\
\hline & 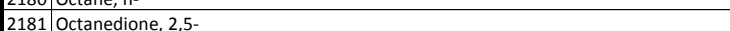 & 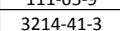 & $\frac{0.0031 .63-3}{0032141-3}$ & $\frac{14.60}{142}$ & $\frac{L}{L}$ & $6.000 E+00$ & $\frac{1.0006 \mathrm{~F}}{7.602}$ & 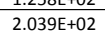 & 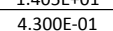 & $\frac{2.300601}{2.50 \mathrm{~F}+01}$ & 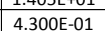 & F \\
\hline & & & $\frac{000124-12-9}{0}$ & 125.24 & & $\frac{-4.5600 \mathrm{E}+01}{1}$ & & $2.052 \mathrm{E}+02$ & $3.900 E-01$ & & $3.9000-01$ & $\begin{array}{l}\text { RIDIICKN,A ET AL. (1986) } \\
\end{array}$ \\
\hline 2184 & 2183 Ooctanethiol, 1 - & $111-88-6$ & $000111-88-6$ & 146.21 & $\mathrm{~L}$ & 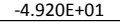 & $7.6000 \mathrm{E}+022$ & $1.981 \mathrm{E}+02$ & $4.245 \mathrm{E}-01$ & $2.500 \mathrm{E}+01$ & $4.245 E-01$ & PAC 27 Listing Table 1 \\
\hline$\frac{2185}{2186}$ & 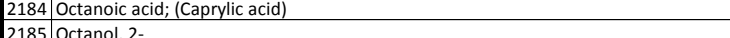 & $\frac{124-07-2}{123-6-6}$ & $000124-07-2$ & $\frac{144.24}{130.26}$ & L & $\frac{1.700 E+01}{-3.165 E+01}$ & $\begin{array}{l}7.600 E+02 \\
7.600 E+02\end{array}$ & $\begin{array}{l}2.400 E+02 \\
1723+502\end{array}$ & $\begin{array}{l}3.710 E-03 \\
2.420=0.01\end{array}$ & $2.500 E+01$ & 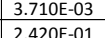 & 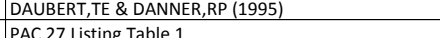 \\
\hline & 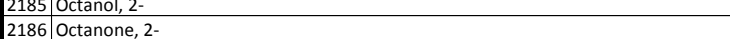 & $\frac{123-96-6}{11113-7}$ & 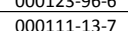 & $\frac{130.6}{12824}$ & & 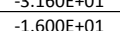 & T. & & 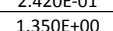 & & $2.420 E-01$ & $\begin{array}{l}\text { PAC } 2 \text { Listing Table } 1 \\
\text { RIDDCKK A ET AL (1986) }\end{array}$ \\
\hline$\frac{2568}{2128}$ & T) & $\frac{1115-57}{546-5-5}$ & $\frac{0.00115-15}{0.65-5}$ & $\frac{126.4}{793.4}$ & $\frac{\mathrm{L}}{\mathrm{s}}$ & $\frac{1.0000 .51}{2005}$ & 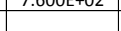 & 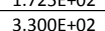 & $1.530 \mathrm{Vt}+00$ & & & PAC C2 \\
\hline 2189 & 2188 Ooctene, 1 - & 1111-66-0 & $\frac{000111-66-0}{0.0}$ & 112.22 & $\mathrm{~L}$ & $-1.0177 \mathrm{E}+02$ & $7.600 E+02$ & 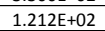 & $1.740 E+01$ & $2.500 E+01$ & $1.740 E+01$ & PAC 27 Listing Table 1 \\
\hline 2190 & 2189 Octyl alcohol; (n-Octanol) & $111-87-5$ & $000111-87-5$ & 130.28 & $L$ & $-1.670 E+01$ & $7.600 \mathrm{E}+02$ & $1.945 E+02$ & 7.940E-02 & $2.500 E+01$ & $7.9400-022$ & DAUBERT,TE \& DANNER,RP (1989) \\
\hline 2191 & $\begin{array}{ll}2190 \mid \text { |ctyl mercaptan, } t \text { - } \\
20101\end{array}$ & $\frac{141-59-3}{83220-95-9}$ & $000141-59-3$ & $\frac{146.32}{2103232}$ & $\frac{1}{s}$ & & 7.600E+02 & 1.540E+02 & $5.200 E+00$ & 2.500E+01 & & 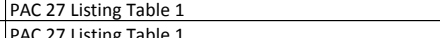 \\
\hline$\frac{2192}{2193}$ & 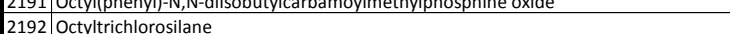 & $\begin{array}{l}832243-956-9 \\
5283-6-9\end{array}$ & 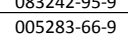 & $\frac{210.0182}{2466.69}$ & & $8.120= \pm 00$ & $76000+022>20$ & $2320=+02$ & 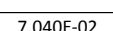 & $2500 \mathrm{~F}+01$ & $7040=-022$ & $\begin{array}{l}\text { PAA } 27 \text { Iisting Table } 1 \\
\text { EPI Suite Mean VP of Antoine and Grain Methods }\end{array}$ \\
\hline$\frac{2504}{2104}$ & 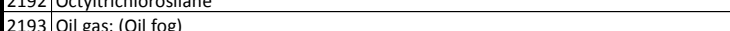 & $\frac{328306-9}{2-035}$ & $000000=0035$ & 119 & $\frac{L}{G}$ & & & & & & & 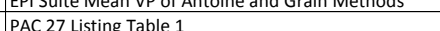 \\
\hline $\begin{array}{ll}2195 \\
2195\end{array}$ & 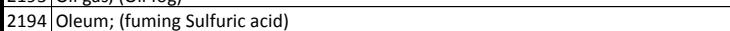 & $8014-95-7$ & $008014-95-7$ & 178.14 & $\mathrm{~L}$ & $1.031 \mathrm{E}+01$ & $7.600 E+02$ & $2.900 E+02$ & $5.930 E-05$ & $2.500 E+01$ & $5.9300-05$ & $\begin{array}{l}\text { EpI Sutite Experimental Data } \\
\end{array}$ \\
\hline 2196 & 2195 | organorhodium complex & z-0036 & $000002-0036$ & & $\mathrm{~s}$ & & & & & & & PAC 27 Listing Table 1 \\
\hline 2197 & $\begin{array}{l}2196 \mid \text { Orthoformic acid, trimethyl sster; (Trimethyl orthoformate) } \\
2197 \text { lossium }\end{array}$ & $\frac{149-73-5}{740-54-2}$ & $000149-73-5$ & $\frac{106.14}{1092}$ & $\frac{L}{S}$ & $\frac{1.5006+01}{3.033}$ & $8.429 \mathrm{E}+01$ & $\frac{1.006 \mathrm{E}+02}{5021502}$ & 3.660E +01 & $2.500 E+01$ & 3.660E+01 & $\begin{array}{l}\text { EPI Suite Mean VP of Antoine and Grain Methods } \\
\text { A C }\end{array}$ \\
\hline$\frac{2198}{2199}$ & $\begin{array}{l}\frac{2197}{2198} \text { | Ossmium } \\
2 \text { osmium tetroxide }\end{array}$ & $\frac{7440-044-2}{20816-12-0}$ & $\frac{007440-04-2}{020816-12-0}$ & $\frac{190.2}{254.23}$ & $\frac{s}{5}$ & $\frac{3033}{4.0605+01}$ & & 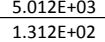 & $1.100 E+01$ & $2.700 E+01$ & & $\begin{array}{l}\text { PAC 27 Listinin Table } 1 \\
\text { PAC 27 i isting Table } 1\end{array}$ \\
\hline 10 & 21999 Otto Fuel (mainly Propylene glycol dinitrate 6423-43-4) & $106602-80-6$ & $106602-80-6$ & 170 & $\mathrm{~L}$ & $-2.770 \mathrm{E}+01$ & & & $8.770 \mathrm{E}-02$ & $2.5006+01$ & $8.770 E-02$ & Data; CDC Toxicological Profile for OTTO Fuel II and Its \\
\hline$\frac{22200}{2201}$ & $2200 \mid$ Ouabain & $630-60-4$ & $000630-60-4$ & 584.73 & $\mathrm{~s}$ & $1.9006+02$ & & & & & & $\begin{array}{l}\text { PComponents, J UN1995 } \\
\text { PAC 27 Listing Table } 1 \\
\end{array}$ \\
\hline & 2201 |oxalic aci & & & & & $1.895 \mathrm{E}+02$ & & & 5.400E-01 & $1.050 \mathrm{E}+02$ & & $\begin{array}{l}\text { PAC } 27 \text { Listing Table } 1 \\
\end{array}$ \\
\hline$\frac{2203}{203}$ & , anhhydrous; (Ethanedioic acid) & 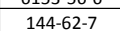 & $\frac{000144-62-7}{0.030}$ & $\frac{2.004}{990.04}$ & $\mathrm{~s}$ & 189.5 dec & & 157 subl & $\begin{array}{l}5.4006-01 \\
5.01\end{array}$ & 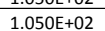 & & PAC 27 Listing Table 1 \\
\hline 2204 & 2203 | Oxalyy chlorine & $79-37-8$ & $000079-37-8$ & 126.93 & $\mathrm{~L}$ & $-1.600 E+01$ & $7.600 E+02$ & $6.350 E+01$ & $1.840 E+02$ & $2.5000++01$ & $1.840 E+02$ & EPI Suite Mean VP of Antoine and Grain Methods \\
\hline 2205 & 2204 |xamide & $471-46-5$ & $000471-46-5$ & 88.08 & $\mathrm{~s}$ & $350 \mathrm{dec}$ & & $419 \mathrm{dec}$ & & & & PAC 27 Listing Table 1 \\
\hline 2206 & 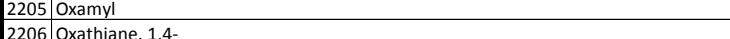 & $23135-22-00$ & 023135-22-0 & 219.29 & $\frac{s}{L}$ & $\frac{100-102}{-17005+01}$ & $7.6006+02$ & $\begin{array}{l}3.100 \mathrm{E}+02 \\
1.487+02\end{array}$ & $\begin{array}{l}2.300 E-04 \\
4.6010+00\end{array}$ & $2.500+101$ & & 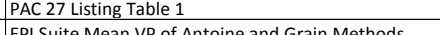 \\
\hline 2208 & 2207 Oxirane, ethenyl-; (3,4-Epoxy-1-butene) & $930-22-3$ & $000930-22-3$ & $\frac{70.10}{70.1}$ & $\frac{L}{L}$ & -1.350E+02 & $7.6006+02$ & 1.40006+01 & 1.5070E+02 & 2.500E+to1 & 1.540E+ +02 & EPI Suite Mean VP of Ante \\
\hline 2209 & 2208 Oxone, monopersulfate compound & $37222-66-5$ & $037222-66-5$ & & $\mathrm{~s}$ & & & & & & & PAC 27 Listing Table 1 \\
\hline 2210 & 22090 Oxybis(N,N-dimethylethanamine), 2,2,'; (Bis(2-dimethylaminoethy)) ether; DMAEE) & 3033-62-3 & 003033-62-3 & 160.2588 & $\mathrm{~L}$ & $-5.780 \mathrm{E}+00$ & $1.500 E+01$ & $8.000 E+01$ & $7.480 \mathrm{E}-01$ & 2.500E+01 & $7.480 \mathrm{E}-01$ & EPI Suite Mean VP of Antoine and Grain Methods \\
\hline & 2210 Oxydiacetic acid; (Oxodiacetic acid) & $110-99-6$ & $000110-99-6$ & 134.09 & $s$ & $1.480 E+02$ & & $\mathrm{dec}$ & & & & PAC 27 listing Table 1 \\
\hline 2212 & $2211 \mid$ |xxydiethlylenedicarbonic acid, diallyl lester & $142-22-3$ & 0000142-22-3 & 274.3 & & $-4.0006+00$ & $7.6006+02$ & $1.620 \mathrm{E}+02$ & $6.4606-05$ & $2.500 E+01$ & . $4600-05$ & EPI Suite Modified Grain Method \\
\hline 2213 & 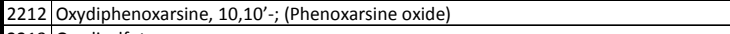 & $58-36-6$ & $000058-36-6$ & 502.24 & $\mathrm{~s}$ & 184-185 & & $380 \mathrm{dec}$ & $1.000 E-05$ & $2.500 E+01$ & & PAC 27 Listing Table 1 . \\
\hline$\frac{2214}{2215}$ & $\begin{array}{l}22213 \text { | } 2 \text { |xdyisulfoton } \\
2214 \text { Oxxyen difluoride; (Fluorine monoxide) }\end{array}$ & 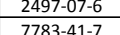 & 002999-07-6 & 290.42 & $\frac{L}{G}$ & $2.238 E+02$ & $7.600 \mathrm{E}+022$ & 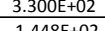 & 3.500-E03 & $2.500+5+01$ & 3.500E-03 & PAC 27 74 \\
\hline $\begin{array}{llll}22216 \\
22\end{array}$ & 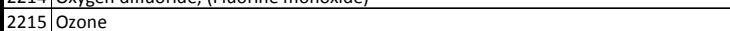 & $10028-15-6$ & $010028-15-6$ & 48 & $\frac{G}{G}$ & $-1.9306+02$ & & $-1.1144+02$ & $7.500 \mathrm{E}+02$ & 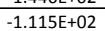 & & PAC 27 \\
\hline 22217 & |l21616 palladium & $7440-05-3$ & & 106.42 & $\mathrm{~s}$ & $1555 E+03$ & & $2963 E+03$ & $7.5 \times 10-3$ & $1.448 E+03$ & & \\
\hline 2218 & 2217 Palladium chloride & $7647-10-1$ & $007647-10-1$ & 1777.33 & $s$ & $678-680$ & & & & & & \\
\hline 2219 & 22218|Palladium hydroxide & $12135-22-7$ & $0121235-22-7$ & 1400.43468 & $\mathrm{s}$ & & & & & & & PAC 27Li $>$ \\
\hline$\frac{2220}{2221}$ & $\begin{array}{l}2229 \text { palalalum nitrate } \\
2220 \text { Palladium oxide }\end{array}$ & $\frac{101202-053-3}{1314-08-5}$ & $\begin{array}{ll}010102-05-3 \\
001314-08-5 \\
\end{array}$ & $\frac{230.42}{122.42}$ & $\frac{5}{5}$ & $\begin{array}{l}\text { dec. } \\
750 \text { dec. }\end{array}$ & & & & & & $\begin{array}{l}\text { PAC/ I Ltsing Iable } 1 \\
\text { PAC 27 Listing Table } 1\end{array}$ \\
\hline 2222 & $\begin{array}{l}\text { treated, reaction products with petroleum white } \\
\text { hanolamine }\end{array}$ & $71808-29-2$ & $071808-29-2$ & & $\mathrm{~s}$ & $1.150 \mathrm{E}+02$ & & $3.600 E+02$ & & & & PAC 27 Listing Table 1 \\
\hline & & $8002-74-2$ & $008002-74-2$ & & $\mathrm{~s}$ & $47-65$ & & & & & & PAC 27 Listing Table 1 \\
\hline
\end{tabular}




\begin{tabular}{|c|c|c|c|c|c|c|c|c|c|c|c|c|c|}
\hline & No. & Chemical Compound & $\begin{array}{l}\text { cass unber } \\
\text { (CASPRN) }\end{array}$ & $\begin{array}{l}\text { Modifid } \\
\text { Format CAS }\end{array}$ & $\begin{array}{l}\text { Molecular } \\
\text { Weight (MW }\end{array}$ & $\begin{array}{l}\text { ats State } \\
\text { ats } \\
25^{\circ}\end{array}$ & 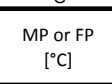 & 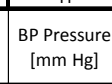 & ${ }_{B P P C l}$ & $\begin{array}{l}\text { vapor } \\
\text { Pressure } \\
\text { [mm HIF }\end{array}$ & $\begin{array}{l}\text { Tfor VP } \\
{\left[\begin{array}{c}\text { Tc] }\end{array}\right.}\end{array}$ & 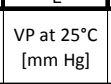 & 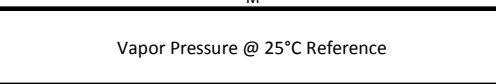 \\
\hline & & 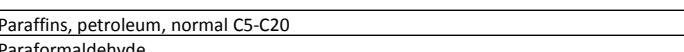 & 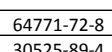 & 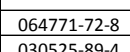 & 189 & & & & $\frac{225-243}{60200}$ & 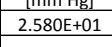 & $3.800 €+01$ & & Zlisting Table 1 1 \\
\hline$\frac{2225}{2226}$ & & $\begin{array}{l}\text { Parartormaldehyde } \\
\text { Paraldehyde }\end{array}$ & 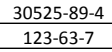 & $\begin{array}{l}030525.599-4 \\
000023-63-7\end{array}$ & 132.16 & $\frac{s}{L}$ & 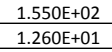 & $7.600 \notin+02$ & $\begin{array}{l}\text { decomp } \\
1.243 E+02 \\
\end{array}$ & 1.1.033+01 & $2.5005+01$ & $1.1035+01$ | $12>0$ & 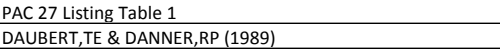 \\
\hline 2227 & 2226 & Paraguat & 4685-147.7 & 004685-14-7 & 186.25 & $\mathrm{~s}$ & & & 175-180 dec & $\sim_{0}$ & $2.000+501$ & & PAC 27 Listing Table 1 \\
\hline & & 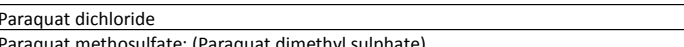 & $\frac{1910-42-5}{2024-5.2}$ & $\frac{001910-42 \cdot 5}{0.027245-2.25}$ & 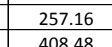 & $\frac{s}{s}$ & 300 dec & & & & & & $\begin{array}{l}\text { PAC 27 Listing Table } 1 \\
\text { PAC }\end{array}$ \\
\hline 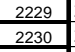 & & 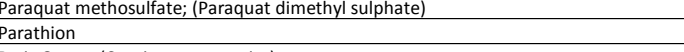 & $\frac{270450-2}{56-38-2}$ & & 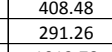 & $\frac{S}{L}$ & 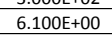 & $7.600 E+02$ & $3.750 E+02$ & 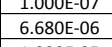 & & $6.680 \mathrm{E}-\mathrm{C}$ & 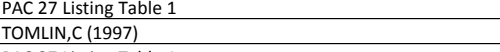 \\
\hline & & 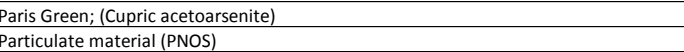 & 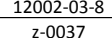 & 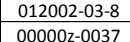 & 1013.78 & & & & & & & & $\begin{array}{l}27 \text { Listing Table } 1 \\
2.2 \text { lisinin Table } 1 \\
\end{array}$ \\
\hline$\frac{2233}{2233}$ & 釷2 & 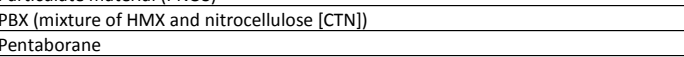 & $\frac{2-0038}{196242-2.7}$ & 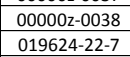 & $\frac{450}{63.14}$ & $\frac{\mathrm{s}}{\mathrm{L}}$ & $-4.660 \mathrm{E}+01$. & $7.600 E+02$ & $6.000 \mathrm{t}+011$ & 1.:000 & $2.500=0+1.10$ & 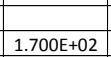 & 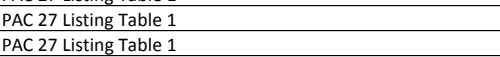 \\
\hline 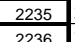 & & 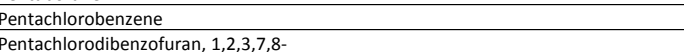 & 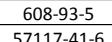 & 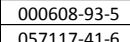 & 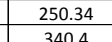 & $\frac{s}{s}$ & $8.600 E+011$ & & $2.7706+02$ & & & & 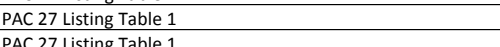 \\
\hline & & 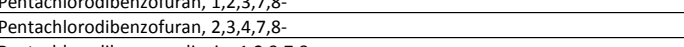 & 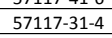 & & $\begin{array}{l}\frac{3.304}{3004} \\
30.4\end{array}$ & $\mathrm{~s}$ & $196-196.5$ & & & $2.63 \times 10.9$ & $2.500 £+01$ & & 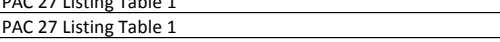 \\
\hline 年238 239 & 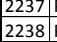 & 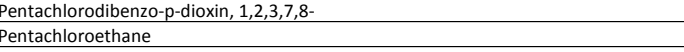 & 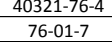 & 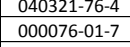 & $\begin{array}{l}\frac{3364}{2029} \\
2029\end{array}$ & $\frac{s}{L}$ & $\frac{2420241}{-2.878+01}$ & $7.600 E+02$ & $1.620 \mathrm{E}+02$ & $3.500+ \pm 00$ & $2.500 E+01$ & $3.500+500$ & 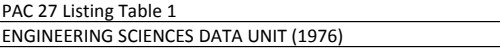 \\
\hline 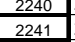 & & $\begin{array}{l}\text { Pentachloronitrobenzer } \\
\text { Pentachlorohenol }\end{array}$ & 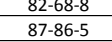 & 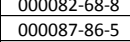 & 295.34 & $\frac{5}{s}$ & 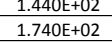 & & 310 dece & 1.10×10-6 & & & $\begin{array}{l}\text { Listing Table } 1 \\
\text { Listing Table } 1\end{array}$ \\
\hline$\frac{2242}{2223}$ & 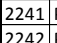 & 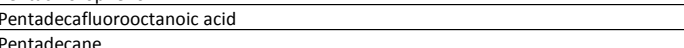 & $\frac{335.67-1}{620.62 .9}$ & $\frac{000335-67-1}{0.00256-92.9}$ & $\frac{44.09}{212097}$ & 5 & $\frac{55-56}{90005+00}$ & 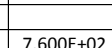 & & $1.5000-013$ & 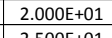 & 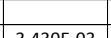 & 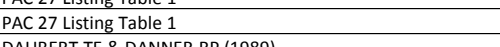 \\
\hline 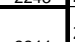 & 22439 & $\begin{array}{l}\text { Pentaceacane } \\
\text { Pentecanoic acid }\end{array}$ & $1002 \cdot 84-2$ & 0001002-84-2 & 242.45 & $\mathrm{~s}$ & $5.305=01$ & & 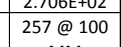 & & & & $\begin{array}{l}\text { DAABERT,TE \& ANNERR,PP (1989) } \\
\text { PAC } 27 \text { Listing Table } 1\end{array}$ \\
\hline 年2445 & $2244 \mid$ & $\begin{array}{l}\text { Pentadecclamine } \\
\text { Penter }\end{array}$ & $\frac{2570-26.5}{210.575}$ & 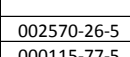 & $\frac{227.49}{12.67}$ & $\mathrm{~s}$ & $\begin{array}{llll}3.5605+01 \\
.2502010\end{array}$ & & $\frac{\mathrm{MM}}{3.076 \mathrm{E}+2 \mathrm{2}}$ & $3.000=04$ & $2.500+501$ & & 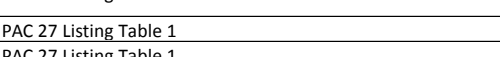 \\
\hline & 2246 & 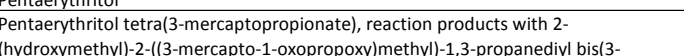 & 95823:35-1. & 0958823-35.1 & 1001.3772 & $s$ & & & & & & & PAC 27 Listing Table 1 \\
\hline 2247 & & 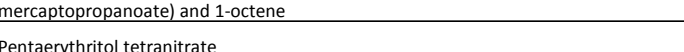 & 70115 & (00070-115 & 21617 & $c$ & 138-100 & & 20032015 & & & & PAC 27 Listing Table 1 \\
\hline 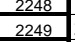 & 2248 & 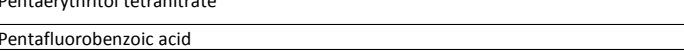 & $602 \cdot 94.8$ & 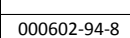 & 212.08 & $\mathrm{~s}$ & 107 & & $2.2000+02$ & & & & 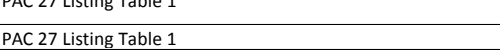 \\
\hline 22250 & 22494] & 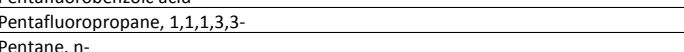 & $\begin{array}{l}460-73-1 \\
\end{array}$ & $0.00460-73-1.10$ & 134.06 & $\sigma^{6}$ & 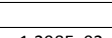 & & $1.530 \mathrm{E}+01$. & & & & 27 listing Table 1 - \\
\hline$\frac{251}{2252}$ & 列 & $\begin{array}{l}\text { Pentane, } n-\text { - } \\
\text { Pentanediol, }, 5-\end{array}$ & 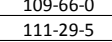 & 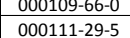 & 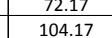 & $\frac{L}{L}$ & 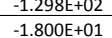 & 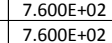 & $\begin{array}{l}3.6 .60+5+1 \\
2.3906+02\end{array}$ & 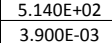 & 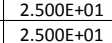 & 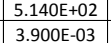 & 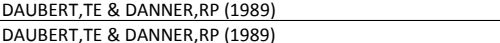 \\
\hline 先2535 & $\begin{array}{lllll}22252 & 2253\end{array}$ & 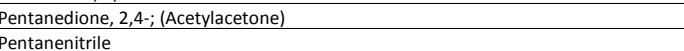 & 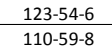 & 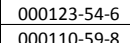 & $\frac{100.13}{8815}$ & $\frac{L}{L}$ & 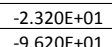 & $\begin{array}{l}7.600+522 \\
7.000+02\end{array}$ & $\begin{array}{l}1.3906+02 \\
1.143+02\end{array}$ & $\begin{array}{l}2.960+500 \\
73000+000\end{array}$ & $\begin{array}{l}2.500+501 \\
25000+01\end{array}$ & 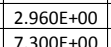 & 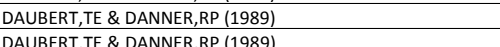 \\
\hline 2255 & 2254 | & 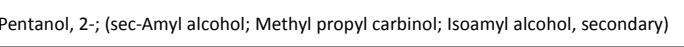 & 60032-29-7 & 006032-29-7 & 88.17 & $\mathrm{~L}$ & -5.000₹+01 & 7.600Е+02 & 1.1.93E+02 & $6.100++00$ & $2.500 \mathrm{E}+01$ & $6.100+500$ & raws,CL (1994A) \\
\hline$\frac{22256}{2257}$ & 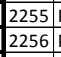 & 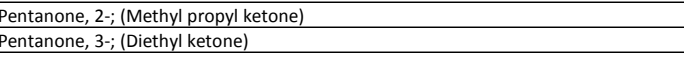 & $\begin{array}{l}109-87-9 \\
96-2-0.0\end{array}$ & 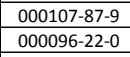 & $\frac{86.15}{86.15}$ & $\frac{L}{L}$ & $-4 \cdot 2000+01$ & 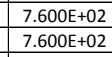 & 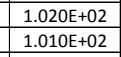 & 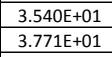 & $\begin{array}{l}2.5000+51 \\
2.5005+01\end{array}$ & 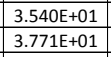 & 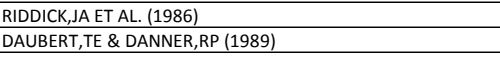 \\
\hline 2258 & 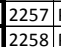 & $\begin{array}{l}\text { Pentatricantane } \\
\text { enenten } 1 \text {. }\end{array}$ & $\begin{array}{l}\frac{630.07 .9}{10.67-1} \\
10.1\end{array}$ & 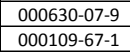 & $\frac{492.9538}{77.13}$ & $s_{\mathrm{L}}^{\mathrm{s}}$ & & $7.600 E+02$ & & & $2.500 E+01$ & & $\begin{array}{l}\text { PAC } 27 \text { Listing Table } 1 \\
\text { PCA } 2 \text { Listing Tatale } 1\end{array}$ \\
\hline 2260 & 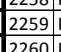 & 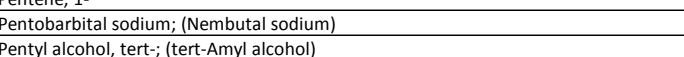 & 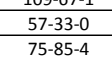 & 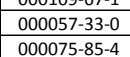 & $\begin{array}{l}299.3 \\
8.37 \\
\end{array}$ & $\frac{t}{L}$ & 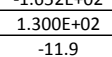 & 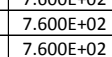 & 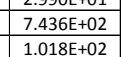 & 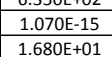 & 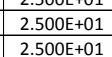 & 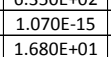 & 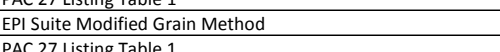 \\
\hline 22262 & 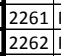 & 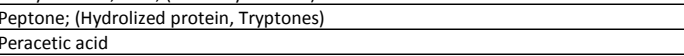 & 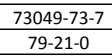 & 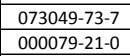 & 76.06 & $\frac{\mathrm{s}}{\mathrm{L}}$ & $1.000=01$ & $7.600+02$ & 1.05060202 & 1.4466+01 & $2.5005+01$ & $1.466+011$ & 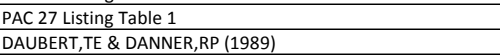 \\
\hline$\frac{22245}{2265}$ & 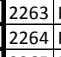 & 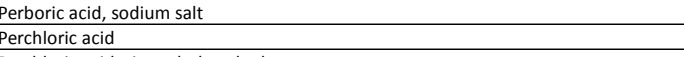 & 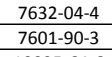 & 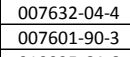 & 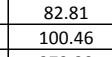 & $\frac{s}{L}$ & 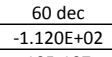 & $7.600 \notin+02$ & $9.0000+01$ & 5.550:-12 & $2.5005+01$ & $5.550-12$ & $\begin{array}{l}\text { PAC C 7 istitis g able } 1 \\
\text { EPP I Sute Modified Grain Method }\end{array}$ \\
\hline$\frac{2266 \mathrm{~T}}{2267}$ & 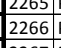 & 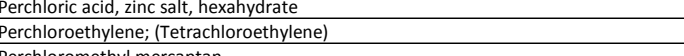 & 12005-184. & 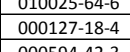 & 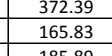 & $\frac{3}{L}$ & 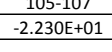 & $7.600+502$ & 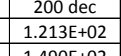 & $1.850 E+01$ & 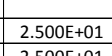 & $1.8500+01$ & 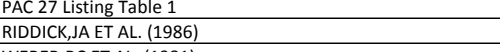 \\
\hline$\frac{22069}{2029}$ & 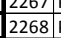 & 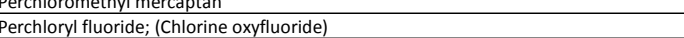 & 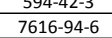 & 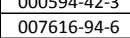 & 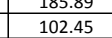 & $\frac{1}{6}$ & -1.46060202 & & 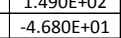 & 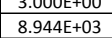 & 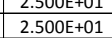 & & 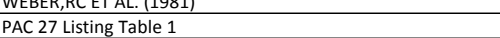 \\
\hline & $22699 \mid$ & Percoll & 65455-52.9. & 065455-52-9 20.9 & & $\mathrm{t}$ & & & & 1.0000:05 & $2.5005+01$ & 1.000E:05 & 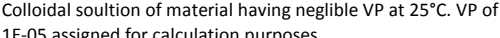 \\
\hline 2271 & 2270 & 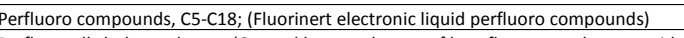 & 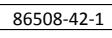 & 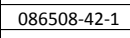 & 332.42 & 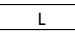 & & $7.600 E+022$ & $1.020 \mathrm{E}+02$ & & $2.0005+01$ & 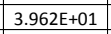 & |Stimated using data from $\mathrm{PAC} 27$ listing Table \\
\hline 2272 | & $\left.2271\right|^{4}$ & 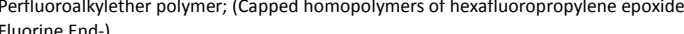 & 60164-51-4 & 060164-51-4 & & $\mathrm{s}$ & & & & & & & PAC 27 Listing Table 1 \\
\hline 2273 & 2272 & 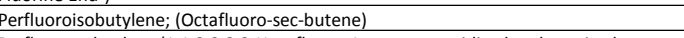 & 382.2.1.8 & c00388-21-8 & 200.03 & & $-1.300=502$ & & 7.000E+00 & $2.56 \times 10+3$ & $2.500 \mathrm{E}+01$ & & \\
\hline 2274 & $\mid 2273$ & 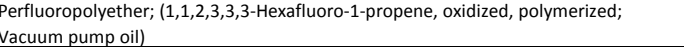 & 69991-67.9 & $0699991-67.9$ & & & & 5.000€ +00 & $2.700 E+02$ & $1.000+500$ & $2.000 €+01$ & $1.060+500$ & Estimated using data from PAC 27 Listing Table 1 \\
\hline$\frac{2275}{2276}$ & 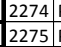 & Periodicacid & $\frac{10450-6.9}{7790-28.5}$ & 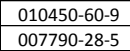 & 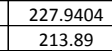 & $\frac{s}{s}$ & 300 & & dec & & & & $\begin{array}{l}\text { Solid material per MSDS } \\
\text { PAC } 2 \text { L Lisinin Table } 1\end{array}$ \\
\hline & & 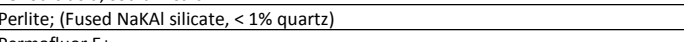 & 93763:-70.3 & & & & & & $x_{0}$ & & & & 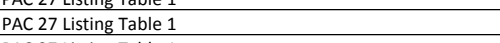 \\
\hline$\frac{278}{2279}$ & & 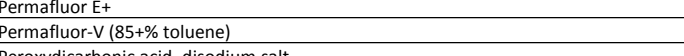 & 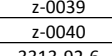 & 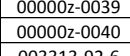 & 85 & & 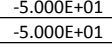 & $\begin{array}{ll}7.600+t \\
7.600 E+t\end{array}$ & 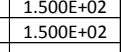 & 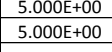 & 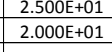 & 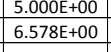 & 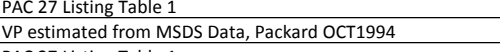 \\
\hline
\end{tabular}




\begin{tabular}{|c|c|c|c|c|c|c|c|c|c|c|c|c|}
\hline & Chemical Compound & 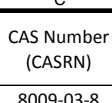 & 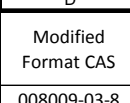 & 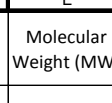 & $\begin{array}{l}\text { avstat } \\
\text { ats } \\
25^{\circ} \mathrm{c}\end{array}$ & 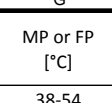 & 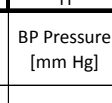 & ${ }_{B P}[\mathrm{CC}]$ & 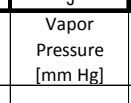 & $\begin{array}{l}\text { Tforvp } \\
\text { racl }\end{array}$ & 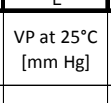 & Vapor Pressure @ $25^{\circ}$ C Reference \\
\hline 2281 & 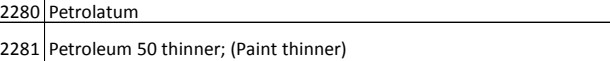 & 2:0041 & 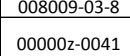 & 130 & t & 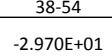 & 7.600E+02 & & & & & 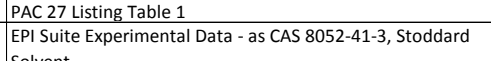 \\
\hline & 2828 Petroleum asphat & 8005-2-2-4b & $008052-42-4 b$ & & s & & & $>700^{\circ} \mathrm{F}$ & $0.0181 / 1 / 5 \mathrm{sin}$ & $2.1005+02$ & & $\begin{array}{l}\text { PAC } 27 \text { Listing Table } 1 \\
\end{array}$ \\
\hline 22864 & 2283 Petrolueum coke, calcined & 647430.05-1 & $066773-05 \cdot 1$ & & s & & & & & & & 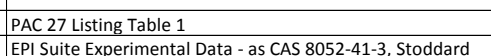 \\
\hline 2285 & 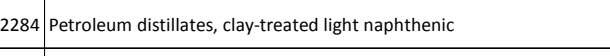 & 64722-45.6 & $066772-45.6$ & & เ & $-2.970+01$ & $7.600 €+02$ & 1.741E+o2 & $1.430 \mathrm{E}+00$ & $2.500+01$ & $1.430+500$ & unte experimental Data as CAS 8052-41. \\
\hline 2286 & 2285 | Petroleum distillates, Iow boling & 68477-31-6 & $068877-31-6$ & 99 & L & $-1.000 £+01$ & 7.600E+02 & $2.5906+02$ & $4.670 E+00$ & $2.500+501$ & $4.670+500$ & 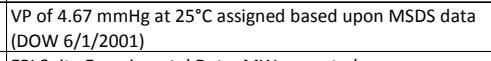 \\
\hline $\begin{array}{lll}22287 \\
2288 \\
\end{array}$ & 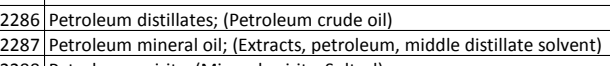 & 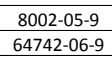 & 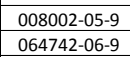 & 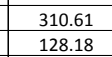 & L & $5.010 \mathrm{E}+00$ & 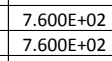 & 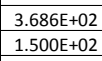 & $\begin{array}{ll}1.2800-06 \\
.0000=02 \\
\end{array}$ & $\begin{array}{l}2.5000+501 \\
2.5000+01\end{array}$ & 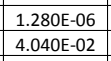 & 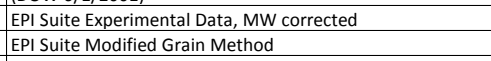 \\
\hline 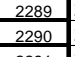 & 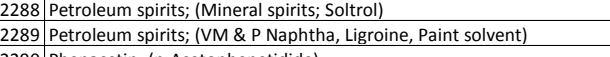 & (60372-32-4 & 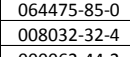 & $\frac{97.26}{111.73}$ & $L$ & -7.300 - $0=12$ & $7.600 \mathrm{E}+02$ & 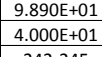 & 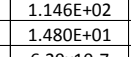 & $\begin{array}{l}3.7 .5000+01 \\
2.500+01\end{array}$ & 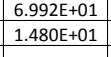 & 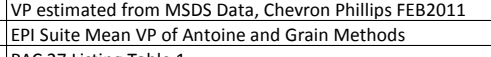 \\
\hline 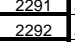 & 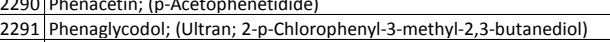 & & 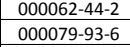 & & & & & & & & & \\
\hline 告2033 & 292] henenanthrene & 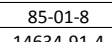 & .000003.01.1. & 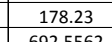 & & 9.924t+01 & & 3.400E+ 022 & $7.5 \times 10-3$ & 5.300E+01 & & 部 Table: \\
\hline$\frac{2205}{2205}$ & 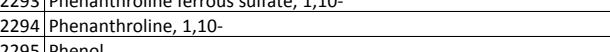 & 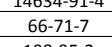 & 0.00066-71. & 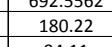 & & 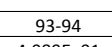 & & 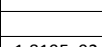 & 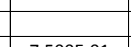 & 2 & & \\
\hline$\frac{2226}{2297}$ & 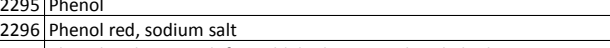 & $\frac{108-95}{34487-6}$ & 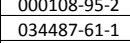 & 34.11 376.36 & & $\begin{array}{llll} & \end{array}$ & & $1.8196+02$ & 7.500E:01 & 3.410E+01 & & 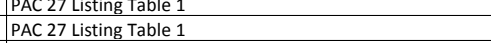 \\
\hline 22298 & 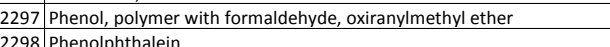 & 28064-14-4. & $\begin{array}{l}028064-14 . \\
0.0070909\end{array}$ & 31834 & & & $7.600 E+02$ & $2.450+502$ & $2.000 E+01$ & $2.000+501$ & $2.3025+t+2$ & ted using data from PAC 27 listing Table 1 \\
\hline 2300 & 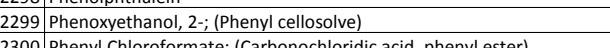 & $\frac{122.99 \cdot 6}{172.60}$ & 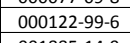 & 138.18 & $i$ & $\frac{1.400 E+01}{1.01}$ & $7.6006+02$ & $2.4206+02$ & $3.000 \mathrm{E}-02$ & $2.500 €+01$ & $3.000=0202$ & 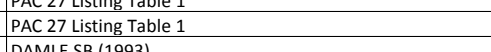 \\
\hline 20302 & 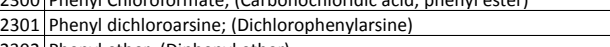 & 1595-28.6 & 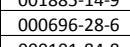 & 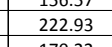 & $\frac{1}{t}$ & $-1.560 \mathrm{~F}+01$ & 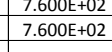 & 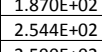 & $\begin{array}{l}5.60901 \\
\text { 1.1306:01 }\end{array}$ & $\begin{array}{l}2.5000+201 \\
2.500+01\end{array}$ & $\begin{array}{l}5.660-01 \\
1.305-0.1\end{array}$ & 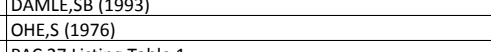 \\
\hline $\begin{array}{l}2303 \\
2304 \\
\end{array}$ & 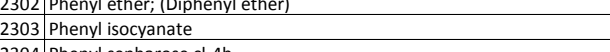 & 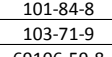 & 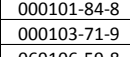 & 年17.22 & $\frac{5}{5}$ & 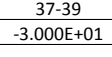 & $7.6005+02$ & $\begin{array}{l}2.509+52 \\
1.500+02\end{array}$ & $2.570 \mathrm{E}+00$ & $2.5005+01$ & $2.570 \mathrm{E}+50$ & 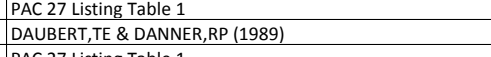 \\
\hline 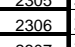 & 305 Phenyl-1,2-2-propanedione, 1 - & 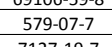 & 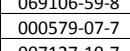 & 148.16 & $\frac{3}{1}$ & 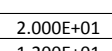 & 7.6000+0202 & $2.22020+022$ & 1.1806:012 & $2.5005+011$ & $\frac{1.1806-01}{1.8003}$ & 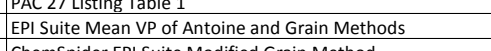 \\
\hline 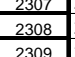 & 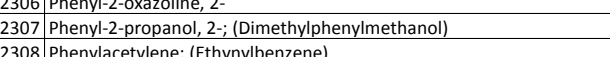 & 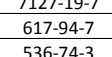 & 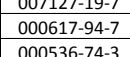 & $\begin{array}{lll}14.11 \\
13.21 \\
10214\end{array}$ & $\frac{5}{5}$ & 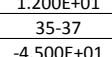 & 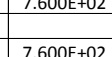 & 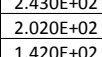 & 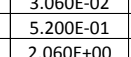 & 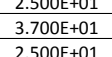 & 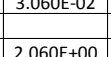 & 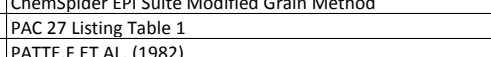 \\
\hline 2330 & 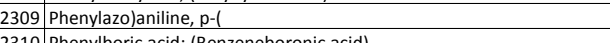 & $60.09 \cdot 3$ & 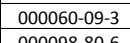 & $\frac{197.26}{10.104}$ & sc & $\frac{1.2060+02}{1.6050202}$ & & & & & & \\
\hline$\frac{2311}{2312}$ & 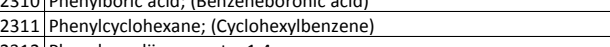 & 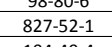 & 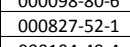 & 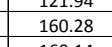 & $e^{3}$ & 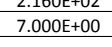 & $7.600+52$ & $2.400 E+02$ & 3.9900:02 & $2.500=+11$ & 3.9906:02 & DAUERT,TE \& DANNER,RP (1989) \\
\hline $\begin{array}{l}23313 \\
22314 \\
\end{array}$ & 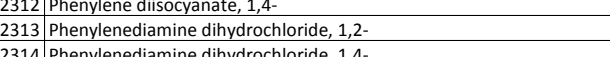 & 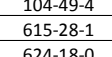 & 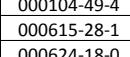 & $\begin{array}{l}16.14 \\
18.106 \\
18.06\end{array}$ & $\begin{array}{c}5 \\
\frac{5}{4} \\
s\end{array}$ & & $7.600 €+02$ & $2.500 E+02$ & $1.4900-02$ & $2.500 €+01$ & $1.4900-02$ & 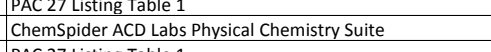 \\
\hline$\frac{2316}{2316}$ & 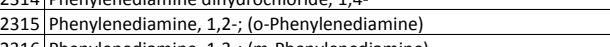 & $\frac{96.54 .5}{950.5}$ & c000095-5.5.5 & $\begin{array}{l}108.16 \\
108\end{array}$ & & 1.04005 +022 & & $2.5700+022$ & & & & \\
\hline & 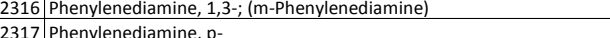 & & 0000108-45-2 & & & & & & $1.000=400$ & $\begin{array}{l}9.9800+01 \\
9.00+01\end{array}$ & & ting Table \\
\hline 22319 & 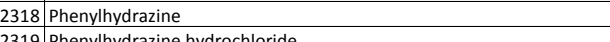 & 100:-63-0. & 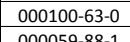 & 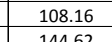 & & 1.960:-51 & $7.6005+022$ & $2.4355+022$ & 2.600502 & $2.5005+011$ & $2.600=022$ & 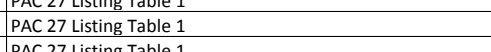 \\
\hline 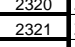 & 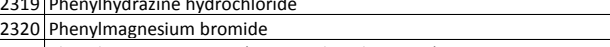 & 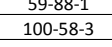 & 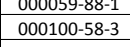 & 148.131 & $\frac{5}{5}$ & $250-254$ & & decom & & & & 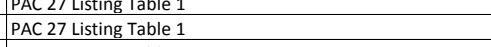 \\
\hline & 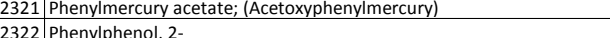 & 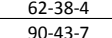 & 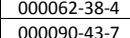 & & & $\begin{array}{l}1.5060202 \\
5.750+01\end{array}$ & & & 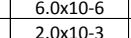 & $\begin{array}{l}2.0000+01 \\
2500+01\end{array}$ & & 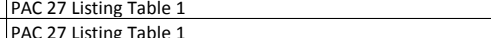 \\
\hline 23244 & 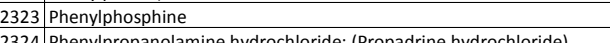 & 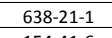 & 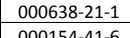 & 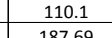 & & & $7.600 E+02$ & $1.600+02$ & $3.094 \mathrm{E}+00$ & $2.500+011$ & $3.094 \mathrm{E}+00 \mathrm{O}$ & Ider AcD Labs Physical Chemistry Suite \\
\hline 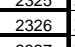 & 2325 Phenplysilatrane & 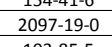 & 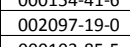 & 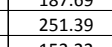 & $\frac{5}{5}$ & $\frac{208209}{20209}$ & & $3.5005+02$ & $1.000=05$ & $2.5005+01$ & & Listing raverte 1 . \\
\hline 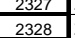 & 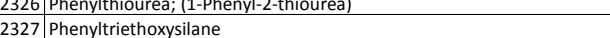 & 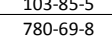 & 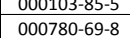 & 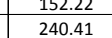 & $\frac{3}{4}$ & & $7.6000+02$ & $2.320 \notin+02$ & $1.0006+00$ & $2.000+01$ & $1.304+500$ & 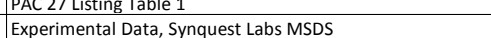 \\
\hline & 2328 Phenyytrimethoxysiline & 2996:-22.1 & 002996-92-1 & 198.32 & $\mathrm{~L}$ & -2.50050101 & $2.000 \leftarrow+01$ & $1.100 E+02$ & 7.2906:02 & $2.500+\div 01$ & $7.200 E-02]$ & 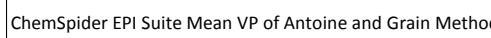 \\
\hline 2330 & 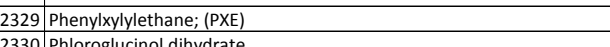 & $\begin{array}{l}6196-95.8 \\
6009027\end{array}$ & $\begin{array}{l}006196-95.8 \\
0.0050907 .7\end{array}$ & $\frac{210.32}{15216}$ & & & $7.600 €+02$ & $3.059 E+02$ & \begin{tabular}{|c|c|}
$6.380 E-04$ \\
\end{tabular} & $2.500+\div 01$ & 6.380E-04 & 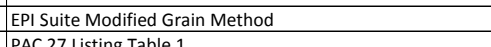 \\
\hline & 2331 phorate & $298-02-2$ & 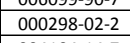 & 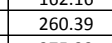 & & $-4.2906+01$ & $7.600 E+02$ & $2.900 E+02$ & 8.400E-04 & $2.500 \mathrm{E}+01$ & $8.400=0.0$ & \\
\hline & 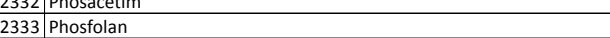 & 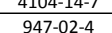 & 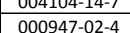 & 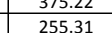 & & 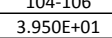 & & 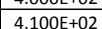 & 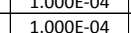 & 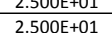 & & 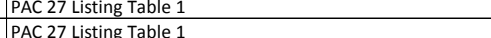 \\
\hline & Phosgene & $75 \cdot 44.5$ & 0.00075-44: & 98.92 & & $-1.278 \&+02$ & & $8.000 E+00$ & 7.5006 +02 & & & \\
\hline & $\begin{array}{l}\text { Ponsegene oxxme } \\
\text { Phosmet }\end{array}$ & 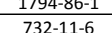 & 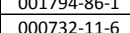 & 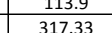 & & 7 1900E01 & & 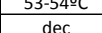 & 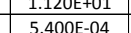 & 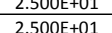 & & 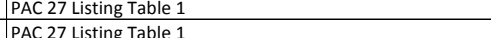 \\
\hline & 2337 - hos shhamididon; (Famfos) & 13171-21-6 & 013171-21- & 299.72 & L & $-4.5000+01$ & $7.600 E+02$ & $3.500 \mathrm{E}+022$ & $2.500=055$ & $2.500 \mathrm{E}+01$ & $2.500=0.05$ & \\
\hline 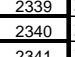 & 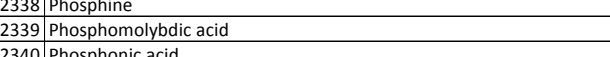 & 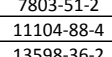 & 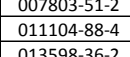 & $\begin{array}{l}159.92046 \\
\end{array}$ & $\begin{array}{l}\frac{6}{5} \\
\end{array}$ & $-1.338 \mathrm{E}+022$ & & $8.7 / 75=0201$ & 7.5000 00202 & $-8.8000+0101$ & & 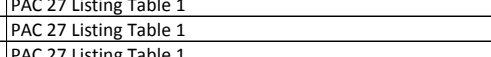 \\
\hline
\end{tabular}




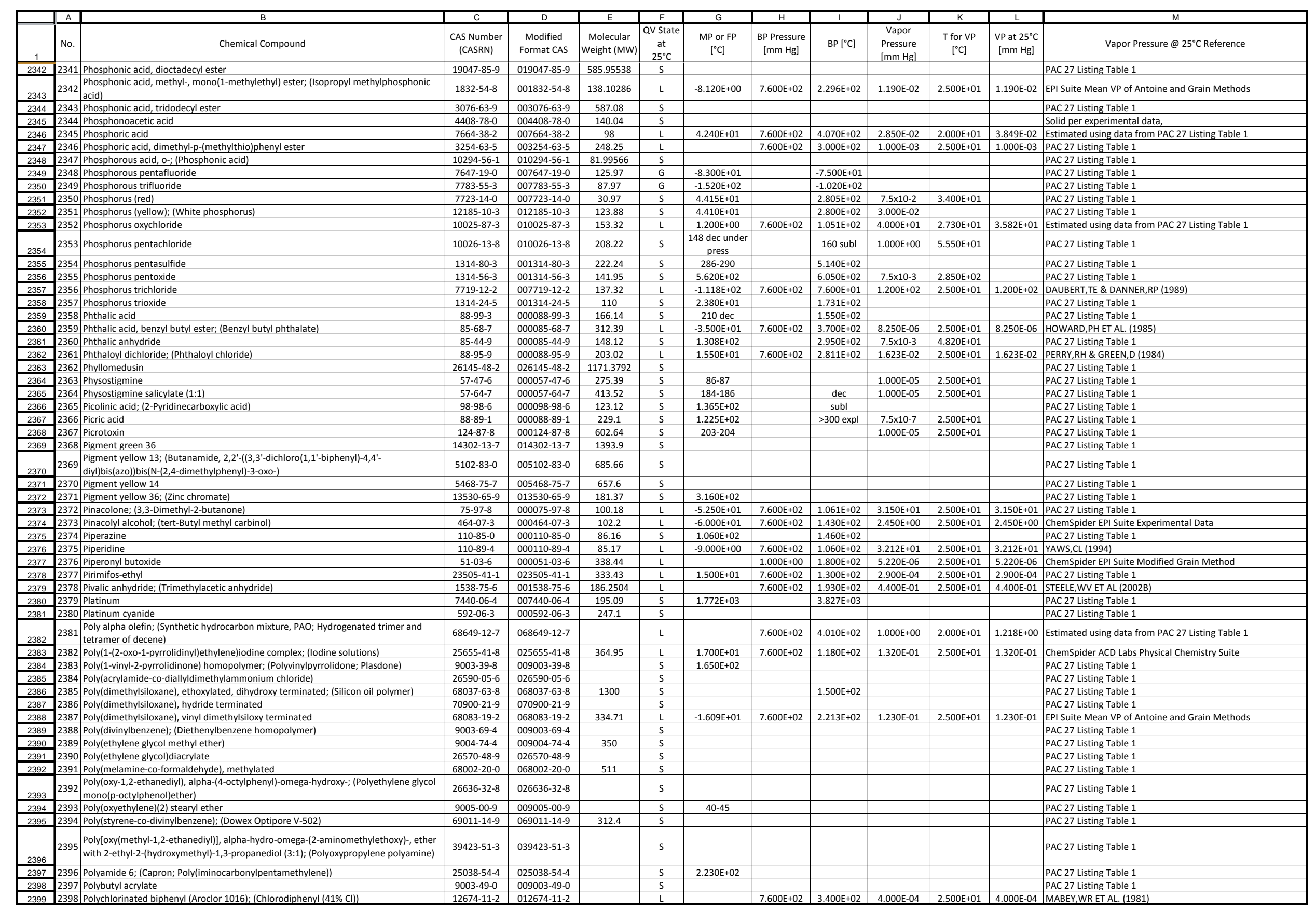




\begin{tabular}{|c|c|c|c|c|c|c|c|c|c|c|c|c|c|}
\hline & & & & & & $F_{F}$ & & Hy & & & $\bar{k}$ & & \\
\hline & No. & Chemical Compound & $\begin{array}{c}\text { CAS Number } \\
\text { (CASRN) }\end{array}$ & $\begin{array}{l}\text { Modified } \\
\text { Format CAS }\end{array}$ & $\begin{array}{l}\text { Molecular } \\
\text { Weight (MW) }\end{array}$ & $\begin{array}{c}\text { Qv State } \\
\text { at } \\
25^{\circ} \mathrm{C}\end{array}$ & $\begin{array}{l}\text { MP of fP } \\
{\left[{ }^{\circ C}\right]{ }^{2}}\end{array}$ & $\begin{array}{c}\text { BP Pressure } \\
{[\mathrm{mm} H \mathrm{Hg}]}\end{array}$ & $B P\left[{ }^{[C}\right]$ & $\begin{array}{l}\text { Vapor } \\
\text { Pressure } \\
\text { [mm HE] }\end{array}$ & $\begin{array}{l}\text { Tfor VP } \\
\text { [C] }\end{array}$ & $\begin{array}{c}\text { VPat } 25^{\circ} \mathrm{C} \\
{[\mathrm{mm} \mathrm{Hg}]}\end{array}$ & 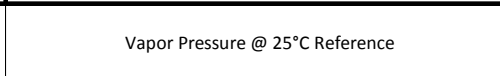 \\
\hline & $\left.2399\right|_{\mathrm{F}} ^{\mathrm{F}}$ & Polychlorinated bipheny (Aroclor 1016/1242): : Chllorodipheny) (37\% CII)) & 2-0042 & $00000 z-0042$ & & L & & $7.600 E+02$ & 3.400E+02 & $8.000 E-04$ & $2.500 E+01$ & $8.000 E-04$ & $\begin{array}{l}\text { Encyclopedia of Environmental Science and Enginering, vol. } \\
\text { (2) }\end{array}$ \\
\hline 2401 & & Polychlorinated bipheny (Arocclor 1221); (Chlorodipheny) (21\% Cl)) & $11104-28-2$ & $011104-28-2$ & 192 & $\mathrm{~L}$ & $1.000 E+00$ & $7.600 E+02$ & $3.400 E+02$ & $6.700 \mathrm{E}-03$ & $2.500 E+01$ & $6.700 \mathrm{E}-03$ & 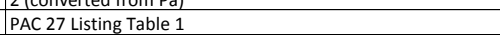 \\
\hline 2402 & 2401 & Polychlorinated biphenyl (Aroclor 1232); (Chlorodiphenyl (32\% CII) & $11141-16-5$ & $011141-16-5$ & & $\mathrm{~L}$ & & $7.600 \mathrm{E}+02$ & $3.400 E+02$ & $4.060 \mathrm{E}-03$ & $2.500 \mathrm{E}+01$ & $4.060 \mathrm{E}-03$ & MABEY, WR ET AL. (1981) \\
\hline 2403 & 2402 & 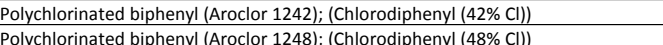 & $\frac{53469-21-9}{12672-29-6}$ & $053469-21-9$ & 261 & $\frac{L}{L}$ & $-1.889 \mathrm{E}+01$ & $\begin{array}{ll}7.600 E+02 \\
7.60 \mathrm{~F}+02 \\
\end{array}$ & $\begin{array}{ll}3.250 \mathrm{E}+02 \\
3.40 \mathrm{E}+02 \\
\end{array}$ & \begin{tabular}{|l|l|}
$1.300 E-03$ \\
$4.940-04$ \\
\end{tabular} & $\frac{2.500 E+01}{2.50 \mathrm{E}+01}$ & $\begin{array}{l}1.3000-03 \\
4.900=04\end{array}$ & \begin{tabular}{|l} 
PAC 27 Listing Table 1 \\
CALAHAN 1 \\
\end{tabular} \\
\hline & 2404 & Polychlorinated biphenyl (Aroclor 1254); (Chlorodiphenyl (54\% C1) & $\begin{array}{l}126 / 2-29-6 \\
11097-69-1 \\
\end{array}$ & $\begin{array}{lll}0126 / 2-2-6 \\
011097-69-1 \\
\end{array}$ & 327 & & & $\begin{aligned} 7.600+020 \\
7.600+02 \\
\end{aligned}$ & \begin{tabular}{|l|l|l}
$3.4050 E+02$ \\
3
\end{tabular} & \begin{tabular}{|l|l|}
$4.940 \mathrm{E}-04$ \\
$7.710 \mathrm{E}-05$ \\
\end{tabular} & $\begin{aligned} 2.500+01 \\
2.500 E+01 \\
\end{aligned}$ & $\begin{aligned} 4.940 \mathrm{E}-04 \\
7.710 \mathrm{E}-05 \\
\end{aligned}$ & 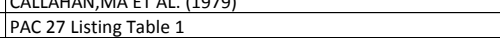 \\
\hline 2406 & 2405 & 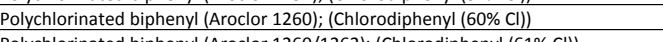 & $11096-82-5$ & $011096-82-5$ & & 5 & & & $340-375$ & & & & PAC 27 Listing Table 1 \\
\hline$\frac{2407}{2408}$ & $\frac{24260}{2007}$ & 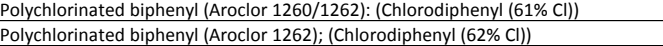 & $\frac{2-0043}{37324-23-5}$ & $\frac{000002-0.043}{037324-23-5}$ & & $\frac{s}{s}$ & & & $\frac{330-35}{340-375}$ & & & & $\begin{array}{l}\text { PAC C } 7 \text { Listinin Table } 1 \\
\text { PAC 27 isting Table } 1\end{array}$ \\
\hline 2409 & 2408 & 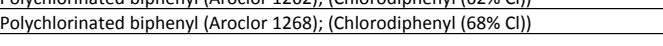 & 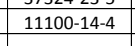 & $011100-14-4$ & & $\frac{5}{5}$ & & & $340-375$ & & & & PAC 27 Listing Table 1 \\
\hline & $\left.2409\right|_{\mathrm{F}} ^{\mathrm{F}}$ & Polychlorinated biphenyl; (Aroclor; PCBs) & $1336-36-3$ & $001336-36-3$ & $291.98-$ & $\mathrm{s}$ & $340-375$ & & $340-375$ & varies & & & PAC 27 Listing Table 1 \\
\hline 24111 & 2410 & Polydimethyl siloxane; (Dimethylpolysiloxane) & $9016-00-6$ & $009016-00-6$ & $\begin{array}{l}36.026 \\
462.95\end{array}$ & $\mathrm{~L}$ & $3.9577+01$ & $7.600 E+02$ & $1.490 \mathrm{t}+02$ & $3.6700-04$ & $2.500 \mathrm{E}+01$ & $3.670 \mathrm{E}-04$ & EPI Suite Modified Grain Method \\
\hline$\frac{2412}{2413}$ & $\frac{2411 \mid}{2421}$ & $\begin{array}{l}\text { Polyyther polyyo ester } \\
\text { Polyythylbenzene residue: (Dowtherm O) }\end{array}$ & $\frac{z-0112}{66987-42-8}$ & $\frac{000002-0112}{0088977-42-8}$ & $\frac{3000}{190}$ & $\frac{\mathrm{S}}{\mathrm{L}}$ & $-4.000 E+01$ & & & & & $1.4900-02$ & \\
\hline & 2413 & 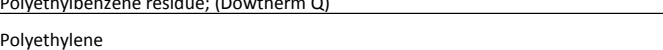 & $90002-88-4$ & $0090002-88-4$ & 1500 & $\mathrm{~s}$ & $2.400 E+02$ & $7.600 E+02$ & $2.670 \mathrm{E}+02$ & $\begin{array}{ll}1.490 E-02 \\
\end{array}$ & $2.500 \mathrm{E}+01$ & $1.4900-02$ & PAC 27 Listing Table 1 1 \\
\hline$\frac{2414}{2415}$ & 2414 & Polyethylene glycol & $25322-68-3$ & $025322-68-3$ & & $\mathrm{~s}$ & $4-8$ & & & & & & 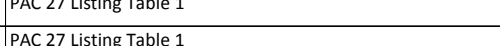 \\
\hline 2416 & 2415 & Polyethylene e glycol $20 \mathrm{M}$ & $37225-26-6$ & $037225-26-6$ & 187.12 & $\mathrm{~L}$ & & $7.600 E+02$ & $4.007 \mathrm{E}+02$ & $1.730 E_{-08}$ & $2.500 E+01$ & $1.730 E-08$ & $\begin{array}{l}\text { PAA 27 listitig Table } 11 \text { Min Method } \\
\text { EPI Suite Modified Grain Methed }\end{array}$ \\
\hline 2417 & 2416 & Polyethylene glycol dimethacrylate & $25852-47-5$ & $025852-47-5$ & & $\mathrm{~s}$ & & & & & & & PAC 27 Listing Table 1 \\
\hline 2418 & 2417 & 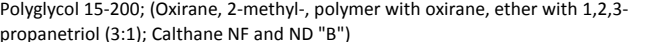 & $9082-00-2$ & 009082-00-2 & 614.82 & $\mathrm{~L}$ & $-2.500 E+01$ & $7.600 \mathrm{E}+02$ & $6.156 \mathrm{E}+02$ & $5.710 \mathrm{E}-18$ & 2.500E+ +01 & $5.710 \mathrm{E}-18$ & EPI Suite Modified Grain Method \\
\hline & 2418 & Polyisocyanate prepolymer (based on MDI) & $2-0115$ & $00000 z-0115$ & & $\mathrm{~s}$ & & & & & & & PAC 27 Listing Table 1 \\
\hline$\frac{2420}{2420}$ & & 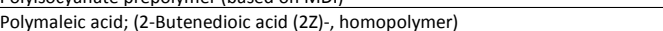 & $26099-09-2$ & 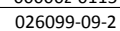 & & & & & & & & & PAC 27 listing Table 1 \\
\hline 2421 & 2420 & Polymerized linseed oil & $67746-08-1$ & $067746-08-1$ & & $\mathrm{~L}$ & $0.000 E+00$ & $7.600 E+02$ & $3.000 E+02$ & $3.000 E+00$ & $2.500 E+01$ & $3.000 \mathrm{E}+00$ & VP estimated from MSDS bounding values \\
\hline 2422 & $2421 / \mathrm{F}$ & Polymethylene polypheny i isocyanate; (Polymeric diphenylmethane dissocyanate) & $9016-87-9$ & 009016-87-9 & & $\mathrm{L}$ & & $5.000 E+00$ & $2.100 E+02$ & $1.000 \mathrm{E}-05$ & $2.500 \mathrm{E}+01$ & $1.000 \mathrm{E}-05$ & PAC 27 Listing Table 1 \\
\hline 2423 & 2422 & Polymethylhydrosiloxane; (Methyl hydrogen polysiloxane) & $63148-57-2$ & $063148-57-2$ & 254.5898 & $\mathrm{~L}$ & $-6.3466+01$ & $7.600 E+02$ & $2.292 \mathrm{E}+02$ & $8.150 \mathrm{E}-02$ & $2.500 E+01$ & $8.150 \mathrm{E}-02$ & EPI Suite Mean VP of Antoine and Grain Methods \\
\hline 2424 & 2423 & Polymethylmethacrylate; (Lucite) & $9011-14-7$ & 009011-14-7 & & 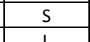 & & 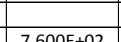 & & 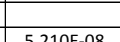 & & & 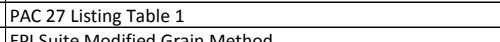 \\
\hline$\frac{2425}{2426}$ & $\frac{24242}{2245}$ & 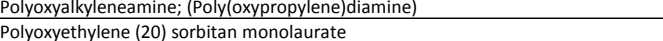 & $\frac{9046-10-0}{99005-64-5}$ & $\begin{array}{ll}009046-10-0 \\
000005-64-5\end{array}$ & 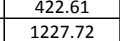 & $\frac{\mathrm{L}}{\mathrm{s}}$ & $-1.000 E+01$ & & $\frac{4.309 E+02}{>100}$ & 5.210E-08 & $2.500 \mathrm{E}+01$ & 5.210E-08 & $\begin{array}{l}\text { EPI Suite Modified Grain Method } \\
\text { PAC 27 Listing Table } 1 \text {. }\end{array}$ \\
\hline & 2426 & Polyoxyethylene monooctylyhenyl ether & 9036-19-5 & $009036-19-5$ & $\frac{1247.12}{426.6}$ & L & & 7.600E+02 & 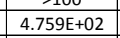 & 1.790E-11 & $2.500 E+01$ & & EPP Suite Modified Grain Method \\
\hline 2428 & 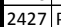 & rbititan monopalmititate; (Tween 40) & 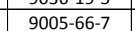 & 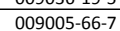 & 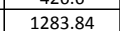 & $\mathrm{L}$ & & 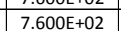 & 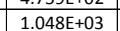 & $\frac{1.16506-34}{1.653}$ & 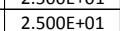 & 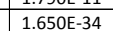 & Modified Grain Method \\
\hline 2429 & 2428 & Polyphosphoric acid & $8017-16-1$ & 008017-16-1 & 257.96 & L & $2.100 E+01$ & $7.600 \mathrm{E}+02$ & $3.000 E+02$ & $2.0600-11$ & $2.500 E+01$ & $2.0600-1$ & EPI Suite Modified Grain Method \\
\hline 2430 & 2429 & Polypropylene & $9003-07-0$ & $009003-07-0$ & & 5 & $1.650 \mathrm{E}+02$ & & & & & & PAC 27 listing Table 1 \\
\hline 2431 & $2430 \mid$ & 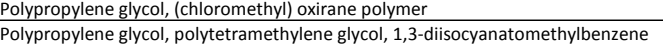 & $9072-62-2$ & $009072-62-2$ & & $\frac{\mathrm{s}}{\mathrm{c}}$ & & & & & & & PAC 27 Listing Table 1 \\
\hline 2432 & $\left.2431\right|^{243}$ & 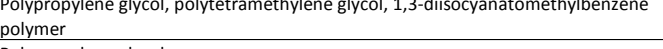 & 65636-36-4 & 065636-36-4 & & $\mathrm{s}$ & & & & & & & PAC 27 listing Table 1 \\
\hline$\frac{2433}{2434}$ & $\frac{2432}{2433}$ & Polypropylene glycols & $\frac{25322-69-4}{9003-11-6}$ & $\frac{025322-69-4}{0.9903-1-6}$ & 528.69 & $\frac{\mathrm{s}}{\mathrm{L}}$ & & $7.600 E+02$ & 5.491E+02 & $8.8900-15$ & & $8.8900-15$ & $\begin{array}{l}\text { PAC C 7 listing Table } 1 \\
\text { EPI Suite Modified Grain Method }\end{array}$ \\
\hline 24355 & 2434 & 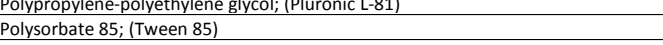 & $9005-70-3$ & $009005-70-3$ & 1177.79 & $\mathrm{~L}$ & $-2.890 \mathrm{E}+01$ & $7.600 \mathrm{~F}+02$ & $2.000 \mathrm{E}+02$ & $9.0600-34$ & $2.500 \mathrm{E}+01$ & $9.060 E-34$ & EPI Suite Modified Grain Method \\
\hline 2436 & 2435 & $\begin{array}{l}\text { Polystyrene resin; (Styrene polymer) } \\
\text { Plowylfor }\end{array}$ & $9003-53-6$ & $009003-53-6$ & & $\frac{s}{c}$ & & & & & & & 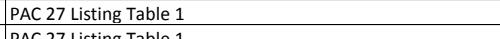 \\
\hline 2438 & $\begin{array}{l}\mid 24367 \\
2437\end{array}$ & $\begin{array}{l}\text { Polysuluone resin } \\
\text { Polytetrafluoroethylene; (Teflon) }\end{array}$ & $\frac{25135-511-1}{9002-84-0}$ & $0009002-84-1-0$ & & $\frac{2}{3}$ & $3.4206+02$ & & & & & & 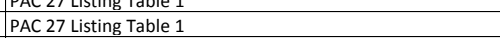 \\
\hline & & 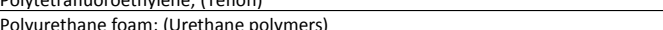 & 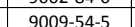 & 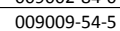 & & & & & & & & & Paç \\
\hline 2440 & 2439 & Polyurethene prepolymer of MDI and PEP & $68092-58-0$ & $068092-58-0$ & & $\mathrm{~L}$ & & $7.600 \mathrm{E}+02$ & $3.793 E+02$ & $5.920 E-06$ & $2.500 \mathrm{E}+01$ & $5.920 \mathrm{E}-06$ & ACD Labs Physical Chemistry Suite \\
\hline 2441 & 2440 & & $9002-89-5$ & $009002-89-5$ & & $\mathrm{~s}$ & $>200$ & & $\tau_{340}$ & & & & PAC 27 listing Table 1 \\
\hline 2442 & $\left.2441\right|_{\mathrm{F}}$ & Polyvinyl chloride & $9002-86-2$ & $009002-86-2$ & $\begin{array}{l}60000- \\
150000\end{array}$ & s & & & & & & & PAC 27 Listing Table 1 \\
\hline 2443 & 2442 & Potassium & $7440-09-7$ & $007440-09-7$ & 39.1 & $\mathrm{~s}$ & $6.350 \mathrm{E}+01$ & & $7.5906+02$ & $7.5 \times 10-3$ & $2.002 E+02$ & & PAC 27 Listing Table 1 \\
\hline 2444 & 2443 & Potassium acetate & $\frac{127-08-2}{7799-29-9}$ & $000127-08-2$ & 98.15 & $\frac{s}{s}$ & $2.920 \mathrm{E}+02$ & & & & & & $\begin{array}{l}\text { PAC } 27 \text { Listing Table } 1 \\
\text { PAA 27 }\end{array}$ \\
\hline 2445 & 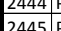 & $\begin{array}{l}\text { Potassium acid fluoride } \\
\text { Potassium aluminate }\end{array}$ & 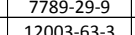 & $007789-29-9.9$ & 78.11 & $\frac{s}{s}$ & & & & & & & \\
\hline & & $\begin{array}{l}\text { Potassium alumininite } \\
\text { Potsits }\end{array}$ & $=2.0044$ & 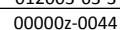 & 134.1 & $\mathrm{~s}$ & & & & & & & PAC 27 \\
\hline 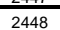 & $|2447|$ & Potassium aluminosilicate & $1327-44-2$ & $001327-44-2$ & 155.16162399 & $\mathrm{s}$ & & & & & & & $\begin{array}{l}\text { PAC } 27 \text { Listing Table } 1 \\
\end{array}$ \\
\hline 2449 & 2448 & Potassium antimonate; (Antimony potassium oxide) & $29638-69-5$ & & 511.89 & s & & & & & & & PAC 27 Listing Ta \\
\hline$\frac{2450}{2451}$ & $\frac{2449}{2450}$ & $\begin{array}{l}\text { Potassium antimonote; ; Potassium hexahydroxyantimone } \\
\text { Potassium antimonite }\end{array}$ & $\frac{12208-13-8}{2-0045}$ & $\frac{012208-13-8}{000000-0045}$ & 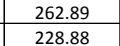 & $\frac{\mathrm{s}}{\mathrm{s}}$ & & & & & & & $\begin{array}{l}\text { PAA } 27 \text { listiting Ta } \\
\text { PAC 2listitg Ta }\end{array}$ \\
\hline & 2451 & 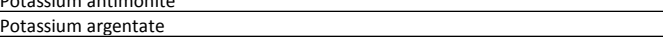 & $2-0046$ & $000002-0046$ & 180.97 & $\mathrm{~s}$ & & & & & & & $\begin{array}{l}\text { PAC 27 Listing Table } 1 \\
\text { Pand }\end{array}$ \\
\hline 2453 & & $\begin{array}{l}\text { Potassium arsenate; ; Monopotassium arsenate) } \\
\end{array}$ & $7784-41-0$ & $007784-41-0$ & 180.04 & $\mathrm{~s}$ & $2.880 \mathrm{E}+02$ & & & & & & PAC 27 Listing Table 1 \\
\hline 2454 & 2453||$^{2454}$ & 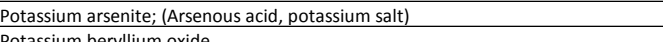 & $13464-35-2$ & 013464-35-2 & $\frac{146.02}{1042}$ & $\frac{\mathrm{s}}{\mathrm{s}}$ & & & & & & & $\begin{array}{l}\text { PAC 27 Listing Table } 1 \\
\text { PAC }\end{array}$ \\
\hline$\frac{2453}{2456}$ & & Potassium bicarbonate & $298-14-6$ & $000298-14-6$ & $\frac{144.2}{100.106}$ & $\frac{s}{5}$ & $\sim 100 \mathrm{dec}$ & & & & & & $\begin{array}{l}\text { PAC 27 Listing Table } 1 \\
\text { Pans }\end{array}$ \\
\hline 2457 & 年 2456 & Potassium bi-iodate & \begin{tabular}{|l|}
$13455-24-8$ \\
\end{tabular} & $\begin{array}{l}013455-24-8 \\
\end{array}$ & 389.90033 & $\mathrm{~s}$ & & & & & & & PAC C L Listing Table 1 \\
\hline
\end{tabular}




\begin{tabular}{|c|c|c|c|c|c|c|c|c|c|c|c|c|c|}
\hline & No. & Chemical Compound & $\begin{array}{l}\text { CAS Number } \\
\text { (CASRN) }\end{array}$ & $\begin{array}{c}\text { Modified } \\
\text { Format CAS }\end{array}$ & \begin{tabular}{|c|} 
E \\
$\begin{array}{c}\text { Molecular } \\
\text { Weight (WW) }\end{array}$ \\
\end{tabular} & \begin{tabular}{|c|} 
F State \\
at \\
$25^{\circ} \mathrm{C}$
\end{tabular} & $\begin{array}{c}\text { MP or FP } \\
\left.{ }^{\circ} \mathrm{C} C\right]\end{array}$ & \begin{tabular}{|c|} 
\\
BP Pressure \\
[mm Hg]
\end{tabular} & $\mathrm{BP}\left[{ }^{\circ} \mathrm{C}\right]$ & $\begin{array}{l}\text { Vapor } \\
\text { Pressure } \\
{[\mathrm{mm} H \mathrm{Hg}]}\end{array}$ & $\frac{\mathrm{K}}{\mathrm{K}}$ & \begin{tabular}{|c|} 
Vp at $25^{\circ} \mathrm{C}$ \\
{$[\mathrm{mm} \mathrm{Hg}]$}
\end{tabular} & Vapor Pressure @ $22^{\circ}$ C Reference \\
\hline 2459 & 2458 & Potassium bisulfate & \begin{tabular}{|l|}
$7646-93-7$ \\
$776-9$
\end{tabular} & $007646-93-7$ & \begin{tabular}{|l|l|}
136.17 \\
\end{tabular} & & $2.140 \mathrm{E}+02$ & & & & & & PAC 27 Listing Table 1 \\
\hline 2460 & \begin{tabular}{|c|c|}
2459 \\
2465
\end{tabular} & \begin{tabular}{|l} 
Potassium bromate \\
Potassium bromide
\end{tabular} & $\begin{array}{l}7758-01-2 \\
7758-02-3 \\
\end{array}$ & $\begin{array}{ll}007758-01-2 \\
007758-2-3-3\end{array}$ & $\frac{167.01}{11901}$ & $\frac{5}{5}$ & $\begin{array}{l}3.500 \mathrm{E}+02 \\
7.30 \mathrm{E}+02\end{array}$ & & $1.435 E+03$ & $10005+000,-3$ & 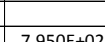 & & $\begin{array}{l}\text { PAC } 27 \text { Listing Table } 1 \\
\text { PaC } 27 \text { istion Thble } 1\end{array}$ \\
\hline 2462 & $\left|\begin{array}{l}\mid 24061 \\
2461\end{array}\right|$ & $\begin{array}{l}\text { Potasssium cadminate } \\
\text { Potumate }\end{array}$ & $2-0048$ & $00000 z-0048$ & $\frac{119.01}{258.62}$ & $\frac{\mathrm{s}}{\mathrm{s}}$ & & & & & & & $\begin{array}{l}\text { PAC27 Lising Gable 1 } \\
\text { PAC 27 Listing Table } 1\end{array}$ \\
\hline 2463 & 2462 & Potassium carbonate & $584-08-7$ & 000584-08-7 & 138.21 & $\mathrm{~s}$ & $8.990 \mathrm{E}+02$ & & dec & & & & PAC 27 Listing Table 1 \\
\hline 2464 & 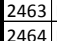 & $\begin{array}{l}\text { Potasssium chlorate } \\
\text { Potasssum chloride }\end{array}$ & $\begin{array}{l}3811-04-9 \\
7447-40-7\end{array}$ & $\begin{array}{l}003811-04-9 \\
007447-4-7\end{array}$ & $\frac{122.55}{7.45}$ & $\frac{s}{s}$ & $\begin{array}{l}3.560 \mathrm{E}+02 \\
7.710 \mathrm{E}+02\end{array}$ & & $\begin{array}{ll}400 \mathrm{dec} \\
1500 \text { subl }\end{array}$ & & & & 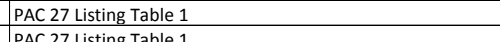 \\
\hline$\frac{2465}{2466}$ & \begin{tabular}{|c|c|}
24464 \\
2465
\end{tabular} & $\begin{array}{l}\text { PPotassium chloride } \\
\text { Potassium chromate(VI) }\end{array}$ & $\begin{array}{l}74747-40-7 \\
7789-00-6\end{array}-20$ & $\begin{array}{l}007477-40-7 \\
007789-0-6\end{array}$ & $\begin{array}{r}74.55 \\
194.19 \\
\end{array}$ & $\frac{\mathrm{s}}{\mathrm{s}}$ & $\begin{array}{l}7.710 E+02 \\
9.70 \mathrm{E}+02 \\
\end{array}$ & & 1500 subl & $0.000 E+00$ & no temp & & $\begin{array}{l}\text { PAC 27 Lising g able 1 } \\
\text { PAC 27 Listing Table 1 }\end{array}$ \\
\hline & & Potassium citrate & & $000866-84-6$ & $\begin{array}{l}194.19 \\
306.41\end{array}$ & & 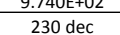 & & & & notemp & & $\begin{array}{l}\text { PAC } 27 \text { Listing rabie } 1 \\
\text { PAable } 1\end{array}$ \\
\hline 2468 & 2467 & Potassium citrate, monohydrate & $600-05-26$ & $006100-05-6$ & $\begin{array}{r}324.41865 \\
32465 \\
\end{array}$ & $\frac{2}{3}$ & & & & & & & $\begin{array}{l}\text { PAC 27 Listing Table } 1 \\
\text { PAC }\end{array}$ \\
\hline & 2468 & Potassium columbate; (Potassium niobate) & $12030-85-2$ & $012030-85-2$ & 1174.26 & $\mathrm{~s}$ & & & & & & & PAC 27 Listing Table 1 \\
\hline 2470 & \begin{tabular}{|c|c|c|c|}
24699 \\
2670
\end{tabular} & 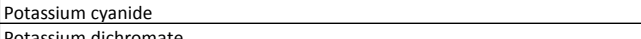 & $\begin{array}{l}151-50-8 \\
7770-8\end{array}$ & $\begin{array}{ll}000151-50-8 \\
00770500\end{array}$ & 65.12 & $s$ & $6.340 \mathrm{E}+02$ & & & & & & PAC 27 Listing Table 1 \\
\hline$\frac{2471}{2472}$ & $\left|\begin{array}{l}\mid 2470 \\
2471\end{array}\right|-100$ & $\begin{array}{l}\text { Potossisimm dichromate } \\
\text { Potassium dideuterium phosphate }\end{array}$ & $\begin{array}{l}7778-50-9 \\
13761-99-0 \\
\end{array}$ & $\begin{array}{l}007778-5-5-9 \\
01376-79-0 \\
\end{array}$ & $\frac{294.19}{139.11}$ & $\frac{s}{s}$ & $3.980 E+02$ & & $\sim 500$ dec & & & & $\begin{array}{l}\text { PAC27 Listing Table } 1 \\
\text { PAC 27 listing Table } 1\end{array}$ \\
\hline & & $\begin{array}{l}\text { Potassumum cideuturerum mhosphnate } \\
\text { Potassium ferricyanide }\end{array}$ & $\frac{13 / 61-9-9-0}{13746-66-2}$ & 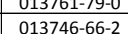 & $\frac{1399.11}{329.27}$ & & 70 loses water & & dec & & & & $\begin{array}{l}\text { PAC 2 Lsting lable } 1 \\
\text { PAC } 27 \text { Listing Table } 1\end{array}$ \\
\hline 2474 & & Potasssium ferrocyanide; (Tetrapotassium hexacyan oferrate) & $13943-58-3$ & $013943-58-3$ & 368.37 & s & & & & & & & $\begin{array}{l}\text { PAC } 27 \text { Listing gable } 1 \\
\text { PAting } 1\end{array}$ \\
\hline 2475 & 2474 & Potassium fluoride & $7789-23-3$ & $007789-23-3$ & 58.1 & $\mathrm{~s}$ & $8.580 \mathrm{E}+02$ & & $1.502 E+03$ & $7.500=-01$ & $8.690 \mathrm{E}+02$ & & PAC 27 Listing Table 1 \\
\hline 2476 & 2475 & Potassium fluoride dihydrate & $13455-21-5$ & $013455-21-5$ & 94.14 & $\mathrm{~s}$ & $4.100 E+01$ & & $1.560 \mathrm{E}+02$ & & & & PAC 27 Listing Table 1 \\
\hline 2477 & |2476] & Potassium formate & $590-29-4$ & $000590-29-4$ & 84.12 & $\mathrm{~s}$ & $1.680 \mathrm{E}+02$ & & dec & & & & PAC 27 Listing Table 1 \\
\hline$\frac{2478}{2479}$ & $\frac{24 / 1}{2478}$ & $\begin{array}{l}\text { Potassium glycolate } \\
\text { Potassium gold cyanide }\end{array}$ & $\frac{1932-50-9}{554-07-4}$ & 001932-50-9 & $\frac{114.1283}{288.0965}$ & $\frac{s}{5}$ & & & & & & & $\begin{array}{l}\text { PAC27 Listing Table } 1 \\
\text { PAC 27 listing Table } 1 \\
\end{array}$ \\
\hline$\frac{2499}{2480}$ & \begin{tabular}{|c|c|}
$\mid 2479$ \\
2479
\end{tabular} & 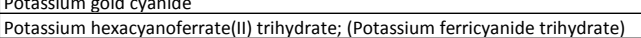 & $\frac{554-0-4}{14459-95-1}$ & $0000554-0 .-4-4$ & $\frac{28.09965}{422.39}$ & $\frac{5}{5}$ & 60 dec & & & & & & $\begin{array}{l}\text { AAC 2 Lsting a able } \\
\text { PAC 27 Listing Table } 1\end{array}$ \\
\hline$\frac{2481}{2481}$ & 2480 & Potassium hexafluorosilicate; (Silicate(2-), hexafluoro, dipotassium) & $16871-90-2$ & $016871-90-2$ & 220.29 & $\frac{5}{5}$ & dec & & & & & & PAC 27 Listing Table 1 \\
\hline 2482 & 2481 & Potassium hydride & 7693-26-7 & $007693-26-7$ & 40.11 & $\mathrm{~s}$ & & & & & & & PAC 27 Listing Table 1 \\
\hline 2483 & 2482 & 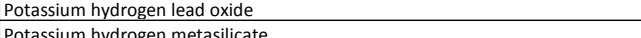 & $2-0049$ & $000002-0049$ & 279.3 & $\frac{5}{5}$ & 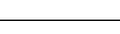 & & & & & & PAC 27 Listing Table 1 \\
\hline 2484 & 年 42483 & 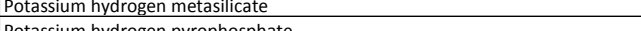 & $\frac{2-00051}{2}$ & 0000002-0051 & 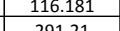 & $\frac{s}{s}, \frac{1}{2}$ & & & & & & & 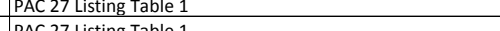 \\
\hline 2485 & $\frac{2484}{2485}$ & 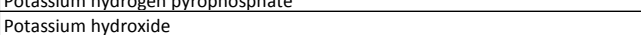 & $\begin{array}{c}2-0000 \\
1310-58-3 \\
\end{array}$ & $001310-58-3$ & $\frac{291.11}{56.11}$ & $\frac{5}{5}$ & $4.060 E+02$ & & $1.327 \mathrm{E}+03$ & $7.5 \times 10-3$ & $5.200 E+02$ & & PaC \\
\hline$\frac{2487}{2487}>>2>0$ & 24886 & Potassium iminodiacetate; (Potassium IDA) & $\frac{2-0052}{2}$ & $0000002-0052$ & 209 & $\mathrm{~s}$ & & & & & & & $\begin{array}{l}\text { PAC } 27 \text { Listing Table } 1 \\
\end{array}$ \\
\hline 2488 & 2487 & Potassium iodate & $7758-05-6$ & $007758-05-6$ & 214 & $\mathrm{~s}$ & $5.6000 \mathrm{E}+022$ & & & & & & PAC 27 Listing Table 1 \\
\hline 2489 & $2488 / 5$ & $\begin{array}{l}\text { Potassium iodide } \\
\text { Potanssumbantanate }\end{array}$ & $7681-11-0$ & $007681-11-0$ & $\frac{166}{22602}$ & $\frac{s}{s}$ & $6.810 \mathrm{E}+02$ & & $1.330 \mathrm{E}+03$ & $1.000 E+00$ & $7.450 E+022$ & & PAC 27 Listing Table 1 \\
\hline & $\begin{array}{l}24890 \mid \\
2490 \mid\end{array}$ & $\begin{array}{l}\text { Potassium lanthanate } \\
\text { Potassium metaarsenite: (Potassium arsenite) }\end{array}$ & $\begin{array}{c}z-00033 \\
10124-50-2\end{array}$ & $\frac{000002-0053}{0100245-2}$ & $\frac{246.02}{399.65}$ & $\frac{s}{s}$ & & & & & & & $\begin{array}{l}\text { PAC } 27 \text { Listing Table } 1 \\
\text { PAC } 7 \text { listing Table } 1\end{array}$ \\
\hline$\frac{2492}{2492}>2>0$ & $\left.\right|_{2491}$ & Potassium metaborate & $13709-94-9$ & $013709-94-9$ & 82.92 & $\mathrm{~s}$ & & & & & & & PAC 27 Listing Table 1 \\
\hline 2493 & 2492 & Potassium molybdate & $13446-49-6$ & $013446-49-6$ & 238.14 & $\mathrm{~s}$ & $9.190 \mathrm{E}+02$ & & & & & & PAC 27 Listing Table 1 \\
\hline 2494 & $2493 \mid \mathrm{t}$ & Potassium nickel oxide (liquids); (Nickel potassium oxide) & 50811-97-7 & 050811-97-7 & 168.8888 & $\mathrm{~L}$ & & & & $1.000 E-05$ & $2.500 E+01$ & $1.000 E-05$ & 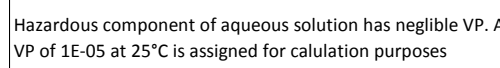 \\
\hline & $2494 \mid$ & Potassium nickelate (liquids) & 2-0055 & $00000 z-0055$ & 204.9 & $\mathrm{~L}$ & & & & $1.000 E-05$ & $2.500 E+01$ & $1.0000-05$ & 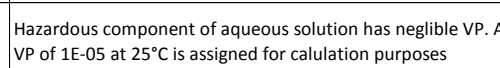 \\
\hline 2496 & $2495 \mathrm{~F}$ & Potassium nickelate (solids) & 2-0056 & $00000 z-0056$ & 204.9 & & & & & & & & PAC 27 Listing Table 1 \\
\hline 2497 & 24966 & & $7757-79-1$ & $007757-79-1$ & 101.1 & $\mathrm{~s}$ & $3.340 \mathrm{E}+02$ & & $400 \mathrm{dec}$ & & & & PAC 27 Listing Table 1 \\
\hline$\frac{2498}{2499}$ & $\begin{array}{l}2499 \mid \\
2498 \mid\end{array}$ & $\begin{array}{l}\text { Potassium nintrilotriacetate; (Potassium NTA) } \\
\text { Potassium nitrite }\end{array}$ & $\frac{2399-859-1}{7758-09-0}$ & $002399-85-1$ & $\frac{305.43}{8.511}$ & $\frac{s}{s}$ & $4.400 \mathrm{~F}+02$ & & 1000 exol & & & & $\begin{array}{l}\text { PAC27 Listing Table } 1 \\
\text { PAC 27 listing Table } 1 \\
\end{array}$ \\
\hline & 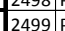 & $\begin{array}{l}\text { Potasssumm intrtite } \\
\text { Potasssium orthovanadate }\end{array}$ & 14293-78-8 & $\begin{array}{ll}0 \\
0\end{array}$ & $\frac{8.511}{232}$ & s & & & & & & & 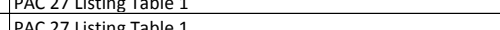 \\
\hline$\frac{2501}{2501}$ & 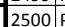 & $\begin{array}{l}\text { Potasssium oxalate } \\
\text { Potacte }\end{array}$ & $\frac{1533-52-8}{583}$ & $\frac{100583-5-8}{0.85}$ & $\frac{252}{168.24}$ & s & & & & & & & PAC 2 L Listing Table 1 \\
\hline 2502 & 2501 & Potassium oxalate monohydrate & $6487-48-5$ & $006487-48-5$ & 184.231 & $\mathrm{~s}$ & $1.600 E+02$ & & & & & & $\begin{array}{l}\text { PAC } 27 \text { Listing Table } 1 \\
\end{array}$ \\
\hline 2503 & 2502 & \begin{tabular}{|l} 
Potassium oxide \\
\end{tabular} & $12136-45-7$ & $012136-45-7$ & 94.1794 & $\mathrm{~s}$ & & & & & & & PAC 27 Listing Table 1 \\
\hline 2504 & $2503 \mid$ & $\begin{array}{l}\text { Potassium perchlorate } \\
\text { P.tassusim periodate }\end{array}$ & $7778-74-7$ & $007778-74-7$ & $\frac{138.55}{23001}$ & ${ }_{c}^{s}$ & $\begin{array}{l}400 \mathrm{dec} \\
\mathrm{502}\end{array}$ & & & & & & 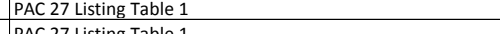 \\
\hline & $\begin{array}{l}2504 \mid \\
2505: \mid\end{array}$ & $\begin{array}{l}\text { Potosassium periodate } \\
\text { Potassium permanananate }\end{array}$ & $\frac{7790-2118}{7722-64-7}$ & $\frac{0079002-21-8}{007722-6-7}$ & $\frac{230.01}{15803}$ & $\frac{3}{5}$ & 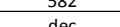 & & & & & & 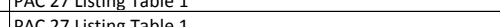 \\
\hline$\frac{2500}{2507}$ & 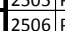 & 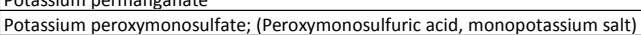 & $10058-23-8$ & $010058-23-8$ & $\frac{150.07}{152.17}$ & $\frac{5}{s}$ & & & & & & & $\begin{array}{l}\text { PaC } \\
\text { PAC Listin Table } 1\end{array}$ \\
\hline 2508 & 2507 & Potassium persulfate; (Dipotassium persulfate) & $7727-21-1$ & 007727-21-1 & 272.34 & $\mathrm{~s}$ & $100 \mathrm{dec}$ & & & & & & PAC 27 Listing Table 1 \\
\hline 2509 & 2508 & Potassium phosphate dibasic trihydrate & 16788-57-1 & $016788-57-1$ & 228.2256 & $\mathrm{~s}$ & & & & & & & PAC 27 Listing Table 1 \\
\hline$\frac{2510}{2511}$ & 2509 & 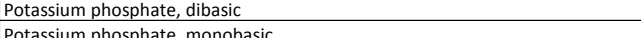 & $\begin{array}{l}7758-11-4 \\
7778-77-0\end{array}$ & $007758-11-4$ & $\frac{174.18}{1369}$ & $\frac{s}{s}$ & & & & & & & $\begin{array}{l}\text { PAC } 27 \text { Listing Table } 1 \\
\text { pac? }\end{array}$ \\
\hline$\frac{2.01}{2512}$ & $\left.2511\right|_{\mathrm{F}}$ & Potassium phosshate tribahic & 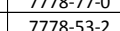 & (2) & $\frac{212.27}{212.27}$ & s & & & & & & & P \\
\hline 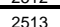 & 2512 & Potassium phosphide & $20770-41-6$ & $020770-41-6$ & 148.24376 & $\mathrm{~s}$ & & & & & & & PaC \\
\hline$\frac{2514}{2514}$ & 2513 & Potassium pyrophosphate; (Tetrapotassium diphospt & $7320-34-5$ & $007320-34-5$ & 330.24 & $\mathrm{~s}$ & $1.800 \mathrm{E}+02$ & & $3.000 E+02$ & & & & PAC 27 Listing Table 1 \\
\hline 2515 & 2514 F & Potassium pyrosulfate; (Disulfuric acid, dipotassium s. & $77900-62-7$ & $007790-62-7$ & 256.32 & $\mathrm{~s}$ & & & & & & & PAC 27 Listing Table 1 \\
\hline 2516 & 20555 & $\begin{array}{l}\text { Potassium pyrosulfite; (Potassium metabisulfite } \\
\text { Potazsium seleante }\end{array}$ & $16733-55-8$ & $0016737-155-8,8$ & $\frac{22.32}{20272}+20$ & $\mathrm{~s}$ & 150 decom & & & & & & PAC 27 Listing Table 1 \\
\hline$\frac{2517}{2518}$ & 2517 & Potassium selenite & $10431-47-7$ & $010431-47-7$ & 205.16 & s & & & & & & & PAC 27 Listing Table 1 \\
\hline$\frac{2519}{5250}$ & 2518 & $\begin{array}{l}\text { Potassium silicate; (Silicic acid, potassium salt) } \\
\text { Potazsium silvercyaide }\end{array}$ & $\frac{1312-76-1}{506-61-6}$ & $001312-76-1$ & 10901 & $\frac{\mathrm{s}}{\mathrm{s}}$ & $1.015 \mathrm{E}+03$ & & & 10005055 & $25005+01$ & & $\begin{array}{l}\text { PAC } 27 \text { Listing Table } 1 \\
\text { PaC } 2 \text { listiog Table } 1\end{array}$ \\
\hline
\end{tabular}




\begin{tabular}{|c|c|c|c|c|c|c|c|c|c|c|c|c|c|}
\hline & No. & Chemical Compound & $\begin{array}{l}\text { CAS Number } \\
\text { (CASRN) }\end{array}$ & $\begin{array}{c}\text { Modified } \\
\text { Format CAS }\end{array}$ & $\begin{array}{l}\text { Molecular } \\
\text { Weight (MW) }\end{array}$ & \begin{tabular}{|c} 
\\
av state \\
at \\
$25^{\circ} \mathrm{C}$
\end{tabular} & $\begin{array}{c}\text { MP or fP } \\
{\left[{ }^{\circ} \mathrm{C}\right]}\end{array}$ & $\begin{array}{c}\text { BP Pressure } \\
{\left[\begin{array}{cc}\mathrm{mm} \mathrm{Hg} \\
\mathrm{mg}]\end{array}\right.}\end{array}$ & $B P\left[{ }^{\circ} \mathrm{C}\right]$ & $\begin{array}{l}\text { Vapor } \\
\text { Pressure } \\
{[\mathrm{mm} H \mathrm{Hg}]}\end{array}$ & 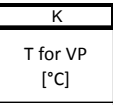 & $\begin{array}{r}\text { vp at } 22^{\circ} \mathrm{C} \\
{[\mathrm{mm} \mathrm{Hg}]}\end{array}$ & Vapor Pressure @ @25 C Reference \\
\hline 2521 & 2520 & Potassium stannate & $12142-33-5$ & $012142-33-5$ & 244.9048 & & & & & & & & PAC 27 Listing Table 1 \\
\hline$\frac{2522}{2523} \mathrm{ral} \mathrm{ll} \mathrm{l}$ & $\mid \begin{array}{ll}2521 \\
2522\end{array}$ & \begin{tabular}{|l} 
Potassium strontium phosphate \\
Potassium suffat (2:1) (ipiotassium sulfatet
\end{tabular} & $\frac{53201-92-6}{7778-80-5}$ & $\begin{array}{ll}053201-92-6 \\
00778-8-5\end{array}$ & 221.68 & $\frac{s}{s}$ & $1067 F+03$ & & $1.689+03$ & & & & $\begin{array}{l}\text { PAC } 27 \text { Listing Table } 1 \\
\text { PAC 27 Listing Table } 1\end{array}$ \\
\hline$\frac{2523}{2524}$ & 2523 & 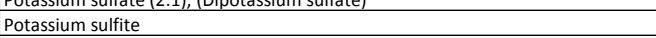 & $10117-38-1$ & $010117-38-1$ & $\frac{1 / 4.46}{158.26}$ & $\frac{5}{5}$ & & & & & & & $\begin{array}{l}\text { PAC C7 Listing a able } 1 \\
\text { PAC } 27 \text { Listing Table } 1\end{array}$ \\
\hline 2525 & 2524 & Potassium tellurate & 15571-91-2 & $015571-91-2$ & 269.78 & $\mathrm{~s}$ & & & & & & & PAC 27 Listing Table 1 \\
\hline 2526 & $2525 \mid$ & $\begin{array}{l}\text { Potassium tellurite } \\
\text { P.tassusum tetrafluoroborate(1-) }\end{array}$ & $\frac{7790-58-1}{14075-53-7}$ & $007790-58-1$. & 253.7782 & $\frac{s}{s}$ & 570 & & & & & & $\begin{array}{l}\text { PAC 27 Listing Table } 1 \\
\text { PAC 27 Listing Table } 1\end{array}$ \\
\hline & $\mid \begin{array}{ll}25267 \\
2527\end{array}$ & $\begin{array}{l}\text { Potassium tetrafluoroborate(e-1-) } \\
\text { Potassium tetraphenylborte }\end{array}$ & $\frac{14075-53-7}{3244-41-5}$ & $\begin{array}{l}014075-53-7 \\
003244-41-5\end{array}$ & $\frac{125.91}{358.31}$ & & 570 & & & & & & $\begin{array}{l}\text { PAA 27 Listing gable } 1 \\
\text { PAC } 27 \text { isting Table } 1\end{array}$ \\
\hline & & & $3433-20-5$ & $\begin{array}{ll}003244-41-5 \\
000333-20-0\end{array}$ & $\frac{358.18}{97.18}$ & & $1.730 \mathrm{E}+02$ & & 500 dec & & & & $\begin{array}{l}\text { PAC C27 Listing a abble } 1 \\
\text { PAC } 27 \text { Listing Table } 1\end{array}$ \\
\hline$\frac{2529}{2530}$ & 年258 & Potassium trihydrogen silicate & $z-0058$ & $\begin{array}{l}0,00002-20058 \\
00030\end{array}$ & 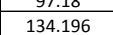 & $\mathrm{s}$ & & & & & & & $\begin{array}{l}\text { PAC 27 Listing Table } 1 \\
\text { Pat }\end{array}$ \\
\hline 2531 & 2530 F & Potassium tungstate (liquids) & $7790-60-5 a$ & $007790-60-5 \mathrm{a}$ & 326.03 & $\mathrm{~s}$ & $9.210 \mathrm{E}+02$ & & & & & & PAC 27 Listing Table 1 \\
\hline 2532 & 2531 & \begin{tabular}{|l|} 
Potassium tungstate (solids) \\
Potacsium wranvicarbiate
\end{tabular} & $7790-60-5 b$ & $007790-60-5 b$ & 326.03 & 5 & $9.210 \mathrm{E}+02$ & & & & & & PAC 27 Listing Table 1 \\
\hline 2533 & 2532 & $\begin{array}{l}\text { Potassium uranny I carbonate } \\
\text { Potassium ziriconate (iotassium zirconium oxide. Dipotassium zirconate) }\end{array}$ & $\begin{array}{c}z-0059 \\
12030-98-7\end{array}$ & $000002-0059$ & 468.2427 & $\frac{\mathrm{s}}{\mathrm{s}}$ & & & & & & & 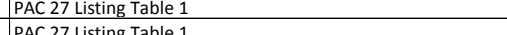 \\
\hline & & & $\frac{120030-98-1}{865-47-4}$ & & $\frac{21,1.4}{112.2}$ & & & & $257 \mathrm{dec}$ & $1.3000-01$ & $2.2006+02$ & & $\begin{array}{l}\text { PAC 27 L Ltsing a able } 1 \\
\text { PAC Listing Table } 1\end{array}$ \\
\hline$\frac{2536}{2536}$ & (2535) & Prasedymium & $7440-10-0$ & 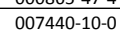 & 140.9077 & s & 935 & & $\frac{3.512 \mathrm{E}+03}{3}$ & & & & $\begin{array}{l}\text { PAC 27 Ltsing labie } \\
\text { PAC } 27 \text { Listing Table } 1\end{array}$ \\
\hline & 2536 & Praseodymium nitrate & $10361-80-5$ & $010361-80-5$ & 326.92 & $\mathrm{~s}$ & & & & & & & PAC 27 Listing Table 1 \\
\hline 2538 & 2537 & Praseodymium oxide & $12036-32-7$ & $012036-32-7$ & 329.8136 & $\mathrm{~s}$ & $2.300 \mathrm{E}+03$ & & & & & & PAC 27 Listing Table 1 \\
\hline$\frac{2539}{2540}$ & 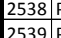 & 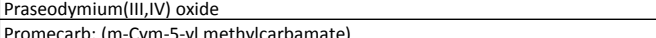 & $\frac{12037-29-5}{2631-37-0}$ & $012037-29-5$ & $\frac{1021.4396}{2073}$ & $\frac{s}{s}$ & & & & & & & $\begin{array}{l}\text { PAC 27 Listing Table } 1 \\
\text { PAC 27 Listing Table } 1\end{array}$ \\
\hline 2500 & 年 & 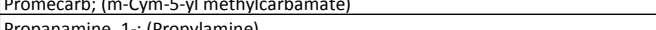 & 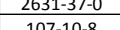 & 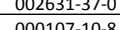 & 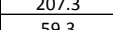 & & 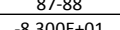 & 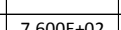 & 3.400t02 & 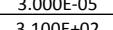 & $2.500+20101$ & (31005+02 & 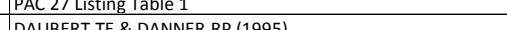 \\
\hline$\frac{2541}{2542}$ & 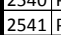 & $\begin{array}{l}\text { Propanamine, 1; (Proplyamine) } \\
\text { Propane }\end{array}$ & $74-108-8$ & 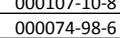 & $\frac{99.3}{44.1}$ & $\frac{L}{G}$ & $\begin{array}{ll}-0.030 \mathrm{E}+01 \\
-1.876 \mathrm{E}+02 \\
\end{array}$ & 1.00uetuz & 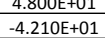 & 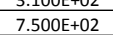 & 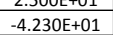 & & $\begin{array}{l}\text { DAUBREI, IE \& DANNER,RP (1995) } \\
\text { PAC } 27 \text { Listing Table } 1\end{array}$ \\
\hline 2542 & 2542 & Propane sultone, 1,3- & $1120-71-4$ & $001120-71-4$ & 122.14 & $\mathrm{~s}$ & $3.1006+01$ & & $180 @ 0.039$ & & & & PAC 27 Listing Table 1 \\
\hline & $2543 \mid \mathrm{F} / \mathrm{F}$ & Propanediamine, 1.2- & $78-90-0$ & $000078-90-0$ & 74.15 & & & $7.600 E+02$ & 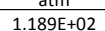 & $1.061 E+01$ & $2.500 E+01$ & $1.061 E+01$ & DAUBERT,TE \& DANNER,RP (1989) \\
\hline 2545 & 2544 & Propanediamine, $1,3-$ & $109-76-2$ & $000109-76-2$ & 74.15 & L & $-1.200 E+01$ & $7.600 E+02$ & $1.350 \mathrm{E}+02$ & $6.410 \mathrm{E}+00$ & $2.500 \mathrm{E}+01$ & $6.410 \mathrm{E}+00$ & EPI Suite Mean VP of Anttine and Grain Methods \\
\hline 2546 & 2545 & 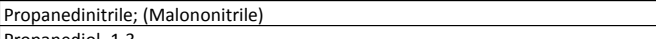 & $109-77-3$ & $000109-77-3$ & 66.07 & $\mathrm{~L}$ & $3.0506+01$ & $7.600 E+02$ & $2.200 \mathrm{E}+02$ & $2.004 E-01$ & $2.500 E+01$ & $2.004 \mathrm{E}-01$ & DAUBERT,TE \& DANNER,RP (1989) \\
\hline 2547 & 2546 & 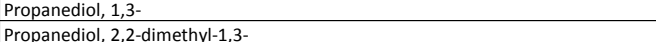 & $\frac{504-63-2}{126-30-7}$ & $000504-63-2$ & $\frac{76.11}{10417}$ & $\frac{1}{5}$ & 129 & 7.600EE+02 20 & $\frac{2.100 E+02}{2.0655+02}$ & 4.410E-02 & 2. $.500 E+01$ & 4.410E-02 & 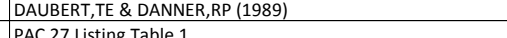 \\
\hline 2548 & $\frac{2547 \mid}{2548}$ & 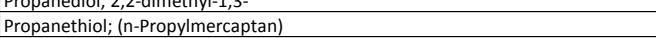 & $126-30-7$ & 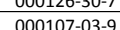 & 76.17 & & 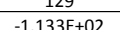 & $76000+022$ & $2.0606+02$ & 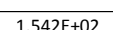 & $2500 \mathrm{~F}+01$ & $1542 F+02$ & 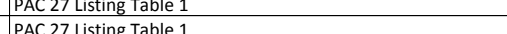 \\
\hline 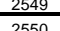 & 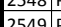 & 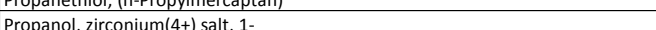 & 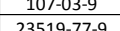 & 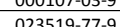 & 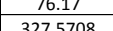 & $\frac{L}{G}$ & 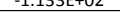 & $7.0006=02$ & & & 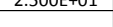 & $1.542 E+02$ & 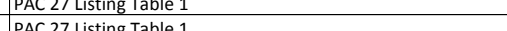 \\
\hline 2551 & 2550 & Propargyl alcohol & $107-19-7$ & $000107-19-7$ & 56.06 & $\frac{3}{L}$ & $-5.180 E+01$ & $7.600 E+02$ & $1.136 \mathrm{E}+02$ & $1.560 \mathrm{E}+01$ & $2.500 E+01$ & $1.560 E+01$ & PAC 27 listing Table 1 \\
\hline 2552 & 2551 & Propargyl bromide & $\begin{array}{ll}106-96-7 \\
\end{array}$ & $000106-96-7$ & 118.97 & $\mathrm{~L}$ & $-6.107 \mathrm{E}+01$ & $7.600 E+02$ & $8.800 \mathrm{E}+01$ & $6.740 \mathrm{E}+01$ & $2.500 \mathrm{E}+01$ & $6.740 \mathrm{E}+01$ & EPI Suite Mean VP of Antoine and Grain Methods \\
\hline 2553 & 2552 & Propiolactone, b- & $57-57-8$ & $000057-57-8$ & $\frac{72.06}{6589}$ & $\mathrm{~L}$ & $\begin{array}{l}-3.3400 \mathrm{E}+01 \\
-8.00501\end{array}$ & $\begin{array}{ll}7.600 E+02 \\
760 \mathrm{~F}+02\end{array}$ & $1.620 \mathrm{E}+02$ & $\begin{array}{l}7.5000=-01 \\
3.6550 .02\end{array}$ & $\begin{array}{lll}8.000 E+00 \\
25050101\end{array}$ & 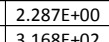 & \begin{tabular}{|l|l|} 
Estimated using data from PAC 27 Listing Table 1 \\
\end{tabular} \\
\hline$\frac{2554}{2555}$ & 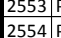 & \begin{tabular}{|l} 
Propionaldehyde \\
Propionic acid \\
\end{tabular} & $\frac{123-38-6}{79-09-4}$ & $\begin{array}{l}0001233-38-6 \\
000079-09-4 \\
\end{array}$ & $\frac{5.88}{74.09}$ & $\frac{L}{L}$ & $\frac{-8.000 E+01}{-2.150 E+01}$ & 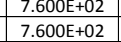 & 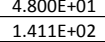 & $\frac{3.1 .68+5 \mathrm{E}+02}{3.50+00}$ & $\frac{2.500 \mathrm{E}+01}{2.500 \mathrm{E}+01}$ & & 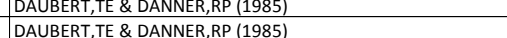 \\
\hline 2555 & 告554] & 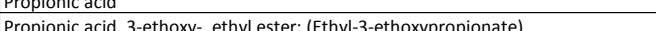 & 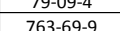 & 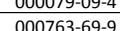 & $\frac{14.09}{14621}$ & & 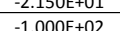 & $\frac{1.60060+02}{7.60502}$ & 1.4141502 & & $\begin{array}{ll}2.30060+11 \\
250 F+01\end{array}$ & 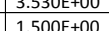 & 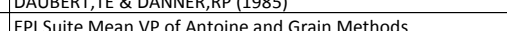 \\
\hline$\frac{2557}{2557}$ & 2556 256 & Propionic acid, sodium salt & $\frac{137-40-6}{137}>0$ & 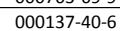 & $\frac{19.607}{96.07}$ & $\frac{5}{\mathrm{~s}}$ & (2) & 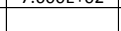 & & & & (2) & $\begin{array}{l}\text { PAC } 27 \text { Listing Table } 1 \\
\end{array}$ \\
\hline 2558 & 2557 & Propionic anhydride & $\begin{array}{ll}123-62-6 \\
\end{array}$ & $000123-62-6$ & 130.16 & $\mathrm{~L}$ & $-4.500 E+01$ & $7.600 E+02$ & $1.670 \mathrm{E}+02$ & $1.360 \mathrm{E}+00$ & $2.5000++01$ & $1.360 \mathrm{E}+00$ & DAUBERT, TE \& DANNER,RP (1989) \\
\hline 2559 & 2558 & 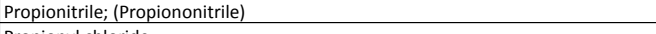 & $107-12-0$ & $000107-12-0$ & 55.09 & $\mathrm{~L}$ & $-1.035 \mathrm{E}+02$ & $7.600 E+02$ & $9.710 \mathrm{E}+01$ & 4.7396+01 & 2.500e+01 & $4.7396+01$ & DAUBERT,TE \& DANNER,RP (1985) \\
\hline 25601 & 25690 & 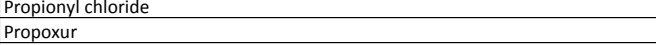 & $\begin{array}{c}71-93-2-8 \\
11-2-1 \\
\end{array}$ & $\begin{array}{l}0.00079-0.0-8 \\
000114-26-1 \\
\end{array}$ & 92.53 & $\frac{\mathrm{L}}{\mathrm{s}}$ & $\begin{array}{ll}\frac{-9.400 E+01}{8.700 E+01} \\
\end{array}$ & $7.600 \mathrm{E}+02$ & $\begin{array}{c}8.000 \mathrm{de}+01 \\
\mathrm{dec}\end{array}$ & $\frac{9.60 \mathrm{~F}+01}{9.68 \times 10-6}$ & $\frac{2.500 E+01}{2.000++01}$ & $9.660 \mathrm{E}+01$ & \begin{tabular}{|l} 
PF I Iutete Mean V V of Antoine and Grain Methods \\
PAC 27 Listing Table 1
\end{tabular} \\
\hline & $\left.2561\right|_{\mathrm{F}}$ & Propoxypropanol, n; (Propylene glycol monpropyl ther) & 30136-13-1 & $030136-13-1$ & 118.2 & $\mathrm{~L}$ & $-8.000 E+01$ & $7.600 E+02$ & $1.498 E+02$ & $7.040 \mathrm{E}-01$ & $2.5006+01$ & $7.040 \mathrm{E}-01$ & chemSpider EPI Suite Mean VP of Antoine and Grain Methoo \\
\hline 2563 & 2562 & Propyl acetate, $n-$ & $109-60-4$ & $000109-60-4$ & 102.15 & $\mathrm{~L}$ & $-9.300 E+01$ & $7.600 \mathrm{E}+02$ & $1.015 E+02$ & $3.5906+01$ & $2.5006+01$ & $3.5906+01$ & PAC 27 Listing Table 1 \\
\hline 2564 & & Propyl alcohol, n; (n-Propanol) & $71-23-8$ & $000071-23-8$ & 60.11 & $\mathrm{~L}$ & $-1.270 E+02$ & $7.600 E+02$ & $9.7196+01$ & $2.0996+01$ & $2.500 E+01$ & $2.0996+01$ & RIDDICK,JA ET AL. (1986) \\
\hline 2565 & 2564||$^{25}$ & $\begin{array}{l}\text { Propyl chloroformate; (Propyl chlorocarbonate) } \\
\text { PProyy nitrate }\end{array}$ & $\frac{109-61-5}{6627-13-4}$ & $0000109-613-5$ & $\frac{122.56}{10511}$ & & $-1.010 \mathrm{E}+02$ & $\begin{array}{l}7.680 \mathrm{E}+02 \\
7.600+502\end{array}$ & $\begin{array}{l}1.140 \mathrm{E}+02 \\
1.105+02\end{array}$ & $\begin{array}{l}2.000 E+01 \\
2.36+0101\end{array}$ & $\begin{array}{l}2.500 E+01 \\
2.500+5+01\end{array}$ & $\begin{array}{l}2.000 E+01 \\
23265+01\end{array}$ & | DAMLE,SB (1993) \\
\hline$\frac{2566}{2567}$ & 25656 & 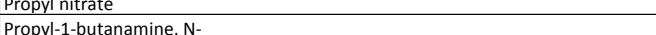 & $\frac{62 / 1-13-4}{20193-21-9}$ & 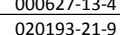 & $\frac{105.11}{115.218}$ & L & 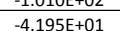 & $\frac{1.00070 \mathrm{~F}}{7.602}$ & 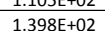 & 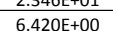 & 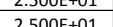 & 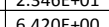 & 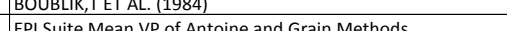 \\
\hline$\frac{2506}{258}$ & $\left.2567\right|_{\mathrm{F}}$ & Propylbenze & $103-65-1$ & $000103-65-1$ & 120.19 & $\mathrm{~L}$ & $-9.9606+01$ & $7.6006+02$ & $1.592 \mathrm{E}+02$ & $3.420 \mathrm{E}+00$ & $2.500 \mathrm{E}+01$ & $3.420 \mathrm{E}+00$ & 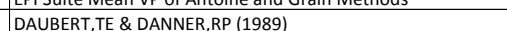 \\
\hline 2569 & $\left.2568\right|_{\mid}$ & Propylene carbonate, $1,2-$ & 108-32-7 & & 102.09 & L & $-4.8806+01$ & $7.600 E+02$ & $2.420 \mathrm{E}+02$ & & & & DAUBERT,TE \& DANNER,RP \\
\hline 2570 & 2569 & Propylene glycol dinitrate & $6423-43-4$ & $006423-43-4$ & 166.11 & $\mathrm{~L}$ & $-7.778 E+00$ & & & $3.7800-01$ & 2.500E+01 & $3.7800-01$ & WYMAN, JF ET AL. (1984) \\
\hline 2571 & $2570 \mid$ & 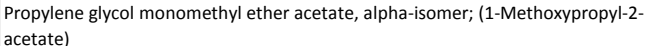 & $108-65-6$ & $000108-65-6$ & 132.18 & $\mathrm{~L}$ & & $7.600 E+02$ & $1.500 \mathrm{E}+02$ & $3.921 E+00$ & $2.500 E+01$ & $3.921 E+00$ & DAUBERT, TE \& DANNER,RP (1994) \\
\hline 2572 & 2571 & $\begin{array}{l}\text { Propylene glycol monomethyl ether actate, beta-isomer; (2-Methoxypropoyl-1- } \\
\text { acectate) }\end{array}$ & 70657-70-4 & $070657-70-4$ & 132.18 & $\mathrm{~L}$ & $-5.031 \mathrm{E}+01$ & $7.600 E+02$ & $1.358++02$ & 7.720E+00 & 2.500E+ +01 & $7.720 E+00$ & EPI Suite Mean VP of Antoine and Grain Methods \\
\hline$\frac{2572}{2573}$ & & Propylene glyycol monomethyl there; (Ucar Triol HG-170) & $107-98-2$ & $000107-98-2$ & 90.12 & $\mathrm{~L}$ & $-9.500 E+01$ & $7.600 E+02$ & $1.190 E+02$ & $1.250 \mathrm{E}+01$ & $2.5006+01$ & $1.250 €+01$ & PAC 27 Listing Table 1 \\
\hline 2574 & 2573 & Propylene Elycol mono-n-butyl ether; (3-Butoxy-1-propanol) & $10215-33-5$ & $010215-33-5$ & 132.23 & $\mathrm{~L}$ & $-8.750 E+00$ & $7.600 E+02$ & $2.0145+02$ & $6.7600-02$ & $2.5000++01$ & $6.760 \mathrm{E}-02$ & EPI Suite Mean VP of Antoine and Grain Methods \\
\hline 2575 & $2574 \mid$ & Propylene glycol; (1,2-Propanediol) & $\frac{57-55-6}{75-569}$ & $000057-55-6$ & $\frac{76.1}{5508}$ & L & 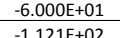 & $\begin{array}{l}7.6006+02 \\
7.6005+02\end{array}$ & $1.876 \mathrm{E}+02$ & 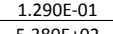 & $\begin{array}{l}2.500 E+01 \\
2.5050 .12\end{array}$ & $\frac{1.2906-01}{5.20050 .02}$ & DAUBERT,TE \& DANNER,RP (1989) \\
\hline 2576 & 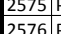 & $\begin{array}{l}\text { Propylene oxide; (Methyl lethylene oxide } \\
\text { Propyene; (1-Pronene) }\end{array}$ & $\frac{75-56-9}{115-7-1}$ & $\begin{array}{l}000075-56-9 \\
000115-07-1\end{array}$ & $\frac{58.08}{4209}$ & $\frac{L}{G}$ & 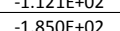 & & $\begin{array}{l}3.435+601 \\
-4770 \mathrm{E}+1\end{array}$ & $\begin{array}{l}3.3500+202 \\
760 F+03\end{array}$ & $2.500+E+01$ & & PAC2 L L Listing \\
\hline$\frac{2578}{2578}$ & 577 & Propoleneiminin 12. & $\frac{1555-1}{77-55}$ & $000075-55-8$ & 5.71 & 5 & - $6.500 \mathrm{~F}+01$ & $7.600 E+02$ & $6.700+101$ & $1400 \mathrm{EF}+02$ & $2500+5+1$ & $1.400 E+02$ & 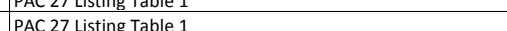 \\
\hline 2579 & 2578 & \begin{tabular}{|l|l|} 
Propyltrichlorosilane \\
\end{tabular} & $141-57-1$ & .000141-57-1 & 177.54 & $\mathrm{~L}$ & & $7.600 E+02$ & $1.235 E+02$ & $2.880 \mathrm{E}+01$ & $2.500 \mathrm{E}+01$ & $2.8806+01$ & 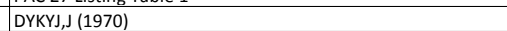 \\
\hline 2580 & 2579 & Prothoate; (Isopropyl diethyldithioph & $2275-18-5$ & $002275-18-5$ & 285.39 & $\mathrm{~s}$ & 2.850E+01 & & $3.300 E+02$ & $1.000 E-04$ & $2.500 E+01$ & & AC 27 Listing Table 1 \\
\hline & $81 / 6$ & & $64742-65-0$ & $\begin{array}{l}0.0442-55-0 \\
0.0020-0.0\end{array}$ & 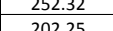 & 5 & $1506=+02$ & $7.600 \mathrm{E}+022$ & 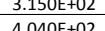 & 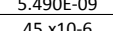 & 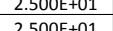 & & AC 27 listing otable \\
\hline
\end{tabular}




\begin{tabular}{|c|c|c|c|c|c|c|c|c|c|c|c|c|c|}
\hline & A) & & & & Meleswar & $\frac{F}{\text { QV State }}$ & & $\mathrm{H}_{\mathrm{H}}$ & 1 & & $\mathrm{~K}$ & & \\
\hline & No. & Chemical Compound & $\begin{array}{l}\text { CAS Number } \\
\text { (CASRN) }\end{array}$ & $\begin{array}{l}\text { Modified } \\
\text { Format CAS }\end{array}$ & \begin{tabular}{|c} 
Molecular \\
Weight (MW)
\end{tabular} & $\begin{array}{c}\text { at } \\
25^{\circ} \mathrm{C}\end{array}$ & $\begin{array}{l}\text { MP or fP } \\
{\left[{ }^{\circ}\right]}\end{array}$ & $\begin{array}{c}\begin{array}{c}\text { BP Pressure } \\
{[\mathrm{mm} \mathrm{Hg}]}\end{array} \\
0\end{array}$ & $\mathrm{BP}\left[{ }^{[\mathrm{C} C]}\right.$ & $\begin{array}{l}\text { Vapor } \\
\text { Pressure } \\
\text { Imm Hg }\end{array}$ & $\begin{array}{l}\text { Tfor VP } \\
\text { ['C] }\end{array}$ & $\begin{array}{l}\text { VP at } 25^{\circ} \mathrm{C} \\
{[\mathrm{mm} \mathrm{Hg}]}\end{array}$ & Vapor Pressure @ $25^{\circ}$ C Reference \\
\hline 2583 & $\left.2582\right|^{5}$ & 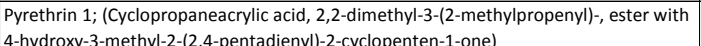 & 121-21-1 & $000121-21-1$ & 328.49 & $\mathrm{~L}$ & & $1.000 E+00$ & $1.700 E+02$ & $2.030 \mathrm{E}-05$ & $2.500 E+01$ & $2.030 \mathrm{E}-05$ & TOMLIN,C (1997) \\
\hline & 2583 & Pyridine & $110-86-1$ & $000110-86-1$ & 79.1 & $\mathrm{~L}$ & $-4.1706+01$ & $7.600 E+02$ & 1.152E+02 & $2.080 E+01$ & $2.500 E+01$ & $2.080 \mathrm{E}+01$ & PAC 27 Listing Table 1 \\
\hline 2585 & 2584 & Pyridinecarboxaldehyde, 4; (Isonicotinaldehydd) & $872-85-5$ & $000872-85-5$ & 107.12 & $\mathrm{~L}$ & $1.200 E+01$ & $7.600 E+02$ & $7.100 E+01$ & 5.680E-01 & $2.500 E+01$ & $5.680 \mathrm{E}-01$ & EPP Suite Mean VP of Antoine and Grain Methods \\
\hline 2586 & $2585 \mid$ & Pyridine-d5 & 7291-22-7 & 007291-22-7 & 84.13 & $\mathrm{~L}$ & $-4.200 E+01$ & $7.600 E+02$ & $1.144 \mathrm{E}+02$ & $2.080 \mathrm{E}+01$ & $2.500 \mathrm{E}+01$ & $2.080 \mathrm{E}+01$ & ChemSpider EP S Suite Mean VP of Antoine and Grain Methods \\
\hline 2587 & 2586 & Pyriminili; (Pyriminyl) & $53558-25-1$ & $053558-25-1$ & 272.29 & $\mathrm{~s}$ & $223-225$ & & & $1.0000-05$ & $2.500 E+01$ & & PAC 27 Listing Table 1 \\
\hline 2588 & 2587 & Pyrogallic acid & $87-66-1$ & $000087-66-1$ & 126.12 & $\mathrm{~s}$ & $131-133$ & & $3.0906+02$ & $1.000 \mathrm{E}+01$ & $1.677 \mathrm{E}+02$ & & PAC 27 Listing Table 1 \\
\hline$\frac{2589}{2590}$ & $\begin{array}{l}2588 \\
2589\end{array}$ & $\begin{array}{l}\text { Pyryomellitic acid } \\
\text { Pyroxxini (Cellulose tetranitrate) }\end{array}$ & 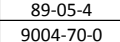 & 000089-05-4 & 254.16 & $\frac{s}{s}$ & $257-265$ & & & & & & $\begin{array}{l}\text { PAC } 27 \text { Listing Table } 1 \\
\text { PAC 7 Listin Table } 1\end{array}$ \\
\hline$\frac{2590}{2591}$ & $\begin{array}{l}\left.25599\right|^{2} \\
2590\end{array}$ & 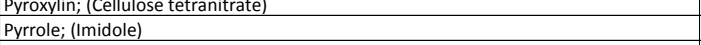 & 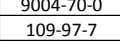 & $\begin{array}{ll}0.090044-0-0 \\
000109-97-7 \\
\end{array}$ & 507.3 & $\frac{3}{L}$ & $-2.340 E+01$ & $7.600 E+02$ & $1.297 \mathrm{E}+02$ & $8.3506+00$ & 2.500E+01 & $8.350 \mathrm{E}+00 \mathrm{O}$ & $\begin{array}{l}\text { PAC27 Listing tabble } 1 \\
\text { PAC } 27 \text { Listing Table } 1\end{array}$ \\
\hline & & & & $000123-75-1$ & & & & $7.600 \mathrm{E}+02$ & & & & 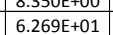 & 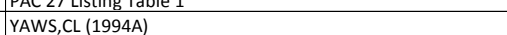 \\
\hline 2593 & 2592 & Pyrrolidininon, 2 - & $616-45-5$ & $000616-45-5$ & 85.12 & $\mathrm{~s}$ & $2.460 \mathrm{E}+01$ & & $2.4506+02$ & $9.4906-03$ & $2.500 \mathrm{E}+01$ & & PAC 27 Listing Table 1 \\
\hline 2594 & 2593 & $\begin{array}{l}\text { Pryuate kinase } \\
\text { Quaterrara yammonium compounds, benzyl-C12-c16-alkyldimethyl, chlorides }\end{array}$ & 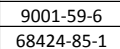 & $\frac{009001-59 \cdot 6}{0.08824-1}$ & & $\frac{s}{s}$ & & & & & & & $\begin{array}{l}\text { PAC 27 Listing Table } 1 \\
\text { PAC C7 Listin Table } 1\end{array}$ \\
\hline & 2595 c & Quaternary ymmonium compounds, coco alkylbishhydroxyethyl|'methyl, chlorides; & 70750-47-9 $>0$ & $070750-47-9$ & & $\mathrm{~s}$ & & & & & & & PAC 27 Listing Table 1 \\
\hline & & (Methyl bis(2--hydroxxyethy)) cocoalkyl quaternary ammonium chlorides) & (0) & & & & & & & & & & \\
\hline 2597 & 2596 & 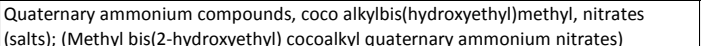 & $71487-00-8$ & $071487-00-8$ & & $\mathrm{~s}$ & & & & & & & PAC 27 Listing Table 1 \\
\hline 2598 & $2597 \mathrm{C}$ & Quinhydrone & $106-34-3$ & $000106-34-3$ & 220.24 & $\mathrm{~s}$ & $1.710 \mathrm{E}+02$ & & & & & & PAC 27 Listing Table 1 \\
\hline$\frac{2599}{2600}$ & 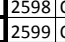 & $\begin{array}{l}\text { Quinoline } \\
\text { Quinolinol, 8- }\end{array}$ & 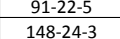 & 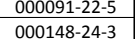 & $\frac{129.16}{145.17}$ & $\frac{L}{S}$ & $\frac{-1.477 \mathrm{E}+01}{7.600 \mathrm{E}+01}$ & $7.600 E E+022$ & $\begin{array}{l}2.372 E+02 \\
2.670+02\end{array}$ & $6.000 \mathrm{E}-02$ & $2.500 \mathrm{E}+01$ & $6.000 \mathrm{E}-02$ & $\begin{array}{l}\text { DAUCERT TLE \& DANERR, } \\
\text { PAC 27 tisting Table } 1\end{array}$ \\
\hline & 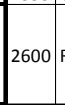 & Red Arrow Insect Spray & z-0117 & 00000z-0117 & & $\mathrm{L}$ & & & & $7.600 \mathrm{~F}+02$ & $2.500 \mathrm{E}+01$ & $7.600 E+02$ & $\begin{array}{l}\text { No data available. As this is a product in a spraycan, it is } \\
\text { pressumed that materiali is under pressure in the a arosol } \\
\text { dispenser; a v of } 760 \text { at } 25^{\circ} \text { Ci sasigned for calculation } \\
\text { puruposes. }\end{array}$ \\
\hline 2602 & 2601 & Resorcinol & $108-46-3$ & $000108-46-3$ & 110.11 & $\mathrm{~s}$ & $1.094 \mathrm{E}+02$ & & $2.765 \mathrm{E}+02$ & $4.89 \times 10-4$ & $2.500 \mathrm{E}+01$ & & PAC 27 Listing Table 1 \\
\hline 2603 & 2602 & 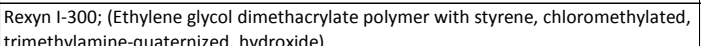 & $69011-49-0$ & 069011-49-0 & 361.4586 & $\mathrm{~s}$ & & & & & & & PAC 27 Listing Table 1 \\
\hline 2604 & 2603 & Rhenium hexafluoride & $10049-17-9$ & $010049-17-9$ & 300.197 & $\mathrm{~L}$ & $1.850 \mathrm{E}+01$ & $7.600 \mathrm{E}+02$ & $3.360 \mathrm{E}+01$ & $8.480 \mathrm{E}-09$ & $2.500 E+01$ & $8.480 \mathrm{E}-09$ & EPI Suite Modified Grain Method \\
\hline & 2604 & Rhenium oxide; (Rhenium(VIII) oxide) & 1314-68-7 & $001314-68-7$ & 484.41 & $s$ & $2.9706+02$ & & $\begin{array}{l}3.6006+02 \\
.7205023\end{array}$ & & & & 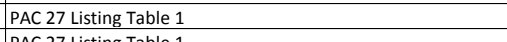 \\
\hline 2606 & 2605 & Rhodium & $7440-16-6$ & $007440-16-6$ & 102.91 & $\mathrm{~s}$ & $1.966 E+03$ & & 3.727₹E+03 & & & & PAC 27 Listing Table 1 \\
\hline & $2606 \mid$ & Rhodium oxide (liquids); (Rhodium(IV) oxide) & $12137-27-8 \mathrm{a}$ & 012137-27-8a & 134.91 & $\mathrm{~L}$ & & & & $7.600 E+02$ & $2.500 \mathrm{E}+01$ & $7.600 E+02$ & $\begin{array}{l}\text { Hazardous component of aqueous solution has neglible VP. A A } \\
\text { vP of } 11-05 \text { a t2 } 25^{\circ} \mathrm{C} \text { is assigned for calulation purposes }\end{array}$ \\
\hline 2608 & 2607 & Rhodium oxide (solids); (Rhodium((IV) oxide) & $12137-27-8 \mathrm{~b}$ & $012137-27-8 \mathrm{~b}$ & 134.91 & $\mathrm{~s}$ & & & & & & & PAC 27 Listing Table 1 \\
\hline & $2608 \mid$ & Rhodium(III) hydroxide (liquids) & 21656-02-0a & 02165-02-0a & 153.93 & $\mathrm{~L}$ & & & & $1.000=-05$ & $2.500 \mathrm{E}+01$ & $1.000 E-05$ & $\begin{array}{l}\text { Hazardous component of aqueous solution has neglible VP.A A } \\
\text { vP of } 11-05 \text { at } 25^{\circ} \text { i is assigned for callutation purposes }\end{array}$ \\
\hline$\frac{2610}{2611}$ & & \begin{tabular}{|l|l} 
Rhodium(IIII) hydroxide (solids) \\
Rhodium(II)
\end{tabular} & $21656-02-0 b$ & $\frac{021656-02 \cdot 0 \mathrm{~b}}{0123250 \mathrm{~s}}$ & $\frac{153.93}{253092}$ & $\frac{s}{s}$ & & & & & & & 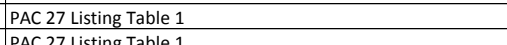 \\
\hline 2611 & & Rhodium(III) oxide (solids) & $12036-35-0$ & $012036-35-0$ & 253.8092 & $\mathrm{s}$ & $1100 \mathrm{dec}$ & & & & & & PAC 27 Listing Table 1 \\
\hline$\frac{2612}{2613}$ & $\frac{2611}{2612}$ & $\begin{array}{l}\text { Riboflavine } \\
\text { Ribonuclease T1 }\end{array}$ & $\frac{83-88-5}{9026-4}$ & $\begin{array}{ll}0.00083-38-5 \\
009026-12-4\end{array}$ & 376.37 & $\frac{s}{s}$ & 282 & & dec & & & & $\begin{array}{l}\text { PAC C7 L Listing Table } 1 \\
\text { PAC 27 isting Table } 1\end{array}$ \\
\hline 2614 & 2613 & Rosin core solder decomposition products; (Colophony Gum) & $8050-09-7$ & $008050-09-7$ & & $\mathrm{~s}$ & & & & & & & PAC 27 Listing Table 1 \\
\hline 2615 & 2614 & Rotenone & $83-79-4$ & $000083-79-4$ & 394.42 & s & $1.7606+02$ & & $\begin{array}{c}215 @ 0.5 \\
\mathrm{mmHg}\end{array}$ & $8.000 E-04$ & $2.000 E+01$ & & PAC 27 Listing Table 1 \\
\hline 2616 & 2615 & Rubber solvent; (Naphtha (petroleum) light aliphatic) & $64742-89-8$ & $064742-89-8$ & 97.26 & $\mathrm{~L}$ & $-9.060 \mathrm{E}+01$ & $7.600 E+02$ & $3.800 \mathrm{E}+01$ & $4.600 E+01$ & $2.500 \mathrm{E}+01$ & 4.600E+01 & EPI Suite Experimental Data \\
\hline$\frac{2617}{2618}$ & $\frac{2616}{2617}$ & $\begin{array}{l}\text { Rubidium } \\
\text { Rubidium bromide }\end{array}$ & $\frac{74409-1-7}{7789-3-1}$ & $\begin{array}{l}007440-17-7 \\
000789-39-1\end{array}$ & 85.47 & $\frac{\mathrm{s}}{\mathrm{s}}$ & 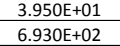 & & $\begin{array}{l}6.808 \mathrm{E}+02 \\
1.30 \mathrm{E}+03 \\
\mathrm{n}\end{array}$ & & & & $\begin{array}{l}\text { PA 27 fisting Table } 1 \\
\text { PAC } 27 \text { Lsting Table } 1\end{array}$ \\
\hline 2619 & & Rubidium chloride & $7791-11-9$ & 007791 & 120.92 & $\mathrm{~s}$ & $7.180 \mathrm{E}+02$ & & $1.390 \mathrm{E}+03$ & & & & isting Table 1 \\
\hline 2620 & 2619 & $\begin{array}{l}\text { Rubidium hydroxide } \\
\text { Rutidium nitroto }\end{array}$ & $1310-82-3$ & $001310-82-3$ & 102.48 & $G$ & $3.820 \mathrm{E}+02$ & & & & & & 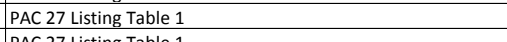 \\
\hline$\frac{2621}{2622}$ & $\frac{2620}{2621}$ & $\begin{array}{l}\text { Rubidium nitrate } \\
\text { Ruthenium }\end{array}$ & $\frac{13126-12-0}{7440-18-8}$ & & $\begin{array}{l}147.48 \\
10.07\end{array}$ & $\frac{\mathrm{s}}{\mathrm{s}}$ & $2.333 E+03$ & & $4.150 \mathrm{E}+03$ & $7.5 \times 10-3$ & $2.315 E+03$ & & $\begin{array}{l}\text { PAA 27 listing Ta } \\
\text { PAC 27 listing Ta }\end{array}$ \\
\hline 2623 & & Ruthenium trichloride & $10049-08-8$ & 01004 & 207.42 & $\mathrm{~s}$ & $500 \mathrm{dec}$ & & & & & & PAC 27 Listing Table 1 \\
\hline 2624 & & Ruthenium(IV) oxide & $12036-10-1$ & 01203 & 133.07 & $G$ & 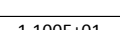 & & $2,2255+07$ & & & & PAC 27 Listing Table 1 \\
\hline$\frac{2625}{2626}$ & 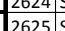 & 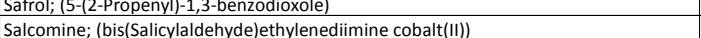 & $\begin{array}{l}94-59-7 \\
1416-18-1\end{array}$ & $000094-59-7$ & $\frac{162.2}{32525}$ & $\frac{i}{s}$ & 1.1.UUEE+U1 & 1. $1.600 \mathrm{E}+02$ & $2.325+02$ & 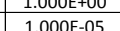 & 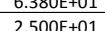 & $7.522 E-0.0$ & ta from PAC 27 \\
\hline 2627 & $2626 \mid$ & Salicylaldehyde & $\frac{90-02-8}{94-18}$ & $000090-02-8$ & 122.13 & $\mathrm{~L}$ & $-7.0000++00$ & $7.600 \mathrm{E}+02$ & $1.970 E+02$ & $5.930 E-01$ & $2.500 E+01$ & $5.9300-01$ & $\begin{array}{l}\text { PAC2 2Lsting a abbe 1 } \\
\text { PAC 27 Listing Table } 1\end{array}$ \\
\hline 2628 & 2627 s & Salicylic acid & $69-72-7$ & $000069-72-7$ & 138.13 & $\mathrm{~s}$ & $1.583 E+02$ & & $\begin{array}{c}211 @ \\
20 \mathrm{~mm}\end{array}$ & & & & PAC 27 Listing Table 1 \\
\hline 2629 & & Salicylic acid, monoammonium salt & $528-94-9$ & $000528-94-9$ & 155.17 & $\mathrm{~s}$ & & & & & & & \begin{tabular}{|l} 
PAC 27 Listing Table 1 \\
\end{tabular} \\
\hline 2630 & $2629 \mathrm{~s} s$ & Salicylic acid, phenyl ester & $118-55-8$ & $000118-55-8$ & 214.23 & $\mathrm{~s}$ & 43 & & 172-173@ & & & & PAC 27 Listing Table 1 \\
\hline$\frac{2631}{2632}$ & $\frac{263015}{25315}$ & \begin{tabular}{|l} 
Samarium \\
Samive to
\end{tabular} & \begin{tabular}{|l|l|}
$7440-19-9$ \\
1201929
\end{tabular} & $\begin{array}{ll}007440-19-9 \\
01201929 \\
\end{array}$ & 150.36 & $\mathrm{~s}$ & $1.072 E+03$ & & $\frac{1.778 \mathrm{E}+03}{1.73}$ & & & & 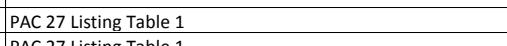 \\
\hline
\end{tabular}




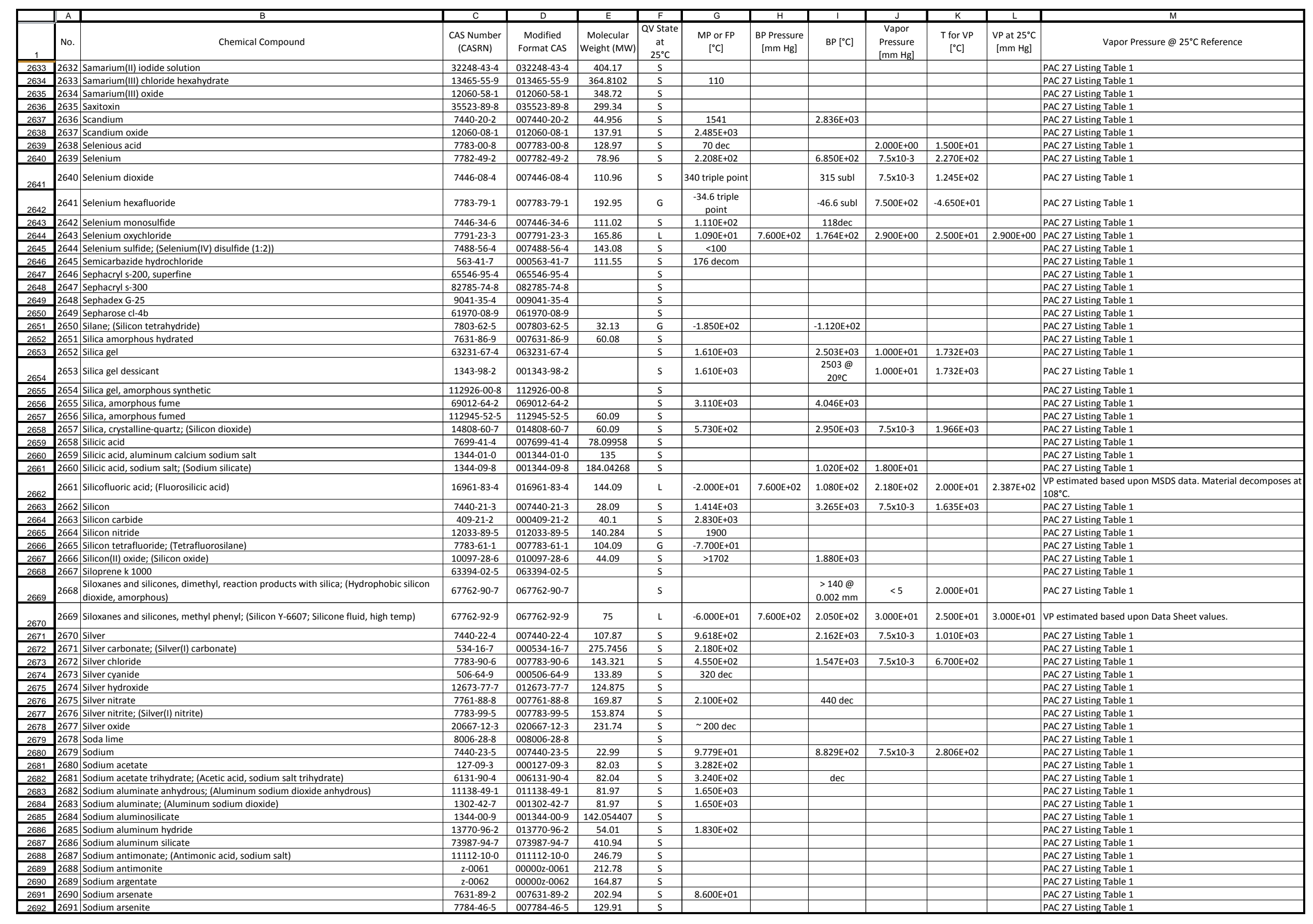




\begin{tabular}{|c|c|c|c|c|c|c|c|c|c|c|c|c|c|}
\hline & AT & & & $\mathrm{D}$ & $E$ & $\frac{F}{Q \text { Qv State }}$ & $G$ & $\begin{array}{rll}\mathrm{H} \\
\mathrm{H}\end{array}$ & 1 & & $\mathrm{~K}$ & & $\mathrm{M}$ \\
\hline & No. & Chemical Compound & $\begin{array}{l}\text { CAS Number } \\
\text { (CASRN) }\end{array}$ & $\begin{array}{l}\text { Modified } \\
\text { Format CAS }\end{array}$ & $\begin{array}{c}\text { Molecular } \\
\text { Weight (MW) }\end{array}$ & $\begin{array}{c}\text { at } \\
25^{\circ} \mathrm{C}\end{array}$ & $\begin{array}{c}\text { MP or FP } \\
{\left[{ }^{\circ} \mathrm{C}\right]}\end{array}$ & 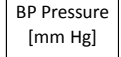 & $\mathrm{BP}\left[{ }^{[\mathrm{C} C}\right]$ & $\begin{array}{l}\text { Vapor } \\
\text { Pressure } \\
\text { Imm Hgl }\end{array}$ & $\begin{array}{c}\text { Tf for vp } \\
{\left[{ }^{\circ} \mathrm{C}\right]}\end{array}$ & $\begin{array}{l}\text { VP at } 25^{\circ} \mathrm{C} \\
{[\mathrm{mm} \mathrm{Hg}]}\end{array}$ & Vapor Pressure @ $2^{\circ} \mathrm{C}$ Reference \\
\hline 2693 & 26925 & Sodium azide & $26628-22-8$ & $026628-22-8$ & \begin{tabular}{|c|c|c|c|}
65.01 \\
11002
\end{tabular} & $\mathrm{~s}$ & $300 \mathrm{dec}$ & & & $7.5 \times 10.3$ & $2.000++01$ & & $\begin{array}{l}\text { PAC } 27 \text { Listing Table } 1 \\
\text { PAC } 7 \text { lictiog TThlo1 }\end{array}$ \\
\hline 2694 & 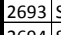 & Sodium berllium oxide & 2-0063 & $0000002-0063$ & 112.0021 & $\mathrm{~s}$ & & & & & & & PAC 27 Listing Table 1 \\
\hline$\frac{2695}{2696}$ & 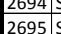 & $\begin{array}{l}\text { Sodulum bicarabonate } \\
\text { Sodium bifluoride; (Sodium hydrogen fluoride) }\end{array}$ & $\frac{14445-8}{1333-83-1}$ & 001333-53-8 & $\frac{84.01}{62}$ & $\frac{s}{s}$ & $\frac{\sim 50 \text { dec }}{\text { decom }}$ & & & & & & $\begin{array}{l}\text { PAC 27 Listing Table } 1 \\
\text { PAC 27litsing Talele } 1\end{array}$ \\
\hline 2697 & 2696 & Sodium bis(2-methoxyethoxy) aluminum hydride & $22722-98-1$ & $\frac{102532-58-1}{0.0272-1}$ & 202.16 & s & & & $396-402$ & & & & $\begin{array}{l}\text { PAC } 27 \text { Listing Table } 1 \\
\text { Pats }\end{array}$ \\
\hline & & & $12232-99-4$ & $012232-99-4$ & 279.97 & $\mathrm{~s}$ & & & & & & & $\begin{array}{l}\text { AC } 27 \text { Listing Table } 1 \\
\text { PA }\end{array}$ \\
\hline 2699 & $2698 \mid s$ & Sodium bisulfate monohydrate & $10034-88-5$ & $010034-88-5$ & 138.08 & $\mathrm{~s}$ & & & & & & & $\begin{array}{l}\text { PAC } 27 \text { Listing Table } 1 \\
\end{array}$ \\
\hline 2700 & 26999 S & Sodium bisulfate; (Sodium acid sulfate) & 7681-38-1 & 007681-38-1 & 120.06 & $\mathrm{~s}$ & >315dec & & & & & & PAC 27 Listing Table 1 \\
\hline 27011 & $2700 \mathrm{~s} s$ & $\begin{array}{l}\text { Sodium bisulfite } \\
\text { Sodium borte decahydrate }\end{array}$ & $\begin{array}{l}7631-90-5 \\
132039-5\end{array}$ & (007361-190-5 & 10.06 & $\frac{s}{c}$ & 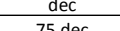 & & & $\sim 0$ & & & 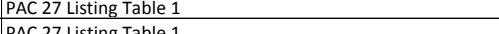 \\
\hline 2703 & 2702: & Sodium borate; (Disodium tetraborate; Borates, tetrasodium salts) & 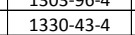 & 010130-03-4 & 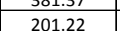 & 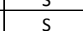 & 743 & & $1.575 E+03$ & neelijiple & 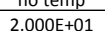 & & $\begin{array}{l}\text { PAC 2/ LLting labbe } 1 \\
\text { PAC 27 Listing Table } 1\end{array}$ \\
\hline & 27003 & Sodium borohydride & $16940-66-2$ & $016940-66-2$ & 37.84 & $\mathrm{~s}$ & $497 \mathrm{dec}$ & & & & & & PAC 7 Listing Table 1 \\
\hline$\frac{2705}{2705}$ & 2704:s & & $\frac{7789-38-0}{778}$ & $\frac{007789-38-0}{0.0}$ & $\frac{150.9}{150.9}$ & $\mathrm{~s}$ & 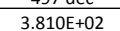 & & & & & & $\begin{array}{l}\text { AC } 27 \text { Listing Table } 1 \\
\text { PA }\end{array}$ \\
\hline 2706 & $2705 / \mathrm{s}$ & Sodium bromide & $7647-15-6$ & $007647-15-6$ & 102.9 & $\mathrm{~s}$ & $7.470 \mathrm{E}+02$ & & $1.3900+03$ & & & & \begin{tabular}{|l|l|l|} 
PAC 27 Listing Table 1 \\
\end{tabular} \\
\hline 2707 & 2706 s & Sodium buty| (2-ethylhexyl)phosphate & z-0064 & $000002-0064$ & 288.299428 & $\mathrm{~s}$ & & & & & & & PAC 27 Listing Table 1 \\
\hline$\frac{2708}{2709}$ & 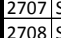 & $\begin{array}{l}\text { Sodium butyl butlyphosphonate } \\
\text { Sodium cacodylate (Sodium dimethylarsinate) }\end{array}$ & $\frac{2-0065}{124-65-2}$ & $000002-0065$ & 望2.19191908 & $\frac{s}{c}$ & $20005+02$ & & & & & & $\begin{array}{l}\text { PAC 27 Listing Table } 1 \\
\text { PAA 27lusting Tate } 1\end{array}$ \\
\hline$\frac{2710}{2710}$ & 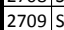 & Sodium cadminate & $2-0066$ & $000002-0066$ & $\frac{226.42}{22.42}>20$ & $\mathrm{~s}$ & & & & & & & PAC L Listing Table 1 \\
\hline 2711 & 2710 s & Sodium carbonate & $497-19-8$ & $000497-19-8$ & 105.99 & $\mathrm{~s}$ & $8.510 \mathrm{E}+02$ & & dec & & & & PAC 7 Listing Table 1 \\
\hline & $2711 \mathrm{~s}$ & Sodium carbonate monohydrate & 5968-11-6 & 005968-11-6 & 124.005 & $\mathrm{~s}$ & $8.510 \mathrm{E}+02$ & & & & & & PAC 27 Listing Table 1 \\
\hline$\frac{2713}{2714}$ & 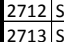 & $\begin{array}{l}\text { Sodium carboxymethyl cellulose; (Dowex 11) } \\
\text { Sodium chloride }\end{array}$ & $\frac{9004-32-4}{77647-14}$ & $000904-32-4$ & 58.44 & $\frac{s}{s}$ & $8.007 €+02$ & & $1.465 E+03$ & $7.5 \times 10-3$ & $6.5306+02$ & & $\begin{array}{l}\text { PAC 27 Listing Gable } 1 \\
\text { PAC 27 isting Table } 1\end{array}$ \\
\hline 271 & 2714 s & Sodium chlorite & $7758-19-2$ & 007758-19-2 & 90.44 & $\mathrm{~s}$ & $2.570 \mathrm{E}+02$ & & $180-200$ dec & & & & PAC 27 Listing Table 1 \\
\hline$\frac{1716}{2716}$ & $2715 \mid s$ & Sodium chromate decahydrate & $13517-17-4$ & $013517-17-4$ & 342.18 & $\mathrm{~s}$ & & & & & & & PAC 27 Listing Table 1 \\
\hline 2717 & $2716 \sqrt{s}$ & Sodium chromate tetrahydrate & $10034-82-9$ & $010034-82-9$ & 303.03 & 5 & dec. & & & & & & $\begin{array}{ll}\text { PAC } 27 \text { Listing Table } 1 \\
\end{array}$ \\
\hline 2718 & $2717 \mid \mathbf{s}: 5$ & Sodium chromate(l(I); (Disodium chromate) & $7775-11-3$ & $007775-11-3$ & 161.97 & $\mathrm{~s}$ & 7.940E+02 & & & & & & PAC 27 Listing Table 1 \\
\hline 2720 & 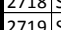 & $\begin{array}{l}\text { Sodum cltrate; (MNonososodum citrate) } \\
\text { Sodium cobaltinitrite }\end{array}$ & 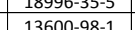 & 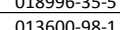 & 214.12 & $\frac{3}{s}$ & & & & & & & 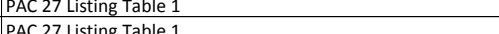 \\
\hline 2721 & 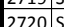 & Sodium cranide & $\frac{13003-30-1}{143-9}$ & $0000143-33-9$ & $\frac{40.930}{49.01}$ & $\frac{\mathrm{s}}{\mathrm{s}}$ & $5.630 \mathrm{E}+02$ & & $14965+03$ & $1000=+00$ & $81705+02$ & & 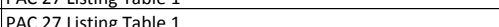 \\
\hline 2722 & $2721 \mathrm{~s}$ & $\begin{array}{l}\text { Sodium cyclopentadienylide; (Cyclopentadienyl sodium) } \\
\end{array}$ & $4984-82-1$ & $004984-82-1$ & 88.08 & $\mathrm{~s}$ & & & 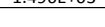 & & > & & PAC 27 Listing Table 1 \\
\hline$\frac{2723}{2723}$ & $|2722| s ;$ & Sodium deuteroxide & $14014-06-3$ & 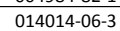 & $\frac{31}{41}$ & $\mathrm{~s}$ & & & & - & - & & $\begin{array}{lll}\text { PAC } 27 \text { Listing Table } 1 \\
\end{array}$ \\
\hline 2723 & $2723 \mathrm{~s}$ & Sodium dichromate dihydrate & $7789-12-0$ & $007789-12-0$ & 298.02 & $\mathrm{~s}$ & loses $\mathrm{H2O}$ at & & 400 dec & & & & PAC 27 Listing Table 1 \\
\hline$\frac{2725}{2725}$ & & sodium dichromate; (Disodium dichromate) & $10588-01-9$ & $010588-01-9$ & 261.98 & $\mathrm{~s}$ & $3.5677+02$ & & 400 dec & & & & PAC 27 Listing Table 1 \\
\hline 2726 & 2725 s & Sodium diethyldithiocarbamate trihydrate & $20624-25-3$ & $020624-25-3$ & 225.33 & $\mathrm{~s}$ & 95 & & & & & & PAC 27 Listing Table 1 \\
\hline 2727 & 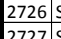 & Sodium diethyldithiocarbamate; (Diethyldithiocarbamic acid, sodium salt) & $\frac{148-18-5}{1004-51-5}$ & $000148-18-5$ & $\frac{171.27}{11988}$ & $\frac{s}{s}$ & 9.500E+01 & & 204 dec & & & & $\begin{array}{l}\text { PAC 27 Listing Table } 1 \\
\text { PACC2 }\end{array}$ \\
\hline$\frac{2728}{2729}$ & 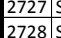 & $\begin{array}{l}\text { Sodium didyrdrogenen phosshhate; (Sodium phosphate monobasic, monohydrate) } \\
\text { Sodium dithionate dihydrate }\end{array}$ & $\frac{10099-21-5}{7631-94-9}$ & 010049-21-5 & $\frac{119.88}{242.14}$ & $\frac{5}{5}$ & $1.100 E+02$ & & 264dec & & & & $\begin{array}{l}\text { PAC 27 Listing Table } 1 \\
\text { PAC27 jesting Table } 1\end{array}$ \\
\hline$\frac{2730}{2730}$ & 2729: & Sodium dodecylbenzenesulfonate; (Dodecyl benzene sodium sulfonate) & $25155-30-0$ & $\frac{025155-30-0}{020}$ & 34.5.52 320 & $\mathrm{~s}$ & & & & & & & $\begin{array}{l}\text { A AC 27 Listing Table } 1 \\
\text { PAC }\end{array}$ \\
\hline$\frac{2731}{2731}$ & $\mid 2730$ s & $\begin{array}{l}\text { Sodium ethoxide } \\
\end{array}$ & $141-52-6$ & $000141-52-6$ & 68.05 & $\mathrm{~s}$ & $260 \mathrm{dec}$ & & & $<0.1$ & $2.000 E+01$ & & $\begin{array}{l}\text { PAC } 27 \text { Listing Table } 1 \\
\end{array}$ \\
\hline 2732 & 2731 s & Sodium ferrocyanide & 13601-19-9 & 013601-19-9 & 303.93 & $\mathrm{s}$ & & & & & & & PAC 27 Listing Table 1 \\
\hline 2733 & 2732 s & Sodium fluoride & 7681-49-4 & $007681-49-44$ & 41.99 & s & $9.960 \mathrm{E}+02$ & & 1.7045+03 & $7.5 \times 10-2$ & $9.200 \mathrm{E}+02$ & & PAC 27 Listing Table 1 \\
\hline $\begin{array}{lll}2134 \\
2725\end{array}$ & 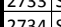 & $\begin{array}{l}\text { Soldum formate } \\
\text { Sodium gluconate }\end{array}$ & 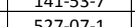 & 年 & 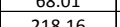 & $\frac{3}{c}$ & & & & & & & 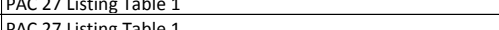 \\
\hline$\frac{2736}{2736}$ & 27735|s & Sodium glycinate & $6000-44-8$ & $006000-44-8$ & 97.06 & $\mathrm{~s}$ & & & & & & & PAC 27 Listing Table 1 \\
\hline$\frac{1737}{2737}$ & $2736 \mid s$ & Sodium glycolate; ; (Sodium hydroxyacetate) & $2836-32-0$ & $002836-32-0$ & 98.04 & $\mathrm{~s}$ & & & & & & & PAC 27 Listing Table 1 \\
\hline 2738 & $27377^{5}$ & 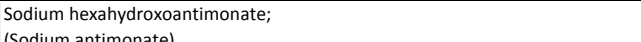 & $33908-66-6$ & $033908-66-6$ & 192.744968 & $\mathrm{~s}$ & & & & & & & PAC 27 Listing Table 1 \\
\hline & $2738 \mathrm{~s} s$ & Sodium hexamethyldisilazane; (Sodium bis(trimethylsily)|amide) & $1070-89-9$ & $001070-89-9$ & 184.38377 & $\mathrm{~s}$ & & & & & & & PAC 27 Listing Table 1 \\
\hline 2740 & 2739 : & & $7646-69-7$ & 007646-69-7 & 24 & $\mathrm{~s}$ & 800 decom & & & & & & PAC 27 Listing Table 1 \\
\hline 2741 & $2740 \mid \mathrm{s} 5$ & Sodium hydrogen lead oxide & z-0067 & $000002-0067$ & 263.2 & $\mathrm{~s}$ & & & & & & & PAC 27 Listing Table 1 P \\
\hline$\frac{2424}{2733}$ & $\begin{array}{lll} & 0\end{array}$ & 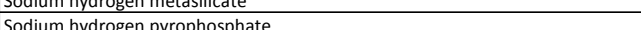 & $z^{20.0068}$ & 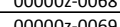 & $\begin{array}{ll}200.081 \\
2392\end{array}$ & $\frac{3}{5}$ & & & & & & & 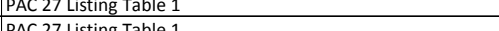 \\
\hline$\frac{2744}{274}$ & 2774]: & & $7775-14-6$ & 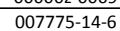 & $\frac{2147.1}{174.1}$ & s & $55 \mathrm{dec}$ & & & & & & $\begin{array}{lll}\text { PAC } 27 \text { Listing Table } 1 \\
\end{array}$ \\
\hline 2745 & $2744 \mid s$ & Sodium hydroxide & $1310-73-2$ & $001310-73-2$ & 40 & $s$ & $3.2306+02$ & & $1.388 \mathrm{E}+03$ & $7.5 \times 10-3$ & $5.130 E+02$ & & PAC 27 Listing Table 1 \\
\hline 2746 & $2745 \mid s$ & Sodium hypobromite & 13824-96-9 & $013824-96-9$ & 119.9 & 5 & & & & & & & \begin{tabular}{|l} 
PAC 27 Listing Table 1 \\
\end{tabular} \\
\hline 2747 & 2746 & Sodium hypochlorite & $7681-52-9$ & $007681-52-9$ & 75.45 & $\mathrm{~s}$ & $1.800 E+01$ & & & & & & PAC 27 Listing Table 1 P \\
\hline & 2747 & Sodium hypochlorite pentahydrate & $10022-70-5$ & $010022-70-5$ & 165.55 & $\mathrm{~L}$ & & & & $1.000 E-05$ & $2.500 E+01$ & $1.000 E-05$ & $\begin{array}{l}\text { Hazardous component of aqueous solution has neglible VP. A A } \\
\text { vP of } 1 \text { - }-05 \text { at } 25^{\circ} \mathrm{C} \text { is assigned for callutation purposes }\end{array}$ \\
\hline & $2748 \mathrm{~s}$ & Sodium hypophosphite & $7681-53-0$ & $007681-53-0$ & 87.98 & & & & & & & & 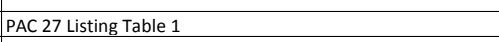 \\
\hline 2750 & 2749 s & Sodium hypophosphite hydrate & $123333-67-5$ & $123333-67-5$ & & $s$ & dec & & & & & & ting Table 1 \\
\hline 2751 & $2750 \mid \mathrm{s} s$ & $\begin{array}{l}\text { Sodium iodate } \\
\text { Sclun natis }\end{array}$ & 7681-55-2 & $007681-55-2$ & 197.89247 & s & dec & & 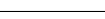 & 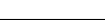 & & & PAC 27 Listing Table 1 \\
\hline 2753 & 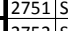 & 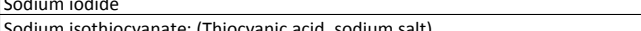 & $\begin{array}{l}7681-82-5 \\
540-72-7\end{array}$ & 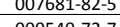 & $\frac{149.89}{8107}$ & $\frac{s}{s}$ & 6.600t地 & & $1.304 t+03$ & $1.000 \mathrm{E}+00$ & 7.670E+02 & & $\begin{array}{l}\text { PAC 27 Listing Table } 1 \\
\text { Pat }\end{array}$ \\
\hline
\end{tabular}




\begin{tabular}{|c|c|c|c|c|c|c|c|c|c|c|c|c|c|}
\hline & A & & & & Molecular & $\overline{\text { QV State }}$ & & & & & Tforyp & & \\
\hline & No. & Chemical Compound & $\begin{array}{c}\text { CAS Number } \\
\text { (CASRN) }\end{array}$ & $\begin{array}{c}\text { Modified } \\
\text { Format CAS }\end{array}$ & $\begin{array}{l}\text { Molecular } \\
\text { Weight (MW) }\end{array}$ & $\begin{array}{l}\text { at } \\
25^{\circ} \mathrm{C}\end{array}$ & $\begin{array}{l}\text { MP or FP } \\
{\left[{ }^{\circ C}\right]}\end{array}$ & $\begin{array}{c}\text { BP Pressure } \\
\text { [mm Hg] }\end{array}$ & $\mathrm{BP}\left[{ }^{[\mathrm{C}]}\right]$ & $\begin{array}{l}\text { Pressure } \\
\text { Pressure }\end{array}$ & $\begin{array}{l}\text { Tfor VP } \\
{\left[{ }^{\circ}\right]}\end{array}$ & 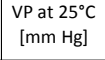 & Vapor Pressurue @ $25^{\circ} \mathrm{C}$ Reference \\
\hline 2754 & 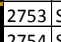 & $\begin{array}{l}\text { Sodium lactate; (Lactic acid, monosodium salt) } \\
\text { Sodium lanthanate }\end{array}$ & $72-17-3$ & $\begin{array}{l}000072-17-3 \\
000002-070\end{array}$ & $\frac{112.07}{229.92}$ & $\frac{s}{s}$ & & & & & & & $\begin{array}{l}\text { PAC } 27 \text { Listing Table } 1 \\
\text { PAC } 2 \text { líting Table }\end{array}$ \\
\hline$\frac{2755}{2756}$ & 2755 s & Sodium laurry sulfifate & $151-21-3$ & $000151-21-3$ & 288.38 & $\frac{2}{2}$ & $2.050 E+02$ & & & & & & $\begin{array}{l}\text { PAC 27 Listing Table } 1 \\
\text { Pand }\end{array}$ \\
\hline 资 2757 & 2756 s & Sodium metabisulfite & $7681-57-4$ & $007681-57-4$ & $\begin{array}{ll}190.11 \\
\end{array}$ & $\mathrm{~s}$ & & & & & & & PAC 27 Listing Table 1 \\
\hline 2758 & 2757 : & Sodium metaborate & 7775-19-1 & $007775-19-1$ & 66.81 & $\mathrm{~s}$ & $9.660 \mathrm{E}+02 \mathrm{~T}$ & & 1.434E+ +03 & & & & $\begin{array}{l}\text { PAC 27 Listing Table } 1 \\
\end{array}$ \\
\hline 2759 & 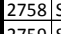 & 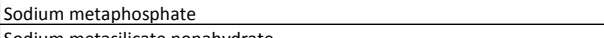 & $10361-03-2$ & $010361-03-2$ & 101.96 & $\mathrm{~s}$ & $6.280 \mathrm{E}+02 \mathrm{C}$ & & & & & & PAC 27 Listing Table 1 \\
\hline 27600 & 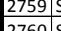 & $\begin{array}{l}\text { SSodium metasisicate nonahyyrate } \\
\text { Sodium metalicticte nentahydrate }\end{array}$ & 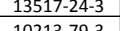 & 0131317-24-3. & 28.622 20. & s & $1.089 \mathrm{E}+03$ & & & & & & 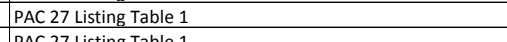 \\
\hline & & 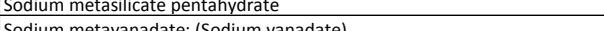 & & 010213-79-3 & 212.15 & & & & & & & & \\
\hline 2762 & 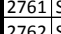 & $\begin{array}{l}\text { Sodium metavanadate; (Sodium vanadate) } \\
\text { SScdium mothlyate }\end{array}$ & $13718-26-8$ & $013718-26-8$. & 121.93 & s & 6.300다02 & & & & & & PAC 27 Listing Table 1 \\
\hline$\frac{2705}{2764}$ & 2763] & Sodium molybdate dihydrate; (Disodium molybdate dihydrate) & $\frac{1.142-40-6}{1010}$ & 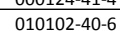 & 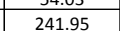 & s & $>300$ & & 121/dec & & & & $\begin{array}{l}\text { PAC 27 L Ltsing a able } 1 \\
\text { PAC } 27 \text { isting Table } 1\end{array}$ \\
\hline 2765 & $\left.2764\right|^{s}$ & Sodium monoxide; (Sodium oxide) & $12401-86-4$ & $012401-86-4$ & 38.99 & $\mathrm{~s}$ & & & 1275 subl & & & & PAC 27 Listing Table 1 \\
\hline 2766 & 2765 s & Sodium nickel oxide (Liquid) & $37367-09-2$ & $037367-09-2$ & 136.6717 & $\mathrm{~L}$ & & & & $1.000 E-05$ & $2.500 E+01$ & $1.000 E-05$ & $\begin{array}{l}\text { Hazardous component of aqueous solution has neglible VP. A A } \\
\text { vP of } 11-\text { - } 5 \text { at } 25^{\circ} \mathrm{C} \text { is assigned for callution purposes }\end{array}$ \\
\hline & 2766 s & Sodium nickelate (Liquids) & $2-0073$ & $00000 z-0073$ & 172.7 & $\mathrm{~L}$ & & & & $1.0000-05$ & $2.500 E+01$ & $1.000 E-05$ & $\begin{array}{l}\text { Hazardous component of aqueous solution has neglible VP.A A } \\
\text { vip of } 11-05 \text { at } 25^{\circ} \mathrm{C} \text { is assigned for callutation purposes }\end{array}$ \\
\hline 2768 & 2767 s & $\begin{array}{l}\text { Sodium nickelate (Solids) } \\
\text { Solim nitat }\end{array}$ & $\frac{-0.074}{762009}$ & $000002-0074$ & $\frac{172.7}{95}$ & $\frac{s}{c}$ & & & & & & & $\begin{array}{l}\text { PAC 27 Listing Table } 1 \\
\end{array}$ \\
\hline$\frac{2769}{2770}$ & $\begin{array}{lll}2108 \\
27695\end{array}$ & $\begin{array}{l}\text { Sodum infree } \\
\text { Sodium nitrite }\end{array}$ & $\frac{76311-99-4}{7632-00-0}$ & $\begin{array}{ll}007631199-4 \\
007632-00-0\end{array}$ & $\frac{85}{69}$ & $\frac{2}{3}$ & $20.050 E+02+02$ & & 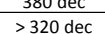 & & & & $\begin{array}{l}\text { PAC 27 Listing Table } 1 \\
\text { Palle } 1\end{array}$ \\
\hline 2771 & 2770 s & 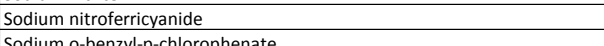 & $14402-89-2$ & $014402-89-2$ & 261.94 & $\mathrm{~s}$ & & & & & & & PAC 27 Listing Table 1 \\
\hline$\frac{2772}{2773}$ & 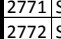 & 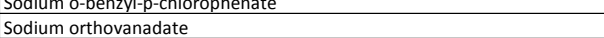 & $\frac{3184-655}{13721-39-6}$ & $003184-65-4$ & 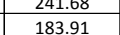 & $\frac{5}{5}$ & $850-866$ & & & & & & $\begin{array}{l}\text { PAC 27 Listing Gable } 1 \\
\text { PAC } 27 \text { isting Table } 1\end{array}$ \\
\hline 2774 & 2773 & $\begin{array}{l}\text { Sodium oxalate } \\
\end{array}$ & $\begin{array}{l}62-76-0 \\
62-0\end{array}$ & $\frac{000062-76-0}{0.002-0}$ & 134 & $\frac{5}{s}$ & $\sim 250 \mathrm{dec}$ & & & & & & PAC 27 Listing Table 1 \\
\hline 2775 & 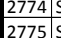 & $\begin{array}{l}\text { Sodium pentacchlorophenate } \\
\text { Sodium perchlorate }\end{array}$ & $\frac{131-52-2}{7701-89-0}$ & $\begin{array}{l}000131-52-2 \\
007601-89-0\end{array}$ & $\frac{288.3}{122.44}$ & $\frac{s}{s}$ & $482 \mathrm{dec}$ & & & & & & $\begin{array}{l}\text { PAC 27 Listing Table } 1 \\
\text { PA 27 Listing Table } 1\end{array}$ \\
\hline$\frac{2466}{2777}$ & & Sodium perchlorate monohydrate & $7791-07-3$ & $007791-07-3$ & 140.46 & $\frac{\mathrm{s}}{\mathrm{s}}$ & 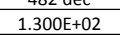 & & & & & & $\begin{array}{l}\text { PAC 27 Listing Table } 1 \\
\text { Pacte } 1\end{array}$ \\
\hline 2778 & 2777 s & Sodium permanganate & $10101-50-5$ & $010101-50-5$ & 141.93 & $\mathrm{~s}$ & & & & & & & PAC 27 Listing Table 1 \\
\hline 2779 & $2778 \mathrm{~s}$ & Sodium peroxide & $1313-60-6$ & $001313-60-6$ & 77.98 & $s$ & $6.750 \mathrm{E}+02$ & & $657 \mathrm{dec}$ & & & & PAC 27 Listing Table 1 , \\
\hline 27800 & 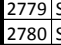 & $\begin{array}{l}\text { Sodium perthenata; (Rhenium(VIII) sodium oxide) } \\
\text { Sodium persulfate }\end{array}$ & $\frac{13472-333-8}{7775-27-1}$ & $\begin{array}{l}0.13472-3-3-8 \\
007775-27-1 \\
\end{array}$ & 2738.19 & $\frac{\mathrm{s}}{\mathrm{s}}$ & 4.200E do2 100 & & & & & & $\begin{array}{l}\text { PAC 27 Listing Gable } 1 \\
\text { PAC 27 Listing Table } 1\end{array}$ \\
\hline 2782 & 2781 s & Sodium phosphate & $7632-05-5$ & $007632-05-5$ & 258.93 & & & & & & & & \\
\hline 2783 & 27825 & Sodium phosphate, dibasic & $7558-79-4$ & $007558-79-4$ & 141.96 & $\mathrm{~s}$ & 240dec & & & & & & PAC 27 Listing Table 1 \\
\hline 2784 & & Sodium phosshate, dibasic, dodecahhydrate & $10039-32-4$ & 010039-32-4 & 358.2 & $\mathrm{~s}$ & & & & & & & PAC 27 Listing Table 1 \\
\hline 2785 & 2784 s & Sodium phosphate, dibasic, heptahydrate & $7782-85-6$ & $007782-85-6$ & 383.05 & s & & & $\begin{array}{l}\text { loses } 5.120 \\
\text { @ } 48 \mathrm{C}\end{array}$ & & & & PAC 27 Listing Table 1 \\
\hline & & Sodium phosphate, monobasic & $7558-80-7$ & $\begin{array}{ll}007558-80-7 \\
0030\end{array}$ & 119.976 & $\mathrm{~s}$ & & & & & & & PAC 27 Listing Table 1 \\
\hline 2787 & & Sodium phosphate, tribasic, dodecahydrate & $10101-89-0$ & $010101-89-0$ & 380.18 & $\mathrm{~s}$ & & & & & & & PAC 27 Listing Table 1 \\
\hline 2788 (2790 & & 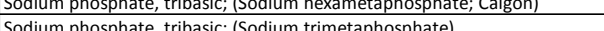 & 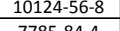 & 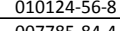 & 611.76 & $\frac{s}{s}$ & & & & & & & 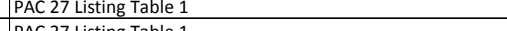 \\
\hline$\frac{2700}{2790}$ & & Sodium phosphate, tribasic;; (Trisodium phosphate) & $7601-54-9$ & $007601-54-9$ & $\begin{array}{lll}105.50 \\
163.94\end{array}$ & $\mathrm{~s}$ & $1.583 E+03$ & & & & & & $\begin{array}{l}\text { PAC 27 Listing Table } 1 \\
\end{array}$ \\
\hline 2791 & $2790 \mid \mathrm{s}: 5$ & Sodium phosphide & $12058-85-4$ & $012058-85-4$ & 99.94 & $\mathrm{~s}$ & & & & & & & PAC 27 L Listing Table 1 \\
\hline$\frac{2792}{2793}$ & $\frac{27915 s}{27925}$ & $\begin{array}{l}\text { Sodium polyphosshhate } \\
\text { Sodium polytungstate }\end{array}$ & $\frac{68915-31-1}{12141-67-2}$ & $068915-31-12$ & 6211.7703836 & $\frac{s}{s}$ & & & & & & & $\begin{array}{l}\text { PAC C } 7 \text { Listing Gable } 1 \\
\text { PAC } 27 \text { listing Table } 1\end{array}$ \\
\hline 2794 & & Sodium potassium tartrate tetrahydrate & $6381-59-5$ & $006381-59-5$ & 282.22 & $\mathrm{~s}$ & $90-100$ & & $2.000 E+02$ & & & & PAC 27 Listing Table 1 \\
\hline 27955 & & 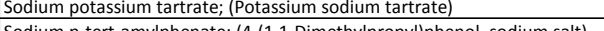 & $\frac{304-59-6}{3067}$ & $0.00304-59-6.6$ & 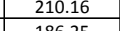 & $\mathrm{s}$ & $90-100$ & & $2.000 \mathrm{E}+02$ & & & & 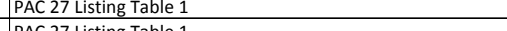 \\
\hline$\frac{21966}{2797}$ & 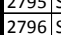 & 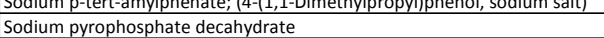 & $\frac{31366-95-1}{13472-36-1}$ & $013472-936-1$ & $\frac{186.25}{446.1}$ & $\frac{5}{5}$ & & & & & & & $\begin{array}{l}\text { PAC/ LIting gable } 1 \\
\text { PAC 27 Listing Table } 1\end{array}$ \\
\hline 2798 & 2797 : & Sodium pyrophosphate, di- & $7758-16-9$ & $007758-16-9$ & 221.94 & $\mathrm{~s}$ & $220 \mathrm{dec}$ & & & & & & $\begin{array}{l}\text { PAC } 27 \text { Listing Table } 1 \\
\end{array}$ \\
\hline 2799 & & Sodium pyruvate; (Pyruvic acid, sodium salt) & $\frac{113-24-6}{55217}$ & $000113-24-6$ & $\frac{110.04667}{16011}$ & $s$ & $3.0006+02$ & & & & & & PAC 27 Listing Table 1 \\
\hline 28000 & 279995 & Sodium salicylate; (Salicylic acid, sodium salt) & $54-21-7$ & $0.00054-21-7$ & 160.11 & $\frac{s}{c}$ & $2.000 \mathrm{E}+02$ & & & & & & | $\mathrm{AAC} 27$ Listing Table 1 \\
\hline$\frac{2801}{2802}$ & $\frac{28004 s}{2801 s}$ & $\begin{array}{l}\text { Sodium selenata; (Disodium selenate) } \\
\text { Sodium selenite }\end{array}$ & $\frac{13410-01-0}{10102-18-8}$ & $\begin{array}{ll}013410-0110 \\
010102-18\end{array}$ & $\frac{188.94}{172.94}$ & $\frac{s}{s}$ & $7.100 E+02$ & & & & & & $\begin{array}{l}\text { PAC27 Listing Gable } 1 \\
\text { PAC 27 Listing Table } 1\end{array}$ \\
\hline 2803 & 28025 & Sodium silicate; (Sodium metasilicate) & $6834-92-0$ & $006834-92-0$ & 122.07 & $\mathrm{~s}$ & $1.089 E+03$ & & & & & & PAC 27 Listing Table 1 \\
\hline 28044 & & Sodium stannate & $12058-66-1$ & $012058-66-1$ & 212.687736 & $\mathrm{s}$ & & & & & & & PAC 27 Listing Table 1 \\
\hline 20305 & 2080455 & 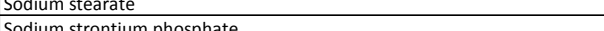 & $\frac{822-16-2}{10252-1}$ & $\frac{0.00222-16-2}{0.053-2}$ & $\frac{306.52}{2052}$ & $\frac{s}{s}$ & & & & & & & 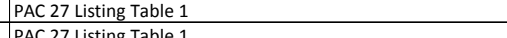 \\
\hline $\begin{array}{l}2807 \\
2807\end{array}$ & 2806 s & Sodium succinate & $\frac{19530-00-1}{150-90-3}$ & 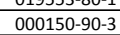 & $\frac{0.350}{162.06}$ & $\mathrm{~L}$ & & $7.600 E+02$ & 4.260E+02 20 & $8.7000=08$ & $2.500 E+01$ & 8.700E -0.0 & 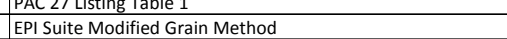 \\
\hline 2808 & 2807 s & Sodium succinate, hexahydrate & $6106-21-4$ & 006106-21-4 & 270.14 & $\mathrm{~s}$ & & 2 & 2 & 2 & & ( & 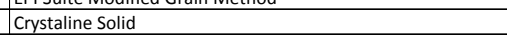 \\
\hline 2809 & 2808 s & Sodium sulfamate & $13845-18-6$ & $013845-18-6$ & 120.08 & $\mathrm{~s}$ & & $7.600 E+02$ & 1.044E E02 & $1.500 E+01$ & & & PAC 27 Listing Table 1 \\
\hline 2810 & $280995 \mathrm{~s}$ & Sodium sulfate, anhydrous & $7757-82-6$ & $007757-82-6$ & 142.04 & 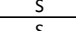 & $\begin{array}{l}8.800 \mathrm{EF} 02 \\
.8202\end{array}$ & & & & & & |PAC 27 Listing Table 1 \\
\hline$\frac{2814}{2812}$ & 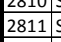 & 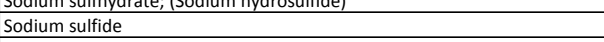 & $\frac{16 / 113-80-5}{1313-22-2}$ & $\frac{001313-82-2}{0.25}$ & $\begin{array}{c}5.060 \\
78.04 \\
\end{array}$ & $\frac{2}{3}$ & $\begin{array}{l}3.500 \mathrm{E}+\mathrm{U} 2 \\
.172 \mathrm{E}+03 \\
\end{array}$ & & & & & & $\begin{array}{l}\text { PAC 27 Listing Iabiel } 1 \\
\text { PAC Table } 1\end{array}$ \\
\hline 2813 & 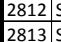 & \begin{tabular}{|l|l} 
Sodium sulfide nonahydrate \\
Sodium sulfie
\end{tabular} & $\frac{1313-84-4}{7757-83-7}$ & $\frac{001313-84-4}{007575-83-7}$ & 240.22 & $\frac{s}{5}$ & $\sim 50$ & & 1124 dec & & & & $\begin{array}{l}\text { PAC } 27 \text { Listing Table } 1 \\
\text { PAC } 7 \text { Listin Table } 1\end{array}$ \\
\hline
\end{tabular}




\begin{tabular}{|c|c|c|c|c|c|c|c|c|c|c|c|c|}
\hline & Chemical Compound & $\begin{array}{c}\text { cas N Number } \\
\text { (CASPN) }\end{array}$ & $\begin{array}{c}\text { Modified } \\
\text { Format CAS }\end{array}$ & $\begin{array}{l}\text { Molecular } \\
\text { Weight MW }\end{array}$ & $\begin{array}{ccc}\text { alstate } \\
\text { at } \\
\text { sor }\end{array}$ & $\begin{array}{c}\text { MP or fP } \\
\text { [cr] }\end{array}$ & $\begin{array}{llll}\text { Bep ressure } \\
\text { [mm M }\end{array}$ & ${ }_{B P}[\mathrm{CC}]$ & $\begin{array}{c}\text { vapor } \\
\text { pressurue }\end{array}$ & $\begin{array}{c}\text { Torverp } \\
\text { crla }\end{array}$ & 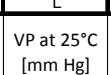 & Vapor Pressure @ $25^{\circ} \mathrm{C}$ Reference \\
\hline 2815 & 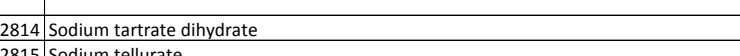 & $\frac{6106-247}{1002393}$ & $\frac{006106-24 \cdot 7}{0102-239}$ & 233.082 & $\frac{25 \mathrm{c}}{\mathrm{s}}$ & 150 dehydrat & & & & & & 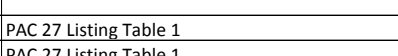 \\
\hline 2817 & $\begin{array}{l}885 \text { Sodulu telurate } \\
816 \text { Sodium tellurite }\end{array}$ & 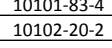 & 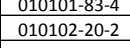 & $\frac{237.58}{221.58}$ & $\frac{5}{5}$ & & & & & & & 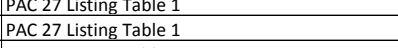 \\
\hline$\frac{2818}{2829}$ & 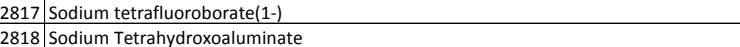 & 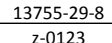 & 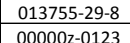 & 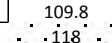 & $\frac{s}{5}$ & 322 & & dec & & & & 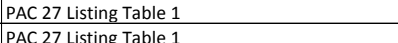 \\
\hline 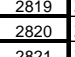 & 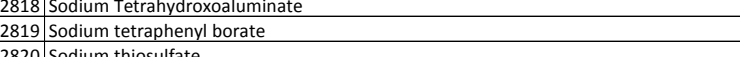 & 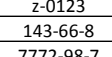 & 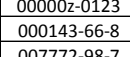 & $\begin{array}{l}-1183 \\
34223 \\
3425\end{array}$ & $\frac{5}{5}$ & & & Idec & & & & 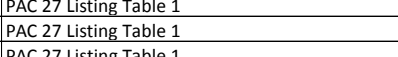 \\
\hline$\frac{2821}{28822}$ & 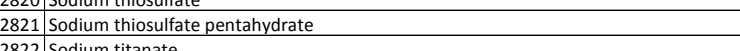 & 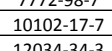 & 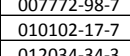 & 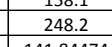 & $\frac{5}{5}$ & $4.800 \mathrm{E}+01$ & & dect & & & & 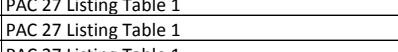 \\
\hline 2083 & 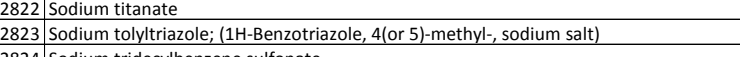 & 1.2034-3.3-3. & 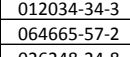 & 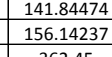 & & $-5.000+ \pm 00$ & $7.600 €+02$ & $1.060 \mathrm{E}+02$ & $1.250-10$ & $2.500+\div 01$ & $1.250=10$ & $\begin{array}{l}\text { Lising Table } 1 \text { 1in Method } \\
\text { emodfifed Grain Metod }\end{array}$ \\
\hline & 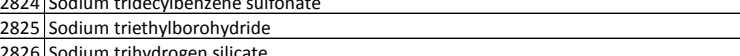 & 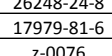 & 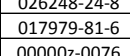 & 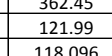 & $\frac{5}{5}$ & & & & & & & 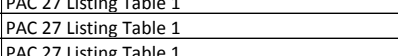 \\
\hline$\frac{2827}{2028}$ & 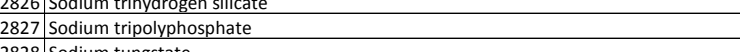 & $7758-29.4$ & (0.7758-2.29.4 & 367.86 & $\mathrm{s}$ & $6.220 \mathrm{E}+022$ & & & & & & 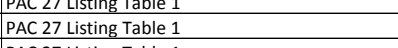 \\
\hline 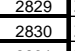 & $\begin{array}{l}828.8 \text { odium tungstate } \\
829 \text { Sodium tungstate dihydrate }\end{array}$ & 13427-45-10-2 & 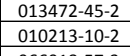 & $\begin{array}{l}293.83 \\
32987 \\
397\end{array}$ & $\frac{5}{5}$ & 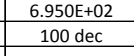 & & & & & & 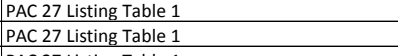 \\
\hline$\frac{2831}{2832}$ & 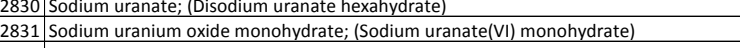 & 1006135-92-9.9 & 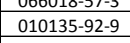 & $\frac{142}{652.049}$ & $\frac{5}{5}$ & & & & & & & 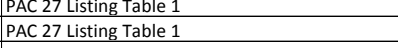 \\
\hline 28834 & 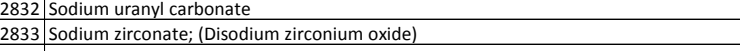 & 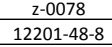 & 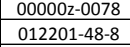 & 428.0.09324 & $\frac{s}{s}$ & & & & & & & 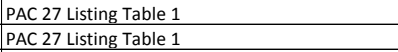 \\
\hline$\frac{2835}{2836}$ & 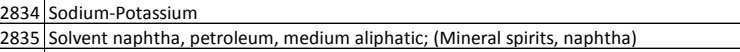 & 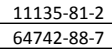 & 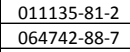 & & & . & $7.600+02$ & $1.500 E+02$ & $1.500++00$ & 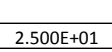 & $1.500 E++$ & 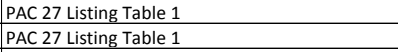 \\
\hline 2837 & 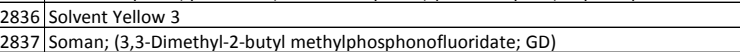 & 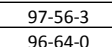 & 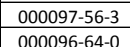 & 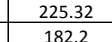 & & 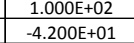 & $7.600+02$ & $1.670 \mathrm{E}+02$ & $\begin{array}{l}7.500 .07 \\
000=01\end{array}$ & $\begin{array}{l}2.500+51 \\
2.500++01\end{array}$ & 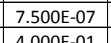 & 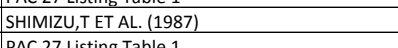 \\
\hline 2839 & 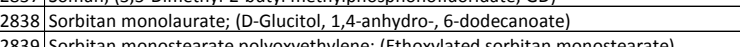 & 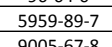 & 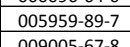 & $\begin{array}{l}346.46 \\
131.9 \\
130\end{array}$ & s & & & & & & & 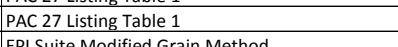 \\
\hline$\frac{2801}{2881}$ & 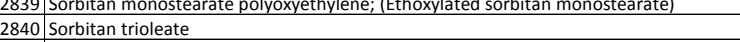 & 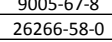 & 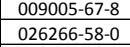 & 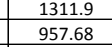 & & & 7.600E+02 & 5.3966+02 & 5.760e-15 & $2.500 E^{+01}$ & & \\
\hline$\frac{2824}{2283}$ & 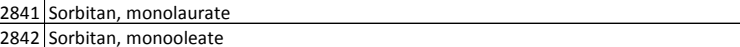 & 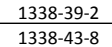 & 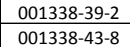 & 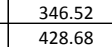 & $\frac{s}{L}$ & & $7.600+02$ & $2.2311+02$. & 1.030E-14 & $2.500 E+01$ & 1.0306-14 & $\begin{array}{l}\text { PAA } 27 \text { i isting Table } 1 \\
\text { EPI Sunte Modified Grain Method }\end{array}$ \\
\hline 2844 & 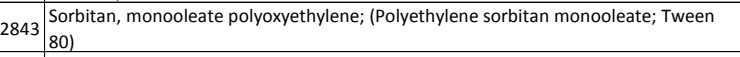 & $9005-55.6$ & $009005-65-6$ & 1089.92 & $\mathrm{~L}$ & & $7.600 €+02$ & $1.075+03$ & $1.670=35$ & $2.500 \mathrm{E}+01$ & $1.670=-35$ & Suite Modified Grain Method \\
\hline & 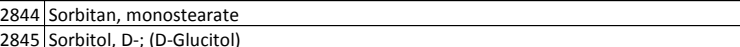 & $\begin{array}{l}1338-41-6 \\
\end{array}$ & .01338-41-6 & 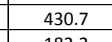 & $\frac{5}{5}$ & 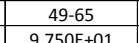 & & & & & & 27 listing Table 1 (2) \\
\hline$\frac{2847}{28949}$ & 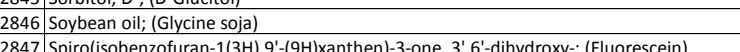 & 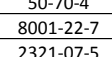 & 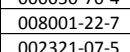 & 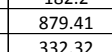 & $\frac{t}{S}$ & $314: 316$ & $7.600+02$ & 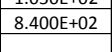 & $\begin{array}{l}5.000: 21 \\
\end{array}$ & $2.500+\div 01$ & $5.0002-21$ & 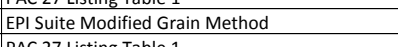 \\
\hline t & 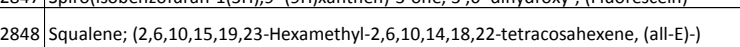 & 111.02.24 & 000011-11-02-3 & $\begin{array}{ll}210.72 \\
410.72\end{array}$ & $\mathrm{~L}$ & $-7.500+\div 01$ & $2.500 \Leftarrow+01$ & $2.850 \mathrm{~F}+02$ & $20000+00$ & $2.400+02$ & $30.091=10$ & 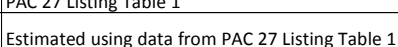 \\
\hline & 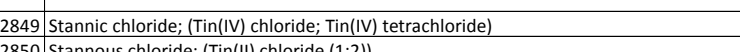 & 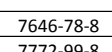 & $\begin{array}{l}007646-78.8 \\
0.77200 .8\end{array}$ & $\frac{260.49}{1099}$ & $\frac{L}{s}$ & 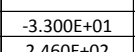 & $7.600+02$ & $1.14141+02$ & $1.000+51$ & $1.000 \mathrm{E}+01$ & $2.299+01$ & Estimated using datat from PAC 27 L Lsting T Table 1 \\
\hline$\frac{20652}{2.522}$ & 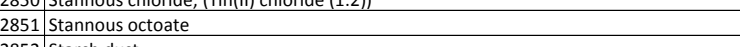 & 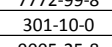 & 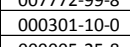 & 405.12 & 5 & & & 0.20verou & & & & $\begin{array}{l}\text { PAA 27 fisting table } 1 \\
\text { PAC } 27 \text { Listing Table } 1\end{array}$ \\
\hline 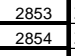 & 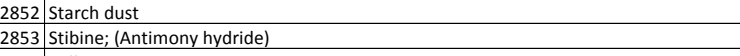 & 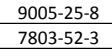 & 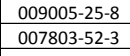 & 124.78 & $\frac{5}{6}$ & $\frac{\text { dec }}{-8.800+01}$ & & 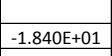 & 7760 & & & 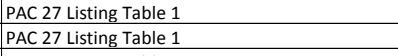 \\
\hline & 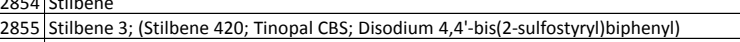 & & & 年56.258 & & & & & & & & \\
\hline$\frac{2657}{2502}$ & 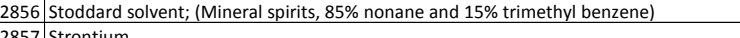 & 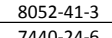 & 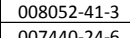 & 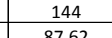 & 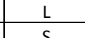 & 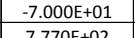 & 7.600E+02 & 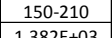 & 1. $1.506+00$ & $2.500 \mathrm{E}+01$. & $.500 \mathrm{E}+0 \mathrm{CC}$ & Sising Table 1 . \\
\hline$\frac{2565}{2859}$ & $\begin{array}{l}28585 \text { Strontium carbonate } \\
280 \text { Stronte }\end{array}$ & 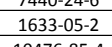 & 0.1433-0.5-2. & $\frac{14.6292}{147.629}$ & $\frac{5}{5}$ & 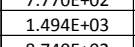 & & dect & & & & 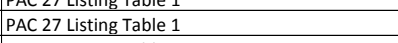 \\
\hline & 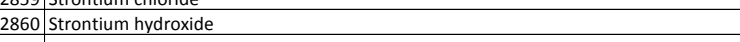 & 18480.07-4.4 & 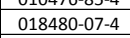 & & & & & & & & & 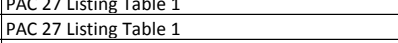 \\
\hline 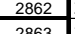 & 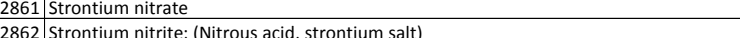 & 1.0024-76-9.9 & 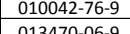 & 年11.63 & $s$ & 5.7.00 $00+02$ & & $6.450=02022$ & & & & Sting Table 1 - \\
\hline 20684 & 2863 Strontium oxalate & 814.959 .9 & 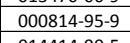 & 175.64 & $\frac{5}{5}$ & & & & & & & PAC 27 L Listing Table 1 \\
\hline 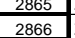 & 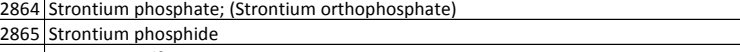 & 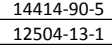 & $\begin{array}{l}014444-409.5 \\
0.12504-13-1\end{array}$ & $\begin{array}{l}42528.8244 \\
324.9\end{array}$ & s & & & & & & & $\begin{array}{l}\text { A AC 27sting Iable } 1 \\
\text { PAC 27 Listing Table } 1\end{array}$ \\
\hline 2867 & 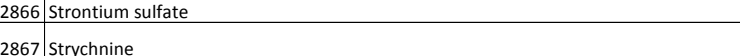 & 7759-22:-6 & 007505-2-22.6. & 33441 & $\frac{\mathrm{s}}{\mathrm{s}}$ & 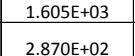 & & $270 @ 5$ & $0.000=0$ & $20000+01$ & & $\mid \begin{array}{l}\text { PAC } 2 \text { L Listing Table } 1 \\
\text { PAC } 2 \text { Listin Table } 1\end{array}$ \\
\hline one & 2868 S Strychnine suffate (2:1) & 60:41-3 & $000060-41-3$ & 383.49 & $\mathrm{~s}$ & $2.000+02$ & & $27005 \mathrm{~mm}$ & 1.000 .08 & $2.500++01$. & & PAC 27 Listing Table 1 \\
\hline 20870 & 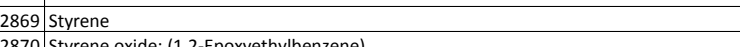 & $100 \cdot 42 \cdot 5$ & $\frac{000100-42-5}{0.000-503}$ & 104.15 & & 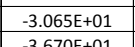 & 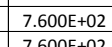 & $\begin{array}{l}1.4506+02 \\
1.0525+02\end{array}$ & $\begin{array}{lll}6.4006+500 \\
3.00501\end{array}$ & $\begin{array}{l}2.5000+01 \\
2.0050101\end{array}$ & $\begin{array}{l}6.400 E+00 \\
3.00501\end{array}$ & CHAO, ET AL. (1983) \\
\hline $\begin{array}{ll}28872 \\
22872 \\
\end{array}$ & 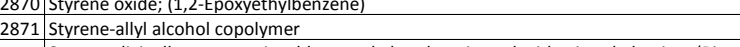 & 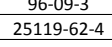 & 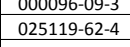 & & $\frac{t}{s}$ & & & & & & & 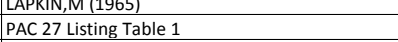 \\
\hline 2873 & 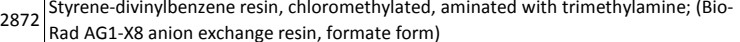 & 60177-39.1 & 060177-39.-1 & & $\mathrm{s}$ & & & & & & & PAC 27 Listing Table 1 \\
\hline 28874 & $\begin{array}{l}2873 \text { Styrenesulfonate), poly(sodium 4- } \\
8274 \text { Succicic acid }\end{array}$ & 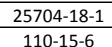 & 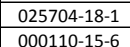 & $\frac{70000}{1118}$ & $\frac{5}{5}$ & 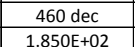 & & $23560+022$ & & & & \begin{tabular}{|l} 
PAC 27 Listing Table 1 \\
PAC 27 Listin Table 1
\end{tabular} \\
\hline
\end{tabular}




\begin{tabular}{|c|c|c|c|c|c|c|c|c|c|c|c|c|c|}
\hline & No. & Chemical Compound & $\begin{array}{c}\text { cas Number } \\
\text { (CASNR) }\end{array}$ & $\begin{array}{c}\text { Modified } \\
\text { Format CAS }\end{array}$ & $\begin{array}{l}\text { Molecular } \\
\text { Weight MW }\end{array}$ & 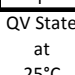 & $\begin{array}{c}\text { MPorfP } \\
\text { [Cc }\end{array}$ & $\begin{array}{llll}\text { Bep ressure } \\
\text { [mm M }\end{array}$ & вр [ст] & $\begin{array}{c}\text { vapor } \\
\text { pressurue }\end{array}$ & $\begin{array}{c}\text { Toryp } \\
\text { prcl }\end{array}$ & 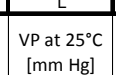 & 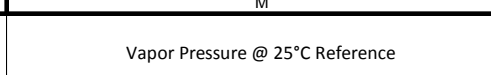 \\
\hline & & Succinic anhydide & $100 \cdot 30.5$ & $\frac{000108-30.5}{00003020}$ & 100.08 & & $1.196 \mathrm{E}+02$ & & $2.610 €+02$ & & $9.2000+01$ & & 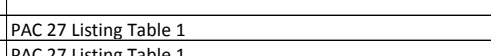 \\
\hline 20878 & & 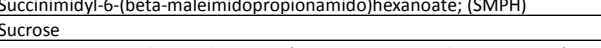 & $\frac{22.020}{57-50-1}$ & 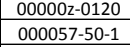 & 342.34 & 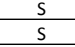 & $1.855+02$ & & & & & & 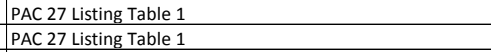 \\
\hline$\frac{2879}{2280}$ & & 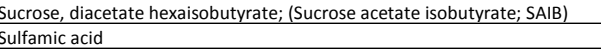 & 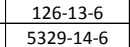 & & & $\frac{s}{5}$ & $\sim 205$ dec & & & & & & 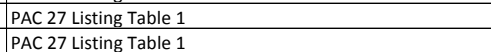 \\
\hline & & 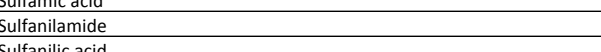 & 63374.1.1. & 0.00063-74-74-1 & $\begin{array}{lll}172.22 \\
172.29\end{array}$ & $\begin{array}{c}2 \\
2\end{array}$ & $\begin{array}{cccc}1.655 \mathrm{et}+02 \\
1.52\end{array}$ & & & & & & 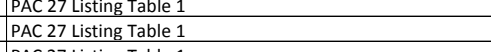 \\
\hline $\begin{array}{lll}2882 \\
2833 \\
\end{array}$ & 2882] & 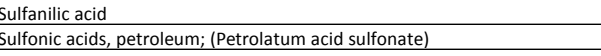 & 61789895:-3. & $\begin{array}{l}000121-175-3 \\
001789-85-3\end{array}$ & 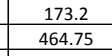 & $\begin{array}{l}s \\
\text { L }\end{array}$ & & 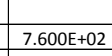 & .5.5106+02 & $2.620-14$ & $2.500=01$ & $2.620-14$ & 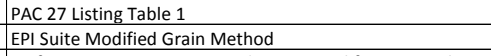 \\
\hline 2884 & 2883 & 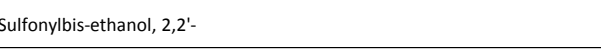 & $2580-77-0$ & $002580-77.0$ & 154.19 & $\mathrm{~L}$ & & $7.600 €+02$ & 3.154 $15 \mathrm{~F}+2$ & $2.350 \mathrm{~F}+01$ & 2.000E+01 & $2.639 \mathrm{E}+01$ & 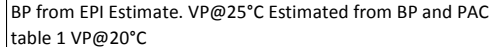 \\
\hline & & 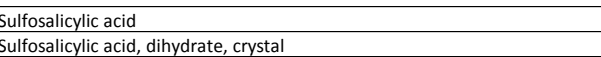 & $\frac{97.052 .2}{556583-3}$ & 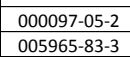 & $\frac{218.19}{25421212}$ & $\frac{s}{s}$ & $\frac{1.200+102}{108-110}$ & & & & & & 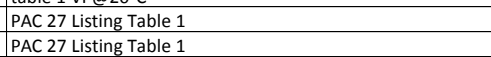 \\
\hline$\frac{2887}{2088}$ & & 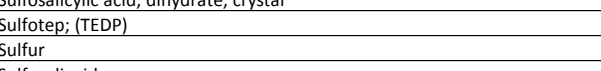 & 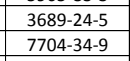 & 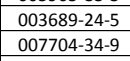 & $\frac{32.234}{32.07}$ & $\frac{s}{5}$ & 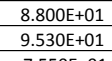 & & 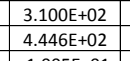 & 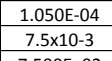 & 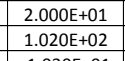 & & 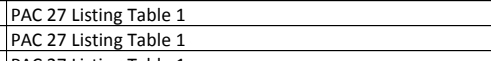 \\
\hline 2889 & & 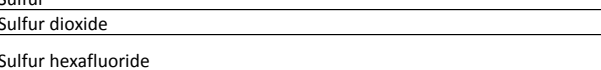 & 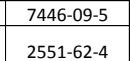 & 002551-62-4 20. & $\frac{64.06}{146.06}$ & $\frac{G}{G}$ & 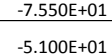 & & $\frac{1.0 .0550 .02}{1000}$ & $1.000+02$ & & & PAC 27 Listing Table 1 \\
\hline$\frac{22890}{2091}$ & & 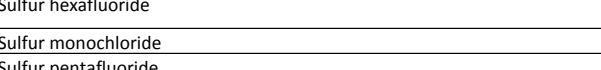 & $10025 \cdot 67.9$ & 010025.67 .9 & $\frac{135.02}{2512}$ & L & 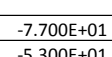 & $\begin{array}{l}7.600+2+0 \\
7.6002+0\end{array}$ & 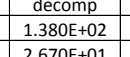 & 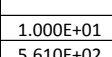 & 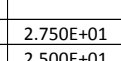 & 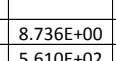 & 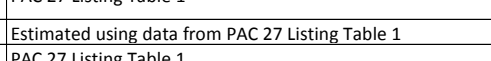 \\
\hline 28992 & $28991]$ & 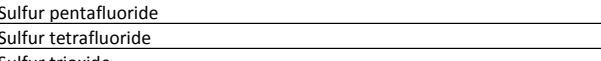 & Th1742-7.7. & 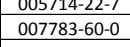 & 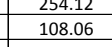 & $\frac{1}{6}$ & 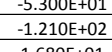 & & 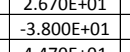 & & & & 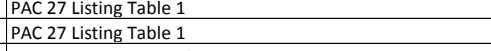 \\
\hline$\frac{2894}{2005}$ & 2893 & $\begin{array}{l}\text { Suffur trioxide } \\
\text { unufuric acid }\end{array}$ & $\begin{array}{l}7446611.9 \\
76-939\end{array}$ & 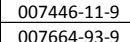 & & & 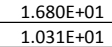 & $\begin{array}{l}7.600+022 \\
7.600202\end{array}$ & & 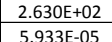 & $\begin{array}{l}2.500+51 \\
2500+511\end{array}$ & 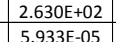 & 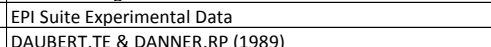 \\
\hline 2096 & 2895 & sulfuric acid-did; ; (Deuterosouffuricic aciid) & 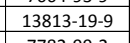 & 018383-19.9.9 & 100.09 & & $\frac{1.0315+01}{1.01}$ & $7.600 \mathrm{E}+02$ & $2.9000+02$ & $5.9300-05$ & 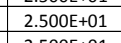 & 5.9300-05 & 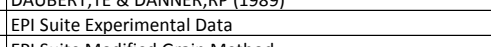 \\
\hline$\frac{2098}{2098}$ & 28997 & 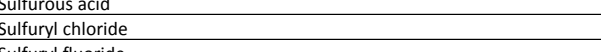 & 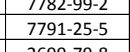 & 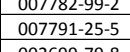 & $\begin{array}{l}\frac{8208}{134.96} \\
1396\end{array}$ & $\frac{L}{L}$ & 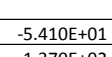 & $\begin{array}{l}7.600+522 \\
7.600+022\end{array}$ & 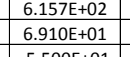 & 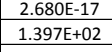 & $\begin{array}{l}2.500+501 \\
2.500+01\end{array}$ & $\begin{array}{l}2.680-17 \\
1.397+02\end{array}$ & 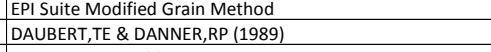 \\
\hline & & & 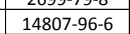 & & & & & & & & & & PAC 27 listing Table 1 \\
\hline 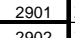 & 30 & 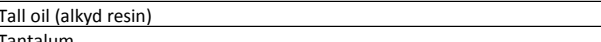 & 683333-62-0. & 0683333.62:- & 412.57 & & & $7.600++022$ & 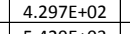 & 3.350E:07 & $2.5005+011$ & 3.350-0.0 & Modified Grain Method; as CAS 8002-26-4 \\
\hline 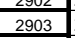 & 然2 & 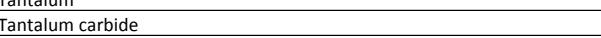 & 年2070.06-3. & 年2070.06: & 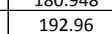 & s & $3.875+03$ & & 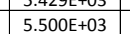 & & & & ing Table 1 - \\
\hline 2004 & & Tatatum(l) enthoxide & 60744846.6 & 0.6077.84.- & 告60.251 & & $\begin{array}{l}2.000+01 \\
2.00+011\end{array}$ & $7.600 E+022$ & $1.5010+0202$ & 8.280E+01 & 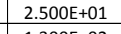 & 8.2805+011 & raco L Labs PI \\
\hline 2005 & 2005 & 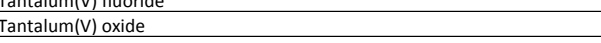 & 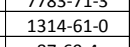 & 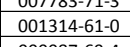 & 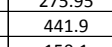 & $\frac{5}{5}$ & 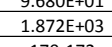 & & & & & & 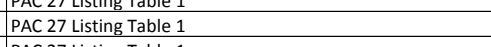 \\
\hline$\frac{2008}{2008}$ & 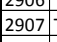 & $\begin{array}{l}\text { artartacicacid } \\
\text { Tartaric acid, monopotasssium salt }\end{array}$ & 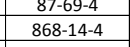 & o00868-14.4 & 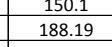 & $\frac{5}{5}$ & 170-172 & & & & & & 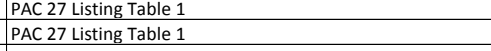 \\
\hline 2099 & 20089 & 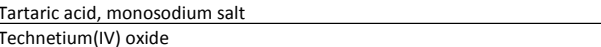 & 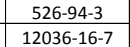 & 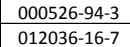 & $\frac{172.28}{130}$ & & & & & & & & 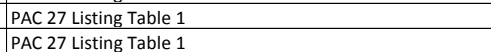 \\
\hline 2991 & 2910 & Tellutium & 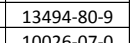 & 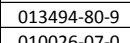 & 127.6 & $\frac{5}{5}$ & 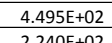 & & $\begin{array}{ll}9.8806+02 \\
3.8005502\end{array}$ & $7.500: 01$ & $5.020 E+02$ & & 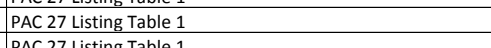 \\
\hline 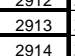 & 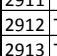 & 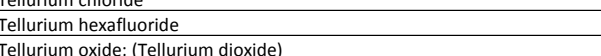 & 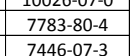 & 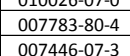 & 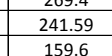 & $\frac{6}{5}$ & 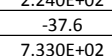 & & 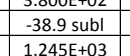 & $7.600+02$ & $2.000 \notin 01$ & & 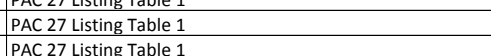 \\
\hline$\frac{2945}{2915}$ & 2914 & Tetlurous acid & 10049-23-7.7 & 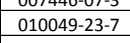 & 177.614 & $\frac{3}{6}$ & & & 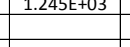 & & & & 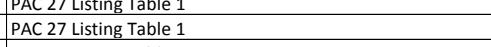 \\
\hline$\frac{2916}{2917}$ & 2915 & Terbium oxide & 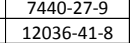 & & & $\frac{5}{5}$ & 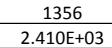 & & 3.230E +03 & & & & \\
\hline $\begin{array}{ll}29219 \\
22919 \\
2\end{array}$ & $\begin{array}{l}2917 \\
2218\end{array}$ & 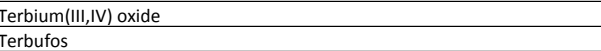 & 1.2037-1.1.3 & 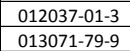 & $\begin{array}{l}777.6974 \\
288.45\end{array}$ & $\frac{S}{L}$ & $\begin{array}{l}\frac{2300}{2.900+01} \\
-201\end{array}$ & $7.600 E+02$ & $3.1500+02$ & 3.000:04 & $2.500 E+01$ & 3.000E-04 & 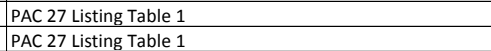 \\
\hline$\frac{2920}{22021}$ & 2019| 2019 & $\begin{array}{l}\text { Terephthalic acid } \\
\text { Terephthaloyl chloride }\end{array}$ & $\frac{10-212-0}{100-2 \cdot 9}$ & 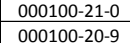 & $\frac{166.14}{20302}$ & s & $83-84$ & & $\begin{array}{l}30 \text { subl } \\
2.6600+02\end{array}$ & & & & $\begin{array}{l}\text { PAC } 27 \text { Listing Table } 1 \\
\text { PAC } 2 \text { Listing gable } 1\end{array}$ \\
\hline 2920 & 2921 & Terphenyl, p- & $92 \cdot 94 \cdot 4$ & $000092-94 \cdot 4$ & 230.32 & $\mathrm{~s}$ & 212-213 & & 259 @ 45 & & & & PAC 27 listing Table 1 \\
\hline 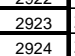 & 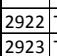 & 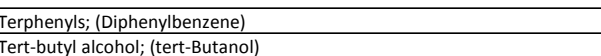 & $\frac{26190.60 \cdot 3}{75-50.5}$ & 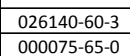 & $\frac{230.32}{74.14}$ & $\frac{\mathrm{s}}{\mathrm{L}}$ & $2.500+0101$ & $7.600 E+02$ & $8.280+01$. & $4.0070 \mathrm{E}+01$ & $2.5005+01$ & $4.0706+010$ & $\begin{array}{l}\text { PAC } 27 \text { Listing Table } 1 \\
\text { DAUUERT }\end{array}$ \\
\hline (2925 & 2024 & 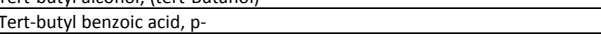 & $98-7 \cdot-7$ & 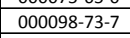 & $\frac{178.25}{10}$ & 5 & 164.5 & & & & & & PAC 27 listing Table 1 T \\
\hline 2926 & 29255| & Tert-butyllithium & $594 \cdot 19 \cdot 4$ & 000599-19-4 & 64.06 & $\mathrm{~s}$ & & & Subl@ @ $0.1 c \mid$ & & & & PAC 27 listing Table 1 \\
\hline $\begin{array}{lll}29272 \\
2298 \\
\end{array}$ & 2026] 2027$]$ & 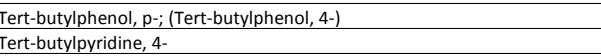 & 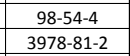 & 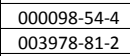 & $\frac{150.24}{135.2084}$ & $\frac{s}{L}$ & $\frac{99}{-4.100+51}$ & $7.600 \mathrm{~F}+22$ & \begin{tabular}{|l}
$233-238$ \\
$1.955+02$ \\
\end{tabular} & 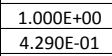 & $\begin{array}{l}7.000+51 \\
2.500+0+1\end{array}$ & $4.290 E-01$ & 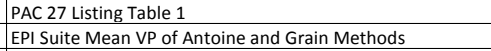 \\
\hline 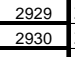 & 2928 & 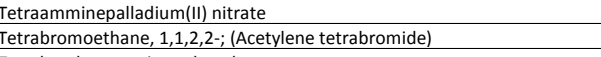 & 136010-8.6 $79-6$ & 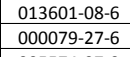 & 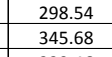 & $\frac{s}{1}$ & $-1.000 E+00$ & $5.400 €+01$ & $1.510 \mathrm{E}+22$ & $2.000: 02$ & $2.500+011$ & $2.000=02$ & 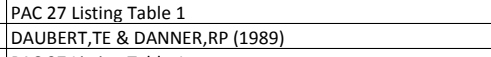 \\
\hline & & 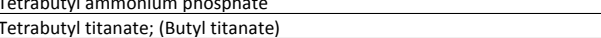 & & & & & -5.500Et+01 & & & & & & \\
\hline 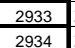 & & 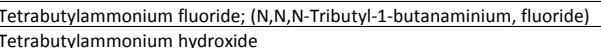 & 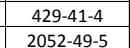 & 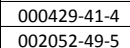 & $\begin{array}{l}261.46 \\
25954\end{array}$ & $\frac{1}{5}$ & $\begin{array}{c}5.600 E+1 \\
2.5000+01\end{array}$ & $7.600+02$ & $4,4888+02$ & 1.410E-08 & $2.500++01$. & 1.410E:08 & offied Grain Nethod \\
\hline 20936 & 29345 & 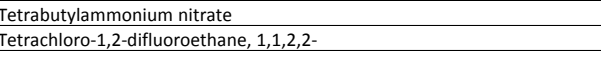 & $\frac{199127-1}{76-12-0}$ & $\begin{array}{l}000914172-1 \\
0.0076-12.0\end{array}$ & 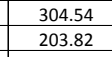 & 5 & $\frac{118.820}{2.455+01}$ & $7.680 \mathrm{E}+022$ & $9.280 \mathrm{E}+01$ & $5.050 E+011$ & $2.500 €+01$ & $5.050+01$ & 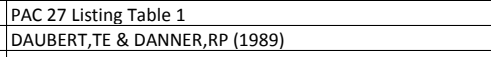 \\
\hline
\end{tabular}




\begin{tabular}{|c|c|c|c|c|c|c|c|c|c|c|c|c|c|}
\hline & o. & chemical Compound & $\begin{array}{c}\text { cas Number } \\
\text { (CASSN) }\end{array}$ & $\begin{array}{c}\text { Modified } \\
\text { Format CAS }\end{array}$ & 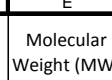 & 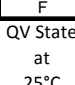 & $\begin{array}{c}\text { Mporfp } \\
\text { [Cc }\end{array}$ & $\begin{array}{l}\text { sp peressure } \\
\text { [mm Mgl }\end{array}$ & $\mathrm{BP}_{\mathrm{P}[\mathrm{c}]}$ & 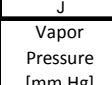 & $\begin{array}{c}\text { Tfor vp } \\
{\left[{ }^{\circ C}\right]}\end{array}$ & 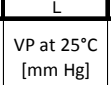 & Vapor Pressure @ $25^{\circ}$ C Reference \\
\hline & 烈 & 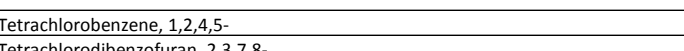 & $\frac{95.943}{55273.9}$ & 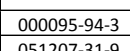 & $\begin{array}{l}215.89 \\
20597 \\
3507\end{array}$ & & $\frac{1.395 E+02}{22025020}$ & & $2.455+02$ & & & & 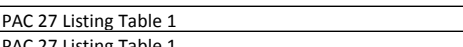 \\
\hline$\frac{2939}{2090}$ & 2939 & 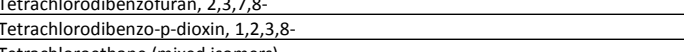 & 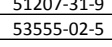 & 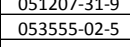 & $\begin{array}{l}30.97 \\
321.96 \\
3\end{array}$ & $s$ & & & & & & & 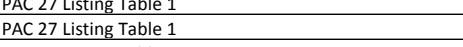 \\
\hline & & 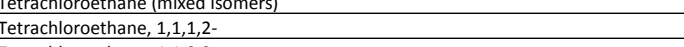 & & & & & & & & & & & UERET, EE \& DAN NER,RP (1989) \\
\hline & 繁2 & Tetatachlorethane, 1,2,2.2. & 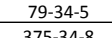 & 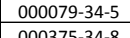 & $\begin{array}{l}167.85 \\
37.84\end{array}$ & & 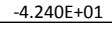 & 7.600E+t & 1.452E+c & 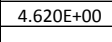 & $2.500 \mathrm{O}+0101$ & $4.620 \mathrm{E}+0 \mathrm{CC}$ & 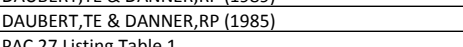 \\
\hline 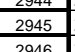 & 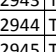 & 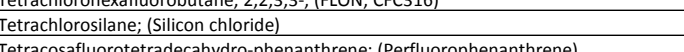 & 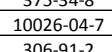 & 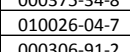 & 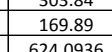 & & 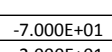 & $7.6800+022$ & 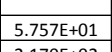 & $2.3644+022$ & 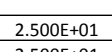 & 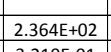 & 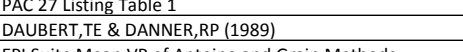 \\
\hline & & 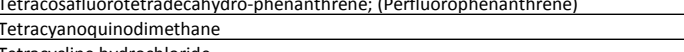 & 150-619-2.-7 & 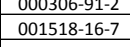 & 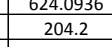 & & 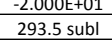 & & & & & & 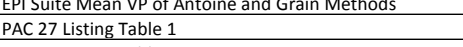 \\
\hline$\frac{2.246}{2099}$ & 948 & & $355-42-0$ & 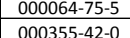 & $\begin{array}{l}20.94 \\
33806\end{array}$ & & - $\frac{170-175}{-8206+01}$ & 7.6806+02 & 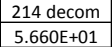 & 2.179e+02 & $2.500 \mathrm{O}+01 \mathrm{1}$ & 2.179eEto & 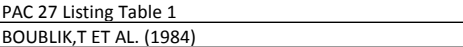 \\
\hline $2950 \mid$ & 9999 & 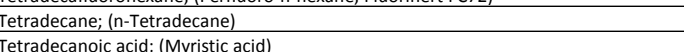 & $\begin{aligned} 629.59 \cdot 4 \\
545-3.8\end{aligned}$ & 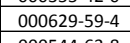 & 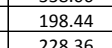 & $i$ & $\begin{array}{l}5.5006+00 \\
5000+501\end{array}$ & $7.600++022$ & 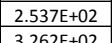 & 1.1606-02 & $2.500++011$ & & 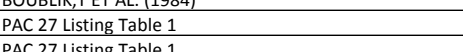 \\
\hline & & tetradectuttimimethlyammonium bromide & 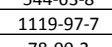 & 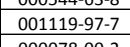 & 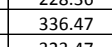 & 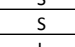 & 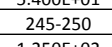 & & & & & & $\begin{array}{lll}A & 1\end{array}$ \\
\hline$\frac{2963}{2054}$ & 953 & 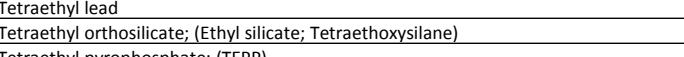 & $\begin{array}{l}78.0 .2 \\
78-10.4 \\
70.02\end{array}$ & 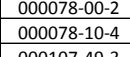 & $\begin{array}{l}323.47 \\
20833 \\
2032\end{array}$ & $\frac{t}{L}$ & 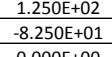 & 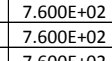 & 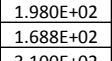 & 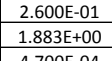 & 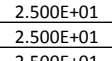 & 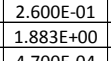 & 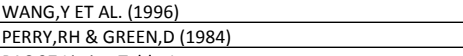 \\
\hline & & 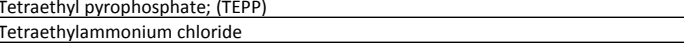 & & & & & & & & & & & \\
\hline$\frac{2055}{\frac{2055}{205}}$ & 956 & Tetrathylene elyycol & 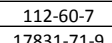 & 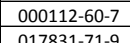 & 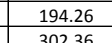 & & $\begin{array}{l}-6.000 E+00 \\
1020+520\end{array}$ & $7.600+5022$ & 3.2.73E+02 & 4.650-05 & $2.500+5011$ & 4.650-0.0: & 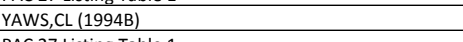 \\
\hline 20599 & 20588 & 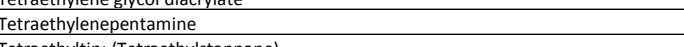 & 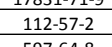 & 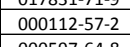 & 189.36 & $\frac{L}{L}$ & 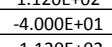 & 7.6.600 0022 & 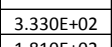 & $\begin{array}{l}8.000=07 \\
0.07\end{array}$ & 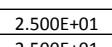 & 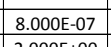 & 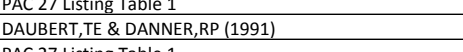 \\
\hline & & 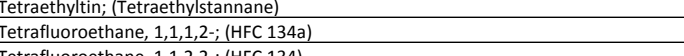 & 811-67-97-2 & & $\begin{array}{lll}2.39 .97 \\
102.04\end{array}$ & & & & 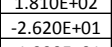 & $\begin{array}{l}2.0000+700 \\
0.600+01\end{array}$ & 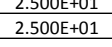 & & 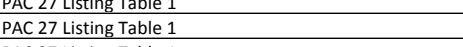 \\
\hline$\frac{2062}{2063}$ & 961 & 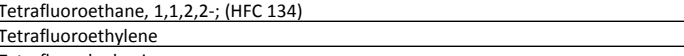 & 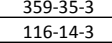 & 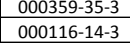 & & & 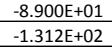 & & 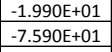 & 7.500E+02 & $-7.580+5011$ & & 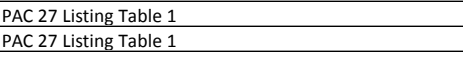 \\
\hline 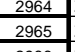 & 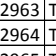 & 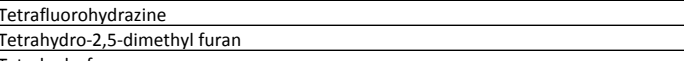 & 1.0036-37-2. & 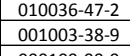 & $\frac{104}{100.18}$ & $\frac{6}{1}+\frac{1}{L}$ & 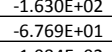 & $7.600 €+02$ & $9.200 E+011$ & 3.640E+ +01 & $2.500 \mathrm{E}+01$ & 3.640E+ +01 | & 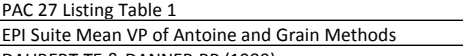 \\
\hline (2007 & 2966 T & 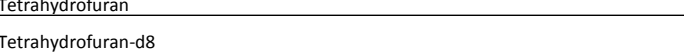 & $1633-749$ & 001693-74.9 & 80.154 & $\mathrm{t}$ & $-1.060+02$ & $7.600 E+02$ & 6.500E+01 1 & $1.620 \mathrm{~A}+02$ & $2.500+501$ & $1.620 \mathrm{E}+02$ & 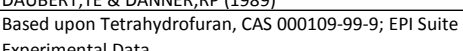 \\
\hline 20068 & $2967 T^{\top}$ & 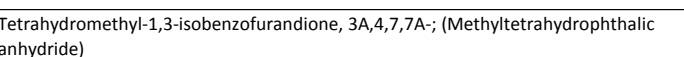 & $26590-20.5$ & 02659-20-5 & 166.19 & $\mathrm{~s}$ & & & & & & & PAC 27 Listing Table 1 \\
\hline 2096 & $\begin{array}{llll}29667 & 7 \\
\end{array}$ & 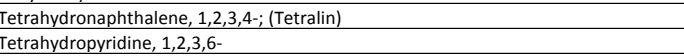 & 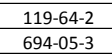 & 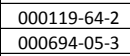 & $\begin{array}{l}132.22 \\
83.13\end{array}$ & $\frac{t}{L}$ & 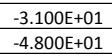 & $\begin{array}{l}7.600+502 \\
7.6005+02\end{array}$ & $\begin{array}{l}2.0726+522 \\
1.080+02\end{array}$ & $\begin{array}{l}3.6 .600-01 \\
.2505+01\end{array}$ & $\begin{array}{l}2.500+5+1 \\
2.500+01\end{array}$ & 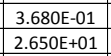 & 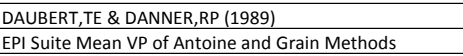 \\
\hline$\frac{2971}{2972}$ & 2970 & 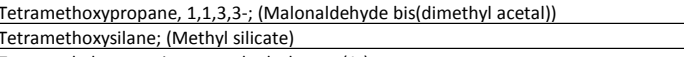 & 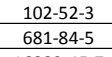 & 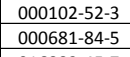 & 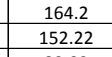 & $\frac{s}{L}$ & $-1.000+00$ & $7.600+502$ & $1.2100+02$ & 7.500E+00 & $1.4005+01$ & $1.376 E+01 \mid$ & 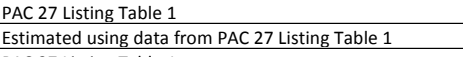 \\
\hline & & 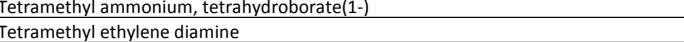 & & & & & . $5.5000+01$ & 7.6006+02 & $1.200 \mathrm{E}+022$ & 1.500e+01 & $2.500+01.10$ & & 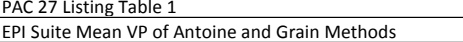 \\
\hline 2975 & 20747] & Tetramethyl lead & 75-74-1 & 0.00075-74-1.1. & 267.35 & & 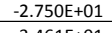 & 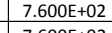 & 1.1006 +02 & $2.000+01$. & $2.500 \mathrm{E}+01.11$ & $2.600 \mathrm{E}+011$ & VANGG, ET AL. (1996) \\
\hline$\frac{2977}{2097}$ & 年 & 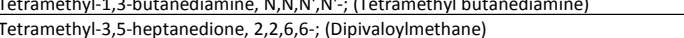 & $\frac{11167 \cdot 7 \cdot 4}{10.4}$ & 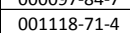 & $\frac{184.28}{18.28}>0$ & $\frac{t}{4}$ & & $\begin{array}{lll}3.5000601 \\
3\end{array}$ & & 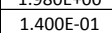 & 2.5000+ & 1.0000-01 & 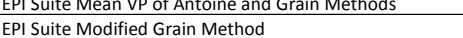 \\
\hline (1070 & $\left.2977\right|_{\mathrm{T}}$ & Tetramethy-5.-decyne-4,7-diol, 2,4,7,9.9- & 122-86-3 & 000126-86-3 & 226.36 & $s$ & 4.700E+01 & & 165@ @ 40 & & & & PAC 27 Listing Table 1 \\
\hline $\begin{array}{lll}22979 \\
2280\end{array}$ & 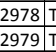 & $\begin{array}{l}\text { Tetramethylammonium chloride } \\
\text { Tetramethylammonium hydroxide }\end{array}$ & $\frac{75.57 \cdot 0}{75-59-2}$ & 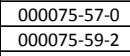 & $\frac{29.18}{91.18}$ & $\frac{S}{L}$ & $\frac{>3.30}{6.505+01}$ & $7.600 E+02$ & $1.020 E+02$ & 2.5800 .07 & $2.500 \mathrm{E}+01$ & 2.580007 & 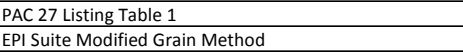 \\
\hline & & Tetramethylammonium myltoxide pentahydrate & $\frac{1024265 .}{55116851}$ & 053116- & $\frac{1812288}{21132}$ & & $6.800 \mathrm{Et}+\mathrm{C}$ & & & & NOEE+C & & 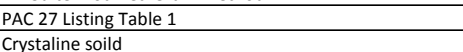 \\
\hline & & Tetramethybenzene, 1, & 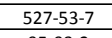 & c000527.53 & $\begin{array}{l}134.24 \\
\end{array}$ & & $-2.4005+0$ & $7.6800+022$ & 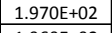 & 4.977 0.0. & $2.500=0+0.0$ & 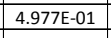 & S.CL (1994) \\
\hline $\begin{array}{lll}20055 \\
2035\end{array}$ & 2084 & 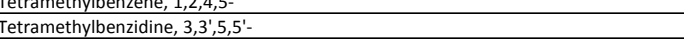 & 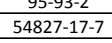 & 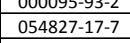 & $\frac{140.38}{248}$ & $\frac{5}{5}$ & 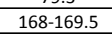 & & & & & & 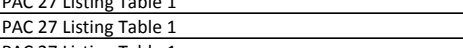 \\
\hline & 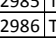 & Tetramethylene sulfone; ; Sulfolane; Tetrahydro & 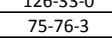 & 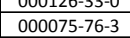 & $\frac{120.18}{88.23}$ & & & 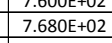 & & & & & \\
\hline & & Fetranitromethane & 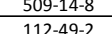 & 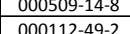 & $\frac{19603}{17936}$ & & $\begin{array}{l}1.300601 \\
-1500501\end{array}$ & $\begin{array}{ll}7.600+0202 \\
7.6020\end{array}$ & 1.26110202 & $8.420 F^{2}+000$ & 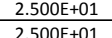 & 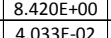 & jOUBLLK, T ET AL. (1984) \\
\hline 200 & $2989 \sigma_{\mathrm{T}}$ & 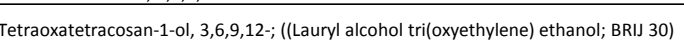 & 5274.68.0 & 00527468.0 & 362.5488 & $\mathrm{t}$ & & $2.470+02$ & $1.0005+01$ & 1.520:09 & $2.500=+01$ & $\mid 1.520=09$ & EPI Suite Modified Grain Method \\
\hline$\frac{2091}{2091}$ & 2990 17 & 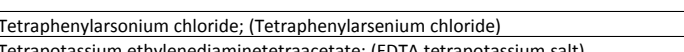 & 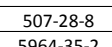 & 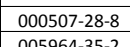 & $\frac{418.81}{41064}$ & & $258-260$ & & & & & & \\
\hline$\frac{2039}{2093}$ & 2092] & 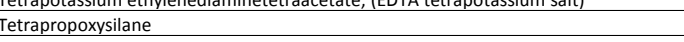 & 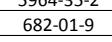 & 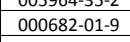 & 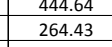 & $\frac{S}{L}$ & & $7.600 E+022$ & $9.000 \mathrm{e}+01.10$ & 5.440E-02 & $2.500+01$ & 5.400E-0.0. & $\begin{array}{l}\text { PAC } 2 \text { L Lisiting Gable } 1 \\
\text { EP Suin Method }\end{array}$ \\
\hline 20995 & 292947 & 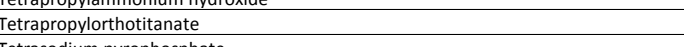 & 3087-37.-4 & 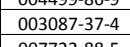 & 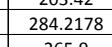 & $\frac{5}{5}$ & $0601000 \mathrm{~mm}$ & & $170 @ 38 C$ & & & & 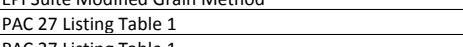 \\
\hline 2097 & 29967 & & $288 \cdot 94.8$ & 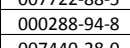 & 70.05 & 5 & $156-158$ & & & & & & \\
\hline$\frac{2009}{2009}$ & 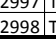 & 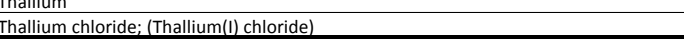 & $\begin{array}{l}7791-12.0 \\
7720\end{array}$ & 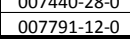 & 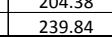 & 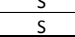 & 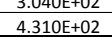 & & $\frac{1.2006+02}{7}$ & $\frac{7.5006+00}{7.500}$ & 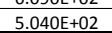 & & 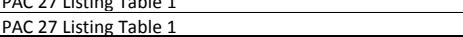 \\
\hline
\end{tabular}




\begin{tabular}{|c|c|c|c|c|c|c|c|c|c|c|c|c|c|}
\hline & A & & & & 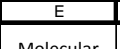 & $\frac{F}{\text { Qv state }}$ & 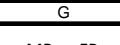 & 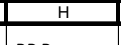 & 1 & & $\mathrm{k}$ & & 1 \\
\hline & No. & Chemical Compound & $\begin{array}{l}\text { CAS Number } \\
\text { (CASRNN) }\end{array}$ & $\begin{array}{c}\text { Modified } \\
\text { Format CAS }\end{array}$ & $\begin{array}{c}\text { Molecular } \\
\text { Weight (MW) }\end{array}$ & $\mid \begin{array}{c}\text { at } \\
25^{\circ} \mathrm{C}\end{array}$ & $\begin{array}{c}\text { MP or FP } \\
{\left[{ }^{\circ} \mathrm{C}\right]}\end{array}$ & $\begin{array}{c}\text { BP Pressure } \\
{[\mathrm{mm} \mathrm{Hg}]}\end{array}$ & $\mathrm{BP}\left[{ }^{[\mathrm{C} C}\right]$ & $\begin{array}{l}\text { Vapor } \\
\text { Pressure } \\
\text { Imm Hgat }\end{array}$ & $\begin{array}{c}\text { Tfor VP } \\
{\left[{ }^{\circ C} \mathrm{C}\right]}\end{array}$ & $\begin{array}{c}\text { VP at } 25^{\circ} \mathrm{C} \\
{[\mathrm{mm} \mathrm{Hg}]}\end{array}$ & 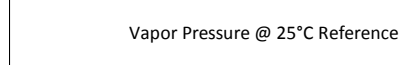 \\
\hline 3000 & 2999 T & Thallium hydroxide & $12026-06-1$ & $012026-06-1$ & 221.39 & & $139 \mathrm{dec}$ & & & & & & PAC 27 Listing Table 1 \\
\hline 3001 & $3000 / \mathrm{T}$ & Thallium nitrate; (Thallium(I) nitrate) & $10102-45-1$ & $010102-45-1$ & 266.39 & $s$ & $2.0606+02$ & & $450 \mathrm{dec}$ & & & & PAC 27 Listing Table 1 \\
\hline 3002 & $\begin{array}{l}3001 \mathrm{~T} \\
3002 \mathrm{~T}\end{array}$ & $\begin{array}{l}\text { Thallium nitrite } \\
\text { Thallium oxide } \\
\end{array}$ & $\frac{\frac{13826-63-6}{1314-12-1}}{2}$ & $\begin{array}{ll}013826-63-6 \\
001314-12-1\end{array}$ & 250.38 & $\frac{s}{s}$ & $5.7906+02$ & & $\sim 1080$ & & & & $\begin{array}{l}\text { PAC 27 Listing Table } 1 \\
\text { PACC2 }\end{array}$ \\
\hline 3004 & 3003 T & Thallium sulfate; ; Sulfuric acid, dithallium(1+1) salt) & $\frac{1134-12-1}{10031-59-1}$ & $00031-59-1$ & 1526.25 & s & 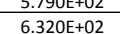 & & decom & & & & 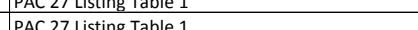 \\
\hline & & & 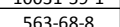 & $000563-68-8$ & 26342 & & 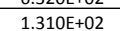 & & & & & & PAC 27 listing arable 1 \\
\hline 3006 & $\left.3005\right|_{17}$ & Thallium(1) carbonate (2:1) & $6533-73-9$ & $006533-73-9$ & 2668.78 & $\mathrm{~s}$ & 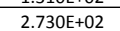 & & & $<1 E-6$ & $2.5006+01$ & & $\begin{array}{l}\text { PAC 27 Listing Table } 1 \\
\text { Pat }\end{array}$ \\
\hline 3007 & 3006 T & Thallium(I) sulfate; (Sulfuric acid, dithallium(1+) salt) & 7446-18-6 & $007446-18-6$ & 504.8 & $\mathrm{~s}$ & $6.3206+02$ & & decom & & & & PAC 27 Listing Table 1 \\
\hline 3008 & 3007T & $\begin{array}{l}\text { Thalllium(IIII) oxide; Thallium sesquioxide; Thallic oxide) } \\
\text { TTanllium(III) perchlorate hexhanyrate }\end{array}$ & $\frac{1314-32-5}{15596-83-5}$ & $001314-32-5$ & 456.77 & $\frac{s}{s}$ & $8.340 E+02$ & & & & & & $\begin{array}{l}\text { PAC 27 Listing Table } 1 \\
\text { PAC }\end{array}$ \\
\hline$\frac{3099}{3010}$ & $\begin{array}{lll}3008 \\
3009 T_{T}\end{array}$ & $\begin{array}{l}\text { Thallium(IIII) perchlorate hexahydrate } \\
\text { Thallous malonate }\end{array}$ & $\begin{array}{r}\frac{15556-83-5}{2757-18-8} \\
\end{array}$ & $\begin{array}{l}015956-8-5 \\
002757-18-8 \\
\end{array}$ & 610.83 & $\frac{\mathrm{s}}{\mathrm{s}}$ & & & & & & & $\begin{array}{l}\text { PAC C7 Listing a able } 1 \\
\text { PAC 27 Listing Table } 1\end{array}$ \\
\hline Pon & 3010 T & Thenoyltrifluoroacetone & $326-91-0$ & $000326-91-0$ & 221.19 & $\mathrm{~s}$ & $4.2806+01$ & & $97 @ 8$ & & & & PAC 27 Listing Table 1 \\
\hline$\frac{3011}{3012}$ & $3011 \mathrm{~T}$ & Thioacetamide & $62-55-5$ & $000062-55-5$ & 75.13 & $\mathrm{~s}$ & $1.155 \mathrm{E}+02$ & & & & & & \begin{tabular}{|l} 
PAC 27 Listing Table 1 \\
\end{tabular} \\
\hline & & & & & & & $-1.700 E+01$ & $7.600 E+02$ & $9.300 E+01$ & & $2.500 E+01$ & & \\
\hline$\frac{2014}{3014}$ & 3013 (3) & Thiobis(4-chloro-6-methyl)phenol, 2,2,-. & $4418-66-0$ & $004418-66-0$ & 315.22 & $\mathrm{~s}$ & & & 4.430E+02 & $1.0006-07$ & $\frac{2.5006+1}{2.501}$ & & $\begin{array}{l}\text { PAC } 27 \text { Listing Table } 1 \\
\text { Pats }\end{array}$ \\
\hline 3015 & 3014 T/ & Thiocarbazide; (Thiocarbohydrazide) & $2231-57-4$ & $002231-57-4$ & 100.17 & $\mathrm{~s}$ & $1.640 \mathrm{E}+02$ & & & & & & PAC 27 Listing Table 1 \\
\hline 3016 & 3015 T & 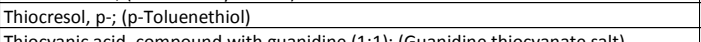 & $106-45-6$ & $000106-45-6$ & 124.21 & $\mathrm{~s}$ & $43-44$ & & $1.950 \mathrm{E}+02$ & $8.070 \mathrm{E}-01$ & $2.500 \mathrm{E}+01$ & & PAC 27 Listing Table 1 \\
\hline 3017 & 每016/T & $\begin{array}{l}\text { Thiocyanic acid, compound with guanidine (1:1); (Guanidine thiocyanate salt) } \\
\text { Triodiegrcol }\end{array}$ & $\frac{593-84-0}{111-48-8}$ & $\frac{000593-84-0}{000111-48-8}$ & $\frac{118.18}{1222}$ & $\frac{s}{L}$ & $\frac{114-117}{-1600 \mathrm{~F}+01}$ & $2000 \mathrm{E}+01$ & $1640 \mathrm{E}+02$ & $32300-03$ & $2500 E+01$ & $3230 \mathrm{E}-03$ & 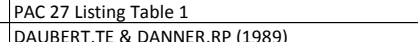 \\
\hline$\frac{3018}{3019}$ & 3018T/T & $\begin{array}{l}\text { Inlodilgycol } \\
\text { Thiofanox; (Dacamox) }\end{array}$ & $\frac{111-18-8-8}{39196-18-4}$ & $00001114-48-8$ & $\frac{1122.2}{218.32}$ & $\frac{1}{5}$ & 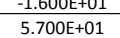 & 2.000Et+01 & & $\frac{3.230 E-03}{1.7 \times 10-4}$ & $\frac{2.500 E+01}{2.500 E+01}$ & $3.230 \mathrm{E}=03$ & $\begin{array}{l}\text { DAABER, II \& E DNANNR,RP (1989) } \\
\text { PAC } 27 \text { listing Table } 1\end{array}$ \\
\hline 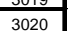 & 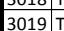 & 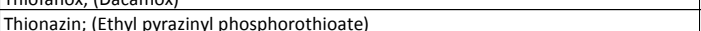 & 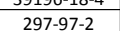 & 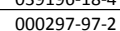 & 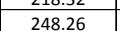 & $\frac{\mathrm{s}}{\mathrm{L}}$ & 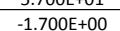 & $7.600 E+02$ & $8.000 E+01$ & 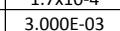 & 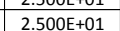 & $3.000 E-0.0$ & BCACCETAL \\
\hline & & & $7719-09-7$ & $007719-09-7$ & 118.96 & $\mathrm{~L}$ & & 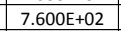 & $7.880 \mathrm{E}+01$ & $1.193 \mathrm{E}+02$ & $2.500 E+01$ & $1.193 \mathrm{E}+0.2$ & $\begin{array}{ll}\text { DAUB ERRT, TE \& DANNER,RP (1989) } \\
\text { (1) }\end{array}$ \\
\hline 3022 & 3021 T & Thiophosphoryl chloride & $3982-91-0$ & 003982-91-0 & 169.38 & G & $-3.500 \mathrm{-}+01$ & $7.600 \mathrm{E}+02$ & $1.250 \mathrm{E}+02$ & $1.635 \mathrm{E}+03$ & $2.5006+01$ & & PAC 27 Listing Table 1 \\
\hline 3023 & 3022 T & $\begin{array}{l}\text { Tlinsomicarbazide } \\
\text { Thiourea }\end{array}$ & $79-19-6$ & $000079-19-6$ & $\frac{91.14}{76512}$ & $\frac{\mathrm{s}}{\mathrm{s}}$ & $\frac{1.830 \mathrm{E}+02}{17025+02}$ & & dec & $28 \times 10-3$ & $25005+01$ & & $\begin{array}{l}\text { PAC 27 Listing Table } 1 \\
\text { PAC27listing }\end{array}$ \\
\hline & 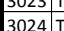 & $\begin{array}{l}\text { Thiourea } \\
\text { Thiram; (Tetramethyythioperoxydicarbonic diamide) }\end{array}$ & 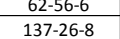 & $\begin{array}{l}000062-56-6 \\
000137-26-8 \\
\end{array}$ & $\frac{77.12}{240.44}$ & & 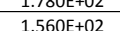 & & 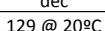 & 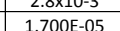 & 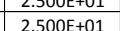 & & $\begin{array}{l}\text { PA 27 Listing Table } 1 \\
\text { PAC 27 isting Table } 1\end{array}$ \\
\hline 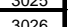 & 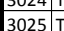 & 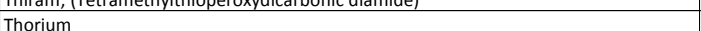 & 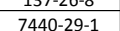 & $\frac{10074-20-1}{0.29-1}$ & 242.424 & $\frac{3}{s}$ & 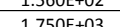 & & 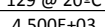 & & & & 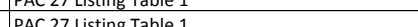 \\
\hline 3027 & 3026 T & Thorium hydroxide & $13825-36-0$ & $013825-36-0$ & 300.07 & $\frac{5}{3}$ & & & & & & & $\begin{array}{l}\text { PAC 27 Listing Table } 1 \\
\text { Pal }\end{array}$ \\
\hline 3028 & 3027 T & Thorium nitrite & z-0079 & $00000 z-0079$ & 416.03 & $\mathrm{~s}$ & & & & & & & PAC 27 Listing Table 1 \\
\hline 3029 & 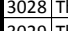 & Thorium oxalate & $\frac{2040-52-0}{1314-20-1}$ & $002040-52-0$ & 444.03 & $\frac{s}{s}$ & 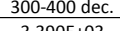 & & 2 & & & & $\begin{array}{ll}\text { PAC } 27 \text { Listing Table } 1 \\
\text { Dac }\end{array}$ \\
\hline 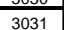 & 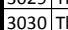 & 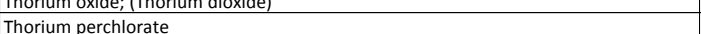 & $\frac{1314-2-10-1}{16045-17-3}$ & 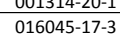 & 332.4887 & $\frac{5}{s}$ & & & 4.4U0UE +03 & & & & $\begin{array}{l}\text { PAC/ Ltsing gable } 1 \\
\text { PAC 27 Listing Table } 1\end{array}$ \\
\hline & $3031 \pi$ & Thorium(IV) nitrate & $13823-29-5$ & $013823-29-5$ & 480.04 & & & & & & & & PAC 27 Listing Table 1 \\
\hline 3033 & $3032 / \pi$ & Thulium & $\frac{7400-30-4}{7}$ & 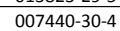 & 168.934 & $\mathrm{~s}$ & $\frac{1545}{1545}$ & & $1.9500+03$ & & & & $\begin{array}{ll}\text { PAC 27 Listing Table } 1 \\
\end{array}$ \\
\hline 3034 & $30337 \mathrm{~T}$ & Thulium chloride & $13537-18-3$ & $013537-18-3$ & 275.28 & $\mathrm{~s}$ & $8.240 \mathrm{E}+02$ & & $1.4406+03$ & & & & PAC 27 Listing Table 1 \\
\hline 3035 & 3034 T/T & Thulium oxide & $12036-44-1$ & $012036-44-1$ & 385.87 & $\frac{5}{5}$ & $2.425 \mathrm{E}+03$ & & & & & & PAC 27 Listing Table 1 \\
\hline$\frac{3036}{3037}$ & 3035T/T & 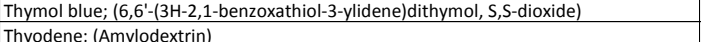 & $\frac{76-61-9}{9905-84-9}$ & $0000076-61-9.9$ & 466.63 & $\frac{s}{s}$ & $2.230 \mathrm{E}+02$ & & & & & & $\begin{array}{ll}\text { PAC } 27 \text { Listing Table } 1 \\
\text { PAC 27lițing Table } 1\end{array}$ \\
\hline 3038 & 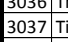 & (IInyouene; (Amylooextrin) & $\begin{array}{cc}9005-84-9 \\
7440-31-5 \\
\end{array}$ & $\frac{009005-84-9}{00740-31-5}$ & 118.71 & $\frac{2}{2}$ & $2.319 \mathrm{E}+02$ & & $2.6022+03$ & $7.5 \times 10-3$ & $1.224 E+03$ & & $\begin{array}{l}\text { PAC 27 Listing Table } 1 \\
\end{array}$ \\
\hline 3039 & 3038 Ti & Tin fluoroborate & $13814-97-6$ & $013814-97-6$ & 292.297224 & & & & & & & & $\begin{array}{ll}\text { PAC } 27 \text { Listing Table } 1 \\
\end{array}$ \\
\hline 3040 & 3039 T 30 & Tin hydroxide & $12026-24-3$ & $012026-24-3$ & 152.73 & $\mathrm{~s}$ & & & & & & & PAC 27 Listing Table 1 \\
\hline 3041 & 3040 T/ & Tin nitrate & 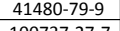 & $041480-79-99$ & 242.68 & $\mathrm{~s}$ & & & & & & & 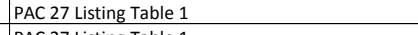 \\
\hline & 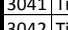 & Tin nitrite & $\frac{10003 / 2-2 /-1}{1020-1}$ & & 210.699 & & 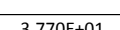 & & 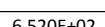 & & & & 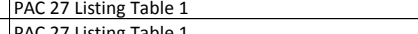 \\
\hline 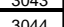 & 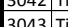 & 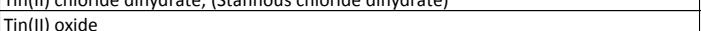 & $\frac{1003-39-1}{132-29}$ & 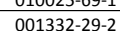 & $\frac{24303}{150.99}$ & $\frac{3}{5}$ & 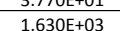 & & 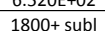 & & & & 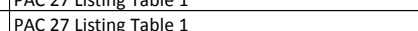 \\
\hline 3045 & 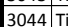 & Tin(III) sulfuete; (Stannous sulfate) & $7488-55-3$ & $007488-55-3$ & 214.75 & s & $378 \mathrm{dec}$ & & & & & & $\begin{array}{l}\text { PAC } \\
\text { PAC L Listing Table } 1\end{array}$ \\
\hline $\begin{array}{llll}3046 \\
30\end{array}$ & $3045 / \mathrm{T} / \mathrm{T}$ & Tin(IV) isopropoxide & 1184-61-8 & $\begin{array}{l}001184-61-8 \\
\end{array}$ & 415.1534 & $\mathrm{~s}$ & & & & & & & \\
\hline 3047 & 3046 T/ & Tin(IV) oxide; (Stannic oxide) & $18282-10-5$ & $018282-10-5$ & 150.69 & $s$ & $1.630 \mathrm{E}+03$ & & $1800 \mathrm{dec}$ & & & & $\begin{array}{l}\text { PAC } 27 \text { Listing Table } 1 \\
\end{array}$ \\
\hline 3048 & 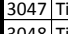 & & 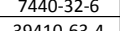 & $0.0740-32-66$ & 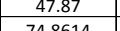 & 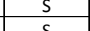 & $1.668 \mathrm{E}+03$ & & $3.287 \mp+03$ & $7.5 \times 10-3$ & $1.709 E+03$ & & 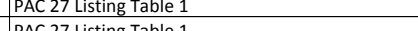 \\
\hline 年3050 & 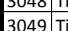 & Thandum auminice & $\frac{34406-534}{1204-5}$ & 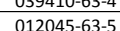 & $\frac{74.8014}{69.5}$ & $\frac{3}{5}$ & $2593-2980$ & & $38996+03$ & & & & 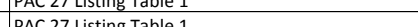 \\
\hline 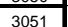 & 3050 & 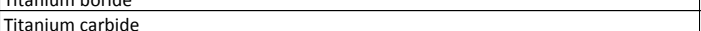 & $12070-08-5$ & $012070-08-5$ & $\frac{0.31}{5991}$ & $\mathrm{~s}$ & $\frac{1.067 \mathrm{E}+03}{3.03}$ & & 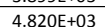 & & & & 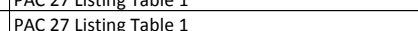 \\
\hline 3052 & 3051 T 3 & Titanium chloride & $7705-07-9$ & $\begin{array}{l}007705-07-9 \\
\end{array}$ & 154.25 & L & $-3.000 E+01$ & $7.600 E+02$ & $1.3644+02$ & $1.000 E+01$ & $2.130 E+01$ & $1.211 \mathrm{E}+0$ & Estimated using dataf from PAC 27 Listing Table 1 \\
\hline 3053 & $30522 \pi$ & Iritanium hydride & $7704-98-5$ & 007704-98-5 & 49.92 & $\mathrm{~s}$ & & & & & & & PAC 27 Listing Table 1 \\
\hline 3054 & 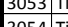 & 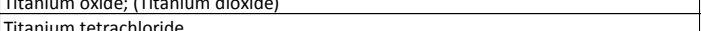 & $\frac{134363-6 /-1}{756}$ & 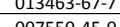 & 19.9 & 5 & $1.843 \mathrm{E}+03$ & 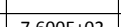 & 25000.300000 & 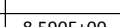 & 2000501 & 1910501 & PACL/2 Lsting lable 1 \\
\hline 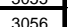 & 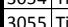 & Tritaiumm lil oxide & 1.15073-20-1 & $012137-20-1$ & $\frac{10300}{638}$ & $s$ & & $1.0000 \mathrm{ctu}$ & & & & & $\begin{array}{ll}\text { PAC 27 } \\
\text { Pisting Table } 1\end{array}$ \\
\hline 3057 & 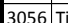 & Titanium(III) fluoride & $13470-08-1$ & $013470-08-1$ & 104.88 & 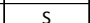 & $12005+03$ & & $1400 \mathrm{E}+03$ & & & & PAC 27 Listing Table 1 \\
\hline 3058 & $\begin{array}{lll}30577 \pi \\
3057\end{array}$ & Titanium-based allovi;(Titanium compounds) & $\frac{2-0081}{2-001}$ & $\frac{000002-0081}{0.0011}$ & & $\frac{\mathrm{s}}{\mathrm{s}} \mathrm{s}$ & & & (n) 50 & & & & $\begin{array}{l}\text { PAC } 27 \text { Listing Table } 1 \\
\end{array}$ \\
\hline 3059 & 3058 & Toluene & $108-88-3$ & $000108-88-3$ & 92.14 & $L$ & 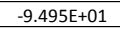 & & & & & & BERT,TE \& DANNER,RP (1985) \\
\hline 3060 & 30599T & Toluene disisocyanate (mixed isomers) & $26471-62-5$ & $026471-62-5$ & 174.16 & & 1.1000E+01 & 7.600E +02 & $2.510 \mathrm{E}+02$ & $2.300 \mathrm{E}-02$ & 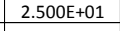 & $2.300 \mathrm{E}-0$ & Ac 27 Listing Table 1 \\
\hline 3061 & 3060 T & Toluene disiscyanate, trimethylolpropane, epsilon-caprolactone polymer, methylated & 71342-93-3 & 071342-93-3 & & $s$ & & & & & & & PAC 27 Listing Table 1 \\
\hline 3062 & 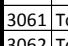 & 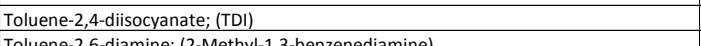 & $\frac{584-849}{8834-9}$ & $000584-84-9$ & $\begin{array}{lll}174.16 \\
12.19\end{array}$ & $\frac{s}{c}$ & $2.050 \mathrm{E}+01$ & & $2.510 \mathrm{E}+02$ & $7.5 \times 10-2$ & $3.900 \mathrm{E}+01$ & & 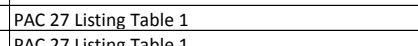 \\
\hline
\end{tabular}




\begin{tabular}{|c|c|c|c|c|c|c|c|c|c|c|c|c|c|}
\hline & & & & & & $F$ & & $x^{4}$ & 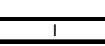 & & $\bar{k}$ & - & \\
\hline & No. & Chemical Compound & $\begin{array}{l}\text { CAS Number } \\
\text { (CASRN) }\end{array}$ & $\begin{array}{l}\text { Modified } \\
\text { Format CAS }\end{array}$ & $\begin{array}{c}\text { Molecular } \\
\text { Weight (MW) }\end{array}$ & $\begin{array}{c}\text { Qv State } \\
\text { at } \\
25^{\circ} \mathrm{C}\end{array}$ & $\begin{array}{c}\text { MP or FP } \\
{\left[{ }^{\circ} \mathrm{C}\right]}\end{array}$ & $\begin{array}{c}\text { BP Pressure } \\
\text { [mm Hg] }\end{array}$ & $B P\left[{ }^{\circ} \mathrm{C}\right]$ & $\begin{array}{l}\text { Vapor } \\
\text { Pressure } \\
\text { Imm Hgl }\end{array}$ & $\begin{array}{c}T \text { for VP } \\
{\left[{ }^{\circ} \mathrm{c}\right]}\end{array}$ & $\begin{array}{c}\text { VPat } 25^{\circ} \mathrm{C} \\
{[\mathrm{mm} \mathrm{Hg}]}\end{array}$ & Vapor Pressure @ $25^{\circ} \mathrm{C}$ Reference \\
\hline 3064 & 3063 T & Toluene-2,6-disocyanate & $91-08-7$ & $000091-08-7$ & 174.17 & 200 & & $1.800 E+01$ & $1.290 \mathrm{E}+02$ & $2.0900-0202$ & $2.500 E+01$ & $2.0900-02$ & DAUBERT,TE \& DANNER,RP (1996) \\
\hline$\frac{3065}{3066}$ & $\begin{array}{l}3004 \mathrm{~T} \\
30655 \mathrm{~T}\end{array}$ & 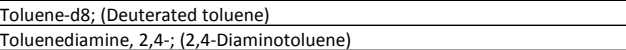 & $\frac{2037-26-5}{95-80-7}$ & $\begin{array}{l}0.02037-26-5 \\
00009-80-7 \\
\end{array}$ & $\frac{100.189}{122.27}$ & $\frac{\mathrm{L}}{\mathrm{s}}$ & $\frac{-8.400 E+01}{9.900 E+01}$ & & 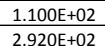 & \begin{tabular}{|l|l|l|l}
$2.840 \mathrm{E}+01$ \\
$7.50 \mathrm{E}-01$ \\
\end{tabular} & $2.5005+011$ & $2.840 \mathrm{E}+01$ & 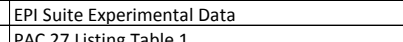 \\
\hline 3067 & 3066 T & Toluenesulfonamide, pi; (Pasam) & 70-55-3 & 000070-55-3 & 171.23 & $\mathrm{~s}$ & $\begin{array}{c}9.9006 \\
138 \\
\text { (anhyl); } \\
105 \text { hydrate }\end{array}$ & & $\begin{array}{c}221 @ 10 \\
\mathrm{~mm} \mathrm{Hg}\end{array}$ & $9.600 E-05$ & $2.500 E+01$ & & PAC 27 Listing Table 1 \\
\hline 3068 & 3067 T & Toluenesulfonic acid, methyl ester, p- & 80-48-8 & $000080-48-8$ & 186.24 & $\mathrm{~s}$ & 27.5 & & $\begin{array}{c}144.5 \\
₫ 5 \mathrm{~mm}\end{array}$ & & & & PAC 27 Listing Table 1 \\
\hline & $\left.\left.\right|_{3068}\right|_{\mathrm{T}}$ & Toluenesulfonyl chloride, $\mathrm{p}$ - & 98-59-9 & $000098-59-9$ & 190.64 & $\mathrm{~s}$ & $69-71$ & & $152 @ 20$ & $1.000 E+00$ & $8.800 E+01$ & & PAC 27 Listing Table 1 \\
\hline 3070 & $3069 T^{\top}$ & 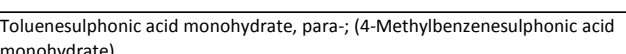 & $6192-52-5$ & $006192-52-5$ & 190.23 & $s$ & $1.070 \mathrm{E}+02$ & & $\frac{m m}{140 @ 20}$ & & & & PAC 27 Listing Table 1 \\
\hline$\frac{30 / 0}{3071}$ & $3070 T^{n}$ & $\begin{array}{l}\text { monohydratel } \\
\text { Toluenethiol, } \mathrm{m}-\text { - }\end{array}$ & $108-40-7$ & $000108-40-7$ & 124.21 & $\mathrm{~L}$ & & & & 5.530E-01 & $2.500 \mathrm{E}+01$ & $5.5300-01$ & CHAO, ET AL. (1983) \\
\hline 3072 & 3071 T & Toluidine, m;; (3-Methylaniline) & 108-44-1 & $000108-44-1$ & 107.17 & $\mathrm{~L}$ & $-4.360 \mathrm{E}+01$ & $7.600 E+02$ & $2.033 \mathrm{E}+02$ & $3.030 \mathrm{E}-01$ & $2.500 E+01$ & $3.030 \mathrm{E}-01$ & \\
\hline$\frac{3073}{3074}$ & 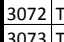 & $\begin{array}{l}\text { Toluidine, o- } \\
\text { Toluidine, (4- (4-Methylbenzenamine) }\end{array}$ & $95-53-4$ & $000095-53-4$ & $\frac{107.15}{107.17}$ & L & & $\begin{array}{ll}7.600 E+02 \\
7600 \mathrm{~F}+2\end{array}$ & $\frac{2.003 E+02}{2004+502}$ & $2.600 \mathrm{E}-01$ & 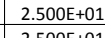 & $2.6000-01$ & 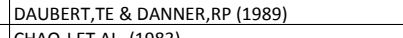 \\
\hline 3074 & 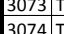 & $\begin{array}{l}\text { Toludinine, } p ;-; \text { (4-Methethylbenzenamine) } \\
\text { Toxaphene; (Chlorinated camphene) }\end{array}$ & 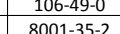 & $\frac{0001066-49-0}{008001-3-2}$ & $\frac{107.17}{441}$ & $\frac{l}{5}$ & $\frac{4.200 E+01}{65.90}$ & & $\frac{2.004 E+02}{\text { dec }}$ & 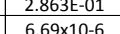 & $\frac{2.5000++01}{2000 E+01}$ & & 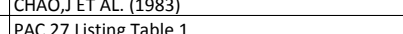 \\
\hline$\frac{3015}{3076}$ & 30757 & 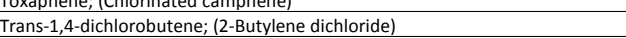 & $\frac{8010-57-2}{110-57-6}$ & $000110-57-6$ & $\frac{414}{125}$ & $\frac{1}{3}$ & $\frac{05-50}{3.580+04}$ & $7.600 \mathrm{E}+02$ & $\frac{\text { eec }}{1.560 \mathrm{E}+02}$ & $\begin{array}{l}0.09510-0 \\
6.000 \mathrm{E}+00 \\
\end{array}$ & $\begin{array}{l}2.20000+01 \\
2.500++01\end{array}$ & $6.0006+00$ & $\begin{array}{l}\text { PAC } 27 \text { Listing Table } 1 \\
\end{array}$ \\
\hline 3077 & $\left.3076\right|^{\top}$ & $\begin{array}{l}\text { Transformer oili, (Mineral oil, petroleum distillates, hydrotreated (mild) light } \\
\text { nathenic) }\end{array}$ & $64742-53-6$ & $064742-53-6$ & & L & & $7.6006+02$ & $1.540 \mathrm{E}+02$ & $3.7500-03$ & $2.000 \mathrm{E}+01$ & 7.2066-03 & MSDS Shell Gravex oil \\
\hline & 3077 T & Tri(2-ethylhexyl) phosphate; (Tris(2-ethylhexyl)phosphate) & $78-42-2$ & $000078-42-2$ & 434.72 & & $-7.400 E+01$ & $7.600 \mathrm{E}+02$ & $2.269++02$ & $8.2500-08$ & $2.500 E+01$ & $8.2500-08$ & HIIICKLEY,DA ET AL. (1990) \\
\hline 3079 & & Triacetin; (Triacety) glycerin) & $\frac{102-76-1}{10}$ & $\frac{000102-76-1}{0.1}$ & 218.23 & $\mathrm{~L}$ & $\frac{-7.8000+01}{-101}$ & $\frac{1.6800+02}{702}$ & 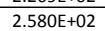 & 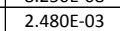 & 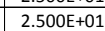 & $2.4800-03$ & 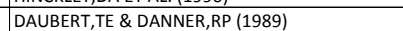 \\
\hline 3080 & 3079 T & Triamiphos & $1031-47-6$ & $001031-47-6$ & 294.34 & $\mathrm{~s}$ & $167-168$ & & $4.000 E+02$ & $1.000 \mathrm{E}-07$ & $2.500 \mathrm{E}+01$ & & PAC 27 listing Table 1 \\
\hline$\frac{3081}{3082}$ & 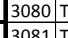 & $\begin{array}{l}\text { Triary sulfonium chloride salts } \\
\text { Trizine }\end{array}$ & $\frac{109037-76-5}{4719-04-4}$ & $\frac{109037-76-5}{00470-04-4}$ & 284.04 & $\frac{s}{s}$ & & & & & & & $\begin{array}{l}\text { PAC } 27 \text { Listing Table } 1 \\
\text { PAC 27| }\end{array}$ \\
\hline$\frac{3082}{3083}$ & 3082 T & 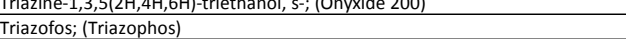 & $\begin{array}{l}4 / 19904-4 \\
24017-47-8 \\
\end{array}$ & $\begin{array}{ll}004 / 199-04-4 \\
024017-47-8\end{array}$ & $\frac{219.33}{313.34}$ & $\frac{1}{2}$ & $5.000 E+00$ & $7.600 E+02$ & $3.500 E+022$ & $1.000 \mathrm{E}-05$ & $2.500 E+01$ & $1.000 E-05$ & 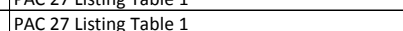 \\
\hline & & Tribenzylamine & $620-40-6$ & $000620-40-6$ & 287.4036 & $\mathrm{~s}$ & $90-92$ & & 380-390 & & & & PAC 27 Listing Table 1 \\
\hline 3085 & 3084 T & Tributyl citrate & $77-94-1$ & $000077-94-1$ & 360.5 & $\mathrm{~L}$ & $-2.000 \mathrm{C}+01$ & $2.330 E+02$ & 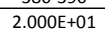 & $3.310 E-05$ & $2.500 E+01$ & $3.310 \mathrm{E}-05$ & EP S Sute Modififed Grain Method \\
\hline 3086 & 3085 T & Tributyl phosphate & $126-73-8$ & $000126-73-8$ & 266.31 & $\mathrm{~L}$ & $\frac{-2.000 E+01}{-801}$ & $7.600 \mathrm{E}+02$ & $2.890 \mathrm{E}+02$ & $1.1300-03$ & $2.500 \mathrm{E}+01$ & $1.1300-03$ & SKENE,WG \& KRZMIEN,ME (1995) \\
\hline 3087 & 3086 T & Tributy|(2,-4-dichlorobenzzy)|phosphonium chloride & $115-78-6$ & $000115-78-6$ & $\frac{397.83}{26013}$ & $\mathrm{~s}$ & $114-120$ & & & & & & PAC 27 Listing Table 1 \\
\hline$\frac{3088}{3089}$ & $\begin{array}{l}3087 \mathrm{~T} \\
3088 \mathrm{~T}\end{array}$ & Tributyly-1-10utanaminium iodide, N,N,N; (Tetrabutylammonium iodide) & $\frac{311-2-28}{998-4-3}$ & $\begin{array}{ll}000311-2-28-4 \\
000998-40-3\end{array}$ & 202.33 & $\mathrm{s}$ & $146-148$ & $7.600 E+02$ & $2.400 E+02$ & $1.600 E+01$ & $1.210 \mathrm{E}+02$ & $7518 E-02$ & $\begin{array}{l}\text { PAC } 2 \text { L Listing Table } 1 \\
\text { Fstimated using data from PAC } 27 \text { Listing Table } 1\end{array}$ \\
\hline$\frac{3099}{3090}$ & $\begin{array}{l}30889 \mathrm{~T} \\
3089\end{array}$ & ITlibutlyhosospine & $\begin{array}{l}998-40-3 \\
81741-28-8 \\
\end{array}$ & $\begin{array}{l}009098-4-4-3 \\
08174-28-8\end{array}$ & & $\mathrm{~s}$ & & 1.600Et02 & $2.400 \mathrm{E}+02$ & & & & 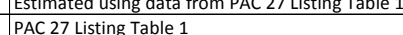 \\
\hline 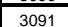 & T3090/T & Trichloramine; (Nitrogen chloride) & $10025-85-1$ & $010025-85-1$ & 120.36 & L & $-4.000 E+01$ & $7.600 E+02$ & $7.100 E+01$ & $1.500 \mathrm{E}+02$ & $2.000 E+01$ & $1.802 E+02$ & Estimated using data from PAC 27 Listing Table 1 \\
\hline 3092 & 3091 T & Trichloroldichlorophenyl) silane; (Dichlorophenyltrichlorosilane) & $27137-85-5$ & $027137-85-5$ & 280.43 & & & $7.600 E+02$ & $2.600 \mathrm{E}+02$ & $7.000 E+01$ & $2.500 E+01$ & 7.000E+01 & PAC 27 Listing Table 1 \\
\hline 3093 & 3092 T & Trichloro-2,2,2,-trifluoroethane, 1,1,1. & $\begin{array}{l}354-58.5 \\
1190130\end{array}$ & $000354-58-5$ & 187.37 & L & 1.420EE+01 & $7.600 E+02$ & 4.580E+01 & $3.600 E \mathrm{E}+02$ & $2.500 E+011$ & 3.600E+ +0 & PAC 27 Listing Table 1 \\
\hline & 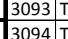 & 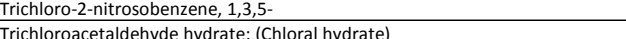 & $\frac{1196-13-0}{302-17-0}$ & $\frac{001196-13-0}{0000302-1-0}$ & $\frac{210.44}{165.4}$ & $\frac{5}{5}$ & 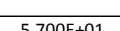 & & asder & 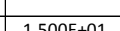 & 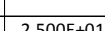 & & 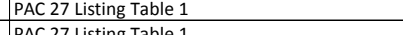 \\
\hline 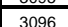 & 3095T & 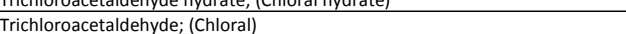 & 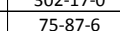 & (0.0075-17-6 & 147.39 & L & 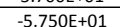 & $7.600 E+02$ & $\frac{9.700 \mathrm{~F}+01}{\mathrm{a}}$ & $5.0006+01$ & $\frac{2.500 \mathrm{E}+01}{2.01}$ & $5.000 E+0$ & $\begin{array}{l}\text { PERRY RH \& GREEN D (1984) } \\
\end{array}$ \\
\hline 3097 & |3096 T & Trichloracectic acid & 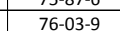 & 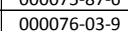 & 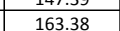 & $s$ & $5.7706+01$ & & $1.975 \mathrm{E}+02$ & $\frac{1.000 E+00}{100}$ & $5.100 \mathrm{E}+01$ & & 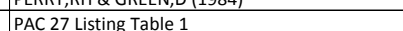 \\
\hline 3098 & 30977T & $\begin{array}{l}\text { Trichloroacetyly chloride } \\
\end{array}$ & $76-02-8$ & $000076-02-8$ & 181.82 & L & $-1.460 \mathrm{E}+02$ & $7.6000+02$ & $1.140 E+02$ & $2.132 E+01$ & $2.500 \mathrm{E}+01$ & 2.132E+01 & $\begin{array}{l}\text { PAC } 27 \text { Listing Table } 1 \\
\end{array}$ \\
\hline 3099 & 3098 T & Trichlorobenzzene, 1,2,3- & $87-61-6$ & $000087-61-6$ & 181.45 & $\mathrm{~s}$ & 5.130EE+01 & (3) & $2.185 E+02$ & $2.100 E-01$ & $2.500 E+011$ & & PAC 27 Listing Table 1 \\
\hline$\frac{3100}{3201}$ & 3099 T & Trichlorobenzene, 1,2,4- & $\frac{120-82-1}{125}$ & 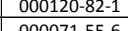 & 181.25 & $L$ & 1.692E E01 & $\begin{array}{l}7.600 \mathrm{E}+02 \\
.602\end{array}$ & 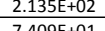 & $\begin{array}{l}4.6000=01 \\
4.600020\end{array}$ & $2.500 \mathrm{E}+01$. & 4.600E-0.11 & \begin{tabular}{|l} 
PAC 27 Listing Table 1 \\
Pate
\end{tabular} \\
\hline$\frac{3101}{3102}$ & $\begin{array}{lll}31300 / T \\
301 / T\end{array}$ & Trichloroethane, $1,1,1 ;$; ; (Methy chloroform) & 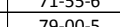 & $\begin{array}{ll}0 & 0\end{array}$ & 13.42 & L & 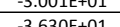 & 1. & 1.409te+U1 & 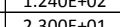 & $\begin{array}{ll}2.3000+01 \\
2500.01\end{array}$ & 1.2440E=02 & 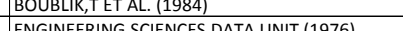 \\
\hline 3.102 & 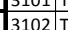 & 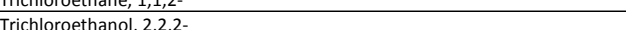 & 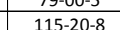 & $000115-20-8$ & 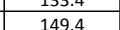 & 5 & 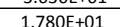 & & $1500 \mathrm{E}+02$ & 1000F+o0 & $20000+01$ & & 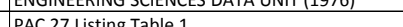 \\
\hline 3104 & $\mid 3103 / \mathrm{T}$ & Trichloroethinlilene & $\frac{79-01-6}{70}$ & $\frac{000079-01-6}{0.0}$ & 131.39 & L & 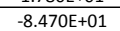 & $7.600 E+02$ & $8.721 \mathrm{E}+01$ & $2.900 E+01$ & 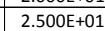 & 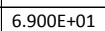 & BOUBGLK, ET ET AL. (1984) \\
\hline 3105 & 3104 T & Trichloroethylsilane; (Ethyl trichlorosilane) & $115-21-9$ & $000115-21-9$ & 163.51 & $\mathrm{~L}$ & $-1.0566+02$ & & $9.790 \mathrm{E}+01$ & $4.718 \mathrm{E}+01$ & $2.500 E+01$ & & PAC 27 Listing Table 1 \\
\hline 3106 & 31055T T & Trichloroffluoromethane; (Fluorotrichloromethane; Freon 11) & $75-69-4$ & $000075-69-4$ & 137.37 & & $-1.104 E+02$ & & $2.370 E+01$ & $7.500 \mathrm{E}+02$ & $2.330 E+011$ & & PAC 27 Listing Table 1 \\
\hline 3107 & $\left.3106\right|_{h} ^{\top}$ & 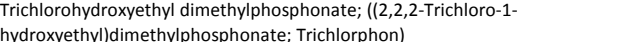 & $52-68-6$ & $000052-68-6$ & 257.44 & $\mathrm{~s}$ & $83-84$ & & $100 @ 1$ & & & & PAC 27 Listing Table 1 \\
\hline & $3107 T$ & Trichlorolisocyanuric acid & $87-90-1$ & $000087-90-1$ & 232.41 & $\mathrm{~s}$ & $2.350 \mathrm{E}+02$ & & & & & & PAC 27 Listing Table 1 \\
\hline 3109 & 3108 T & Trichloronaphthalene & $1321-65-9$ & $001321-65-9$ & 231.5 & $\mathrm{~s}$ & $9.278 \mathrm{E}+01$ & & $304.44-$ & & & & PAC 27 Listing Table 1 \\
\hline & 3109 T 3 & Trichloronatte; (Ethyl trichlorophenylethyl|phosphonothioate) & 327-98-0 & $000327-98-0$ & 333.6 & & & $7.600 E+02$ & & $2.000 E+01$ & $2.500 \mathrm{E}+01$ & $2.0006+01$ & PAC 27 Listing Table 1 \\
\hline$\frac{3111}{3111}$ & 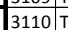 & Trichloronitrobenzzene, 2,4,5- & $89-69-0$ & $0000089 \cdot 69-0$ & 226.44 & $\mathrm{~s}$ & $5.700 E+01$ & & $2.080+5+02$ & & & & 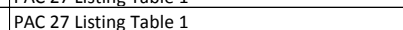 \\
\hline 3112 & 3111] T & Trichlorophenol, 2,3,6- & 933-75-5 & $000933-75-5$ & 197.44 & $\mathrm{~s}$ & $5.8000 \mathrm{E}+01$ & & $2.530 E+02$ & & & & PAC 27 Listing Table 1 \\
\hline 3113 & 3112|T T & Trichlorophenol, , $2,4,5-$ & 95-95-4 & $000095-95-4$ & 197.45 & s & 6.900E +01 & & $2.470 \mathrm{E}+02$ & 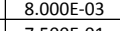 & $2.5000 \mathrm{E}+01$ & & PAC 27 Listing Table 1 \\
\hline$\frac{3144}{3315}$ & (3144T & 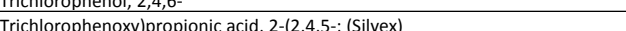 & 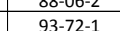 & 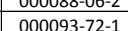 & 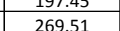 & & 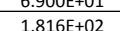 & & & 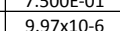 & 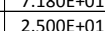 & & $\begin{array}{l}\text { PAC 2 L Listing lable } 1 \\
\text { PAC 27 Listing Table } 1 \\
\end{array}$ \\
\hline 3116 & 3115 T/T & Trichlorophenoxyacetic acid, $2,4,5 ;$; $2,2,4,5-\mathrm{T})$ & & $0000093-76-5$ & 255.48 & $\mathrm{~s}$ & $1.5306+02$ & & dec & $<0.01 \mathrm{mPa}$ & $2.000 E+01$ & & $\begin{array}{ll}\text { PAC } 27 \text { Listing Table } 1 \\
\end{array}$ \\
\hline 3117 & 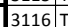 & Trichlorophenylsialane & 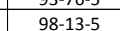 & $000098-13-5$ & 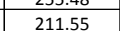 & t & & $7.680 \mathrm{E}+02$ & $2.010 \mathrm{E}+02$ & $4.260 \mathrm{E}-01$ & $2.500 \mathrm{E}+01$ & $4.260 \mathrm{E} \mathrm{C}-\mathrm{C}$ & BOULLK TIT AA. (1984) \\
\hline 3118 & $31177 \mathrm{~T}$ & Trichloropropane, 1,2,3- & $96-18-4$ & $000096-18-4$ & $\begin{array}{lll}147.43 \\
\end{array}$ & & $-1.470 E+01$ & $7.600 E+02$ & $1.5706+02$ & $3.6906+00$ & $2.500 E+01$ & $3.690 E+00$ & DAUBERT,TE \& DANNER,R \\
\hline 3119 & 31181 T T & Trichlorosiline & $10025-78-2$ & $010025-78-2$ & 135.45 & & $-1.282 E+02$ & 7.600E+02 & $3.300 E+01$ & 5.936E+02 & $2.500 E+01$ & $5.936 E+02$. & DAUBERT,TE \& DANNER,RP (1 \\
\hline$\frac{3120}{31212}$ & 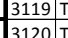 & $\begin{array}{l}\text { Trichlorotrifluoroethane; }(\mathrm{Fr} \\
\text { Tridecane }\end{array}$ & $76-13-1$ & $000076-13-1$. & $\frac{187.38}{18441}$ & 1 & $\begin{array}{l}-3.622 E++1 \\
-5.5005+00\end{array}$ & & $\frac{4.7704+01}{2.354++02}$ & 600E- 02 & $\begin{array}{l}2.5000+01 \\
2.500 \mathrm{E}+01\end{array}$ & 5600E- 0 & BOUBLIK, ET AL. (1984) \\
\hline
\end{tabular}




\begin{tabular}{|c|c|c|c|c|c|c|c|c|c|c|c|c|c|}
\hline & No & Chemical Compound & $\begin{array}{c}\text { cas N Number } \\
\text { (CASPN) }\end{array}$ & $\begin{array}{c}\text { Modfied } \\
\text { format as } \\
\text { fas }\end{array}$ & $\begin{array}{l}\text { Molecurar } \\
\text { Weight MWW }\end{array}$ & at & $\begin{array}{c}\text { MP or FP } \\
\text { [ic }\end{array}$ & 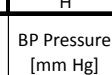 & $\mathrm{BP}[\mathrm{pc}]$ & $\begin{array}{c}\text { vapor } \\
\text { Pressure }\end{array}$ & $\begin{array}{c}\text { Tforvp } \\
\text { crca }\end{array}$ & 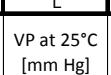 & Vapor Pressure @ $95^{\circ}$ C Reference \\
\hline$\frac{3122}{3222}$ & & 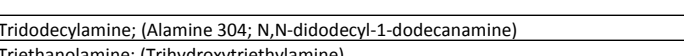 & $\frac{102-27}{10271}$ & 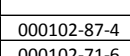 & $\frac{521.952}{511992}$ & & $\frac{1.606 E+01}{1.200+21}$ & $7.600 E+02$ & 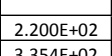 & 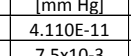 & 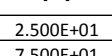 & 4.110E-11 & $\begin{array}{l}\text { EPI Suite Modified Grain Method } \\
\text { PAC Thl }\end{array}$ \\
\hline 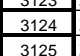 & & 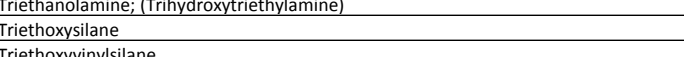 & 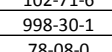 & 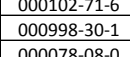 & 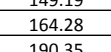 & $\frac{L}{L}$ & $\begin{array}{l}2.50601 \\
-1.005+02\end{array}$ & $\begin{array}{l}7.600 \mathrm{E}+22 \\
2.000+51\end{array}$ & 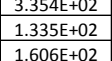 & 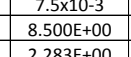 & $\begin{array}{l}7.5000+201 \\
2.500+01\end{array}$ & $\begin{array}{l}8.5005+0 \\
.2835+0\end{array}$ & 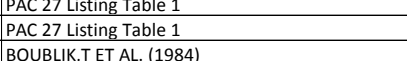 \\
\hline $\begin{array}{lll}3126 \\
3.127 \\
\end{array}$ & & 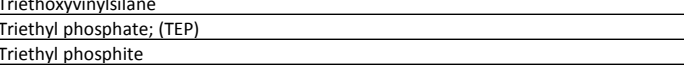 & 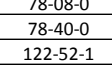 & 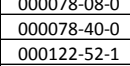 & $\begin{array}{l}\frac{19.35}{18.218} \\
166.18\end{array}$ & $\frac{L}{L}$ & -5.6006 +01 & 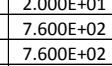 & 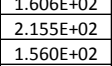 & 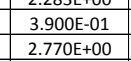 & 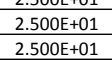 & 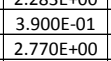 & 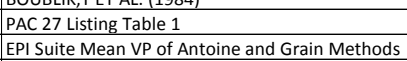 \\
\hline$\frac{\frac{3128}{3129}}{3}$ & & $\begin{array}{l}\text { Iriethalulumium } \\
\text { riethylyamine }\end{array}$ & 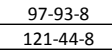 & 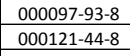 & $\frac{10.122}{101.22}$ & $i$ & 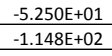 & 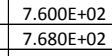 & 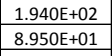 & $\begin{array}{l}2.560-02 \\
5.707+51\end{array}$ & 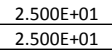 & $\begin{array}{l}2.5 .560 .02 \\
5.707+01\end{array}$ & 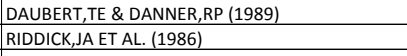 \\
\hline & & 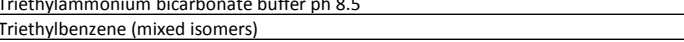 & & 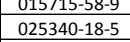 & 162.3 & & 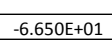 & 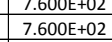 & & 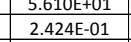 & 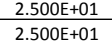 & & $\begin{array}{l}\text { AcD Labs Physical Chemistry Suite } \\
\text { CHAO, ET ALL (1983) }\end{array}$ \\
\hline & & 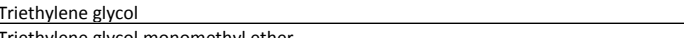 & 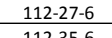 & & & & 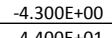 & 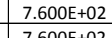 & $2.850 \mathrm{E}+\mathbf{E}+22$ & 1.3200:033 & & & AAUBERT,TE \& DANNER,RP (1989) \\
\hline $\begin{array}{lll}3133 \\
3134 \\
\end{array}$ & & 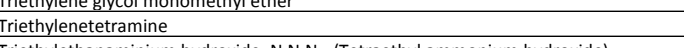 & $\frac{112-30}{112-24.3}$ & 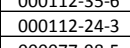 & 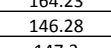 & $\frac{1}{t}$ & $\begin{array}{l}-4.4000+201 \\
1.2005+01\end{array}$ & 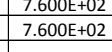 & $\begin{array}{l}2.9460+02 \\
2.720+02\end{array}$ & 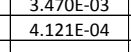 & $\begin{array}{l}2.55000+501 \\
2.500+01\end{array}$ & 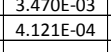 & 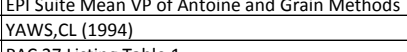 \\
\hline & & 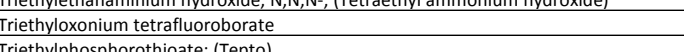 & 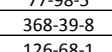 & 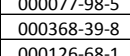 & 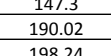 & $\frac{5}{5}$ & & 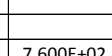 & 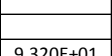 & 8780:502 $>0$ & & & $\begin{array}{l}\text { PAC } 27 \text { Listing Table } 1 \\
\text { EP SSTite Mean }\end{array}$ \\
\hline 31373 & & 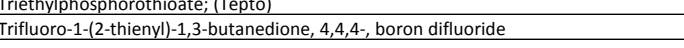 & & & 2012.24 & s & & & & $8.1800-02$ & & & $\begin{array}{l}\text { EAP I Pute Mean VP of Ant } \\
\text { PAC } 27 \text { Listing Table } 11\end{array}$ \\
\hline & B138| & 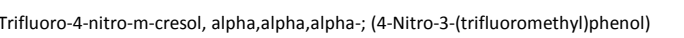 & 88:30-2 & 000088-30-2 & 207.12 & $\mathrm{~s}$ & $76-79$ & & ${ }_{135-138}$ & & & & PAC 27 Lsting Table 1 \\
\hline 3.400 & & 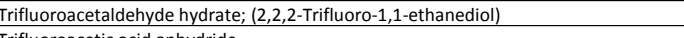 & & 0.00421.5.5. & $\frac{116.05}{16 .}$ & L & 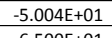 & 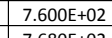 & 1.05 & 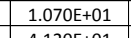 & $\begin{array}{l}2.500 \mathrm{E}+\mathrm{t} \\
\mathrm{n}\end{array}$ & 1.0700E+ & Pil suite Mean VP of Antoine and Grain Methods \\
\hline 3.432 & & 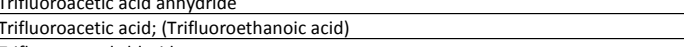 & & 0000076-25.5 & $\begin{array}{l}211.04 \\
114.03\end{array}$ & $\frac{t}{L}$ & 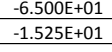 & $\begin{array}{lll}7.608 \mathrm{E}^{+} \\
7.680 \mathrm{E}\end{array}$ & 7.106t+ & 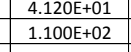 & 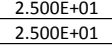 & 1.100et+ & 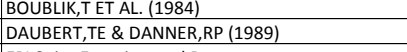 \\
\hline$\frac{324}{3144}$ & & 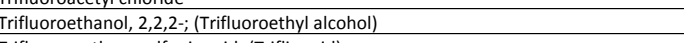 & & 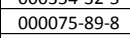 & 100.05 & & $-4.400 E+011$ & 7.680 E & 1.040E & 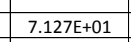 & (2) & & 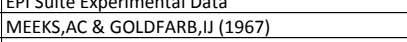 \\
\hline$\frac{3145}{3146}$ & & 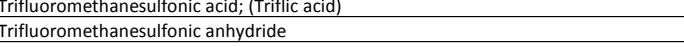 & - $\frac{1939-13 \cdot 6}{358-23}$ & 001933-1. & $\frac{15.08}{282.14}$ & $\frac{L}{L}$ & & $\begin{array}{l}7.680+52 \\
7.6005+02\end{array}$ & 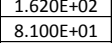 & 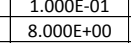 & $\frac{2.500+501}{2.0005+01}$ & 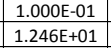 & 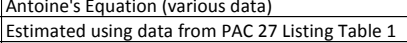 \\
\hline$\frac{3147}{3148}$ & & 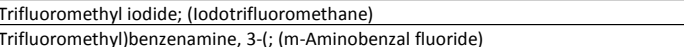 & 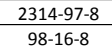 & 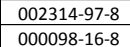 & $\frac{195.91}{161.14}$ & $\frac{6}{4}$ & $\frac{<.78}{3.000+00}$ & & $1.800+0202$ & 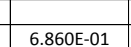 & 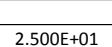 & & 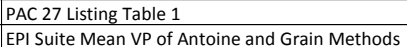 \\
\hline 21199 & 3148 & 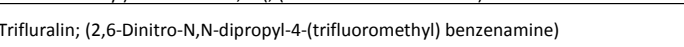 & $1582-09.8$ & $001582-09 \cdot 8$ & 335.28 & $\mathrm{~s}$ & 4.900e+501 & & 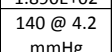 & 4.585800.5 & $2.500 \mathrm{E}+01$ & & PAC 27 Listing Table 1 \\
\hline 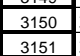 & $\begin{array}{l}3149 \\
3150\end{array}$ & Trisisbuttlaluminum & 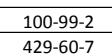 & 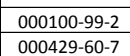 & $\frac{198.3}{28.281}$ & $\frac{t}{s}$ & $6.000+500$ & 1.000E+01 & & $1.000+500$ & 4.700E+01 & 2.09250 .0 & 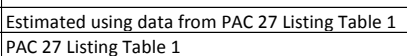 \\
\hline$\frac{3152}{3.52}$ & & 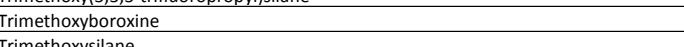 & $\frac{102-249}{20890.9}$ & 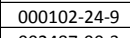 & 173.55 & & & & & & & & 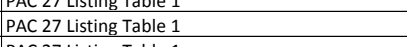 \\
\hline & & 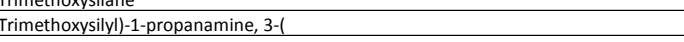 & 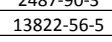 & 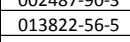 & $\frac{12.22}{179.29}$ & $\frac{t}{L}$ & 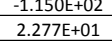 & 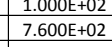 & 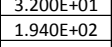 & . & & & 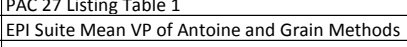 \\
\hline $\begin{array}{lll}31556 \\
3156 \\
\end{array}$ & & 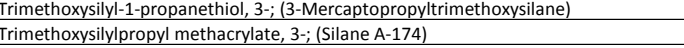 & 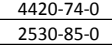 & 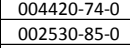 & 248.379 & L & $-5.000+01$ & 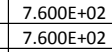 & 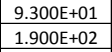 & $\begin{array}{l}1.5600 .01 \\
1.270-02\end{array}$ & $\begin{array}{l}2.5000+501 \\
2.500+01\end{array}$ & $\begin{array}{l}1.5600-0.0 \\
1.270-0.0\end{array}$ & 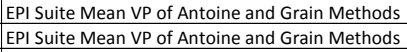 \\
\hline & & 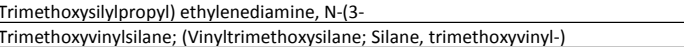 & & & 148.26 & & & & 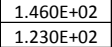 & 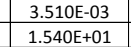 & & & 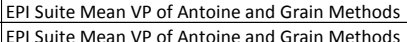 \\
\hline 31599 & & Trimethy borate & 121-4. & 0000121-43:- & 103.93 & L & $-2.900 E+011$ & 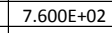 & 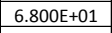 & $1.3706+022$ & $2.500 \mathrm{E}+01.10$ & 1.370Et+ & PAC 27 listing Table 1 i \\
\hline$\frac{3100}{3161}$ & & 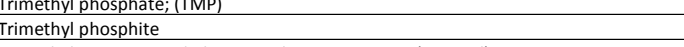 & 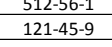 & 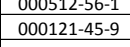 & 1420.09 & $\frac{t}{L}$ & $\begin{array}{l}-7.8000+01 \\
\end{array}$ & $7.6005+02$ & 1.1100+02 & 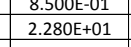 & 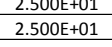 & 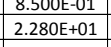 & 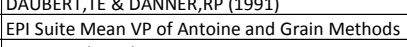 \\
\hline & & 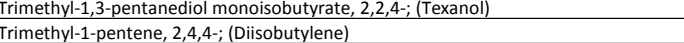 & & 000107-39.1 & 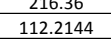 & & $-9.350 \mathrm{E}+01$. & & 1.1044+02 & & .5006 + +0.0 & & \\
\hline$\frac{3164}{3165}$ & & & 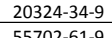 & 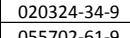 & 264.36 & s & $73665+5019$ & 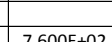 & 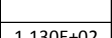 & 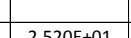 & & & 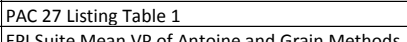 \\
\hline & 3165 & 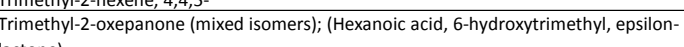 & 60404730.9.9 & 066047-30.9 & 156.25 & $\mathrm{~s}$ & & & & & & & 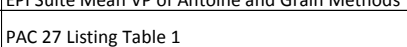 \\
\hline$\frac{3167}{367}$ & & 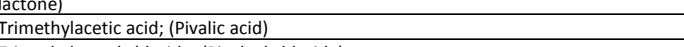 & $75.98 \cdot 9$ & 0.00075.989.9 & 102.15 & $s$ & 35 & & & & & & $\mathrm{~L}_{\mathrm{L}} \mathrm{C}$ \\
\hline & & 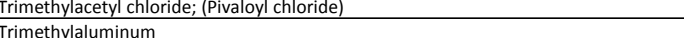 & & 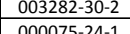 & $\frac{120.59}{10209}+20$ & & & & & 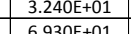 & & & 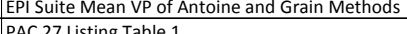 \\
\hline & & frimethlyamine & & 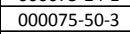 & (5011 & & -2 & & & & & & 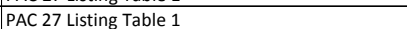 \\
\hline 年1717 & & 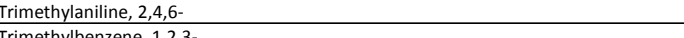 & & & & & 然 & & & 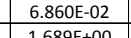 & & & 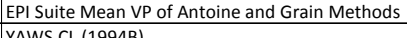 \\
\hline 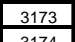 & & 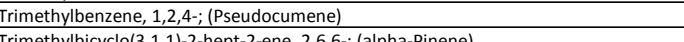 & 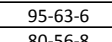 & 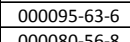 & $\frac{120.19}{10.19}$ & 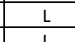 & 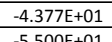 & 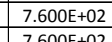 & 1.6964 & 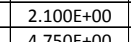 & 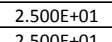 & 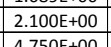 & 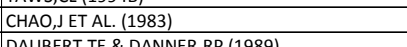 \\
\hline & & 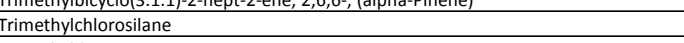 & 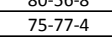 & & $\begin{array}{ll}108.66 \\
\end{array}$ & & (0) & & & & & & \\
\hline & & 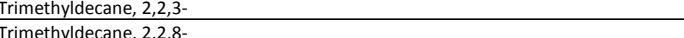 & 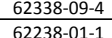 & 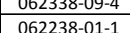 & $\begin{array}{l}184 \\
184\end{array}$ & & & 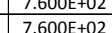 & & $6.970 \mathrm{~S}$ & & & 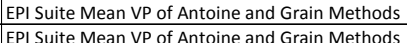 \\
\hline & & 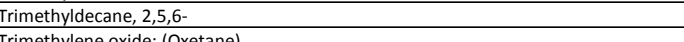 & & & $\frac{184}{5509}$ & & 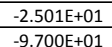 & $\begin{array}{l}7.600 \mathrm{E}_{\mathrm{t}} \\
7.600 \mathrm{E}\end{array}$ & 1.1937E+t & 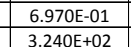 & 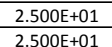 & 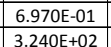 & $\begin{array}{l}\text { EPI Sute Mean VP of Anttine and Grain Methods } \\
\text { Pac }\end{array}$ \\
\hline$\frac{13100}{3180}$ & & 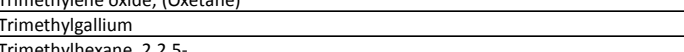 & $\frac{1445-9.0}{1.352019}$ & 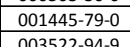 & $\frac{114.8}{120.89}$ & L & $\frac{-1.500 \mathrm{E}+01}{-101}$ & 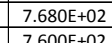 & 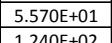 & 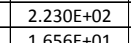 & 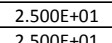 & 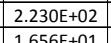 & DABPRT \& ANNER PP (10989) \\
\hline & & 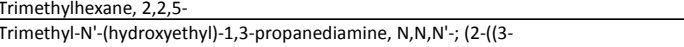 & 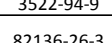 & 0.03252-949-9 & & & & & & & & & DAUBRR, TE\& DANNRR,RP (1989) \\
\hline $\begin{aligned} 3182 \\
31383 \\
\end{aligned}$ & $\begin{array}{l}3181 \\
3182 \\
3.182\end{array}$ & 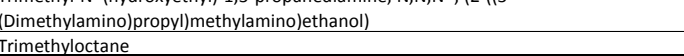 & 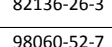 & 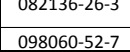 & 156.31 & $\frac{s}{G}$ & & & $1.0706+01$ & $7.600+02$ & $1.070 \div 01$ & & 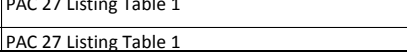 \\
\hline
\end{tabular}




\begin{tabular}{|c|c|c|c|c|c|c|c|c|c|c|c|c|c|}
\hline & & & & & & $F$ & & & & & $\bar{k}$ & -4 & \\
\hline & No. & Chemical Compound & $\begin{array}{l}\text { CAS Number } \\
\text { (CASRN) }\end{array}$ & $\begin{array}{l}\text { Modified } \\
\text { Format CAS }\end{array}$ & $\begin{array}{l}\text { Molecular } \\
\text { Weight (MW) }\end{array}$ & $\begin{array}{c}\text { Qv State } \\
\text { at } \\
25^{\circ} \mathrm{C}\end{array}$ & $\begin{array}{l}\text { MP of fP } \\
{\left[{ }^{\circ C}\right]{ }^{2}}\end{array}$ & $\begin{array}{c}\text { BP Pressure } \\
{[\mathrm{mm} \mathrm{Hg]}}\end{array}$ & $B P\left[{ }^{[C}\right]$ & $\begin{array}{l}\text { Vapor } \\
\text { Pressure } \\
\text { [mm HE] }\end{array}$ & $\begin{array}{l}\text { Tfor VP } \\
\text { [C] }\end{array}$ & $\begin{array}{c}\text { VP at } 25^{\circ} \mathrm{C} \\
{[\mathrm{mm} \mathrm{Hg}]}\end{array}$ & 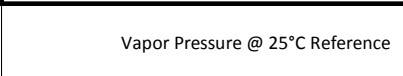 \\
\hline 3184 & 3183 T & Trimethyloctane, $2,2,6-$ - & $62016-28-8$ & $062016-28-8$ & 156.31 & & $-4.860 E+01$ & $7.6006+02$ & $1.515 E+02$ & & $2.5006+01$ & $\begin{array}{l}4.800 E+00 \\
.005000\end{array}$ & EPI S Sute Mean VP of Antoine and Grain Methods \\
\hline 3185 & $3184 \pi / T$ & Trimethyloctane, $2,3,7-7$ & $62016-34-6$ & $062016-34-6$ & 156.31 & $\mathrm{~L}$ & $-4.860 E+01$ & $7.600 E+02$ & $1.515 E+02$ & $4.800 E+00$ & $2.5000++01$ & 4.800E+ +00 & EPI Suite Mean VP of Antoine and Grain Methods \\
\hline 3186 & 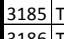 & $\begin{array}{l}\text { Trimethyloctane, } 2,4,6- \\
\text { Trimenthlyctane } 2,66-\end{array}$ & $\frac{62016-37-9}{5416-33-4}$ & $\begin{array}{l}062016-37-9 \\
054266-32-4\end{array}$ & $\begin{array}{l}156.31 \\
15631\end{array}$ & $\mathrm{~L}$ & 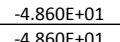 & $\begin{array}{ll}7.600 E+02 \\
7.600 F+02 \\
\end{array}$ & $\frac{1.515 E+02}{1515502}$ & $4.800 \mathrm{E}+00$ & $2.5006+01$ & 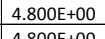 & EPP Suite Mean VP of Antoine and Grain Methods \\
\hline$\frac{3187}{3188}$ & $\begin{array}{l}3186 \mathrm{~T} \\
3187 \mathrm{~T}\end{array}$ & $\begin{array}{l}\text { Trimethyloctane, 2,6,6- } \\
\text { Trimethlololoropane ethoxylate }\end{array}$ & $\frac{54166-32-4}{50586-5-9}$ & $054166-32-4$ & $\frac{156.31}{79498}$ & 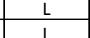 & & $\begin{array}{ll}7.600 \mathrm{E}+02 \\
7.600 \mathrm{E}+02\end{array}$ & $\frac{1.515 E+02}{8.197+502}$ & $\begin{array}{l}4.800 E+00 \\
3.605-26\end{array}$ & $\frac{2.500 E+01}{2.500+0101}$ & $\begin{array}{l}4.800 E+00 \\
3.60-26\end{array}$ & $\begin{array}{l}\text { EPP Sute Mean n P of Antoine and Grain Methods } \\
\text { EPI Suite Modified Grain Method }\end{array}$ \\
\hline$\frac{3188}{3189}$ & $\begin{array}{l}3188 \\
3188 \mathrm{~T}\end{array}$ & $\begin{array}{l}\text { IImethinglopropane ethoxxlyate } \\
\text { Trimethylopropane phosphite }\end{array}$ & 5058-59-9 & $\begin{array}{l}050086-59-9 \\
00082411-3 \\
\end{array}$ & $\begin{array}{l}194.98 \\
162.14\end{array}$ & $\frac{\mathrm{L}}{\mathrm{s}}$ & $5.600 E+01$ & & $\begin{array}{l}8.199 t+02 \\
2.000 E+02\end{array}$ & $\begin{array}{ll}3.6010 E-26 \\
2.000 E-01\end{array}$ & $2 \frac{2.500+E+01}{2.500++01}$ & & $\begin{array}{l}\text { EPPS Sute Moditied Grain Method } \\
\text { PAC } 27 \text { Listing Table } 1\end{array}$ \\
\hline 3190 & 3189 T & Trimethylphosphine & $594-09-2$ & $000594-09-2$ & 76.08 & $\mathrm{~L}$ & $-8.500 E+01$ & $7.600 E+02$ & $3.750 E+01$ & $4.750 \mathrm{E}+02$ & $2.500 E+01$ & $4.750 \mathrm{E}+02$ & EPI Suite Maan VP of Antoine and Grain Methods \\
\hline 3191 & 3190 T & Trimethylpyrididine, 2,4,6- & $108-75-8$ & $000108-75-8$ & 121.2 & $\mathrm{~L}$ & & & & $1.987 \mathrm{E}+00$ & $2.500 \mathrm{t}+01$ & $1.987 \mathrm{~F}+00$ & CHAO, ET AL. (1983) \\
\hline$\frac{3192}{31903}$ & 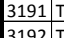 & $\begin{array}{l}\text { Trimethylsilianol } \\
\text { Trimethylsilymethyllithium }\end{array}$ & $\frac{1066-40-6}{182200-0}$ & $001066-40-6$ & 90 & $\frac{L}{L}$ & $\begin{array}{l}-6.6011 \mathrm{E}+01 \\
1.3015+01\end{array}$ & 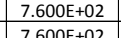 & $\frac{1.071 E+02}{3.5055+01}$ & $\frac{1.380 \mathrm{E}+01}{90.01050 .07}$ & $2.5006+01$ & $1.3800+01$ & EPI Suite Mean VP of Antoine and Grain Methods \\
\hline$\frac{3193}{3194}$ & $\begin{array}{l}3192 \mathrm{~T} \\
3193 \mathrm{~T}\end{array}$ & $\begin{array}{l}\text { Trimethhyslilymethhllithium } \\
\text { Trimethythin chloride: (Chlorotrimethylstannane) }\end{array}$ & $\begin{array}{l}1822-00-0 \\
1066-45-1\end{array}$ & $001822-00-0$ & $\frac{94.16}{199.26}$ & $\frac{L}{s}$ & 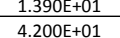 & & $\begin{array}{l}3.5006+01 \\
54-156\end{array}$ & $\begin{array}{l}9.490 E=-07 \\
3000+00\end{array}$ & $\begin{array}{l}2.500 E+01 \\
2550+5+01\end{array}$ & & 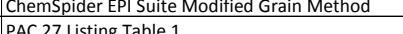 \\
\hline$\frac{3194}{31955}$ & 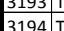 & 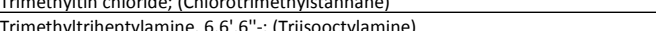 & $\begin{array}{l}1006-45-1 \\
275-28\end{array}$ & 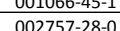 & $\begin{array}{l}195.66 \\
35376\end{array}$ & $\frac{3}{s}$ & & & & & & & 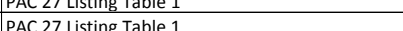 \\
\hline 3196 & 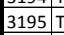 & Tri-n-butylamine & $102-82-9$ & $000102-82-9$ & 185.4 & $\frac{\mathrm{L}}{\mathrm{L}}$ & $-7.000 E+01$ & $7.600 E+02$ & $2.165 \mathrm{E}+02$ & $9.340 \mathrm{E}-02$ & $2.500 E+01$ & $9.340 \mathrm{E}-02$ & $\begin{array}{l}\text { PAC 27 Listing Table } 1 \\
\text { Pal }\end{array}$ \\
\hline 3197 & 3196 T & Trinitrobenzene, 1,3,5- & $99-35-4$ & $000099-35-4$ & 213.1 & $\mathrm{~s}$ & $1.229 \mathrm{E}+02$ & & $3.150 \mathrm{E}+02$ & $6.44 \times 10-6$ & 2.500E+01 & & PAC 27 Listing Table 1 \\
\hline 3198 & 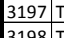 & 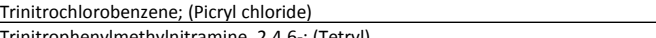 & 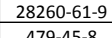 & $028260-61-9$ & 247.56 & $\frac{s}{s}$ & 131132 & & & & & & $\begin{array}{l}\text { PAC } 27 \text { Listing Table } 1 \\
\text { PAA 27lusting Taple } 1\end{array}$ \\
\hline$\frac{3199}{3200}$ & 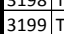 & Trinitrotoluene, $2,4,6-$ - alme, $2,4,0$, , (letry) & 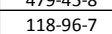 & $000118-96-7$ & 227.15 & $\frac{s}{s}$ & 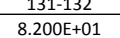 & & $\begin{array}{ll}18 / \operatorname{expl} \\
240 \text { expl }\end{array}$ & & & & $\begin{array}{l}\text { PAC 2/ IItsing Iable 1 } \\
\text { PAC 27 Listing Table } 1\end{array}$ \\
\hline me & $3200 \mid \mathrm{T}$ & Tri-n-octy|phosphine oxide; (Triocty|phophine oxide) & $78-50-2$ & 000078-50-2 & 386.64 & $s$ & $5.2006+01$ & & $201 @ 2$ & & & & PAC 27 Listing Table 1 \\
\hline$\frac{3201}{3202}$ & $3201 \mathrm{~T}$ & Trioctylamine; (N,N-Diocty-1-1-octanamine) & $1116-76-3$ & $001116-76-3$ & 353.76 & $\mathrm{~L}$ & & $7.600 E+02$ & $\frac{\operatorname{minfB}}{3.650 \mathrm{E}+02}$ & $6.860 \mathrm{E}-06$ & $2.500 \mathrm{E}+01$ & $6.8600-06$ & EPI Suite Modified Grain Method \\
\hline 3203 & 3202 T & 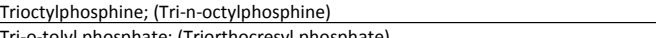 & 4731-53-7 & 004731-53-7 & 370.72 & $\mathrm{~s}$ & & & & 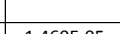 & & & PAC 27 listing Table 1 \\
\hline$\frac{3244}{3205}$ & 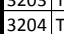 & Tripentary prosphritol & $\frac{18-50-8}{78-24-0}$ & $\begin{array}{l}000078-30-8 \\
000078-24-0\end{array}$ & 30.399 & $\frac{2}{5}$ & $\frac{2.700 E+01}{225-240}$ & 7.680EF+02 & 4.100E+02 2020 & $1.4600=-05$ & $2.500 E+01$ & $1.460 E=-05$ & 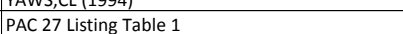 \\
\hline ent & 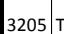 & Triphenyl phosphate & $115-86-6$ & $\begin{array}{ll}000115-86-6 \\
\end{array}$ & 326.3 & $\mathrm{~s}$ & $49-50$ & & $245 @ 11$ & $1000 F+00$ & $1935 \mathrm{E}+02$ & & PAC 27 Listing Table 1 \\
\hline 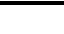 & $3206 \mathrm{~T}_{\mathrm{T}} \mathrm{C}, \mathrm{C}$ & Triphenyl phosphite & $101-02-0$ & $000101-02-0$ & 310.3 & $\mathrm{~s}$ & $21-23$ & & $\lim _{183-184}$ & & & & PAC 27 Listing Table 1 \\
\hline 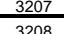 & $3207 \mathrm{TI}$ & Triphenylborane & $960-71-4$ & $000960-71-4$ & 24214 & $\mathrm{~s}$ & $1.420 \mathrm{E}+02$ & & & & & & \begin{tabular}{|l|l} 
PAC 27l listing Table 1 & \\
\end{tabular} \\
\hline 3209 & 32087 & Triphenylethoxysilane & $1516-80-9$ & $001516-80-9$ & 304.458 & $\frac{\mathrm{s}}{\mathrm{s}}$ & 65 & & $3.440 E+02$ & & & & PAC 27 Listing Table 1 \\
\hline 3210 & $3209 / \pi$ & $\begin{array}{l}\text { Triphenylolmethane triglycidyl ether } \\
\text { Trinhenyluhbshoine }\end{array}$ & $66072-38-6$ & $\begin{array}{lll}0.06072-38-6 \\
00023250\end{array}$ & 460.5256 & $\frac{5}{s}$ & & & & & & & PAC 27 Listing Table 1 \\
\hline & & & $603-35-0$ & $000603-35-0$ & 262.3 & & 7.900E+01 & & 2360 & & & & PAC 27 Listing Table 1 \\
\hline 3212 & 3211 T & Triphenyltin chloride; (Chlorotriphenylstannane) & 639-58-7 & $000639-58-7$ & 385.47 & $\mathrm{~s}$ & $1.060 \mathrm{E}+02$ & & 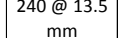 & & & & PAC 27 Listing Table 1 \\
\hline 3213 & $3212 \mathrm{~T}$ & Tripotassium (2-hydroxyethyl)ethylenediaminetriacetate; (Tripotassium HEDTA) & $62029 \cdot 50-9$ & $062029-50-9$ & 395.5549 & $\mathrm{~s}$ & & & & & & & PAC 27 Listing Table 1 \\
\hline & 3213 T & Tripotassium arsenate & 13466-36-3 & $013464-36-3$ & 256.22 & $\mathrm{~s}$ & & & & & & & PAC 27 Listing Table 1 | \\
\hline 3215 & 3214|T & Tripropyl phosphate; (TPP) & 513-08-6 & $000513-08-6$ & 2224.23 & $L$ & e & 1.000E+01 & 1.200E+ +02 & $2.310 \mathrm{E}-02$ & 2.500E+01 & $2.310 \mathrm{E}-02$ & EPI Suite Modified Grain Method \\
\hline$\frac{3216}{3217}$ & $\frac{521516}{3216 \mathrm{~T}}$ & Tripropylamine & $\frac{102-6-6-2}{25498-49-1}$ & 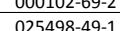 & $\frac{14.31}{206.32}$ & $\frac{L}{L}$ & 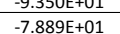 & $\frac{7.600+U 2}{7.680 E+02}$ & 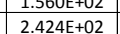 & $\begin{array}{l}1.5010+00 \\
5.990-03\end{array}$ & 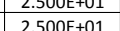 & $5.9900-03$ & 作 \\
\hline 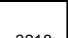 & $32177^{\top}$ & 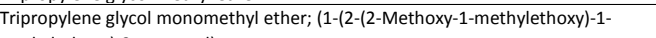 & $20324-33-8$ & 020324-33-8 & 206.32 & $\mathrm{~L}$ & & $7.600 E+02$ & $2.430 \mathrm{E}+02$ & $2.000 E-02$ & $2.500 E+01$ & $2.000 E-02$ & DOW CHEMICAL COMPANY (1990) \\
\hline$\frac{3218}{3219}$ & $\frac{m}{3218} \mathrm{~T}$ & 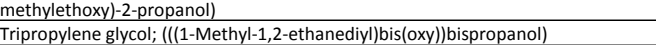 & $24800-44-0$ & $024800-44-0$ & 192.29 & $\mathrm{~L}$ & $-3.000 E+01$ & $7.600 E+02$ & $2.670 E+02$ & $1.000 E+00$ & $9.600 E+01$ & $6.808 E-03$ & Estimated using data from PAC 27 Listing Table 1 \\
\hline 1 & $32199^{T^{\top}}$ & Tris hydrochloride; (Trizma hydrochloride; Trishthydroxymethyl) aminomethane & $1185-53-1$ & $001185-53-1$ & 157.6 & $\mathrm{~s}$ & $150-152$ & & & & & & PAC 27 listing Table 1 \\
\hline 3221 & 3220 Th & 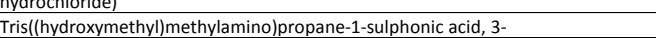 & $29915-38-6$ & $029915-38-6$ & 243.2744 & $\mathrm{~s}$ & 240 & & & & & & \begin{tabular}{|l} 
PAC 27 Listing Table 1 \\
\end{tabular} \\
\hline 3222 & $3221] \mathrm{T}$ & Tris(2-aminoethylamine & $4097-89-6$ & $004097-89-6$ & 146.28 & $\mathrm{~s}$ & 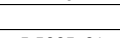 & & & & & & PAC 27 Listing Table 1 \\
\hline$\frac{3223}{32024}$ & & 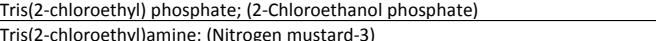 & $\frac{115-96-8}{555-77-1}$ & $000115-96-8$ & $\frac{285.5}{2045}$ & $\mathrm{~L}$ & $-5.500 E+01$ & $\begin{array}{l}7.6800+02 \\
20005+00\end{array}$ & $\begin{array}{l}3.3006+02 \\
0.8005+01\end{array}$ & $6.1300-02$ & $2.5006+01$ & 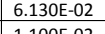 & DOBRY, A \& KELLER, R (1957) \\
\hline$\frac{3224}{3225}$ & 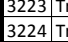 & 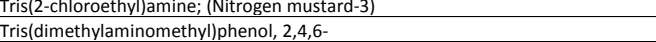 & $\frac{555-77-1}{90-72-2}$ & $\frac{0000555-77-1}{00009-72-2}$ & 2045.57 & $\frac{L}{S}$ & $-4.000 \mathrm{E}+00$ & & & & & & $\begin{array}{l}\text { MUNNRO,NB E E ALL (1999) } \\
\text { PAC 27 Listing Table } 1\end{array}$ \\
\hline 3226 & 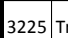 & Trishydroxymethylaminomethane; (THAM) & $77-86-1$ & $000077-86-1$ & 121.16 & $\mathrm{~s}$ & 171-172 & & $219-220$ & & & & PAC 27 Listing Table 1 \\
\hline$\frac{3260}{3227}$ & 3226 T & Trisodium arsenate & $13464-38-5$ & $013464-38-5$ & 207.89 & $\mathrm{~s}$ & & & & & & & PAC 27 Listing Table 1 \\
\hline 3228 & $\left.3227\right|_{11} ^{T}$ & $\begin{array}{l}\text { Trisodium arsenate, heptahydrate; (Arsenic(V) acid, trisodium salt, heptahydrate } \\
(11: 3) \text { ) }\end{array}$ & 64070-83-3 & 064070-83-3 & 334.03 & s & & & & & & & PAC 27 Listing Table 1 \\
\hline$\frac{3200}{3229}$ & $3228 \pi$ & Trisodium citrate & $68-04-2$ & $000068-04-2$ & 258.08 & $\mathrm{~s}$ & & & dec & & & & PAC 27 Listing Table 1 \\
\hline 2320 & $\left.3229\right|^{\top}$ & Trisodium N-hydroxyethylethylenediaminetriacetate; (Trisodium N-2-2- & 139-89-9 & 000139-89-9 & 344.22 & $\mathrm{~s}$ & $2.880 \mathrm{E}+02$ & & & & & & PAC 27 Listing Table 1 \\
\hline 年3230 & $3230 \mathrm{Th}$ & 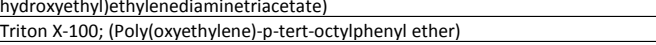 & $9002-93-1$ & $009002-93-1$ & 514.35 & $\mathrm{~L}$ & $-4.0000+00$ & $7.600 \mathrm{E}+02$ & $1.200 E+02$ & $6.990 \mathrm{E}+00$ & $2.500 \mathrm{E}+01$ & $6.9906+00$ & EPI Suite Mean VP of Antoine and Grain Methods \\
\hline 3232 & 32331|T & Trypan blue & & & 960.81 & $\mathrm{~s}$ & 3.000E+02 & & & & & & PAC 27 Listing Table 1 \\
\hline 3233 & 323232T T & Trypsin; (Parenzyme; Chymotrypsin inhibitor) & 9002-07-7 & $009002-07-7$ & & $\mathrm{~s}$ & & & & & & & PAC 27 Li: \\
\hline 3234 & [3233]T & Trypsinogen & 9002-08-8 & 009002-08-8 & & $s$ & & & & & & & PAC 27 Lis \\
\hline$\frac{3235}{3236}$ & 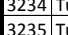 & $\begin{array}{l}\text { Tungsten } \\
\text { Tungsten boride }\end{array}$ & 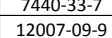 & $\begin{array}{ll}0 \\
00072007-3-7-7 \\
01200-9\end{array}$ & $\frac{183.84}{194.65}$ & $\frac{s}{s}$ & $3.426+03$ & & 5.555E +03 & $7.5 \times 10-3$ & 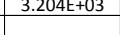 & & $\begin{array}{l}\text { PAC C L Lising tabble } 1 \\
\text { PAC 27 Listing Table } 1\end{array}$ \\
\hline & $3236 \sqrt{\mathrm{T}}$ & Tungsten carbide & $12070-12-1$ & $012070-12-1$ & $\frac{194.05}{195.9}$ & $\mathrm{~s}$ & $2.7855+03$ & & & & & & $\begin{array}{l}\text { PAC } 27 \text { Listing Table } 1 \\
\text { Pall }\end{array}$ \\
\hline 3238 & 32337T/T & Tungsten hexafluoride & $7783-82-6$. & $007783-82-6$ & 297.85 & $G$ & $2.3006++00$ & & $1.750 \mathrm{E}+01$ & & & & \\
\hline & 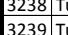 & 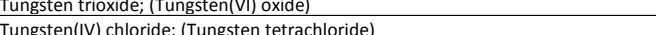 & $\frac{1314-35-8}{13470-13-8}$ & $001314-35-8$ & 231.84 & $\frac{s}{s}$ & & & & & & & $\begin{array}{l}\text { PAC 27 Listing Table } 1 \\
\text { Pac C2 listing Tatle } 1\end{array}$ \\
\hline
\end{tabular}




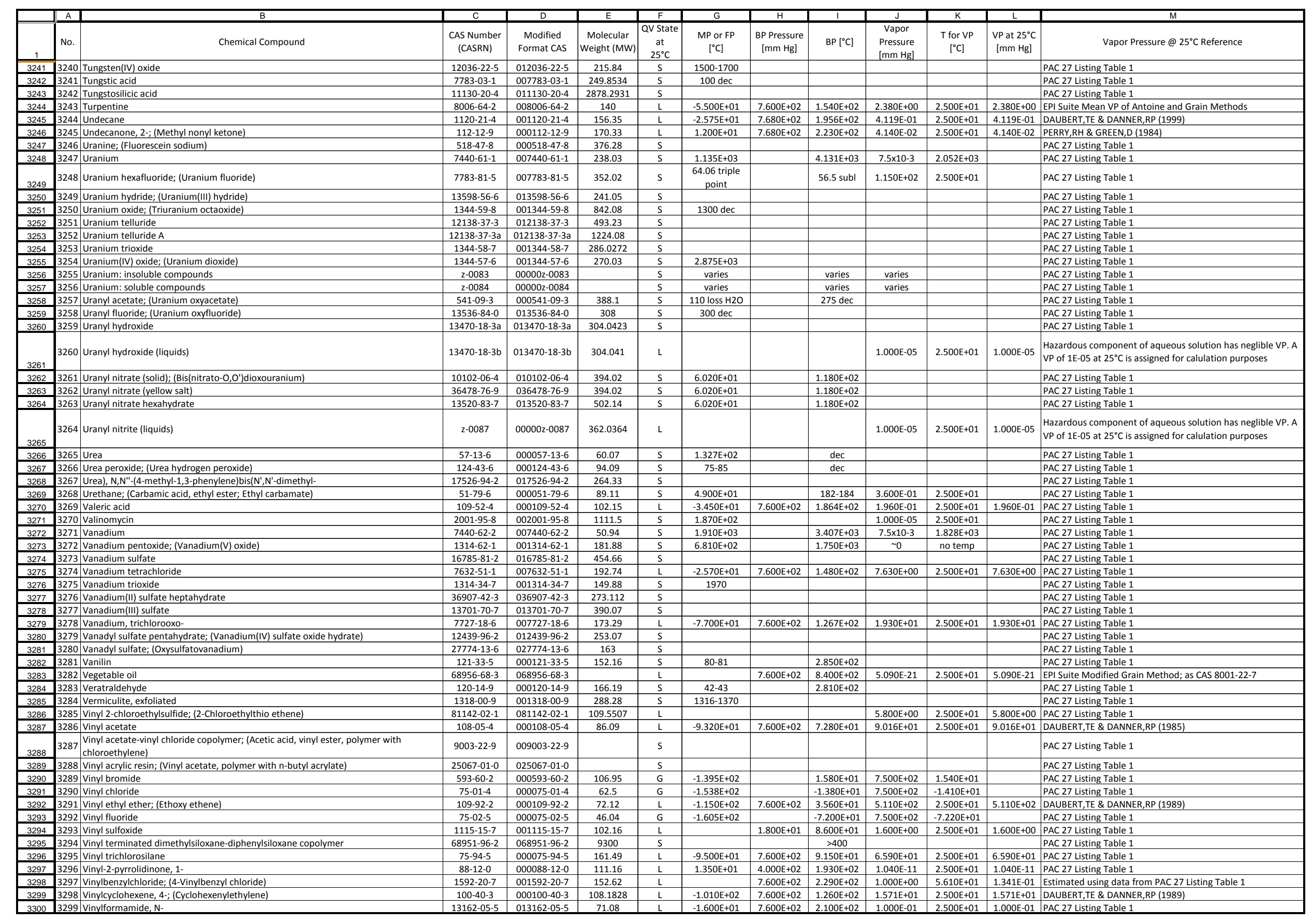




\begin{tabular}{|c|c|c|c|c|c|c|c|c|c|c|c|c|c|}
\hline & No. & Chemical Compound & $\begin{array}{c}\text { cas N Number } \\
\text { (CASPN) }\end{array}$ & $\begin{array}{c}\text { Modified } \\
\text { Format CAS }\end{array}$ & $\begin{array}{l}\text { Molecular } \\
\text { Weight MW }\end{array}$ & 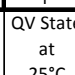 & $\begin{array}{c}\text { MPorfP } \\
\text { [Cc }\end{array}$ & 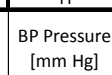 & вр [ст] & $\begin{array}{c}\text { vapor } \\
\text { pressurue }\end{array}$ & $\begin{array}{c}\text { Toryp } \\
\text { prcl }\end{array}$ & 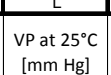 & Vapor Pressure @ $95^{\circ}$ C Reference \\
\hline 33015 & 3300 & Vinvilidene chloride; (1,1-1.-ichloroerethyene) & 75.35 .4 & $000075-35-4$ & 96.94 & & $-1.226 \mathrm{-1}+02$ & $7.600 \mathrm{E}+02$ & $3.1606+01$ & $6.000 \mathrm{E}+02$ & $2.500 €+01$ & $6.000 £+02$ & UKK, E ET AL. (1984) \\
\hline 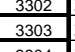 & 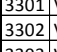 & 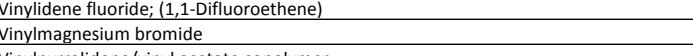 & 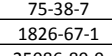 & $\begin{array}{l}00075-387 \\
001826-67-1\end{array}$ & 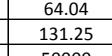 & $\frac{G}{L}$ & $\begin{array}{l}-1.406+52 \\
-1.735+01\end{array}$ & $7.600+02$ & 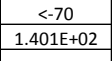 & 7.9906 +00 & $2.500+01$ & $7.9406+50$ & 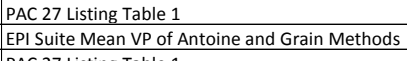 \\
\hline 3304 & 330334] & 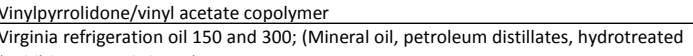 & 25086-89.9.9. & 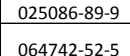 & & $\frac{s}{4}$ & & $7.6005+02$ & $35000+02$ & 2.490604 & $2.500+01$ & $24900=04$ & 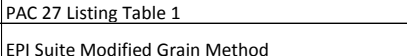 \\
\hline 33056 & & Whald haray naphithenic) & $\frac{81.812 .2}{6100.69}$ & $\frac{00081.81 .2}{0.028-2}$ & 30835 & s & $\frac{1.6106+02}{1.100 .155}$ & & $3.560 \mathrm{E}+02$ & $\frac{1.000=05}{1.0005}$ & $\frac{2.500+501}{200501}$ & & 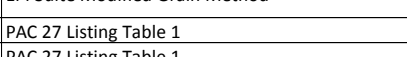 \\
\hline & & & $\frac{7440 \cdot 63-3}{770-3}$ & 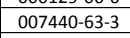 & $\frac{13.29}{131.29}$ & 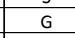 & $\frac{1.12006+02}{-1.20}$ & & & & & & \\
\hline 3309 & 309 & 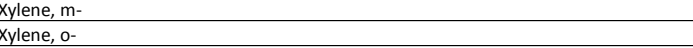 & $\frac{103-388.3}{95-47-6}$ & 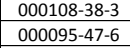 & $\frac{160.17}{106.17}$ & $\frac{\mathrm{L}}{\mathrm{L}}$ & 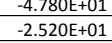 & 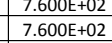 & $\frac{1.3919+52}{1.445+02}$ & 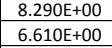 & $\frac{2.500+51}{2.005+01}$ & $\begin{array}{l}8.290+6000 \\
6.6160+00\end{array}$ & 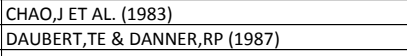 \\
\hline 3311 & 310| & Xylene, p- & 106-42-3.3 & $000106-42 \cdot 3$ & & $\mathrm{~L}$ & $1.325 \mathrm{E}+01$ & $7.600 E+022$ & 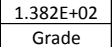 & $8.8406+00$ & $2.500=0.01$ & & HAO, ETALL(1983) \\
\hline 3312 & 3311: & Xylenes & 1330-20-7 & 001330-20-7 & 100.17 & $\mathrm{t}$ & & & $\begin{array}{l}\text { Dependent } \\
\text { Depent }\end{array}$ & 7.990E +00 & $2.500 \mathrm{E}+01$ & 7.990E $+50 \mid$ & DAUBERT,TE \& DANNER,RP (1989) \\
\hline 33134 & 3113: & 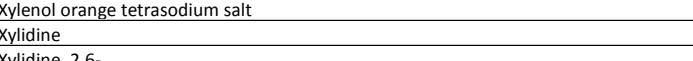 & $\begin{array}{l}3618.43 .7 \\
130-73.8\end{array}$ & 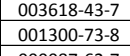 & $\begin{array}{l}764.63 \\
121.18 \\
121\end{array}$ & $\frac{s}{L}$ & $-3.600 €+01$. & 7.68060+0202 & 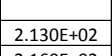 & 1.3835-011 & $2.500+5+11$ & 1.:383E-0 & 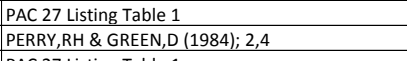 \\
\hline 年3315 & $\begin{array}{l}3.145 \\
3150\end{array}$ & 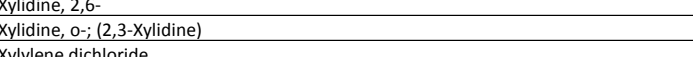 & 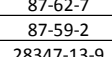 & 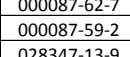 & 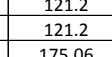 & $\frac{1}{4}$ & 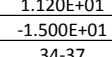 & $\begin{array}{l}7.6000+52 \\
7.600+02\end{array}$ & 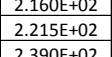 & 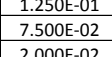 & 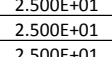 & 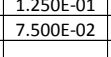 & 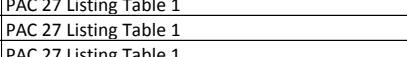 \\
\hline $\begin{array}{ll}\frac{2318}{33318} \\
3319 \\
\end{array}$ & $\begin{array}{l}317 \\
381\end{array}$ & 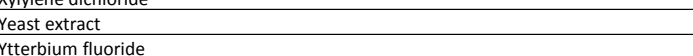 & 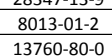 & 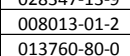 & 230.04 & $\frac{s}{s}$ & $1.1577 \pm+03$ & & $2.200 E+03$ & & & & 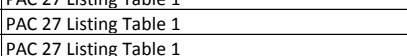 \\
\hline 3320 & 319 & $\begin{array}{l}\text { Ytterbium oxide } \\
\text { Yly }\end{array}$ & $\begin{array}{l}1314-37-0 \\
7143-5\end{array}$ & 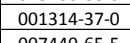 & $\begin{array}{l}394.08 \\
30.01\end{array}$ & 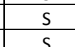 & 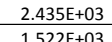 & & & & & & 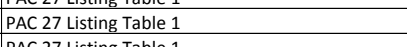 \\
\hline$\frac{-3232}{3322}$ & 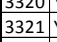 & 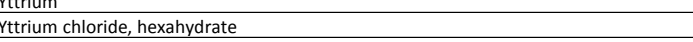 & 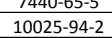 & & $\begin{array}{l}20358 \\
30338\end{array}$ & & & & $3.345 E+03$ & $7.5 x 10-3$ & 1.610E +03 & & \\
\hline $\begin{array}{lll}33323 \\
3324 \\
\end{array}$ & 322] & $\begin{array}{l}\text { trtrium oxide } \\
\text { tride }\end{array}$ & 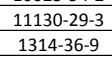 & 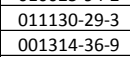 & $\frac{10491}{225.82}$ & $\begin{array}{cc}\frac{s}{s} \\
\end{array}$ & $2.410 \div+03$ & & & & & & $\begin{array}{llll}\text { Listing Trable } 1 \\
\text { Listing }\end{array}$ \\
\hline $\begin{array}{l}3325 \\
3326 \\
\end{array}$ & 32425: & 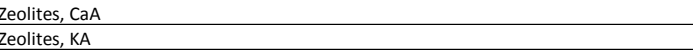 & 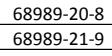 & 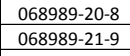 & 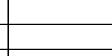 & $\frac{5}{5}$ & & & & & & & 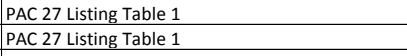 \\
\hline 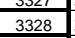 & 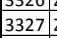 & 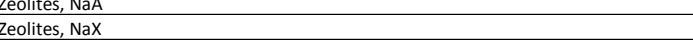 & 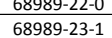 & 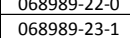 & & & & & & & & & \\
\hline 3329 & 3282] & Zlich & $7440-66-6.6$ & 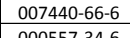 & $\frac{65.41}{60972}>0$ & 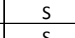 & 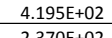 & & $9.0070+02$ & $7.5 \times 10=3$ & $3.370 \mathrm{E}+02$ & & \\
\hline 3331 & 330 & Zinc acetate dihydrate & 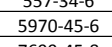 & 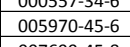 & 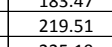 & $\frac{5}{5}$ & 2.370 & & 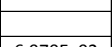 & & & & 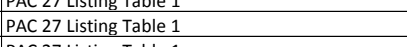 \\
\hline$\frac{2.333}{3.333}$ & 每32] & 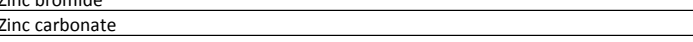 & 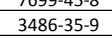 & 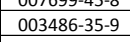 & $\frac{225.38}{1.258}$ & & & & $6.970 \mathrm{E}+02$ & & & & 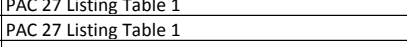 \\
\hline 33345 & 33333 & $\begin{array}{l}\text { Zizcc cabbonate hydroxide } \\
\text { Zinc chloride }\end{array}$ & $\begin{array}{l}5263-2.25 \\
7646-85-7\end{array}$ & 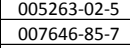 & $\frac{142.4}{136.27}$ & $\frac{s}{5}$ & $2.9005+02$ & & $7.320 E+02$ & $1.000+50$ & 4.2806+02 & & 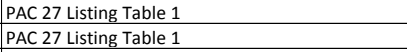 \\
\hline \begin{tabular}{l|l}
33367 \\
3377 \\
\end{tabular} & 333565 & $\begin{array}{l}\text { Zizcc vanide } \\
\text { Zinc fluoride }\end{array}$ & $\begin{array}{l}55-212-1 . \\
7783-49.5\end{array}$ & $\begin{array}{l}0.00552-21.1 \\
0.07738-9.5\end{array}$ & 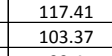 & $\frac{5}{5}$ & $\begin{array}{l}8.80 \mathrm{deccm} \\
8.220+0202\end{array}$ & & $1.5005+03$ & $1.000+500$ & $9.700 E+02$ & & 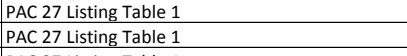 \\
\hline & 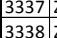 & 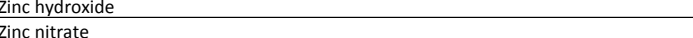 & & & $\begin{array}{l}\frac{99.4}{18.42} \\
18.25\end{array}$ & & $4.2505+01$ & & & & & & \\
\hline$\frac{3300}{3201}$ & 3939] & 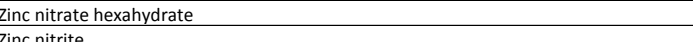 & 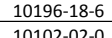 & 0101096-18.6. & 297.51 & $s$ & $36 \mathrm{dec}$ & & & & & & 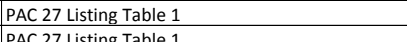 \\
\hline 3342 & 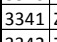 & Zinc oxide & 1314-13:-2. & .013131413.2. & 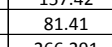 & 5 & $19974+03$ & & & $\therefore$ & notemp & & $\begin{array}{lll}2 & 1\end{array}$ \\
\hline & 33433] & 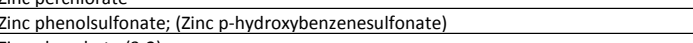 & & & 413.73 & $s$ & & & & & & & \\
\hline 3345 & 33445 & $\begin{array}{ll}\text { zincec hosphatet (3:2) } \\
\text { zinc phosphide }\end{array}$ & 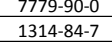 & 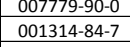 & $\begin{array}{l}38.05 \\
258.1 \\
258\end{array}$ & $\frac{5}{5}$ & 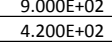 & & $1.1000+03$ & $1.000: 07$ & $2.500 E+01$ & & 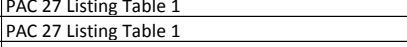 \\
\hline 年3448 & 3347] & 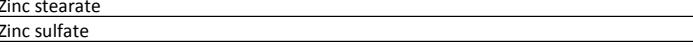 & 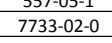 & 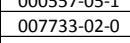 & 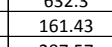 & $\frac{5}{5}$ & 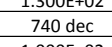 & & & & & & 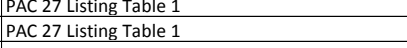 \\
\hline $\begin{array}{lll}33399 \\
3350 \\
3\end{array}$ & 33489][ & 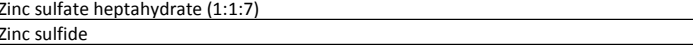 & $\begin{array}{l}7466-2-2-0 \\
1314-98-3\end{array}$ & 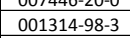 & $\begin{array}{l}20.73 \\
97.43\end{array}$ & $s$ & $\begin{array}{l}1.7006+03 \\
1.7063\end{array}$ & & sublime & & & & 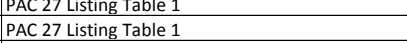 \\
\hline 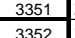 & 望50: & 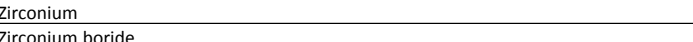 & 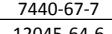 & 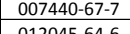 & $\frac{91.22}{91.24}$ & $s$ & 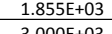 & & 4.409E +03 & $7.5 \times 10 \cdot 3$ & $2.366 E+03$ & & 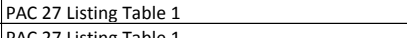 \\
\hline 3353 & 33352] & Zirconium carbide & & 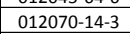 & & $\mathrm{s}$ & $340-3540$ & & $5.100 E+03$ & & & & \\
\hline $\begin{array}{ll}33545 \\
3355\end{array}$ & 33533|: & $\begin{array}{l}\text { Zizconium chloride } \\
\text { Zirconium chloride oxide octahydrate }\end{array}$ & 1.00252-1.1-62-8 & 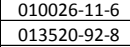 & 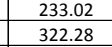 & $\begin{array}{c}\mathrm{s} \\
\mathrm{s}\end{array}$ & 437s subl. & & 3.310E+02 & $1.000 E+00$ & 1.900E+ +02 & & $\begin{array}{l}\text { able } 1 \\
\text { able } 1\end{array}$ \\
\hline 年3567 & 3356 & 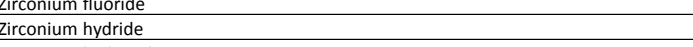 & 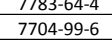 & 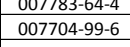 & 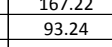 & $\frac{6}{5}$ & & & $>300$ dec & & & & 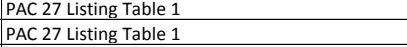 \\
\hline & 年3518 & 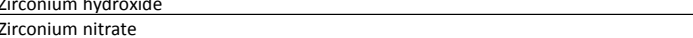 & 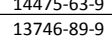 & & 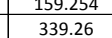 & & aec & & & & & & 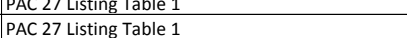 \\
\hline 3360 & 3359: & Zirconium nitride & $25655-42-8.8$ & $0256558-42.88$ & 105.2267 & $s$ & $2.9606+03$ & & & & & & Sising Table 1 _ \\
\hline 年3612 & 3361] & Iriconium oxide & 1314-23-4.4 & 001314-23-4-4 & 123.22 & s & & & & & & & 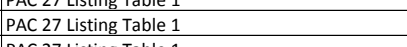 \\
\hline 年3334 & 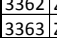 & 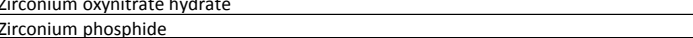 & 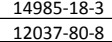 & 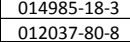 & 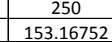 & 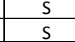 & & & & & & & 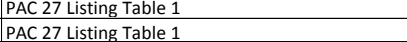 \\
\hline
\end{tabular}




\begin{tabular}{|c|c|c|c|c|c|c|c|c|c|c|c|c|c|}
\hline & №. & Chemical compound & $\begin{array}{l}\text { CAS Number } \\
\text { (CASRN) }\end{array}$ & $\begin{array}{l}\text { Modified } \\
\text { Format CAS }\end{array}$ & $\begin{array}{l}\text { Molecular } \\
\text { Weight (MW) }\end{array}$ & \begin{tabular}{|c|} 
aV state \\
at \\
$25^{\circ} \mathrm{C}$
\end{tabular} & $\begin{array}{c}\text { MP or FP } \\
{\left[{ }^{\circ} \mathrm{C}\right]}\end{array}$ & $\begin{array}{c}\text { BP Pressure } \\
{[\mathrm{mm} \mathrm{Hg}]}\end{array}$ & $B P\left[{ }^{[C}\right]$ & $\begin{array}{l}\text { Vapor } \\
\text { Pressure } \\
{[\mathrm{mm} H \mathrm{Hg}]}\end{array}$ & $\begin{array}{l}\text { Tfor vp } \\
\text { ['c] }\end{array}$ & $\begin{array}{l}\text { VPat } 25^{\circ} \mathrm{C} \\
{[\mathrm{mm} \mathrm{Hg}]}\end{array}$ & Vapor Pressurue @ $2^{\circ} \mathrm{C}$ Reference \\
\hline 3365 & $\begin{array}{ll}3364 \\
3355\end{array}$ & 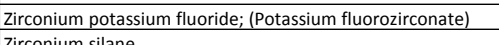 & $16923-95-8$ & $016923-95-8$ & 288.4 & \begin{tabular}{|l|l}
$s$ \\
$s$
\end{tabular} & & & & & & & $\begin{array}{l}\text { PAC 27 Listing Table } 1 \\
\text { DA 27 listing Table } 1\end{array}$ \\
\hline $\begin{array}{ll}33667 \\
3367\end{array}$ & 33665 & $\begin{array}{l}\text { ZZiriconium silane } \\
\text { Ziconium silicate; (Zirconium silicon oxide; Zircon) }\end{array}$ & $\frac{2-0089}{10101-52-7}$ & 0000002-00089 & $\frac{147.391}{187.337}$ & $\frac{s}{s}$ & & & & & & & $\begin{array}{l}\text { PAC 27 Listing Table } 1 \\
\text { PAC27 }\end{array}$ \\
\hline$\frac{3507}{3368}$ & (3367 & Zirconium sulfate tetrahydrate & $7446-31-3$ & 007446-31-3 & $\frac{10.5594}{355.4}$ & 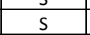 & 410 decom & & & & & & $\begin{array}{l}\text { PAA 27 Listing Table } 1 \\
\text { PAC } 27 \text { isting Table } 1\end{array}$ \\
\hline 3369 & 3368 & Zirconyl chloride; (Zirconium oxychloride) & $7699-43-6$ & $007699-43-6$ & 178.12 & $\mathrm{~s}$ & 150 & & $2.1006+02$ & & & & PAC 27 Listing Table 1 \\
\hline 3370 & 3369 & Zirconyl nitrate; (Bis(nitrato-O)oxozirconium) & $13826-66-9$ & $013826-66-9$ & 231.24 & $\mathrm{~s}$ & & & & & & & PAC 27 Listing Table 1 \\
\hline 3371 & 3370 & Zonyl fsn fluorosurfactant & $65545-80-4$ & $065545-80-4$ & 114.066 & $\mathrm{~L}$ & $-7.795 \mathrm{E}+01$ & $7.600 E+02$ & $8.000++01$ & $2.060++01$ & $2.500 €+01$ & $2.060 \mathrm{E}+01$ & chemSpider EPI Suite Mean VP of Antoine and Grain Methods \\
\hline 72 & 3371 & 2zAcrylic latex & z-0090 & 00000z-0090 & & L & & & & $1.000 E=-05$ & $2.500 \mathrm{E}+01$ & $\begin{array}{l}1.000 \mathrm{E}-05 \\
\end{array}$ & 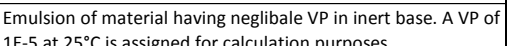 \\
\hline 3373 & 3372 & zzAlumination 301 & z-0091 & $000002-0091$ & & $\mathrm{~s}$ & & & & & & & 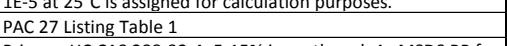 \\
\hline & 3373 & zhHydranal coulomat / AG & z-0092 & 00000z-0092 & & $\mathrm{L}$ & & $7.600 E+02$ & $6.470 \mathrm{E}+01$ & 1.200E+ +02 & $2.5006+01$ & $1.200 E+02$ & $\begin{array}{l}\text { Primary H H CAA } 288-32-4 ; \text {; } 5-15 \% \text { in methanol. As MSOS BP for } \\
\text { mixture is that of methano; contribution of Imidazole is } \\
\text { presumed to be 5\%. }\end{array}$ \\
\hline 3375 & 3374 & 2 z2Hydrocarbon polymer & 2-0093 & $000002-0093$ & & $\mathrm{~s}$ & & & & & & & 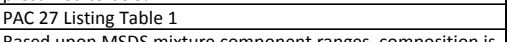 \\
\hline & 3375 & zzHydrocount(R), LSC cocktail & z-0094 & 00000z-0094 & & L & & & & $4.8006+00$ & 2.500E+01 & $4.800 E+00$ & 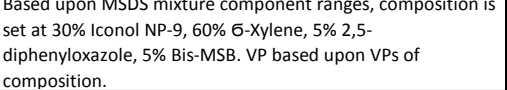 \\
\hline 3377 & 3376 & 221 conol(R) & 2-0095 & $000002-0095$ & & $\mathrm{~L}$ & & & & $1.000 E-02$ & $2.5006+01$ & $1.000 \mathrm{E}-02$ & 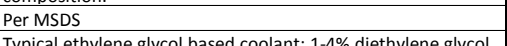 \\
\hline 3378 & $\left.3377\right|^{2}$ & zzMachine coolant 1 & $z-0096$ & 00000z-0096 & & $\mathrm{L}$ & $-5.6006+00$ & $7.600 E+02$ & $1.000 E+02$ & $1.406 \mathrm{E}+01$ & $2.500 E+01$ & $1.406 E+01$ & 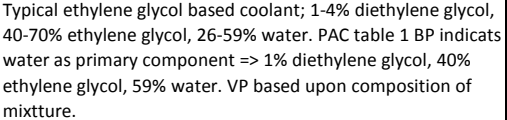 \\
\hline 3379 & 3378 & zzMonophase-s & $z-0097$ & 00000z-0097 & & L & & & & $2.100 E+00$ & $2.5006+01$ & $2.100 E+00$ & $\begin{array}{l}\text { Per MSDS, mixture is } 60.80 \% 1,2,4 \text {-4trimethylbenzene + other } \\
\text { components having neglipible VPs. }\end{array}$ \\
\hline 3380 & 3379 & zzMornar & z-0098 & $000002-0098$ & & $\mathrm{~s}$ & & & & & & & $\begin{array}{l}\text { No data on material or analogs. Assigned physical state of "s" } \\
\text { for calcultion purposes. }\end{array}$ \\
\hline $\begin{array}{llll}3381 \\
\end{array}$ & 3380 & 220 ptiFFluor; (Alkyl benzene blend, 3\% tributylphophate) & $z-0099$ & $00000 z-0099$ & & $\mathrm{~L}$ & & $7.600 E+02$ & $2.915 \mathrm{E}+02$ & $1.000 E-01$ & $2.000 E+01$ & $1.366-01$ & VP estimated based upon MSDS data, Packard JUN1996 \\
\hline 3382 & 3381 & 22 Propanol (-2) aluminum derivative & z-0101 & 00000z-0101 & & $\mathrm{s}$ & & & & & & & $\begin{array}{l}\text { Nodata on material or analogs. Assigned physical state of " } \\
\text { for calcultion purposes. }\end{array}$ \\
\hline \begin{tabular}{l|ll}
3383 \\
3383
\end{tabular} & 33822 & 2zScintillation cocktail, Ultima Gold XR & 2-0102 & $000002-0102$ & 212.36 & $\mathrm{~L}$ & $-3.0005+01$ & $7.600 E+02$ & $2.900 E+02$ & $2.8006+00$ & $2.500 E+01$ & $2.800 E+00$ & Estimated using data from PAC 27 Listing Table 1 \\
\hline $\begin{array}{r}33845 \\
3385 \\
\end{array}$ & $\frac{3383: 2}{\left.3384\right|_{2}}$ & $\begin{array}{l}\text { 2zScicapent } \\
\text { 2isynthetic resins }\end{array}$ & $\begin{array}{c}z-01033 \\
z-0104 \\
\end{array}$ & $\begin{array}{ll}0.000000-0103 \\
000002-0104 \\
\end{array}$ & & $\frac{S}{L}$ & & $7.600 E+02$ & $9.800 E+01$ & $1.000 E+01$ & $2.000 E+01$ & $1.413 \mathrm{E}+01$ & $\begin{array}{l}\text { Solid per manuffacture data sheet } \\
\text { Vip estimated based upon MSDS and other data sources }\end{array}$ \\
\hline 3386 & 3385 & $2 z$ Total sequestrant reagent $\# 5$ & z-0105 & $00000 z-0105$ & & $\mathrm{~L}$ & $-5.000 E-01$ & $7.600 E+02$ & $9.800 E+01$ & $8.080 E-10$ & $2.500 \mathrm{E}+01$ & $8.080 \mathrm{E}-10$ & $\begin{array}{l}\text { Based upon Nitric Acid, CAS 7697-37-2; EPI Suite Modified } \\
\text { Grain Method }\end{array}$ \\
\hline 3387 & 3386 & 2ZTriffluoroacety)|-N,0,0,0-tetrakisi(TMS)norepinephrine, N-l & z-0106 & $00000 z-0106$ & & s & & & & & & & $\begin{array}{l}\text { No data on material or analogs. Assigned physical state of "S" } \\
\text { for calculation purposes. }\end{array}$ \\
\hline 3388 & $\mid 3387$ & zzWaste oil & $z-0107$ & $00000 z-0107$ & & L & varies & & varies & $3.750 \mathrm{E}-03$ & 2.500巨+01 & $3.750 \mathrm{E}-03$ & $\begin{array}{l}\text { Assigned VP for calculation purposes based upon Transformer } \\
\text { oil, cAS 6472-53-6; MSOS Shell Gravex Oil }\end{array}$ \\
\hline
\end{tabular}

